\title{
Expanding the understanding of language mapping
}

by

\author{
Adam Stone
}

A thesis submitted to the Faculty of Graduate and Postdoctoral Affairs in partial fulfillment of the requirements for the degree of

Doctor of Philosophy

in

Applied Linguistics and Discourse Studies

Carleton University

Ottawa, Ontario

(C) 2020, Adam Stone 
Expanding the understanding of language mapping.

Adam Stone

\begin{abstract}
Language mapping is a cluster of practices surrounding the spatial representation of linguistic information, which has a variety of traditions, associated perspectives, and purposes. As maps are inherently political acts that contain their creators' subjectivities and biases, the way they are made can determine how users view a geographic region and the people within it, which is particularly relevant given that language maps are first and foremost maps of people (Thun, 2010, p.507). While language maps are becoming increasingly widespread in our societies, a marked lack of consensus remains on what language mapping is, who does it, and how it is done. Furthermore, Luebbering, Kolivras, and Prisley (2013, p.383) identify a general lack of guidelines and related infrastructure that enable those involved in the mapping process to reflect on language mapping practices, which is becoming all the more important at a time when technologies have increased the spheres language maps are present in. To address these issues while accounting for emerging language mapping practices and technologies, this dissertation contains two main parts, followed by a section identifying potential future directions for research of this kind. Part 1 is an extended literature review that develops a unified definition of language mapping and its practices by exploring what maps and human language are, and how language and physical space interact with one another. Part 2 is a collection of three studies which propose and demonstrate the use of specific tools for fostering reflection within language mapping, followed by an essay that identifies a particular framework relevant to language mapping - cybercartography - as being of use to Indigenous Language Education. Together, these parts define what language mapping is, offer the means to study its practices more systematically, and identify some future directions in research of this kind, with the intention of advancing language mapping as a cohesive, reflective, and conscientious collection of practices.
\end{abstract}

Keywords: language mapping; geolinguistics; GIS; applied linguistics; linguistic geography 


\section{Acknowledgments}

The completion of any degree program is no easy feat and is made all the more difficult with the added responsibilities and pressures that come with doctoral studies, both inside and outside of the university. While my responsibilities as a doctoral student have been mine alone, I would like to take this limited space to gratefully acknowledge some of those who have worked tirelessly to teach and support me, and in alleviating many of these pressures, have helped me succeed in my path:

The faculty of the department of Applied Linguistics and Discourse Studies. Thank you for involving me in interesting and rewarding research projects that have enabled me to contribute to fields that explore interactions between language and space, while allowing me to explore my strengths and interests. I appreciate the wisdom you have shared and look forward to collaborating with many of you in years to come.

My supervisors, Erik Anonby and Marie-Odile Junker. I have greatly enjoyed working with the both of you, whose different research projects and perspectives toward language mapping issues have given me the opportunity to approach my own research from different angles. Erik, aside from being my first linguistics professor during my undergraduate studies, you encouraged me to apply for this doctoral program, and over the years have taught me the value of the work we do. Marie-Odile, in addition to showing me what it takes to orchestrate language mapping projects, you have helped me think like a researcher and think critically about our work as language mappers.

My doctoral committee. It has been a pleasure to get to know and work with each and every one of you at the most crucial stages of my doctoral studies. Your commitment to my education and level of detail in advice have helped me in ways I cannot express, and you have served as models for the academic I wish to become.

Rita Richardson. Aside from being the best grandmother a lad could ever hope for, your proofreading assistance, challenging research debates, suggestions, financial contributions, and moral support (to name a few) helped me stay on track in what can otherwise be a very complicated and challenging four years.

Michaela Stone and Adam Gaffen. Thank you, Adam, for your skills, time, and patience in teaching me to write effectively and concisely. Mom, thank you for the endless hours of guidance and commiseration over the grad school experience, and for always telling me I could become a PhD in anything I wanted. Perhaps not engineering. I could have killed people, mom.

Jim Stone and Nadia Stuewer. Thank you, Nadia, for your stimulating discussions and tough questions. They've kept me thinking about what it means to do research, and what place this research has in the world. Dad, your jokes, stories, camping trips, and considerate advice have kept me sane during my studies, and have helped shape my thinking about the practical aspects of study, work, and life. 
Table of Contents

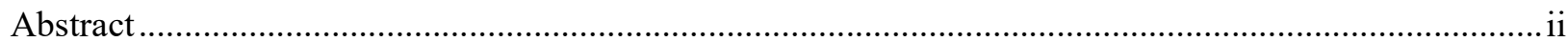

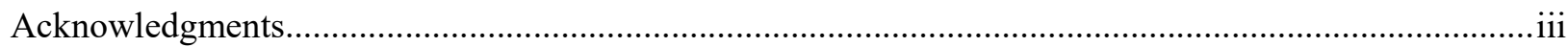

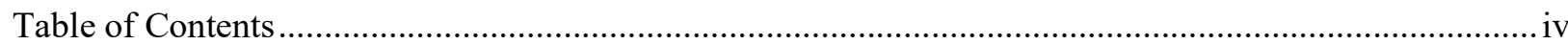

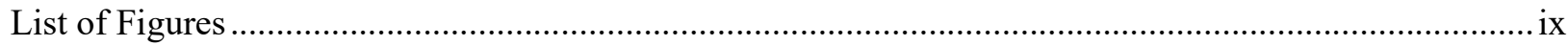

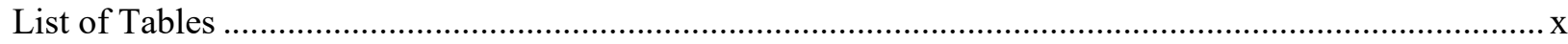

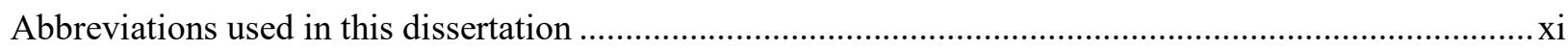

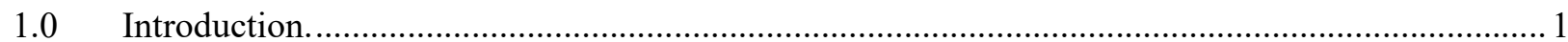

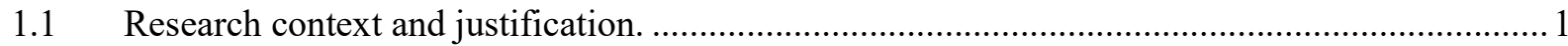

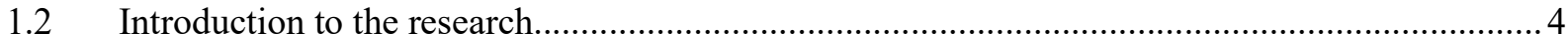

1.3 A mixed methods approach to studying language mapping. …..............................................5

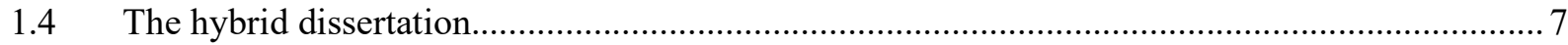

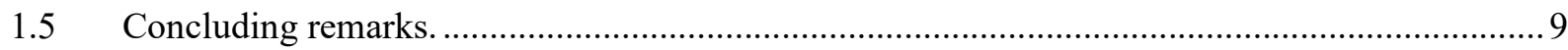

Part 1: Understanding language mapping: Content, techniques, and contexts ............................ 12

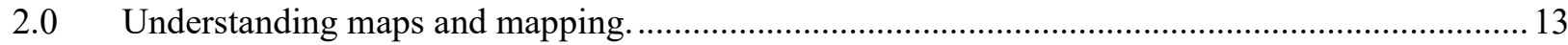

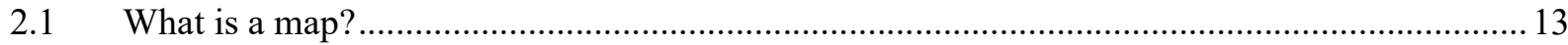

2.2 What maps made of: Internal and external components and the map field. ........................... 17

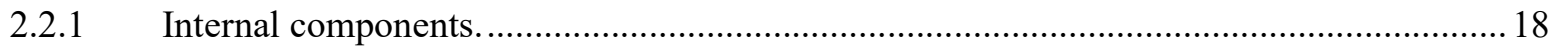

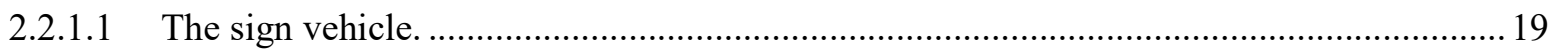

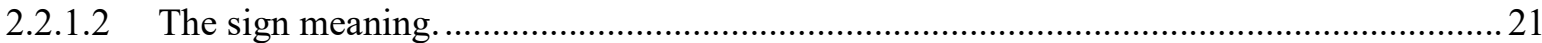

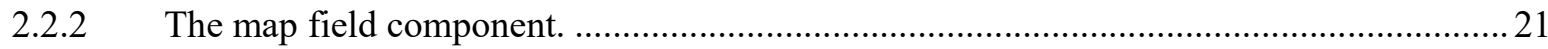

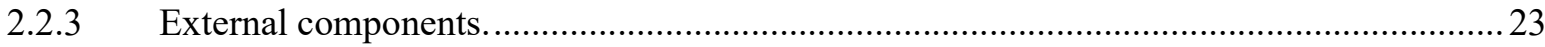

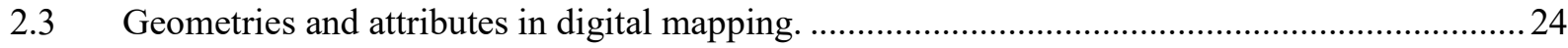

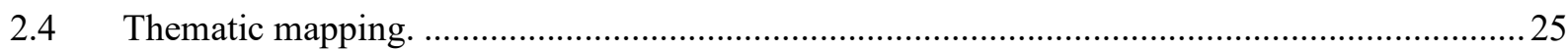

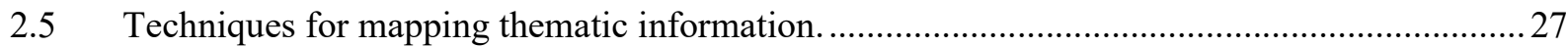

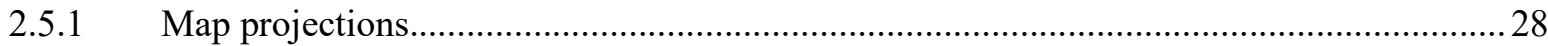

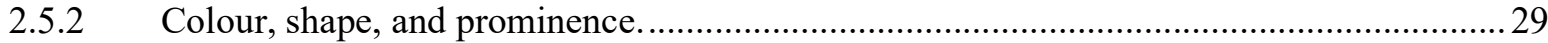




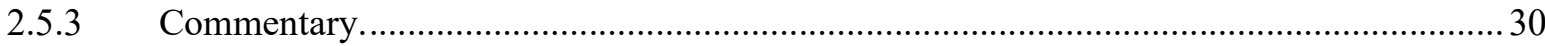

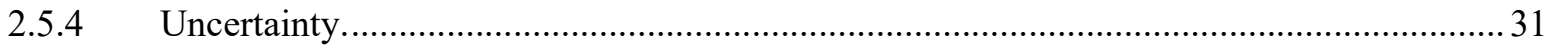

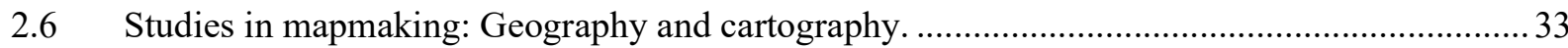

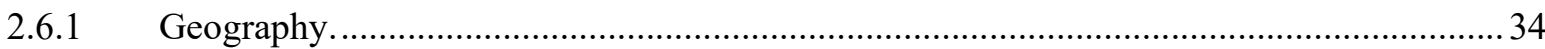

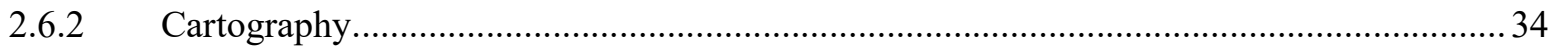

2.7 Techniques in mapmaking: Paper maps to the digital sphere................................................... 36

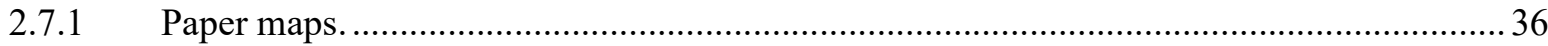

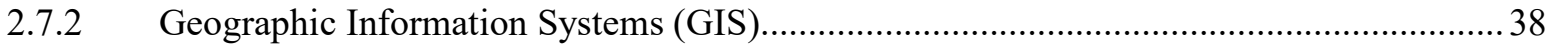

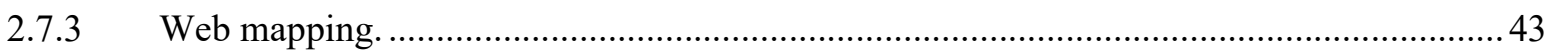

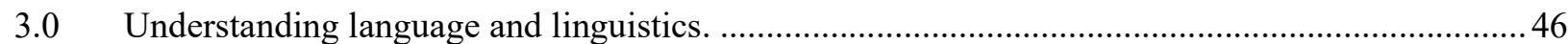

3.1 Language

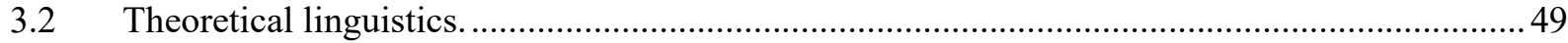

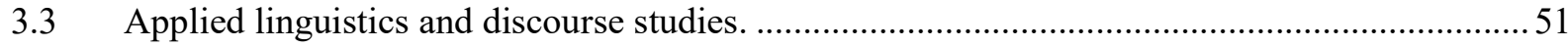

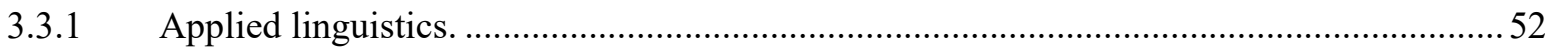

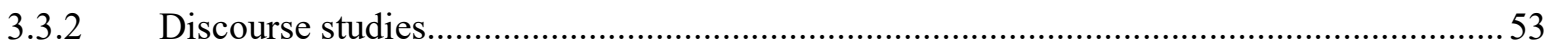

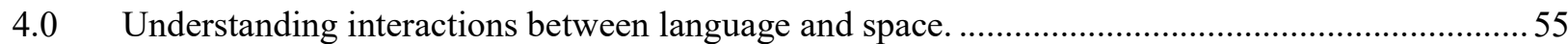

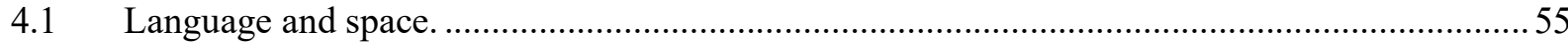

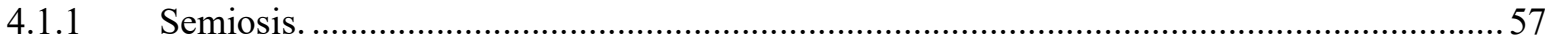

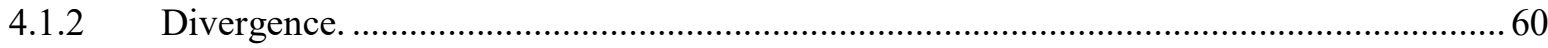

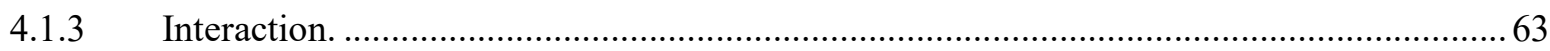

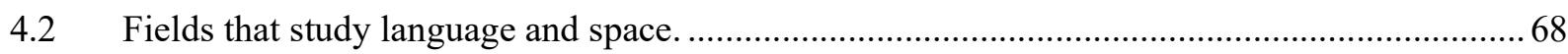

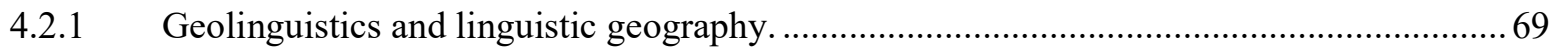

4.2.2 Dialectology, dialect geography, and dialectometry................................................... 72

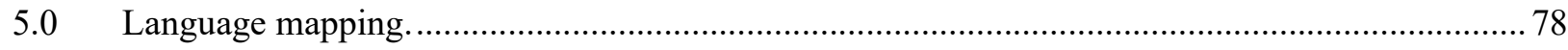

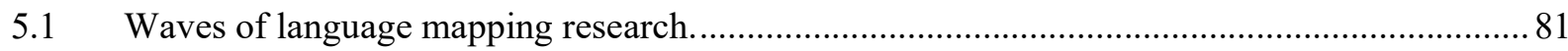

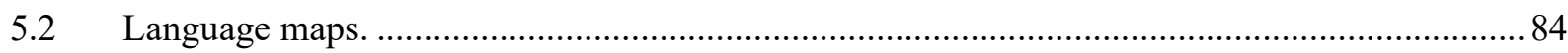

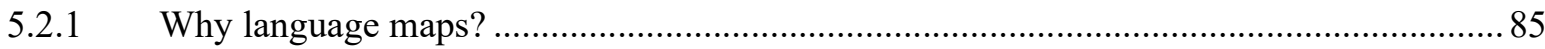




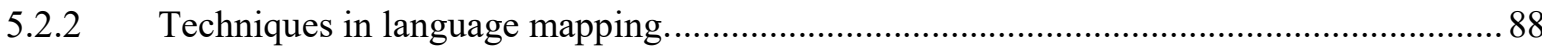

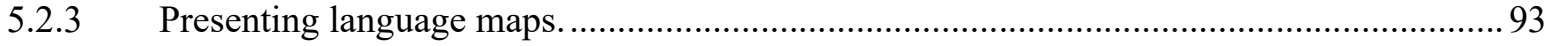

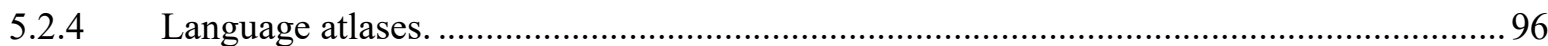

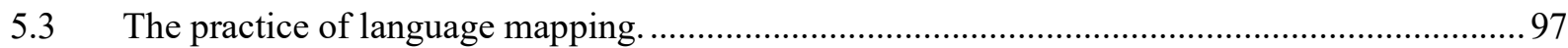

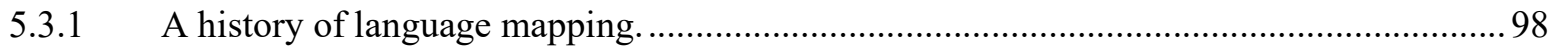

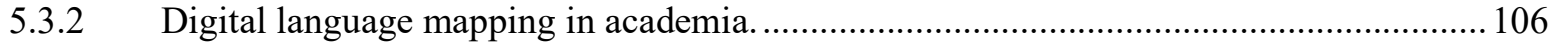

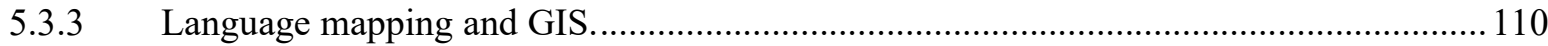

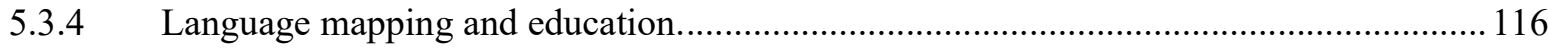

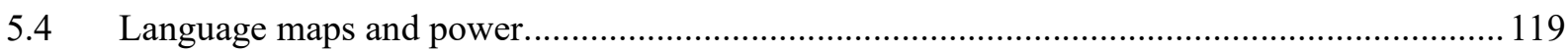

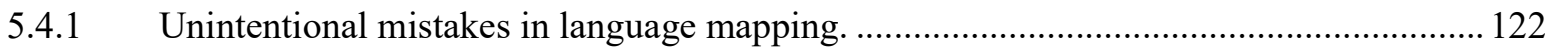

5.4.2 Consequences of language mapping techniques..................................................... 123

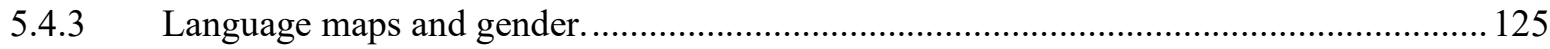

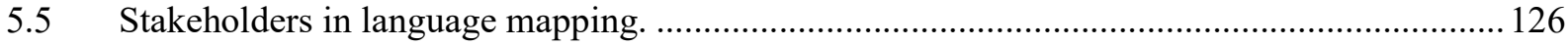

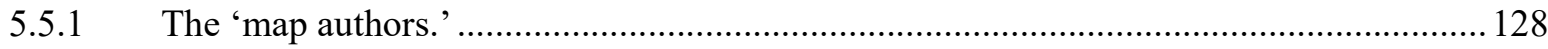

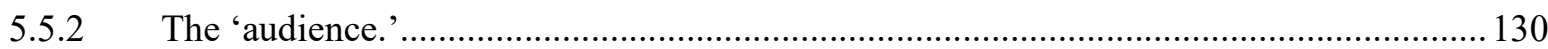

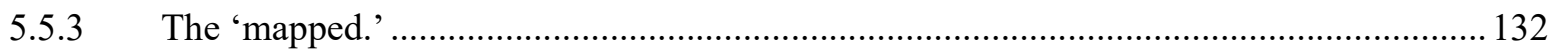

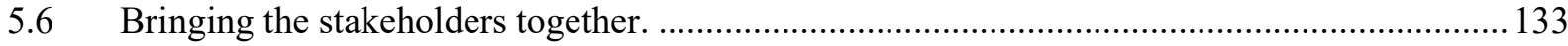

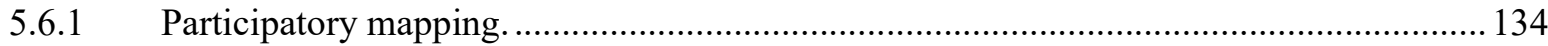

5.6.2 Participatory Geographic Information Systems. ...................................................... 136

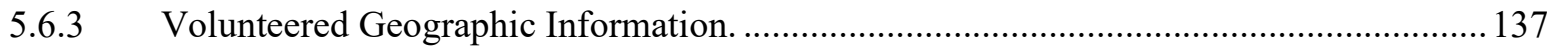

5.6.4 Cybercartography and language empowerment........................................................... 139

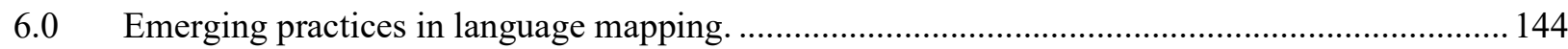

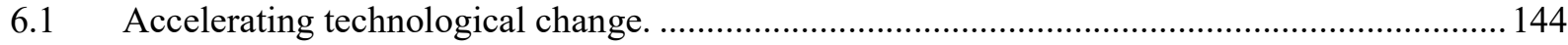

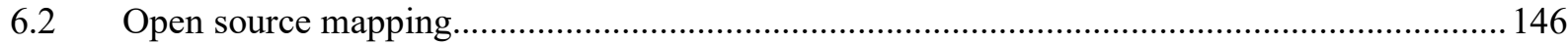

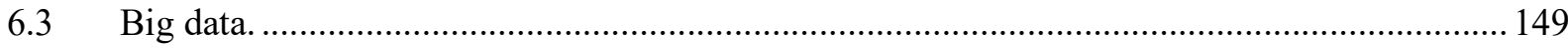

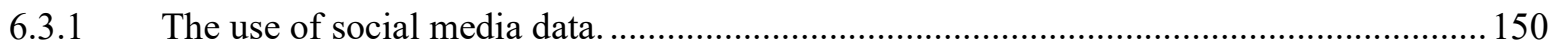

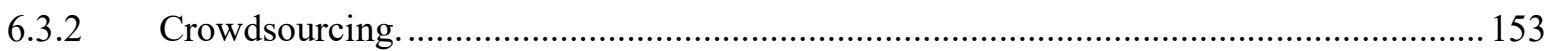




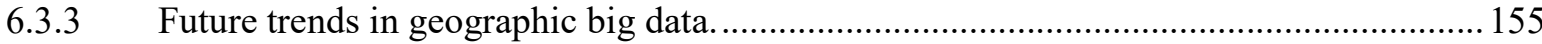

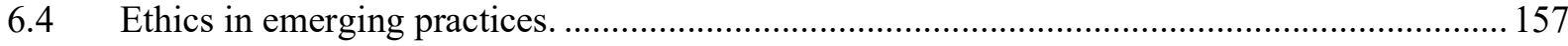

6.4.1 Ten questions for ethical practices in language mapping. ......................................... 159

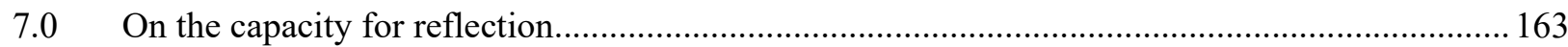

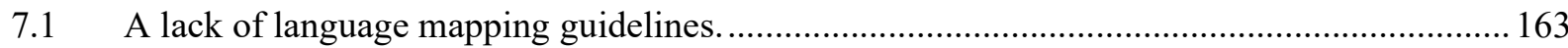

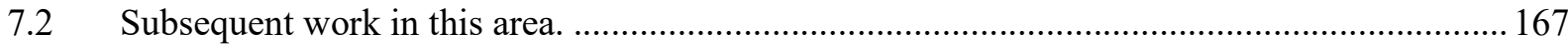

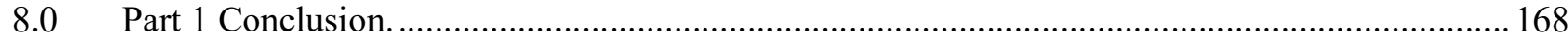

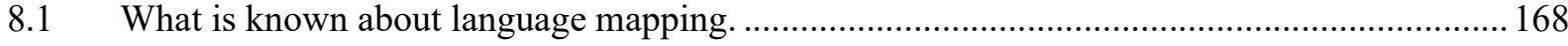

8.2 What is still not known about language mapping. ............................................................... 170

Part 2: Critically reflecting on language mapping practices ........................................................ 173

$9.0 \quad$ Preface

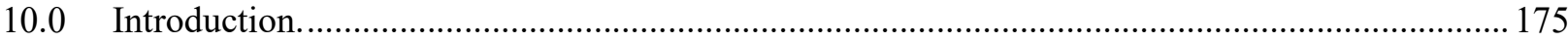

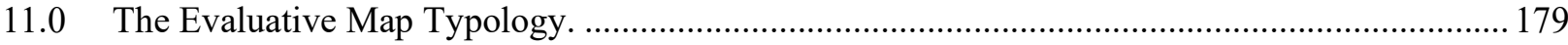

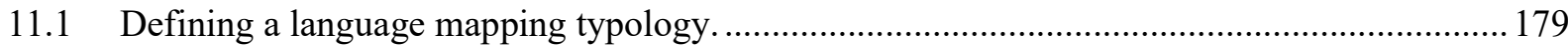

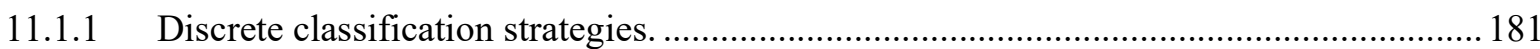

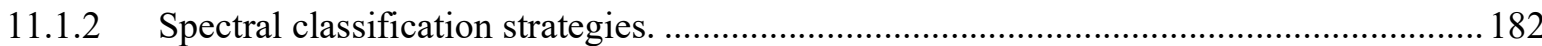

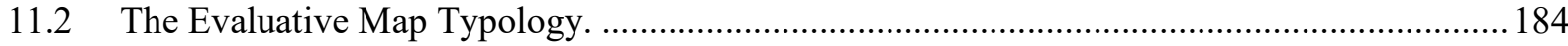

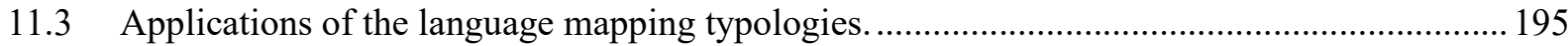

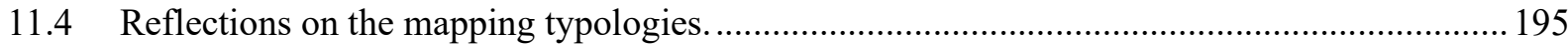

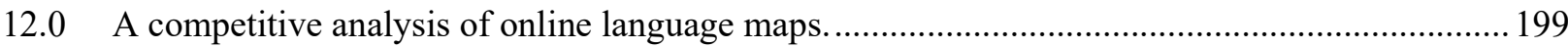

12.1 A competitive analysis performed using the EMT. ................................................................201

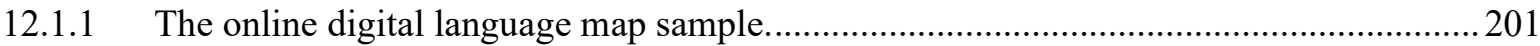

12.1.2 An analysis of the language map sample's relative strengths and weaknesses...............204

12.1.3 A comparison of language map features using the competitive analysis method............207

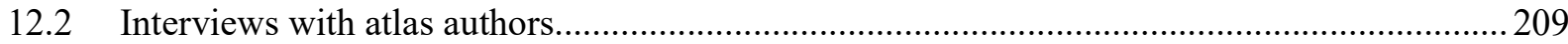

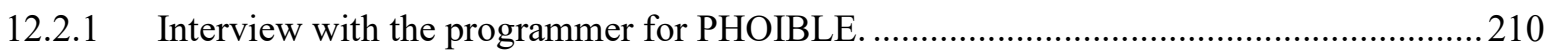

12.2.2 Interview with the project lead of NativeLand.ca.....................................................2 212 
12.2.3 Interview with the director and the programmer of the Algonquian Linguistic Atlas......214

12.3 Reflecting on the competitive analysis and semi-structured interviews. ...............................219

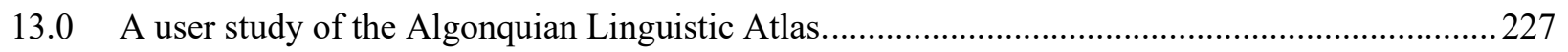

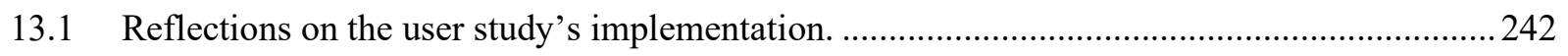

13.1.1 Looking back at the Algonquian Linguistic Atlas user study...................................... 242

13.1.2 Future directions for studies of language atlas user experience ....................................244

14.0 Indigenous Language Education and Cybercartography. ......................................................247

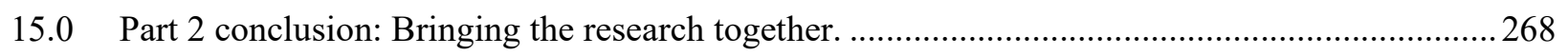

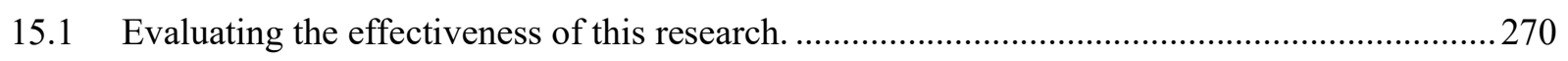

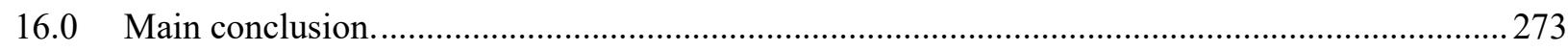

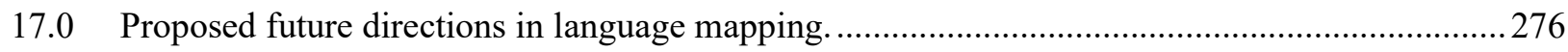

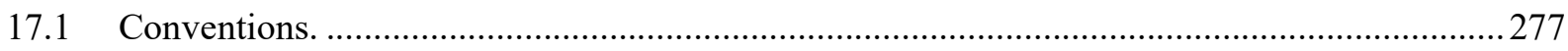

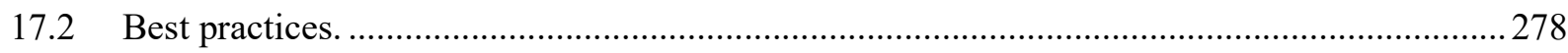

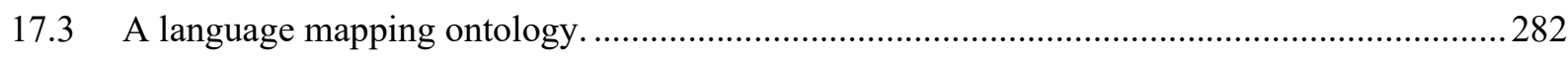

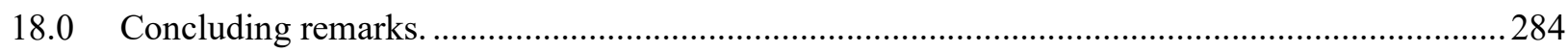

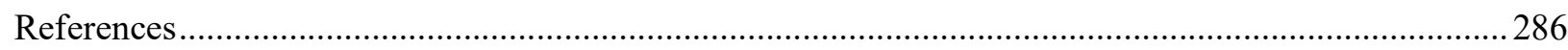

Appendix I: Accompanying Guide to the Evaluative Map Typology (Studies 1 and 2) .........................334

Appendix II: The Evaluative Map Typology tool as applied to Study 2 ............................................. 337

Appendix III: Anonby and Stone’s (2016-2019) typology for analyzing language maps...................... 392

Appendix IV: First observations of digital language atlases, tools, and technologies (Study 2).............401

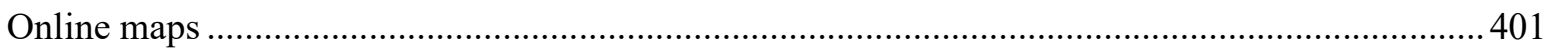

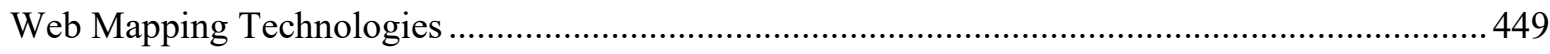

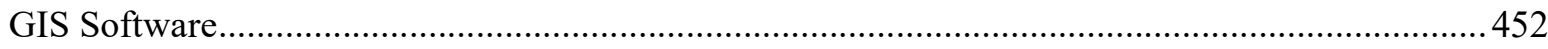

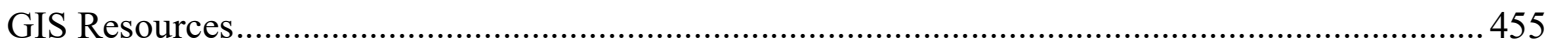

Appendix V: Codes used in the strengths/weaknesses analysis of language atlases (Study 2)..............458

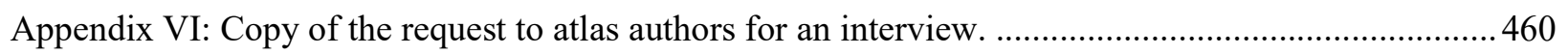




\section{List of Figures}

Figure 1: A map of dialect regions in the United States based on aggregated studies 13

Figure 2: By interpreting signs that model reality, people make predictions about that reality. 14

Figure 3: Maps without physical space: Examples from Object Relations and Genetics......................... 16

Figure 4: Example internal components as they are found on a map (own work) ................................. 18

Figure 5: Star in a circle, often used to indicate a capital city on a map.............................................. 19

Figure 6: The sign vehicle and its relations (Morris, 1971, p.45, in Keates, 1982, p.64)........................ 20

Figure 7: Example external components as they are found on a map (own work), ............................... 23

Figure 8: Thematic maps as an integration of variables of interest within a geographic unit...................26

Figure 9: Three strategies used to indicate uncertainty in language mapping. ....................................... 32

Figure 10: A hand-drawn paper map of the battle of Valcour Island. ....................................................... 37

Figure 11: GIS rendering of the "abeille" term in the Atlas Linguistique de la France............................ 42

Figure 12: Saussure's linguistic sign has an abstract concept and tangible sound image........................ 47

Figure 13: Fields of theoretical linguistics as they pertain to aspects of human language. .......................49

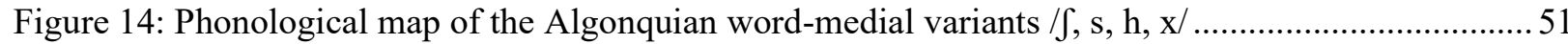

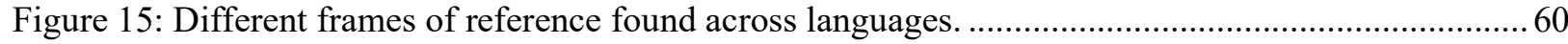

Figure 16: Rotation task involving speakers of languages that use different frames of reference............. 62

Figure 17: Frequency of use of [eI] among friendship groups in a Tower Hamlets (London) youth club. 65

Figure 18: Trilingual sign in Welsh, Irish, and English outside my grandmother's town...................... 67

Figure 19: A visual representation of a geolinguistic framework intended for language mapping. .......... 70

Figure 20: Levenshtein distances between regional language varieties in the Netherlands and Standard

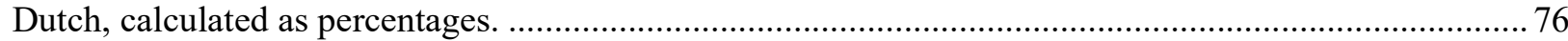

Figure 21: Four different digital language map presentation styles..................................................... 94

Figure 22: An early map depicting languages and their writing systems in Africa (Hensel, 1741)..........98

Figure 23: "plough" as mapped across England in the Atlas of English Sounds (Kolb, 1979).............. 102

Figure 24: Isoglosses reflecting the east-west dialectal divide in early twentieth-century Japan............. 105 
Figure 25: An uploaded linguistic and ethnographic atlas of Italy and southern Switzerland. 107

Figure 26: Online version of the term "abeille" (bee) from the Atlas Linguistique de la France. 109

Figure 27: Language distribution map of French in Manitoba made using Microsoft Paint. 110

Figure 28: Abstract Levenshtein distances of an Iranian province computed with Gabmap. 112

Figure 29: Language of Twitter usage in Montreal in 2013. 152

Figure 30: Number of languages per sign in Luxembourg: 1 (blue), 2 (green), 3 (yellow), 4 (red) 154

Figure 31: Fluid typological approach to cartography. 183

Figure 32: An interactive Google Form of Anonby and Stone's (2016-2019) typology. 194

Figure 33: Favourable and unfavourable attributes of the surveyed maps .206

\section{List of Tables}

Table 1: Levenshtein distance for the word "schedule" between Canadian and British English. 75

Table 2: Summary of the debate on Lexicostatistics by Geisler and List $(2010$, p.2). ............................ 77

Table 3: Number and proportions of maps found in the interpretive analysis.......................................200

Table 4: The 11 interactive language maps and atlases selected for analysis......................................202

Table 5: Summary of an impressionistic survey of digital interactive language maps..........................204

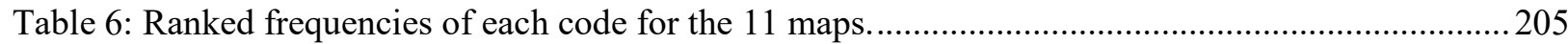

Table 7: Supported interaction operators found in a sample of 11 language maps...............................208 
Abbreviations used in this dissertation

Part 1

FEL Foundation for Endangered Languages

LL Linguistic Landscape

ALDS Applied Linguistics and Discourse Studies

GIS Geographic Information Systems

OSM Open Street Maps

ESRI Environmental Systems Research Institute

MLA Modern Language Association

NILLA NEXOLNEW Indigenous Language Learning Atlas

PGIS Participatory Geographic Information Systems

VGI Volunteered Geographic Information

GCRC Geomatics and Cartographic Research Centre

FOSS Free and Open Source Software

OSGeo Open Source Geospatial Foundation

IoT Internet of Things
Part 2

EMT Evaluative Map Typology

ANAE Atlas of North American English

WALS World Atlas of Language Structures

WATP World Atlas of Transitivity Pairs

ILE Indigenous Language Education 


\subsection{Introduction.}

While thinking about this dissertation, I cannot help but be reminded of the forest in C.S. Lewis' sixth book of the Chronicles of Narnia-The Magician's Nephew-known as the Wood between the Worlds. In this forest, a seemingly endless number of pools sit below a deep canopy. They are shallow in appearance, but in fact provide a portal to other worlds - once the appropriate ring is used. I imagine each subsection in the first part of my dissertation forming a pool that contains a specific facet of what is known about language mapping. Some of these pools lead to language mapping 'worlds' of which much is already known, such as the history of how language maps came to exist in academia. Other pools lead to places about which we know very little, such as how we can look back and reflect upon our own mapmaking practices.

This dissertation, perhaps analogous to the magic ring, strives to bring us into important pools that involve language mapping. Part 1 brings what is known about the familiar pools to the surface and discusses them alongside each other, while also identifying many of the pools we know less about. By contrast, part 2 takes three less understood pools and explores them through the lens of original peer-reviewed research to contribute to the body of knowledge surrounding language mapping. In keeping with this analogy then, this introductory section before the dissertation's two main parts seeks to describe this Wood between the Worlds; that is, the nature of how and why these pools collect the way they do and how they are to be explored.

1.1 Research context and justification.

This research was conducted from the campus of Carleton University, situated in Canada's capital region. The Ottawa-Gatineau area is a crossroads of linguistic and cultural diversity and thus an ideal nexus from which to pursue this line of inquiry. For millennia, the Algonquin 
people have lived along the shores and in the watershed of the Ottawa river, interacting and trading with Iroquoian peoples. Following the arrival of European settlers, the linguistic and cultural landscape changed dramatically. To the north of Ottawa and the Ottawa River now lies Quebec, a province with a French-speaking majority. To the east of Ottawa, a sizeable population of Franco-Ontarians speak distinct varieties of French. To the northwest, in an area known as the Ottawa Valley, many residents speak a variety of English with prominent Scottish, Irish, and French influences (Cheshire, 1991, p.134). To the south and west, dialects more closely approaching Standard Canadian English are heard more often. Importantly, Ray and Gilbert (2020, p.1489) note that Ottawa-Gatineau is among the most important sites of FrenchEnglish contact in Canada, and observe that while the region is in many ways exemplary in its approach to bilingualism, it is also a complex and often confusing myriad of both marked boundaries and transition zones (p.1491). Similarly, as Gilbert and Ray (2020, p.1493) reflect, many recent arrivals to Canada are introducing new languages to the cultural landscape: As they begin to add English or French to their lives, they also have an identifiable impact on English and French in turn.

Having grown up in a foreign service family, I spent my early years immersed in this at times perplexing landscape of language. The later years of my childhood were spent living in and visiting other rich zones of language contact such as Belgium, Singapore, Malaysia, and India. During these formative years, I witnessed first-hand the many ways languages, their use, their prestige, and their vitality can change markedly in the spaces they occupy. I also spent many summers with my grandmother in a small hamlet in a part of Northwest Wales known to be a stronghold of the Welsh language. There I grew up witnessing the struggles and triumphs of a local minority language in the face of a large and monolithic outside linguistic force. Because of 
this, I grew increasingly passionate about the promotion of linguistic diversity and the empowerment of minority languages. During my undergraduate and graduate studies, I was fortunate enough to participate in research assistantships focusing on the documentation, description, and pedagogical tool development for minority and Indigenous languages in Finland, Iran, and Canada. Through my work I was offered a glimpse of some of the current hardships faced by minority and Indigenous languages across the world and began to understand just how little is known by the general public about language endangerment.

It is because of these opportunities I witnessed firsthand some of the many different ways physical and social geographies affect language distribution and use, how power balances and politics across landscapes affect how languages exist, and how we see them. In this dissertation, I take stock of what is known about the relationships between human language and physical space. Furthermore, I look at how this knowledge is being formalized and communicated to others. One of the best-known ways of doing so is with the map, which displays relationships between information about languages and the spaces they are found in. For many, maps of languages present one or two windows into a world people would otherwise not have any access to. For most, these maps are a learning opportunity through a medium that is both familiar and largely intuitive. For some, maps are a means through which they can empower and promote agency over their own knowledge, and for others, maps are tools that can be used to subjugate others and exercise power.

I am thus interested in how language maps exist and are used; the techniques utilized to make them; who makes and accesses them; why they are made; and the consequences they have on our knowledge. However, looking into the processes of language mapping is not an easy task. While maps of linguistic information are being made in ever-increasing numbers, little has been 
done to look inward and reflect upon what goes into their existence and use. Those who do look at language mapping have different things to say and look at the process in wildly different ways. As it stands, a systematic and reflective approach is needed to improve our knowledge of language maps as a medium, and language mapping as a practice.

\subsection{Introduction to the research.}

This dissertation asks two main questions: First, what do we already know about language mapping, and what do we not? And second, how can we advance the way we look at language mapping so that we can further this knowledge? To answer these questions, this dissertation is split into two parts, which each address their respective question. Part 1 approaches what is already known about language mapping from multiple perspectives, including linguistics, cartography, Geographic Information Systems (GIS), history, and education. Think of it as a series of literature reviews that integrate disciplines with the goal of surveying the language mapping landscape. On the other hand, Part 2 suggests and demonstrates how evaluative techniques found in other disciplines can be adapted and refined so that language maps and the practice of language mapping can be studied in a more controlled, consistent, and constructive manner. In addition, an exploratory article highlights an important area of work that can be addressed by responsibly implemented language maps, namely Indigenous Language Education. Finally, a section devoted to exploring future directions in the study of language mapping proposes future work into the development of a cohesive set of conventions, best practices, and a language mapping ontology.

Language mapping is an area of study and a practice that is by necessity multidisciplinary. As such, there is no one theoretical framework that can be applied. Instead, special detail is given 
to many of the constituent parts that make up language mapping, to account for varied perspectives and knowledge that form the basis for language mapping.

Each proposed tool in the second part of this dissertation makes use of investigative methods found elsewhere that have been adapted for language mapping. In this way—despite lengthy discussions of abstract and theoretical concepts in Part 1-I would argue that the second part of this dissertation is an exercise in application, as it offers some tools for reflection and demonstrates their use in practical settings. The absence of a specific theoretical framework notwithstanding, this research follows the mixed-methods methodology, which incorporates elements from qualitative and quantitative research to create a novel set of approaches that are greater than the sum of their parts.

1.3 A mixed methods approach to studying language mapping.

Broadly defined, mixed-methods research is an approach to knowledge that considers and incorporates multiple viewpoints, positions, and perspectives (Johnson, Onwuegbuzie, \& Turner, 2007, p.113). Borne out of disputes lasting over a century between those following qualitative (interpretivist) and quantitative (constructivist) methods and thought, mixed-methods incorporates elements from both camps to establish a 'third' perspective (Johnson \& Onwuegbuzie, 2004, p.14). For the purposes of this dissertation, qualitative research can be thought of as that which uses detailed and empathic writing styles to capture subjective and contextually rich experiences, while quantitative research uses detached writing styles and numerical analyses to reduce bias and promote rhetorical neutrality (Johnson \& Onwuegbuzie, 2004, p.14). Importantly, Johnson, Onwuegbuzie, and Turner (2007, p.113) note that instead of just combining qualitative and quantitative methodologies, mixed-methods should be thought of 
as occurring somewhere in the middle of a spectrum that holds quantitative values on one end and qualitative on the other. Furthermore, Tashakkori and Creswell $(2007$, p.4) stress integration, whereby data are collected and analyzed, the findings are integrated, and conclusions can be made using qualitative and quantitative methodologies. Importantly, mixed-methods research need not be situated directly in the middle of this spectrum, and can instead draw from those methods best suited the subject of study, such as with qualitatively driven or quantitatively driven mixed-methods research (Creswell, Shope, Plano Clark, \& Green, 2006, p.2).

Such an integrative approach is important as it eliminates many of the limitations associated with performing research from only one angle (McLean, 2006, p.iii). While qualitative research does much to make contextual factors more explicit in a study's results, its critics are quick to note that it lacks evidence-based research (Creswell et al., 2006, p.2). Similarly, while quantitative research makes precise, data-driven, and objective analyses possible, the resulting knowledge risks being too abstract and difficult to understand without the appropriate context (Johnson \& Onwuegbuzie, 2004, p.19). By incorporating qualitative and quantitative research into a mixed-methods design, qualitative information such as interview questions can give quantitative statistical analyses needed context, while at the same time, those quantitative analyses can provide numerical evidence that further supports the conclusions reached by qualitative analyses.

The investigations in this dissertation that collect and analyze information—namely the Algonquian Linguistic Atlas user study and the Evaluative Map Typology—are mixed-methods studies as they harness the interactions between qualitative and quantitative methodologies. The Evaluative Map Typology is qualitatively driven, and while it uses descriptive statistics, those statistics are based on previous analyses of coded textual material. On the other hand, the user 
study is an example of a relatively balanced mixed-methods design which employs statistical analyses of questionnaire results, qualitative reporting of semi-structured interview questions, and the numerical interpretation of participants' written responses. In addition to the interaction of multiple techniques used in the research for this dissertation, the structure of the dissertation also draws on multiple perspectives and techniques.

\subsection{The hybrid dissertation.}

Currently, most doctoral research completed in a supervised university setting is done so in the form of a monograph, which is usually a single large research project covering a particular issue (Remenyi, 2015, p.238). Typically, the objective of a monograph is to delve deeply into the theoretical aspects of a field of study — the underlying concepts and frameworks—-while also to perform an empirical analysis and include a study design, data collection and analysis processes, and a discussion thereof (Fridlund, 2010, p.144). Furthermore, Paltridge and Starfield (2007, p.67) note that many monographs follow an Introduction-Methods-Results-Discussion (IMRAD) format, which is well-suited to research that asks a single research question and performs one study to answer it. However, they continue to note that many dissertations also have a more complex structure, driven both by the nature of the research and decisions made by the doctoral candidate in conjunction with their supervisors.

While the monograph is often a default medium for a doctoral dissertation, many universities hold in their regulations an additional option to incorporate research published during the course of the doctoral program, though this option is often not as readily advertised (Remenyi, 2015, p.239). Described by Remenyi (2015) as a papers approach, a compilation thesis by Fridlund (2010), and as a manuscript thesis by the Applied Linguistics and Discourse 
Studies (ALDS) program at Carleton University, this format is appropriate for research that performs multiple studies to answer a research question or series of questions (Paltridge \& Starfield, 2007, p.67). By the standards of the ALDS program this doctoral research is a part of, doctoral candidates are able to undertake the manuscript dissertation if they can include three or more academic works published through a peer review process during the course of their doctoral studies, provided they are the first or sole author of each (ALDS, n.d). Essentially, the manuscript dissertation is an anthology of relevant published works that address one or more research questions, make a contribution to a field's body of knowledge, and demonstrate the candidate's competence as a scholar (Remenyi, 2015, p.329). Importantly, Remenyi (2015) also notes that the body of these articles typically needs to be precluded by a narrative that introduces and reviews the nature of the research, and followed by a summary and conclusion, which are in turn followed by a discussion of limitations and future directions.

The structure of this dissertation follows a third, less charted option, and combines elements from monograph and manuscript dissertation styles to create a hybrid dissertation style of research. Such a strategy has been approved by my doctoral committee and has been done for two reasons. First and foremost, this research is conducted in a largely unexplored area with sparse internal consistency and communication. This means that a review of existing literature, technologies, and methods - more typical of the first sections of a monograph thesis - must occur before any meaningful empirical research can be undertaken. Part 1 is devoted to this role. Part 2, on the other hand, not only proposes new tools for the field but also demonstrates their use by carrying out their intended research. These tools and their uses are presented as published and unpublished articles woven together through reflections and narratives. Second, I believe the 
multifaceted structure of this dissertation reflects the multidimensional nature of all that goes into language mapping, which to be studied in detail, needs multiple angles and perspectives.

While this hybrid dissertation style is perhaps a bit unorthodox, especially in the ALDS department at Carleton University, I believe it to be a necessary format for the study of language mapping. In my opinion, it is as effective in demonstrating primary research at the doctoral level as the monograph and manuscript options. Hybrid dissertations are found elsewhere, in other fields and departments. In educational leadership for example, Lotulelei (2012, p.vi) introduces a dissertation that includes an extended literature review and methods section, but is still centred around publishable material and appended resources. In addition, and similarly in education, Enriquez (2012, p.ii) separates his dissertation into three sections, which comprise two studies and a single unifying discussion which also features as an extended literature review. Though this dissertation discusses at length theoretical considerations for language mapping as an area and a practice, it is also very much a working dissertation, which — ideally — is intended as a resource in fields that make use of language maps.

\subsection{Concluding remarks.}

Coming back to the Wood between the Worlds analogy, we have with us the ring we need to explore the known and unknown pools alike, and we have a plan we can follow through with to use it. From here, Part 1 explores what is already known about language mapping, in terms of its histories, stakeholders, practices and techniques, and the consequences of their implementation. To make this possible, Part 1 introduces and discusses the main fields that can be thought of as contributing to language mapping, namely cartography, linguistics, and studies of language and physical space. To do so in detail, more foundational questions that ask what maps are and what 
language is need asking. Subsequently, Part 2 accounts for this knowledge and important issues therein by performing several analyses of technologies, users, and the maps themselves, while proposing tools that can make the practice of language mapping more self-reflective in nature and identifying an emerging area that requires further investigation. 


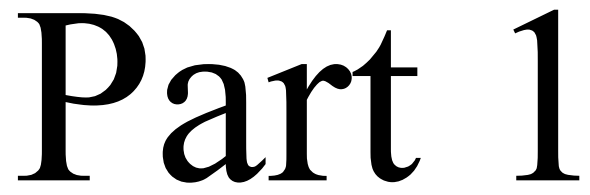

Understanding contemporary language mapping 


\section{Part 1: Understanding language mapping: Content, techniques, and contexts}

This part explores existing (and often conflicting) ideas about what a language map is, what it does, how it may be used by those doing the mapping, and how this usage affects those being mapped. While this is by no means an exhaustive account of what maps and mapping are, it acts as a basic foundation before exploring more specific language mapping processes. Many of the ideas in this part are not necessarily new or the output of doctoral research per se. However, they may very well be ideas that have never been paired side-by-side, leading the reader to conclusions that are not readily graspable in linguistics, geography, or applied linguistics alone. 
2.0 Understanding maps and mapping.

Instead of being something straightforward, what a map is depends on who you ask, and why it was made. As such, this section explores what maps and mapping are in relation to the following general questions: What exactly is a map? What are maps made up of? What are some techniques that are used to make maps? And finally, what are the disciplines that focus on mapmaking?

2.1 What is a map?

Figure 1: A map of dialect regions in the United States based on aggregated studies.

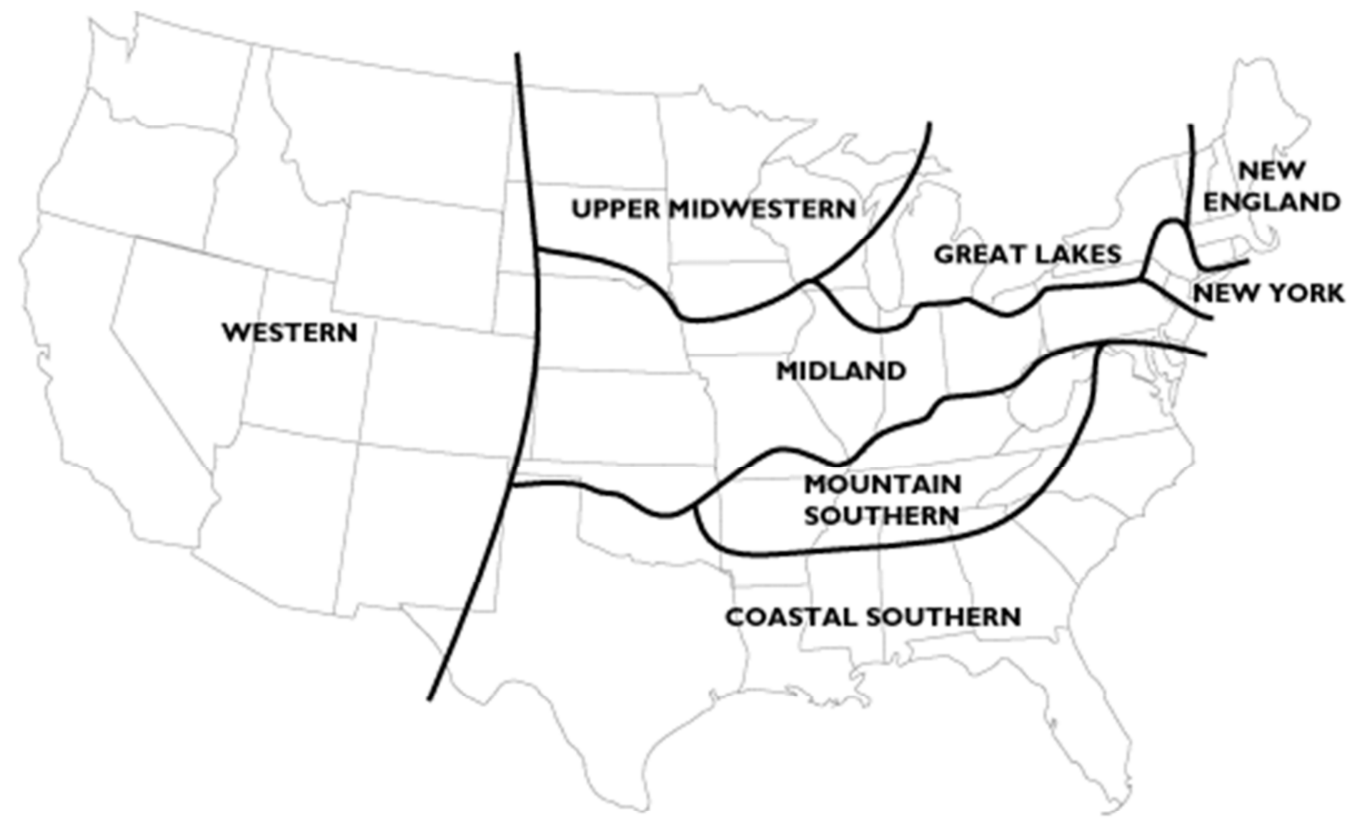

SOURCES: Kurath 1949. Thomas 1958, Kurath \& McDavid 1961, Cassidy 1985, Carver 1987, Labov 1997.

We tend to know maps such as Figure 1 when we see them. They can be found on classroom walls, in textbooks, in cars as navigational tools, on subways, and in many other parts of everyday life. Indeed, such a prevalence of maps in many societies leads those such as Upton 
(2010) to describe maps as "something to which very many people seem instinctively to be drawn, of which they feel they have some immediate understanding..." (p.144). Relatively speaking, it is perhaps easiest to understand a map by what it does. For example, MacEachren (1995, p.1) starts not by defining maps but by explaining how they act as representations, which can either be used to communicate a direct message about a reality to an implied audience, or perform a more artistic purpose. Indeed, Darkes (2017, p.287) explains that maps are as important for decoration as they are for navigation and delineation, and can be used to point out what is still unknown (Monmonier, 1993, p.17), making them a strikingly versatile artefact in human societies. In other words, in its deepest sense the map is a medium of communication (Thrower, 2008, p.3) and a mediator between our inner mental world and the outer physical world (Harley, 1987, p.1).

Maps use graphic symbols to model reality and recognize, measure, and analyze the distribution of things (Ormeling, 2010, p.21). By looking at maps, audiences make conscious and unconscious predictions about the reality being mapped and come to conclusions about places they have never been to and may never see in person (see Figure 2).

Figure 2: By interpreting signs that model reality, people make predictions about that reality.
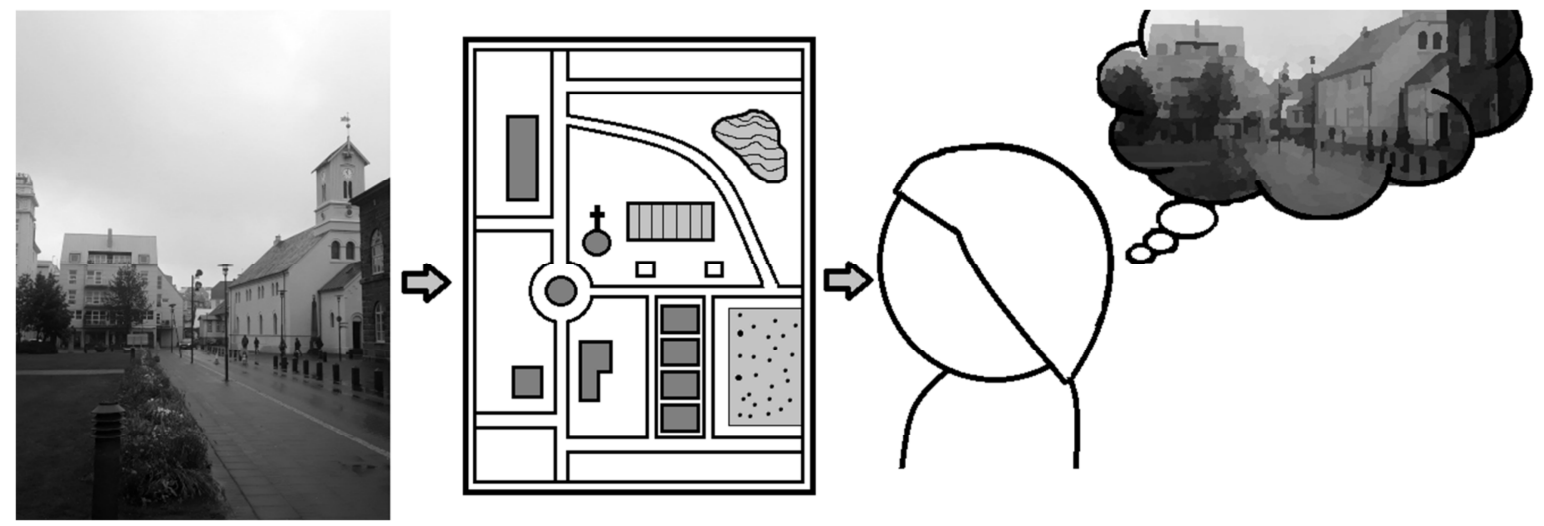

Own figure, derived from Ormeling (2010, p.29) 
In defining the essence of what maps are, Kehrein, Lameli, and Rabanus (2010) summarize a map as a "visual representation of spatial objects" (p.xiii). Furthermore, Ormeling (2010) describes maps as "two-dimensional graphic models of (parts of) the Earth's surface or of geospatial phenomena related to that surface produced to scale for decision-making purposes" (p.22). Herlihy and Knapp (2003) oppose Ormeling's (2010) notion of maps as strictly visual and necessarily practical, and note how many Indigenous communities have "maintained cognitive maps that are delineated verbally using place names that convey place and spatial orientations" (p.304). As a notable example, "songlines" are used by Aborigines throughout Australia, which are songs that describe the creation of the land and, using mnemonics linked to both the land and sky, make navigation possible (Norris \& Harney, 2014, p.7). Furthermore, Hendry (2014, p.111) and Gersmehl (1991, p.1) cite Marshall Islanders' traditional use of 'stick-maps,' which are specific arrangements of sticks representing ocean swells and currents that needed to be memorized before travel, and thus exist in their readers' minds.

In a literal sense, Pacheco and Velez $(2009$, p.274) note that the term "map" originated from a Medieval Latin term meaning "sheet" of the world. While it is important to understand that many maps in Western scholarly traditions are indeed two-dimensional, any definitions here should be broad enough to account for differing cartographic traditions and not limit what maps could be. In opposition to Ormeling's (2010) strictly two-dimensional maps, Salischev (1986, p.167) observes the potential for maps to be three-dimensional because of altitudinal changes in topography or, in the digital sphere, four-dimensional, as they can make use of time. Indeed, a principal assumption within Geographic Information Science (GIS) - a discipline based on the digital creation, visualization, and analysis of geographic information (Goodchild, 2009, 
p.251) - is that the Earth's surface is a "spatio-temporal continuum in which processes of different kinds take place" (Molenaar, 1998, p.1).

The above definitions presuppose that maps reflect a reality based in physical space in some way. This begs an important question: must the map reflect physical space to truly be called a map? Vasilev (2007) explains that maps do not need to be limited to geographic phenomena, and can also represent concepts outside of a physical reality (p.1), such as abstract notions involved in workflow (Deelman et al., 2003, p.27) or representing patterns in natural language (Head, 1984, p.8). Skupin and Fabrikant (2003) identify how cartographic research has been applied to non-geographic information, such as self-organizing maps (p.100) and tree maps (p.101). Similar to geographic maps that model relationships between things in physical space (Ormeling, 2010, p.23), non-geographic maps strive to show and preserve relationships between concepts in a more abstract space. As shown in Figure 3, such maps can look quite different from one another depending on their purpose and discipline.

Figure 3: Maps without physical space: Examples from Object Relations and Genetics.

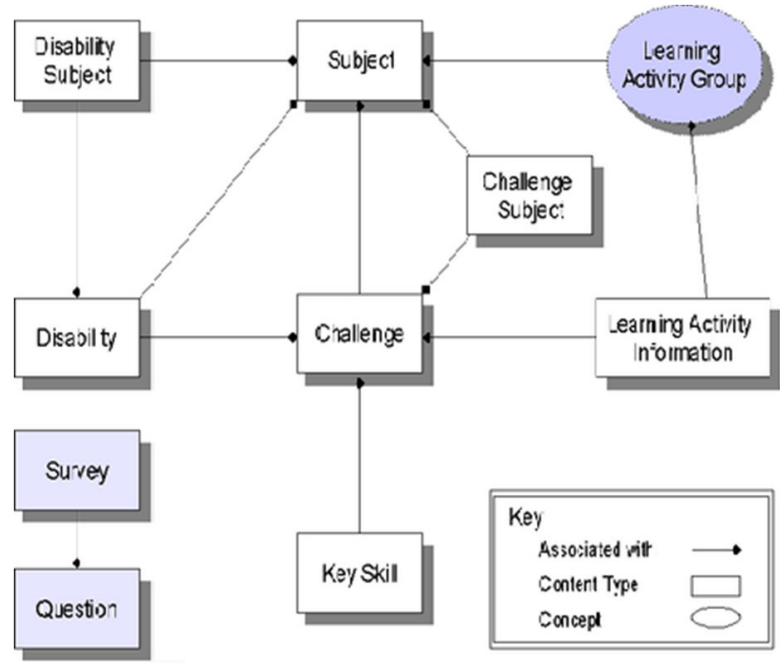

Accessed from: https://files.asset-web.co.uk/

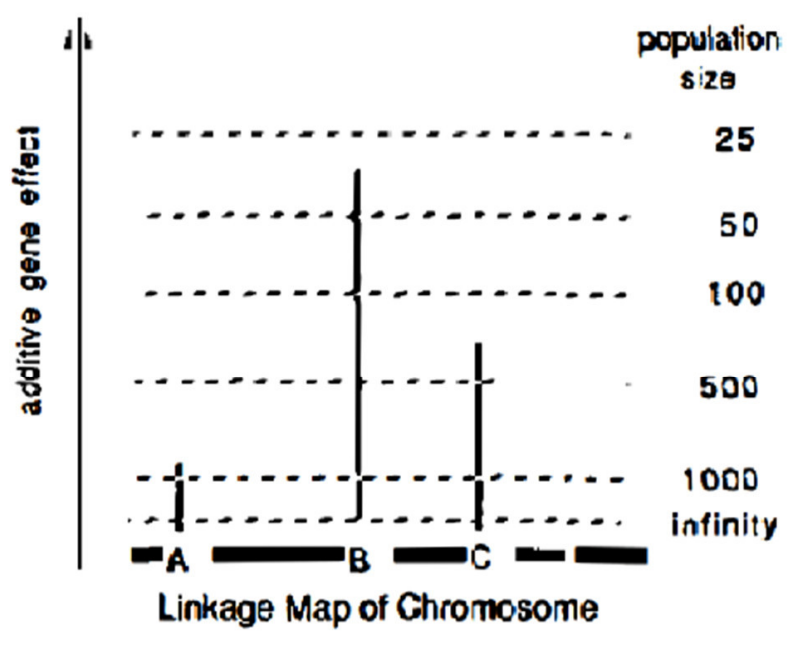

Accessed from: Tanksley (1993, p.217)

[both images have been made more narrow to fit the width of this page] 
However, Keates (1982, p.73) mentions that maps are at best highly artificial representations that are at the mercy of various decisions and intentions. Furthermore, Wood (2020, p.342) claims that if maps are indeed representations of reality, they are not very good at what they do, as they select such specific elements to represent that a model of reality is no longer feasible. Here, Wood (2020, p.342) describes maps as a kind of discourse, but cautions that any such definition should not go so far as to describe maps as a language; maps are the content of what is said, but not the language used to communicate them. Furthermore, though Tyner (2020, p.334) notes that the "communication model" of cartography—where maps are considered to be a language of sorts - has been largely discredited, she does explain that maps do make use of a language that employs techniques such as "lines, colours, marks, and patterns" (p.338). In addition to trying to describe what a map is in the first place, it is just as important to discuss the basic components that make up a map, as these determine what can be mapped and how.

2.2 What maps made of: Internal and external components and the map field.

Broadly speaking, the term components when applied to maps in this dissertation refers to key parts of a map that are used in some way to communicate information about a thing or a relationship in physical space. It is important to note here that these characteristics apply to maps that focus on physical space, and not to non-geographic maps. Vasilev (2007,p.1) separates maps' components into three main categories: internal components, external components, and the map field. 


\subsubsection{Internal components.}

Internal components are cartographic signs depicting objects from a reality. Figure 4 features four examples of internal components: labels, lines, points, and polygons. In addition, researchers such as Christophe et al. (2016, p.61) include textures and colours as key internal components.

Figure 4: Example internal components as they are found on a map (own work).

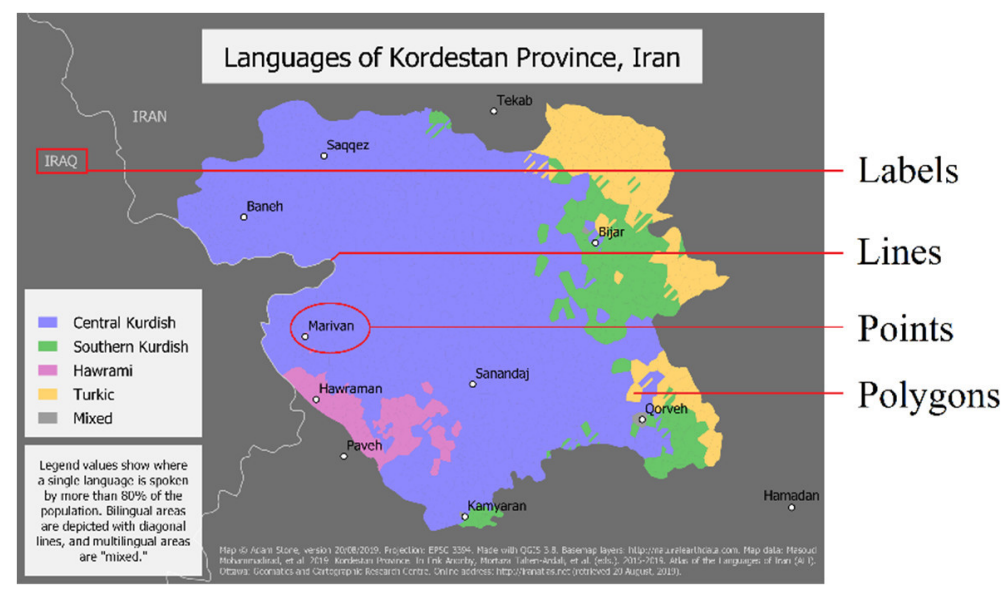

Vasilev (2007) describes internal components as "cartographical signs that represent definite objects from reality" (p.1), which can be further separated into the sign vehicle (a visual form) and the meaning it conveys (p.7), as in Figure 5. ${ }^{1}$ In support of this, Girnth (2010, p.99) applies Pierce's (1931) theory of semiotics to define a language map as the triadic interaction of the representamen (here, sign vehicle), interpretant (meaning), and object (reality which the map reflects). Vasilev's (2007) analysis is the basis for the discussion of language maps' internal characteristics here, which is separated according to sign vehicle and meaning.

\footnotetext{
${ }^{1}$ Such a distinction reflects notions of the signifier and the signified found in decodification semiotics (e.g. Petrilli \& Ponzio, 2002, p.72).
} 
Figure 5: Star in a circle, often used to indicate a capital city on a map.

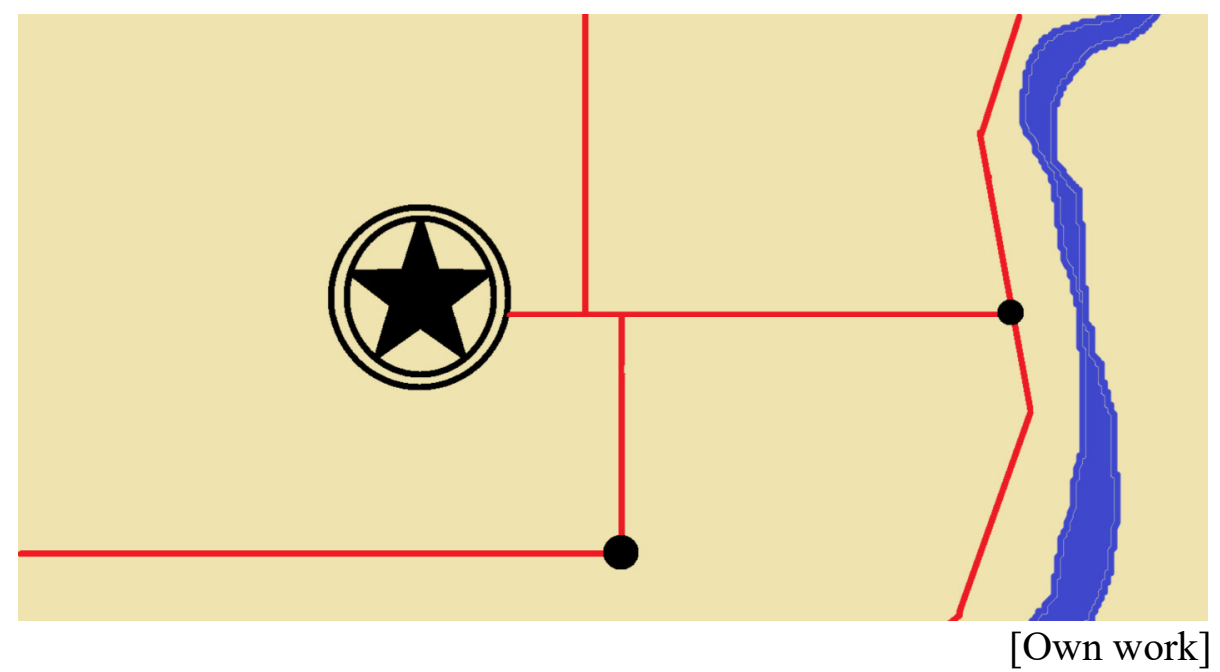

One sign vehicle on this map is a visual depiction of a star within a circle.

The meaning conveyed is that this object of interest is a capital city.

\subsubsection{The sign vehicle.}

In their most basic sense, sign vehicles are things that represent a certain piece of information about the world (Morris, 1938, p.4). In cartography, the sign vehicle is an object on a map — such as a point, line, or polygon — that relays information from map to reader (Vasilev, 2007, p.7). Misra, and Ramesh (1989, p.331) note that each symbol (sign vehicle) on a map is an entire component in of itself. As such, it is a way for maps to communicate a reality about something to their audiences (Ormeling, 2010, p.26). While a sign vehicle can be abstract or concrete, in the latter case it is also called a "spatial object" by researchers such as Peuquet (1988, p.383) and Molenaar (1998, p.xi). Keates (1982, p.64) visualizes Morris' (1971, p.45) understanding of what a sign vehicle consists of in specific regard to mapmaking, shown in Figure 6. 
Figure 6: The sign vehicle and its relations (Morris, 1971, p.45, in Keates, 1982, p.64).

\begin{tabular}{|c|c|c|c|}
\hline $\mathrm{m}$ & Designation & The sign & Connotation \\
\hline maker & Semantics & vehicle & Pragmatics \\
\hline
\end{tabular}

On a map, a sign vehicle has two basic components: its location ${ }^{2}$ (realized by its placement on the map and/or geographic co-ordinates) and a set of properties (a specific language, lexical item, speaker population, etc.) (Vasilev, 2007, p.7). Location refers to information that situates spatial objects on a map (Geoscience Australia, 2012). These attributes commonly come in the form of geographic co-ordinates in digital mapping, with values for latitude and longitude (Stevens, Smith, \& Bianchetti, 2012, s.1.3). In digital mapping, locationally-attributed spatial objects are said to be georeferenced, and the distinction between location and attributes becomes less relevant (Gregory et al., 2015, p.3). While such characteristics have usually referred to objects as they are located in a particular place (Orford, 2002, p.106), the added dimension of time made possible by digital mapping means that locational attributes may change over time as information (such as a speaker population) is updated (Andrienko et al., 2010, p.1546).

Goodchild (1989, p.107) discusses another mapping model where points, lines, and polygons are replaced by continuous fields such as a heat map, which uses continuous colour gradients over a surface. Here, locational attributes are part of a spatial continuum instead of being plainly identifiable from one another (Goodchild, Haining, \& Wise 1992, p.412).

Non-locational attributes refer to a spatial object's information not related to its position (Stevens, Smith, \& Bianchetti, 2012, s.1.3). Depending on the map, these could be a placename,

\footnotetext{
${ }^{2}$ Also referred to as a locational attribute and its properties as a non-locational attribute in some digital mapping conventions (e.g. Geoscience Australia, 2012).
} 
population, or geographic feature, which make spatial objects measurable and comparable to one another (Vasilev, 2007, p.7). Neither locational nor non-locational attributes are more necessary than one another and are instead more like logical duals (Peuquet, 1988, p.383); if such attributes have no corresponding location, the point of having a spatially oriented representation is lost. If, on the other hand, spatial objects have no non-locational attributes, no information would be available about what makes their location important and the object would be meaningless.

\subsubsection{The sign meaning.}

A sign's meaning is a common understanding of an object on a map that connects it to what is modelled in reality (Vasilev, 2007, p.7). Girnth (2010, p.99) states that as maps cannot directly depict a reality, they attempt to model it through the meaning conveyed by sign objects. This meaning is invoked by both the cartographer and audience. It is thus important for what the audience understands to resemble the intended meaning faithfully, as such depictions may be the only interpretation people have of a map's content (Ormeling, 2010, p.29). Though a map's meaning ultimately depends on what individual sign objects represent (Blumson, 2010, p.1), a map itself is also a sign (Wood \& Fels, 1986, p.54), as well as a genre (Spinuzzi \& Zachry, 2000, p.175). Maps also act as one object to "immediately convey a good deal of information without the interference of text" (Upton, 2010, p.144).

\subsubsection{The map field component.}

The map field refers to conceptual models and notions that go into framing the reality the map attempts to reflect (Vasilev, 2007, p.6). In many maps, the field is decided before a single 
line has been drawn (Tyner, 2010, p.18). In practice, parts of the map field can include the following:

- Scale: Size of the map compared to the real world (Tyner, 2010, p.73). As maps depict parts of the world that are significantly larger than the surface they are viewed on, certain details must be sacrificed to make the map legible.

- Projection: The systematic way the Earth's spherical proportions are flattened onto a two-dimensional surface (Snyder, 1989, p.vii). As objects (such as the Earth's surface) with a curved surface cannot be directly flattened, authors must choose which systems of distortion are most appropriate for the specific map (Snyder, 1989, p.8), of which there are endless kinds possible (Snyder, 1997, p.1).

- Co-ordinate system: A set of values (such as numbers) that is used to determine the location of anything on the Earth's surface in Euclidian space (Woods, 1922, p.1). A common example of a co-ordinate system is the use of longitude (x-axis) and latitude (yaxis) values on a rectangular plane (Bernhardsen, 2002, p.117).

- Scope of the field: What elements of reality the map chooses to focus on. 


\subsubsection{External components.}

Figure 7: Example external components as they are found on a map (own work).

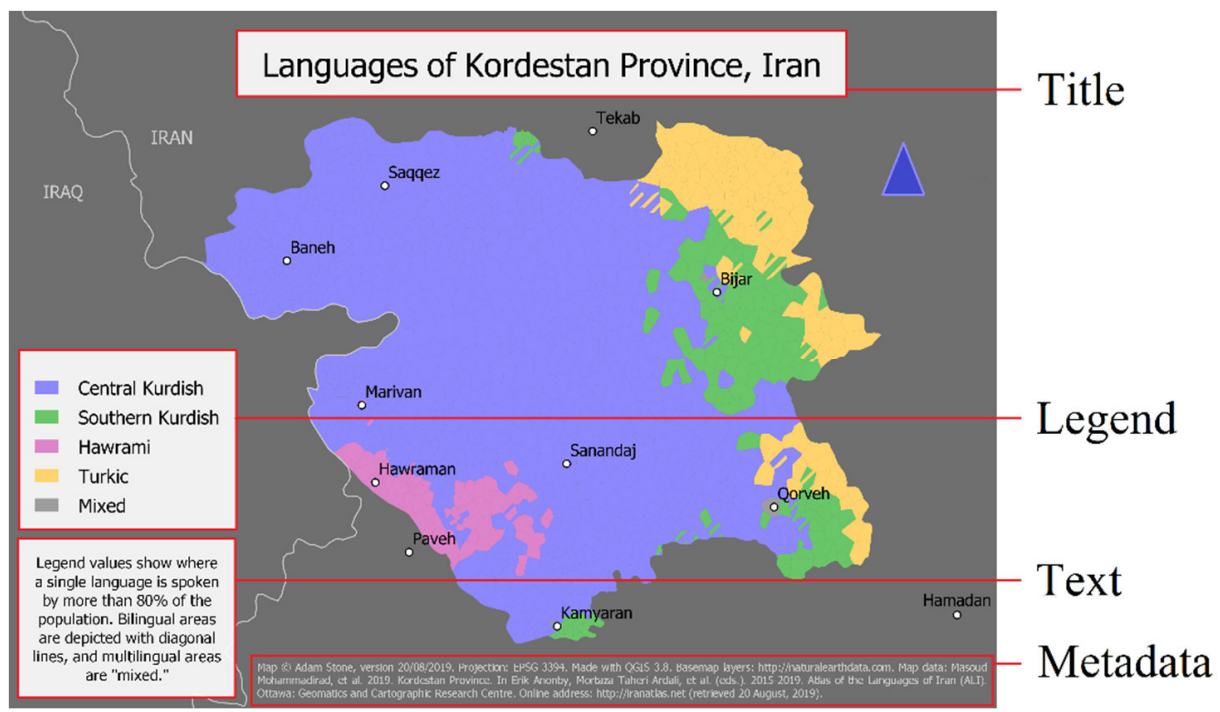

Also referred to as a layout element by Peterson (2014, p.25), Vasilev (2007) describes external components as "signs from some non-cartographical sign system" (p.7), which are used to describe the map's subject matter, internal components such as symbols, parts of the map field such as the projection system or the scale, and ultimately the map itself, as in Figure 7 above. Such a set of possible external components ${ }^{3}$ according to Vasilev $(2007$, p.8) may include:

- Map heading: also known as the Title.

- Legend: a guide to what the internal component symbols on the map mean.

- Scale description: a number and accompanying graphic explaining what a single unit of measurement on the map is equivalent to in the 'real world.'

- Map field description: for example, a description of the projection used on the map.

\footnotetext{
${ }^{3}$ While Vasilev (2007, p.7) also included Advertisements in his list, I have excluded them here as I argue they are not there to enhance maps' communicative power.
} 
- Map object description: a description of the content the map focuses on.

- Map description: a description of the map's author(s), producers, date of production, etc.

- Area of interest: the physical area focused on, which can be an adjacent graphic.

- Illustrations: accompanying images, diagrams, or decorative elements.

- Index of the objects: an account of what the symbols refer to in greater detail than the legend.

However, it should be noted that Girnth (2010, p.106) specifies language maps to have six external components (map face, graticule, border, title, legend, and scale), suggesting disagreement within mapmaking regarding key components. In addition, there are other conceptualizations in the cartographic literature that rival notions of internal and external components that are necessary to discuss.

2.3 Geometries and attributes in digital mapping.

While internal and external components may adequately capture the layout of maps found on paper and thus incapable of interaction, the situation is more complicated when interactive maps are involved, such as those on computers and the internet. While the goal of 'static' paper maps is mainly to visualize a cartographic image, interactive and digital maps integrate information of interest, allow for alteration based on updated information, and allow audiences to see the underlying basis of the maps (Sandinska, 2016, p.288). In the context of digital mapping using GIS, Ammar, Behairy, and Habibi (2016, p.707) describe what Vasilev (2007) refers to as internal components as but one small element of a total map that are interrelated on the same level with other elements such as the title, legend, metadata, and scale. As symbols in digital maps are representations of data that exist elsewhere (such as in a database), they and their attributes (attached information in nominal, ordinal, interval, or ratio form) are linked to other 
elements that enhance their display such as the legend or scale (Ammar, Behairy, \& Habibi, 2016, p.709). As such, the boundary between internal and external components becomes fuzzy, as these different mechanisms are different manifestations of the same information.

\subsection{Thematic mapping.}

As maps can serve an almost infinite number of purposes and represent anything with a spatial (or relational) component (Tyner, 2017, p.7), there is a seemingly endless number of different kinds of maps that could be made. However, if one were to group maps based on what they do (as in Tyner, 2010), the following categories appear:

1. General purpose: Created for a broad spectrum of uses and users, such as a regional map.

2. Special purpose: Made for particular uses such as navigation or resource management.

3. Thematic: Represent specific phenomena, whether visible or invisible, such as language.

While general and special purpose maps are important in any analysis of mapping and cartography in general, it is this third category which is of particular interest due to the thematic nature of language maps. In keeping with the previously mentioned tendency to describe maps first by what they do, Pisati (2004) explains that thematic maps "illustrate the spatial distribution of one or more variables of interest within a given geographic unit" (p.361). In an illustration seen in Figure 8, Ormeling (2010, p.22) considers language maps to be thematic as they portray geospatial information (i.e. linguistic phenomena) against a topographic map (i.e. a physical map of the land). 
Figure 8: Thematic maps as an integration of variables of interest within a geographic unit.

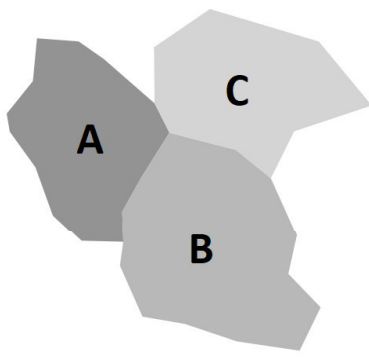

Thematic content

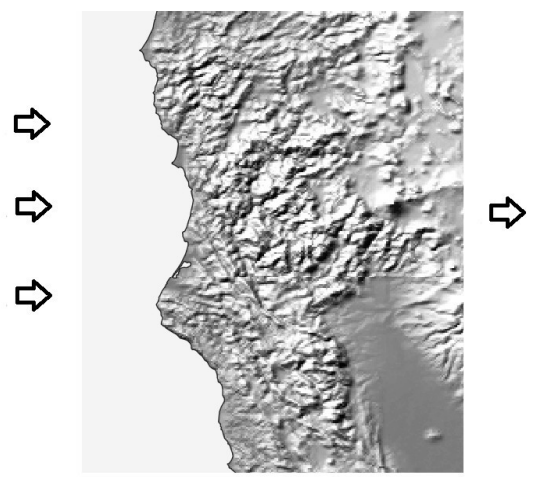

Physical world map

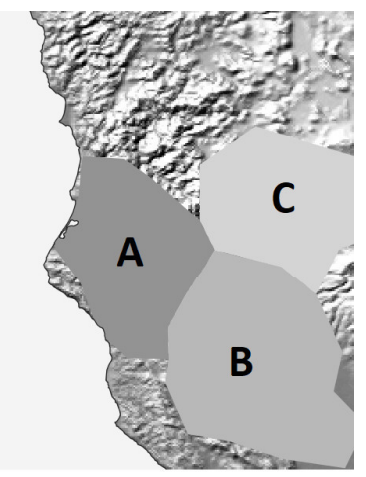

Thematic map

[own work]

Thematic maps typically result from a collaboration between cartographers making the maps and thematic content experts, who are knowledgeable about the field (Vozenilek, 2012, p.1).

This is important to consider as the cartographer's field of expertise is often different and separate from that of the thematic content expert, meaning that informed and adequate communication between the two camps is important and necessary.

As more in-depth definitions of maps and their parts are outside the scope of this dissertation and will not be discussed further, it is important to acknowledge that the above accounts only skim the surface of what maps are. For an expansive introduction to design features and considerations in mapmaking, see Ormeling (2010) and Darkes (2017). Similarly, for a detailed analysis of thematic mapmaking, see Tyner (2017). To better understand maps and mapmaking, it is also important to look at what mapmakers do, and how their actions and beliefs are influenced by theory and knowledge. 
2.5 Techniques for mapping thematic information.

Maps act as scale models of reality, in that they are almost always smaller than the spaces they represent (Monmonier, 1993, p.21). Because of this, maps require specific techniques to render their information of interest legible to their users. Representational techniques, especially when applied to language mapping, make visualizing thematic (such as linguistic) information possible (Girnth, 2010, p.107) and help authors unpack information for audiences (Ormeling, 2010, p.144). As they are often the sole experience an individual might have with a particular area, Rankin (2016, p.2) notes that maps' power lies in their ability to stand in for an original landscape. Tyner (1982, p.140) claims that any persuasive map is created by the manipulation of cartographic elements through distortion, selection, symbolization, and the choice of text and title. In addition, Rabanus (2020, p.104) notes that the results of the mapping process can either be topographically faithful, (superimposed over a physical map of a region), or topographically unfaithful (abstract in design). While Western cartographic traditions started in the late Middle Ages with the designing of property maps, attempts at transforming representational mapping into a scientific pursuit only began in the 1950s (Kitchin, Perkins, \& Dodge, 2009, p.5). Formalized techniques for representing information on maps are found in many other disciplines that visually represent information (such as architecture, Farrelly, 2008, p.184), and are said by Pauwels (2006, p.vii) to touch upon the very essence of scientific activity. Within these so-called representational techniques, there are many ways a map's message can be communicated. While only four are mentioned below, more of these representational techniques can be found in Ambrose and Williams (1991), Wood and Fels (2008), Girnth (2010), and Luebbering (2011), to name a few. 


\subsubsection{Map projections.}

Map projections attempt the seemingly impossible task of transposing the surface of the earth, a slightly irregular spheroid, onto a flat surface (Deetz \& Adams, 1921, p.9). Such flat projections cannot be true representations of the globe as some areas on the map need to be expanded, distances need to be changed, and entire landmasses need to be distorted (Mulcahy \& Clarke, 2001, p.167). When raster images (akin to photographs) are used to make a background in digital maps — which is essential in thematic mapping (Ormeling, 2010, p.22)—pixels in some places will multiply, squeeze, or fade out entirely depending on the chosen projection system (Mulcahy \& Clarke, 2001, p.167). Even when designed with the best of intentions, Ormeling (2014, p.354) regards projections as blatant distortions of reality that promote some information at the massive expense of others. ${ }^{4}$

Ormeling (2010, p.30) notes that different projection choices affect how audiences perceive the distribution of language data. Quite often, the location and size of the area of interest-a large island in the Arctic or a small country along the Equator-requires a specific projection to approximate its actual proportions (p.31). Depending on the projection system used, one map could indicate a sparse distribution of speaker communities, while another using a different projection would display them as clustered together (p.30). While neither map is "right" or “wrong” in how it depicts communities' distributions, these different projections have farreaching implications regarding how people see the information thus arranged (p.22). As such, projections reflect particular viewpoints: a projection that makes a region of interest look large

\footnotetext{
${ }^{4}$ A discussion of specific projection systems is outside the scope of this section and is indeed already the focus of many textbooks (e.g. Bugayevskiy \& Snyder, 2013). For example, Maling (2013, p.47) describes how projections are created, Tobler (1963, p.59) offers insights into how such projections can be analyzed, and Ormeling (2010, p.31) discusses projection systems relevant to language mapping along with an assortment of related implications.
} 
may cause the distribution of linguistic information to appear vast or diffuse, while a projection that makes a region look small may cause information to appear localized or dense. Similarly, surrounding areas may appear insignificant by comparison, or large and imposing.

Quite understandably then, detailed accounts of the projection system help contextualize information on a map (Mulcahy \& Clarke, 2001, p.180). In many GIS programs, projection systems can be chosen easily to fit a project's needs, which in theory makes the use of projections in language mapping a straightforward process. In practice however, as it is difficult to predict how much audiences are versed in cartographic theory (Upton, 2010, p.143), many publishers are unclear on the relationships between projection systems (Maling, 2013, p.xiii). Therefore, map authors may fail to account for such information or do so inconsistently (Maling, 2013, p.122).

\subsubsection{Colour, shape, and prominence.}

If used appropriately, an arrangement of colour (along with hue and saturation) on a map corresponds to the logical organization of mapped information (Brewer, 1994, p.123). While the use of colour in maps may at first seem trivial, it is essential for categorizing features (Misra \& Ramesh, 1989, p.481), reflecting how data are related to each other (Girnth, 2010, p.110), and indicating such features' prominence (Gill, 1988, p.36). As Tufte (1990, p.81) describes humans as able to distinguish between up to 30 colours that are used to classify abstract information, maps can be coloured in a number of versatile ways. For example, while maps use colour to measure a mapped region's altitude, others use colour to reflect the population density of a language's speakers. Similarly, colour may be used to imitate reality, such as the use of blue lines for a river, or white blotches to reflect glaciers (Tufte, 1990, p.81). However, colour can 
also be used in ways that transcend simple representation. In the context of propaganda maps, Tyner (1982, p.144) considers colour to attract attention or evoke emotional responses and considers any clarificatory use to be secondary. Here, colours that attract the audiences' attention are used for whatever spatial concepts need to be "sold," while areas deemed less significant, important, or desirable are shown with unpleasant colours or weaker shades (p.144).

While prominence can be achieved through symbol shape or size (Gill, 1988, p.47), it is also notably harnessed — whether intentionally or by mistaken implication — through colour (Johnson, 2001, p.13). By making something on a map more prominent, one distinguishes its visual dominance over other information (Hopkin, 1992, p.192), which in language mapping would increase the priority of certain linguistic phenomena over others. Data become prominent when assigned a more 'dominant' colour—such as red—or a stronger saturation (Johnson, 2001, p.13). While this can be useful for prioritizing specific information, the resultant map can be confusing if colour choices have been overlooked (Brewer, 1994, p.123), or misleading when used to satisfy power-related agendas (Johnson, 2001, p.13). Such is the efficacy of the intentional use of colour - along with projection systems - that two maps using the exact same information can convey two very different realities (Ormeling, 2014, p.342).

\subsubsection{Commentary.}

While a commentary can be thought of as a supplementary text that informs audiences of the map's purpose and context (Lameli, 2010, p.572), commentaries are also a response to the limitations of thematic maps in explaining phenomena using symbols alone (Ormeling, 2010, p.37). Girnth (2010, p.106) expands on this, noting that the commentary typically found on the map (as opposed to a page elsewhere in an atlas) provides additional information about the 
subject, the process behind the research, or significant issues in the research process. Furthermore, Lameli (2010, p.572) describes a noteworthy sentiment among mapmakers that maps are not sufficiently self-explanatory, and without question language maps require a supplementary commentary.

It is evident from these two differing accounts that commentary is regarded in the literature as either useful in extending the scope and ease-of-use of language maps, or as a way of compensating for shortcomings in the mapping process. While they can indeed be helpful additions, it is my assessment that a descriptive commentary is an indispensable part of any thematic map. This echoes Tyner (1982, p.143) who states that the cartographer's intent is most easily identifiable in a map's text. However, even still, the biases and motivations for mapping techniques are often not plainly evident, and there are a host of contextual factors, both on and off the map, that need to be accounted for.

\subsubsection{Uncertainty.}

As a basic definition, uncertainty in mapping can be thought of as "any cause for a mismatch between reality and the user's understanding of reality" (Roth, 2009, in Roth, Quinn, \& Hart, 2015, p.7). Noted by Roth (2009, p.315), uncertainty is inherent to all geographic information, and therefore is an issue that can be managed but not eliminated. In identifying what uncertainty consists of in general mapping, MacEachren, Roth, O’Brien, Li, Swigley, and Gahegan (2012, p.2496) identify three main components:

1. Accuracy: Correctness and/or freedom from mistakes; conformity to a standard or model.

2. Precision: The level of detail with which a measurement is expressed or performed.

3. Trustworthiness: Predicted confidence the user has in the information provided. 
In language mapping, the term uncertainty perhaps best refers to any linguistic information that the language mappers, contributors, and/or thematic content experts are unsure about or do not wish to have shown. Examples include one language's genealogical relatedness to another, the extent of a linguistic boundary, or the extent to which a phenomenon is present in a region. Furthermore, Luebbering (2011, p.56) notes that uncertainty is one of the most difficult parts of language mapping (along with linguistic complexity) and describes a number of ways language maps have attempted to fix the issue in the past. Figure 9 shows three examples ${ }^{5}$ of such techniques, as outlined by Luebbering (2011, p.73):

Figure 9: Three strategies used to indicate uncertainty in language mapping.

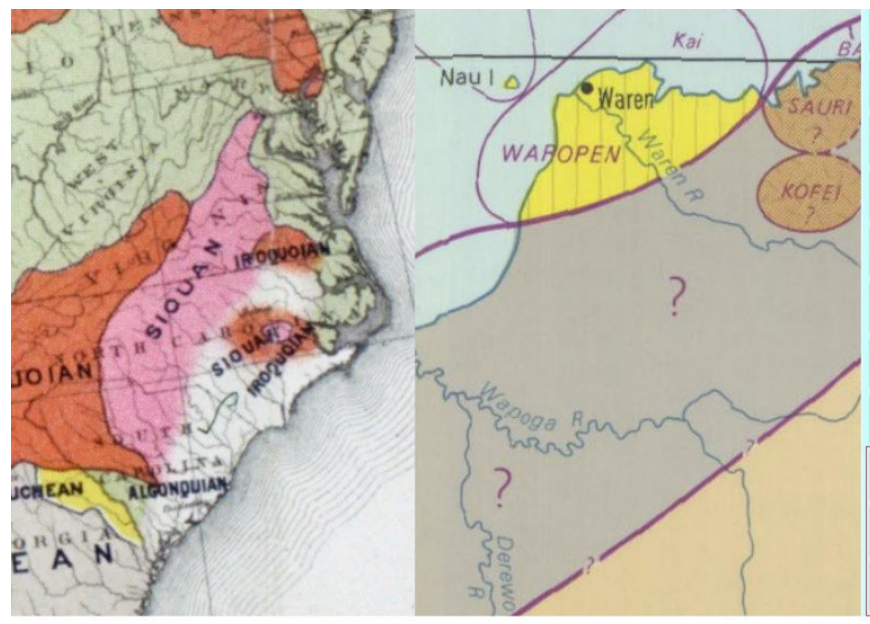

Undefined Boundaries (Powell, 1891)
Question Marks

(Wurm \& Hattori, 1981)

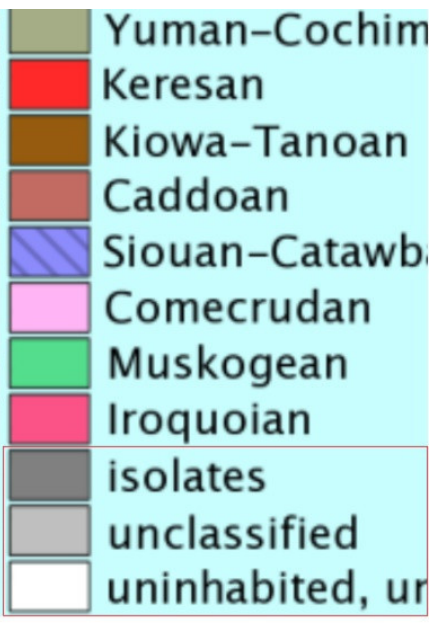

Legend Items

(Ish Ishwar, 2005)

Importantly, Luebbering (2011, p.57) notes that many language mappers choose an additional strategy, which is to not admit that such uncertainty exists in the first place. In this,

${ }^{5}$ Luebbering (2011, p.73) also outlines a fourth strategy, including a zipper-like boundary to denote uncertain linguistic borders. 
Luebbering (2011) mentions that this is typically done out of fear that the admission of uncertainty will make the map appear less authoritative, and thus would reduce the degree of trustworthiness users perceive the map to have. Agumya and Hunter (2002, p.405) note that this perception arises from a long-standing cartographic aversion to admission of uncertainty, which I argue has not diminished in emerging thematic mapping practices. However, Luebbering (2011) argues that, as uncertainty is an inevitable part of any map, the admission of data limitations paradoxically makes the map appear more informed and realistic and thus more trustworthy. Finally, Luebbering (2011, p.61) expresses a need for further research, reflection, and refinement in the area of mapping uncertainty due to digital mapping practices becoming more commonplace within mapping. This is not to say that there are no existing attempts at understanding mapping practices, and the following subsections describe two areas that are perhaps most relevant.

2.6 Studies in mapmaking: Geography and cartography.

Though a description of what maps are in the first place allows us to understand the tools that attempt to depict reality, it is arguably equally important that the fields of study devoted to mapmaking also receive attention. The following fields are a core subset of what could be applied to the study of physical space: both cartography and geography are most central to a study of language of space. However, by no means do they comprise an exhaustive account. 


\subsubsection{Geography.}

Pask (2006, p.6) puts it simply: geography is an area of research that focuses on how human and/or physical features are linked and interact with one another. Indeed, a principal assumption within geography — known as Tobler's First Law of Geography—states that "everything is related to everything else, but near things are more related than distant things" (Tobler, 1970, p.236). ${ }^{6}$ As humans have come to influence every part of the globe, down to the planet's very biological fabric and machinery (Steffen, Crutzen, \& McNeill, 2007, p.614), geography is now as much a study of the spaces humans occupy as it is a study of the spaces found on earth.

In this sense then, geography sets out to study the relationships between and within human and non-human phenomena and does so by looking at how such phenomena exist in physical and imagined spaces (Crampton, 2011). In addition, Borchert (1987) defines geography to study the location of "places, regions, and routes within aggregated geographical patterns of resources, places, spaces, and routes" (p.387). Here, maps are important for communicating large amounts of spatial information of interest, but they are tools and resources (along with tables and diagrams) rather than objects of study (Pask, 2006, p.10).

\subsubsection{Cartography.}

Cartography is the art, science, and technology of mapmaking (Kent \& Vujakovic, 2017, p.1; Pápay, 2017, p.20) that overlaps strongly with geography and many other disciplines (Thrower, 2008, p.2) but focuses on studying maps instead of just their use as tools (Thrower,

\footnotetext{
${ }^{6}$ It should be noted that Li, Sen, and Hecht (2014) find a limit to the extent to which this law holds true in practice and demonstrate that some neighbouring spatial phenomena can, in fact, be unrelated. In addition, Goodchild (2020, p.3679) notes that this law has its shortcomings, especially given increases in globalization, travel, and migration in the $21^{\text {st }}$ century.
} 
2008, p.xii). As another way of looking at it, mapmaking is the act of cartographic visualization (Meng, 2003, p.1888). Furthermore, Harvey (2015, p.6) explains that while geography focuses on human and environmental phenomena on the Earth's surface, cartography focuses on the theories, skills, and processes used to describe the phenomena.

Cartography can be less than straightforward. Thrower (2008, p.1) notes that knowing how maps function is not necessarily automatic or easy, despite maps being important to many areas of modern life. Indeed, cartography only arose in the late nineteenth century as a way of developing theoretical principles that could inform best practices in mapmaking, while accounting for techniques and map use in different parts of society (Kent \& Vujakovic, 2017, p.1). Since then, many cartographic frameworks have been developed that focus on the processes occurring within map authors and audiences (e.g. who they are and why they make particular choices), as well as between authors and audiences (i.e. the entire process of transferring knowledge) (Board, 2017, p.29).

Such an area of study is important, given that Tyner (1982, p.140) claims that any persuasive map necessarily manipulates cartographic elements through distortion, selection, and symbolization. Any choices of what map elements should be manipulated impact author and audience alike. Harley (1988) infers that as opposed to being "passive reflections of the world of objects," maps are "refracted images [that contribute] to dialogue in a socially constructed world" (p.277). In support of this idea, Peterson $(1999$, p.5) cautions that it would be unethical for a map to be created that purposefully does not communicate a specific message. However, mapmakers do not simply communicate a reality, but instead actively try to analyze and discover (Monmonier, 1993, p.12) realities that, as opposed to existing at one point in time, constantly change as parts of a rich orchestra of interacting forces. Finally, Woodward and Lewis (1998, 
p.1) claim that any definition that does not regard maps as social constructs fails to grasp that maps are more than just wayfinding devices.

It is important to understand that cartography has many goals as well as many different perspectives surrounding how maps are made, who makes them, what they are used for, and the consequences of their uses. However, cartographic study is theoretically abstract in many ways, and just as cartographic research often does not directly involve making maps (e.g. see Thrower, 2008, p.xii), many of those creating the maps are not cartographers themselves (Darkes, 2017, p.287). It is important to take this into account when looking at maps that currently exist, especially when they are the result of projects involving those knowledgeable in their own thematic area, but not necessarily in mapmaking.

2.7 Techniques in mapmaking: Paper maps to the digital sphere.

As a brief and non-exhaustive overview of mapmaking techniques that are currently (and historically) in use, we take a look here at the kinds of mapping most relevant to this dissertation. Throughout the past millennium, the most commonly used and most feasible modality with which to present a map has been print. This century, there has been a growing production of maps in the digital sphere, such as with computer programs like GIS and web mapping.

\subsubsection{Paper maps.}

While paper maps have been used for millennia, they still play an important role in mapping as they are generally easy to use, transport, and store (Hurst \& Clough, 2013, p.48). Similarly, 
though paper maps have been painstakingly hand drawn for much of mapping history (for example, see Figure 10, many maps are now drawn and printed with computer assistance.

Figure 10: A hand-drawn paper map of the battle of Valcour Island.

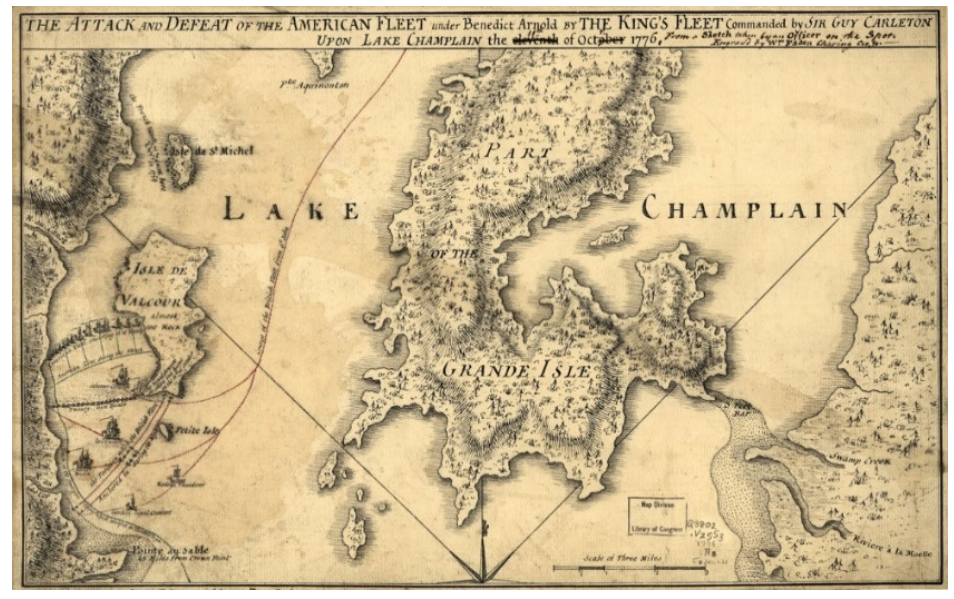

Reproduced from Faden (1776).

Paper maps are static in nature: their components do not move and cannot be altered or changed at will by the map user. These maps are found in books, on classroom walls, or in tourist pamphlets, to list a few. While paper maps are accessible to many, there are important considerations regarding what being static means for their ability to communicate information. Because they are pictorial representations of specific phenomena, paper maps are only able to represent a snapshot of what a language or linguistic structure distribution fixed in time looks like (Hanewinkel \& Losang, 2010, p.416). As many kinds of thematic content neither exist on their own, nor are static and unchanging, an unfathomable wealth of knowledge about a thematic phenomenon's social and geographical situations is lost to audiences who are unable to reflect on the temporal complexity important to developing a better situational understanding. To address these issues, internal components such as symbols need to be adapted to show the most amount 
of information with the least amount of effort, or use repeated but slightly different versions of the map (Hanewinkel \& Losang, 2010, p.416) in a body of text or a collection such as an atlas.

\subsubsection{Geographic Information Systems (GIS).}

Geographic Information Systems (GIS) are currently some of the most well-known digital mapping techniques and can be found in almost all fields, work areas, and disciplines (Goodchild, 2020, p.3674). They involve computer-assisted systems that capture, store, retrieve, analyze, and display spatial data (Clarke, 1986, p.175) for a variety of purposes, including mapping particular locations, finding distances and routes, monitoring and predicting changes, and mapping quantities and densities (Cotfas \& Diosteanu, 2010, p.45). Furthermore, GIS incorporates scientific approaches to understanding spatial phenomena (Mark, 2003, p.2) using "software packages providing a range of functions for creating, [...] modeling, and archiving information about the surface and near-surface of the earth" (Goodchild, 2009, p.251).

Jack Dangermond, founder of the Environmental Systems Research Institute (ESRI) that has helped define the course of proprietary GIS software, describes GIS as the very language of geography (Dangermond, 2004). Though GIS was originally developed in the $1960 \mathrm{~s}^{7}$, it did not become widely available until the 1980s (Kessler \& Slocum, 2020, p.297), likely in tandem with an increasing availability of personal computers. Though GIS information was originally designed to be installed and run on individual workstations, advancements in Internet and serverbased technologies mean that maps no longer need to exist in a physical location, and can now be

\footnotetext{
${ }^{7}$ There is disagreement whether GIS was first invented by the United States Geological Survey (USGS), or the Canadian Geological Survey and Natural Resources Canada, which is located less than 2 kilometres away from Carleton University. As an Ottawa local, I am inclined to vie for the latter possibility.
} 
accessed via the Internet (Rabanus \& Kehrein, 2010, p.377), as with other computer generated maps. Because they are presented through the digital medium, researchers and software developers alike have a greater opportunity to present information in different ways, depending on what sorts of presentations fit the data. What follows are just a few examples of common GIS (and related) software that are available for use among the public:

QGIS http://www.qgis.org

[from the website] "QGIS is a user-friendly Open Source Geographic Information System (GIS) licensed under the GNU General Public License. QGIS is an official project of the Open Source Geospatial Foundation (OSGeo). It runs on Linux, Unix, Mac OSX, Windows and Android and supports numerous vector, raster, and database formats and functionalities."

\footnotetext{
ArcGIS http://www.arcgis.com

Made with ESRI technologies, ArcGIS is a collection of 2-D and 3-D analytical tools that allow users to identify patterns and trends in geospatial occurrences and phenomena. While it is considered by many in the field to be an authoritative source of GIS capability, its use requires a paid subscription that can be expensive for individuals.
}

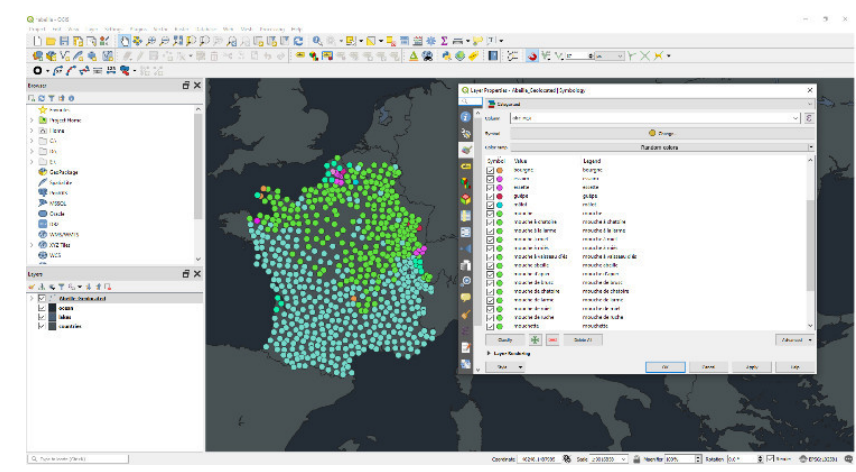

Own screenshot of the QGIS interface

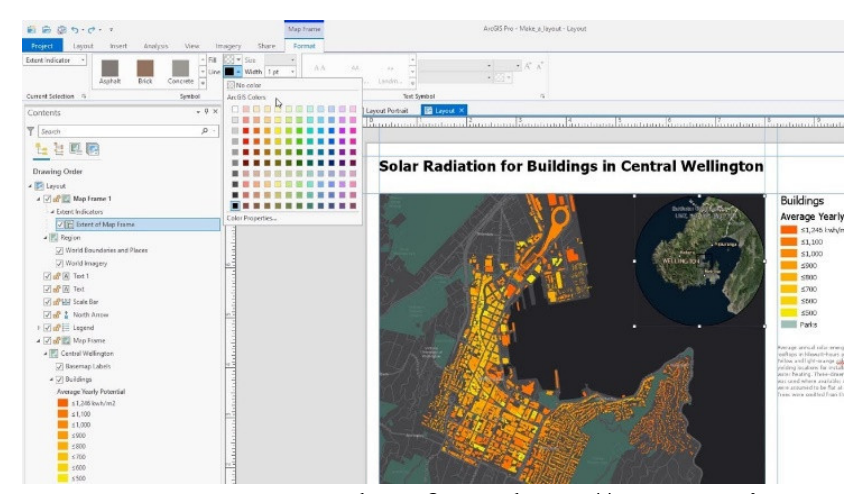

Screenshot from http://pro.arcgis.com 
SagaGIS http://www.saga-gis.org [from the website] Used to edit spatial data, it is free and open-source software, developed originally by a small team at the University of Göttingen and is now being maintained and extended by an international developer community.

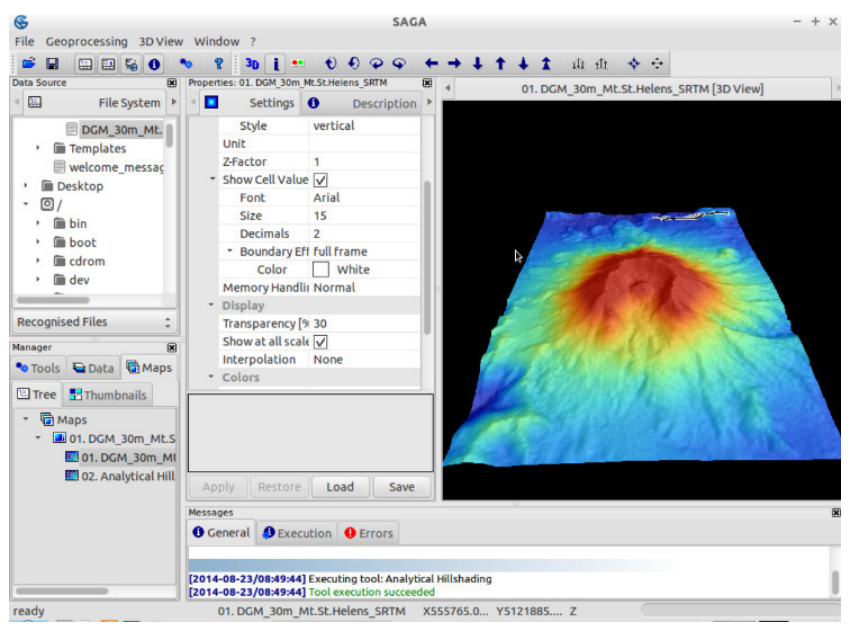

Screenshot from http://live.osgeo.org

\section{GRASS GIS https://grass.osgeo.org/}

[from the website] GRASS GIS, commonly referred to as GRASS (Geographic Resources Analysis Support System), is a free and open source Geographic Information System (GIS) software suite used for geospatial data management and analysis, image processing, graphics and maps production, spatial modeling, and visualization. GRASS GIS is currently used in academic and commercial settings around the world, as well as by many governmental agencies and environmental consulting companies. It is a founding member of the Open Source Geospatial Foundation (OSGeo).

gvSIG http://www.gvsig.com/en

[from the website] gvSIG is known for having a user-friendly interface, being able to access the most common formats, both vector and raster ones. It features a wide range of tools for working with geographic-like information (query tools, layout creation, geoprocessing, networks, etc.).

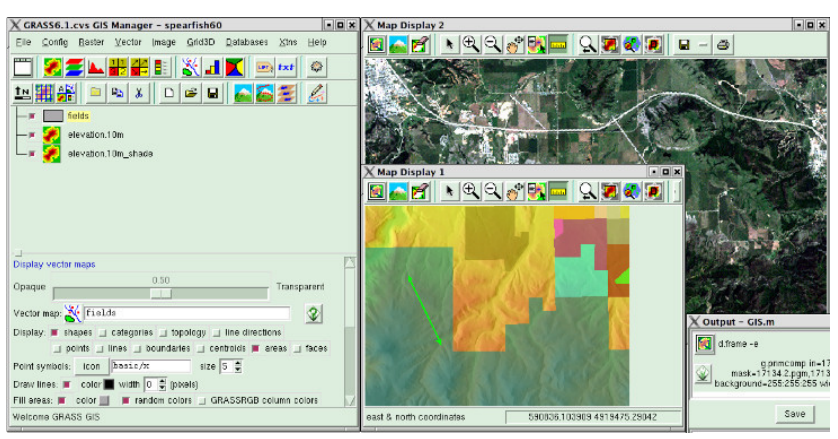

Screenshot from http://grass.osgeo.org

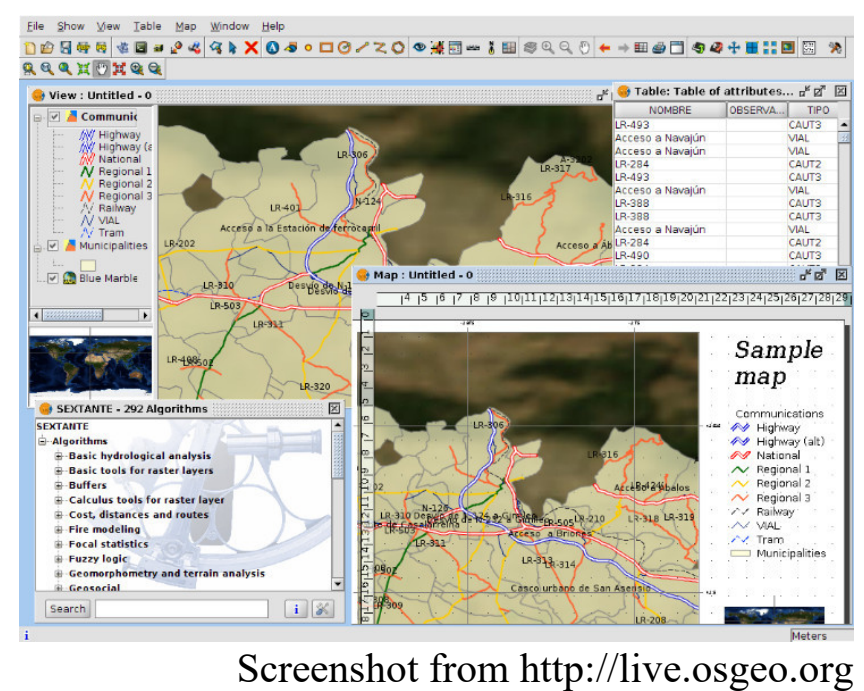


OpenJUMP http://www.openjump.org/

[from the website] OpenJUMP is an open source Geographic Information System (GIS) written in the Java programming language. It is developed and maintained by a group of volunteers from around the globe. OpenJUMP started as JUMP GIS designed by Vivid Solutions.

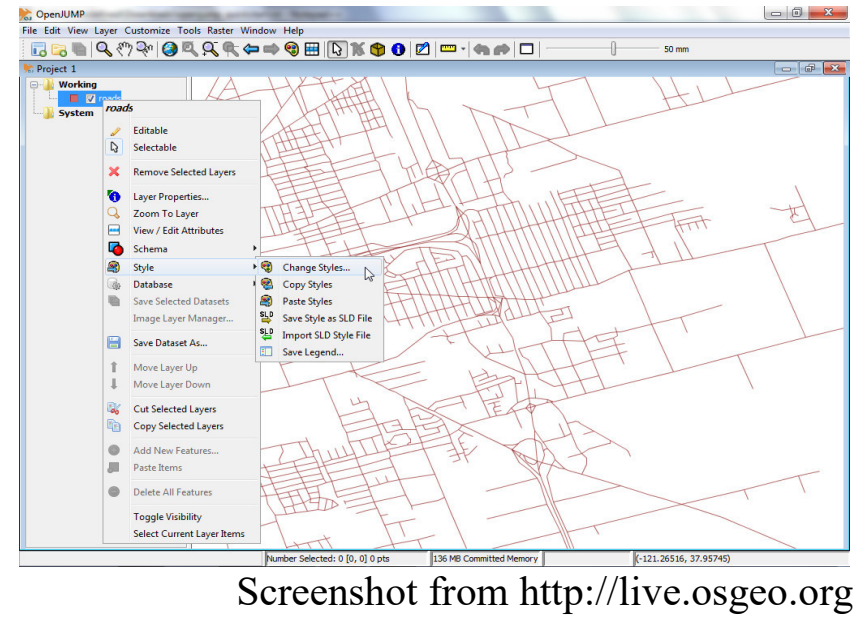

Unlike paper maps that cannot easily display the data behind the illustrations, maps displayed digitally can enable users to select what they wish to see and what data they want shown alongside it (Kunst \& Barbiers, 2010, p.401). Such maps can also avoid overloading users with information, which strengthens the map's communicative potential. Different themes, levels of interactivity, and features mean that the experience, instead of being limited by the medium in which the map is being presented, is enhanced if done properly. As demonstrated in Figure 11, GIS can be applied to data from already existing maps to bring out trends which may otherwise be unnoticed. 
Figure 11: GIS rendering of the "abeille" term in the Atlas Linguistique de la France.

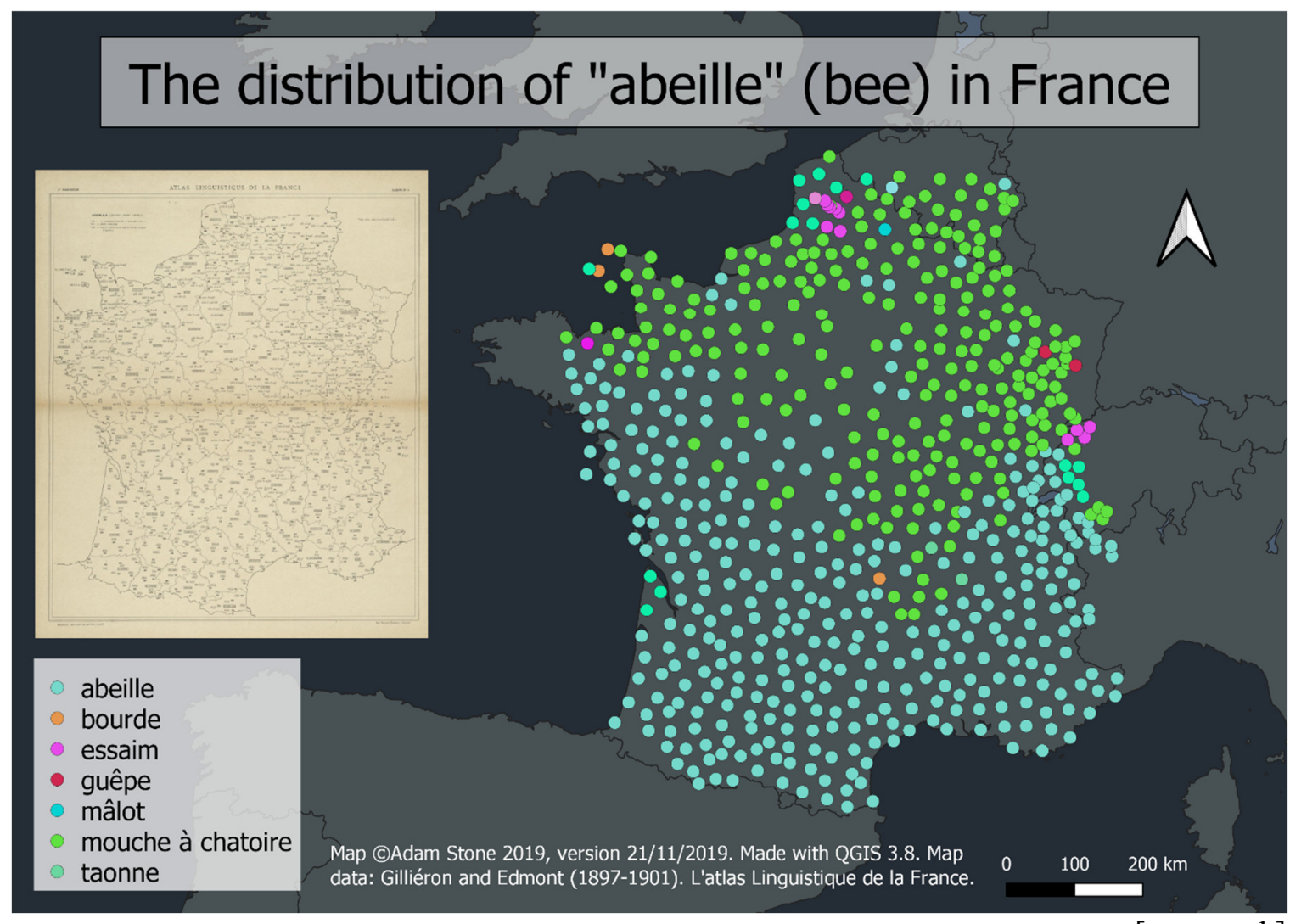

[own work]

One of the primary benefits of displaying maps through digital interfaces are the improvements to access the availability of maps on the web can grant. Instead of requiring subscriptions to map producers or access to a library, maps hosted on a web platform can be accessed from anywhere with an internet connection, affording opportunities to those not otherwise able to access maps. Aside from ensuring that researchers' map viewership is not constrained by geographical proximity or subscription, this improved access means that maps can be better integrated into the classroom, and that a greater variety of maps is available to the public.

Another important factor regarding GIS, in the context of this dissertation, is the degree to which it lends itself to mixed-methods research. While GIS offers numerous quantitative and 
statistical approaches (Briscoe, 2009, p.19), Pacheco and Velez (2009, p.275) observe that there is also a creative capacity to work with qualitative data and subjective experience. In practice, such a mixed-methods GIS approach was demonstrated by Brown, Strickland-Munro, Kobryn, and Moore (2017), where participants identified Indigenous marine and coastal elements in Northern Australia through qualitative semi-structured interviews and the quantitative identification of areas of interest by creating polygons on a map. In this, Brown et al. (2017, p.165) demonstrated that GIS maps are useful in ensuring the validity of both quantitative and qualitative methods for identifying the range and place-based values within a geographical region of interest. However, GIS comprises just a handful of mapping approaches, and with everincreasing improvements in digital connectivity come new opportunities for map-related work and research.

\subsubsection{Web mapping.}

A major realization of real-time spatial data portrayal comes in the form of web mapping, which is defined by Neumann (2011) as "the process of designing, implementing, generating, and delivering maps on the World Wide Web" (p.567). Despite considerable overlap, web mapping currently differs from GIS — which emphasizes spatial analysis — by the kinds of data presented. Assuming maps can be generated from databases, they can display large amounts of information in near-real-time (Neumann, 2011, p.570). However, Neumann (2011, p.571) considers several major limitations in web-based mapping, including cost, unrealistic bandwidth requirements, and software platforms that are difficult for inexperienced users. It should be noted that at the time of this dissertation's publication, Neumann's (2011) analysis is nine years old. 
What follows are some examples of web mapping platforms that have been used in linguistic research:

OpenStreetMap

openstreetmap.org/

[from the website] OpenStreetMap powers map data on thousands of web sites, mobile apps, and hardware devices. OpenStreetMap is built by a community of mappers that contribute and maintain data about roads, trails, cafés, railway stations, and much more, all over the world. OpenStreetMap emphasizes local knowledge. Contributors use aerial imagery, GPS devices, and low-tech field maps to verify that OSM is accurate and up to date.

\section{Google Maps}

maps.google.com/

[from the website] Google Maps is a web mapping service developed by Google. It offers satellite imagery, aerial photography, street maps, $360^{\circ}$ panoramic views of streets, real-time traffic conditions, and route planning for traveling by foot, car, bicycle and air, or public transportation. Google maps differs from the related downloadable app, Google Earth, as it is based primarily on the internet.

\section{Mapbox}

https://www.mapbox.com/

[from the website] Mapbox is a developer platform used across industries to create custom applications that solve problems with maps, data, and spatial analysis. Mapbox's tools are building blocks that support every part of the web and mobile map-making process.

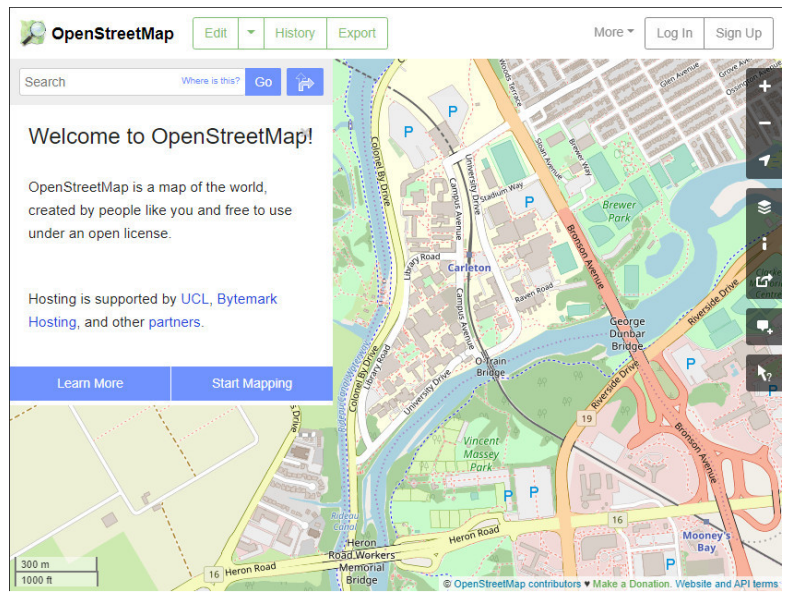

Screenshot from: http://openstreetmap.com

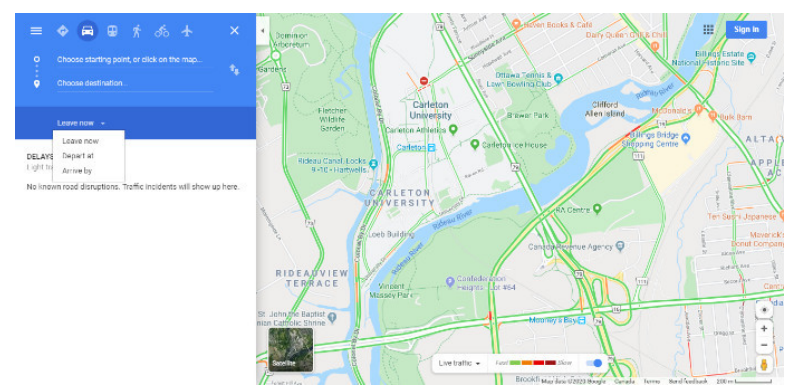

Screenshot from: http://google.com/maps

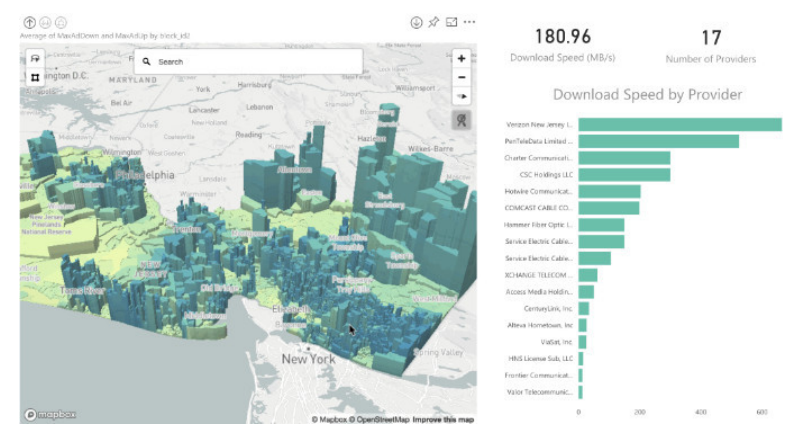

Screenshot from: http://blog.mapbox.com 
WorldMap

http://worldmap.harvard.edu/

[from the website] Harvard WorldMap is an Open Source mapping platform developed to lower barriers for scholars who wish to explore, visualize, edit, and publish geospatial information.

(since 2011)

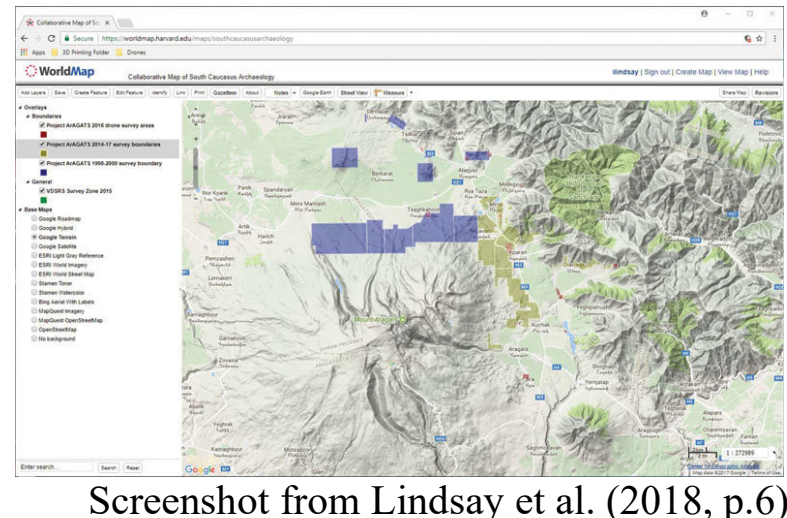

\section{D3JS}

https://d3js.org/

(since 2011)

[from the website] d3.js is a JavaScript library for manipulating documents based on data. D3 helps you bring data to life using HTML, SVG, and CSS. D3's emphasis on web standards gives you the full capabilities of modern browsers without tying yourself to a proprietary framework, combining powerful visualization components and a data-driven approach to DOM manipulation.

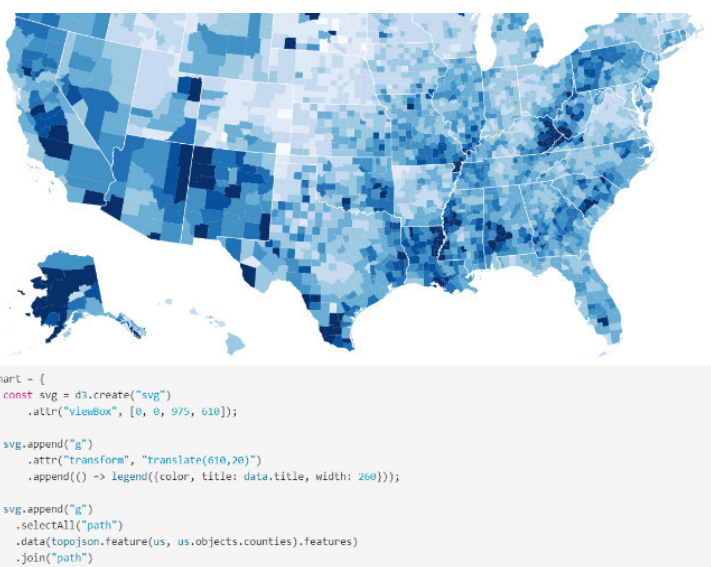

Screenshot from https://observablehq.com/ 
3.0 Understanding language and linguistics.

Having focused on defining maps, their fields, and techniques of study, this section discusses the other half of the topic of this dissertation (and just as essential a component): language. To do so, this section first identifies what language is, and then discusses fields that investigate the structures and usage of language in a variety of human spheres.

\subsection{Language.}

We tend to know language when we see it. As expressed artfully by Fromkin, Rodman, and Hyams, (2018), "We live in a world of language. [...] Hardly a moment of our waking lives is free from words, and even in our dreams we talk and are talked to" (p.3). Language is found in all cultures; indeed, it is one of the very things which defines our humanity. However, attempting to come up with a single, tangible definition of language can be difficult, as there are many different ways one could approach the issue. ${ }^{8}$ What follows is a basic overview of how language has been described in the literature, which will likely not yield many surprises to readers with a background in linguistics.

At its most basic, a language can be thought of as a system of meaning (Halliday, 2003, p.2). In a modern English translation of Saussure's 1916 Course in General Linguistics (Saussure, 2011), language is a formal system of signs organized by grammatical rules for the purpose of communicating meaning. Saussure (2011, p.66) argues here that these signs are things that link a concept in our minds to something produced by us and received by others (called the sound image), such as a combination of sounds or hand signs to make a word, as in Figure 12.

\footnotetext{
${ }^{8}$ For the purposes of this dissertation, any reference made to language will refer to human language and not animal or computer languages, unless specified otherwise.
} 
Language is seen in this Structuralist perspective to be a complex and closed system (Trask, 2007, p.93), meaning that the relationships between the concepts and their surface realizations are independent of any external influences such as events, cultural change, or geography. While this assumption has guided much of twentieth century linguistic research, it should be noted that this dissertation does not treat language as a system free of influence from external forces; in contrast, the interactions between language and space are of central importance here. ${ }^{9}$

Figure 12: Saussure's linguistic sign has an abstract concept and tangible sound image.

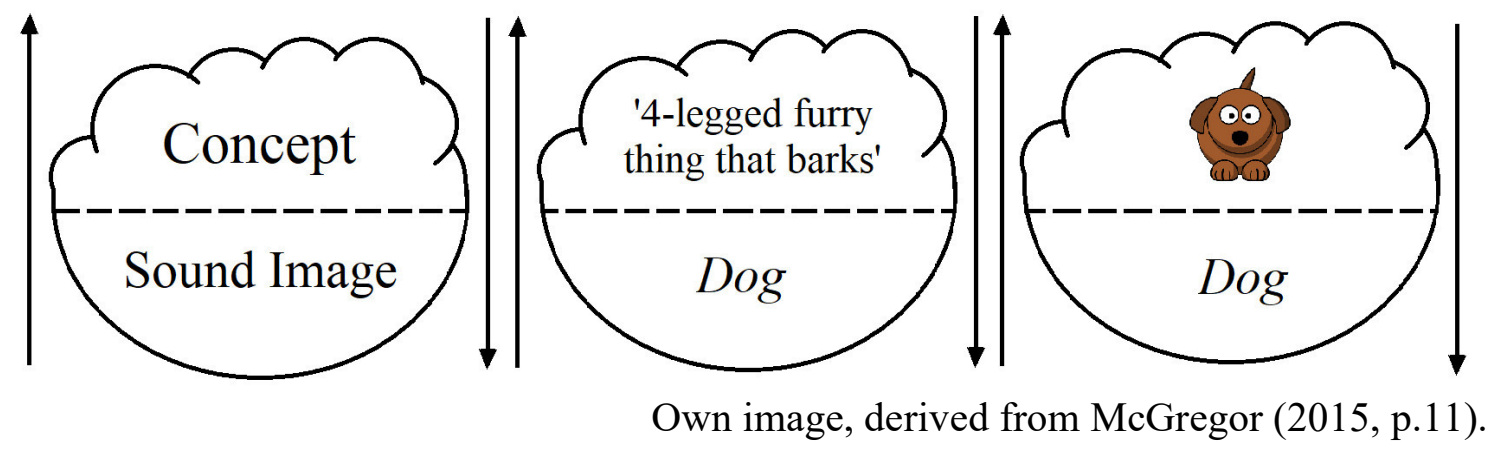

Fromkin, Rodman, and Hyams (2018, p.4) understand language as a system of knowledge shared by a community of speakers and/or signers that allows them to express ideas and be understood. In this body of knowledge, speakers are aware of what sound or sign is a part of their language, and which is not. Similarly, speakers know that combinations of such sounds in specific orders convey a specific meaning which differs from other sound arrangements (Fromkin, Rodman, \& Hyams, 2018, p.5), and have a knowledge of rules that determine how such words can be combined to create more complex words and sentences (p.11).

\footnotetext{
${ }^{9}$ The idea that language is a closed system is a hotly debated topic. For arguments against this notion, see Hall (1997) for example.
} 
Pinker and Jackendoff (2009, p.126) see language as a part of a human biological system related to other human systems through interactions with the environment and the specific composition of the brain. Hauser, Chomsky, and Fitch (2002, p.1576) postulate an “independently-evolved mechanism" which had originally developed for other reasons that allows children acquiring language to learn and remember concepts' meanings, the forms, and the links between the two. Language thus is a way of understanding and communicating the world and its realities, made possible by an ability we have evolved to perceive differences, often tiny, between speech sounds, organize words into specific arrangements, and remember and interpret links between these forms and meaning (Pinker \& Jackendoff, 2009, p.143).

Finally, Fromkin, Rodman, and Hyams (2018, p.27) summarize facts they believe to apply to all human languages:

1. Language exists wherever humans do.

2. All languages are equally complex ${ }^{10}$ and capable of expressing any idea.

3. All languages change over time.

4. The relationships between form and meaning are almost always arbitrary.

5. While humans make a limited number of sounds, these sounds' combinations into words and sentences are infinite.

6. Every language has discrete sound or gestural segments, such as vowels and consonants.

7. Grammatical categories such as nouns and verbs are found in every language.

8. Every language can issue commands and negate something.

\footnotetext{
${ }^{10}$ This claim is often disputed. Karlsson, Miestamo, and Sinnemäki (2008, p.vii) list many different ways that languages can be complex, and note how large second-language learner communities reduce a language's relative complexity. Similarly, Dixon (1997, p.23) gives examples for how a language's relative complexity can influence its decline in speakers.
} 
9. Any neurotypical child can learn any language they are exposed to sufficiently.

In addition to a definition of what language is, a number of fields approach language from different angles in an attempt to understand how languages exist and differ from one another.

3.2 Theoretical linguistics.

Figure 13: Fields of theoretical linguistics as they pertain to aspects of human language.

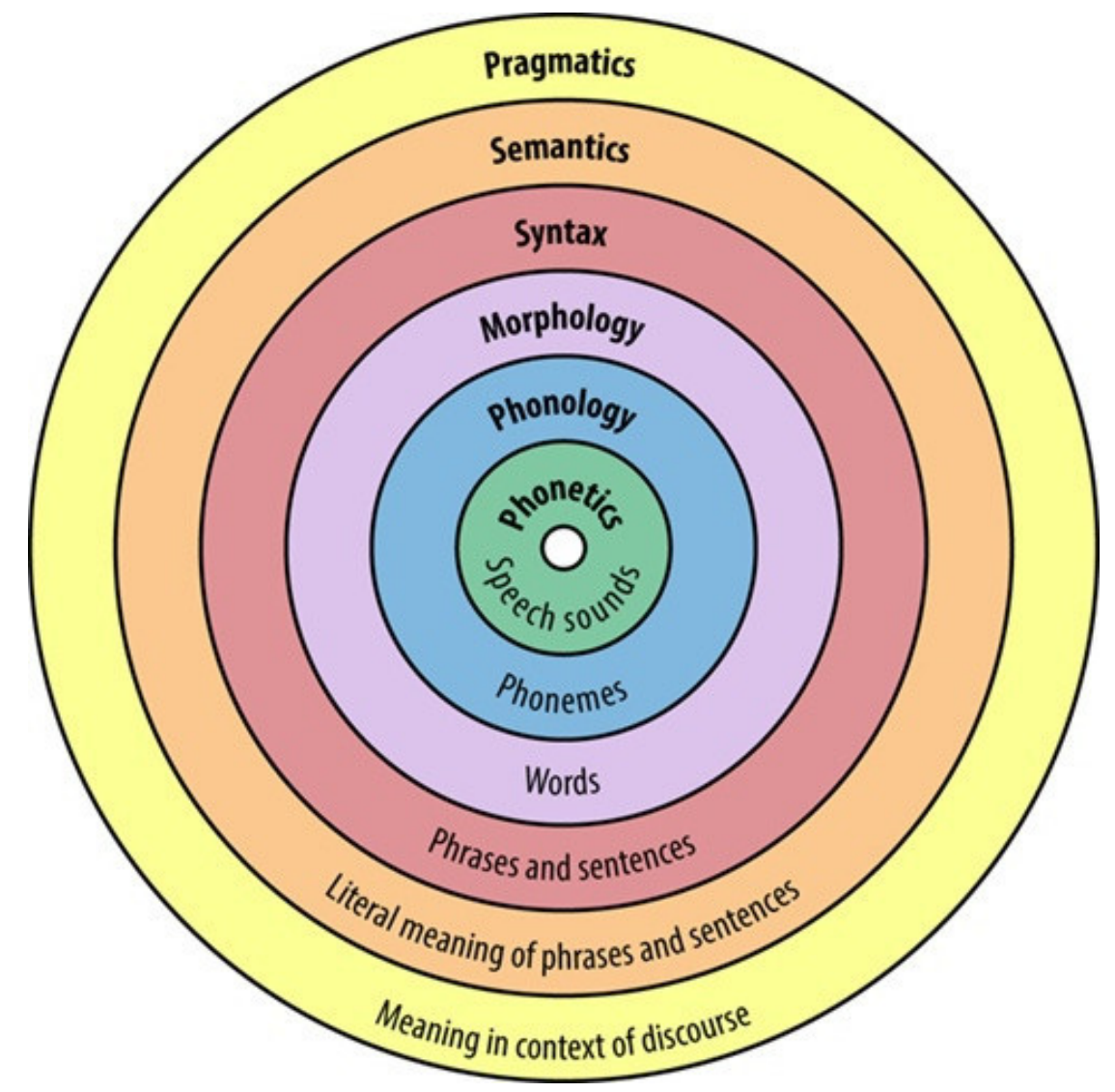

Bloom (2007)

Linguistics is the scientific study of language (Halliday, 2003, p.vii; Lyons \& John, 1968, p.1), which aims to understand the components of language that build outward and tangible forms/signs, as well as the components of language involved in the meaning that comes from 
mental representations in the mind (Martinet, 1960, p.15). Importantly, much of linguistics strives to be as objective as possible and avoid the biases and prejudices which come with familiarity with any one language (Lyons \& John, 1968, p.2). Similarly, much of linguistics is descriptive rather than prescriptive, meaning that linguists attempt to study language as it is actually occurring, and do not develop rules that dictate how elements within language should exist (McGregor, 2015, p.4). As indicated in Figure 13 on the previous page, there are a number of fields within theoretical linguistics that approach different aspects of language. The following non-exhaustive account of main fields are listed as they are defined by McGregor (2015, p.6-7):

- Phonetics: The physics of sound waves and how speech sounds are produced and perceived.

- Phonology: How sounds pattern differently and interact in various languages.

- Morphology: The structure of words, and the meaningful units they are composed of.

- Syntax: The ways words relate to each other and combine to form sentences and phrases.

- Semantics: Meaning, and how meaning is encoded in words and grammar.

- Pragmatics: Meaning, and how meaning is encoded in utterances within different contexts.

- Historical Linguistics: How languages change over time; reconstructing protolanguages.

Maps are of use in many of these fields of linguistics to display the areal distributions of particular features. For example, phonological maps (such as in Figure 14) display the distribution of speech sounds over a given area, while syntactic maps show how the arrangement of word forms differ (Girnth, 2010, p.105). Found less commonly, pragmatic maps showcase speech act patterns, such as the directionality of a word such as 'into' as being toward or away from the speaker (Girnth, 2010, p.104). Finally, historical linguistic maps may show the distribution of any one of these linguistic features, either synchronically (at one point in time) or diachronically (across a period of time, where language is a temporal phenomenon) (Haynie, 
2014, p.344). Taking this into account, a focus on the structural aspects of language is only one perspective of what language is and how it exists within its speakers, leading us to need to look at other dimensions of language as well.

Figure 14: Phonological map of the Algonquian word-medial variants $/ \int, \mathrm{s}, \mathrm{h}, \mathrm{x} /$

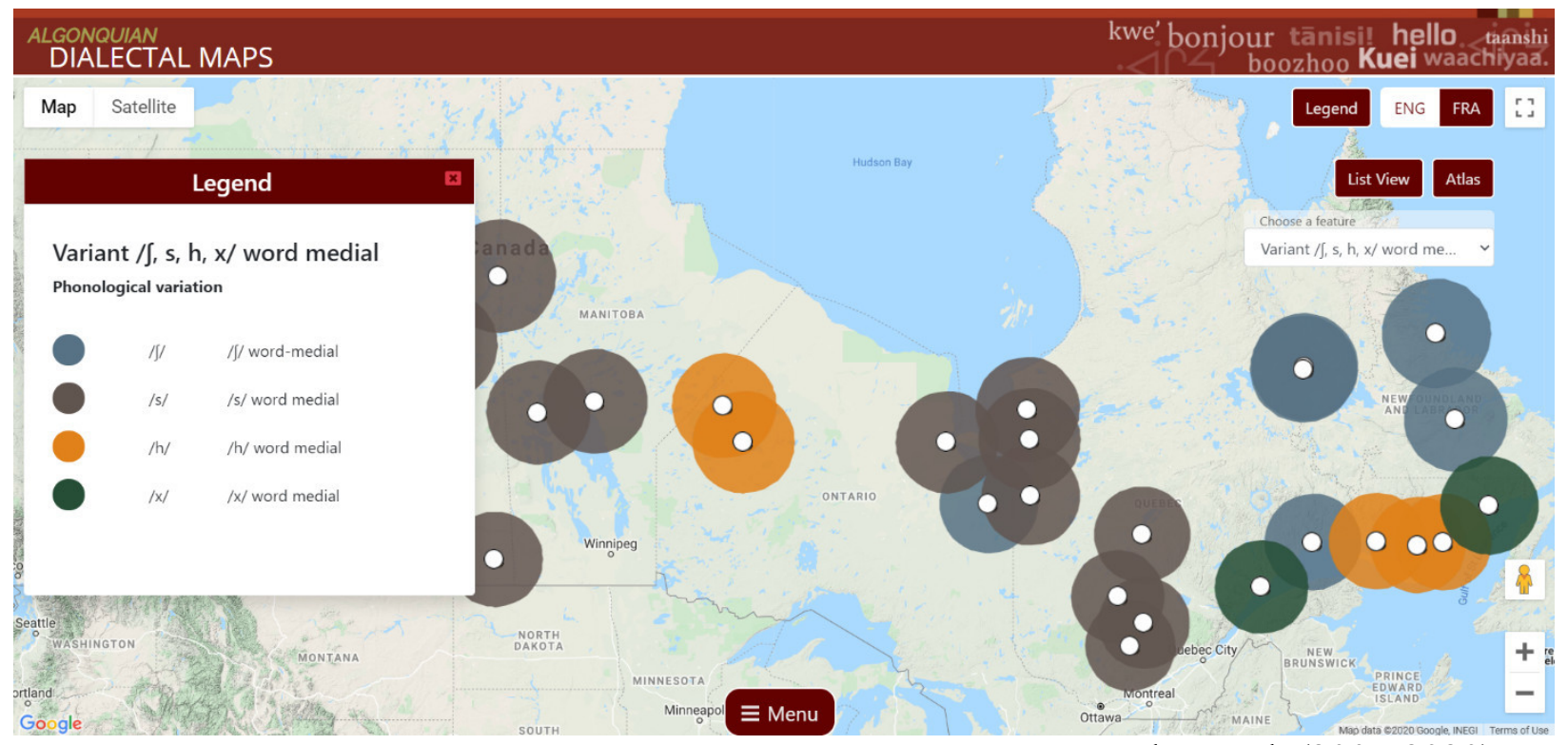

Junker et al. (2005-2020)

\subsection{Applied linguistics and discourse studies.}

This subsection focuses on two closely related fields that take the study of language out of the proverbial laboratory and apply it to real-life issues. While applied linguistics focuses on how a knowledge of language is used to achieve social ends, discourse studies look at how language is used in the real world and what the consequences of such usage are. These two fields have been placed in this subsection together for a few reasons. First, each field informs the other: while discourse studies might focus on how language is used within institutions, for example, applied linguistics might focus on how problems with language use could be solved within said 
institutions. Second, these interrelations are a key component of the ALDS doctoral program at Carleton University. Finally, Davies and Elder (2004) and Trappes-Lomax (2004, p.133) view discourse studies as being a part of applied linguistics, while still existing outside of the field due to its multidisciplinary nature and diversity in its range of interests.

\subsubsection{Applied linguistics.}

As opposed to theoretical linguistics, which can be readily described in terms of what it focuses on, applied linguistics is noted by researchers such as Davies (2007, p.1) to mean many different things to different people. Cook (2003, p.20) argues that while the scope of applied linguistics continues to be vague, a central task of the field is arguably to mediate between the study of language and its application in the real world. Here, as according to the Carleton University homepage for the ALDS program, ${ }^{11}$ language is considered in applied linguistics to be a system of communication and social action, as opposed to theoretical linguistics, which focuses on the structure of language. Davies and Elder (2004, p.1) add that applied linguistics is concerned with solving or at least helping to address social problems that involve language in some way. Here, Davies and Elder (2004, p.2) note that much of applied linguistics research focuses on areas such as language learning and education, translation and interpreting studies, or language policy and planning.

11 This information was accessed on 26/11/2019 from: https://carleton.ca/slals/applied-linguistics-discourse-studies/ 


\subsubsection{Discourse studies.}

As in this dissertation, introductions to studies of discourse often begin with a description of discourse as language as it occurs beyond the sentence level (that is, beyond syntax and semantics), and how it occurs within greater contexts and practices with society (Tannen, 2019). In this way, the study and analysis of discourse is the study of language in use (Gee, 2010, p.8). Trappes-Lomax (2004) poetically describes discourse studies as "the study of language viewed communicatively and/or of communication viewed linguistically” (p.134). Additionally, Trappes-Lomax (2004, p.133) notes that as members of a linguistic community, people notice and study the patterns and agents of language use instinctively and unconsciously. It is the job of the discourse analyst to study such language use and participation consciously and systematically.

Van Dijk (2011, pp.3-4) introduces discourse studies by first defining discourse as language as it occurs in many different areas of the human experience:

- Social interaction: Situational language use forms the basis of human societies' social order.

- Power and domination: Language acts as a tool of both oppression and empowerment.

- Communication: Expression and communication of beliefs and knowledge among speakers.

- Contextually situated: Language is a part of social situations and people's lived experiences.

Gee (2010, p.9) identifies that discourse can be analyzed from different angles and with different intentions. Researchers in descriptive discourse studies, for example, focus on instances of language use across a variety of situations to understand how language is used and why. While such analyses may be applicable practically, this practical application is not the primary motivation for research. Those in critical discourse studies, on the other hand, focus on how language is used within social and political issues, controversies, and problems, and find 
strategies to remedy and intervene in such issues. As an example, Van Dijk (2011, p.5) describes a situation where scholars of discourse focus on how the use of argumentative fallacies affects potential voters' opinions during federal elections. Using Gee's $(2010$, p.9) two distinct types of discourse studies, descriptive discourse analysts would be interested in which argumentative fallacies voters are most susceptible to and why, while considering other information related to broader contexts and society. Critical discourse analysts would be more interested in how such argumentative fallacies impact different communities of voters, and might focus on developing strategies to either reduce politicians' use of fallacies or insulate vulnerable communities from the strategies' negative impacts.

Together, endeavours within applied linguistics and discourse studies do not generate information that can be directly mapped per se. Instead, issues within applied linguistics and discourse, such as the distribution of minority language communities that need access to education in a city or region, contribute to the bases for which the mapping of language-related information is pertinent. In other words, while applied linguistics and discourse studies provide the contexts for which language maps are of use, theoretical linguistics make the display of linguistic features possible. While such methods are integral to any contextual analysis of language in use, I argue that the physical world is a crucial yet often overlooked element of the human linguistic experience. What follows is a discussion of approaches that integrate language and space. 
4.0 Understanding interactions between language and space.

Given the above discussions of some of the basic components and issues within language and space, there are some parallels between the two that deserve exploration. This section looks at some of the things human languages and physical space have in common, how they intersect one another, how we as linguistic and spatial human beings use these intersections in our lives, and how those interested in such intersections go about studying them.

\subsection{Language and space.}

Language is spatial in nature, and physical space, at least for humans, is linguistic in nature. This is first and foremost because language is spoken by people, and people exist in and influence countless physical and social spaces during their lifetime. Landau, Dessalegn, and Cohen-Goldberg (2011, p.3) claim that knowledge of language and space are two of our most fundamental ways of understanding the world. Indeed, Kracht (2002, p.157) mentions that we prefer the use of spatial concepts even when dealing with abstract notions, as space is what we understand best. As a result of this, language in general contains countless spatial metaphors, which are used constantly without speakers' conscious awareness (Kracht, 2002, p.157).

Similarly, as humans have begun to dominate many (if not all) aspects of physical and environmental systems across the planet in an age referred to as the 'Anthropocene' by Crutzen (2002), any study of the physical landscape has become by definition a study of the human world (Goudie, 2017, p.23). Because language is an important factor in coordinating human behaviour and initiatives (Miller, 1951, p.v), it is reasonable to suggest that language has played a significant role in shaping the modern physical world. 
This is, however, where a general discussion of language and space ends. From here, authors such as Levinson (1996, p.355) focus on how languages' features and structure communicate physical space, authors such as Bryant (1997) discuss how linguistic knowledge influences speakers' perceptions of physical space, and authors such as Johnstone (2011) focus on the relationships between language use and the social mediation of physical spaces. In support of these three perspectives, Britain (2010) defines spatiality in of itself as an ongoing process of the "tridimensional realization of space" (p.72), whereby people see space through three lenses: physical (distance from point A to B), psychological (how we react to the physical and social spaces we encounter), and social (how we interact with each other in these spaces). ${ }^{12}$ Finally, Lucy (2000, pp.ix-x) identifies three levels of how language organizes thought, including semiotic (how the structure of natural language influences thought), structural (how a particular language influences thought), and functional (how using language in a particular way/setting influences thought). Therefore, the following discussion covers a proposed amalgamation of the different perspectives of these three different levels involved in the relationship between language and humans' conceptualizations of physical space:

1. Semiosis: How space is manifested in the structure of language.

2. Divergence: How different languages' treatment of space influence the way speakers orient themselves.

3. Interaction: How space influences the way language is used and vice versa.

${ }^{12}$ Importantly, such spatiality is viewed by Britain (2010, p.76) to be an inseparable component of human interaction, making it essential to any study of language therein. 


\subsubsection{Semiosis.}

While linguistic expressions - unlike images or maps - do not convey spatial information by default, they can communicate any spatial aspect of an environment with a considerable level of detail (Bierswisch, 1999, p.31) using fixed forms that serve specific purposes (Talmy, 1983, p.229). This is possible, Bierswisch $(1999$, p.31) argues, because language is primarily symbolic, not iconic, meaning that it can describe colour, distance, or altitude, even though no parts of language have a particular colour, distance, or altitude. All languages have specialized terms for space in the form of nouns (e.g. tree, river, or village) or verbs (e.g. to leave somewhere) (Bierswisch, 1999, p.31, own examples), which interact in different ways to create syntactic and semantic forms such as the agent, patient, object, and subject (Landau, Dessalegn, \& CohenGoldberg, 2011, p.6).

When focusing on events that involve motion in space of some kind, different mechanisms come into play that exist cross-linguistically but vary from language to language (Landau, Dessalegn, \& Cohen-Goldberg, 2011, p.8), as shown by Talmy (1985) in English:

- Figure: The subject or object that is doing the moving, often in the form of a noun.

- Motion: The action the figure performs, often in the form of a verb.

- Manner: The way in which the figure performs the motion, often an adjective or adverb.

- Path: The directions the figure takes to perform the motion, often a preposition.

- Reference Object: Something the figure moves in relation to, often a noun.

Importantly, these mechanisms come together in a Path Expression, which Jackendoff (1983, p.161) classifies into three main forms that occur in some way cross-linguistically: 
- FROM path: The figure moves from a reference object to somewhere (e.g. 'to,' into,' etc.).

- TO path: The figure moves to a reference object from somewhere else (e.g. 'off,' 'out,' etc.).

- VIA path: The figure moves past a reference object (e.g. 'by,' 'past,' 'through,' etc.).

In many languages, the position of a figure in relation to something else is either marked by prepositions or case markers (Meakins, 2011, p.44). While these above English examples are a first glance at how movement may be encoded using prepositions, they are used differently in other languages. For example, in Finnish and Estonian, a locative (that is, dealing with location) case system takes the place of prepositions that occur in many Indo-European languages, meaning that prepositions such as to, on, off, or into are instead suffixes that attach mostly to nouns (see Karlsson, 2018). In Hungarian, the same is true with an added dimension of time (i.e. when the motion occurred), which is similarly embedded in the case system (Kracht, 2002, p.229).

All languages also follow specific protocols known as frames of reference for describing figures (including the speakers themselves). In many languages, these features are used to refer to a point of view or a hypothetical observer of the scene (Cresswell, 1978, p.1), known as a relative frame of reference (Majid, Bowerman, Kita, Haun, \& Levinson, 2004, p.109). In English, such commonly used examples for describing where something is in relation to something else include right, left, on top of, or behind (Cresswell, 1978, p.1). While cardinal directions (such as north, south, east, and west) are common in English, they are typically reserved for navigational purposes, and not to casually describe a figure's location in space. In languages such as Bak ${ }^{w}$ əmkala, spoken in British Columbia, Canada, the intrinsic location of an object can be more important than the object's properties (Wilson \& Hemphill, 2017, p.2). While an English speaker may ask a listener for a pencil, for example, a Bak ${ }^{\mathrm{w}}$ əmk kala speaker would be 
more likely to ask a listener for "a long horizontal thing near you that is visible to me" (Wilson \& Hemphill, 2017, p.2). Additionally, many other languages have different strategies for communicating point of view or orientation, and in some notable cases, such strategies are defined by the physical environment in which speakers live.

The Guugu Yimithirr speaking people in North Queensland, Australia, for example, orient themselves and objects in space using cardinal directions, ${ }^{13}$ (Haviland, 2002, p.25), which is known as an absolute frame of reference (Majid et al., 2004, p.109). Here, the objects' locations do not change relative to the subject, meaning that regardless of the direction the speaker is facing, a tree (or some other object) will remain to the west of them. In another Indigenous Australian language, Gurindji, each cardinal direction was historically reflected in 28 specifically inflected forms, indicating the language's overt grammaticalization of cardinal directions (Meakins, 2011, p.45).

In the case of the Papuan language Yupno, speakers orient themselves according to slope, meaning that figures are described as being either uphill or downhill from the subject, even when on flat ground (Núñez, Cooperrider, Doan, \& Wassmann, 2012, p.25). Similarly, in Denaakk’e (Koyukon), whose speakers live almost entirely along the banks of the Koyukuk and Yukon rivers in Central Alaska, figures' locations are described not only in relation to a river, but in relation to that river's flow (Henry \& Henry, 1969, p.138). Instead of having English-style forms such as 'upriver on the left' or 'downriver on the right,' spatiality is encoded in the language's structure, such that the following locational affixes are possible:

${ }^{13}$ These cardinal directions are oriented differently to what is commonly understood by outsiders. For example, "west" in Guugu Yimithirr is roughly oriented toward magnetic north (Haviland, 2002, p.30). 
/-tli/ $\quad$ Something in front of something else, facing a river

/-nte/ Upriver, towards the general area of a tributary

These examples suggest that the very structure and composition of linguistic forms can be influenced by the surrounding physical environment, causing the language to encode aspects that are reflective of the spaces its speakers inhabit. Such a frame of reference is known as an intrinsic frame of reference (Majid et al., 2004, p.109). While these 'fixed bearing' orientation methods are useful for describing one's placement within an environment, they are also often generalized to smaller-scale spaces, including distinguishing sides of the body (Meakins, 2011, p.44). For a visual depiction of languages' different ways of regarding space, see Figure 15 below.

Figure 15: Different frames of reference found across languages.

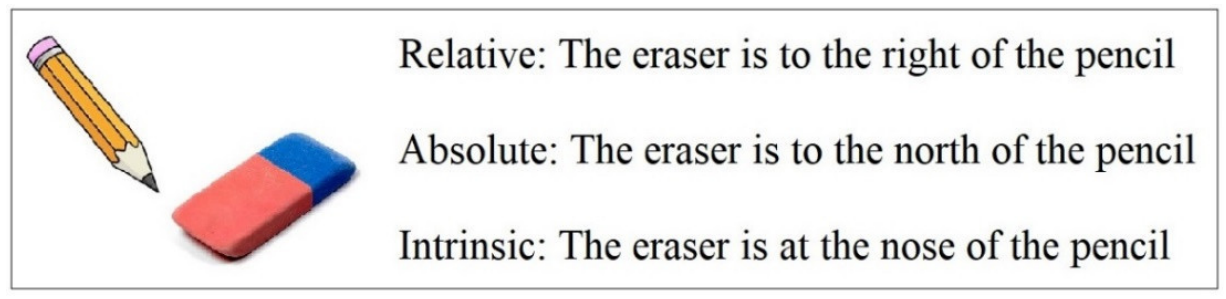

Adapted from Majid et al. (2004, p.109)

\subsubsection{Divergence.}

Through the uniquely human ability to communicate spatiality through discourse, we can convey environments to others that shape their thoughts and behaviour (Bryant, 1997, p.239). While a dedicated discussion of the merits of linguistic universalism and relativism is outside the scope of this dissertation, it is important to acknowledge that numerous theorists since Whorf 
(1941, p.210) have described what humans understand to be physical space as a fundamentally linguistic concept (Patrão, 2018, p.32). That being said, this dissertation operates under the assumption that the language(s) an individual speaks will at least in part affect how they perceive and understand the physical and social spaces that surround them. In support of this notion, Levinson, Kita, Haun, and Rasch (2002, p.156) suggest that there is a meaningful amount of cross-cultural variation in semantic relations and categories of spatial language, which play a role in non-linguistic reasoning and memory tasks.

Referring back to the Guugu Yimithirr example, Haviland (1998, p.18) and Deutscher (2010, p.7) argue that any speaker of a language that primarily uses cardinal directions must likely be aware of their absolute (that is, not relative) placement at all times. As these speakers see themselves as small parts of a greater system, the position of figures of interest do not change relative to what direction the speaker or hypothetical observer is facing (Haviland, 1998, p.2). While the same can be said of Guridji, speakers of Guridji Kriol (a heavily Anglicized counterpart) prefer deictics (e.g. here and there) and gestures, requiring both the speaker and listener to be visible to one another (Meakins, 2011, p.44). Though such changes are an example of language loss, they also serve as an important illustration of how intergenerational changes in a language's encoding of space can change how that space is communicated, which may in turn affect different generations' perceptions of that space.

In a study by Majid et al. (2004) that analyzed the relationship between frames of reference and spatial cognition, speakers of Tzeltal (an absolutist language) performed differently on an object rotation task than speakers of Dutch (a relativist language). Here, participants were required to look at an object on a table, turn 180 degrees, and then identify the same orientation of one object out of an array of identical objects on a separate table, as shown in Figure 16. 
Figure 16: Rotation task involving speakers of languages that use different frames of reference.

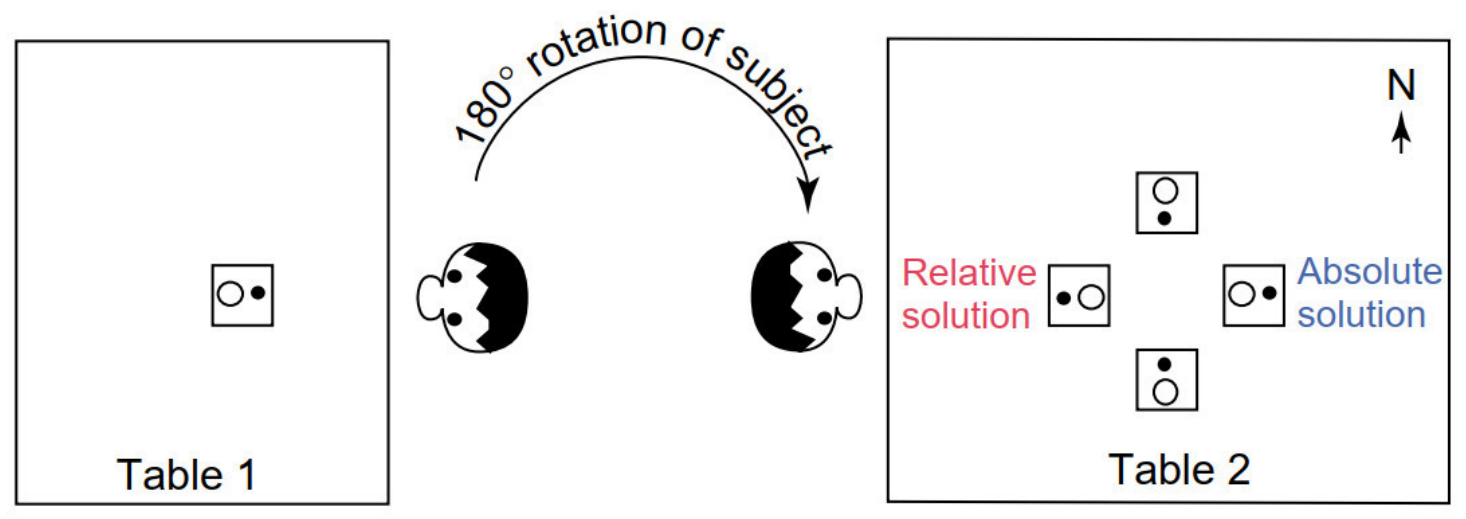

Adapted from Majid et al. (2004, p.110).

In Figure 16, Majid et al. (2004)'s rotation task suggests that speakers of Relative languages rotate the position of objects they see relative to their own position, while speakers of Intrinsic languages will not choose an object whose position has rotated, as to them the position of the object is fixed in place. Reflectively, Majid et al. (2004, p.109) suggest different underlying computations involved in spatiality; while Relative languages require speakers to parse and subsequently project themselves into planes of front, back, left, and right, Intrinsic languages require speakers to run a 'mental compass' computation in the background at all times. While most languages prefer one type of frame of reference over another, it is important to note that very few (an exception being Guugu Yimithirr) languages exclusively use one.

In summarizing the above findings, we can conclude the following about how language determines a speaker's perception of physical space: Languages that primarily use cardinal directions to communicate spatiality likely cause the speaker to view their own placement as incidental and unimportant to the orientation of objects or places in the surrounding environment. On the other hand, languages that primarily use egocentric techniques to communicate spatiality likely cause the speaker to unconsciously view the orientation of objects and places in their surrounding environment to be dependent on their own position, making the speakers more 
integral and less incidental parts of a space. However, accounting for links between the individual and spatiality within language is only part of a greater picture. To better understand the intimate relationships between language and space, its social usage (Deckert \& Vickers, 2011, p.2) should also be accounted for, as that is a defining feature of language.

\subsubsection{Interaction.}

While the previous two levels of language and spatial thought focus on language as an abstract entity that exists in isolation, Malinowski (1935, p.9) and Austin (1975, p.76) acknowledge that "language is not only a way of encoding knowledge but also a way of acting in the world" (Duranti, 2011, p.17). In assuming that acting in the world is predicated on interactions with the world, Britain (2010) notes that "all interaction is spatialized" (p.76). Similarly, Jordan (2014, p.33) notes that language is a social phenomenon with an intrinsic relation to space first and foremost, because speakers are spread over space. As such, this third level focuses on how the social uses of language determine our understanding of the physical and social spaces we inhabit, and how different spaces in turn influence our use of language.

The study of variation in language corresponding to social factors-namely sociolinguistics - is by no means a new field of study, and is attested by Johnstone (2011, p.203) to be one that is concerned with place. When referring to place in the context of language, there are many different perspectives one can take. Johnstone (2011, p.203) identifies several ways language and space interact within social spheres of existence: ${ }^{14}$

${ }^{14}$ An additional field of perceptual dialectology is identified by Preston (2010), which is an area of research that focuses on speakers' perceived distribution of language varieties within a given area. 
1. Speakers' locations within social networks, and their likelihood of being linguistic leaders or followers.

2. The distributions of language varieties and change across a given geographic region.

3. How physical environments affect patterns of variation and change by shaping speakers' social environments.

4. Linguistic landscapes - patterns of signage and other visible manifestations of language.

While most of these research areas are discussed below, the distribution of language varieties fits in most appropriately elsewhere and is thus given attention in later sections.

\section{Speakers' locations within social networks}

In an analysis of how placement within social networks affects language use and form in Cockney English, Fox (2007) illustrates patterns of use of the [eI] diphthong outward from interlocking friendship groups of Bangladeshi boys at a youth club to other and more distantly related friendship groups in the area (Figure 17). In doing so, Fox (2007) illustrates that place need not solely refer to a geographic notion of physical space but can exist in many ways within varying spheres of human existence. 
Figure 17: Frequency of use of [eI] among friendship groups in a Tower Hamlets (London) youth club.

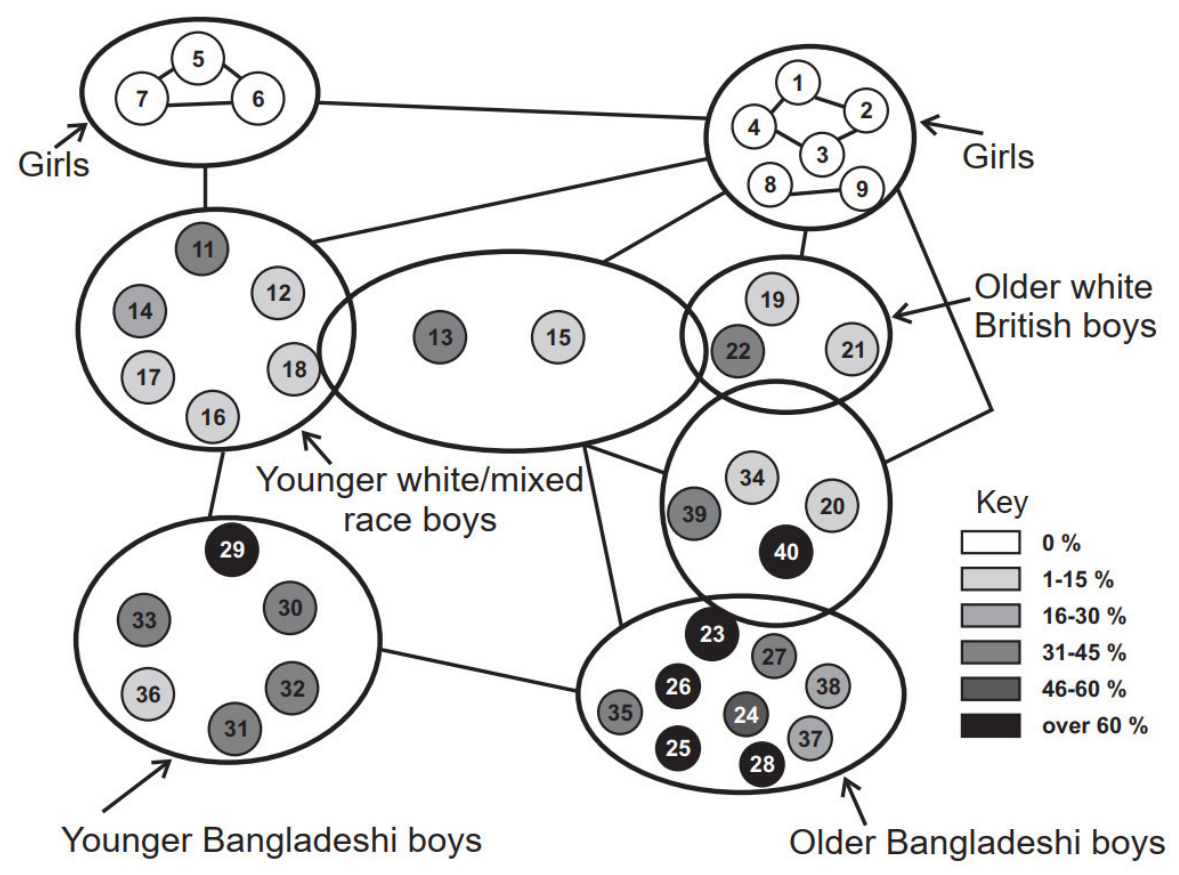

Figure from Fox (2007, p.192) as found in Britain (2010, p.86)

Such social networks influence who adopts a linguistic innovation first and the extent to which an innovation takes hold. For example, Milroy and Milroy (1985, p.380) suggest that more insular social groups whose members are close to one another tend to be resistant to linguistic change and innovation. Individuals with weak ties to different groups tend to be the catalysts in linguistic change, which in turn means that communities with many weak links — such as those facing migration pressures or social instability — tend to experience rapid linguistic change (Milroy \& Milroy, 1985, p.380).

How physical environments shape social and linguistic environments

A second perspective in the study of the interaction between social language and space focuses on how physical environments affect patterns of variation and change by shaping 
speakers' social environments. According to Johnstone (2011, p.209), physical environments affect linguistic distributions not only because of direct physical barriers such as mountains or rivers, but because they affect social factors such as political borders and income-related settlement patterns. Subsequently, Boberg (2000, p.3) suggests that larger urban centres tend to adopt innovative forms earlier than rural areas, and national borders can block or delay the spread of innovative forms as they prevent face-to-face interaction. Such factors are important to consider in any analysis of language and space, given that these social and political divisions may not be readily apparent at a first glance of a geographic area.

As an applied example, Ramisch (2010,p.163) mentions how early research focusing on language and space tended to only look populations deemed to be the most resistant to social aspects of dialectal change. Chambers and Trudgill (1998, p.29) and Lameli (2010, p.583) define such a group as Non-mobile Older Rural Males (NORMs), who Ramisch (2010, p.163) notes were believed to be least influenced by social, educational, or geographic factors. Here, ruralness was suggested to protect contributors from social factors of linguistic change, but by only studying them, researchers ignored other indispensable sources of linguistic data (Romaine, 2003, p.99). ${ }^{15}$ While researchers such as Hickey (2018, p.35) believe NORMs have been abandoned completely in linguistic research, the use of these individuals is still considered by Yang (2018, p.40) to be standard practice. ${ }^{16}$

\footnotetext{
${ }^{15}$ For some examples of spatial linguistic research that involves NORMs, see Klemola and Jones (1999), van Oostendorp (2014), and Yang (2018).

16 There are instances when the use of NORMS is beneficial, such as when working with endangered languages, where the only available speakers are elderly and based rurally, or in studies of historical structural diversity.
} 


\section{Linguistic Landscapes}

In describing an additional field of study that focuses on how language is present within social spaces, Gorter (2006, p.1) assigns two meanings to the study of linguistic landscapes (LLs): the study of languages as they appear on public signs, and more generally, the overall written representation of languages in the community. By offering specific examples, Landry and Bourhis (1997, p.25) view LLs as coming from the culmination of the languages used in public road signs, place names, advertisement posters, commercial signs on shops, and others (see Figure 18). Essentially, the study of LLs is based on the idea that you can tell a lot about languages and their vitality by how they occur in public.

Figure 18: Trilingual sign in Welsh, Irish, and English outside my grandmother's town.

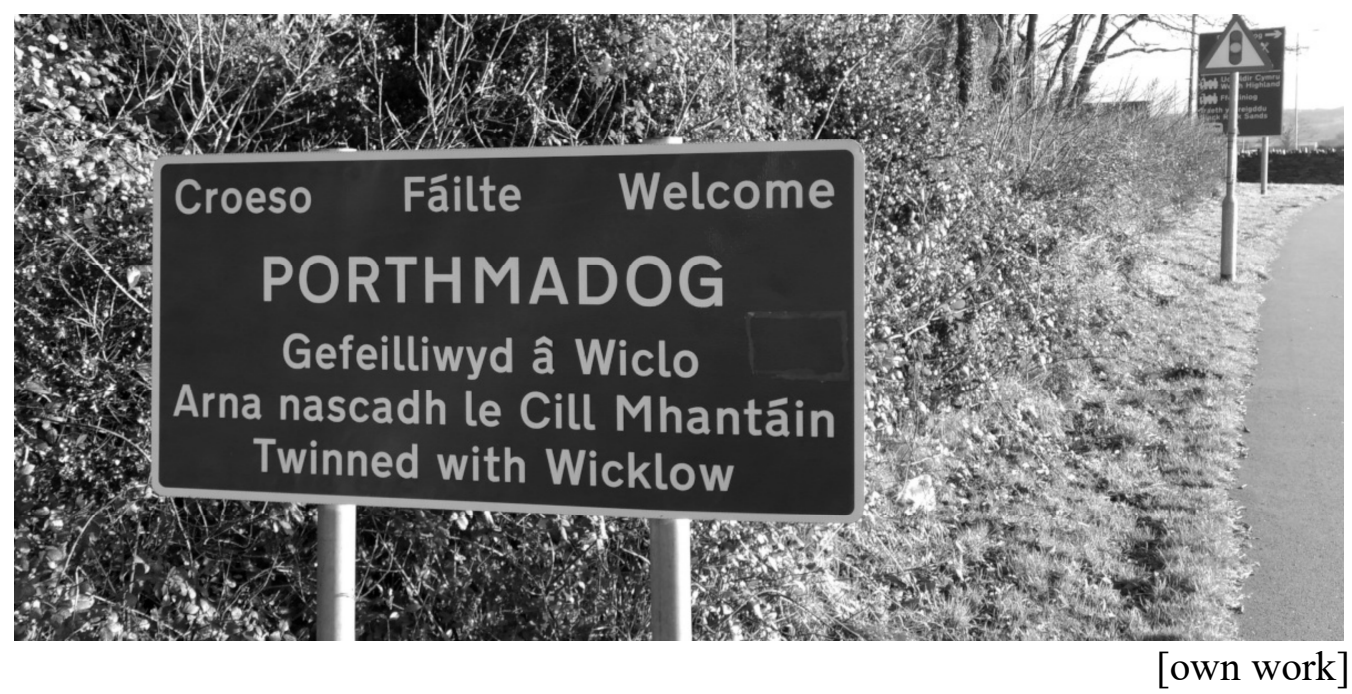

A view of the LL often suggests what languages are present and to what extent they are prevalent in a socio-linguistic/geographic space. Cenoz and Gorter (2006, p.67) view the relationship between LL and sociolinguistic contexts as bidirectional: On one hand, the LL reflects power balances, statuses, and relationships between different languages and 
sociolinguistic contexts. On the other, the LL contributes to sociolinguistic contexts, as people's understanding of languages' status and vitality are shaped by visible evidence. Such issues are most relevant in multilingual settings, especially when two or more languages enjoy different levels of prestige. While national and/or majority languages typically dominate visible signage and other displays of language, Bourhis and Landry (2012, p.39) suggest that the use of a minority language in the public domain can contribute to its increased usage within social institutions and augment the perceived vitality of the minority community. Finally, Cenoz and Gorter (2006, p.68) note that studies of LL can shed light on top-down official language policies (such as street names) and bottom-up acts instigated by individuals, such as shop names or announcements on public notice boards.

4.2 Fields that study language and space.

While the aforementioned research studies, in part, relationships between language and space, they are not necessarily fields which were originally intended for this purpose. As such, this subsection outlines several existing disciplines whose principal purposes are to study the nuanced relationships between language and space. A recurring observation one may make here is the seemingly endless terminological murkiness surrounding each research area, specifically regarding the boundaries between disciplines. I am certainly not the first person to acknowledge this, and Williams (1988, p.1) discusses inconsistencies surrounding naming conventions in this area, which Briscoe $(2009$, p.8) explains is due to these fields' diffused outlines and origins. 


\subsubsection{Geolinguistics and linguistic geography.}

In a nutshell, geolinguistics is an interdisciplinary research area that studies the relationships between languages and land as they are used by various cultures and peoples. While Luebbering (2011, p.11) views geolinguistics as an integration of geography and linguistics, Breton (1991, p.19) notes how geographic thought is valuable for linguists interested in spatial phenomena such as language contact and change, phonology, lexical choices, language policy, and history. The American Society of Geolinguistics' mission statement ${ }^{17}$ defines geolinguistics as an academic discipline that studies language varieties' geographical locations, distributions, and structures within a temporal framework, either in isolation or in relation to one another. Furthermore, Hoch and Hayes (2010, p.23) describe geolinguistics as a field which makes extensive use of maps to describe and analyze spatial patterns of the location of languages or the results of processes that lead to language change. Conversely, Sibata (1969, p.11) views geolinguistics to be just one of many methods associated with historical linguistics. However, Williams (1980, p.208) states that the most fundamental task of geolinguistics is to analyze the distribution patterns and spatial structures of languages in contact with one another, and makes no reference to the field being intended only for diachronic study. In a straightforward illustration, Van der Merwe and Van der Merwe (2007, p.1) created a graphic that divides geolinguistics into different elements based on the level of research occurring and the specific perspective one wishes to take (Figure 19).

${ }^{17}$ Accessed from the American Society of Geolinguistics (https://sites.google.com/view/geolinguistics-information/mission-statement) on January 5, 2020. 
Figure 19: A visual representation of a geolinguistic framework intended for language mapping.

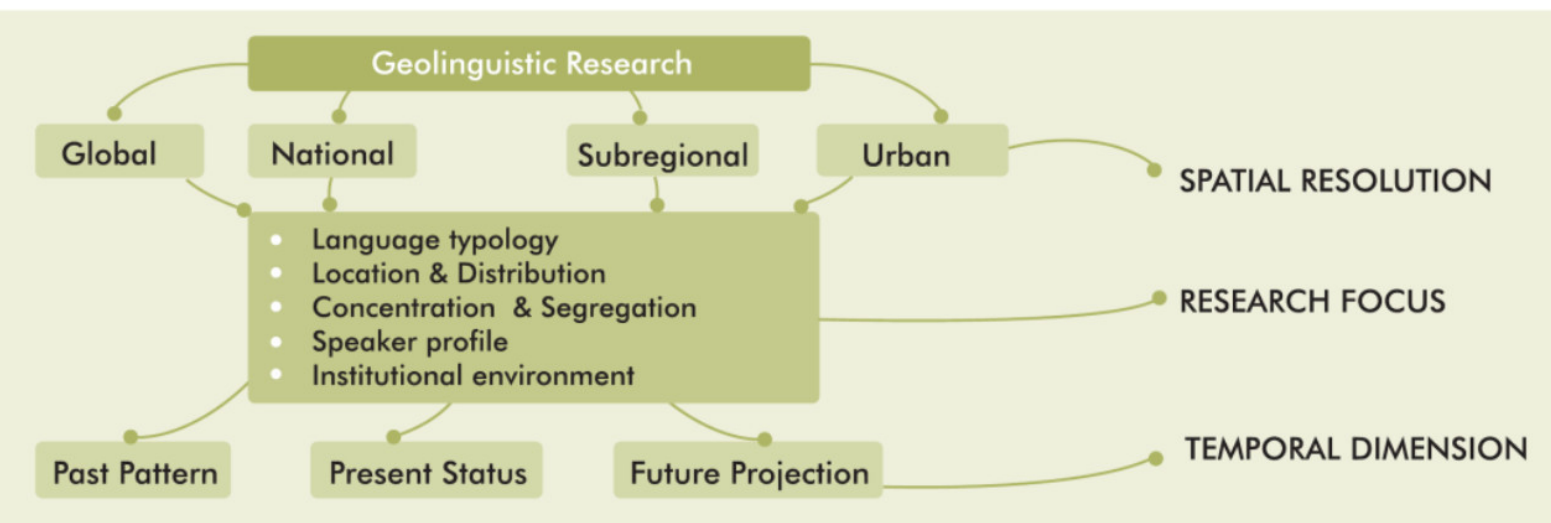

Van der Merwe and Van der Merwe (2007, p.1)

Within the field, Mackey (1988, p.22) differentiates between micro and macro geolinguistics, which Dell'Aquila (2010, p.459) summarizes as focusing on dialectal studies and more broad geographical studies on language respectively. Put into the language of this dissertation then, micro geolinguistics is a subfield that looks at the distribution of language varieties and their features, while macro geolinguistics looks at more general relationships between language and associated geographical spaces.

Despite three geolingusitic societies and their respective academic journals, ${ }^{18}$ there is still considerable disagreement regarding where geolinguistics is situated in relation to other fields that study language and space (Williams, 1988a, p.1). Olariu and Olariu (2014, p.77) and Gawne and Ring (2016, p.202) use the terms geolinguistics and linguistic geography interchangeably, Williams (1980, p.207; 1988, p.2) understands geolinguistics to be a developing branch of human geography, and Trudgill (1974, p.232) believes linguistic geography to have originated

${ }^{18}$ The names of these societies are followed by the names of their journals in parentheses: The American Society of Geolinguistics (Geolinguistics), The Asian Geolinguistic Society of Japan (N/A), and The International Society for Dialectology and Geolinguistics (Dialectologia et Geolinguistica). 
from human geography. At the same time, Hoch and Hayes (2010, p.24) and Breton (1991, p.19) consider geolinguistics to have arisen from linguists making use of geographic thought, Jordan (2014, p.34) sees geolinguistics as most closely affiliated with linguistics, and Gunnemark (1991, p.12) goes as far as considering linguistic geography to be a subfield of geolinguistics.

Following this vein of thinking, researchers such as McDavid Jr. and O'Cain (1973) consider linguistic geography to be a "branch of historical linguistics [... that $]$ provides a framework for interpreting studies of varied populations" (p.137), while Trudgill (1974, p.215) claims that linguistic geography is best paired with sociolinguistic methodologies instead. Similar to geolinguistics, linguistic geography studies the geographic distribution of languages and their phenomena, from which two subfields can be drawn that differ in scope. What has thus far been referred to as linguistic geography is a research area that both describes and explains the geographical distribution of language varieties and linguistic features (Trudgill, 1974, p.216).Language geography, on the other hand, is a distinct yet related area that "restricts itself on studying the spatial variation of words and pronunciations within a given language" (Jordan, 2014, p.34). It is important to note that despite its interdisciplinary nature, linguistic geography has been and continues to be practiced primarily by linguists and not by geographers, contributing to a fractured state within the field (Haynie, 2014, p.344).

While the terminological differences for geolinguistics and linguistic/language geography are by no means agreed upon in the literature, I would argue that micro geolinguistics would be roughly analogous to linguistic and language geography. Researchers in both fields study the distribution of languages and their features, while those in macro geolinguistics seek to explain why such distributions occur and what the associated consequences are. In essence, what can be agreed upon in the study of language and space is that there are three major foci: the 
identification, defining, and labelling of language varieties, the distribution of linguistic features, and the underlying contexts for distribution. Within this first focus - the identification, defining, and labelling of these varieties - there are other subdisciplines of interest here.

\subsubsection{Dialectology, dialect geography, and dialectometry.}

As aptly stated by Auer (2013), dialectology refers to "the study of linguistic variants within the space of one single language or a group of historically closely related languages" (p.1) such as the dialects ${ }^{19}$ of Welsh, or languages within the Brythonic subfamily of the Celtic language family. Though much of early dialectology was purely observational in nature - to describe and catalog linguistic phenomena-it has recently begun to seek to explain why linguistic phenomena are dispersed the way they are across space and time (Watt, 2018, p.17). Typically, dialectology performs a scholarly investigation of dialects (e.g. see Maguire, McMahon, \& Dediu, 2010, p.69) that studies patterns of diffusion (dialectal spread across regions and social groups), the nature of borders between dialects, language contact, transitional forms, and new dialects resulting from migration and relocation (Boberg, Nerbonne, \& Watt, 2018, p.10). Importantly, a central assumption within dialectology is that "Dialect differences are caused by two forces operating in tandem: language change and the expansion of speech communities" (Boberg, Nerbonne, \& Watt, 2018, p.6). By understanding how geographical, social, political, and cultural factors contribute to the spread and interactions of language varieties, dialectology

\footnotetext{
${ }^{19}$ What distinguishes "language" from "dialect" is hotly contested in the literature (e.g. see Haugen, 1966, Hickey, 2018, and Boberg, Nerbonne, \& Watt, 2018). In this dissertation, the term "language variety" is used in their place except when specified otherwise.
} 
seeks to answer the following main questions outlined by Boberg, Nerbonne, and Watt (2018, p.12):

1. How do language varieties vary across the regions they are spoken in?

2. What are some common patterns of variations observed in different language varieties?

3. How do non-linguistic factors like urbanization affect language varieties' distribution?

4. What is the nature of borders or transition areas between language varieties?

5. How do new linguistic features spread across a linguistic region?

6. Is a language variety receding, stabilizing, or growing over time?

Of particular importance, many investigations which regard geography to be a significant factor in the classification of dialects have focused on the differences between dialects, as opposed to their similarities. In this, Röthlisberger and Szmrecsanyi (2020, p.133) describe the commonly used Fundamental Dialectology Principle, in which a null hypothesis states that the geographical proximity of language varieties predicts their linguistic similarity. Given the similarity of this null hypothesis to Tobler's First Law of geography (Tobler, 1970, p.236), Goodchild's (2020, p.3679) recent observation that increasing global connectivity and migration can raise the distinctiveness between neighbouring dialects - while merging others - points to the fact that dialectology is still a rapidly changing and evolving discipline.

As in geolinguistics, there are terminological inconsistencies evident within this field of research. While Auer (2013, p.1) uses the terms dialectology and dialect geography interchangeably, Hickey (2018) uses dialectology in the context of modern research that accounts for complex geopolitical contexts (p.33), and dialect geography in the context of the earlier Neogrammarian perspective, which viewed sound change as rule-governed and 
exceptionless (p.32). However, Boberg, Nerbonne, and Watt (2018, p.6) view dialectologists' role as observing and interpreting dialectal distribution generally, while dialect geographers specifically look at the influence of geographic features such as water bodies or mountains. In all likelihood, some combination of these distinctions is the most realistic.

While there are many approaches to understanding how language varieties relate to one another that employ historical or political information, one particular perspective uses numerical classification methods to measure the degree of relatedness between language varieties. Referred to as synonymous with the European approach to dialectology ${ }^{20}$ by Hickey (2018, p.33), dialectometry attempts to quantify dialect differences and measure language change over time (Nerbonne \& Kretzschmar, 2003, p.245), as well as create numerical taxonomies that organize language varieties (Goebl, 1993, p.278). Furthermore, Goebl (2018, p.123) adds that through numerical analyses of concrete linguistic patterns, dialectometry seeks to uncover abstract patterns that would otherwise remain hidden. As such, dialectometry can be thought of as a quantitative approach to linguistic variation.

Though an in-depth discussion of dialectometry is outside the scope of this dissertation, one dialectometric method, known as lexicostatistics, is of interest here. ${ }^{21}$ As a basic definition, lexicostatistics attempts to ascertain the dates of earlier stages of languages as well as relationships between closely related language varieties (Gudschinsky, 1956, p.1) through the statistical manipulation of lists of core lexical items, or words (Zhang \& Gong, 2016, p.1). One such lexicostatistic technique, the Levenshtein distance, helps illustrate what kinds of tests can

\footnotetext{
${ }^{20}$ Notable pioneering works include the Atlas Linguistique de la Gascogne (Séguy, 1973).

${ }^{21}$ Another related method, glottochronology, acts in much of the same way as lexicostatistics, but makes assumptions regarding language varieties' rates of word retention or loss (Zhang \& Gong, 2016, p.1). For more information, also see Gudschinsky (1956).
} 
be done. Simply put, the Levenshtein distance calculates the number of changes needed for one string to be transformed into another, in what Heeringa (2004, p.121) refers to as a sequence comparison. When dealing with languages, this would refer to how many phonetic substitutions would need to occur for a word in one language variety to become the same (but differently pronounced) word in another language variety ${ }^{22}$, as in Table 1 below.

Table 1: Levenshtein distance for the word "schedule" between Canadian and British English.

\begin{tabular}{|c|c|c|}
\hline String & Operation & Cost \\
\hline /skedzjuət/ & delete $\mathrm{k}$ & 1 \\
\hline /sedzjuət/ & substitute $\mathrm{s} / \int$ & 1 \\
\hline / $\varepsilon \mathrm{dg}^{\mathrm{j} u \partial \mathrm{L} /}$ & delete ə & 1 \\
\hline$/ \int \varepsilon \mathrm{d}^{\mathrm{j}} \mathrm{JuL}^{\mathrm{j}} /$ & & \\
\hline
\end{tabular}

Total Levenshtein Distance: 3

In the above example in Table 1, the calculated Levenshtein distance between the forms for "schedule" in two related dialects of English is relatively small, especially compared to the distance that one would find between the English "schedule" and the Spanish "horario." While a comparison of two words may not be very useful in the latter analysis, a more comprehensive depiction of language change becomes evident when applied to a list of corresponding words in each language variety, as in Figure 20 below:

${ }^{22}$ Importantly, the language varieties of interest do not need to be related to one another. In this way, this method is agnostic regarding varieties' historical relationships. 
Figure 20: Levenshtein distances between regional language varieties in the Netherlands and Standard Dutch, calculated as percentages.

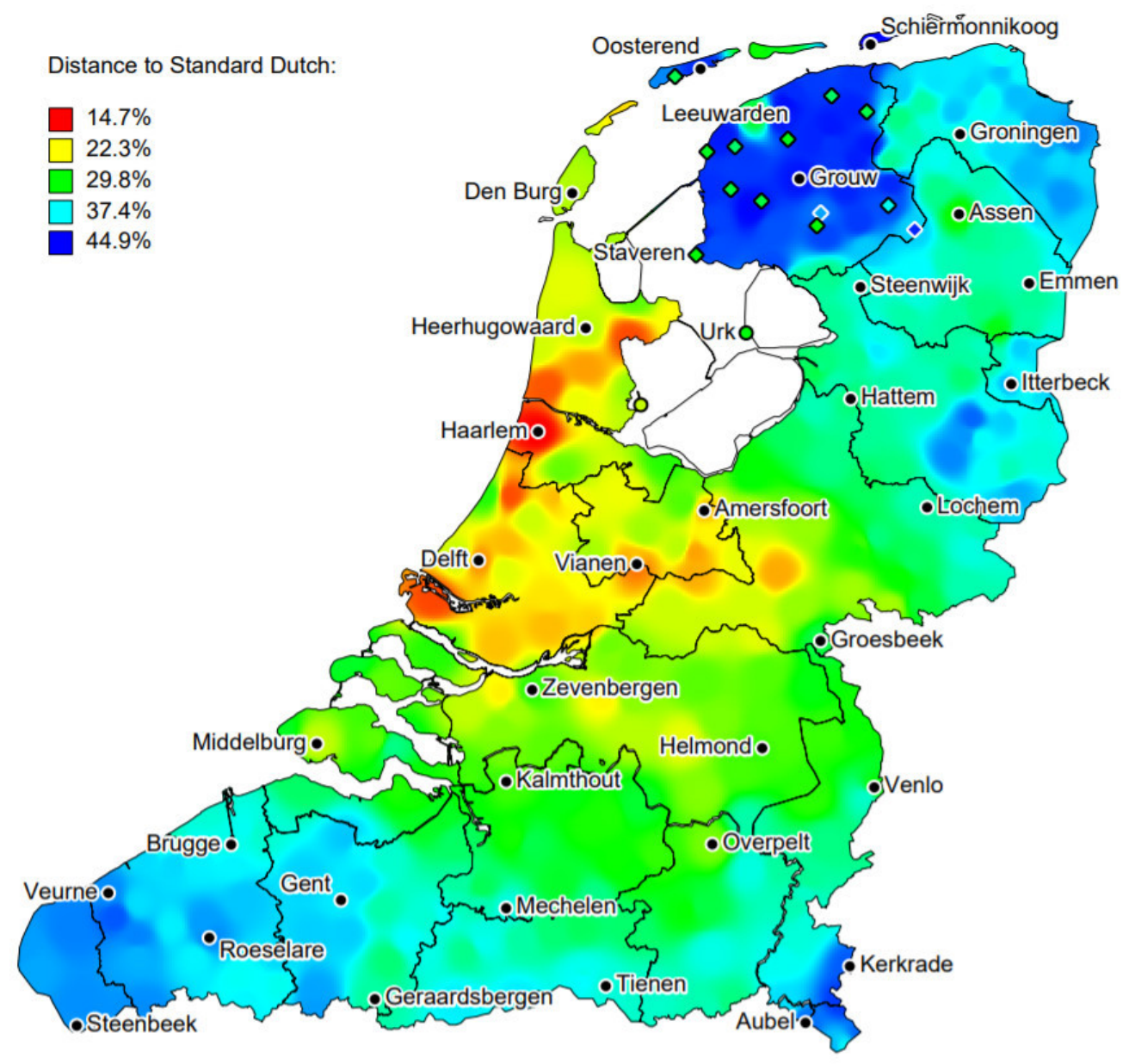

Heeringa (2004, p.276)

However, it is important to note that the use of numerical calculations in determining relatedness between language varieties can at times be controversial, as Geisler and List (2010, p.2) summarize: 
Table 2: Summary of the debate on Lexicostatistics by Geisler and List (2010, p.2).

\begin{tabular}{||l||l|}
\hline \hline Critic & Reply \\
\hline \hline $\begin{array}{l}\text { Distances don't tell us anything about language } \\
\text { history (cf. e.g. Blust 2000). }\end{array}$ & $\begin{array}{l}\text { Our methods are character-based (Atkinson \& } \\
\text { Gray 2006). }\end{array}$ \\
\hline $\begin{array}{l}\text { Borrowing will make the results unreliable (cf. } \\
\text { e.g. Bergsland \& Vogt 1962) }\end{array}$ & $\begin{array}{l}\text { Not within basic vocabulary (Atkinson \& Gray } \\
\text { 2006). }\end{array}$ \\
\hline $\begin{array}{l}\text { Basic vocabulary is not resistant to borrowing } \\
\text { (cf. e.g. Sagart \& Lee 2008). }\end{array}$ & In most cases it still is (Starostin 1999). \\
\hline $\begin{array}{l}\text { The method and its data basis is subjective and } \\
\text { inconsistent (cf. e.g. Hoijer 1956, Rea 1973). }\end{array}$ & NO REPLY SO FAR \\
\hline
\end{tabular}

These arguments hold important points for dialectology as well as lexicostatistics, which more generally quantifies levels of similarity between languages' core vocabulary using statistical methods (Hymes, 1960, p.4). Though a more in-depth discussion of the strengths and weaknesses associated with dialectometry and lexicostatistics is outside the scope of this dissertation, this glance here serves as a reminder that quantitative and authoritative-seeming methods that fall within studies of language and space should always be viewed with a critical lens, as the realities they attempt to understand are quite often nuanced and unpredictable. Coming full circle then, a key focus of this dissertation becomes the study of the mechanisms with which spatio-linguistic reality can be replicated and communicated. 


\subsection{Language mapping.}

I do not currently consider language mapping, as defined in this dissertation, to be a fullyfledged discipline or field of study. Indeed, the very term mapping as a gerund form of the present progressive verb 'map' suggests action and process. In support of this notion, Goodchild (2020, p.3673) describes language mapping as a non-replicable cartographic practice, while Rabanus (2020, p.104) defines language mapping as an act that includes "the visualization of linguistic and language-related data in geographic space and, hence, the representation of correlations between geographic and linguistic facts" (p.104). For the purposes of this dissertation, practice is understood to not only be a series of isolated acts of studying the distribution of linguistic phenomena and making maps of language, but as noted by Simonsen (2007), “continuous flows of conduct which are always future-oriented" (p.170) and purposeful.

While these descriptions are not unique to language mapping, they are important aspects that are directly relevant to language mapping processes. It is important to acknowledge a considerable amount of overlap between language mapping and other mapping practices, both in this dissertation and in mapping more generally. As language mapping is, structurally, a kind of thematic mapping, many existing discussions of mapping techniques and technologies applicable elsewhere are relevant to language maps (Ormeling, 2010, p.28). There is little difference, in form, available features, and appearance, between a map that shows the distribution of languages and a map that shows the distribution of historical territories across a selected region. ${ }^{23}$ In addition, because of a lack of literature involving critical cartographic and ethics-based approaches to language mapping, I assume provisionally that many of the discussions surrounding language mapping issues are applicable more generally to fields such as

\footnotetext{
${ }^{23}$ For an illustration of such similarities, see Temprano (2015-2020) at http://www.native-land.ca
} 
cartography, linguistics, and applied linguistics, while issues in these fields are also found in language mapping.

Instead of form and appearance, language maps differ from other maps because of the kind of information they use. Language is a unique - and as far as we know, uniquely humanphenomenon that permeates all spheres of human existence. As an inseparable part of our identities and cultures (Bucholtz \& Hall, 2004, p.369), languages and information about them are in many ways as intimate as they are universal, and as specific as they are generalizable. While not everyone knows the same language, thankfully, everyone knows at least one language and has a stake in the politics surrounding its use and representation. As such, language maps hold a number of unique roles in representing, displaying, and communicating information that defines and occupies countless parts of our lived experiences. There are thus many areas of language mapping that are fruitful areas of discussion and investigation - many of which have yet to be explored thoroughly.

As demonstrated in the previous section, this is not to say that there are no attested disciplines devoted to studying language and space, and representations thereof. Instead, I consider language mapping to be the collective actions through which linguistic information is transferred from the real world to maps in an attempt to convey the real world's linguistic realities to audiences. In addition, language mapping can be thought of as a means to communicate research and work in language and space, which can be abstract and difficult to grasp without an effective mechanism.

Notably, Thun (2010, p.506-507) does call the study of the acts that go into making language maps geolinguistic cartography. In this so-named proposed area, Thun (2010, p.507) notes that the central purpose is not to create new techniques for making maps of language but 
analyze and deconstruct existing ones as to identify more appropriate ways to apply them. However, aside from describing it as a recent offshoot of thematic mapping, Thun (2010) does not infer that geolinguistic cartography is an established field of study, and I have been unable to find any other works in English-language literature that mention this name. When it does appear once in Spanish-language literature, Quesada Pacheco (2014) treats la cartografía geolingüística as the actual process through which maps are made, which is more in-line with my understanding of what language mapping is: "el fin primordial de la dialectología perceptual es tratar la cartografía geolingüística desde la percepción del hablante [...the first purpose of perceptual dialectology is to treat geolinguistic cartography from the perspective of speakers,...]" (p.258, own translation).

While geolinguistic cartography should be the subject of future investigation and refinement, it is outside the scope of this dissertation, not because its focus is irrelevant but because it is simply too narrow. Firstly, Thun (2010, p.507) understands the primary interest of geolinguistic cartography to be maps that look at a language's structure (e.g. phonetics), not maps of the distribution of languages, which I argue are equally important. Secondly, geolinguistic cartography is mentioned only within the context of dialectology, which is but one of a diverse set of language mapping applications in and outside of academia. Geolinguistic cartography is thus a potentially important field. It may well need to be accounted for, or perhaps incorporated into, language mapping practices. Until then, it is but one piece of the proverbial language mapping puzzle, which I argue along with Luebbering (2011, p.1), also contains many other areas.

As observed elsewhere in studies of physical space, and especially in the context of cartography (Bollmann, 2010, p.43), geographers are slowly beginning to look inward at the 
language and discipline surrounding the creation of general maps (Borchert, 1987, p.389).

However, Lameli (2010, p.567) and Luebbering (2013, p.54) note a lack of such reflection specific to language mapping. In addition, Briscoe (2009, p.11) was unable to find critical reflections of how linguistic data are used in GIS mapping. That being said, a lack of critical reflection (or conventions ${ }^{24}$ ) has not negatively impacted the number of maps being produced (Luebbering, Kolivras \& Prisley, 2013, p.383), and does not mean that there have not been any past conventionalization attempts. While Nadal (2015, p.40) infers little difference between the linguist and the cartographer and Nash (2017, p.37) argues that linguistic placename research is inseparable from cartography, Luebbering $(2013$, p.40) considers cartographers' roles to be entirely separate from linguists'. In viewing such incongruence, one can assume that different mapping projects will be approached by individuals with different scholarly backgrounds, roles, and responsibilities, meaning language maps will vary in design and techniques. As the resultant maps construct audiences' knowledge of a phenomenon (Ormeling, 2010, p.29), such differing techniques have far-reaching consequences.

\subsection{Waves of language mapping research.}

An interesting observation made by one of my supervisors-Erik Anonby — and I is that language mapping research appears to come in waves. Observed elsewhere in research, such as in the social sciences (e.g. Moldaschl \& Weber, 1998), medicine (e.g. Delgado-Escueta, Wilson, Olsen, \& Porter, 1999), and mental health geography (Philo \& Wolch, 2001) (to name a few),

\footnotetext{
${ }^{24}$ Keates (1982) refers to conventions in mapmaking as "an agreement between the creator and the user" (p.66), while Harvey (2015, p.5) describes conventions as common knowledge about how something is done that has evolved from procedures agreed upon by experts.
} 
research in an area of study often does not occur continuously, but instead in chunks or spurts that are identifiably separated over time. Indeed, readers of this dissertation may notice that sources that directly discuss language mapping typically either come from the early nineties, or the ends of the first and second decades of the twenty-first century. Importantly, the following research waves are based upon my observations of the existing literature and are not the result of a specific devoted analysis. As such, they should be regarded as simple observations of trends in the literature, not, as of yet, a definitive conclusion of how research into language mapping exists.

In research that looks at the practice of language mapping (as opposed to studies of particular language maps), I have been able identify three such waves, starting in the early nineties with the proposed language mapping typologies of Ambrose and Williams (1991), which aimed to act as a system that deconstructs maps and catalogues their basic components. In addition, Ormeling (1992) published a paper that proposes and discusses a series of methods that can be applied to language mapping, and identifies new areas of research that should be focused on in more detail. This first research is exploratory in nature, and many of their ideas function as a starting point from which future research could be — and has been — based.

Following this groundbreaking research, the study of language mapping remained quiet until a second wave in approximately the year 2010, starting around the time Briscoe (2009) published a master's thesis on the use and consequences of GIS in language mapping and geolinguistics. It appears, according to Luebbering, Kolivras, and Prisley (2013, p.386), that research about language mapping began to decline in tandem with an increase in research about GIS. A year later, a monumental three-volume compilation of theories, practices, and applications of studies 
of language and space was published. ${ }^{25}$ A year after this, Luebbering (2011) published a seminal dissertation that focused on techniques and research in language mapping, language maps' applications, the proposal of a new language mapping typology, and the need to reflect upon language mapping practices. In 2013, her doctoral research was transformed into two articles that focused on similar themes. Research in this second wave can be thought as more comprehensive in nature and, building upon the ideas in the first wave, identifies major issues in language mapping, proposes solutions, and identifies concrete avenues for future investigation.

The third wave, which I would like to think my research is a part of, started toward the year 2020, and included the publication of a handbook of dialectology by Boberg, Nerbonne, and Watt (2018), as well as chapters in a handbook of cybercartography (Taylor, Anonby, \& Murasugi, 2019) that focus on language mapping and online bottom-up mapping practices. This year, a handbook of language mapping, research, and practices (Brunn \& Kehrein, 2020a) was published online, and includes research and insights into language mapping practices that are more twenty-first century and digital in nature. Building upon the second wave, this third wave could be thought of as 'action-oriented,' as it accounts for changing technologies and demonstrates concrete solutions, practices, and technologies that address previously identified issues.

Separating these spurts of language mapping research into waves is important because the technological landscapes are so different for each of them. While the Internet was virtually unheard of during the first wave and many individuals had yet to purchase a personal computer, they were nearly ubiquitous among academic circles by 2010 and are almost the sole domain of

${ }^{25}$ Volume 1: Theories and methods (Schmidt \& Auer, 2010). Volume 2: Language mapping (Lameli, Kehrein, \& Rabanus, 2010). Volume 3: Dutch (Hüning, 2015). 
language mapping work in 2020. Along with each new technological innovation and domain of usage come new capabilities, as well as new ethical considerations. Because of this, it is important to be aware that while each wave has considerable similarities and can be threaded together as an evolving field of study, the nature of the research can differ dramatically.

The following sections and subsections of Part 1 focus on bringing these issues to light in a manner similar to what has been identified in this dissertation as first-wave and second-wave language mapping research. Part 2 is much more 'third-wave' in its practice and demonstrates the use of tools that can be of benefit to language mapping. Before this third-wave research can be covered, an in-depth discussion from multiple perspectives of what language maps are in the first place is necessary. What follows in this section then is an attempt at grasping the multidimensional nature of language maps and mapping.

\subsection{Language maps.}

Girnth (2010) explains that linguistic maps "connect topographic and linguistic information and document the distribution of linguistic features in space" (p.98), and distinguishes them from language maps, which "present information on the number and distribution of the languages in a region” (p.99). In this way, linguistic maps focus on internal features of languages (such as the sound system), while language maps focus on external features (such as languages' names) (Luebbering, 2013, p.45). In addition, Thun (2010, p.507) describes language maps as the older form of mapping that effectively focuses on the distribution of people, while linguistic maps focus on linguistic phenomena. Though Swiggers (2010) stresses that that linguistic and language mapping are separate (sub)disciplines with differing aims, methods, and implications (p.272), he also notes that both use the map as a medium, are based off geographic views of 
language, and assume that linguistic and spatial variation are correlated (p.273). Finally, Rabanus (2020) describes language maps as important in the visualization of the geography of linguistic features (p.105), the geography of languages (p.106), linguistic distances between varieties (p.107), language classification (p.110), processes in language history (p.111), mental maps (p.112), and correlations of linguistic and non-linguistic features (p.113).

For the purposes of clarity in this dissertation, linguistic maps will be referred to as "linguistic structure maps" while language maps will be referred to as "language distribution maps" when the distinction is necessary, as in Anonby, Murasugi, and Domínguez (2018, p.59) and Anonby and Sabethemmatabadi (2019, p.415). Such a distinction is perhaps unique to language mapping, due to the many and varied ways languages can be viewed and studied. When both are referred to collectively, they will both be called "language maps," as has been done in Luebbering (2011).

\subsubsection{Why language maps?}

To properly understand what language maps are and how they are used, I argue it is important that we look at what their purposes are, what potential they have for improving the disciplines of language and space, and how their application benefits certain areas of life. As such, language maps are of central interest to this dissertation for their potential to enable new perspectives on language which incorporate multiple dimensions and points of view. In both academia and the real world (to the extent that such a dichotomy exists), language maps function as a window to a more holistic and encompassing understanding of what it means for a language to exist in space, and what this existence means for its speakers. 
Perhaps best summarized by Montello (2002), "maps re-present the world by providing versions of truth for human minds to apprehend" (p.238). More specifically regarding language, Girnth (2010, p.101) states that the central purpose of language mapping is to document how linguistic phenomena are distributed across a given area. While Wood (2020, p.343) describes language maps as the content and not the tool (analogous to what is said over the telephone, not the telephone itself), I argue that they are also tools that tell a story and communicate a reality about languages and people.

Following this more general notion of maps as tools, Wikle and Bailey (2010, p.253), Upton (2010, p.144), and Luebbering, Kolivras, and Prisley (2013, p.383) all describe language maps as mechanisms through which novel comparisons can be made, and with which patterns in distributions - difficult to show with words alone — can be revealed. Girnth (2010, p.101) believes that language maps are more of a research tool than a product, as the amount of content communicable to an audience increases while the actual amount of information does not change (Bollmann, 2010, p.41). Ambrose and Williams (1991) support this notion by referring to language maps as "useful servants in linguistic study" (p.305). From the perspective of academia then, the map is something which linguists and other researchers can use to make geolinguistic information accessible to those outside the field (Upton, 2010, p.144).

Language maps are equally important outside of academia as well. In a practical sense, language mapping can directly impact non-linguists by enabling greater representation among communities whose languages are being mapped (Cenerini, Junker, \& Rosen, 2017, p.320) or by being a language-learning resource (Stone \& Anonby, 2019, p.447). As opposed to directly displaying linguistic information per se, an important consideration is that non-aggregated 
language maps first and foremost show the distribution of people, to the extent that languages are found within the people who live in a particular place (Thun, 2010, p.507).

As a means of representing linguistic data spatially (Girnth, 2010, p.98), language maps are important in spatio-linguistic research such as the study of dialectal distribution (Kirk \& Kretzschmar, 1992), features within dialects (Cenerini, Junker, \& Rosen, 2017), Indigenous placenames (Harmsworth, 1999, p.3), and pronunciation differences between speakers of different ages across different regions in Japan (Fukushima, 2014, p.40).

An important application to consider (as in Stone \& Anonby, 2019) is the potential for language maps to be used as pedagogical tools in the classroom. In what could be considered part of a typical education for primary or secondary school students in much of the world, language maps are often used as materials to illustrate linguistic and cultural distribution and diversity (Luebbering, 2011, p.8). However, Stone and Anonby (2019, p.447) identify a specific application of digital language maps in Indigenous Language Education (ILE) and revitalization. They argue that map-based multimedia such as sound recordings, images, and videos permit a more interactive learning environment, through which spatially-encoded linguistic information can be displayed and transmitted more appropriately than from text alone, which is important both in ILE and elsewhere in education ${ }^{26}$ (Olariu \& Olariu, 2014, p.76).

Outside the educational sphere, Luebbering (2011, p.1) identifies a variety of possible language mapping applications more generally, including emergency services, marketing, and political discussions related to immigration. In addition, Van der Merwe and Van der Merwe (2007, p.4) claim that the information in their digital Linguistic Atlas of South Africa is intended

\footnotetext{
${ }^{26}$ Education here and elsewhere in this dissertation is referred to in a very broad sense-as in Henze and Davis (1999, p.7) - and encompasses consciousness raising, learning in the home, informal apprenticeships, and even school programs.
} 
for use for educational purposes, as well as for decision makers, policy planners, marketing analysts, and any people, local or foreign, who might be interested. However, I have been unable to find any works in the literature which elaborate on any of these applications in significant detail. Suffice to say, an in-depth study of where and how languages maps can be applied in various dimensions of society is a fruitful area for future research but is lamentably outside the scope of this dissertation. One important aspect which has to date received more interest and attention in the literature involves the kinds of language maps that currently exist.

\subsubsection{Techniques in language mapping.}

Throughout the brief century and a half in which language maps have existed, numerous types of language maps have addressed different questions and needs in studies of linguistic spaces. Many maps differ simply due to the cartographic knowledge and tools available at the time of investigation (Luebbering, 2011, p.12). The account below showcases some of the kinds of language maps used in linguistic research but is by no means exhaustive. For existing summaries of language mapping techniques, see Ambrose and Williams (1991), Ormeling (1992), Girnth (2010), Luebbering (2011), and Rabanus (2018). 


\section{Large-scale chorochromatic map:}

All areas are coloured and classified. Linguistic regions do not generally overlap, meaning that only one language/ language family is shown per area. This form of mapping is qualitative, meaning that the different regions are directly generated by the map authors, not by numerical calculations (Klawe, 1973, p.48).

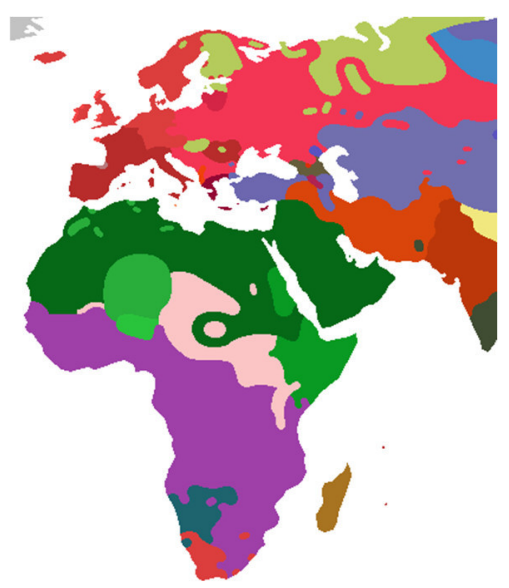

Constant (2011)

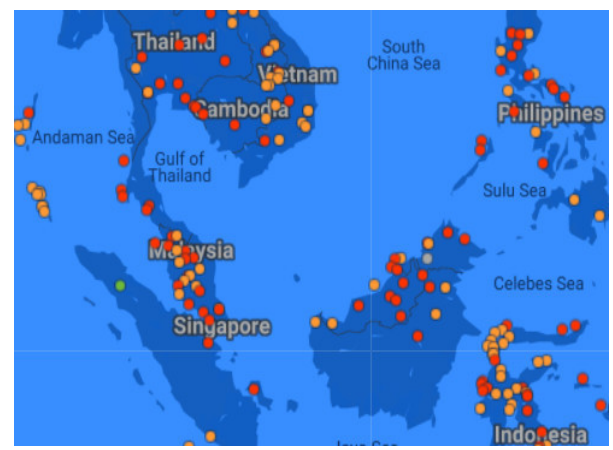

Endangered Languages Project

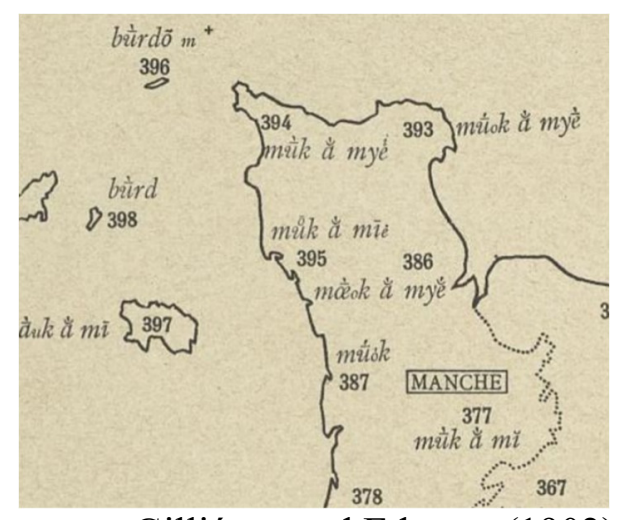

Gilliéron and Edmont (1902)

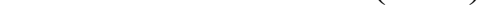

\section{Point-based map with labels:}

While this type of map is in principle the same as a dot map in that a linguistic phenomenon is represented with a single point, this map employs labels along with numbers that are linked to an index elsewhere. This is a style especially common to early language maps. 


\section{Point-symbol map with isogloss:}

In this specific type of point symbol map, data points are differentiated by colour, size, and quantity, and are paired with an isogloss to illustrate regional distinctiveness.

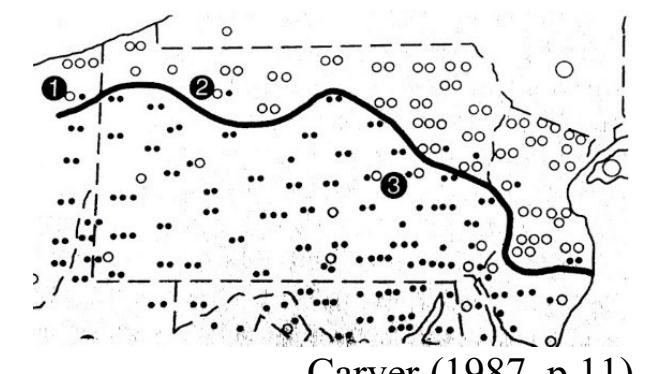

\section{Simple isoline map:}

Isoline maps use isoglosses, which are lines on a map that depict the boundaries of regions where a particular language or linguistic feature is used (Luebbering, 2011, p.12). In practice, isoglosses are often used to mark absolute differences between language varieties or between linguistic phenomena such as speech sounds (where they are called isophones), morphemes (isomorphs) or word choices (isolexes) (Girnth, 2010, p.122).

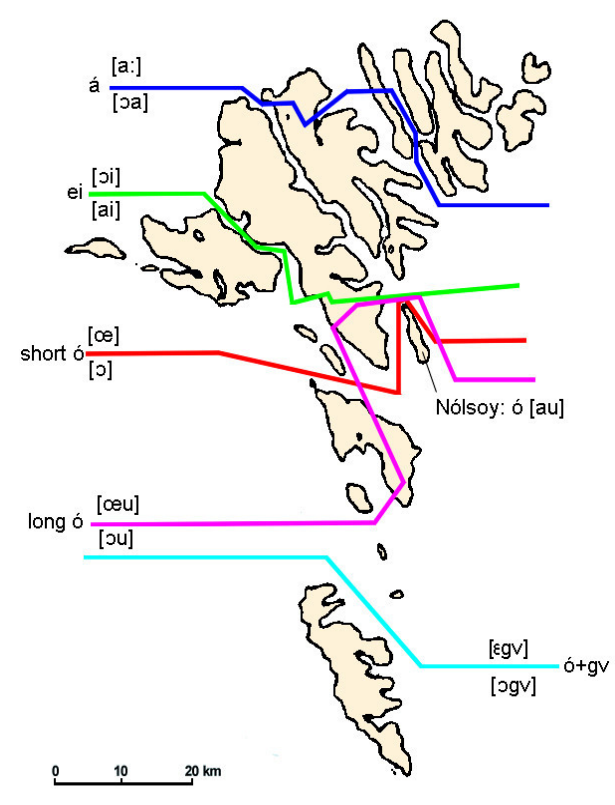

\section{Isogloss bundle map:}

As single isoglosses can suggest a region's homogeneity along with an overtly clear break between varieties (Luebbering, 2011, p.15), bundling isolines together can reflect regions' degrees of separation more effectively (Girnth, 2010, p.114).

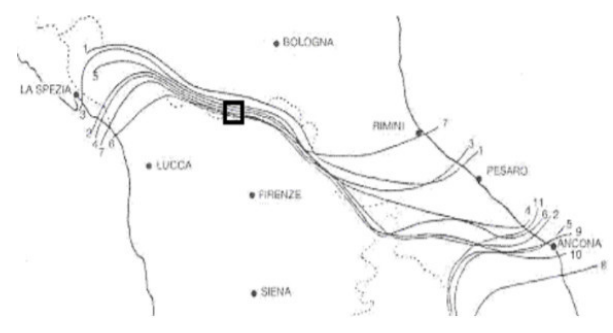

Filipponio and Nocchi (2010, p.5) 


\section{Mutual intelligibility maps:}

These maps are more abstract in nature, though they can in theory partially reflect language varieties' actual relative placements. The goal of these maps is to emphasize gradients of relatedness or intelligibility.

\section{Directed line map:}

A principal objective of the directed line map is to indicate the direction movement of a language variety or phenomenon. This form of mapping is useful in historical linguistics, where researchers wish to visualize linguistic spread over a given area (Rabanus, 2018, p.354).

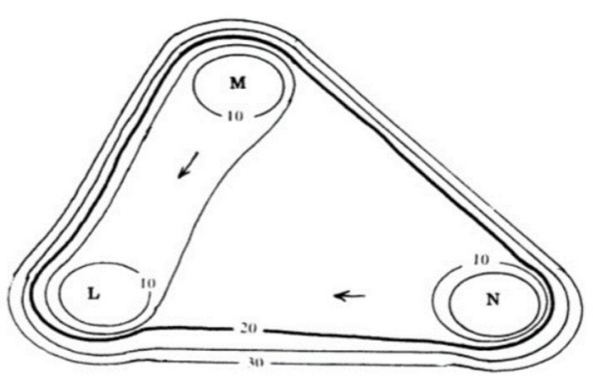

Egland et al. (1978)

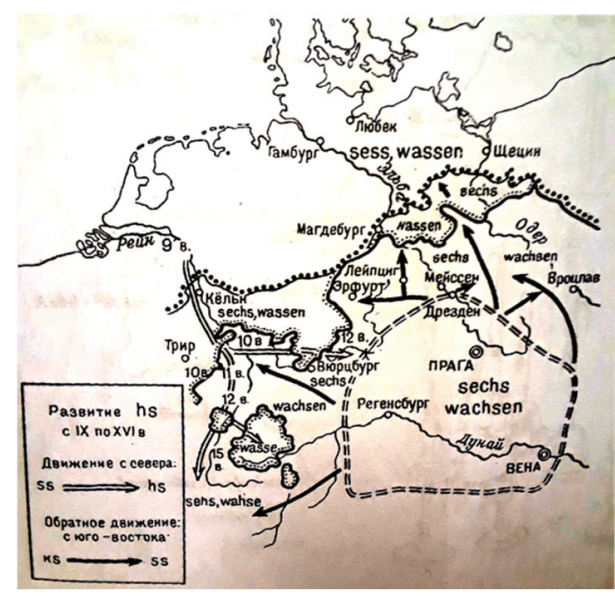

Schirmunski (1956, p.374)

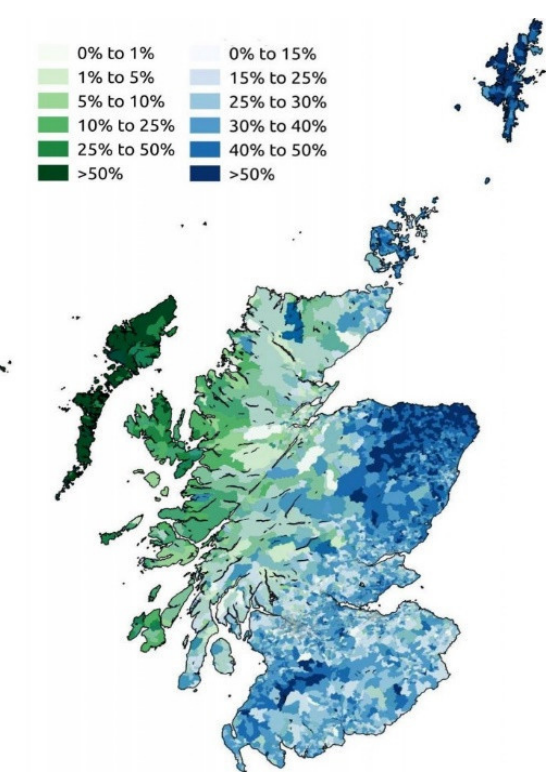

Cafolla (2011) administrative and geographic boundaries, or can be generated from points using Voronoi polygons. 
Heat map / Intensity map:

A quantitative map that, similar to a quantified choropleth map, can display the frequency or prevalence of a particular linguistic behaviour. Unlike the choropleth map, heat maps depict linguistic phenomena and their placement (originally in the form of point data) as continuous and gradated (DeBoer, 2015, p.39).

\section{Qualitative area map:}

In this form of mapping, regions that have identical data form continuous areas. The end result is often a chorochromatic map, which also contains accompanying data (Rabanus, 2018, p.355).

\section{Interpoint map (Honeycomb mode):}

This kind of quantitative map consists of multiple polygons with sides whose darkness, colour, and thickness increase with similarity (or dissimilarity, depending on the author's choice) to a language variety or phenomenon. (Goebl, 2007, p.78). Here, this digital map is applied to data from Gilliéron and Edmont (1902).
BALTIMORE

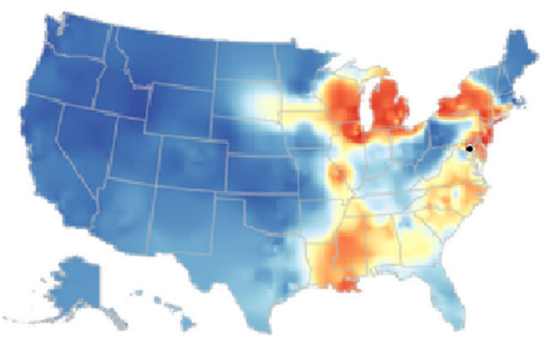

Do you pronouce cot and caught the same?

different

Vaux and Golder (2003)

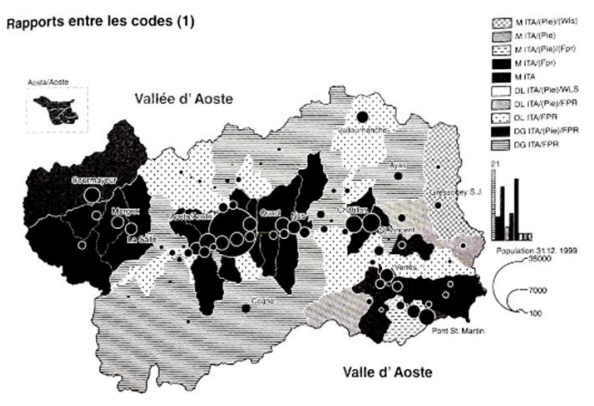

Dell'Aquila (2010)

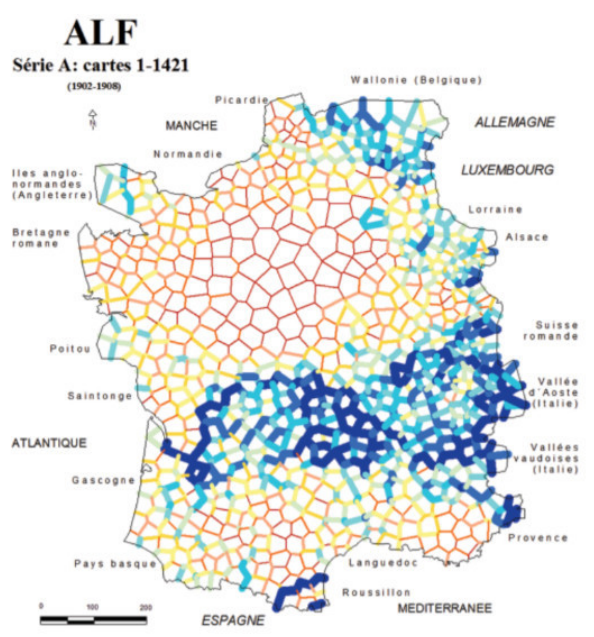

Goebl (2018, p.146) 


\section{Contour plot:}

Similar to a heat map or an interpoint map, a contour plot displays the degree of distinction from a sample (in this case the aggregated pronunciation distance from Standard Dutch) as a function of longitude and latitude. The black lines (isoglosses) represent calculated aggregated dialectal boundaries. The lighter the colour, the greater the distance (Wieling, 2012, p.90).

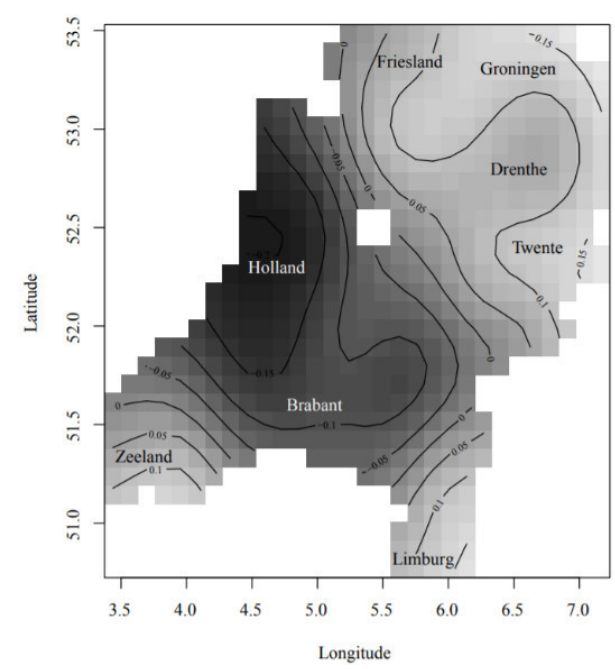

Wieling (2012, p.90)

\subsubsection{Presenting language maps.}

It is important to note that while many of these above maps readily occur in paper form, others have been generated and - in the case of the Endangered Languages Map — are primarily accessed online. Notably, many previous accounts of language mapping styles mostly involved paper maps, as they were developed at a time before the digital production of language maps was as ubiquitous as it is now. ${ }^{27}$ While map viewing computer programs continue to exist, the use of web services on the internet enable users to view and interact with maps on their smartphones, tablets, and laptops, in addition to desktop personal computers (Kretzschmar, 2015, s.3.3.2). It is thus important that we account for different ways such above maps may be presented in the digital sphere, as their medium of presentation can have important effects on how the linguistic data is perceived and used by audiences (Figure 21).

${ }^{27}$ See Ambrose and Williams (1991), Ormeling (1992), Girnth (2010), Luebbering (2011) specifically. 
Figure 21: Four different digital language map presentation styles.

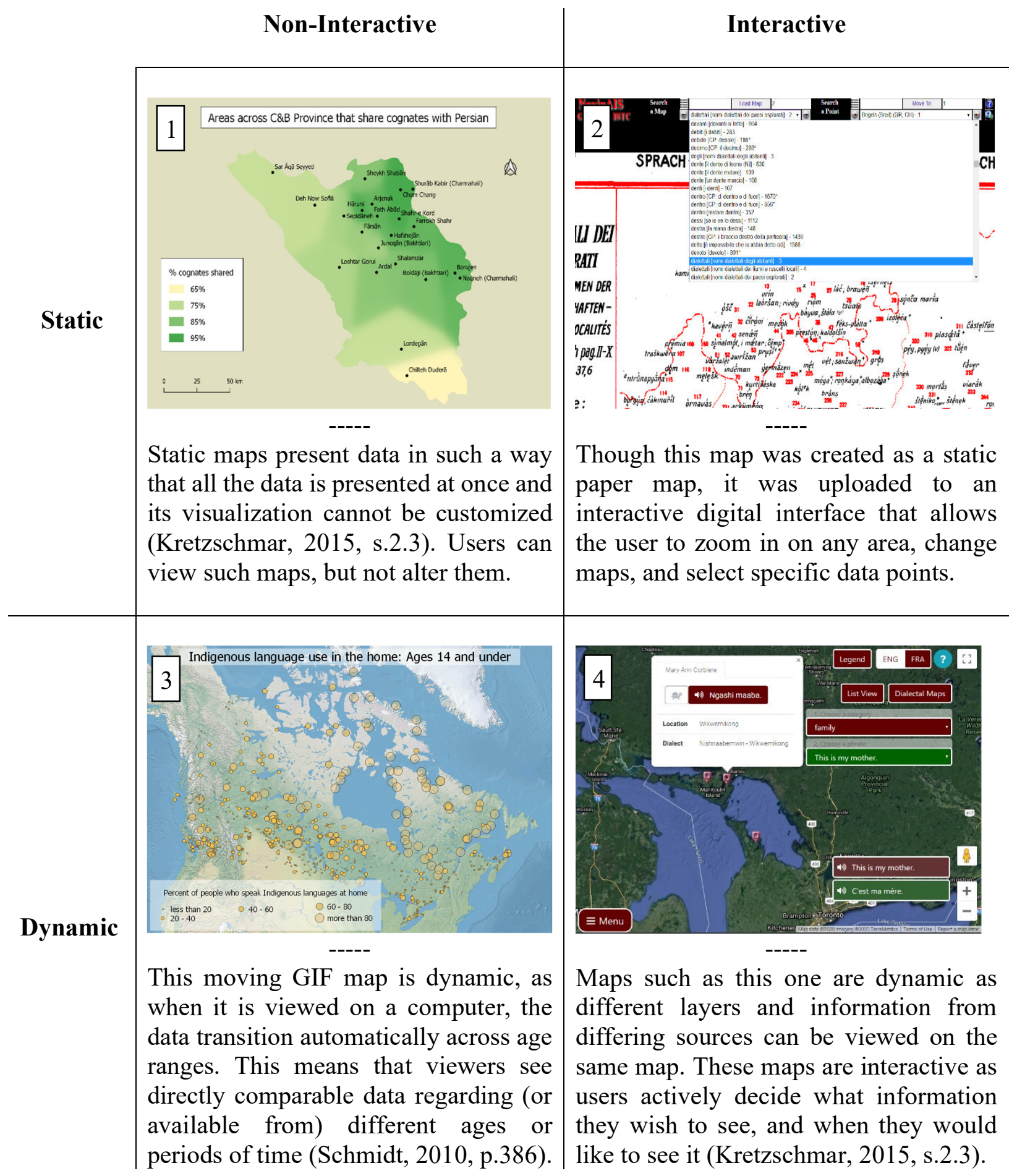

1. Own work, data acquired from Anonby, Taheri-Ardali, et al. (2015-2020).

2. Atlas linguistique et ethnographique de l'Italie et de la Suisse méridionale (Tisato, 2018).

3. Own work, data acquired from the 2016 Canadian Census.

4. The Algonquian Linguistic Atlas (Junker et al., 2005-2020). 
Because research and development tools are increasingly versatile in how they are used to design and implement maps, they can present information in a number of different modalities that suit the data. In contrast to the temporally static snapshots of language characteristic of paper maps, GIS and other digital forms of mapping allow for animations, differing modalities (such as audio recordings, videos, etc.), and enhanced interactivity to help researcher and viewer alike gain insights into spatial linguistic phenomena (Hanewinkel \& Losang, 2010). Language maps can use animations that depict a change over time, patterns of movement, or internal dispersions of characteristics within languages that may change over a geographic area or through social aspects. Recordings of language samples can both show the reader what the language sounds like and indicate how both preferred forms and the languages themselves change over a given geographic area (for example, see Junker et al., 2005-2020).

The ability for digital interfaces to superimpose layers of maps and relate them to each other means that, as opposed to more traditional forms of aggregate mapping that compared maps conveying different information side by side, different kinds of information can be placed alongside others at the viewer's discretion. For more information on how digital maps can use animations and interactivity to convey dynamic aspects of language, see Hanewinkel and Losang (2010). Importantly, this technique can be applied either to the creation of new maps, or the enhancement of older maps originally created in paper form (Rabanus \& Kehrein, 2010, p.375), meaning that previous linguistic research is not necessarily invalidated by more current digital methods. Quite often, these maps do not occur alone, but are instead part of a greater collection of works, which aim to showcase a particular issue. 


\subsubsection{Language atlases.}

In general mapmaking, Kraak and Ormeling (2013) define an atlas as “[an] intentional combination of maps or data sets, structured in such a way that specific objectives are reached" (p.159). In language mapping more specifically, Lameli (2010) defines language atlases as "a collection of thematic maps showing the regional distribution of language" (p.572). In the case of early atlases such as the Atlas Linguistique de la France (Gilliéron \& Edmont, 1902), paper maps have often been compiled in book format in large and multiple volumes (Bollmann \& Koch, 2001, p.39), and are then shelved and available to those with physical access to the archives or libraries where they were stored. Following the widespread adoption of computers and other digital technologies, Lameli (2010, p.585) notes that virtually every atlas has been realized using computers, including those which have previously existed in paper form but have since been transferred to a digital modality.

While atlases have been a driving force in much of the monodimensional geolinguistic research $^{28}$ to date, atlases have also facilitated pluridimensional geolinguistic research (Thun, 2010, p.509), which controls for multiple sociolinguistic and extralinguistic variables such as the distribution of age, sex, and profession (p.508). Notably, Thun (2010, p.523) argues that pluridimensional research strives to avoid simplifying unwieldy levels of variation found in spatial studies of language to more closely reflect the reality of the phenomena in question. When in physical (typically book) form, Thun (2010, p.506) argues that atlases can host a logical series of maps which each focus on a different aspect or dimension of a linguistic phenomenon in order to tell a story about the data. Creating new maps or uploading existing ones into any variety of digital atlases further facilitates such pluridimensionality (Murasugi, 2019, p.393)

\footnotetext{
${ }^{28}$ That is, looking at only one aspect of language at a time.
} 
through accompanying statistics, images, and sound recordings (Peterson, 2007, p.45). The process of telling a story about a language or linguistic phenomenon is of key interest in language maps, and as such, deserves special attention in this dissertation.

\subsection{The practice of language mapping.}

In a most general sense, language mapping is the act of making a map that displays information about a language, its parts, or its distribution. Girnth (2010, p.101) states that the central purpose of language mapping is to document how linguistic phenomena is distributed across a given area. Upton (2010, p.144) observes that language mapping is a valuable means for linguists to make complicated linguistic information understandable to those without a background in linguistics, making it useful in mobilizing information and making it accessible. ${ }^{29}$ In this way, Girnth (2010, p.101) infers that language maps are more of a research tool than a product, as the amount of content communicable to an audience increases while the actual amount of information does not change (Bollmann, 2010, p.41). Language mapping can also directly impact non-linguists, such as by enabling greater representation among communities whose languages are being mapped (Cenerini, Junker, \& Rosen, 2017, p.320) or as a languagelearning resource (Stone \& Anonby, 2019, p.451). What follows is a brief account of how language maps have been made in the past and what they have been used for.

\footnotetext{
${ }^{29}$ The term accessibility is specified here as in Cartwright (2003) to refer to the "convenience in accessing data anytime and from anywhere" (p.45).
} 


\subsubsection{A history of language mapping.}

While putting languages on maps is a relatively new act, visual representations of space date back to ancient times (Lameli, 2010, p.568). The first known instance of putting languages on a map comes from Scultetus (1593), who attempted to indicate regional differences between German and Sorbian using coloured lines in his map Lusatia Superior (Lameli, 2010, p.572). A century and a half later, Hensel (1741) produced a variety of hand-drawn maps focusing on the distribution of contemporaneously known languages, language families, and writing systems in Europe, North America, Africa, and Eurasia (Figure 22).

Figure 22: An early map depicting languages and their writing systems in Africa (Hensel, 1741).

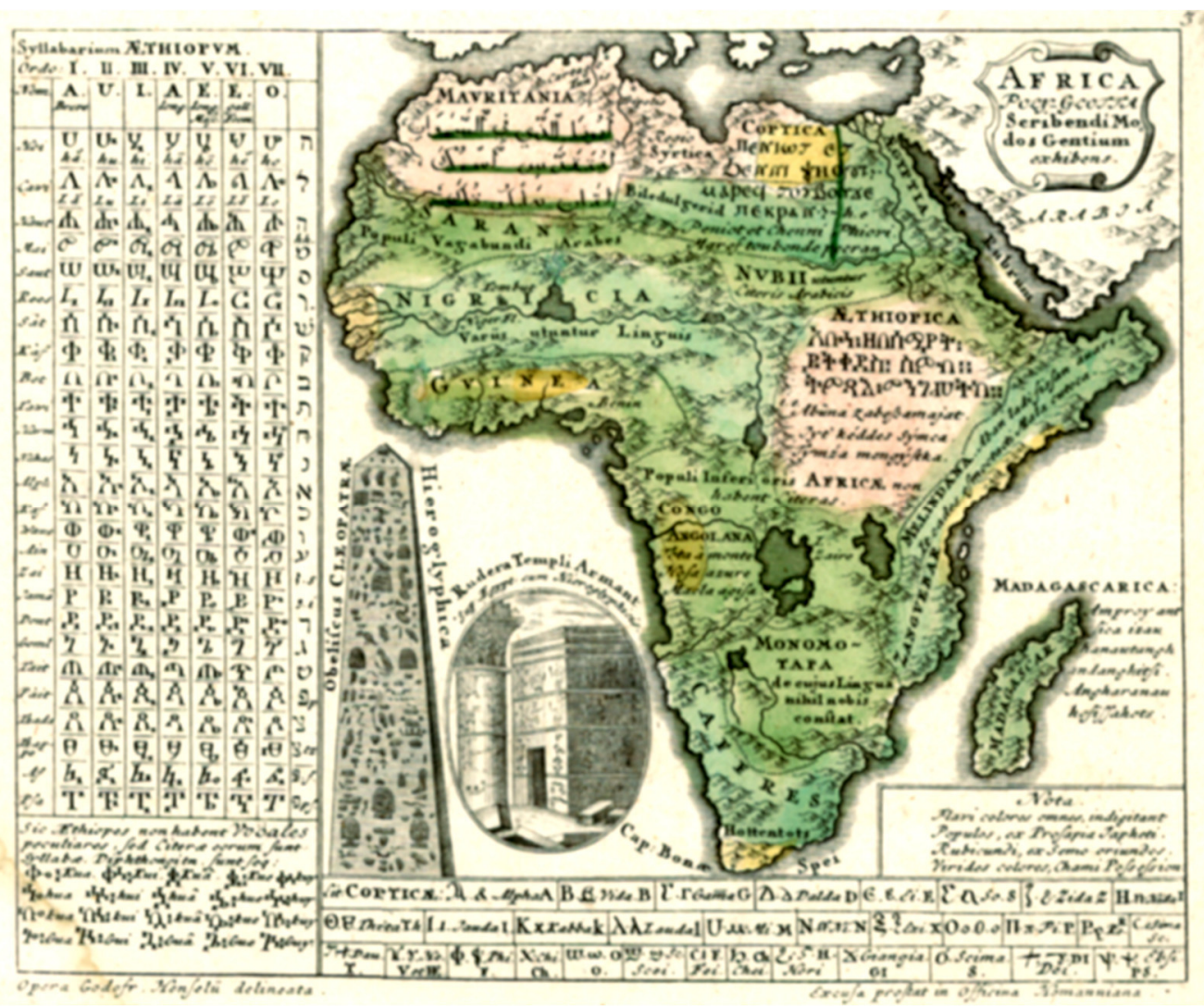


Maps covering languages remained the exception. Recognizable language maps as part of serious endeavours only started in the late nineteenth century and mainly focused on the distribution of dialects in Germany and France (Luebbering, Kolivras, \& Prisley, 2013, p.384; Swiggers, 2010, p.273). Nearly a century after Hensel (1741), Schmeller (1821) published Kärtchen zur geographischen Uebersicht der Mundarten Baierns (A little map on the geographic overview of the Bavarian dialects), which shows an exhaustive distribution of dialects across the Kingdom of Bavaria (Lameli, 2010, p.159) and may be the first example of a modern dialect map (Scheuringer, 2010, p.158). Twenty years later, Bernhardi (1849) developed a map of linguistic boundaries between German-speaking areas and surrounding linguistic regions with the goal of fostering a geographic understanding of where 'German' people lived (Scheuringer, 2010, p.159). Importantly, Bernhardi was perhaps one of the first to consider atlases useful in creating logical sets of maps of linguistic regions (Scheuringer, 2010, p.160). While Bernhardi's vision of a comprehensive linguistic atlas was never realized, it did set the stage for others to continue in his place.

The first such notable linguistic atlas was made by Georg Wenker, who in 1877 created a map called Sprachkarte der Rheinprovinz, which displayed variations of 42 Standard German target sentences throughout settlements and villages in an area known at the time as the Northern Rhine province (Scheuringer, 2010, p.160). From here, Wenker conducted more studies from 1889 to 1923, culminating in the Sprachatlas Des Deutschen Reichs, which was the first linguistic atlas to display a country's dialectal variation across space (Rabanus \& Kehrein, 2010, p.376). This earliest example of an atlas solely devoted to the distribution of language varieties (Lameli, 2010, p.574) is especially remarkable given that it was the first to make use of 
isoglosses to denote the boundaries of language varieties and their features (Scheuringer, 2010, p.158).

During this time, emerging fields such as Romance studies focused on the interconnections between ethnolinguistic groups. Within these studies, a major debate arose surrounding the feasibility of drawing boundaries between language varieties, which was often exacerbated by local and political conceptions of how closely (or distantly) related linguistic groups were to each other (Swiggers, 2010, p.273). It is from these debates that refinements to isogloss techniques began to take place, including Gauchat's (1903) use of bundles of isoglosses to denote more gradual linguistic changes and 'nuclear linguistic landscapes' (Swiggers, 2010, p.274). Importantly, many of the debates surrounding the use of line boundaries in language mapping continue to this day. In some of the first attempts to resolve this issue objectively and classify language varieties according to 'exceptionless phonetic laws' (Swiggers, 2010, p.273), the Swiss researcher Gilliéron (1881) developed the Petit atlas phonétique du Valais roman, while Millardet (1910) developed the Petit atlas d'une région des Landes.

In 1880, two years after Wenker had begun planning his Sprachatlas, Gilliéron independently began planning an atlas of a similar nature regarding the distribution of Romance language varieties across France, Belgium, the Channel Islands, and Switzerland (Lameli, 2010, p.574). Unlike his previous work in phonetic mapping, the Atlas Linguistique de la France (ALF) (Gilliéron \& Edmont, 1902) shifted his focus from sounds to words (Swiggers, 2010, p.275) and language mapping from a simple curiosity to a rigorous endeavour which made use of first-hand linguistic data that had been gathered following strict guidelines (p.274). In their study between 1897 and 1902, Gilliéron and Edmont (1902) focused on 1920 different lexical items and sentences. Each item was shown with 639 data points representing communities where the 
information originated, obtained from 735 contributors of varying age and gender. While Gilliéron had originally intended on visualizing simple dialectal variation, he and Edmont began to understand that the large number of lexical items they had gathered could also be used to demonstrate degrees of genealogical relatedness between individual varieties, thus creating the possibility for empirically-derived family trees (Swiggers, 2010, 275).

Though large-scale language mapping initiatives started much later in the United Kingdom than elsewhere in Europe, the country's dialectal diversity made research an appealing prospect (Ramisch, 2010, p.238). Originally, much of the dialectal research in Britain attempted to study varieties spoken in the past to understand why forms existed the way they did (Ramisch, 2010, p.240). In perhaps one of the most noted examples of twentieth-century English dialect surveys (Ramisch, 2010, p.240), the Survey of English Dialects (Dieth \& Orton, 1952) focused on nonstandard varieties of English, which were considered by the researchers to be manifestations of more antiquated speech. The Survey of English Dialects employed rigorous techniques to ensure the consistency and reliability of the data in ways that differed from the ALF; namely, a team of specially-trained linguists interviewed 955 contributors controlled to be NORMs (Ramisch, 2010, p.241). As a result, few pluridimensional conclusions can be drawn from this study that involve gender, social class, or age. That being said, the data that were used achieve a degree of uniformity that is rarely seen in other contemporaneous language atlases. As a pioneering study, this survey created the base from which many different atlases could be produced, such as the Atlas of English Sounds (Kolb, 1979), which is exemplified in Figure 23 below: 
Figure 23: "plough" as mapped across England in the Atlas of English Sounds (Kolb, 1979).

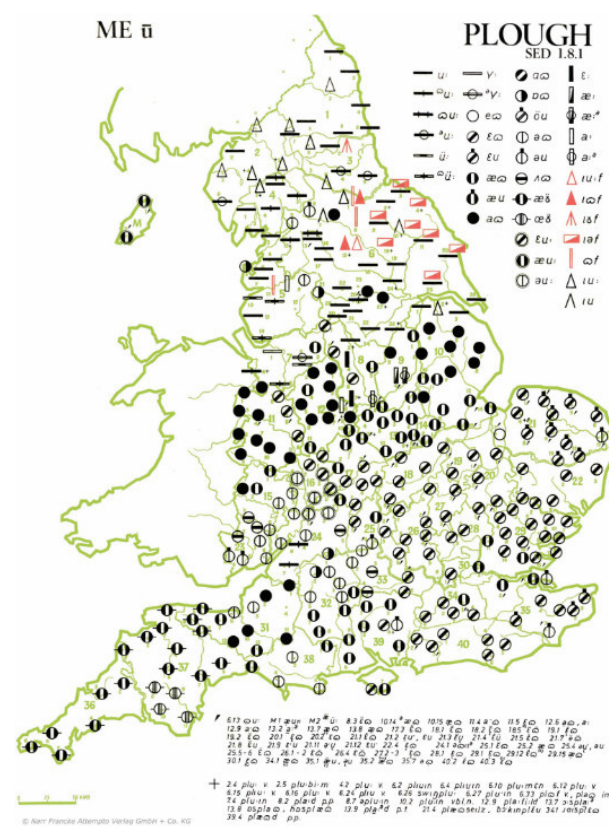

Across the ocean in the United States, the twentieth century saw a number of varied language mapping projects that focused primarily on the distribution of dialects and dialectal features. In addition to the numerous English and Irish dialects spoken by early settlers, contact with African peoples brought over as slaves, Indigenous peoples, and successive waves of immigration from different parts of Europe contributed to many unique linguistic features that developed in particular areas of the United States (Wikle \& Bailey, 2010, p.254). In the nineteenth century, considerable westward expansions spread many of these dialectal variants to new regions, where contact with influences such as Spanish in the American Southwest contributed to further variation. Interestingly, Wikle and Bailey (2010, p.255) note that the emerging European language atlases_namely Wenker's (1887) Sprachatlas and Gilléron and Edmont's (1902) ALF - attracted the attention of American language scholars, who founded the American Dialect Society in 1889. 
While numerous projects resulted from such a collaboration (see Wikle and Bailey, 2010 for more information), one noteworthy example includes the Word Geography of the Eastern United States (Kurath, 1949), which made use of isogloss boundaries of varying thickness to portray specific dialectal regions and boundaries in the eastern United States. Reminiscent of the Romance scholars' debates half a century earlier in Europe, a major source of disagreement that began to emerge in the wake of this map as well as others of the time revolved around the accuracy of isogloss boundaries and bundles, which were increasingly acknowledged to be both arbitrarily placed and defined by the map authors (Wikle \& Bailey, 2010, p.257). In response to this, researchers such as Shuy (1962) developed a counting method that relied on a grid, whereby a honeycomb pattern could be achieved by drawing lines between the placements of data points corresponding to individual contributors. Following the second world war, American cartography began to gravitate toward statistical analyses, which quickly became adopted into language mapping practices. In the latter half of the twentieth century, the Dictionary of American Regional English (Cassidy \& Hall, 1985) gathered lexical data through face-to-face interviews using 1847 questions divided into 41 categories. Importantly, this mapping project was one of the first to employ the use of computers in the data analysis and map production process, signaling a dramatic innovation in language mapping (Wikle \& Bailey, 2010, p.260).

Outside of the Anglosphere, investigations into the regional distribution of language varieties in the Netherlands date back to as early as Junius (1567). However, the first geographical investigation into Dutch dialects involving maps did not occur until 1898, when te Winkel (1898) published a survey map of Dutch dialects (Kruijsen \& Sijs, 2010, p.180). In a similar tradition to Gilliéron and Edmont (1902), Schrijnen (1917), along with colleagues van Ginneken and Verbeeten, conducted a large-scale survey in the southern Netherlands which 
obtained approximately 350000 pieces of data from 194 different locations (Kruijsen \& Sijs, 2010, p.184). From this data, Schrijnen (1917, p.34) developed a map of the lexical item "butterfly," which is regarded by Kruijsen and Sijs (2010, p.184) to be the first lexical map of Dutch-speaking regions. After 1925, other dialect surveys were initiated, leading to multiple atlases of the Dutch language area through a variety of media. ${ }^{30}$

Following a similar time frame, the Language Research Commission in Japan carried out two studies between 1905 and 1906, which focused on phonetic and grammatical dialectal differences across the country and culminated in 66 maps (Onishi, 2010a, p.334). During this time, researchers such as Shimmura (1904) identified a boundary between eastern and western dialects of Japanese, which were reflected using isoglosses as shown in Figure 24 (Onishi, 2010b, p.124). As described by Onishi (2010a, p.334), a Japanese interest in dialectology appears to have originated from within Japan, starting with a collection published in the year 1775 by Gozan Koshigaya of over 4000 dialectal words from across Japan.

\footnotetext{
${ }^{30}$ Kruijsen and Sijs (2010) outline several atlases and provide links to those available online.
} 
Figure 24: Isoglosses reflecting the east-west dialectal divide in early twentieth-century Japan.

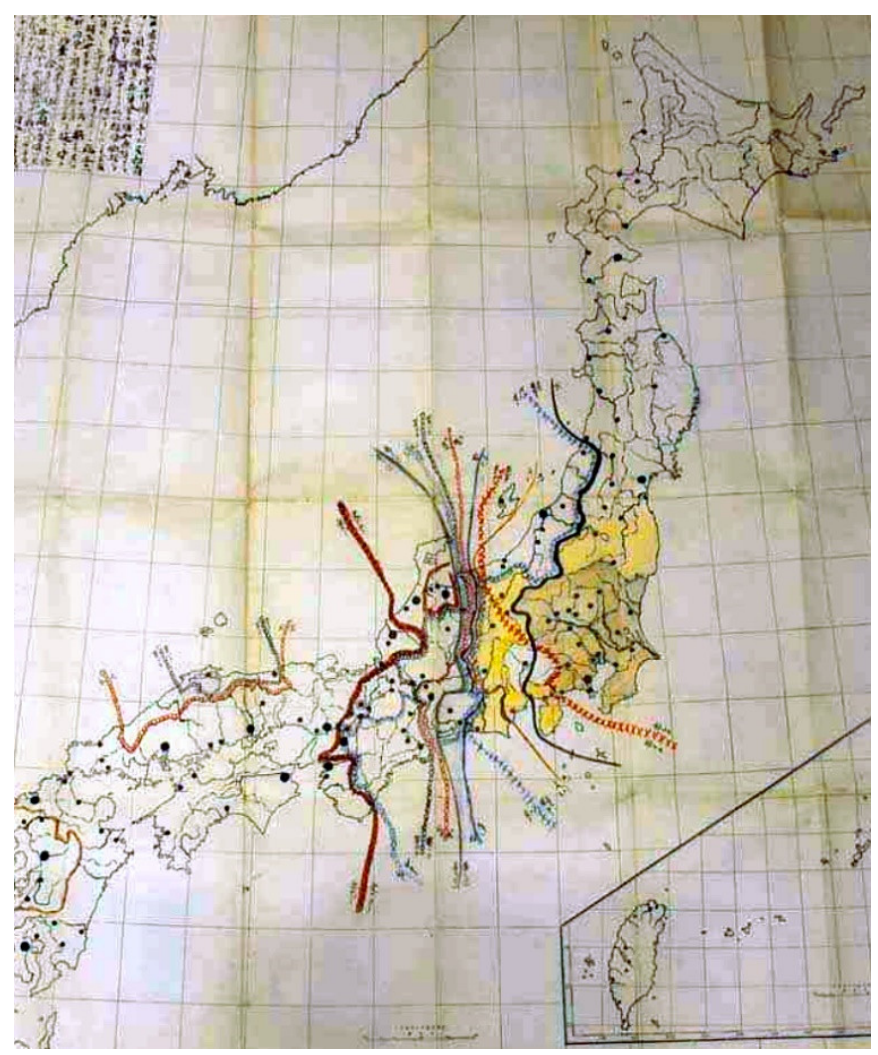

Shimmura (1904), in Onishi (2010b, p.124)

Half a century later, in 1966, the National Research Institute of Japan published the Linguistic Atlas of Japan, which upon completion of the sixth volume in 1974, included 300 maps covering 2400 specific locations (Onishi, 2010a, p.336). In the following decade, the same research institute conducted a series of surveys to investigate the distribution of grammatical items, such as adjectival forms (Onishi, 2010a, p.336). In the most-recent volume in 2006, 350 maps covered 807 locations, along with 120 small-area linguistic atlases.

At the turn of the $21^{\text {st }}$ century, advances in digital technologies such as GIS meant that quantitative methods such as large-scale frequency analyses of lexical items became feasible in dialectal research (Lee \& Kretzschmar, 1993, p.543). Improvements in digital devices' 
performance meant that linguistic data could be represented through modalities such as sound recordings and hosted on the Internet (Peterson, 2007, p.45). Furthermore, topographies not traditionally associated with the study of language, such as traffic, altitude, rainfall, and demography, could be paired more easily with linguistic data (Onishi, 2010b, p.129), which introduced new possibilities for pluridimensional mapping, as demonstrated in Thun (2010). As noted in Section 2.7.2, GIS and other digital technologies have created new possibilities for restoring and extending older atlas projects, as well as creating new studies that employ multimedia and real-time data.

\subsubsection{Digital language mapping in academia.}

In the $21^{\text {st }}$ century, dynamic and interactive digital maps are increasingly popular ways of mapping many kinds of information and their functionality is expanding (Trbovich, Lindgaard, \& Dillon, 2019, p.36). Through rapid, detailed, interactive displays that can be accessed from a computer interface, the digital sphere offers new possibilities for mapmaking and map reading (Monmonier, 1991, p.3). As highlighted by DiBiase, MacEachren, Krygier, and Reeves (1992, p. 201), while quantitative data about the environment used to be in short supply, they are increasingly available in quantities which cannot be reasonably be worked with in their raw form. In light of this plethora of available data, digital machines in turn allow for new ways of processing, summarizing, visualizing, and identifying patterns in data. Digital technologies are thus useful and important tools in language mapping and can occur in different ways.

For the purposes of this dissertation, computer generated maps refer to static maps that are either created using photo-editing software, or are previously existing paper maps which have been digitally uploaded and manipulated in some way to enhance clarity or emphasize additional 
features (for more information, see Rabanus \& Kehrein, 2010). Though many early works in language mapping that made use of digital technologies still released text-based publications, improvements in home workstations as well as server-based technologies have allowed for information to be republished directly on the internet (Rabanus \& Kehrein, 2010, p.375). A notable and perhaps most basic example of this kind of work includes the scanning and uploading of previously existent paper atlases onto a digital interface, as in Figure 25 below:

Figure 25: An uploaded linguistic and ethnographic atlas of Italy and southern Switzerland.

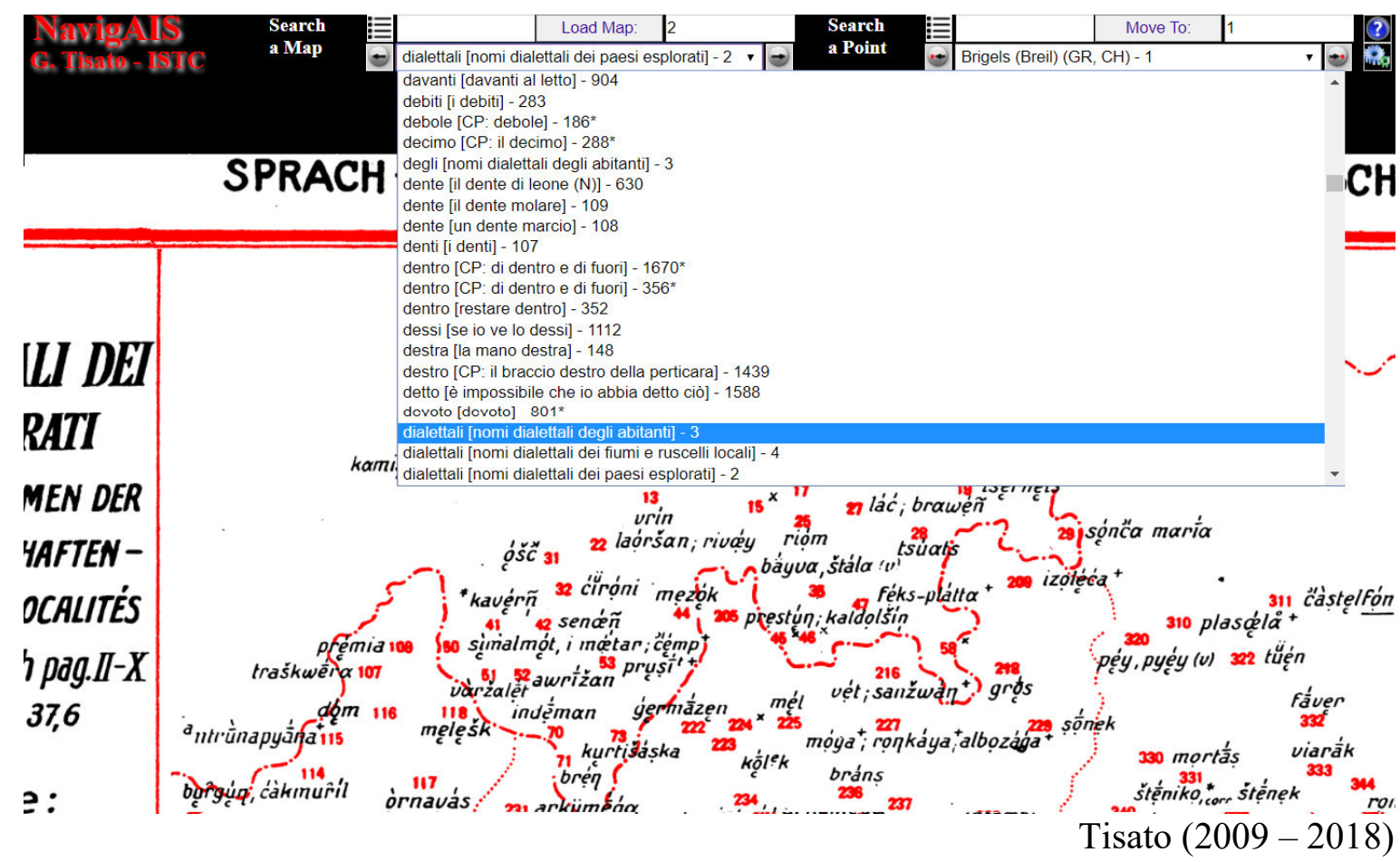

In making digitized maps of this kind, the basic configuration of the map remains the same while each datapoint is highlighted and made more visible when selected from a drop-down menu at the top of the interface. In addition, users have the option of clicking on any part of the map to zoom in to specified areas and can easily shift through different maps with the use of a 
menu. Unlike paper maps, their digital existence means they do not deteriorate physically with repeated use, and are not limited by the size of an available paper surface (Rabanus \& Kehrein, 2010, p.376), and can be accessed anywhere with an internet connection (p.377). Similarly, entire collections of maps can be displayed side-by-side on a computer, whereas paper maps would require large physical atlases (Rabanus \& Kehrein, 2010, p.380).

Importantly, previously existing paper maps can be archived, preserved, and hosted on an online server, making them easily accessible as part of a database. One such example includes the Atlas Linguistique de la France (Gilliéron \& Edmont, 1902). Due to the astounding level of detail in this atlas, the paper maps are large, numerous, and expensive, meaning they are typically held in libraries and archives. As a result, access to the atlas has historically been limited to those with a membership to one of these centres. However, over 1920 of the original maps were uploaded by the GIPSA (http://www.gipsa-lab.grenoble-inp.fr/) and PERSYVAL (https://persyval-lab.org/) labs to the online CartoDialect database (http://ligtdcge.imag.fr/cartodialect3/index.html), making them available for download free of charge, as in Figure 26 below. 
Figure 26: Online version of the term "abeille" (bee) from the Atlas Linguistique de la France.

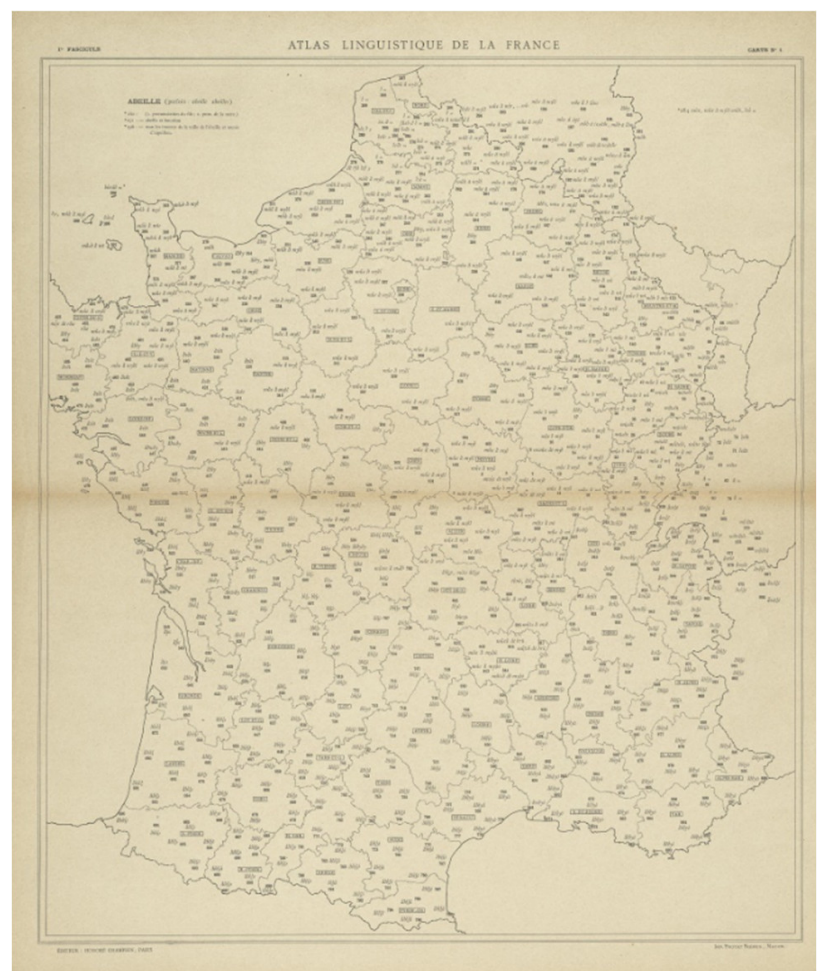

Gilliéron and Edmont (1902)

In addition, computer generated maps be created through a number of different picture manipulation programs. While this dissertation does not aim to promote any program over another, programs such as Microsoft Paint allow for the manual construction of basic maps with hand-drawn points, lines, and polygons, as in Figure 27 below. While detailed and beautiful maps can be produced with considerable effort, these maps likely do not follow a specific projection system, as all elements found on the map are created at the map author's discretion, meaning that errors on the map can be difficult to control for. That being said, the added freedom of being able to create graphic images from scratch means that authors can put exactly what they wish to on a map. 
Figure 27: Language distribution map of French in Manitoba made using Microsoft Paint.

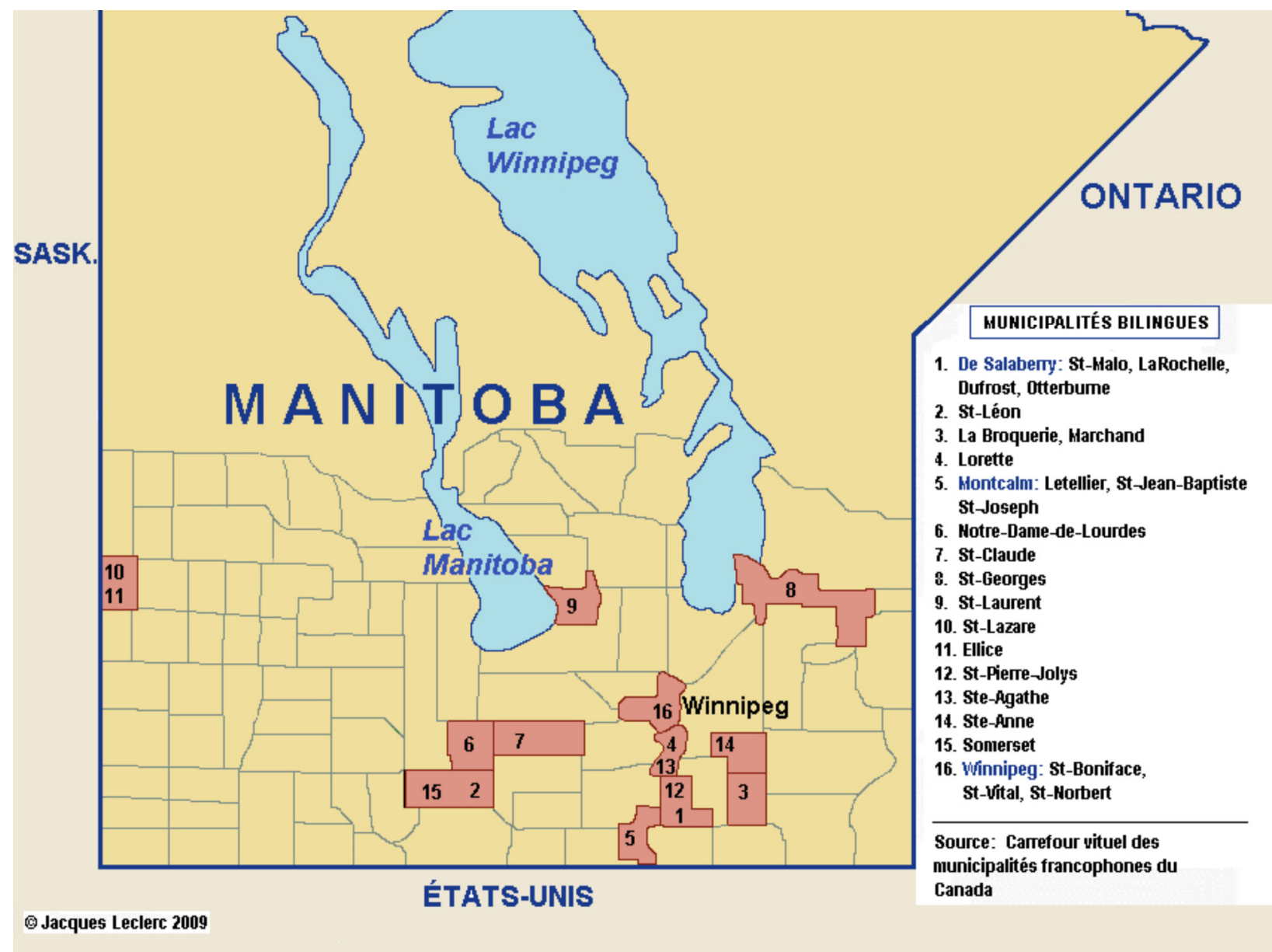

Leclerc (2009)

\subsubsection{Language mapping and GIS.}

First introduced in Section 2.7.2, GIS and similar quantitative software approaches have allowed for a more systematic integration of previously existing and newly gathered linguistic information into digital and online mapping interfaces. While presenting exciting new opportunities for language mapping projects and research, Goodchild (2020, p.3674) describes GIS as disruptive to specific patterns of thought surrounding what it means to map languages. As such, GIS not only opens up new applications and methods in language mapping but also 
changes the methods that language mappers already utilize. At the same time, GIS also renders other methods obsolete, such as hand-drawn maps.

As perhaps one of the earliest efforts to integrate dialectology and GIS, Briscoe (2009, p.9) discusses the Generalized Linguistic Atlas Printing System (GLAPS), which is a computer program developed by Ogino (1980) that assists in data processing for studies of dialectal distances and similarities, such as in a perceptual dialectological study of subjective dialect division in Great Britain by Inoue (1999). More recently, an online program with a similar premise, Gabmap, has made the process of performing map-based quantitative dialectology more accessible to those with little computational experience (Nerbonne, Colen, Gooskens, Kleiweg, \& Leinonen, 2011, p.65). As shown in Figure 28, such a program can be used to calculate degrees of congruity between language varieties based on how similar word forms are to one another. 
Figure 28: Abstract Levenshtein distances of an Iranian province computed with Gabmap.

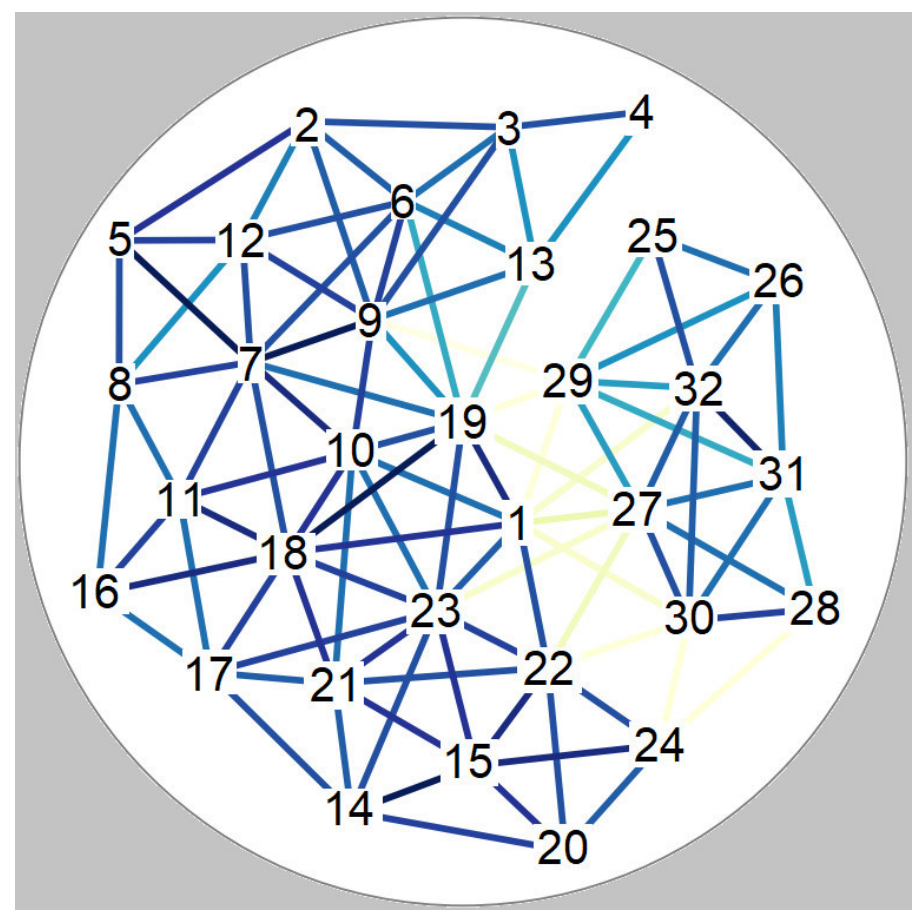

[Own work, data from Anonby, Taheri-Ardali, et al., 2015-2020.]

Each number corresponds to a particular language variety, while deeper blue colours correspond to stronger relatedness. Here we can observe two dialect clusters entirely unrelated to each other.

Despite the opportunities digital mapping techniques provide researchers and cartographers, challenges remain that compromise their ability to reliably impart information to audiences. Though the challenges in (1), (2), and (3) below are similar to those encountered in paper language maps, those found in (4), (5), and (6) are added issues that become evident with the use of digital information systems.

1. As is the case with paper maps (Luebbering, Kolivras, \& Prisley, 2013, p.384), digital language maps do not have a general set of rules and standards available to them (Hanewinkel \& Losang, 2010, p.431) despite recurring conventions. This means some 
maps will account for and communicate language-related information better than others, and that users will be required to spend time and energy adapting to new mapping frameworks. Though increased connectivity through global information networks means that researchers and cartographers have more opportunities to connect and communicate with one another, developing a standard system for creating maps becomes more difficult due to added factors associated with software development methods, digital atlas layouts, and sets of features available to the viewer.

2. In both paper and digital language mapping, most atlas projects are created by an individual or group holding an authoritative position for an intended audience (Ormeling, 2010, p.37), and are intended as a product to be used by the audience, who are held akin to consumers (Taylor, 2005, p.5). Though an ample body of research has been devoted to developing optimal ways through which the maps' information is presented (such as the use of symbols, legends, scales, etc.), the intended audience may not have the opportunity to offer any input during the production process. They are thus required to assume a more passive role in learning how to interpret the cartographical conventions for the map or atlas.

3. Though the medium of mapping may have changed, traditional assumptions that confuse national or regional political boundaries with linguistic ones may prevail, as they are determined more by researcher bias and disciplinary background than the medium being used. Though digital mapping techniques such as GIS have changed the way in which maps can be produced, orchestrated, and presented to a more public audience, emerging technologies do not necessarily influence the attitudes of the mapmakers. In other words, the nature of the instrument will not necessarily influence the nature of its interoperability 
(Monmonier, 2005a, p.19). As mentioned in (2) and by Ormeling (2010, p.22), prevailing attitudes towards how information should be plotted occur in part due to conceptual and power distances between author and audience. In turn, these distances have the potential to stifle communication and feedback between author and audience.

4. Though communication technologies make accessing digital language maps easier, one must possess some level of competency to use them in the first place. While many of us in the academic community take the digital technologies we use on a daily basis for granted, there are also many for whom computers, tablets, and smartphones are a source of confusion or apprehension. A lack of familiarity with contemporary digital technologies could be due to a variety of factors, such as age, education level, socioeconomic status, devices' difficulties with displaying information, or perceived irrelevance of said technologies. As is characteristic of many societies, there is a gap in knowledge between older generations and younger generations, often simply because members of the older generations have had more time to learn and experience their world. On the other hand, having grown up with a (comparatively) greater immersion in digital technologies, members of younger generations can perhaps intuitively grasp how digital systems work more easily than their elder counterparts (Schnürer, Sieber, \& Çöltekin, 2015, p.2).

5. Based in software, digital language maps require their developers to have a high degree of technical expertise in software development alongside a competency in data gathering and linguistic research. This results in either a large and expensive research team, or arduous and long projects for a few exceptionally trained specialists. This means that 
fewer people are able to contribute information and data to maps, so that the knowledge is in essence controlled by a powerful few.

6. Users of digital technologies in general may prioritize simply being engaged over the accuracy of the information or content. In what Odell (2019, p.xi) refers to as the attention economy, many of the digital technologies available to us are designed to capture our attention (and thus our data). Many authors of technology see the fact of our engagement as more important than our critical appraisal of what we see. Similarly, users often may not have the ability to devote their attention fully to the content, as they frequently experience information overload (Odell, 2019, p.18). As a result, users of digital maps as a collective may not be in the habit of assessing and analyzing the information they encounter in a careful and deliberate manner.

The main issues characteristic of both paper and digital maps identified here could thus be summarized as issues of voice and access. As atlases and their maps have typically been created by a particular authority as a product for a particular audience, map audiences tend to assume the passive role of receiving the information, adapting to the maps' layouts, and coming to a particular conclusion based on how the information has been presented. Being part of an audience is made possible through knowledge of how to access maps and related materials and sufficient money, connections, and subscriptions to obtain them. The issues of being located close enough to a source to physically obtain maps decrease when atlases are presented through a digital medium, provided one has a reliable broadband connection or computer equipment.

Without a voice, language communities risk either not being represented fairly and accurately, or not being represented at all. Sometimes, such exclusion may appear trivial or 
simply insulting to the affected groups, but may become more sinister when such maps are used for convincing governing bodies to instate new policies or actions. As maps in recent years have generally become more oriented towards serving specific purposes (Ormeling, 2010, p.32) such as cataloging information or as educational models, the need for different groups-especially those who have historically been marginalized — to be able to effectively represent their knowledge through maps in ways appropriate to themselves has become ever more important. Emerging paradigms in the mapping of knowledge and language may offer new and different insights into the people and processes involved in the production of language maps.

\subsubsection{Language mapping and education.}

As language maps are communication tools that can engage audiences from different backgrounds (Upton, 2010, p.144), they have potential as pedagogical resources inside and outside of post-secondary education. Indeed, Luebbering (2011, p.8) notes that language maps are often part of introductory textbooks throughout a variety of subjects, while Wiegand (2006, p.1) comments that maps are low cost and engaging classroom options that increase engagement. In addition, maps have been demonstrated to promote learning behaviour and success (Dagenais, Moore, Sabatier, Lamarre, \& Armand, 2008, p.293). Furthermore, Wiegand (2006, p.1) notes that maps are important enough in classroom settings that researchers such as Balchin and Coleman (1966, p.23) consider a knowledge of how to read maps — or graphicacy — to be a fundamental skill in education. It comes as a surprise then that I have been unable to find any works in the literature specifically dedicated to the use of language maps in the classroom. As such, the development of language maps as a pedagogical resource is a subject that rightfully 
deserves ample further investigation, and while it is discussed in part 2 of this dissertation, future research into this area is required.

This lack of related research notwithstanding, Luebbering $(2011$, p.20) identifies several online language maps intended for education. The Modern Language Association (MLA) map, for example, is an online and interactive language distribution map created using ArcGIS that describes itself as a resource designed for "students, teachers, and anyone interested in learning about the linguistic and cultural composition of the United States" (Modern Language Association, 2020). ${ }^{31}$ Additionally, LL-Map, created by Linguist List, ${ }^{32}$ enables both linguists and the general public to view linguistic information on an online interactive world map, as well as the original materials from which the information was derived. Importantly, a user-friendly GIS environment is welcoming and easy to use for those without a background in online mapping (Luebbering, 2011, p.20). Outside of Luebbering's (2011) list (and outside of academia as a matter of principle), Native-Land.ca (Temprano, 2015-2020) is a map produced by an Indigenous-led nonprofit organization devoted to raising awareness and engagement about the distribution and states of existence of Indigenous languages across the world. While the interactive map has layers that show Indigenous lands and treaties, the distribution of languages is an important component with a devoted map layer. Importantly, the website that hosts the Native Land map offers resources that structure the map's information into lesson plans and activities for classroom use.

\footnotetext{
${ }^{31}$ For more information, visit https://www.mla.org/Resources/Research/MLA-Language-Map

${ }^{32}$ Linguist List is an online resource and network of linguists, which offers information related to language analysis and infrastructures that allow linguistics to be used in the digital sphere. For more information, visit https://linguistlist.org/
} 
While many online language maps are designed to showcase geolinguistic research for general interest or highly specific linguistic research projects, Native Land is a language mapping project with classroom-based education in mind. While I argue that a classroom setting is not (and should not be) the distinguishing criteria for a language map to be considered educational, it is a means for those who would otherwise never encounter the map to do so in a controlled and predictable way. As well as acting as a guide to using the map and understanding challenges and successes Indigenous languages face, a teacher's guide provided by the Native Land website (Native Land, 2019) facilitates critical discussions surrounding the presentation of Indigenous languages through maps in the first place. As a move that can only augment students' and educators' grasp on graphicacy, such a teacher's guide also demonstrates a marked and applied effort to facilitate a capacity for reflection in practices that involve language mapping.

Additionally, Purschke (forthcoming, p.15) demonstrated the use of linguistic landscape maps in classrooms to promote language learning, digital literacy, and discussions of cultural and linguistic diversity. In an implementation of the linguistic landscape methodologies, two groups of Namibian secondary school students collected over 200 photographs, which ultimately indicated a landscape filled with signs displaying colonial languages, with English in the lead followed by German and Afrikaans. In addition to this activity, students also drew mind maps ${ }^{33}$ based on how they viewed multilingualism in their country. While providing students and teachers alike with a new perspective on the use of various languages in Namibian society, the linguistic landscape and mapping activities also encouraged class discussions regarding the use of ethnic languages and English in Namibian society.

\footnotetext{
${ }^{33}$ Mind maps are defined by (Davies, 2011, p.280) as a visual technique that individuals can use to explore and imagine relationships between concepts through the use of diagrams, pictures, colours, or line thickness, which can aid in free form and spontaneous thinking (p.281).
} 
Aside from a classroom tool, language maps can also act as a platform from which other linguistic educational resources can be shared. Though not a language map per se, the NILLA (NETOLNEW Indigenous Language Learning Atlas) platform aims to act as a network of Indigenous language learning resources, programs, and initiatives designed for adult learners and educators (McIvor \& Jacobs, 2017, p.1). Currently in its third year of a six-year Social Sciences and Humanities Research Council (SSHRC) grant, NILLA is a project resulting from partnerships between a research team at the University of Victoria in British Columbia and language educators, learners, and activists in Indigenous communities across Canada. Through an online interactive map which displays Indigenous Language Revitalization (ILR) programs across the country, NILLA will act as a portal that assists educators, communities, learners, and researchers in finding each other, sharing language learning resources, and reflecting upon which ILR strategies have worked well in the past. Perhaps most notably, every effort within the production and implementation of the NILLA map is the result of partnerships between the university and communities, which is important given the power that maps can reinstate-or exert upon - those involved in some way in the mapping process.

\subsection{Language maps and power.}

Mapping has a troubled legacy of exploitation and colonialism, arguably because of its communicatory strength. While those creating physical geographic maps tend to see objectivity as a desirable goal, maps that focus on language are inevitably embedded with emotional attachments and prejudices (Briscoe, 2009, p.14). Furthermore, Kitchin, Perkins, and Dodge (2009, p.10) cite property ownership, national identity, race, military power, bureaucracy, and gender as playing key roles in cartographic practice. Historically, only those who controlled an 
area could map it, and quite often, such maps were instrumental in exercising additional control (Ormeling, 2010, p.28). More specifically, colonialism as a regime of power was (and still is) organized through spatiality: "to capture the land, it had to first be explored and mapped" (Garuba, 2002, p.87). In addition, Aporta et al. (2014, p.230) discuss how European explorers exercised claims of ownership over land through naming, which imposed their own frames of reference. Harley (1988) mentions that maps legitimized realities of conquest by propagating myths that aided in maintaining the "territorial status quo" (p.280). Finally, Wood (1992, p.1) argues that the power of such maps is found in the interests being mapped, and through such logic, maps work because they serve specific interests.

As an example of this, Garuba (2002, p.87) describes mapping as defining the lands in terms of physical containment - while colonies and territories were plotted, existing communities were compartmentalized into tribes and areas that were seldom faithful to their previous existences. In establishing territories through this model, demarcation lines drawn between such areas would create new popular myths about how these people exist (Wood, 1992, p.2). In this case, such a myth could be the inherent ownership of land that the explorers had 'discovered.' Furthermore, Kitchin, Perkins, and Dodge (2009, p.9) describe a situation from Wood (1992) where colonial entities claimed sovereignty over marginalized groups by pushing them "off the map" and not including their information in spatial discourse. By generalizing this notion, Ormeling (2014, p.342) identifies the exclusion of otherwise relevant information as an unfortunately all-toocommon practice among mapmakers, who are already known to distort rigorously collected statistics to suit their requirements. Instead of being localized to language mapping, these observations are found throughout broader mapmaking practices. 
However, there are also observations specific to language mapping. Throughout early foundational research, language maps were effectively as much maps of people as they were of languages (Thun, 2010, p.507). Processes of obtaining information from people play an integral role in the development of language maps, and strongly impact those whose lands and knowledge are represented (Johnson, Louis, \& Pramono, 2006, p.89). Many traditional top-down and academic language mapping approaches, such as dialectology, begin by identifying target populations that possess traits, such as a particular age, sex, or location (Macaulay, 2018, p.242). Historically, information about languages was either collected through interviews between a field worker and informant — as in the Survey of English Dialects (Dieth \& Orton, 1952)—or in the form of telephone interviews or postal questionnaires addressed to potential informants in a designated area (Llamas, 2018, p.253; Macaulay, 2018, p.249), such as Wenker's (1877) Sprachkarte der Rheinprovinz (Scheuringer, 2010, p.160). With methods such as these, there is a clear distinction between the individual(s) gathering the information and the individuals(s) contributing information to a project. In most of these historical approaches to linguistic research, those conducting the research are not from the linguistic communities of focus and have objectives which to not keep informants' interests in mind (Czaykowska-Higgins, 2009, p.20). While observations such as these should not infer that a researcher-centred approach is inherently harmful, and indeed such research can play an important role, an imposed one-sided lack of agency can mean that investigators and mapmakers risk taking advantage of those contributing information, and may misrepresent or underrepresent key points.

Ormeling (2010, p.28) notes that twentieth-century French dialectal research assumed that all linguistic groups followed the French model of living in towns, which led to the underrepresentation of Celtic and Basque communities who tend to reside in hamlets or 
dispersed houses. While one country’s historical language mapping strategy may at a glance appear as a single drop in the proverbial ocean of language cartography, it is important to note that French dialectologists (e.g. Gilliéron \& Edmont, 1902) were foundational in establishing maps in linguistic research (Lameli, 2010, p.577). Here, such underrepresentation more likely stemmed from judgment errors and misunderstanding than intentional colonial acts. However, an absence of nefarious intent ought not detract from the significance of such impacts, and for many groups, representation on a map acknowledges their existence (Luebbering, 2011, p.17).

\subsubsection{Unintentional mistakes in language mapping.}

Unintentional mistakes are important to consider, both in language mapping practices and the effects of language mapping practices, because they tend to occur in mapmaking more often than in intentional deceit or propaganda (Monmonier, 2005b, p.216). As those developing maps necessarily commit "white lies" (such as omitting irrelevant visual information) to make maps legible, there is wide margin of error in deciding what to include, and those accessing the map often take such decisions for granted (Monmonier, 1996, pp.1-2). Mapmaking decisions can come in several forms - such as choosing what to include or exclude, deciding how to separate and organize information, and determining what visual symbols refer to - which means that any number of subtle, yet impactful, mistakes can also occur (Monmonier, 2005b, pp.216-220; Luebbering, Kolivras, \& Prisley, 2013, p.383). As a nuanced and transdisciplinary approach to understanding people, language maps are especially vulnerable to significant and unanticipated errors in the representation of language-related information because of a lack of communication: Cartographers often do not make maps, while mapmakers are not required to study cartography (Monmonier, 1996, p.1), those developing language maps tend to have more experience with 
language than with maps (Haynie, 2014, p.344), and those experienced with maps tend to ignore system-external factors of language, such as contextual and social dimensions (Luebbering, 2013, p.56). Most notably, such mistakes are impactful in language mapping initiatives that aim to account for and describe power imbalances, as they risk creating and repeating the issues they attempt to address.

\subsubsection{Consequences of language mapping techniques.}

Instead of being simple representations of contexts and places, maps are infused with meaning through complex, contested, and interrelated sets of socio-spatial practices (Del Casino Jr. \& Hanna, 2006, p.36). Moreover, the complex and intricate stories language maps tell are highly dependent on how their information is communicated. Importantly, Wikle and Bailey (2010) note that "unlike photographs, which show all things within a field of view, maps are innately selective" (p.253). As such, the techniques used to present linguistic information on a map determine the conclusions those viewing the map can make about a given subject. In support, Luebbering (2011) warns that "the data and design decisions made to compile language maps can undermine their utility if the end product disguises more about language than it reveals" (p.1). More specifically, Luebbering (2011, p.17) notes that conventional language maps using administrative boundaries tend to assign one (or perhaps with crosshatching, two) languages per area. Almost inevitably, the languages appearing on such maps are official and exercise a degree of dominance over nearby minority languages (Luebbering, 2011, p.17). On such maps, regions of interest risk being depicted as overly homogenous (Ormeling, 2014, p.348), falsely indicating a majority's ubiquitous presence. As logic would then dictate, if a map only represents minority languages in certain administrative divisions, audiences may falsely 
presume that majority languages are not found in that region, and underestimate dominant forces' influences.

While such mapping techniques can and have been used to exercise claims over an area (Ormeling, 2014, p.342), monolingual (or bilingual) maps made with the best of intentions may still present information in this way, due to what Luebbering (2011, p.17) calls a symbology limitation. When in paper form, maps showing overlapping language distributions can lead to poorly-defined regions (Girnth, 2010, p.116) which do not represent the relative strength of each language variety, especially in relation to other variables such as population size (e.g. see Ormeling, 2010, p.35).

Digital maps may offer solutions through the added options users have to interact with spatial information (Ormeling, 2010, p.39). While projects approach interactivity differently, GIS can enable users to select which languages or regions they wish to see, as well as the data behind the map (Hoch \& Hayes, 2010, p.27). In this way, a map can contain multiple layers of information, which decreases the need to maintain precision by eliminating data, an issue that has plagued conventional forms of mapping (Ormeling, 2014, p.345).

The access to such maps through a digital and/or online medium is something that many take for granted. Both Haight, Quan-Haase, and Corbett (2014, p.504) and Section 5.6.4 refer to the digital divide which separates those who have internet access (typically those in urban, connected, and wealthy regions) from those who do not, such as remote communities in rural and Northern Canada (Sawada, Cossette, Wellar, \& Kurt, 2006, p.454), who conversely, are often the ones being mapped. Here, elements of power outside the map are of concern and involve many of the same groups who have historically been on the losing side of cartography. 


\subsubsection{Language maps and gender.}

Thun (2010, p.507) describes many classic language mapping projects as only focusing on a single dimension, meaning that contributors with identical general characteristics were chosen whose sole differences were locational. Many such projects focused on NORMs (Lameli, 2010, p.583), who were believed to be least influenced by social, educational, or geographic factors (Ramisch, 2010, p.163). Both Lameli (2010, p.583) and Ramisch (2010, p.163) then note that the exclusive use of such contributors does not constitute a representative sample of any linguistic population. Such practices have not only historically excluded females from language mapping but also continue to do so.

There is a pronounced lack of language mapping literature that discusses gender, and much of the current discourse surrounding cartography and women in general is sparse (Dowler, Carubia, \& Szczgiel, 2005, p.1). Monk (1992, p.123) stresses the necessity of understanding gender in geographic practices, as gender assumes a subtlety distinct from issues such as race or socioeconomic inequality. What literature exists focuses on the sex of those involved in the mapmaking industry (Varanka, 2005, p.223) and criticisms of cartography as "masculinist enterprises" (e.g. Huffman, 1997, p.256).

Most recently, Clark, Johnson, and Mathews (2020) conduct what is perhaps the first indepth investigation into the subtle relationships between geography, discourse, and gender. In a diachronic analysis of how gender is conveyed and represented on gravestones on two sites across the Appalachian region in the eastern United States, the kinds of language used on gravestones were found to be important in indicating contemporaneous social attitudes about gender roles and expectations. While this research focuses on how language is used, I have been unable to find any investigations that focus on languages, space, and gender. This is perhaps 
reflected in a recent observation made in a foreword by Brunn and Kehrein (2020b, xi) that gender is a lesser studied topic in their 215-chapter handbook of language mapping. As such, this is an area of language mapping that deserves increased attention.

\subsection{Stakeholders in language mapping.}

Maps are always made for a purpose, and never just for the sake of producing a map (Johnson, 2001, p.12). A map's design depends on its purpose (Ormeling, 2014, p.341), which is ultimately to communicate a truth about something (Kitchin, Perkins, \& Dodge, 2009, p.5). However, what constitutes the "truth" depends on who defines it, and such truths are bound within the author's ideological frame (Kitchin, 2010, p.7). While this subsection discusses some main groups of stakeholders in many language mapping processes, there are some important factors that need to be accounted for. First, while I attempt to identify everyone involved, I cannot claim that this account is exhaustive, and as new mapping applications and situations arise, this account is going to need updating. Second, I consider myself to fall into specific roles such as the researcher or the map author. As a result, I will naturally understand these perspectives more than minority or Indigenous community ${ }^{34}$ perspectives, or those of anyone else whose specific community has historically been the subject of a map. This means that my accounts of these perspectives should be viewed with a critical eye. Finally, the names identifying the stakeholder groups occur in single quotation marks, which is a conscious effort to

\footnotetext{
${ }^{34}$ For the purposes of this research, I use the terms minority and Indigenous communities in the same way as Czaykowska-Higgins (2009): "all members of a language-speaking community, from the grass-roots language speakers, language learners, and language teachers, to various decision-making bodies" (p.19).
} 
recognize that what on the outside appears to be a cohesive and unified grouping may in fact be too internally variable to truly be considered a single category.

Much of mapping to date in and outside of language mapping follows top-down processes, whereby an "expert" imparts information on a comparatively "inexpert" audience (Perkins, 2018). Until the twenty-first century, maps were overwhelmingly organized by bodies such as governments agencies and executed by civil servants (Ormeling, 2010, p.28), who were seen as having both the right and responsibility to make authoritative maps (Rankin, 2016, p.4). A scan of the literature suggests that while top-down mapping is still more prevalent than the more democratized bottom-up mapping (e.g. Perkins, 2007, p.136), it is distrusted in critical cartography, being referred to as 'monolithic' by Wood (2010, p.179), 'politically-conservative' by Hoefle (2006, p.238), and ignorant of audiences' interests by Sobel (1998). Along a similar vein, Dowler, Carubia, and Szczgiel $(2005$, p.1) cite cartographic practice in general, which is historically representational, as elitist in nature.

Here then, it is important to note that much of the existing critical literature on cartography somewhat over-simplistically refers to two opposing forces in the mapping process. Modelled in a largely antagonistic sense, mapping authorities responsible for developing and creating language maps are depicted as cold and impersonal controllers of knowledge who selectively impart others' information on audiences they deem appropriate. On the other hand, their audiences are modelled as naïve and impressionable members of a greater society who are at the general whim of the mapping authorities. While this general picture may not be entirely inaccurate, it ought to be recognized that those considered to be "mapping authorities" will in fact quite often refer to selfless and hard-working researchers, activists, and community members whose self-sacrificing goals include language representation and empowerment. Similarly, 
audience members, though not linguistically trained, are quite often knowledgeable of the mapping topics and are competent critical thinkers.

\subsubsection{The 'map authors.'}

In a basic and encompassing definition what map authorship has meant throughout much of history, Ercegovac (1990, p.24) quotes a 1973 report by the International Cartographic Association:

Map author: Individual, group of individuals or agency who conceives the idea of a map, participates in its design and in the decision about its definitive presentation and who is primarily responsible for its informational content. (Spiess, 1973, p.56).

In reflecting on the power of mapmakers in cartography generally, Harley (1988, p.278) posits that the map author — consciously or otherwise — not only replicates the spaces being mapped but also the perspectives of the political systems they subscribe to. As experts in some relevant field, authors often have ultimate discretion in deciding where to place 'culture regions,' 'zones of linguistic collapse,' or 'bilingual districts,' as well as the linguistic information ultimately represented (Ambrose \& Williams, 1991, p.305). While such discretion may lead some authors to make decisions in the interest of empowering minority groups, others may be motivated by the interests of a majority (Luebbering, 2011, p.17) in claiming territory (Garuba, 2002, p.87) or for strategic purposes (Ormeling, 2010, p.27). Regardless of motivation, such decisions are often arbitrarily made without consulting those depicted in the map, meaning that quasi-random processes determine whether certain regions benefit or lose from language policies (Ambrose \& Williams, 1991, p.305). Those making such decisions have historically had little 
interest or involvement in the mapped groups (Aporta et al., 2014, p.230), and their eligibility to make such decisions has typically been based on their own academic achievements or professional experience.

Importantly, Ercegovac (1990, p.42) notes that in many mapping projects, those such as surveyors, researchers, topographers, technicians, and publishers who contribute to authorship have very different roles, fields of expertise, and positions of authority in the mapping process. Thus, talking about the map author as a monolithic entity that makes unanimous decisions is problematic. Similarly, mapmakers can represent many different bodies. While some may work for academia, a multinational corporation or a department in the local or state government, others may be employed by grassroots organizations or act alone. Given descriptions of monolithic authorship in much of the conventional mapmaking discourse, ${ }^{35}$ this consideration is rarely accounted for but ought to be remembered.

in linguistic archiving for the purposes of language revitalization, Good (2011) not only describes a marked difference between the linguist (a thematic content expert) and the technician (analogous to the mapmaker) but bases an entire paper on such a distinction. Furthermore, Good (2011, p.112) notes that such a distinction is not only evident but beneficial to a digital archiving project, as linguists who employ technicians' services can devote more time and energy to the project's content, and not technical details. As language mapping is increasingly based on similar interactions between language and digital technology and can involve language archiving (e.g. The Algonquian Linguistic Atlas, Junker, 2005-2020), it is a reasonable conclusion that a similar distinction between linguist and technician applies here.

\footnotetext{
${ }^{35}$ I regard 'conventional' here to refer to representational mapmaking, which is a top-down process most common before the $21^{\text {st }}$ century by which experts impart information on an audience (Perkins, 2007, p.127) assumed to be less knowledgeable (Upton, 2010, p.144).
} 


\subsubsection{The 'audience.'}

As a prototypical definition, Park (1982, p.247) cites the Aristotelian notion of an audience as the final cause for which something — a language map perhaps — exists. However, any attempt to dissect this term further is met with confusing results. While researchers from Thrower (1959, p.12) to Kiik (2015, p.1) discuss making maps available to "wider" or "broader" audiences, they do not explain who these audiences are, or what makes them wide and broad. Here then, a number of distinctions need to be made: is a broad audience one that contains every possible individual, or almost every possible individual? Does a broad audience mean everyone with an advanced degree of some description, or everyone within a specific substrate of North American society? While Upton (2010, p.143) describes audiences as being passive recipients of geolinguistic information, Taylor (2019b, p.63) regards audiences as playing not only an active but an indispensable role in the mapping process.

Similar to the discussion of authors, it is problematic to investigate audiences as a single, identifiable, and stable group. In academic GIS language mapping, for example, Briscoe (2009, p.4) defines the intended audience as those who use GIS themselves, meaning they are also authors of other maps. On the other hand, Upton (2010, p.142) describes the audience of language maps as anyone with an interest in language mapping and the distribution of language varieties more generally. However, I would argue that those who count as “anyone” in Upton's context have an inclination - and more importantly, access - to language maps that are presumably academic in nature, meaning that "anyone" does not mean "everyone." Here, it is important to remember, as noted by O'Raghallaigh, Sammon, and Murphy (2010, p.5), that the intended audiences of any map-related research can be stakeholders that are either internalthose involved in similar research or related affairs — or external members of other populations. 
In essence, those considered to be members of an 'audience' may or may not have prior knowledge or a vested interest in what the map displays.

While a systematic way of understanding the intended audience is important in language mapping and cartographic practices in general (Harvey, Kwan, \& Pavlovskaya, 2005, p.4), I have been unable to find any works in the literature that discuss the general nature of audiences in language mapping. Keeping the above considerations in mind, perhaps language mapping audiences can be thought of generally as anyone who encounters any information conveyed through the medium of language maps and come to conclusions based on what they see. While this definition is overly broad, I believe it to be a good starting point, as it does not specify the audiences' degree, reason of interest, or role played in the mapping process.

As digital technologies become more ubiquitous within mapmaking, specific terms such as 'user,' 'viewer,' or 'reader' are seen more readily to refer to individuals who in some way encounter a map of some description. While the terms map viewer or reader suggest that the audience plays a passive role in absorbing information imparted on them by a map they see, the term user — especially when applied to GIS as in Obermeyer (1995, p.78)—evokes conceptions of audience members playing a more active role, such as manipulating what information is visible, directly altering what information exists, or applying the information to their own ends. At a time when direct manipulation through a digital interface is not only possible but quite often preferable in language mapping (Trbovich, Lindgaard, \& Dillon, 2019, p.36), audiences' conventional passivity associated with top-down should be called into question. 


\subsubsection{The 'mapped.'}

As a group that is perhaps less ambiguously arranged than in the previous two subsections, I understand the 'mapped' to be anyone whose linguistic information or lands are present on a language map. Historically, those whose languages are the subject of a map have either been a majority society whose presence is emphasized to promote sovereignty (Ormeling, 2010, p.28), or minority communities who have rarely played an active part in the mapping process and whose presence is downplayed (Kitchin, Perkins, \& Dodge, 2009, p.9). In this way, the language mapping process has served to either put the 'mapped' at an advantage or a disadvantage, depending on who they are and what circumstances surround their mapping.

Taking this into account, it is important to acknowledge that grouping these individuals and communities into such a discrete and encompassing category is problematic, given that they may not have anything in common aside from the fact that their information appears on a language map. However, provisionally grouping individuals according to this trait while acknowledging some overarching internal differences serves as a basic means to conceptualize general top-down historical mapmaking practices, in which the authorities developing the maps have more control over the ensuing knowledge visible to others than those from whom this knowledge originated.

In support of this notion, quite often in the more traditional top-down mapping, those whose languages are represented on the map do not have agency over what information gets represented (Ambrose \& Williams, 1991, p.305), while those doing the mapping have little interest in their affairs (Aporta et al., 2014, p.230). Such a situation translates into a lack of control on part of the mapped over what aspects of their contributed knowledge get used or omitted, and what the knowledge is used for. In the $21^{\text {st }}$ century, such an identifiable and clear-cut exclusion of the 
mapped has begun to diminish (Chapin, Lamb, \& Threlkeld, 2005, p.620), in part due to new mapping practices that blur the lines between the author, the audience, and the subjects.

5.6 Bringing the stakeholders together.

While a strict separation of stakeholders is perhaps most readily observable in traditional top-down mapmaking (Ormeling, 2010, p.28), many emerging practices aim to create a comparatively democratized (Perkins, 2007, p.136) and bottom-up paradigm, often through digital means (Wiegand, 2006, p.1). In support of this notion, Meng (2003, p.1888) suggested as early as 2003 that the cartographer's role has become increasingly interwoven with that of the user due to changes in technology which affect the production of and access to maps. Ideally, with bottom-up mapping, which enables those possessing the knowledge to also map it (Taylor, 2005, p.4), communities can become authors, and audiences can play an active role in informing the mapping process. Similarly, those traditionally considered the 'authorities,' who once assumed control of the information, can assume advisory roles instead, enabling the work to be guided with and by communities, instead of for or on communities (Czaykowska-Higgins, 2009, p.15). Indeed, as early as 2006 , Wiegand (2006, p.1) noted in his introduction that the use of community-based mapping has begun to increase among members of younger generations.

It is important to note that democratized forms of mapping will be more important in certain contexts. In this, the needs and wishes of stakeholders will differ depending on where the research is conducted, why it is conducted, who it involves, and how it is implemented. For example, the associated risks and benefits to stakeholders in a community mapping and language revitalization initiative in Australia will be very different from those involved in a study of the distribution of dialectal features in rural France. However, as maps are by nature a political act 
(Harley, 1988, p.277), any potential consequences of language mapping always ought to be accounted for.

On a similar vein, it is important to note that much of what is recognized as democratized mapping is still either spearheaded by or benefits companies that develop mapping technologies for proprietary gain. For example, Roberts $(2012$, p.1) provides a humorous example of two roommates, who while using free Google services to create their own maps, begin spying on themselves. While democratized mapping practices have much to offer in way of facilitating collaboration and involving audiences in the mapping process, something labelled 'bottom-up' or 'democratized' is not automatically community-led or grassroots by definition. Indeed, mapping projects spearheaded by proprietary organizations will often secure funding more easily than non-proprietary projects, such as those who follow cybercartographic principles (Erik Anonby, personal communication, 2020). What follow in this discussion are practices that are not specific to language mapping, but are at the same time important practices that I believe are compatible with and necessary in the mapping of language-related information.

\subsubsection{Participatory mapping.}

A common form of democratization observable in many mapping practices generally is known as participatory mapping. Broadly defined, participatory mapping refers to a wellestablished system of community-based approaches built upon intimate collaborations between all stakeholders in a mapping project (Di Gessa, Poole, \& Bending, 2008, p.1). Having emerged in the late 1980s, participatory mapping slowly grew from a set of information exchange values into an important tool for communities to map their knowledge while retaining control and agency over their own resources in a culturally meaningful way (Di Gessa, Poole, \& Bending, 
2008, p.1). Through the introduction of GIS technologies, the overall potential and number of applications of participatory mapping have increased, which has enabled communities who wish to do so to make their knowledge available through new and different media (Chambers, 2006, p.8). As a brief outline of important values within participatory mapping, Di Gessa, Poole, and Bending (2008, p.3) provide a list of lessons learned through their work and research with communities:

1. Develop relationships with political authorities for the purposes of securing rights.

2. Engage in mapping that secures many kinds of rights in addition to fixed land rights that are typically presented on maps.

3. Emphasize the mapping process, not just the finished product.

4. Use advanced technologies that are inclusive to all stakeholders, not just a privileged few.

5. Understand that the map is not the end result of empowerment, but an early stage in empowerment.

6. As maps can challenge power relations in society, mapping projects should be implemented in such a way as to be sensitive to and mitigate any associated risks.

While participatory mapping is at its core a set of values in research and community-based initiatives, Di Gessa, Poole, and Bending (2008, pp.6-8) do offer some examples of different applied methods that have begun to appear with the introduction of digital technologies: 
No-name method 1. Rivers and other features are digitized and extracted from the best topographical maps available, while political features remain absent. This creates a temporary map with which to work.

2. Trained community teams interview community members and employ GPS units to link knowledge to place.

3. Field data are placed on the temporary map and are transferred to a basemap with political features included.

Sketch mapping Community members draw maps of the area of interest featuring the knowledge they wish to present.

GIS mapping Information about an area of interest is gathered and linked with geographic coordinates. The resulting data are then analyzed by community members using GIS programs in order to create a map.

Image maps Satellite maps of a region of interest are obtained and community members superimpose the knowledge they wish to present on the images, either by hand or with a digital device.

In addition to these general procedures, a number of separate methods and frameworks have evolved that borrow from general participatory mapping but diverge and specialize in specific ways.

\subsubsection{Participatory Geographic Information Systems.}

Though GIS has revolutionized how languages are studied and mapped, there are some issues that prevent projects from functioning outside academia, including their expertcenteredness and inability to accommodate marginalized groups (Ndzabandzaba, 2020, p.3825). While many GIS programs are user-friendly and intuitive to those familiar with them, they can be confusing and difficult to understand for those approaching them for the first time. As such, Ndzabandzaba (2020, p.3827) proposes Participatory GIS (PGIS), which emphasizes the participation of those being mapped, and the inclusion of their local knowledge into "official" 
GIS data. As such, approaches within the PGIS framework are issue-driven and context-led, as opposed to being driven by technology.

In a fashion similar to participatory mapping more generally, projects that employ PGIS aim to blend individuals' and communities' qualitative information and experiential knowledge, with the ultimate goal of empowering people to successfully participate in political decision-making processes. PGIS differs from these more historical participatory practices as it uses GIS to create maps and atlases that integrate local knowledge with photographs, video clips, sound recordings, or mental maps (Ndzabandzaba, 2020, p.3831). This form of mapping can promote the knowledge communities wish to communicate in ways they believe most impactful, while at the same time making use of a technology which for many is as prestigious as it is inaccessible. However, Ndzabandzaba (2020, p.3832) cautions that facilitating community participation with tools designed for precision and scientific inquiry can often create data whose accuracy or reliability are uncertain, at least when such community data are used in systems that are rigidly structured by comparison. While the potential of this participatory method is emphasized by Ndzabandzaba (2020), more research and use in communities is required to gain a stronger understanding of how PGIS can be of benefit to language communities and studies of language.

\subsubsection{Volunteered Geographic Information.}

While not a technology per se, Volunteered Geographic Information (VGI), which Ndzabandzaba (2020) relates to PGIS, has influenced many digital mapping practices in recent years. Hardy, Frew, and Goodchild (2012) define VGI in terms of how it works: “...[U]sers can share and pool geographic information via the Internet at lower costs, in effect creating a global digital commons of geographic knowledge that is released from traditional mechanisms of 
production and distribution" (p.1191). Software, hardware, and social media advances have made VGI more feasible, and in turn VGI opens mapping practices up to becoming bottom-up and user-centered (Engler, Scassa, \& Taylor, 2014, p.45) while also being a time and costeffective means to collect data (p.48). Following Sui's (2008, p.5) inference that VGI coupled with non-traditional GIS techniques will create "geography without geographers," I argue that such user-oriented map development can enable linguists without a background in geography or cartography to have greater access to the means of making digital language maps, provided there are systems of guidance in place.

To date, much of the literature focusing on linguistics and VGI discusses computational languages (e.g. McKenzie, Janowicz, \& Adams, 2014) and geoparsing (e.g. Adams \& McKenzie, 2012). At the same time, Stone and Anonby (2019, p.450) discuss the role of VGI in Indigenous language education, and Cenerini, Junker, and Rosen (2017, p.307) reflect on gathering information through collaborators working directly with volunteer contributors. In these areas, VGI allows for a flexible approach to information upload that accounts for many kinds of users' needs and data and can accommodate new relationships between different kinds of information (Engler, Scassa, \& Taylor 2014, p.50).

While the name implies that individuals consciously volunteer their information, it is important to note that laws surrounding privacy and consent for bottom-up designs are much less clearly defined than their more conventional top-down counterparts (Engler, Scassa, \& Taylor 2014, p.54). While consent is implied for anyone uploading information about themselves in many VGI projects, volunteering someone else's information without their knowledge or consent can complicate matters (p.54). Similarly, while data acquired in this method are typically 
anonymous or anonymized, such data can in theory be used to obtain other, more personal information (p.53).

\subsubsection{Cybercartography and language empowerment.}

Originally introduced in in a keynote speech by Taylor (1997), cybercartography is a holistic cartographic discipline focused on mapping as a process, and not just a product (Taylor, 2005, p.3; 2019a, p.27). Unlike forms of digital mapping that attempt to transfer the art of paper mapmaking into digital media (see Rabanus \& Kehrein, 2010, p.375), cybercartography is based on the assumption that, as more than just wayfinding devices, maps serve as social constructs (Woodward \& Lewis, 1998, p.1), can benefit not only from extensive use of people's diverse narratives and perspectives, but also the careful consideration of how atlases are developed (Taylor, 2019b, p.57).

One of cybercartography's primary goals is to foster meaningful maps that act as storytelling devices through which narratives can be communicated, deconstructed, and exposed (Caquard, 2013, p.136) to reflect and communicate alternative viewpoints from historically marginalized groups. Cybercartographic maps recognize and attempt to address the idea that their audiences will have different learning preferences, demands, interpretations, and cultural backgrounds that influence how they interact with any information presented (Taylor, 2019b, p.57). Because audiences are also an integral part of the mapping experience and process, according to cybercartographic thought understanding and catering to their needs is fundamental to developing informative and competent maps. As maps often cover information that is open to subjective interpretation (such as the geographic extent of the area a language is spoken in), differing viewpoints need to be considered with equal merit, so that the viewers can make 
informed decisions. Importantly, cybercartographic atlases strive to integrate both qualitative and quantitative data into multisensory formats easily understandable by a variety of audiences (Taylor, 2019c, p.4).

Unlike many conventional paper and digital maps, cybercartographic atlases are designed to be part of an information or analytical package, while also being available for research partnerships with academia, civil society, and public and private sectors (Taylor, 2019b, p.26). To engage their audiences, cybercartographic atlases take advantage of the multimodal nature of digital mapping. As humans, like many organisms, are beings that employ loosely definable senses to understand the world around them, cybercartography too must represent elements of the world in a multisensory nature. So far, digital technologies allow maps to convey information using vision, hearing, and touch through telecommunications technologies to better engage the viewer.

In this way, cybercartography has the potential to present knowledge in a variety of modalities while offering communication systems that are culturally relevant for audiences and account for their needs and potential for contribution. Systems such as these are especially important given a major limitation to accessing maps, which is that the way maps display information is often different from the way audiences share and transmit knowledge. This is particularly relevant considering that members of many Indigenous societies that traditionally employ oral literacies have long been required to adopt outsiders' viewpoints and ways of thinking to engage with map-related (and other educational) materials. The multimodal nature of cybercartography thus has important applications in the mapping of minority and Indigenous knowledge with specific regard to language, which, as a vehicle through which ideas can be transmitted, is integral to supporting cultures, identities, and worldviews (Henze \& Davis, 1999, 
p.16). Here, worldviews are defined as in Hart (2010, p.2) as perceptual, cognitive, and affective maps developed throughout one's lifetime to make sense of their social landscape, while Wanderley (2020, p.3854) adds that worldviews are expressed by language and culture.

Opposed to more conventional mapmaking strategies, cybercartographic projects involve the group(s) they work with at every stage of the atlas' development and implementation (Taylor, 2019b, p.67). Moreover, map users are considered to be learners and part of the mapmaking process (Eddy \& Taylor, 2005, p.37). To make this possible, any cybercartographic atlas development framework needs to be made completely accessible to members, meaning that any development-related material must be presented using technologies available to the group, and be structured in such a way that members can contribute knowledge and information without an extensive background in linguistics or programming. In such a framework, communities can not only contribute information to an atlas that becomes their own, but decide how to communicate such information and to what extent they become involved in the process.

It is important to note that the use of the term cybercartographic is not always straightforward when applied to mapping projects. Cybercartography at its core is an evolving theoretical construct (Taylor, 2005, p.2), meaning that a mapping project can be considered cybercartographic if it follows the same integrative processes and principles. In practice however, almost all projects that refer to themselves as cybercartographic are involved in some way with the GCRC, ${ }^{36}$ meaning that in its current circumstances, the term cybercartography is functionally synonymous with GCRC initiatives. This means that, official definitions notwithstanding, the terms cybercartography and cybercartographic risk being mistakenly used by those wishing to engage in research of this kind as a brand for the GCRC.

\footnotetext{
${ }^{36}$ A notable exception being Ali (2017).
} 
Serving as an excellent example of what a cybercartographic framework may look like, the Nunaliit Cybercartographic Atlas Framework (http://nunaliit.org) functions as a flexible data management system responsible for linking different kinds of information and presenting narratives in such a way that the resulting information available for display is put into context for the audience (Hayes \& Taylor, 2019, p.206). Though maps are likely the first application that come to mind given this dissertation, Nunaliit can also present information in other ways, including graphs and timelines that use text, audio, video, graphic information, and interactive activities (Taylor, 2014). For those with minimal programming experience, the framework is built to allow files and data from multiple locations to be uploaded and integrated with one another without first being converted and embedded into some form of application logic. After being uploaded, files containing data inside of the framework can be accessed and edited directly, meaning that those involved can easily continue to make changes to their atlases even after the first versions have been published. The servers that are responsible for hosting the majority of Nunaliit processes are primarily located in the Geomatics and Cartographic Research Centre (GCRC), based in Ottawa, Canada.

To ensure that the framework is accessible, Nunaliit has been developed with the intent of becoming available on multiple types of devices, including smartphones, tablets, and computers. Aside from giving users greater freedom and flexibility in choosing how to access information, device compatibility can also facilitate atlas development, as devices equipped with cameras and microphones can create and then transmit data to the atlases. In the absence of such devices, specialized kiosks can be set up that enable audiences in northern communities to access the atlases. Such alternatives are essential to many communities in Canada that still have slow broadband connections and sparing access to communication devices such as computers and 
phones (Haight, Quan-Haase, \& Corbett, 2014, p.505). Along with Nunaliit, many other technologies are emerging that promise to change the way language maps are produced and accessed, along with the very nature of linguistic research. The following section showcases some recent developments within language mapping, along with accompanying ethical issues that arise when new technologies are introduced. 
6.0 Emerging practices in language mapping.

Before looking at specific examples of recent and ongoing innovations within studies of language and mapping, there are some important factors related to contemporary technological innovation that need to be considered.

\subsection{Accelerating technological change.}

To begin with, technological advancement in these early decades of the twenty-first century has occurred extraordinarily quickly and continues to accelerate. As early as 2004, the futurist and inventor Ray Kurzweil referred to a double exponential growth rate, in which the rate of exponential technological growth ${ }^{37}$ is itself growing exponentially (Kurzweil, 2004, p.2). Kurzweil (2004, p.3) noted that such growth can be explained using the Law of Accelerated Returns, which describes a positive feedback model where the more capable methods arising from one stage of a technological evolutionary process are used to create the next stage, causing exponential growth. From here, an additional positive feedback loop is created when a specific technology needs fewer resources to function (e.g. a new silicon chip requires less room and power in a computer), while more resources are simultaneously applied to its use (e.g. more silicon chips can be added), which creates a second layer of exponential growth.

In addition, some technologies periodically get introduced that change how technology can evolve. Through long-distance communication afforded by the telephone or the internet for example, anyone with a connection can collaborate on research projects, activism, and information sharing over vast distances. As a marked example, a significant majority of this

\footnotetext{
${ }^{37}$ Here, technological growth is defined as any rise in the aggregated measure of technologies' value to society, impact on quality of life (Starr \& Rudman, 1973, p.360), as well as the quantity and sophistication of technologies in existence (p.363).
} 
dissertation was written using resources such as maps and academic works that were either directly accessed online or found in a library with the help of an online catalogue. Additionally, the studies found in Part 2 of this dissertation use digital resources found online, and the successive drafts of this text were made possible through e-mail correspondence. Even twenty years ago, when the internet was young compared to its current state, such methods of research and data gathering would have been nearly impossible. Indeed, the common use of the internet has made entirely new fields such as web mapping and cybercartography possible.

In support of this idea, Küng, Picard, and Towse (2008, p.1) describe how the music industry had perceived the early internet as a serious threat, as customers would have the option of freely downloading digital files online, instead of purchasing albums in a store. However, since the time Küng, Picard, and Towse (2008, p.1) noted file sharing technology became available, roughly around 2003, license fees have become a major part of online music access, while copyright laws have changed accordingly. What this inability to predict future advancement on the part of major media actors may indicate is that it is difficult to truly predict what technological changes will occur in coming decades.

Because of this, understanding how existing technologies will be compatible with emerging ones is a concern, especially in areas such as language mapping that make use of linguistic information that is often stored for long periods of time. As early as 2003, Bird and Simons (2003, p.557) noted that language documentation and description resources became inaccessible within a decade of their creation, as many digital technologies' software versions, file formats, and system configurations were often functionally obsolete after three to five years. Though I have been unable to find more recent research that investigates these issues of portability in fields related to language mapping, Roth, Donohue, Sack, Wallace, and Buckingham (2014, 
p.25) note that web mapping technologies have begun to display improved flexibility and interoperability standards, meaning that, at least in part, a solution to this issue is being sought.

Such rapidly accelerating and unpredictable technological growth begs an important question for language mapping: how can we as researchers understand and reflect upon emerging language mapping technologies and practices when keeping pace is so problematic? Section 10.0 in Part 2 will introduce ways this question can be answered.

\subsection{Open source mapping.}

One solution, adopted into many recent language mapping projects, involves the use of open source technologies, which are a subset of technologies within broader Free and Open Source Software (FOSS) and Free and Open Source Software for Geoinformatics (FOSS4G) (Brovelli, Mitasova, Neteler, \& Raghavan, 2012, p.65). Once the domain of hackers and enthusiasts, notable examples of open source technologies currently in use include the Linux and Android operating systems (Levine \& Prietula, 2014, p.1414). A project is open source when the software it uses is free, it has a network of contributors who do not outrank each other, and it does not restrict users based on their intentions or who they are (Perens, 1999, p.1). In addition, open source software is described by McGowan (2001, p.241) as developed by communities of computer programmers from anywhere in the world who submit contributions to the software's overall code, which is then distributed freely and available for download by users. Typically, in open source initiatives and programs, there is a general understanding among programmers that others are free to make changes to their code but cannot make any private claims to the resulting changes. In addition, there are various online source code repositories, mailing lists, and Wikis that act as forums for programmers to share and discuss code, projects, and methods (Brovelli et 
al., 2012, p.65). Because numerous unrelated programmers can contribute to a single project, there are no scheduled times for program updates, and with sufficient support, open source programs can be in a constant state of change that resembles an ongoing process.

However, software does not become open source just because it is developed by a community of independent programmers working together for a common cause. To officially be called open source and not just source available, a software development project must have an Open Systems Interconnection (OSI)-approved license (Brasseur, 2018), of which there are over sixty that differ according to their intended use (Gomulkiewicz, 2009, p.264). Without such licenses, projects can be subjected to penalties related to copyright infringement, which are often resolvable, but can add pressure to the project's undertaking (Brasseur, 2018).

Notably, such software is often of a comparable quality to proprietary software, meaning projects and firms have become able to avoid purchasing expensive programs without sacrificing performance (McGowan, 2001, p.242). Importantly however, while numerous coders’ aggregated works are intended to be freely available, those coders are still able to sell fragments of their originally authored code outside of the project it was intended for (St. Laurent, 2004, p.9).

An important strength of open-source mapping is that it allows for any remote contributors to contribute to the development of mapping projects with relative ease (McGowan, 2001, p.241). Multiple open source mapping initiatives responsible for the production of numerous GIS programs have been available for over a decade now. Perhaps most notably for the purposes of language mapping, the Open Source Geospatial Foundation (OSGeo) serves as an umbrella 
organization that provides a shared infrastructure ${ }^{38}$ and common forum which facilitates the collaboration and development of open source and FOSS4G geospatial technologies (Brovelli et al., 2012, p.65). By associating a project with OSGeo, developers gain access to organizational support, licensing guidance, and open standards (Brovelli et al., 2012, p.66).

Such support, cost-effectiveness, and diversity in the production process is useful to many GIS language mapping projects as it enables those who would not otherwise have access to expensive software packages. On the one hand, this can mean that poorly funded organizations or communities have added opportunities to access and control the mapping process. On the other, linguists and other researchers whose departments may not be accustomed to geographic research can avoid the time, money, and hassle often required in obtaining departmental support for proprietary programs. As access to an open source program's code and architecture is unrestricted and those in the development process cannot outrank each other (Perens, 1999, p.1), those in the mapping process can contact those creating the codes to give feedback or ask for advice, or can modify the code themselves, if able. Furthermore, a wide availability of forums and free online tutorials ${ }^{39}$ mean that, with a little practice and patience, those unfamiliar with GIS can develop the knowledge to create basic maps.

There are, however, still several barriers those wishing to create language maps face, which are true of open source programs as well as proprietary software. For instance, while prospective mappers can teach themselves open source GIS and other FOSS4G software, the amount of time needed can be prohibitive, especially for those with full-time jobs, specific deadlines, and other

\footnotetext{
${ }^{38}$ For the purposes of this dissertation, an infrastructure is defined as an adaptive system consisting of many parts interacting through technical and social processes, which is not specifically engineered per se, but in some cases may arise organically (Borgman, 2015, p.33).

${ }^{39}$ For a list of online tutorials for the open source QGIS, visit https://www.qgistutorials.com/
} 
pressing duties. In situations where tutorials or classes on how to use the software are available, they are not typically designed with linguists in mind and do not feature linguistic data. In my own experience with open source GIS at Carleton University, I found that until recently, the majority of GIS learning resources were available only to geography and geomatics students, which led me to rely on online discussion forums outside the university. While I was able to teach myself enough to make basic language maps using this method, I required three months of extensive trial-and-error map building to do so. Additionally, open source GIS programs tend to be very large and require computers with adequate processing power and space. While this is likely less of an issue for those in academia or proprietary mapping platforms such as Google, practical barriers such as this can be an obstacle for grassroots initiatives.

\subsection{Big data.}

As big-data projects become more feasible, the availability of VGI and other linguistic information has become an increasingly salient issue. Broadly defined, big data refer to information that is huge in volume, diverse, exhaustive in scope, detailed, and high in velocity, meaning that it is created in real or near-real-time (Kitchin, 2013, p.262). In addition, Li, Yu, Xu, Yang, Sha, Liu, and Yang (2020, p.326) add and describe four more broad features, including big data's variable reliability and accuracy, shifting meaning, often vulnerable nature, and high value of information.

It is important to note that some researchers define 'real-time' differently, such as the immediate display of data as it is collected (Wade \& Sommer, 2006, p.176), or the study of sequential sets of data as they were available at a past point in time (Croushore, 2011, p.72), similar to diachronic data. Real-time data as they are understood by Croushore (2011) already have applications in sociolinguistics, where they have been gathered from longitudinal studies of 
language change within linguistic communities (Turell, 2003, p.8). While such studies differ from other conventional studies of language change only in the amount of time they require, realtime (and big data) projects have potential in digital language mapping.

According to Kitchin (2013, p.262), big data can be obtained from directed sources (a device of some sort that records surrounding information), automated sources (a device such as a smartphone that automatically transmits transaction history), or volunteered sources. It is important to note that Kitchin (2013, p.263) uses the term volunteered liberally; users are releasing information such as interactions across social media (Li et al., 2020, p.330) but may not explicitly know they are doing so. While Engler, Scassa, and Taylor (2014, p.45) offer a scenario in which interested parties actively and willingly contribute information to a map, Rubinstein (2013, p.4) warns that organizations, governments, and scholars are more than happy to use data voluntarily submitted on social media for their own purposes—-such as predicting and monetizing online behaviour (Georgiadou, Kounadi, \& de By, 2020, p.788)—which are not always faithful to those the data originated from. In this way, one's data are only as safe as the privacy policies protecting them (p.5), and what is considered the 'norm' for data usage is considered problematic to establish in an age of big data where information exchange between known collaborators is difficult to control or replicate in unknown groups (Borgman, 2015, p.33).

\subsubsection{The use of social media data.}

Big data quite often implicitly or explicitly contain information that is spatial in nature and occur in a variety of fields (Graham \& Shelton, 2013, p.256), such as urban growth simulation (e.g. Pijanowski et al., 2014) and energy management (Zhou, Fu, \& Yang, 2016). Agarwal et al. 
(2011, p.30) describe a real-time linguistic project involving non-spatial geography that classified Twitter posts ('Tweets') according to whether they contained positive, neutral, or negative sentiments. Similarly, Eisenstein, O’Connor, Smith, \& Xing (2012) performed an aggregate and diachronic analysis of the shifting frequencies of common colloquialisms using Twitter, and in doing so, constructed a network of linguistic influence across the United States. By pairing such social media data with census data, Eisenstein et al. (2012) identified demographic and geographic factors within the creation and adoption of new colloquialisms (p.11). Furthermore, Mocanu et al. (2013, p.2) studied languages' spatial distributions on global and national levels by focusing on the language used in individual tweets at a resolution as fine as a single digital device. While such individual data needed to be aggregated for the purposes of privacy and consistency, analyses of Montreal (Figure 29) found clear areal trends regarding locations English and French were spoken (Mocanu et al., 2013, p.6). As the accuracy of GPS technologies increases, such spatial accuracy in linguistic analyses involving social media can only be expected to improve (Mocanu et al, 2013, p.7). 
Figure 29: Language of Twitter usage in Montreal in 2013.

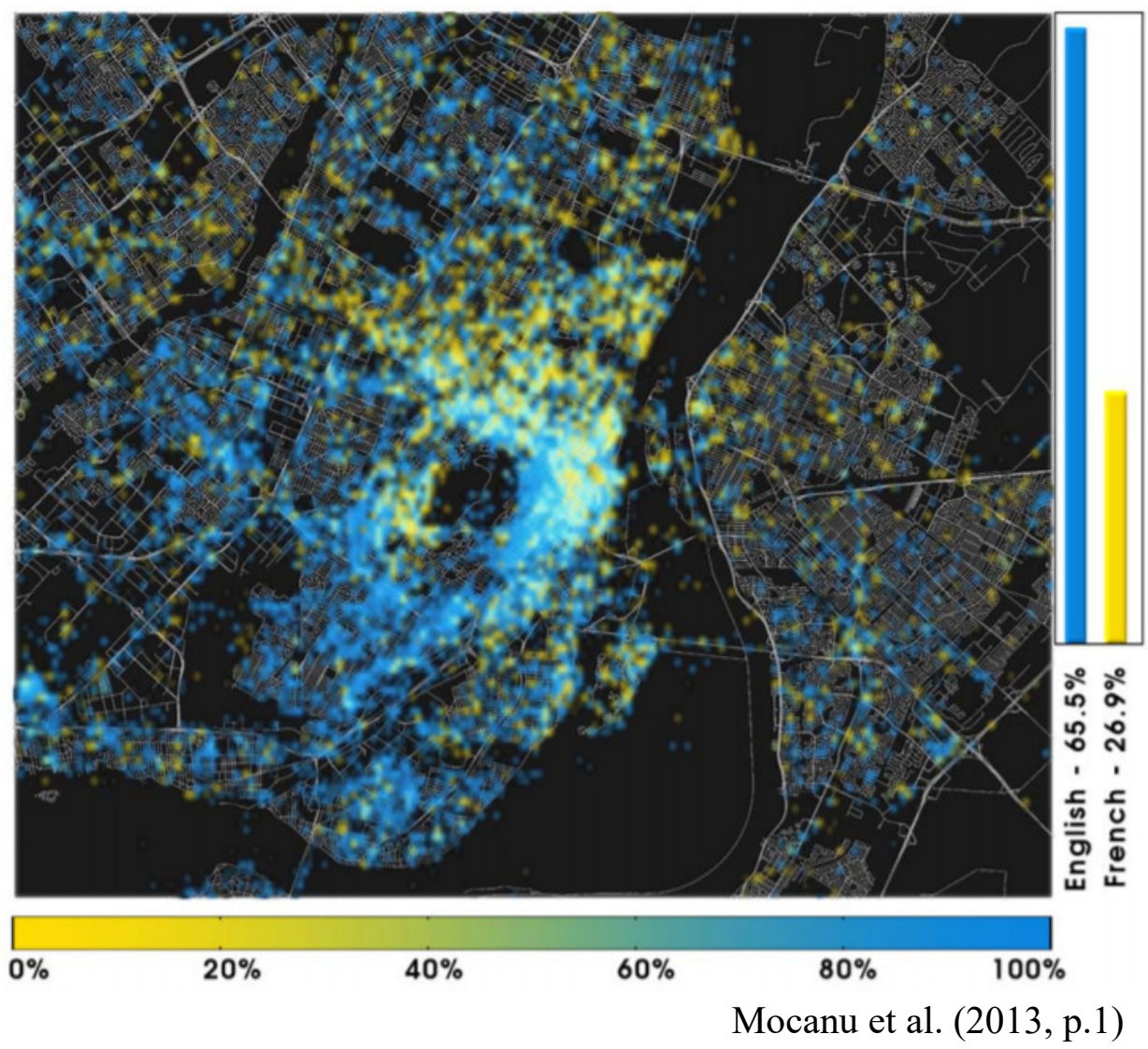

In a more recent study, Hovy, Rahimi, Baldwin, and Brooke (2020) collected 95 million Tweets by superimposing a grid system over a map of Europe and filling each cell with a combination of three colours, each representing a variation of an instance of a particular lexical item (e.g definitely vs. definately vs. defnitly) (p.3727). Importantly, such added flexibility in lexical analyses of big data has helped free language use from spelling conventions, meaning that more accurate pictures of distributions both between and within language varieties are possible. While Najafbadi, Villanustre, Khoshgoftaar, Seliya, Wald, and Muharemagic (2015) discuss the use of big data in audio- and image-based forms in addition to text, I have been unable to find linguistic studies that also use big data in these forms. 


\subsubsection{Crowdsourcing.}

While linguistic information is perhaps implicitly or unknowingly volunteered through social media, infrastructures are possible where data can be gathered with the explicit consent of large groups of people. With greater smartphone usage, sophistication, and metrological accuracy, analyses that use extremely dense temporal and spatial readings have become more feasible (Picaut, Fortin, Bocher, Petit, Aumond, \& Guillaume, 2019, p.20). As a blending of the words crowd and outsourcing, crowdsourcing refers to outsourcing that, instead of being directed to other companies or organizations, is directed instead to the general public by means of an open call through social media and the internet (Schenk \& Guittard, 2011, p.94). Importantly, a crowd is considered to be an anonymous and heterogeneous grouping of individuals who are likely novices in the field but likely also include experts and scientists. While participating individuals in some crowdsourced projects do not receive any compensation for contributing information, other projects offer substantial compensation (Schenk \& Guittard, 2011, p.95). As many crowdsourced projects take advantage of not requiring payment to contributors, many also make use of open source software (such as Picaut et al., 2019), greatly reducing the fees for undertaking and completing a project.

While Cotfas and Dosteanu (2010, p.46) argue that crowdsourcing with GIS leads to less accurate data, I suspect that this assumption has become outdated, given the widespread adoption of smartphones and social media since the publication of their paper. In geolinguistic research, Purschke (forthcoming, p.4) performed a study of the linguistic landscape (or crowdscape) of Luxembourg City and Vienna. Through the use of mobile apps available for download on a smartphone, more than 900 participating contributors uploaded over 16000 publicly available 
photographs, which were then used to calculate the distribution, frequency, and presence of written German, Luxembourgish, English, and French on public signs (Figure 30).

Figure 30: Number of languages per sign in Luxembourg: 1 (blue), 2 (green), 3 (yellow), 4 (red).

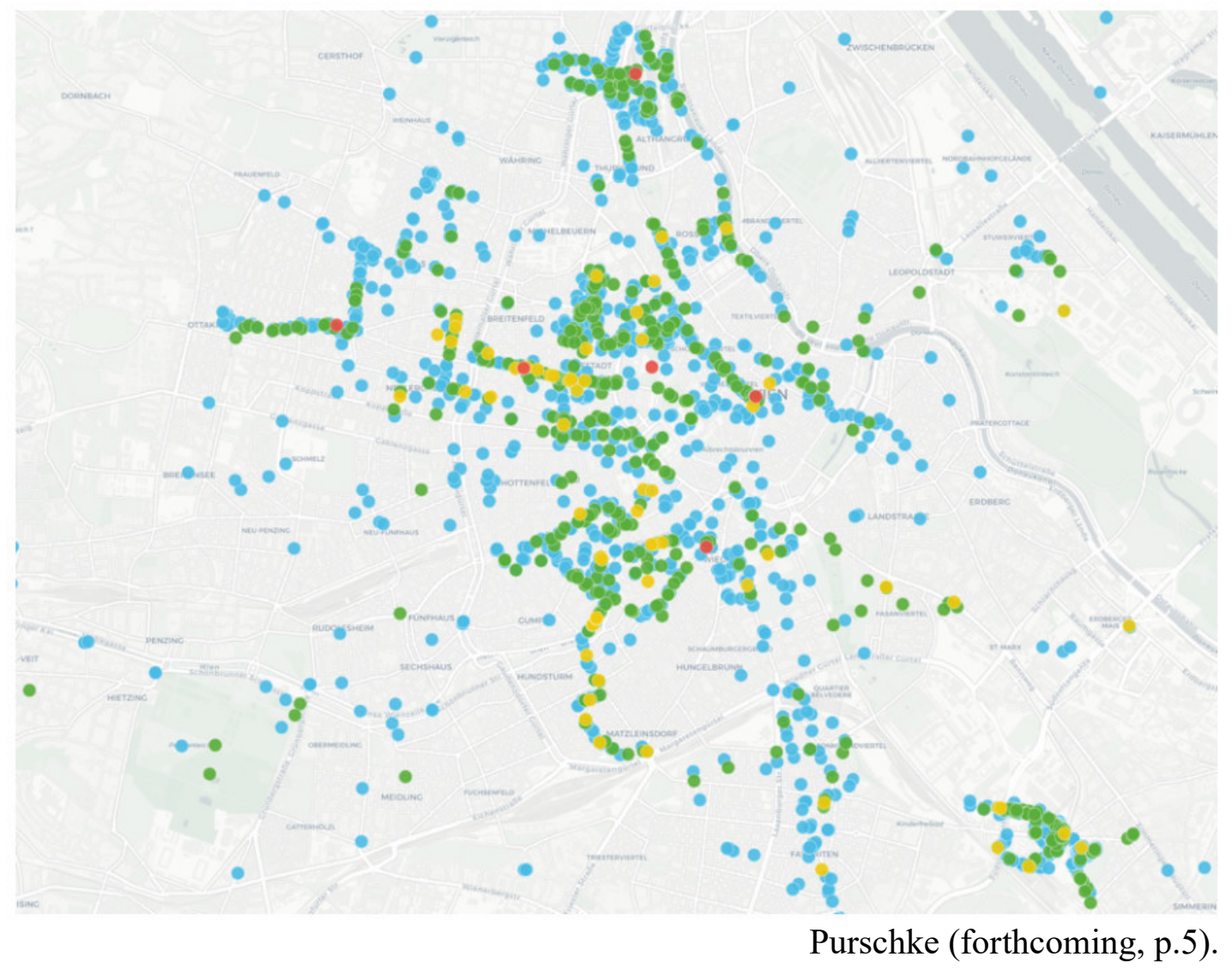

Though most contributors submitted information on only one or two occasions, there were also smaller groups who contributed images over several months (Purschke, forthcoming, p.16). Importantly, Purschke (forthcoming, p.10) notes that while such participatory action helped acquire large amounts of data at nearly no cost, it also benefited contributors, who came away from the study with a changed perspective of the linguistic and cultural diversity surrounding them. Though Purschke (forthcoming) does not explicitly state it, I believe that this reciprocal 
effect toward the contributors serves as an overt example of how the act of language mapping can directly impact those whose communities are the focus of analysis. Because of the impacts language maps can have on communities - both positive and negative - the ethics behind the use of emerging technologies in language mapping deserve the utmost attention and analysis if such technologies are to be used.

\subsubsection{Future trends in geographic big data.}

There are several other emerging technologies found in digital mapping which have potential applications in language mapping. In a discussion of future trends in the web mapping platform Digital Earth, van Genderen, Goodchild, Guo, Yang, Nativi, Wang, \& Wang (2020, p.818) identify an array of new technologies, which are discussed in brief below:

IoT

A commonly accepted abbreviation for Internet of Things, IoT refers to networks of smart devices, including smart refrigerators and home security systems, which can interact with devices such as smartphones via an internet connection to cooperate with one another and reach common goals (Atzori, Iera, \& Morabito, 2010, p.2787). As it is quickly becoming a global infrastructure, IoT has the potential to create a digital nervous system facilitating distributed cognition across the globe (van Genderen et al., 2020, p.818). By connecting sensor networks, information can be gathered and coordinated instantly through multiple means and devices. What this means, is that in theory (and privacy concerns notwithstanding), household language use in a city or geographic area could be ascertained with programmed refrigerators, dishwashers, or ostensibly passive smart speakers listening in on conversations. 


\section{Artificial Intelligence}

Van Genderen et al. (2020, p.818) claim that the real strength of artificially intelligent (AI) systems - that is, those which can independently interact with their environment (Jackson, 2019, p.xxii) — lie their ability to predict things, and not observe or explain phenomena. In conjunction with IoT, artificially intelligent systems can make inferences from enormous amounts of data gathered from any location that has at least one smart device. In an interconnected and globalized world with ever-increasing migrations and cultural contact, such systems could potentially be used to monitor and predict patterns of language contact with an astounding degree of spatial and temporal resolution. However, Van Genderen et al. (2020, p.819) observe that the role of GIS and other digital mapping technologies in AI (and vice-versa) remains unclear.

\section{Hyper-Connectivity}

As the volume of data that can be transferred over networks and the number of connected devices (including wearable technologies such as smartwatches) continue to grow, the sheer amount of information about humans is increasing at an unprecedented rate (van Genderen et al., $2020, p .819)$. What this may mean for future language mapping projects that use big data is that new ways of obtaining, accessing, and retrieving massive amounts of information about languages, their use, and their distribution may be made available at an increasingly personal level.

The benefits and risks of this growing convergence between language mapping, big data, and visualization are profound and necessary to consider. As technologies become more distributed and capable of extracting information about us, we gain new opportunities for developing deep insights into patterns of language use. Such opportunities may be beneficial. For 
example, live information extracted from smartphones about languages spoken in a city's immigrant communities could provide researchers with invaluable knowledge about language contact and shift, and inform community outreach programs and services. However, placing the same information into the hands of an authoritative or government agency could enable more invasive targeting of communities deemed to have high populations of undocumented migrants — imagined or not—which would increase the risk of human rights violations, arrests, and deportations. In a more extreme (yet feasible) case, real-time and only partially anonymized personal information about language use could be used as ammunition by a government with malicious intentions to locate and categorize ethnic and linguistic groups that support or oppose the specific regime. Because of such potential benefits and issues in language mapping, it is important to discuss ethical considerations surrounding advancements in digital technologies that make use of information about people and the languages they speak.

\subsection{Ethics in emerging practices.}

While there is a great opportunity growth and refinement in language mapping projects that take advantage of emerging practices, language is a human phenomenon, meaning that privacy and ownership of information should be considered important topics. Such considerations will vary according to whether directed, automated, or volunteered strategies are used to collect big data. While microphones placed in key locations may help researchers pinpoint what languages are being spoken in an area such as a city, or while using smart appliances as listening devices may help inform researchers on what languages are spoken in the home, the ethics related to data collection and use are still largely unexplored. 
Similar to observations found throughout language mapping disciplines and applications, Graham and Shelton (2013, p.256) mention that little has been done to reflect upon how big data fits in with previous theories and structures of scholarly and geographic knowledge. In the late twentieth century, Harley (1991, p.9) noted that cartography was generally uncritical of its own practices, and while research has focused on the ethicality of precision in mapmaking, less attention has been paid to the moral obligations of mapmakers. As language and linguistic information are intimate aspects of the human experience, the moral obligations and implications of mapmaking should thus be central to any discussion of language mapping ethics. In light of Harley’s (1991) observations, Georgiadou, Kounadi, and de By (2020) discuss (geo)privacy in the context of digital mapping, which they define as a "claim of individuals to determine for themselves when, how, and to what extent location information about them is communicated to others" (p.785). Importantly, Georgiadou, Kounadi, and de By (2020, p.787) caution that norms surrounding acceptable levels of privacy change over time, especially regarding information shared by users on public platforms such as social media. Although Mark Zuckerberg predicted in 2010 that societal attitudes toward sharing information online would relax over the subsequent decade, numerous scandals such as Cambridge Analytica have meant that online privacy has become an even more important issue among users (Georgiadou, Kounadi, \& de By, 2020, p.789).

Van Genderen et al. (2020, p.823) mention that artificially intelligent systems are currently limited in the value they have to society, mostly as a reflection of their human creators' imperfections and biases. However, privacy and ethical usage concerns are expected to increase along with the sophistication of AI. Neither van Genderen et al. (2020) nor Georgiadou, Kounadi, and de By (2020) delve into specific detail regarding what these ethical considerations 
may be, not because they are neglectful in their pursuit of this area, but simply because they, like the rest of us, do not know what the future holds.

This does not mean that we cannot determine what our values are with respect to ethical digital language mapping. Indeed, while van Genderen et al. (2020, p.823) do not describe specific ethical concerns, they do highlight the need for engagement with civil society in informing technological development and use. While desiderata such as not misusing personal information or violating privacy are a good start, they need to be cultivated and operationalized in a meaningful way for the practice and those involved. Within language mapping, the 'civil society' maps can engage is as diverse as the purposes the maps are made for, meaning that engagement would need to take many different forms.

\subsubsection{Ten questions for ethical practices in language mapping.}

As developing a comprehensive list of ethical desiderata in language mapping would require collaboration between all stakeholders, the process is woefully outside the scope of this dissertation. Based on the above discussions and related research in cartography as well as issues highlighted by Gawne and Ring (2016) and Georgiadou, Kounadi, and de By (2020), I can at this time propose ten questions which I understand to lie at the heart of language mapping, which can ideally help stimulate such a discussion: 
1. If the mapping is not led by those from whom the information originated, how can the contributors be included as fully as possible in the mapping process?

2. How can contributors' privacy be respected at all times during the mapping process?

3. What measures can be put in place to safeguard personal and/or thematic information from theft?

4. How are the data stored, and for how long?

5. What processes are in place to encourage contributors' information to be represented in ways they deem appropriate?

6. Are there locations that should not be mapped?

7. What level of spatial resolution can be used to respect contributors' privacy (e.g. city block)?

8. What are the processes through which contributors consent to having their data displayed?

9. In an age of big data and instant visualization, what would it mean for large-scale, detailed, and potentially personal linguistic information to become visible and accessible to a variety of users with different interpretations and motives?

10. How can language maps be produced as to accommodate for accessibility issues such as colour blindness or partial sightedness? ${ }^{40}$

\footnotetext{
40 There is emerging research on how to teach partially sighted or blind people how to read maps (see Kitchel \& Otto, 2020). However, I have thus far been unable to find any research that focuses on language maps and accessible technologies.
} 
Though these questions are intended for stimulating discussion outside of this dissertation, the answers to some of these have, in part, been started in this doctoral research. For example, potential strategies that address question 1 may lie in the use of participatory mapping (Sections 5.6.1 and 5.6.2), volunteered geographic information (Section 5.6.3), and open source mapping (6.2) to make various aspects of the research process available to language communities who are unable or do not wish to lead an initiative of their own. Additionally, responsible practices that respect privacy and security of information outlined by questions 2 and 3 are already actively sought-after, and an example of current practices can be found in interviews with atlas authors and developers in Part 2 of this dissertation (Section 12.2.3). However, I believe the nature of the answers to these questions will continue to change as new technologies and situations arise. Finally, while question 4 is not explicitly answered by the interviews administered in Part 2 (Section 12.2), I expect that answers to this question would vary according to the technology used, the goals of the language mapping initiative, and the contexts the initiative is implemented in. As such, interviews of a similar manner to Section 12.2 that actively account for data storage, along with competitive analyses framed with an evaluative map typology (Sections 11.0 and 12.0), would provide a means to ascertain answers to question 4.

In addition, there are questions outlined here (such as 5, 6, 7, and 8) for which answers could be sought through increased dialogue between those involved in any part of the mapping process. While there are many such strategies to increase this dialogue, one such approach is listed in the following section. Finally, I see questions 9 and 10 as being answerable through ongoing observation and documentation of the state and impacts of technological changes on digital techniques in language mapping. It is my hope that the tools I demonstrate in Part 2 will play a role in specifying and systematizing how we can observe these technological changes to come. 
Technological changes can be exciting, destructive, confusing, and can dramatically affect the societies in which they take place (Küng, Picard, \& Towse, 2008, p.2). Importantly, the introduction of a new technology, such as a smartphone, will have a significant impact on those who engage with it, as well as those who do not. What is perhaps typical of technological change, according to Küng, Picard, and Towse (2008, p.2), is that it tends to be adopted by wealthy nations sooner than less wealthy ones, and by younger generations more readily than older. While such uncertainty will surely make research in this area exciting and fruitful, the implications of such technologies may also have unintended consequences such as (but not limited to) the loss of privacy or misuse in the hands of malevolent entities. To more deeply understand how language mapping can occur ethically, I believe we need to first look at what is already being done, and how all stakeholders are affected. 
7.0 On the capacity for reflection.

As mapping technologies transition from textbooks and classroom walls to computers, GIS, and the Internet, linguistic data can increasingly be manipulated, stored, and analyzed (Luebbering, 2013, p.50), new spheres have evolved where such information can be applied, and larger audiences can access and contribute to maps. While Bollmann (2010, p.43) mentions that cartographic research by nature looks inward at its own function, impact, audience, and audiences' needs, Lameli (2010, p.567) and Luebbering (2013, p.54) note a lack of such reflection in language mapping. For the purposes of this dissertation, reflection is a process through which past and present techniques for visualizing linguistic data spatially are evaluated for their efficacy in imparting an intended message (Briscoe, 2009, p.10), how such techniques influence the power of those creating or using the maps (Peeters, 1992, p.8), and their potential to address current issues, research, and evolving technology. Importantly, the presence of mechanisms through which reflection could be made possible would set the stage for the development of guidelines and established conventions that would help regulate and standardize language mapmaking practices.

\subsection{A lack of language mapping guidelines.}

To advance as a field of study that accounts for differing perspectives, technological change, and all those involved in the mapping process, language mapping requires consistent guiding principles to help define its parameters, methods, and potential (Ambrose \& Williams, 1991, p.313). However, Lameli (2010, p.567) and Luebbering (2013, p.54) lament a general tendency against practice-based introspection in language mapping altogether. Similarly, researchers as early as Agumya and Hunter (2002, p.406) cite a general failure among mapmakers to 
understand the consequences of displaying (or not displaying) uncertainty on maps. This translates into an ostensible lack of desire for introspection on the behalf of researchers, as well as a lack of the means for reflection that hinders any would-be attempts to evaluate the mechanisms and consequences of language mapping techniques. In support of this notion, Kent (2017, p.194) laments that cartography has remained largely acritical of its own practices, and that cartographic theory and practice have become largely divorced from one another.

This is not to say that values and guidelines that could make an identifiable impact in language mapping do not exist; they simply need to be brought into the practice. In exploring contributing factors to low rates of post-secondary enrollment among Indigenous students in Canada for example, Kirkness and Barnhardt (1991, p.6) outline four core values—-known as the '4 R's' - that help facilitate collaboration that not only includes Indigenous and minority groups, but ensures they remain active contributors in a two-way dialogue. Here, I outline these four values, while speculating as to how they would relate to language mapping:

1. Respect: Understanding that the frames of knowledge found within academic and professional spheres are but a few of the diverse ways people approach and understand their world. To be respectful, initiatives that involve Indigenous and minority knowledge and actors must hold these cultural values and traditions as integral pieces of the language mapping process.

2. Relevance: Initiatives that involve Indigenous and minority knowledge and groups must be in a position to institutionally legitimize knowledge and skills that lie outside the conventionalized spheres of academia and industry. Making a language mapping initiative more relevant means considering information sources and data procedures 
outside the bounds of empirical research, so that knowledge can be imparted in ways that are more meaningful to those from the contributing communities.

3. Reciprocity: Recognizing collaboration and involvement as two-way processes, where all involved parties can jointly build knowledge and benefit from the process. In language mapping practices where those contributing knowledge are different from those developing maps, reciprocal approaches would help enable the development of language maps that contribute to the well-being and goals of each group in ways that respect and emphasize positive relationships between them.

4. Responsibility: Fostering an infrastructure through which Indigenous and minority groups - as well as anyone else in a position to contribute knowledge - are responsible for many of an initiative's processes. In such a manner, these groups' participation is not only accepted, but crucial to the initiative's success, be that in language mapping or elsewhere.

While these values (and others like them) are in of themselves not formal guidelines for approaching Indigenous and minority systems of knowledge per se, they are important principles that can inform better and more involved language mapping practices.

Without systems of and values for reflection that account for differing perspectives and knowledges, the risks are great: Those involved in the mapmaking process cannot fully grasp the impact of techniques on their audiences, whether that be creating maps that are overly confusing or boring (Williams and Ambrose, 1992, p.11), or imposing (and continuing) a legacy where maps exert power over others (Kitchin, Perkins, and Dodge, 2009, p.9). While an increased capacity to reflect on past and current language mapping practices would not likely halt those 
with ill intent, Kitchin (2010, p.7) argues that maps are inevitably bound within their author's ideological frame, meaning that authors — often unconsciously_replicate the perspectives of their-often unconscious_political ideologies (Harley, 1988, p.278).

The lack of critical reflection cited by Luebbering (2013, p.54) in Section 5.0 has not negatively impacted the number of maps being produced (Luebbering, Kolivras, \& Prisley, 2013, p.383). Indeed, Griffin and Fabrikant (2012, p.298) warn that such unprecedented map creation poses new challenges to cartography, including ensuring that maps created for new purposes accurately communicate information in meaningful ways. Language maps use a variety of techniques based on the authors' objectives (Ormeling, 2014, p.341), perspectives (Kitchin, 2010, p.7), arbitrary decisions (Girnth, 2010, p.101), and backgrounds (Mühlhäusler, 2010, p.359). As a result, two language maps that focus on the same phenomena may look entirely different from one another and convey two very different realities. While GIS and other digital mapping practices have increased in prevalence and potential, Luebbering (2011, p.43) notes that research on complications involved in communicating linguistic messages through maps has decreased in recent years, meaning that little is still known about the implications of language maps in the digital sphere.

Briscoe (2009, p.11) was unable to find critical reflection of how linguistic data is used in GIS mapping. In this, Briscoe (2009) cites a "relatively young relationship" (p.1) between GIS and linguistics, specifically in the area of data quality, which is more established in other GIS fields. This is also evidenced by Olariu and Olariu (2014, p.77) who note that the digitization of language maps is not uniform and occurs through varying means. 
7.2 Subsequent work in this area.

Keeping this information in mind, the second part of this dissertation focuses on making such a capacity for reflection in language mapping possible. This is done through the inclusion of a survey of existing online language mapping projects, followed by two peer-reviewed academic articles that demonstrate the use of two tools that deconstruct users' experiences and the structure of maps. Finally, a peer-reviewed chapter identifies an important area of cybercartography in language mapping that requires further exploration. 


\subsection{Part 1 Conclusion.}

While a growing body of research focuses on human language, physical space, and the maps that display many intricacies of their relationships, there are still major gaps in our knowledge about the consequences of the techniques used to make maps, as well as the impacts of the technologies used to make them. This Part 1 focused on taking stock of what is known about the constituent parts of language mapping and identified some areas of language mapping practices that require further exploration.

8.1 What is known about language mapping.

As opposed to a single cohesive discipline with known boundaries and divisions, language mapping is a practice which incorporates research, techniques, and attitudes from linguistics, geography, geolinguistics, cartography, sociolinguistics, and many other related fields. As we humans are intrinsically drawn to visual representations of our world, language maps are an important tool and form of discourse we use to understand the intricate and complex relationships between the languages we use and the spaces we navigate. The practice of making them, which is constantly evolving due to advancing technologies and new avenues of use, is growing and becoming more widespread, despite still being quite young.

Language mapping has its origins not only as travelers' curiosities but also as mechanisms that helped claim sovereignty, reinforce perceived ties between peoples and regions, and mark the differences from other places and outsiders. In the twentieth century, language mapping gained a distinct direction in research. Through many scholarly traditions, investigations, and debates the world over it developed a sense of rigor and validity that made language maps part of studies in a number of fields. In the twenty-first century, language mapping has become an 
important aspect of linguistic and cartographic research while gaining new applications in community-led initiatives, language empowerment, and education. During these early decades of the new century, as maps move from paper to the digital sphere, many involved in language mapping are beginning to question the methods and consequence of their practice. While such a questioning has existed in general cartography for much of the twentieth century, a marked rift between different mapmaking practices has meant that little of this reflection washed over into language mapping.

Language maps can look either at the distribution of languages over an area, or the distribution of phenomena within those languages. To do so, there are numerous techniques that can be used to display information, including points, lines, polygons, colours, projections, links, and embedded media. When part of a collection, either with other maps or with accompanying documents and information, language maps have the power to tell a story about linguistic phenomena in ways that text alone cannot. However, this story can differ markedly depending on who tells it and why.

As technologies change, new areas open up where language mapping can be of use. As smartphones become more widespread, maps can be accessed from and made just about anywhere. As digital mapping software becomes open-source and easier to use, more and more people have the power to make publicly available and authoritative appearing maps. While techniques such as GIS and big data have made larger language mapping projects feasible, participatory mapping, cybercartography, and crowdsourcing have enabled others to reap the benefits of these changes too. We know that most people who would either traditionally fall into the category of 'an audience member' or 'an author' are knowledgeable in some way about some kind of linguistic information that could be mapped, but that this knowledge, like intentions, 
varies from person to person. If there is one thing that language maps cannot do, it is plot a known course for where it is going from here.

8.2 What is still not known about language mapping.

Language maps seem to be available in a greater number than ever seen before. While the origins of language mapping are known and well-documented, little is known about how those involved in the mapping process can influence and contribute to them. Additionally, scant research investigates how these stakeholders and academic disciplines are in turn impacted by the language mapping process. I understand these issues to be present as a result of a lack of systematic and critical reflection in language mapping.

It is not well-known, for example, what role gender plays in the distribution of language varieties, though it is ascertainable that the authors and subjects of language mapping have historically been predominantly male. Additionally, while language mapping in education has been mentioned sparingly in the literature, that does not indicate a lessened need for investigation. Furthermore, despite some preliminary research into appropriate methods used to address uncertainty in maps, there is much that can be done to streamline the process. Finally, and most centrally to this dissertation, numerous language mappers have noted that a capacity for critically reflecting upon language mapping as a practice has not been explored or taken advantage of. While some tools have been proposed to address this, I remain unaware of any that have been put into practice.

To fill these gaps in our knowledge, I propose that such reflection needs to occur in a way which involves thoughtfully prepared tools that systematize the way we look at language maps. Part 1 discusses major elements that contribute to language mapping from a number of different 
perspectives; Part 2 demonstrates the use of tools that make systematic reflection in language mapping possible. 


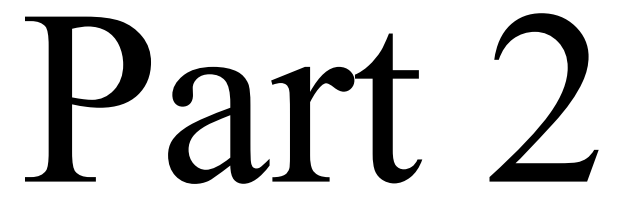

Critically reflecting on language mapping practices 


\section{Part 2: Critically reflecting on language mapping practices}

9.0 Preface.

In this second half of my dissertation, I demonstrate the use of tools that facilitate the reflection of practices in language mapping in ways that engage multiple domains of knowledge. Importantly, I intend for my proposed tools to function in a similar way to the notion of 'tools' described by Rankin (2016, p.18): tools which do tangible intellectual and conceptual work by acting as a way of thinking, making decisions, and opening up new questions. To do so, this part features a collection of four different studies I have carried out during my doctoral program, each in a dedicated chapter. Out of the four studies, three have been accepted and published through a peer-review process, and one has not been published, but instead was carried out during the dissertation writing process. The Algonquian Linguistic Atlas user study (Stone, 2018a) is in the journal Cartographica, the Evaluative Map Typology (Stone, 2018b) is in the proceedings of the $22^{\text {nd }}$ annual conference of the Foundation for Endangered Languages (FEL), and the Cybercartography in Indigenous Language Education book chapter (Stone \& Anonby, 2019) is found in Further Developments in the Theory and Practice of Cybercartography: International Dimensions and Language Mapping (Taylor, Anonby, \& Murasugi, 2019). These three published works are included in their entirety and are unchanged from their original format. As stipulated by the Applied Linguistics and Discourse Studies (ALDS) manuscript option guidelines (ALDS, n.d.), the published works can be found with the following citations: 
Stone, A. (2018). Mapping Experience: Age and Indigeneity as mediating factors in users’ experiences with the Algonquian Linguistic Atlas. Cartographica: The International Journal for Geographic Information and Geovisualization, 53(4), 229-240.

Stone, A. (2018). A typology for understanding and evaluating maps of Indigenous languages. In S. Drude, N. Ostler \& M. Moser (Eds.), Endangered languages and the land: Mapping landscapes of multilingualism, Proceedings of FEL XXII/2018 (Reykjavik, Iceland), 1925. FEL \& EL Publishing.

Stone, A., \& Anonby, E. J. (2019). The utility of cybercartography in Indigenous language education. In D. R. F. Taylor, E. Anonby, \& K. Murasugi (Eds.), Further developments in the theory and practice of cybercartography (pp. 441-460). Elsevier. 


\subsection{Introduction.}

Research which includes critical reflections on language mapping is sparse; thus, I identify the fostering of critical reflection as a challenge to be addressed within the study of language mapping practices. The role of the research here is in part observational, and in part a proposal of new tools for practice. As language mapping is multifaceted and transdisciplinary by nature, I argue that challenges associated with the practice must be approached from a variety of angles and viewpoints; indeed, this perspective is found elsewhere in the field of mapping. When addressing overarching challenges to the discipline of cartography for example, Griffin and Fabrikant (2012, p.298) highlight intersecting domains of knowledge that are central to understanding the field:

1. Mapping technologies and cartographic practices (representation).

2. How authors and users work, think, and reason (cognition).

3. The tasks that the technologies and practices are meant to support (behaviour).

Many different endeavours in the literature seek to address one or two of these domains at a time. For example, in a perception study of differently-sized squares that denote magnitude on thematic maps, Crawford (1973) focuses on how aspects of visual symbols reflect gradated concepts (akin to representation), and investigates the limits of human perception in discerning between squares (cognition). However, the roles these maps play in the authors' and audiences' realities are left out. Similarly, in a study of historic mapping practices in Japan, Botsman (2016) discusses the use of naming conventions and the inclusion/exclusion of information on a map (representation), as well as the roles and consequences the maps of interest had in shaping audiences' beliefs about historical events (behaviour). However, discussions regarding the mechanisms through which users mentally perceived the information are absent. 
Griffin and Fabrikant (2012, p.298) observe that cartographic approaches to addressing issues in mapmaking typically do not account for all three domains of knowledge in the same investigation, especially in the context of digital mapping. Though this dissertation and language mapping in general involve as much linguistics as they do cartography (and varying blends of the two), a cartographic approach that accounts for all three domains is vital, given the expansive critical reflection desired by Crawford (1973), Lameli (2010), Luebbering (2011), and Luebbering, Kolivras, and Prisley (2013). As such, to facilitate critical reflection in a holistic manner, each of my published and unpublished works addresses a different kind of knowledge. First, a published study demonstrates the application of a language mapping typology for deconstructing maps into their basic components, to facilitate comparison to other maps (Section 11.0). Following this, I discuss a second iteration of the typology that is currently in progress in relation to how it differs from the first iteration, and what further directions it can take (Section 11.2).

Second, an unpublished competitive analysis of existing digital language atlases uses the Evaluative Mapping Typology (EMT) in addition to existing competitive analysis practices and an unstructured initial survey to provide a basic picture of what kinds of digital language maps are currently in use, what they are capable of, and where they fall short of their potential (Section 12.0). Out of a sample of 47 maps and atlases, 11 are selected based on their level of interactivity and are the subject of analysis. Corresponding to Griffin and Fabrikant's (2012, p.298) representation domain of knowledge, this study, along with Study 1, offers glimpses into the technologies and practices that are currently found in language mapping. In addition, three semistructured interviews accompany the competitive analysis in order to justify the use of features in 
the atlases selected for analysis (Section 12.2), which correspond to Griffin and Fabrikant's (2012, p.298) cognition domain.

Third, a published study analyzes users' experiences with an exemplary language mapping atlas, the Algonquian Linguistic Atlas (Junker et al., 2005-2020) (Section 13.0). Importantly, this user study proposes a mixed-methods manner of assessing user experience with language maps. By necessity, the built-in reflective component focuses both on the atlas and on the methods used to understand its users' experiences. This study corresponds to the cognition domain of knowledge outlined by Griffin and Fabrikant (2012, p.298).

Finally, an article published as a chapter in Further Developments in the Theory and Practice of Cybercartography: International Dimensions and Language Mapping (Taylor, Anonby, \& Murasugi, 2019) identifies an important future direction language mapping can take that is of benefit to language revitalization strategies (Section 14.0). Being exploratory in nature, this article addresses Griffin and Fabrikant's (2012, p.298) third identified form of knowledge, behaviour, which looks at what maps do and how they can be applied. Following these studies, I identify important next steps in refining language mapping to be a more cohesive field and set of practices (Section 17.0). However, as a fruitful area of future research, subsequent and more detailed investigations into the prospective aspects of this area will follow and build upon the doctoral research presented here. 
Study 1

The Evaluative Map Typology 


\subsection{The Evaluative Map Typology.}

\subsection{Defining a language mapping typology.}

Since the typology proposed by Ambrose and Williams (1991), there have been numerous attempts to create a language mapping typology. In an age of big data and digital mapping, ideas of what typologies can focus on have changed and continue to change at a breathtaking pace. In a most basic sense, Whaley (1996, p.7) describes typologies as classification systems that look at phenomena or components of phenomena based on shared formal characteristics. In turn, Read (1989) defines a classification system as "an assertion about how phenomena in some domain are structured" (p.158). Furthermore, to analyze components of something (such as a map), typologies both describe their characteristics in detail and compare them to other similar characteristics (Croft, 2002, p.1; Read, 1989, p.159). Structurally, every typology is ultimately based on an attribute space that results from the comparison of particular components and their dimensions (Kluge, 2000, p.2).

While Ambrose and Williams' (1991) typology catalogs ways symbols (p.303), colour, and multiple languages (p.311) are shown in language maps, they themselves do not offer a definition of what a typology is. In reference to Ambrose and Williams (1991), Luebbering (2011) states and expands the terminology by referring to a map classification typology, which she defines as a "a survey of map components" that functions as a "methodological approach for assessing map composition" (p.46). Kessler and Slocum (2011, p.294) use a typology of a similar description to qualitatively and quantitatively measure changes in thematic map design during the twentieth century. While Girnth (2010) does not claim to develop a typology, his analysis of language mapping production steps (p.100), research areas (p.102), language map 
types (p.104), external characteristics (p.107), and mapping methods (p.108) functions as an indepth typology of language maps. Finally, Rabanus (2020) introduces a typology that accounts for several kinds of maps that are defined by the techniques used to make them, including point related maps (p.116), area related maps (p.117), line related maps (p.118), surface maps (p.119), and topographically unfaithful maps (p.121), which are referred to elsewhere in this dissertation as abstract maps. While such classification systems already exist throughout academic disciplines, it is important to understand why typologies are useful for language mapping in the first place.

The lack of critical reflection cited by Luebbering (2013, p.54) has not negatively impacted the number of maps being produced (Luebbering, Kolivras \& Prisley, 2013, p.383). Additionally, language maps use a variety of techniques depending on authors' objectives (Ormeling, 2014, p.341), perspectives (Kitchin, 2010, p.7), arbitrary decisions on how they want the maps to look (Girnth, 2010, p.101), and personal and academic backgrounds (Mühlhäusler, 2010, p.359). As a result, two language maps that focus on the same phenomena may look entirely different from one another and send two different messages. As GIS and other digital mapping practices become increasingly prevalent and capable in a wide range of applications, Luebbering (2011, p.43) notes that research on complications involved in communicating messages about languages through maps has decreased in recent years. In light of this, I argue that the ability for us to take a step back and think about these practices remains as important as ever and needs to evolve alongside pertinent social and technological changes.

A typology is a useful tool in deconstructing language maps. Deconstruction enables us to comprehend the purpose of a map, what is being mapped, techniques used to make the map, and the resulting understanding the map generates (Girnth, 2010, p.100). Once isolated, these 
components can then be compared in meaningful ways that review language mapping practices for individual or multiple maps while also offering opportunities for general reflection.

Typologies corroborate researchers' claims about issues in language mapping and uncover issues that have received less attention in the literature, while also revealing authors' creative attempts to overcome such issues and observe what strategies have worked well (Luebbering, 2011, p.61). Typologies can also summarize which strategies are employed more often than others, and in evaluating the extent to which they have been successful can form the foundation from which a set of best practices can emerge.

Such typologies come in different forms and employ different categorization strategies (Hruby, Miranda, \& Riedl, 2009, p.3), such as discrete lists with clear boundaries between representation forms (Moellering, 2007, p.2) or spectra of representational possibilities that are associated with a central conception of a prototypical map (MacEachren, 2004, p.161).

\subsubsection{Discrete classification strategies.}

Moellering (2007, p.3) proposes the discrete usage of clearly defined characteristics in a typology to systematically classify components of maps, which he argues is a necessary step in expanding the definition of a map in an age of rapid technological advancement. Here, Moellering (2007) argues that such a classification style is a basic strategy in science to properly understand "diverse collection[s] of things" (p.3). Though this perspective reflects approaches found elsewhere in science — and Moellering (2007) smugly cites Linnaeus (1735) as evidenceit should be noted that the value Moellering (2007) assigns to the "strictly scientific" typological approach is reminiscent of the late twentieth century superpositivist trend discussed by Rabanus (2011, p.41). Such a scientific perspective produces expansive and precise accounts of complex 
systems (Agassiz, 1859, p.303), but can cause disagreements on how typologies most closely reflect reality (p.2).

In prioritizing the medium through which the map is presented, Moellering (2007, p.4) makes a major distinction between "real" and "virtual" maps, citing significant structural differences between computer and non-computer-generated maps. On the other hand, Luebbering (2011, p.47) prioritizes symbology strategies in her analysis and explicitly does not distinguish between computer and non-computer maps. As an intermediary perspective (perhaps due to technology constraints relative to today), Ambrose and Williams (1991, p.304) classify computer-generated language mapping as one of many possible techniques (along with choropleth and chorochromatic maps) but devote little attention to differences between the two modalities. Such classification decisions guide the general structure (and ensuing differences) in the rest of their typologies. As a result, their proposed typologies differ to the point of requiring a typology of their own! After describing discrete typologies, Hruby, Miranda, and Riedl, (2009, p.3) account for an alternative form of classification that regards map components as less rigidly defined and quantifiable.

\subsubsection{Spectral classification strategies.}

MacEachren $(2004$, p.169) argues that many concepts in cartography—such as a map —are not concretely defined, which prevents them from being readily and consistently classified. Similarly, in linguistics integral concepts such as "language" and "dialect" also lack cohesive definitions (Whaley, 1996, p.4), causing disagreements between where and how languages should be classified (Haugen, 1966, p.922). Quite understandably, different interpretations of linguistic and cartographic terms have led to different techniques for providing rigid 
classifications for fluid terms. MacEachren (2004, p.177) argues that while particular schemata for fluid concepts may be fixed (e.g. the vocal tract is used in spoken languages), other schemata may be variable in nature (e.g. what parts of the vocal tract are used to produce speech sounds).

In representing this fluid classification scheme, MacEachren (2004, p.161) arranges concepts not by their relatedness to each other per se, but on a spectrum that indicates how closely they resemble features part of an abstract 'prototypical map' according to different 'map' dimensions (Figure 31).

Figure 31: Fluid typological approach to cartography.

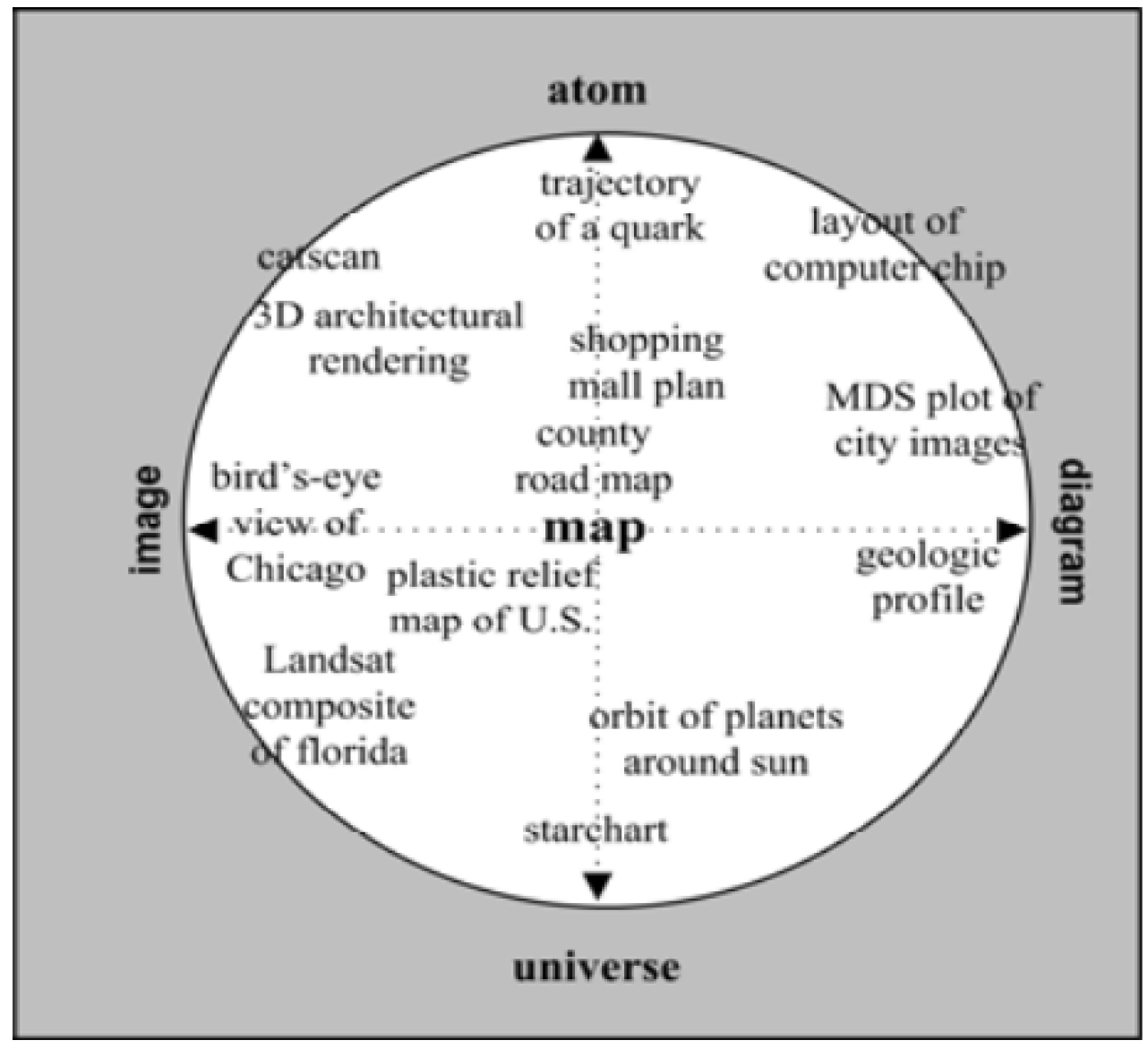

(MacEachren, 2004; in Hruby, Miranda, and Riedl, 2009, p.4) 
While Hruby, Miranda, and Riedl (2009, p.4) use examples from many different cartographic disciplines, such dimensions need to be reconsidered in language mapping. To date, I have been unable to locate any fluid typology in the literature specifically focusing on language maps. A spectrally based language mapping typology is an interesting area for future research but is outside the scope of this dissertation. The two language mapping typologies that follow here use overtly discrete classification strategies, and as with the user study, are designed with the intention of being part of an ongoing process of successive iterations that each become better at successfully deconstructing and comparing language maps.

\subsection{The Evaluative Map Typology.}

Luebbering (2011, p.60) notes that learning from actual practices is the only possibility for reflection in the absence of established guidelines and rules. What follows in this subsection is an article published in the conference proceedings of the Foundation for Endangered Languages (FEL) in 2018. This article demonstrates the Evaluative Map Typology (EMT) as it was applied to a variety of maps in preparation for the design of an atlas with the University of Victoria. Along with this article are supporting documents, such as an accompanying guide to the application of the EMT (Appendix I) and the forms used to deconstruct maps into their basic components (Appendix II). 


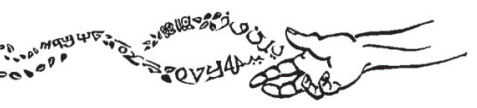

\section{A typology for understanding and evaluating maps of Indigenous languages}

\section{ADAM STONE}

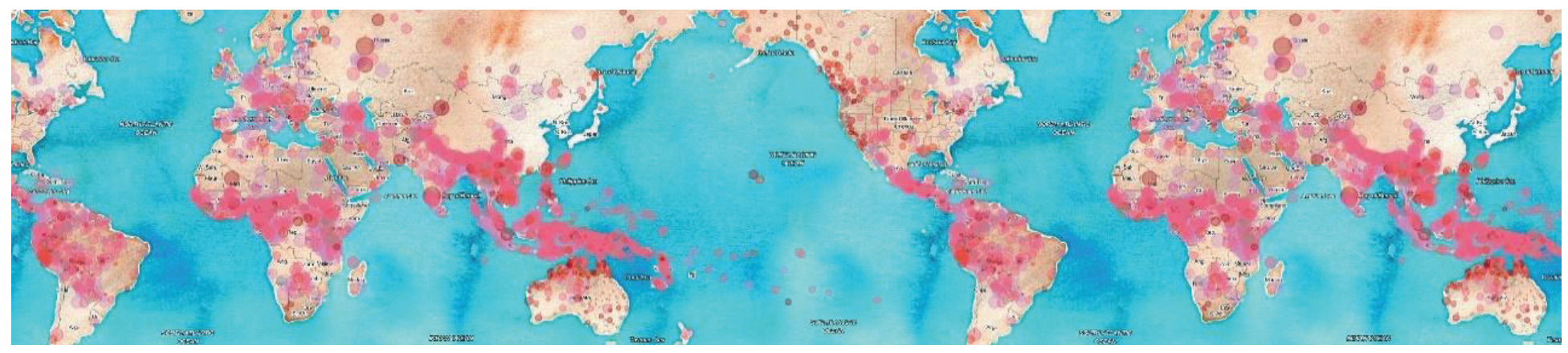

This article appears in:

Endangered languages and the land: Mapping landscapes of multilingualism

Proceedings of the 22nd Annual Conference

of the Foundation for Endangered Languages (FEL XXII / 2018)

Vigdís World Language Centre, Reykjavík, 23-25 August 2018

Editors: Sebastian Drude, Nicholas Ostler, Marielle Moser

ISBN: 978-1-9160726-0-2

Cite this article:

Stone, Adam. 2018. A typology for understanding and evaluating maps of Indigenous languages. In S. Drude, N. Ostler \& M. Moser (eds.), Endangered languages and the land: Mapping landscapes of multilingualism, Proceedings of FEL XXII/2018 (Reykjavík, Iceland), 19-25. London: FEL \& EL Publishing.

First published: December 2018

Link to this article: http://www.elpublishing.org/PID/4007

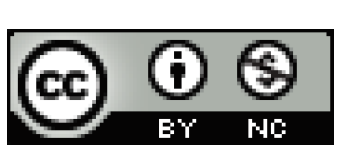

This article is published under a Creative Commons License CC-BY-NC (Attribution-NonCommercial). The licence permits users to use, reproduce, disseminate or display the article provided that the author is attributed as the original creator and that the reuse is restricted to non-commercial purposes i.e. research or educational use. See http://creativecommons.org/licenses/by-nc/4.0/

Foundation for Endangered Languages: EL Publishing:

http://www.ogmios.org http://www.elpublishing.org 


\title{
A typology for understanding and evaluating maps of Indigenous languages
}

\author{
Adam Stone \\ Applied Linguistics and Discourse Studies, School of Linguistics and Language Studies \\ Carleton University • 1125 Colonel By Drive • Ottawa K1S 5B6 • Canada \\ [adam.stone@carleton.ca]
}

\begin{abstract}
Maps are valuable tools for presenting large amounts of complex information that resonate with audiences in ways text alone cannot. European colonization stimulated the use of maps as explorers and colonists tried to understand and control the peoples and lands they encountered, defining what was known about them. Even well-researched linguistic maps designed with the best of intentions can still be confusing or misleading, and Luebbering, Kolivras, and Prisley as well as Monmonier mention that there are no established standards set in place to define how linguistic maps present their information. The Evaluative Map Typology (EMT) is a Structured Observation survey tool intended to compare linguistic maps systematically to one another by identifying and describing techniques used to display linguistic information. This paper describes the research involved in producing the EMT followed by a discussion of the features it evaluates, and an account of its current application in the NETOLNEW project based at the University of Victoria, Canada. As the EMT is continuing to be developed, a discussion of necessary future directions is provided.
\end{abstract}

Keywords: language maps; evaluation; evaluative map typology

\section{Our spaces, our languages, ourselves}

Our understanding of the world in which we live, be it physical, social, or based on intangible aspects such as language, is strongly based on our spatial relationships with where we and those we relate to are in some fashion situated. As spatial beings, understanding how we relate to spaces and are distributed throughout them is of great importance to our knowledge of how we relate to ourselves, each other, and our environments. With a strong drive to make sense of a large and complex world, we as linguistic beings have attempted to study and represent notions of space and the ideas contained within it long before quick and reliable communication in the present day (Lameli 2010).

\section{Space is both physical and social}

As much as space is a physical phenomenon pertaining to an actual location of objects in a particular area, our understanding of space is also deeply social (Britain 2010). How we build and alter the spaces around us will in turn affect how we understand such places to exist. By determining where and how we build roads, bridges, villages or camps in a given location (such as a territory, province, or traditional land), we as humans have the ability to determine which spaces we visit and interact with. Our understanding of how a particular space such as a river - exists in relation to our needs, will determine how we interact with it such as building a bridge, setting up a launching point for boats, or using it as a spot for fishing. How we interact with such a space will in turn change it: fishing sites or bridges may attract houses, roads, or services. Our perceptions of the space will in time also be altered by this change, as we may begin to perceive such a space through what it has become: a village instead of a point along a river, or a reststop on a road before a bridge instead of a part of the ri- ver known to be narrow. One of the most common ways we humans have represented such spaces and the changes that happen within them is with the use of maps.

\section{Maps and language}

Language has been fundamentally oral in our evolution as humans and societies, rather than textual. Maps are an intrinsically pictorial means of communication and can impart significant amounts of complex information intuitively, without the interference of text (Upton 2010). Here, a map can refer to any 'graphic model of parts of (or phenomena related to) a physical area produced to scale for decision making purposes, and graphical representations of relationships between concepts, ideas, or knowledge' (Stone, 2019, in press: 13). A lesser emphasis on text is not to say that maps are without language. Indeed, Gersmehl (1991) infers that like spoken language or text, maps consist of symbols which fully convey meaning only when observed in the context of all other symbols surrounding them. For the purposes of this paper, maps that are linguistic in nature can either focus on the distribution of languages across a given space, or the distribution of linguistic features, such as speech sounds or vocabulary items. Furthermore, atlas will refer to a collection of maps and other geographically-related materials. For more information on specific terminology, see Luebbering (2013). Such maps can occur in print or digital formats and can be either interactive or static (simply an image) in nature. Upton (2010) states that linguistic maps are important and meaningful, and viewers are often instinctively drawn to them. In contrast to many other media of instruction, viewers often feel they have some immediate understanding of the concepts represented. For many, a single linguistic map can convey notions of the distributions of cultures and languages in ways that far outpace text-based counterparts (Luebbering, Kolivras, \& Prisley 2013). 
Such maps have a variety of applications in (and are not limited to) linguistic research (Cenerini, Junker \& Rosen 2017), education (Luebbering 2011), and language revitalization (Stone 2019, in press). In the context of Indigenous societies in particular, the visual nature of maps as a medium echoes the traditionally oral structure of communication, knowledge and education in a way that text-based communication is not capable of doing. Herlihy and Knapp (2003) note that Indigenous communities have 'maintained cognitive maps that are delineated verbally using place names that convey place and spatial orientations,' (304) and that these maps provided a wealth of knowledge to Indigenous peoples in those areas as well as explorers who arrived in those areas (303). As people continue to move to new spaces, such as Indigenous individuals from their traditional communities to urban areas (Norris \& Clatworthy 2003), linguistic maps will play an ever-important role in accounting for changes in the distribution and vitality (Luebbering, Kolivras, \& Prisley 2013) of Indigenous languages, which in most parts of the world are experiencing unprecedented levels of decline and endangerment (Krauss 1992). Increases in human migration across lands and continents will only further complicate relationships between language and space (Mackey 1988), meaning that continuing research and development into linguistic map making practices and technologies is essential to ensuring that linguistic maps can do more for users and communities than act as what Luebbering (2013: 40) refers to as 'generalized snapshots in time.'

\section{Maps are tools of power}

Because of their communicatory strengths and propensity to model a particular reality to their viewerswhatever their motivation, basis or factuality-maps have been and continue to be used as tools of power (Herlihy \& Knapp, 2003). Luebbering (2013: 49) extends this notion by inferring that 'language maps can both convey power and be used for power.' Whoever controls depictions of a given geographical, political or linguistic territory has the means of shaping a society's thoughts regarding that territory, insofar as the maps are accepted as 'top-down' representations of the group's claims to sovereignty (Ormeling, 2010: 28). In colonial contexts, maps were and are used as tools of ownership, in which European groups assigned names and borders to newly-created territories, hence 'claiming' them (Aporta et al., 2014; Ormeling, 2010). Garuba (2002) explains that colonialism as a regime of power was orchestrated through spatiality and subjectivity. Language distribution maps (see Girnth, 2010) produced by scholars perpetuate the association of language communities with European nation-state or territory-like political entities (Ormeling, 2010). This may lead to the view that territory is limited to permanent settlements rather than an approximate and constantly-shifting area, and that societies speaking different languages do not share territory for the same or different land uses (Garuba
2002). In the same way that tools are actualized in the hands of those who use them, maps also have great potential for restoration and rehabilitation in the face of a colonial legacy of damage, provided they are used sensitively and appropriately in efforts toward reconciliation (Stone 2019, in press). However, even with the best of intentions, no map can be completely objective. Any map is the product of an author or authors who have their own histories, opinions, and biases, and is received by audiences that too have their own sets of expectations (Breton 1992). Linguistic maps will in some way inevitably be controversial, as no single language map will satisfy all audiences (Peeters 1992). In any practice of mapmaking then, it is important to consider how a map has been made, why the map has been created and designed the way it has, and who the intended audiences of the map are. However, many barriers remain to understanding these fundamental characteristics due to a number of issues in this emerging discipline.

\section{Pervasive issues in linguistic mapping}

While linguistic mapping is by no means a new endeavour (Luebbering, Kolivras, \& Prisley 2013), Monmonier (2005a) and Luebbering, Kolivras, and Prisley (2013) note a lack of standardization surroundding how linguistic information is presented geographically, and by whom. Monmonier (2005a) infers that maps are at the mercy of their producer's skill and public expectations in part because such producers are reluctant to impose standards on what is considered an endeavour as artistic as it is scientific. One of the reasons cited explains that rules can pose a risk to a map's design if followed blindly and applied in areas where they conflict with higher objectives. Luebbering, Kolivras, and Prisley (2013) cite an absence of common conventions surrounding linguistic mapping as being problematic due to such maps' usefulness in geography, anthropology, and linguistics. As researchers from a variety of disciplines and research backgrounds make use of linguistic maps in their work, they will often approach similar kinds of data in entirely different ways, which risks leading their readers (or users) to come to different and unpredictable conclusions. Such inconsistencies can be exemplified in map makers' treatment of uncertainty in their maps. Luebbering, Kolivras, and Prisley (2013: 392) note that while some map makers mention uncertainty in a text-based commentary, others indicate uncertainty through symbollogy, such as nonsolid boundary lines, zipper-like boundary transition zones, or an 'unknown' category for language information. Such disciplinary discrepancies are further complicated by the fact that while language is in many ways dynamic and intangible, maps and mapping practices tend to rely on discrete points, lines, polygons, and distances. Maps can risk becoming too unspecific if they are rigid in their design constraints, or too unreliably produced if they are made with little or no consideration for practices in linguistics or cartography. In addition, Luebbering (2013) 
notes an absence of research on the cartographic composition of linguistic maps from recent literature as a major hindrance in the discipline's ability to look inward at itself as it progresses in a time of rapid technological development:

'Research on the cartographic composition of language maps however, is noticeably absent from recent literature and the lack of cartographic guidelines for language mapping construction remains. [...] With new tools at our disposal, we are able to quickly produce language maps, but the effectiveness of those maps and the transmission of their intended messages would benefit from a thorough understanding of their cartographic composition as well as efforts to improve it.' (Luebbering 2013: 54-5)

Finally, Monmonier (2005b) states that as maps use discrete techniques to measure and reflect fluid concepts, the truths that are being mapped must necessarily be distorted for a meaningful amount of information to be conveyed to users. Without a set of guidelines in place, researchers with different disciplinary backgrounds risk varying greatly in how they choose to distort reality to deliver a message, and how distorted that message becomes. Any guidelines that attempt to regulate and standardize linguistic maps need to account for researchers' backgrounds, the conceptual gap between language and cartography, and the fact that maps produced to date have not been subjected to any guiding criteria in the first place.

\section{The Evaluative Map Typology}

The Evaluative Map Typology (EMT) is a Structured Observation survey tool created by the author of this paper which contains a number of questions related to the design and implementation of a linguistic map (for more information on Structured Observation, see Creswell 2003). With this tool, individuals answer a series of questions on a fillable-PDF survey form, but the form can also be printed as a physical copy. Survey questions are categorized into five dimensions according to defining questions in linguistic mapping posed by Girnth (2010):

Purpose: The map's goals, audience, function, author, context, and metadata.

Content: What is being mapped, variables involved; where the data comes from.

Symbols: What kind of information is presented (e.g. the use of data points).

Features: The map's layout, language, use of terminology, and commentary.

Result: Messages conveyed - Power, truthfulness, and general appearance.
In addition, the EMT form contains a section devoted to metadata, which allows for a detailed account of where the map was obtained and who produced it. Most questions in the Typology are answered first in sentence-form, followed by a representative code (a one or two-word summary that categorizes features), as often used in qualitative research projects. This provides subsequent summary analyses with efficient means to convert qualitative and subjective observations into meaningful comparable values. This form of survey allows maps to be compared to one another in multiple ways for a variety of purposes: to focus on comparing structural aspects of the chosen maps, or understand how the maps differ in the implications they have for their audiences.

\section{Current use in the NETOLNEW project}

\section{The NETOLNEW project}

The NETOLNEW 'one mind, one people' project, based at the University of Victoria, Canada, is a Social Sciences and Humanities Research Council (SSHRC) funded project which aims to serve as a Canada-wide network that shares adult Indigenous language learning resources, initiatives, and programs. Such a network will act as a platform where Indigenous people can build and lead their own education and revitalization efforts in ways that are appropriate for their own communities.

An important part of the NETOLNEW Project is the creation of the NILLA (NETOLNEW Indigenous Language Learning Atlas) project that shows where different Indigenous language revitalization and educational programs, initiatives, or projects are being held across Canada. As specified by McIvor and Jacobs (2017: 1):
'...[I]nformation gathered [from the documentation of significant sites of language revitalization across Canada] will ... be translated into an interactive, online map and repository to facilitate learning from and combining efforts across various types of immersion and bilingual Indigenous language revitalization projects in Canada.'

\section{The application of the current EMT analysis}

The analysis in this paper originates from a report created as part of the author's research assistantship duties with NETOLNEW, which aimed to identify what kinds of functions and features of maps should be available to users, and how future maps created by the project should be structured. Such recommendations are based upon a survey of existing digital maps and atlases that for the most part focus on Indigenous knowledge, but other kinds of maps have also been included.

The maps in this study primarily involve Indigenous knowledge, be it language, place names, natural resour- 
ces, or the geographic location of (and in) traditional stories. These maps are analyzed to show how previous mapping projects have approached the representation of Indigenous knowledge. However, other maps focus on a variety of subjects, including the distribution of scientific research projects, and are necessary in such an analysis as they indicate how other forms of knowledge can be represented in digital maps as well.

\section{The map selection process}

The first exploratory way maps were first gathered for the Typology with convenience sampling: the EMT was applied to digital maps and atlases that were already known to the author. Once those maps were exhausted, additional maps were then selected based on a cursory search of digital atlases and maps found on Google. Here, keywords such as 'digital atlas,' 'digital Indigenous atlas,' and 'online Indigenous atlas' were used.

Following this method, digital maps were then accessed more methodically through larger map and atlas repositories, such as the University of Victoria's Ethnographic Mapping Lab and the Nunaliit Cybercartographic Framework. In addition, some maps were referred to the researcher by others working in similar areas. A total of 15 maps were used in this analysis.

While this Typology is designed to evaluate both digital maps and those found in other media (such as globes or paper maps), the maps chosen for this current analysis are all digital online, as this is the medium of choice for the NILLA map. In addition to maps and atlases, other accompanying information or features on a website where the maps are featured is also explored. This additional information can also be important in determining how users interact with the maps and situate themselves in relation to the information they convey.

\section{The EMT analysis process}

The maps' positive and negative features were organized into tables of coded attributes Each code was ranked according to how frequently it appeared across maps, as to quantify this qualitative information in a way that is meaningful in determining which features are most important to consider in map development (for more information on Magnitude Coding, see Saldaña 2015).

\begin{tabular}{lr}
\hline Strengths & \# occurrences \\
\hline Intuitive & 10 \\
Minimalist & 7 \\
Layers/transparency & 5 \\
Professional & 4 \\
Community input & 4 \\
Interactive & 3 \\
\hline
\end{tabular}

Table 1: Ranked Frequencies of positive map attributes

\begin{tabular}{lc}
\hline Weaknesses & \# uses \\
\hline Missing information & 3 \\
Not engaging & 3 \\
Tricky interface & 3 \\
Dead links & 2 \\
No context & 2 \\
Difficult access & 2 \\
\hline
\end{tabular}

Table 2: Ranked Frequencies of negative map attributes

From this ranked frequency table, a series of positive and negative attributes found in the map sample were acquired that will inform the NETOLNEW project's future atlas development. Some outstanding attributes are mentioned below.

\section{Positive attributes of the map sample}

Intuitive maps have minimal amounts of features immediately visible, ensuring that the user/reader does not become overwhelmed. If too much information is present on a map, users will spend too much time and energy learning how to navigate the interface, and too little time actually exploring the information. As a result, the NILLA map should strive to present as much information as possible through the fewest mechanisms possible.

While maps are good tools of communication that allow users to access large amounts of information (Ormeling 2010), issues with how well a digital map functions arise when information is embedded directly in the map. Digital maps, especially interactive ones, require large amounts of processing power to run the way they were intended to (Marie-Odile Junker 2017, personal communication). Storing large amounts of information directly on maps thus can cause interactive functions to become slow or stop working altogether. Connecting geolocated information to an easily accessible but separate database would be a powerful tool in both referencing information better and making information easier to find.

Many legends were observed to not only indicate to the user what symbols are used to represent information, but also serve as a control mechanism for determining what appears on the map and what is rendered invisible. Here, a legend refers to a guide found on many maps with a list of symbols and their meanings. This is more of a relevant concern in maps where users are free to explore different features and manipulate particular elements, as opposed to in a more structured tour-based interface. Finally, as a way of controlling how much appears on the map at any given time, legends are especially important when researchers have little choice but to include large amounts of information on the interface. 


\section{Negative attributes of the map sample}

Many maps rely on existing interfaces such as Google to be displayed. Though this does allow for a consistent experience on both the developers' and users' ends, does this create issues of ownership and intellectual property rights when applied to Traditional Knowledge? What if Google discontinues the service in the future, or changes the software in such a way that the maps are no longer able to function as they once did?

Much like making an open-source map and database would allow individuals with diverse skills and knowledge to make important contributions to the map's inner workings over a theoretically infinite amount of time, allowing users to directly upload information would greatly improve the map and database's sustainability. However, doing so would greatly compromise the project's ability to control the kind, quality, and relevance of information being uploaded. While improving sustainability and representation, the map portion of the project would risk becoming inconsistent and fraught with incomplete data. However, by not providing a simple and convenient means to upload information, the project could risk sacrificing a number of ways of reaching out to communities and directly sharing their voices.

\section{Recommendations for map design}

As a result of these findings, the following suggestions can be established:

1. Information on the map should be represented with multiple layers, and users should be able to easily access and turn on or off such layers.

2. Accompanying commentary or information should be included in the map and should either be located in accompanying web pages that are easy to access, a pop-up window that appears at the beginning of each use of the map, or in a panel adjacent to the map. In the case of a pop-up window or adjacent panel, the user should have the option of being able to either move the item or delete it (and be able to easily access it again).

3. Users should have the option of either navigating the map with the help of a structured and introductory guided tour, or freely explore the map and all the features it has to offer.

4. A hideable horizonal slider at the bottom of the screen that contains all the necessary accompanying information or materials would allow users to more readily and easily access the information they are looking for. Features such as a horizontal data slider could be made available in a 'Tools' menu.

5. A link should be available and easily apparent to users that allow them to learn more about how they too could contribute information to the map.

6. Basemaps upon which information is plotted should come from a source which is stable and will not change too drastically in the future as to not be able to support a current mapping system.

\section{Discussion}

\section{How the EMT addresses pervasive issues}

In its current state, the EMT offers a means through which maps produced in different ways by different researchers can be compared to one another in a systematic fashion. As observed here, such comparisons are important in the design phase of new maps and atlases, but could also find relevance in the development of formal training curricula for students of linguistic mapping, which is lacking (Williams 1988).

The EMT's systematic comparisons address Monmonier's (2005a) observations that the map producer ultimately controls the map's design, as a map's information is reduced to individual (and categorized) aspects that can then be integrated into figures and tables. While the EMT cannot control how a map producer designs a map, those who are using the EMT can deconstruct design features to critically analyze how space is used to convey meaning. In doing so, the map producer's techniques and intentions become more transparent.

Though the EMT itself is not a set of guidelines for the production of new maps, it does have the potential to lay the groundwork for a future set of formal conventions. Such a groundwork can only be established by understanding first what tools and features can enable linguistic maps to clearly communicate meaningful messages while avoiding techniques that promote the author's bias. In understanding the different ways linguistic maps use points, lines, and polygons to convey messages of power, ownership, and authority, future guidelines can encourage map producers to account for marginalized perspectives that may otherwise not be heard.

When reflecting on the role of cartography in changing how we think about certain topics, Bollmann (2010: 43) notes that cartographic research by nature looks inward at 'the function and impact of the various cartographic media,'; 'the perceptive and cognitive capabilities of the potential media users, as well as their communicative needs,' and; 'the informational gain while and from using the media'. At a time when digital mapping practices such as Cybercartography (e.g. Taylor 2014) and Geographic Information Systems (GIS) offer unprecedented opportunities in the creation of interactive and multimedia maps, reflecting on standardization is important to ensuring new maps can responsibly and ethically communicate stories of knowledge and language. However, linguistic cartography has missed much of the introspective discussion surrounding standards that is typical of other disciplines that rely on mapmaking, such as geology (Luebbering, Kolivras, \& Prisley 2013). Because of the way individual maps' designs are compared to one another, the EMT has the potential to offer the discipline an introspective window 
into current practices and techniques that comprise linguistic mapping.

\section{The EMT and Indigenous mapping}

As demonstrated in preliminary research conducted with NETOLNEW project, the EMT may have a specific application in the assistance of designing maps of Indigenous knowledge and language that restore the balance of ownership and power to the rightful communities. As the EMT questions the techniques used to display spatial information and the consequences thereof, consideration can be devoted to understanding how maps can be made that reflect Indigenous communities' worldviews. Such a feature is important to account for in any project similar to NETOLNEW, whose primary objective is to build capacity among Indigenous people and ensure the control of educational resources remains in the hands of the communities that need them (McIvor \& Jacobs 2017). It is important to note that such a tool should not be used in isolation, but in integration with emerging practices such as the use of Volunteered Geographic Information (Engler, Scassa, \& Taylor 2014) and projects that are created for, with, and by Indigenous communities (Czaykowska-Higgins 2009).

\section{Limitations of the current EMT}

As the EMT is still very much in a preliminary stage of development, there is ample room for improvement. Though not exhaustive, this paper will account for several outstanding issues that will need to be resolved in order for such a tool to prove useful.

A major limitation in the context of Indigenous languages and mapping is that the EMT has been developed with little Indigenous input aside from the careful considerations of Dr. Onowa McIvor from the NETOLNEW project. As the author does not have Indigenous ancestry, the EMT will require ample Indigenous input and collaboration if it is to be useful in Indigenous contexts.

While the EMT's design has been informed and reviewed by multiple researchers experienced in the fields of linguistics, mapping, and education, it has solely been implemented and reported on by the author. As a result, much of the current EMT has been developed with the single researcher's bias and opinions, and the results obtained from the NETOLNEW collaboration are but one individual's interpretation of the EMT's dimensions. This issue is especially salient in the use of coded attributes and the magnitude coding technique, which ultimately is the result of subjective interprettation. An immediate solution to this issue would be to have multiple researchers (or research assistants) apply the EMT to an identical assortment of maps.

In addition, the majority of the EMT's questions and layout have been informed by Girnth's (2010) five defining questions in linguistic mapping, and could stand to be informed by additional sources. Any future develop- ments of the EMT would greatly benefit from the collaboration of Indigenous and non-Indigenous scholars with a variety of perspectives, training, and backgrounds.

\section{Future directions for the EMT}

While the first application of the EMT with NETOLNEW indicates that the Typology can be of use in determining guidelines for more responsible map and atlas production, further research and refinement needs to occur in order for such a typology to make sizeable impacts in cartography, linguistics, and the lives of those who can benefit from its services. Further refinement will strive to eventually adapt the EMT to act as a set of guidelines and conventions in the production of linguistic maps, both in the contexts of Indigenous language revitalization and in linguistics and cartography more generally. In response to Monmonier's (2005) concerns related to blindly following design rules, such formal conventions ought to be flexible and receptive to producers' interpretations, while still offering a sound framework from which to operate. Such a set of conventions will ideally be accessible to cartographers and linguists alike and assist in narrowing the disciplinary gap that exists between linguistics and cartography. Necessarily, such a set of conventions will need to include the continuous input of many scholars and enthusiasts with varied perspectives, perhaps through similar ways as the Open Language Archives Community (OLAC) (www.language-archives.org/), which relies on multiple stakeholders to reach consensuses on best practices in documentation.

\section{Conclusion}

While linguistic maps are used widely in multiple fields for conveying large amounts of information in easy-tounderstand ways, linguistic mapping as a discipline has remained largely underdeveloped and continues to lack a system of guidelines and conventions. Such a system is especially important when linguistic maps focus on Indigenous knowledge, as cartographic practices have historically been employed (and continue) to control knowledge and shift the balance of power in favour of colonial forces. Though still in its preliminary stages of development, the Evaluative Map Typology (EMT) has demonstrated through its implementation in the NETOLNEW project that it can be used to catalogue cartographic techniques in linguistic maps, indicate how they are used to communicate notions of power and authority, and provide recommendations regarding both userfriendliness and ethical considerations. Though there is a great need to continue to research and extend the tool's applicability, a future form of the EMT could have the potential to act as a set of conventions, allowing for linguistic maps to be made more consistently and thoughtfully. 


\section{Acknowledgments}

I would like to thank my supervisors Dr. Erik Anonby and Dr. Marie-Odile Junker at Carleton University for providing me with the insights, skills and direction to make this research possible. In addition, I would like to thank Dr. Onowa McIvor at the University of Victoria for allowing me the opportunity and patience to implement the EMT in the NEXOLNEW project, while providing me with guidance in working responsibly with Indigenous knowledge and worldviews. Finally, I would like to thank Rita Richardson for assisting me in making attendance at the FEL XXII (2018) conference possible.

\section{References}

Aporta, Claudio, Ingrid Kritsch, Alestine Andre, Kristi Benson, Sharon Snowshoe, William Firth \& Del Carry. 2014. The Gwich'in Atlas: Place Names, Maps, and Narratives. In Modern Cartography Series (Vol. 5). 229-244. Academic Press.

Bollmann, Jürgen. 2010. 3. Maps and cognition. In Language Mapping (Vol. 2). 40-69. De Gruyter Mouton.

Breton, Roland. 1992. Easy geolinguistics' and cartographers. Discussion Papers in Geolinguistics 19. 68-70.

Britain, David. 2010. 4. Conceptualizations of geographic space in linguistics. In Language Mapping (Vol. 2). 69-102. De Gruyter Mouton.

Cenerini, Chantale, Marie-Odile Junker \& Nicole Rosen. 2017. Mapping dialectal variation using the Algonquian Linguistic Atlas. Language Documentation and Conservation 11. 305-324.

Creswell, John W. 2017. Research design: Qualitative, quantitative, and mixed methods approaches. Sage publications.

Czaykowska-Higgins, Ewa. 2009. Research models, community engagement, and linguistic fieldwork: Reflections on working within Canadian indigenous communities. Language Documentation \& Conservation 3(1). 15-50.

Engler, Nate J., Teresa Scassa \& DR Fraser Taylor. 2014. Cybercartography and volunteered geographic information. In Modern Cartography Series (Vol. 5). 43-57. Academic Press.

Garuba, Harry. 2002. Mapping the land/body/subject: Colonial and postcolonial geographies in African narrative. Alternation 9(1). 87-116.

Gersmehl, Philip J. 1991. The Language of Maps. Pathway in Geography Series, Title No. 1. Indiana University of Pennsylvania, Indiana, PA.

Girnth, Heiko. (2010). 5. Mapping language data. In Language Mapping (Vol. 2, pp. 98-145). De Gruyter Mouton.
Herlihy, Peter H. \& Gregory Knapp. 2003. Maps of, by, and for the peoples of Latin America. Human organization 62(4). 303-314.

Krauss, Michael. 1992. The world's languages in crisis. Language 68(1). 4-10.

Lameli, Alfred. 2010. Linguistic atlases-traditional and modern. Auer and Schmidt 2010. 567-592.

Luebbering, Candice R. 2011. The Cartographic Representation of Language: Understanding language map construction and visualizing language diversity (Doctoral dissertation, Virginia Tech).

Luebbering, Candice R. 2013. Displaying the geography of language: the cartography of language maps. Linguistics Journal, 7(1). 39-67.

Luebbering, Candice R., Korine N. Kolivras \& Stephen P. Prisley. 2013. The lay of the language: surveying the cartographic characteristics of language maps. Cartography and Geographic Information Science 40(5). 383-400.

Lund, Arnold M. 2001. Measuring usability with the use questionnaire. Usability interface 8(2). 3-6.

McIvor, Onowa \& Peter Jacobs. 2017. NETOLNEW Environmental Scan (pp. 1-2, Issue brief). Victoria WSÁNEĆ \& Lekwungen territories, B.C.: University of Victoria.

Monmonier, Mark. 2005a. POMP and circumstance: Plain old map products in a cybercartographic world. Cybercartography: Theory and practice, pp.15-34.

Monmonier, Mark. 2005. Lying with maps. Statistical science 20(3). 215-222.

Mühlhäusler, Peter. 2010. Mapping linguistic typology. In Language Mapping (Vol. 2). 355-387. De Gruyter Mouton.

Norris, Mary Jane \& Stewart Clatworthy. 2003. Aboriginal mobility and migration within urban Canada: Outcomes, factors and implications. Not strangers in these parts: Urban Aboriginal peoples. $51-78$

Ormeling, Ferjan. 2010. 2. Visualizing geographic space: The nature of maps. In Language Mapping (Vol. 2). 21-43. De Gruyter Mouton.

Peeters, Yvo J.D. 1992. The political importance of the visualisation of language contact. Discussion Papers in Geolinguistics 19. 6-8.

Saldaña, Johnny. 2015. The coding manual for qualitative researchers. Sage.

Simons, Gary \& Steven Bird. n.d. Open Language Archives Community. (www.language-archives.org) (Accessed 2018-07-27). 
Stone, Adam. 2019, in press. Mapping experience: Age and Indigeneity as mediating factors of users' experiences with the Algonquian Linguistic Atlas. Cartographica 52(4).

Taylor, D.R. Fraser. 2014. Some Recent Developments in the Theory and Practice of Cybercartography: Applications in Indigenous Mapping: An Introduc- tion. In Modern Cartography Series (Vol. 5). 1-15. Academic Press.

Upton, Clive. 2010. 7. Designing maps for nonlinguists. In Language Mapping (Vol. 2). 142-166. De Gruyter Mouton.

Williams, Colin H. 1988. An introduction to geolinguistics. Language in geographic context. 1-19. 
Following the development and implementation of the EMT, I assisted Dr. Erik Anonby in a subsequent iteration, which, instead of being in the form of a fillable PDF with devoted sections to enter text, occurs in list form (Appendix III). From this list, other interactive forms can be made that are designed for their specific purposes using third-party form building platforms. For example, as part of an upper-year introductory language mapping course taught by Dr. Anonby and assisted by $\mathrm{me}^{41}$, students were instructed to critique a language map of their choice using the typology, which had been transformed into an interactive form using Google Forms (Figure $32) .{ }^{42}$

Figure 32: An interactive Google Form of Anonby and Stone's (2016-2019) typology.

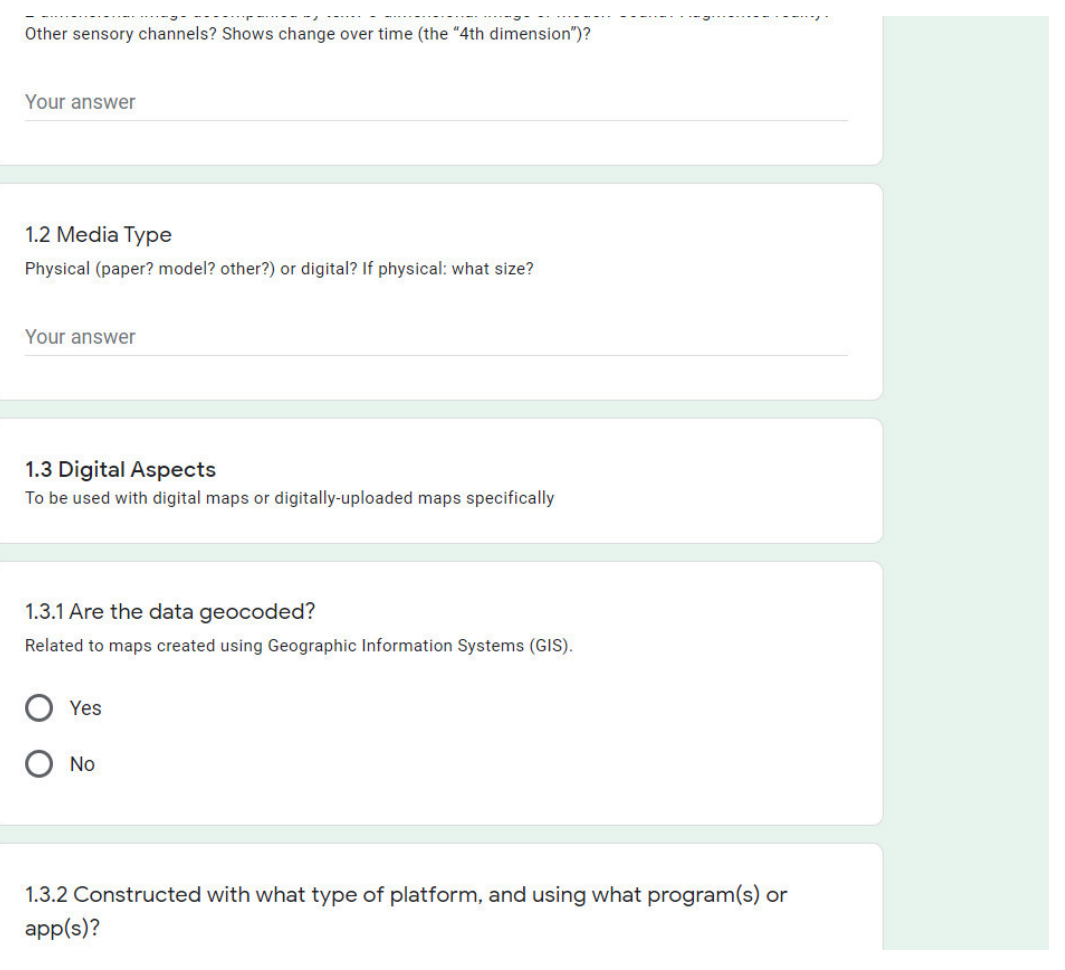

\footnotetext{
41 The course is titled "Language Mapping" and in its first implementation in the Fall semester of 2019, involved fourth-year undergraduate and first-year graduate students from geography, linguistics, and applied linguistics departments.

42 As of July 26, 2020, this online form is accessible at https://forms.gle/vwMYwPnwGQULYHNRA
} 
Using this form, students could then generate a PDF document containing the answers to the items listed in the typology, and I as the teaching assistant received an e-mail with their responses listed.

\subsection{Applications of the language mapping typologies.}

It is important to note that the typologies introduced in this dissertation are not just hypothetical classification systems, but demonstrations of how such classification systems are meaningful and can make a tangible impact in language mapping. While the use of a language mapping typology in the development of a new atlas is showcased with the EMT, the utility of Anonby and Stone's (2016-2019) typology is demonstrated in Indigenous Language Education. While these two typologies are not the first to attempt to address issues within language mapping, they do mark a departure from previous endeavours by authors such as Ambrose and Williams (1991) as they are developed while simultaneously being put into practice. Ideally, future research will follow similar lines of inquiry, whereby subsequent iterations of a language mapping typology are informed by other and emerging language mapping areas.

\subsection{Reflections on the mapping typologies.}

The two versions of the language mapping typologies presented here suggest that refinement can indeed be borne out of the typology process. While Stone's (2018b) evaluative map typology is a basic form whose methods of analysis are demonstrated, Anonby and Stone's (2016-2019) language mapping typology identifies more components of maps that can be deconstructed. An 
arguable strength they share is that they were both being put into practice while being designed and refined, meaning that they could make use of feedback from both users and results.

A trait relevant to them both is that they take ample time to complete when done carefully, meaning large-scale and comprehensive analyses can take many hours. This is due to the numerous dimensions that the typologies identify and is a practical consideration that must be managed in their implementation. For example, in the upper-year language mapping course, the students chose which elements of the typology were most relevant to their maps and analyses and omitted irrelevant sections. Therefore, innovations in the use of the typology arose organically, and greater flexibility in the typologies' implementations will likely come from improvements in the ways the typologies are used. If users of a typology can make use of an artificially intelligent program that scans the maps, isolates their components, and automatically creates summaries — social elements such as power would still need to be added manually—more analyses will be possible in a smaller time frame. Such an innovation would certainly bring with it a new and complex set of challenges and ethical dilemmas, such as the concern that it would be highly unlikely that any computer program that cannot think for itself would be unable to deconstruct the very human map to the extent that humans can.

Furthermore, while these typologies have been made by those with prior knowledge of and experience in language mapping, they nonetheless build on the expertise of specialized researchers across academia. As emerging disciplines related to language mapping such as Cybercartography aim to democratize the mapping process and link stakeholders (as in Section 5.6.4), further development of typologies should logically also include the input from a variety of those who relate in some way to language mapping. One example of how this has already begun to happen involves the use of the Anonby and Stone's (2016-2019) typology in the classroom 
environment. During their work with the typology, the students had the opportunity to give us feedback regarding the typology from the perspective of someone using it for the first time and describe what they liked and thought needed improvement.

It is important to note that I did not use Anonby and Stone's (2016-2019) version of the typology in my doctoral research. Studies 1 and 2 used the EMT instead for three main reasons. First, Anonby and Stone's (2016-2019) typology was not in its current form when the EMT study (Stone, 2018b) took place. Second, the competitive analysis in Study 2 expands upon the research in Study 1 in such a way that the basic tool of analysis remains the same but is extended and made more precise in scope. In this way, the consistent use of the EMT makes Studies 1 and 2 more comparable to one another. Third, the use of the EMT over Anonby and Stone's (20162019) typology is an added measure to ensure that the dissertation makes use of tools of which I am a principal author. ${ }^{43}$ From here, Study 2 takes the EMT and adds a known method of analysis in cartography for the purpose of studying language maps based on their available features.

\footnotetext{
${ }^{43}$ This should not underscore the pivotal role Erik Anonby played in conceiving of and informing the nature and structure of the EMT, as well as the support and guidance he provided during the planning, implementation, and reflection stages in Study 1.
} 


\section{Study 2}

A competitive analysis of online language atlases 
12.0 A competitive analysis of online language maps.

This section includes a competitive analysis study of contemporary online digital language maps ${ }^{44}$ which are currently accessible on the internet. In addition to the methods used by Roth et al. (2015) discussed in Section (10.0), this study analyzes digital language atlases using the Evaluative Map Typology (EMT), reflecting a competitive analysis-type investigation that is tailor-made to studies of language and space. Following the competitive analysis, I interview three atlas authors to contextualize the decisions made in the creation of digital language atlases. Considering the rate at which new technologies become available, maps go offline, and others are introduced, it should be noted that this general assortment of available language maps online is expected to have changed and to continue changing in the coming years.

Originally framed by Nielsen $(1992$, p.3), a competitive analysis is a comparative study that focuses on the relative strengths and weaknesses of competing products' and technologies' approaches to user interface designs. Map developers can avoid 'reinventing the wheel,' as competitive analyses assume that similar existing products work as well or better than a wouldbe prototype. This means that developers can obtain a lot of information and recommendations related to general design, performance, and functionality while respecting copyrighted material and intellectual property laws. Furthermore, Roth, Quinn, and Hart $(2015$, p.1) describe a competitive analysis as a content analysis of secondary resources, especially when applied to fields and archiving practices found within the social sciences. In the context of online language mapping, Roth, Quinn, and Hart $(2015$, p.1) are quick to indicate that a competitive analysis is a good preliminary survey, but cannot replace user-based evaluations. Therefore, the competitive

\footnotetext{
${ }^{44}$ The terms map and atlas often occur interchangeably in language mapping discourse of this nature. As such, map will be used to refer to both maps and atlases in this part, unless otherwise specified.
} 
analysis here is followed by the Algonquian Linguistic Atlas user study in Part 3 to add a human perspective to the investigation.

The first unstructured component of this competitive analysis includes a total of 47 online and web-based language maps from sources in English, French, German, Portuguese, Japanese, and Italian. I categorize each online map according to whether it is interactive or not, and as to whether it had originally been developed online or digitized and uploaded to the internet from paper form. A summary of the medium these maps are found in is shown Table 3 below. In addition, I list 12 web mapping applications, 10 GIS software packages, and 8 resources for GIS programs relevant to language mapping in the analysis. While there are countless other maps, software packages, and emerging platforms on the internet that have not been included here, the list provided in this analysis functions as a first necessary foray into what can be available online.

Table 3: Number and proportions of maps found in the interpretive analysis.

\begin{tabular}{|l|c|c|}
\hline & Static & Interactive \\
\hline Digitized & 8 & 1 \\
\hline Web based & 14 & 24 \\
\hline
\end{tabular}

While the preliminary observations of each map and technology are found in Appendix IV, I chose 11 interactive web-based maps for the subsequent analyses that involve the EMT, competitive analyses, and semi-structured interviews, the results of which are shown below. 
12.1 A competitive analysis performed using the EMT.

Unlike the application of the EMT outlined in the previous article, this investigation has been conducted specifically for the purposes of this dissertation, and only involves digital maps that focus on languages, as opposed to more general maps and maps of cultural knowledge. In doing so, this analysis aims to gain more specific insights into language mapping practices, and combined methods found in Roth et al. (2014) and Roth et al. (2015) with the EMT, taking beneficial attributes from both.

12.1.1 The online digital language map sample.

The 11 maps in this analysis focus on the distribution of language varieties, language status, and structural features such as speech sounds and lexical items. Maps and atlases such as NativeLand or the Atlas of North American English cover a relatively large geographic area, such as a province or country, while the UNESCO Atlas, WALS, and WATP display more global distributions. While the majority of the maps and atlases selected for analysis primarily involve language distribution information, ANAE, PHOIBLE, and WATP display the distributions of phonological and other structural linguistic features. Each map was also present in the initial unstructured survey of 47 maps. The 11 maps used in this analysis are as follow in Table 4: 
Table 4: The 11 interactive language maps and atlases selected for analysis.

First Peoples Language map of BC https://maps.fpcc.ca/

Language and Location (LL) Map http://lmap.org/

Modern Language Association

(MLA) Map

https://www.mla.org/Resources/Research/MLA-

Language-Map

NativeLand

https://native-land.ca/

PHOIBLE

https://phoible.org/

Sprachatlas Niederbayern

https://sprachatlasniederbayern.bayerischelandesbibliothek-online.de/

Atlas of North American English (ANAE)

UNESCO Atlas of the World's Languages in Danger

The World Atlas of Language

Structures (WALS)

https://www.ling.upenn.edu/phono_atlas/home.html

The World Atlas of Transitivity

Pairs (WATP)

The Algonquian Linguistic Atlas http://www.atlas-ling.ca

http://www.unesco.org/languages-atlas/

https://wals.info/

http://watp.ninjal.ac.jp/en/

Along with the other maps found in Appendix $I V$, I used convenience sampling to gather these 11, whereby I focused on maps already known to me. Once those maps were exhausted, additional maps were then selected based on a cursory search of digital atlases and maps found on Google using keywords such as "digital language map", "online linguistic map" and "language distribution map." From here, the 11 maps were selected for further analysis due to their digital and interactive nature, as well as their relative prominence in language mapping. In addition to maps and atlases, I also explored other accompanying information or features on a 
website where the maps are featured, as this additional information can also play an important role in understanding how users interact with the maps and situate themselves in relation to the information they convey.

In a similar manner to the EMT implemented by Stone (2018b), this investigation employed qualitative coding practices that look for patterns, whereby features and aspects are summarized into one- or two-word codes which are then counted to determine which are the most prevalent (Saldaña, 2013, p.4). As such, each of the 11 maps have been summarized below in Table 5 according to their strengths and weaknesses, which is then followed by Table 6, which ranks these codes by how often they occur: 
Table 5: Summary of an impressionistic survey of digital interactive language maps.

\begin{tabular}{|c|c|c|}
\hline Map Name & Strengths & Weaknesses \\
\hline $\begin{array}{l}\text { First Peoples } \\
\text { Language map of BC }\end{array}$ & $\begin{array}{l}\text { Interactive. } \\
\text { Visually appealing. } \\
\text { Navigable. } \\
\text { Freedom to explore. }\end{array}$ & $\begin{array}{l}\text { Missing information. } \\
\text { System errors. }\end{array}$ \\
\hline LL-Map & Minimalist. & $\begin{array}{l}\text { Dead links. } \\
\text { High bandwidth necessary. }\end{array}$ \\
\hline MLA Map & $\begin{array}{l}\text { Objective. } \\
\text { Attractive. } \\
\text { Interactive. }\end{array}$ & Not maintained. \\
\hline NativeLand & $\begin{array}{l}\text { Indigenous narratives. } \\
\text { Self-aware. } \\
\text { Honest. }\end{array}$ & Distorted projection. \\
\hline PHOIBLE & $\begin{array}{l}\text { Verifiable information. } \\
\text { Downloadable data. }\end{array}$ & $\begin{array}{l}\text { Biased politically. } \\
\text { No jargon definitions. }\end{array}$ \\
\hline $\begin{array}{l}\text { Sprachatlas } \\
\text { Niederbayern }\end{array}$ & $\begin{array}{l}\text { Minimalist. } \\
\text { Multimedia. }\end{array}$ & $\begin{array}{l}\text { Flash outdated. } \\
\text { Crowded. }\end{array}$ \\
\hline $\begin{array}{l}\text { Atlas of North } \\
\text { American English } \\
\text { (ANAE) }\end{array}$ & $\begin{array}{l}\text { Minimalist. } \\
\text { Simple. }\end{array}$ & $\begin{array}{l}\text { Dead links. } \\
\text { Unclear symbology. }\end{array}$ \\
\hline $\begin{array}{l}\text { UNESCO Atlas of the } \\
\text { World's Languages in } \\
\text { Danger }\end{array}$ & $\begin{array}{l}\text { Detailed commentary. } \\
\text { Simple symbology. }\end{array}$ & $\begin{array}{l}\text { Dead links. } \\
\text { Not maintained. } \\
\text { Over-generalized data. }\end{array}$ \\
\hline WALS & $\begin{array}{l}\text { Jargon definitions available. } \\
\text { Accessible database. } \\
\text { Good search feature. }\end{array}$ & Difficult to find. \\
\hline WATP & $\begin{array}{l}\text { Reliable data. } \\
\text { Clear features. } \\
\text { Accompanying graphics. }\end{array}$ & Difficult to contribute to. \\
\hline $\begin{array}{l}\text { The Algonquian } \\
\text { Linguistic Atlas }\end{array}$ & $\begin{array}{l}\text { Verifiable information. } \\
\text { Attractive. } \\
\text { Self-aware. }\end{array}$ & High bandwidth necessary \\
\hline
\end{tabular}

12.1.2 An analysis of the language map sample's relative strengths and weaknesses.

The ranked frequencies of each code are displayed below, in Table 6. As some maps had more than one trait coded, the number of codes below do not add up to eleven. In addition, these 
codes are based on the aggregation of other similar codes used in the EMT, a summary and description of which is found in Appendix V:

Table 6: Ranked frequencies of each code for the 11 maps.

\begin{tabular}{l|c}
\multicolumn{1}{c|}{ Strengths } & \# uses \\
\hline VERIFIABLE & 4 \\
\hline MINIMALIST & 3 \\
\hline ATTRACTIVE & 3 \\
\hline CLEAR & 2 \\
\hline SELF-AWARE & 2 \\
\hline OBJECTIVE & 1
\end{tabular}

\begin{tabular}{l|c}
\multicolumn{1}{c|}{ Weaknesses $^{\mathbf{4 5}}$} & \# uses \\
\hline DEAD LINKS & 3 \\
\hline OUTDATED & 3 \\
\hline NOT MAINTAINED & 2 \\
\hline BANDWIDTH ISSUES & 2 \\
\hline MISSING INFO. & 1 \\
\hline POLITICAL BIAS & 1
\end{tabular}

Visual summaries of the ranked frequencies for each code are as follow, in Figure 33:

${ }^{45}$ Most of the maps reviewed were exceptional outputs of years of hard work, and map developers have paid a lot of attention to creating good maps. This makes criticisms of maps more difficult to make. 
Figure 33: Favourable and unfavourable attributes of the surveyed maps
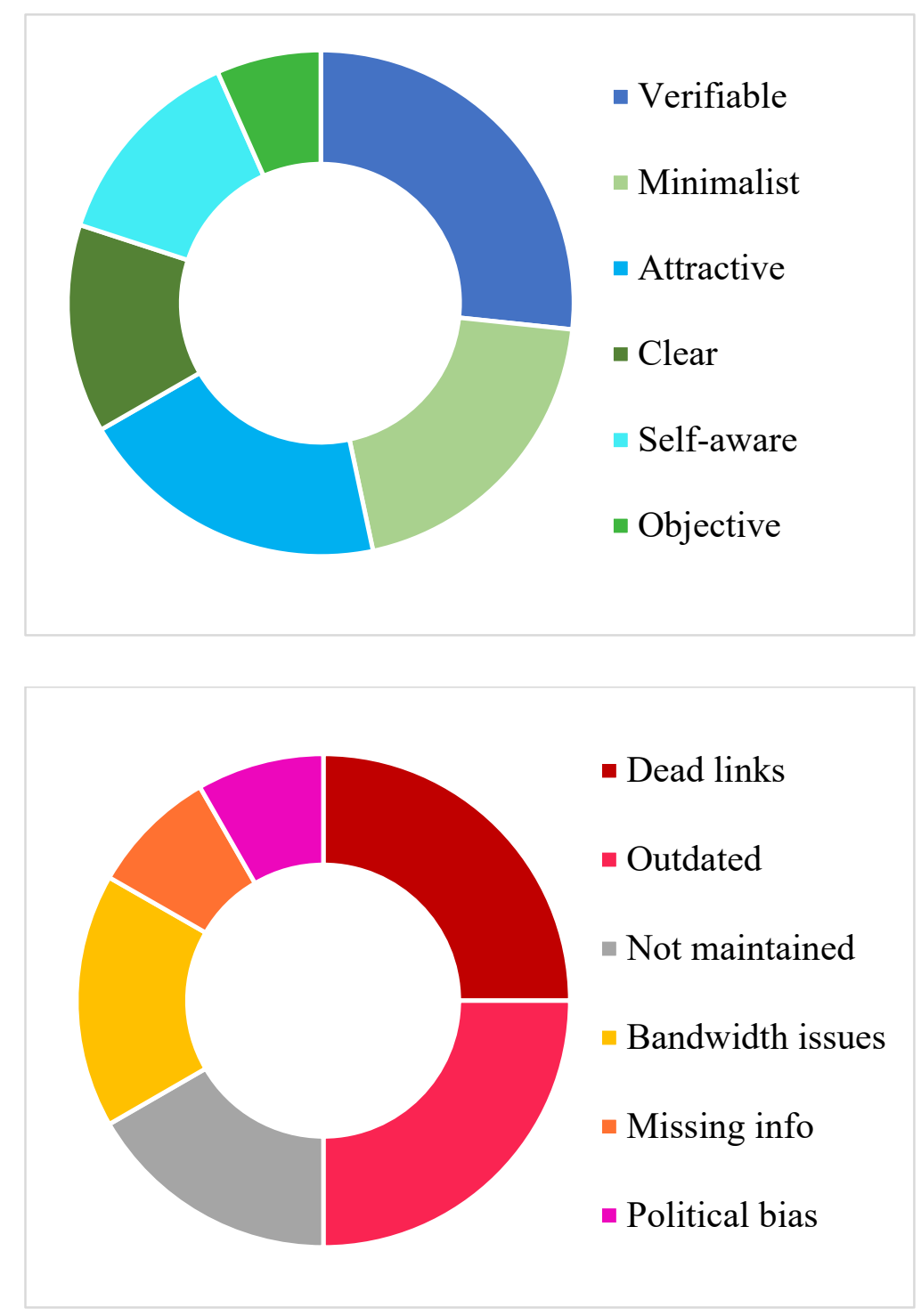

These observations tell us that in a sample of 11 online digital language maps, some of the most common positive traits ${ }^{46}$ of language maps observed by me include a minimalist layout and the use of information which can be verified, either via a database or links to the sources from

\footnotetext{
${ }^{46}$ It should be kept in mind that what is considered positive or negative here is subjective, and indicative of my own biases toward language maps and their features. Others performing similar analyses may have different attributes organized in a different way.
} 
which the information originated. At the same time, I observed the sample of language maps to be plagued most by dead links, outdated software, and a lack of maintenance. However, looking at their relative strengths and weaknesses only sheds light on one aspect of language maps, and while a larger sample analyzed by more people would add detail to this analysis, there are other dimensions that need to be focused on as well. As such, a visual analysis following the methods of Roth, Quinn, and Hart (2015) below focuses on the features gathered by the EMT that the sample of language maps offer.

12.1.3 A comparison of language map features using the competitive analysis method.

The below table (Table 7) displays the features that are available for the sample of 11 online digital language maps. While feature categories such as zoom, pan, search, and filter are reused from Roth, Quinn, and Hart (2015, p.11), I have also added features I believe to be more appropriate to a study of language maps. These are based upon the insights provided in Part 1, sections within the EMT forms, and in the above strengths/weaknesses analysis. As the use of a feature is often the result of an (un)informed choice on part of those making the map and guided by the nature of the mapping, the presence of a feature should not necessarily be thought of as positive, while the absence of a feature is not necessarily negative. 
Table 7: Supported interaction operators found in a sample of 11 language maps

\begin{tabular}{|c|c|c|c|c|c|c|c|c|c|c|c|c|c|}
\hline Map/Atlas & \multicolumn{13}{|c|}{ Supported Feature } \\
\hline $\begin{array}{l}\text { First Peoples } \\
\text { Language } \\
\text { map of BC }\end{array}$ & & & & & & & & & & & & & \\
\hline LL-Map & & & & & & & & & & & & & \\
\hline MLA Map & & & & & & & & & & & & & \\
\hline NativeLand & & & & & & & & & & & & & \\
\hline PHOIBLE & & & & & & & & & & & & & \\
\hline $\begin{array}{l}\text { Sprachatlas } \\
\text { Niederbayern }\end{array}$ & & & & & & & & & & & & & \\
\hline ANAE & & & & & & & & & & & & & \\
\hline UNESCO & & & & & & & & & & & & & \\
\hline WALS & & & & & & & & & & & & & \\
\hline WATP & & & & & & & & & & & & & \\
\hline $\begin{array}{l}\text { Algonquian } \\
\text { Linguistic } \\
\text { Atlas }\end{array}$ & & & & & & & & & & & & & \\
\hline $\begin{array}{l}\text { Present } \\
\text { Partial } \\
\text { Absent }\end{array}$ & $\begin{array}{l}\tilde{\Xi} \\
\stackrel{0}{\circ} \\
N\end{array}$ & Е & 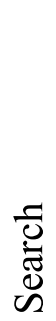 & 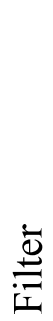 & 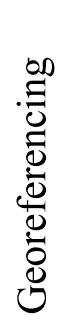 & 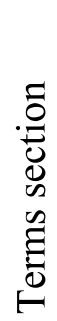 & 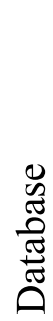 & 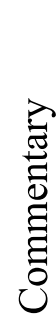 & 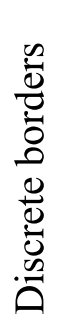 & 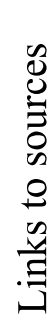 & 㐫 & 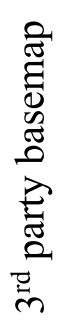 & 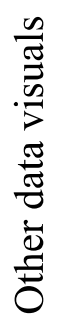 \\
\hline
\end{tabular}

In this analysis, filters and links to sources ( $\mathrm{n}=9$ each) and the zoom, pan, and $3^{\text {rd }}$ party basemap features $(n=8$ each) appear the most frequently, while georeferenced information $(n=4)$ is the least frequent. In addition, the MLA map had the most surveyed functions $(n=13)$, and the 
Atlas of North American English (ANAE) had the fewest $(n=4)$, though in practical terms the UNESCO atlas had even fewer available due to the outdated software it runs on.

While some academic maps such as in the WATP make full use of their surveyed features, maps whose information is not intended primarily for academic research, like those in NativeLand, have more rudimentary versions of some features, such as an available database. As evidenced by the UNESCO atlas, maps that have not been maintained or contributed to for some time have functions which cannot be used, due to their outdated features and resources. Here, it is relevant to note that these features of interest may be more academic in nature. ${ }^{47}$ Were this analysis conducted in the context of secondary education, for example, the features I selected to be analyzed could be very different. In addition, these selected features are but a small set of possible features from language mapping typologies with which language maps could be evaluated. This hypothesized discrepancy highlights the need for analyses such as this, as well as the coding of strengths and weaknesses, to be carried out by multiple people from across varied aspects of the mapping process. This broad spectrum should produce a process which is more inclusive and responsive to differing perspectives. Furthermore, any comparative and reflective language mapping studies would also benefit greatly from understanding why maps and atlases are built the way they are.

\subsection{Interviews with atlas authors.}

While the above observations, especially regarding online language maps, highlight some notable features and options currently available, they do not explain why commonplace options

\footnotetext{
${ }^{47}$ Reflectively, this is likely due to my academic background and inclination, as well as the academic setting this research is conducted in.
} 
are commonplace, or why some map authors made seemingly arbitrary decisions. As such, I sent out interview requests via e-mail to six online language mapping projects and received three responses. Of special interest are language atlases which have been designed to visualize language data found in a back-end database, as they give me the opportunity to study decisions being made in both the language map and database. With the permission of the interviewees, their answers are listed below, while the original request for an interview can be found in Appendix VI.

12.2.1 Interview with the programmer for PHOIBLE.

The following interview took place via e-mail on Friday, September 27, 2019 with Robert Forkel, who is the programmer for PHOIBLE, the repository of cross-linguistic phonological inventory data. His answers to the interview questions are found below, and quoted verbatim from the e-mails:

Let me start by highlighting that in the last years the focus of much of my work was "getting away" from online databases, or at least minimizing their role in actual linguistic research to showcasing some data (i.e. helping with window-shopping for data), while the actual data access is to long-term archived packages of CLDF (https://cldf.clld.org) data, (e.g. from https://zenodo.org/communities/cldf-datasets). The reason is mainly that the maintenance cost of code running on a server is quite high (the web still moves quickly, and security must always be a concern). This is particularly true for code implementing any "fancy" functionality, going into the direction of data analysis. 
Analysis of the data happens locally, using R, Python, spreadsheet software, or whatever a researcher is most comfortable with. We found that most people who will actually do analyses with our datasets will have specific needs which are typically not covered by the web app functionality. Instead, most of them have tools in their analysis pipeline, like $R$ or python, which will support any data manipulation they wish. So rather than trying to compete with desktop analysis tools in a web app, we encourage people to download the full data and use local tools.

\section{What kind of database do you use for your project?}

We use PostgreSQL - a relational database - as backend for data served by a web application built on top of our clld (https://github.com/clld/clld) framework.

\section{What is the name and version of the specific database software you are using?}

PostgreSQL 9.x, accessed from Python 3.5 via SQLAlchemy. All this within clld 4.x.

\section{Why did you choose this form of database to work with?}

PHOIBLE is one of many (https://clld.org/datasets.html) online databases maintained by the same small technical group. Thus, homogeneity across all these databases is vital for maintainability. That said, PostgreSQL can surely deal with most workloads/data models/usage scenarios one could encounter with linguistic data, and clld is quite flexible. So not much lock-in is caused by this choice. 
Why did you choose this specific database program to work with?

Is this about relational vs. no-SQL? Or relational vs. graph-db? Anyway,

PostgreSQL is certainly among the most mature and stable products out there, and seems to have recovered quite a bit of market share from more specific $D B$ (database) products

(e.g. the JSON support is now really good, so no need to look for document-oriented databases).

Is this the first database software and version you have used in this project, or did you use a previous version or program at the beginning of the project?

In other (similar) projects I've used sqlite, mysql, a triple store, or plain file-system backend. Now I am firmly settled on PostgreSQL.

What do you see the benefits of using this form of database over others being?

Well understood performance characteristics, ease of administration, and scalability.

Are there any shortcomings of this database you have encountered in the development and implementation of your atlas?

No.

12.2.2 Interview with the project lead of NativeLand.ca.

The following interview took place over the phone on Thursday, October 3, 2019 with Victor Temprano, who has run and administered the NativeLand.ca project since 2015. The project relays and displays information related to the distribution of Indigenous lands, treaties, 
and languages. His answers are paraphrased into full sentences, as they were originally copied down in point form.

\section{What kind of database do you use for your project?}

We do not use formal databases per se for the maps, we use an online platform called MapBox instead, which just uses computer files. The closest thing to a database we have is the dataset in MapBox. Everything is in GEOJSON format, which is the easiest format for mapping libraries to use.

For the main website, we use a Wordpress site, but it does not house the geographic information. Instead, I have built a plugin that helps to manage the MapBox repository. We have three different datasets in our MapBox account, which makes it easier for research assistants to work with all the information.

Overall, we enjoy using the MapBox platform, as it can handle large amounts of data without any significant amount of lag. That being said, the maps need to render frequently once thousands of points are there. While most MapBox projects of this size require a paid subscription, we are sponsored by them, meaning that we ourselves currently do not need to pay.

\section{Why did you choose this form of database to work with?}

We had originally used Google Maps, but switched to the open-source program Leaflet. However, we switched back to Google Maps because Leaflet gave us issues with updating our data once we started getting them in large amounts. I tried MapBox out one day, and once I got deeper into the platform's interface, I enjoyed using it. It's the closest thing I have to running my own tile server. 
Is this the first version you have used, or did you use a previous version at the beginning of the project?

This is not the first version we have used. MapBox does a lot of feature and interface changes and constantly updates the way it looks. Some of these features improve over time, such as better heat maps, more search filters, and new apps.

\section{What do you see the benefits of using this form of database over others being?}

Its ease of use and customizability, as well as a lot of community support and an online course on Udemy.

\section{Are there any shortcomings of this database you have encountered in the development and implementation of your atlas?}

The interface's complexity is difficult to start out with. It can be quite hard working with lots of layers, especially when it is not made obvious how things work. While a basic map with MapBox is free to begin with, the interface's costs rise with the number of views your atlas gets.

MapBox was originally designed for use by experienced developers, and so has fewer help supports than Google Maps. That being said, this is changing, and there is an increasing amount of support for general users as well.

12.2.3 Interview with the director and the programmer of the Algonquian Linguistic Atlas.

The following interview took place via the video chat application Zoom on Tuesday, May 26, 2020 with Dr. Marie-Odile Junker, project director of the Algonquian Linguistic Atlas, and Delasie Torkornoo, technical director. The answers here are paraphrased from the interview. 


\section{What kind of database do you use for your project?}

While the actual database we use is MySQL, we run it through a database management system. Due to the sensitive nature of our linguistic information and related security issues, the database management system makes security easier and more reliable. The database management system we use utilizes CakePHP for its front end, and TurboGear in the background. Importantly, it should be noted that the Algonquian Linguistic Atlas is a database first and foremost, whose data are presented to viewers via an interactive map, a series of linguistic feature maps, and interactive lessons.

\section{Why did you choose this form of database to work with?}

This kind of database is the most publicly available, and to make the most out of funding, it made sense to code the atlas and use a publicly available server. However, the front end could run on anything really, such as Apache or PHP.

\section{Why did you choose this specific database management program to work with?}

Sustainability and affordability are important to the project.

Is this the first database software and version you have used in this project, or did you use a previous version or program at the beginning of the project?

We originally used Flash, but not for our database system. Originally, in 2004, our database was essentially a CSV, just a table with columns. The first version of this atlas was managed using TurboGear 0.9 and Python 2.4. While the back end still runs TurboGear, the front end does not. Now, we make sure to duplicate our database whenever we make changes, and work on the admin interface. 
When we first built the atlas and database, we emphasized on finding ways for collaborators to contribute directly to the atlas. However, this turned out to be overly idealistic, as unless they engaged with the atlas interface routinely, even academics who had received specific training on how to upload information found themselves unable to do so. It ended up being more efficient for collaborators to focus on the data and have a dedicated employee enter the data for them. Collaborators could still request changes to the data at any time but did not need to spend time learning how to use the back-end interface.

Since the beginning, we designed the original interface to be user friendly, however, an issue of time and control remains within engaging with the back end.

\section{Are there any shortcomings of this database you have encountered in the development and implementation of your atlas?}

Some issues do remain. For example, we do not have a lot of computational power compared to more high-end database software. To get around this, we process the data elsewhere and then return them to the atlas. While this involves extra steps and time, it allows us to ensure that we retain rights to the data and code, which is a priority.

As newer versions of software get developed, an ongoing challenge is migrating the data. Quite often, migrating the data is a different process each time, meaning that we wait until we absolutely need to migrate data over to newer versions, and always stay with the long-term release editions. This is true of our CakePHP, for example, and with Python. When a new version of Python emerged which rendered an older version obsolete, we needed to write a lot of the code again from scratch. In addition, while we 
started off our database entries as items in a table, we now compute data as objects, meaning that the way we structure things has needed to change completely.

Obsolescence of the software used to power our atlas is an ongoing challenge, as well as the threat of our atlas itself becoming obsolete. While an application that promises a new atlas with fresh ideas and solutions can be attractive to funding bodies, it is much more difficult to obtain comparable funding for the maintenance of an atlas already in existence.

\section{What are some of the benefits you have found to using an interactive map format for your information?}

The original goal of this research in 2004 was to create a conversational manual on CD-ROM for Cree educators. Early on however, these Cree educators in conjunction with Marie-Odile pointed out the utility of basing these recordings geographically, to learn more about how different language varieties related to one another, and to show how language exists independent of newly-imposed political borders. As such, the main idea was linking language and healing, and separating languages from provincial boundary notions, and was followed later by more academic applications.

In more recent times, community-based and academia-based people alike enjoy using the atlas for both learning and teaching. Those who describe the atlas to us tend to say that the atlas is fun to use and informative and does not overwhelm them with information. The geographic component allows them to see relationships between languages and phrases that many of them, who are first-or second-language speakers of an Indigenous language, had not seen previously. 
It should be mentioned that the goal was never to make an atlas in the same way that many cybercartographic atlases are made through Nunaliit. The data come first, and the map is essentially a graphical way of visualizing databases.

\section{What are some of the challenges you have found with using an interactive map format?}

Because of the basemap we use, we are in many ways at the mercy of Google, and the decisions the company makes. For example, if they make changes to their pricing or privacy policies, the atlas will be impacted directly. Furthermore, if Google makes fundamental changes to their maps, which they have done on occasion, everything will need to be re-registered. Thankfully however, we are not married to Google, and while we use their map as a basemap, we host our own data. Were our data to be hosted with Google as well, we would be at risk of having it used for other purposes. While some major companies such as Google are changing (and ostensibly improving) their policies surrounding the protection of Indigenous knowledge, there is still a long way to go. Our data could still be appropriated for promotion purposes, for example.

In addition, our maps include some languages which do not have a standard orthography, meaning that the same word spelt three different ways will have appeared as three different words. This can be confusing practically but may also cause contention among users by prioritizing one orthography over another. As such, the sound recordings are now considered to be the primary medium of communication, followed by the written forms, which are secondary. This solves the orthography challenge, but also more closely reflects Indigenous languages, which historically have been spoken, not written. 
We tried working with linguistic symbology such as isoglosses at the beginning of the project, but it was not pretty. There is a fair amount of contention surrounding the placement of these would-be isoglosses, considering that many linguistic boundaries are too fuzzy to be adequately captured by isoglosses, and considering that many Indigenous groups whose information is on the maps moved around historically. So now, for the dialectal maps public interface, we automatically generate colour-coded circles, after analyzing and coding the sound data in the admin interface.

We tried making a mobile version that displayed maps, but they were difficult to visualize due to too many features being present for a small screen. In addition, every device is different, and would visualize the map differently-some better than others. As such, we do have a mobile version available, but to date it makes use of our interactive language learning platform, which is in list form. This language learning platform importantly can also act as an offline resource, which allows users to download tracks for later, and then save and reorganize phrases.

12.3 Reflecting on the competitive analysis and semi-structured interviews.

The analyses carried out here yield some important general observations about online language maps and the process of studying them, which are elaborated upon and contextualized by the semi-structured interviews of the authors and developers of three online digital language maps. The findings here come from both the competitive analyses and interviews: 


\section{- Language maps and mapping technologies are often made with linguists in}

mind. Language maps, especially those spearheaded by linguists, are often difficult for those without a background in linguistics to understand. Undefined jargon, references to similar yet equally indecipherable studies, and specialized maps whose data are not thoroughly explained risk making the atlases inaccessible to many potential audience members and even other linguists who wish to build similar maps.

- Less is (often) more. Online language maps that have too many functions, features, and layers risk being overwhelming and difficult to navigate for first-time users. Some of the clearest and easy-to-navigate maps either reduced the number of features to only those the creators deemed most critical, or had features which could be toggled on or off by the user at their discretion. However, there is a difficult balance to strike: users may assume that if they do not see something on a map, it may not exist or be noteworthy enough to be included. One possible option could be to make some data accessible to casual users of a map, while more sensitive or personal information requires additional permission from contributors and/or map authors.

- Sustainability is an issue. Some maps, even when designed with the intent of being usable for a long time, have been created using platforms such as Flash, which will soon become obsolete. What this means is that even projects that are regularly supported and maintained need to be rebuilt from scratch over regular time periods. As such, it is important for online mapping projects to focus on long-term archiving solutions, including the use of mature open-source platforms that have a smaller server footprint (Junker \& Torkornoo, 2017, p.7) and consistent guidelines regarding file naming, file formats, and folder structures (p.6). 
In addition, it is typically easier to obtain funding and support to create a new language map than it is to maintain one. While new maps shed light on linguistic/cultural phenomena and showcase important initiatives or research, already existing maps contribute knowledge much more slowly, update existing information, or add knowledge in less visible ways, making them less appealing to funding bodies. This is an issue that can be encountered throughout digital data systems more generally, which tend to emphasize releasing data instead of finding ways to reuse them or maintain access (Borgman, 2015, p.39). As a result, many projects that are dependent on external funding cease to be maintained, meaning that they may crash and become unavailable, or slowly lose their functionality and continue to exist as 'zombie maps.'

- Many atlases are buried. On particular atlas sites, seemingly especially those whose research is academic in nature, the maps are often accessible only after venturing deep into the hierarchy of the site's web pages. During this analysis, some maps required multiple attempts to be found. As such, a language map may technically be present in a research project, but nonetheless risks being passed over by audiences.

- Browser/device incompatibility is an issue. Many online maps could not be viewed properly in certain internet browsers such as Microsoft Edge or Firefox. This risks limiting access to those who know which browsers to use, or those who can use more than one browser. In addition, many maps cannot be visualized properly (or at all) on smartphone devices. This is an important issue to consider given that many in the developing world, as well as those with less access to public resources in developed 
nations, can only access the internet with smartphones (Glushkova, Belotserkovich, Morgunova, \& Yuzhakova, 2019, p.11).

- Maps tell a story which is often not acknowledged. While all the linguistic data and information about the projection system and metadata may be available, many of the surveyed maps failed to provide a detailed rationale for why the maps were being made or for whom. In some cases, this prevented the information being discussed from being properly situated in the broader context of its field, meaning that much of that information remained ambiguous.

- Security is a constant and expensive issue. A constant threat of hackers stealing information from databases or corrupting projects means that, along with the linguistic data being presented, there are other design features which need consideration from the project's inception. An additional server if often required to maintain site security and preserve backups of linguistic data in the event of damage or loss; this server is frequently dedicated to only these purposes. Maintaining effective security can thus take up a significant portion of a project's funding and can be an especially delicate issue when involving community knowledge and information.

- Many GIS programs are free. The majority of the surveyed GIS software was opensource or at least free to use. This could come from the dominance of Esri technologies such as ArcGIS in the area of proprietary GIS software. However, while a mapmaking technology may be free, it still costs time and resources to make a map, it can also be expensive to run and maintain secure servers that host the maps and their data. Importantly, while maintaining the servers and platforms which host and 
run the atlas are one of the most expensive aspects of many projects, it is also difficult to secure funding for this aspect of projects, as this area does not directly contribute to knowledge. ${ }^{48}$

- For many, choosing the right framework is trial-and-error. Some projects appear to change the platform or framework they use multiple times during their initial development and maintenance phases. Some platforms become more advanced and gain more functions, while others may become obsolete and fall behind. In addition, some platforms that are appropriate for small projects may no longer be appropriate once the project grows in scope, size, or data requirements. Language mapping projects can save time and money by planning and accounting for how a platform will perform in the future, preparing for increases in scale and scope, as well as surveying existing technologies through techniques such as a competitive analysis.

- Know where the data are hosted and who can access them. While large tech companies may offer easy-to-use and affordable platforms to host data and maps, it is important to know the extent to which the data are owned by authors and contributors, and what rights the companies supporting the platforms gain. While this may not be an issue for academic research involving majority languages or information that is already in the public domain, this can be a serious issue for communities and others who wish to retain agency over what they are mapping.

\footnotetext{
48 There are a number of bodies in Canada that do focus on infrastructure funding. These include and are not limited to the Canada Foundation for Innovation (https://www.innovation.ca/), CANARIE (http://canarie.ca), and the Social Sciences and Humanities Research Council (https://www.sshrc-crsh.gc.ca/).
} 
- Are the features reliable? An atlas may describe itself as accepting contributions of information from users, but in practice the contribution process may be prohibitively difficult or require the information to be gathered in a very specific way. More generally, the maps' features may technically exist, but not function in such a way as to be useful.

This preliminary competitive analysis is a good place to start in terms of assessing what atlases and technologies are currently accessible in online language mapping, but there is more that can be done in this area. First, while I surveyed 47 maps, technologies, and platforms, I only included a small number of maps $(n=11)$ in the EMT, strengths/weaknesses assessment, and competitive analysis. Though focusing only on interactive digital maps was a conscious choice, adding more maps would make my assessments more representative of existing language mapping techniques. Second, due to my background in the academic study of language and space, I am predisposed to focus on some features over others. Therefore, I believe that a competitive analysis of the same maps carried out by someone from education, data science, or language communities would differ considerably from my own. The conclusion drawn from this inference is that dialogue and collaboration is important in competitive analyses of language maps, especially when the intended users have other objectives and interests. Third, other features should be explored to compare maps to one other, and multiple applications of each form of assessment found here would make comparisons more detailed. For example, Roth et al. (2014) include two competitive analysis tables, while Roth, Quinn, and Hart (2015) have five, that each focus on a different dimension of atlas functionality. 
This study contributes to the existing body of language mapping knowledge by providing a proof of concept through which an evaluative typology can compare multiple aspects of language maps to one another. Furthermore, the inclusion of semi-structured interviews with those involved in atlas development helps contextualize the analyses' findings and provides more detailed explanations for mapmaking decisions.

While my analysis summarizes maps, atlases, and online technologies, its focus does not extend to the equally important element of those who the maps are used by, namely the audience of users, viewers, and readers. As such, the following section focuses on how a variety of individuals engage with an online language map interface and analyzes not only how maps are constructed to impart information, but how they end up doing so in practice. 
Study 3

A user study of the Algonquian Linguistic Atlas 


\subsection{A user study of the Algonquian Linguistic Atlas.}

As web mapping practices become more ubiquitous, those who might not otherwise encounter particular kinds of maps can now do so with greater ease. However, as noted by Ooms, De Maeyer, and Fack (2015, p.3), such 'novices' to dynamic and interactive maps may have difficulty processing their content, meaning even carefully constructed messages may not be accessible. This is important to consider, given that Blades and Spencer $(1987$, p.19) consider map use to be a complex skill that requires extensive learning. Thus, understanding users' strengths and needs is arguably just as important as designing a persuasive and informed map. Numerous user studies of digital maps have yielded some important insights into how both novice and experienced map users process and understand what they see.

For instance, Thorndyke and Stasz (1980) investigated the strategies employed by both experienced and inexperienced users in acquiring knowledge from maps. Users viewed a map of a fictional region for two minutes, and using any technique that came to mind, were then asked to redraw the map to the best of their ability (Thorndyke \& Stasz, 1980, p.12). Importantly, experienced users were able to pick and choose which information they wished to pay attention to, while less experienced users attempted to interpret and memorize all the information they saw (Thorndyke \& Stasz, 1980, p.34). Similarly, in the face of what was ultimately an unstructured task, experienced users were better adept at formulating plans that involved memorization and could reflect on both what they did well and what they were unable to do.

Ooms, De Maeyer, and Fack (2013) designed an eye-tracking experiment involving digital maps to study both experienced and inexperienced users' abilities to cope with and interpret maps of unfamiliar regions. These maps had been manipulated to include visual deviations, such as being mirrored on either horizontal or vertical axes, as well as differently coloured geographic 
features such as land and water. Eye-tracking analyses suggested that experienced users looked at each map for an average of two minutes longer than inexperienced users (Ooms, De Maeyer, \& Fack, 2013, p.43). In addition, experienced users fixated on individual points for less time during the task, meaning that they were able to view and interpret more information than their less experienced counterparts (Ooms, De Maeyer, \& Fack, 2013, p.52).

What these studies suggest is that users are by no means a uniform group, and that previous exposure to the digital map as a medium is an important factor in determining how well they will be able to engage with an interface. It is important to acknowledge that these previous studies focused on maps in general. I have been unable to find any user studies in the literature that focus on language maps in particular. As such, the following user study, which I performed in 2018 , is an early if not first analysis of how users interact with language maps. Instead of looking solely at general user experience, this user study focuses on two other variables of interest, namely users' age, and if they are a member of an Indigenous community.

I decided to focus on these two variables for two reasons. First, while age may not necessarily be a strong predictor of competence with maps, mapping technology, or spatiality in general (Bronzaft, Dobrow, \& O’Hanlon, 1976, p.589), it has been demonstrated by Schnürer, Sieber, and Çöltekin (2015) to be a factor in predicting familiarity and competence with emerging digital interfaces. Second, as the digital atlas I chose to work with is designed and operated by both Indigenous and non-Indigenous scholars and contributed to by members of Indigenous communities, it was important that both Indigenous and non-Indigenous perspectives and approaches could be accounted for. ${ }^{49}$ However, as demonstrated in the following paper,

${ }^{49}$ Between 2017 and 2019, I had the privilege of working as part of a research team for the Algonquian Linguistic Atlas and participated in the ongoing design and knowledge gathering for the atlas interface. Because of my connection with the atlas and those working tirelessly to 
while there were some meaningful differences between how younger and older users interacted with the atlas, there were some practical issues related to engaging with Indigenous users that affected cross-cultural analyses, and are themselves an important finding that requires extensive future exploration.

What follows is my user study of the Algonquian Linguistic Atlas in its entirety as it has been published in 2018 in the journal Cartographica, which is in turn followed by a post-hoc reflection of its efficacy, results, and implementation. I contend that this adjunct reflection is a necessary additional step in the research process as it helps situate the user study in the broader course of this doctoral research. In addition, it is an important part of the reflective process I aim to reinforce, and highlights a few of the many and intricate factors in research involving Indigenous knowledge that deserve further exploration and discussion.

advance it, and under the auspices of the program lead, Dr. Marie-Odile Junker, I was given permission to use the atlas for my own research. 


\title{
ARTICLE \\ Mapping Experience: Age and Indigeneity as Mediating Factors in Users' Experiences with the Algonquian Linguistic Atlas
}

\author{
Adam Stone \\ Applied Linguistics and Discourse Studies / Carleton University / Ottawa / ON / Canada
}

\begin{abstract}
To understand how effectively digital maps of Indigenous languages engage a variety of audiences, a mixed-methods user study focused on the experiences of 23 Indigenous and non-Indigenous users aged under and over 30 from a Canadian university as they navigated an online Canadian Indigenous language atlas by completing a series of tasks. An evaluative component assessed the efficacy of the study itself in measuring such experiences. Indigenous participants found the atlas more relevant and useful and focused more on its linguistic content, while non-Indigenous participants focused on the layout and structure of the atlas's framework. Digital language atlases can better address Canadian Indigenous populations by emphasizing multimodal representations of linguistic content, with easily accessible links to additional resources from the communities represented. While the study did capture multiple dimensions of user experience, low Indigenous participation decreased the efficacy of comparative statistical analyses. Future research can improve Indigenous representation by focusing on recruitment methods that engage and are relevant to Indigenous populations.
\end{abstract}

Keywords: language education, digital atlas, map use, language revitalization, Indigenous languages, multimodal mapping

\section{RÉSUMÉ}

Pour comprendre avec quelle efficacité les cartes numériques des langues autochtones rejoignent divers publics, l'auteur mène, auprès d'utilisateurs, une étude faisant appel à une méthodologie mixte portant sur les expériences de navigation en ligne de 23 usagers autochtones et non autochtones, âgés de moins de 30 ans et de plus de 30 ans, recrutés dans une université canadienne et appelés à s'acquitter d'une série de tâches dans le cadre de la consultation d'un atlas de langues autochtones canadiennes. Un élément d'évaluation permet de jauger l'efficacité avec laquelle l'étude elle-même permet de mesurer ces expériences. Les participants autochtones ont jugé l'atlas plus pertinent et utile et se sont concentrés davantage sur son contenu linguistique, alors que les participants non autochtones se sont plus particulièrement intéressés au modèle et à la structure du cadre conceptuel de l'atlas. Les atlas linguistiques numériques permettent de mieux cerner les populations autochtones canadiennes en insistant sur les représentations multimodales du contenu linguistique et en proposant des liens facilement accessibles vers des ressources supplémentaires provenant des communautés représentées. Bien que l'étude circonscrive bel et bien les multiples dimensions des expériences des utilisateurs, la faible participation autochtone réduit l'efficacité des analyses statistiques comparatives. II serait opportun, dans des recherches ultérieures, d'améliorer la représentation autochtone en mettant en œuvre des méthodes de recrutement persuasives, adaptées aux populations autochtones.

Mots clés : atlas numérique, cartographie multimodale, formation linguistique, langues autochtones, revitalisation linguistique, utilisation de cartes

\section{Introduction}

\section{THE NECESSITY OF MAPS IN LANGUAGE EDUCATION}

More than just a means of representing geographic spaces in formats palatable to viewers, maps are important resources in storing and transmitting inexhaustible arrays of knowledge without the interference of text (Upton 2010) and can provide social empowerment due to their ability to communicate large amounts of information easily. When constructed well, maps can reflect relationships between the subjects and factors being explored, in essence contextualizing them (Ormeling 2010). Furthermore, Upton 
(2010) states that language maps are important, as viewers often seem instinctively drawn to them, and in contrast to the case with other media of instruction, viewers feel that they have some immediate understanding of the information displayed. With the increasing sophistication of digital and Internet-based technologies, maps presented digitally are in the novel position of being able to convey information interactively in ways unthinkable with traditional print atlases: with audiovisual information, photo albums, interactive activities, and relevant Web links, to name a few options. In education, maps are powerful tools in teaching learners about the physical spaces they inhabit and the issues connected to those spaces. Learners can engagewith, encode, and interpret visuospatial information (Bollman 2010) in ways they could not with text alone without making learning more demanding on their mental processes (Roberts, Parush, and Lindgaard 2005). Even a single map can communicate ideas of cultural and linguistic dispersion in ways that written texts cannot (Luebbering, Kolivras, and Prisley 2013), as learners can simultaneously view multiple aspects of the subject at hand. Educational theory suggests that learners learn more effectively in both formal and informal settings when actively engaged than when passively involved (Taylor 2005). As well as extending the modalities through which information can be presented, digital maps offer unique accessibility in the classroom, as they do not need to be physically acquired and cared for or replaced. Atlases can be presented through classroom projectors or interactive computer programmes or on mobile devices, allowing unprecedented accessibility in the classroom and the hands of learners.

As tools of power (Aporta and others 2014), convincingly produced maps allow their creators to dictate how their viewers experience reality, as they inspire confidence in their viewers (Caquard and Taylor 2005). Those who produce the maps become authorities in the subjects they are mapping and will often create the maps to further their own interests (Ormeling 2010). In colonial contexts, maps became tools of ownership, in which European groups assigned names and borders to newly conceived territories, hence "claiming" them (Aporta and others 2014; Ormeling 2010). The idea that languages, peoples, and places can be represented on maps in the first place is a product of European nationalism attempting to categorize languages into particular tribal, ethnic, and social groups (Mühlhäusler 2010). Colonialism provided a strong motive for the development of maps, as explorers and colonists attempted to understand and ultimately control the new places, ideas, and lands they encountered. When representing the dispersion of ethnic groups in a given area, the mapping authorities in place created new names for the peoples and languages being mapped, and their ethnic and linguistic boundaries were decided for them. The "reality" of Indigenous peoples in North America thus began to exist outside of themselves, and risked becoming in part the domain of European society.
As digital and information technologies have the perspectives and values of the society from which they came embedded in them (Dyson 2004), researchers such as Pannekoek (2001) argue that while digital technologies have the potential to work as a powerful tool in the empowerment and education of Indigenous communities, the reality is that they bombard these communities with English-language and Eurocentred media, which undermine Indigenous values and culture. Furthermore, Pannekoek (2001) posits that access to digital technologies has served to separate more affluent and middle-class Indigenous people from those with fewer resources. Because of this, the use of maps in North America has a troubled legacy as a tool for exerting control and power over others, but just as tools are actualized in the hands of those who use them, maps also have great potential for restoration and rehabilitation, provided they are used sensitively and appropriately.

In one direction, digital maps have a great potential in the mapping and intergenerational transmission of Indigenous traditional knowledge (TK). TK is described by Oguamanam (2011) as follows:

aspects of [. . .] self determination, human rights, culture, [. . . ] lifestyles, and innumerable aspects of social processes that undergrid a people's worldview. (p. 46)

This definition can be further extended to include unique understandings of "place and space" (as in Aporta and others 2014, 241) and language when applied to the context of map use in education. For more information on this definition and an account of other definitions in this article, see Appendix 1. Because digital maps can present information through the use of videos, photographs, and audiorecordings such as songs, stories, and spoken language samples, TK can be presented in ways that respect intergenerational legacies of oral traditions. The benefits of culturally appropriate and accessible maps in the empowerment of communities and their TK are twofold: atlases can act as a common space where members collect and contribute their own information and data as a form of distributed cognition (see Hutchins 1995) and as a "facilitator of learning among younger generations" (Aporta and others 2014, 242).

For many, however, computers, tablets, and smartphones are a source of confusion or apprehension due to unfamiliarity stemming from a variety of factors such as age, education level, socio-economic status, or perceived irrelevance. There is a knowledge gap between the older and younger generations, in which, having grown up immersed in digital technologies, younger generations can intuitively grasp how digital systems work more easily than their elder counterparts (Schnürer, Sieber, and Çöltekin 2015). When applied to TK, a paradox arises: on one hand, elders, who 
possess a wealth of knowledge regarding the land, culture, and language, have at best a fraction of the digital experience younger generations have, who, on the other hand, possess at best a fraction of the TK elders have (Keith, Crockatt, and Hayes 2014). Remote areas such as much of northern Canada experience hindrances to connectivity such as low bandwidth, a lack of nearby retailers, and prohibitively high product shipping costs. A lack of access to the technologies creates an array of design challenges that require special consideration when working with these populations, such as ensuring that users are not overwhelmed with information, are able to intuitively grasp the atlas's layout and features, and do not get disoriented when using the software (Roberts, Parush, and Lindgaard 2005).

Nevertheless, digital language atlases do have the potential to act as a valuable store of TK as an educational medium that can span Canada's vast geographical distances (Payne, Hayes, and Ellison 2014). As information can be displayed in a variety of more culturally appropriate multimedia alternatives to large bodies of text, users from a variety of backgrounds and ages have the potential to become engaged in ways never before imagined. Understanding how people fit into this system is thus paramount to understanding how successful the atlases are, and chow they an be maximized as an educational resource.

\section{USER STUDY}

A user study will investigate how individuals experience a digital atlas that represents Indigenous languages across a geographic space. Though many digital atlases that map Indigenous knowledge and languages are now produced in partnership with Indigenous communities, I was unable to find any post-production assessments of how effective the atlases are when put into practice. This is particularly troubling because digital atlases require regular maintenance and funding to exist and remain active. If digital atlases contain inadequacies that interfere with learning or do not capture the interest of their viewers, they run the risk of becoming defunct or not satisfying funding criteria. Being able to investigate what makes atlases successful in terms of both information delivery and user experience is thus of great importance. The main objectives of the research conducted here are threefold:

(1) to assess the success of an online linguistic atlas in effectively engaging users and providing them with information they deem as relevant and useful;

(2) to understand how people interact with and experience Indigenous digital language mapping technologies and how personal factors such as age and the presence or absence of an Indigenous background may affect or mediate these experiences;

(3) to better understand the strengths, limitations, and factors involved with conducting user studies on digital Indigenous atlas projects.

\section{The Algonquian Linguistic Atlas}

The Algonquian language family is a large grouping of related languages that spans much of the North-American continent from Mi'kmaw in Nova Scotia, to Blackfoot (Siksiká) in Alberta (Goddard 1978). The Algonquian language family is not to be confused with the Algonquin language, which comprises a cluster of dialects of Ojibwe in the Ottawa River watershed and is part of the Algonquian language family. The atlas used in this study geographically represents a number of languages belonging to the Algonquian language family with individual points (or "dots") on a digital map of Canada (Junker and Stewart 2008). Produced by Junker (2005-17), the Algonquian Linguistic Atlas aims to

co-create an on-line, multimedia linguistic atlas of Algonquian languages [... which] allows [the Atlas] to offer many training opportunities for sound editing and linguistic description training to aboriginal students [and] create contacts between curriculum developers, language specialists and lexicographers of Algonquian languages, with a focus on on-line language resources and dictionaries. (From the "About the atlas" section, Junker 2005-17. For full credits, see https://resources.atlas-ling.ca/about/)

The atlas is presented online and uses satellite maps provided by Google Earth as a base for geographical representations, as seen in Figure 1. Language information is presented on the map using specific points that, when clicked on, display speaker recordings of specific words or phrases selected from a drop-down menu. Links to dictionaries, learning resources, and classroom activities are provided in the atlas, not only allowing users to explore samples of the languages themselves, but also providing the opportunity to learn more about them.

The atlas is in many ways exemplary, as it was created with involved and regular input from members of the linguistic communities it represents. However, no formal assessments focus on how the atlas is experienced by users since it became available to the public. Because the atlas is a product of collaboration with Indigenous communities and represents Indigenous knowledge primarily for these communities, it is of great importance that Indigenous perspectives and experiences be taken into consideration in every aspect of this study's design and implementation.

Users' Backgrounds as an Influential Factor in Their Experiences

Users of different ages differ in both their needs in and their approaches to using digital interfaces (Prensky 2001; Schnürer, Sieber, and Çöltekin 2015). Prensky (2001) uses the term "digital natives" to distinguish those who grew up surrounded by more contemporary technologies such as smartphones and the Internet from "digital immigrants," 


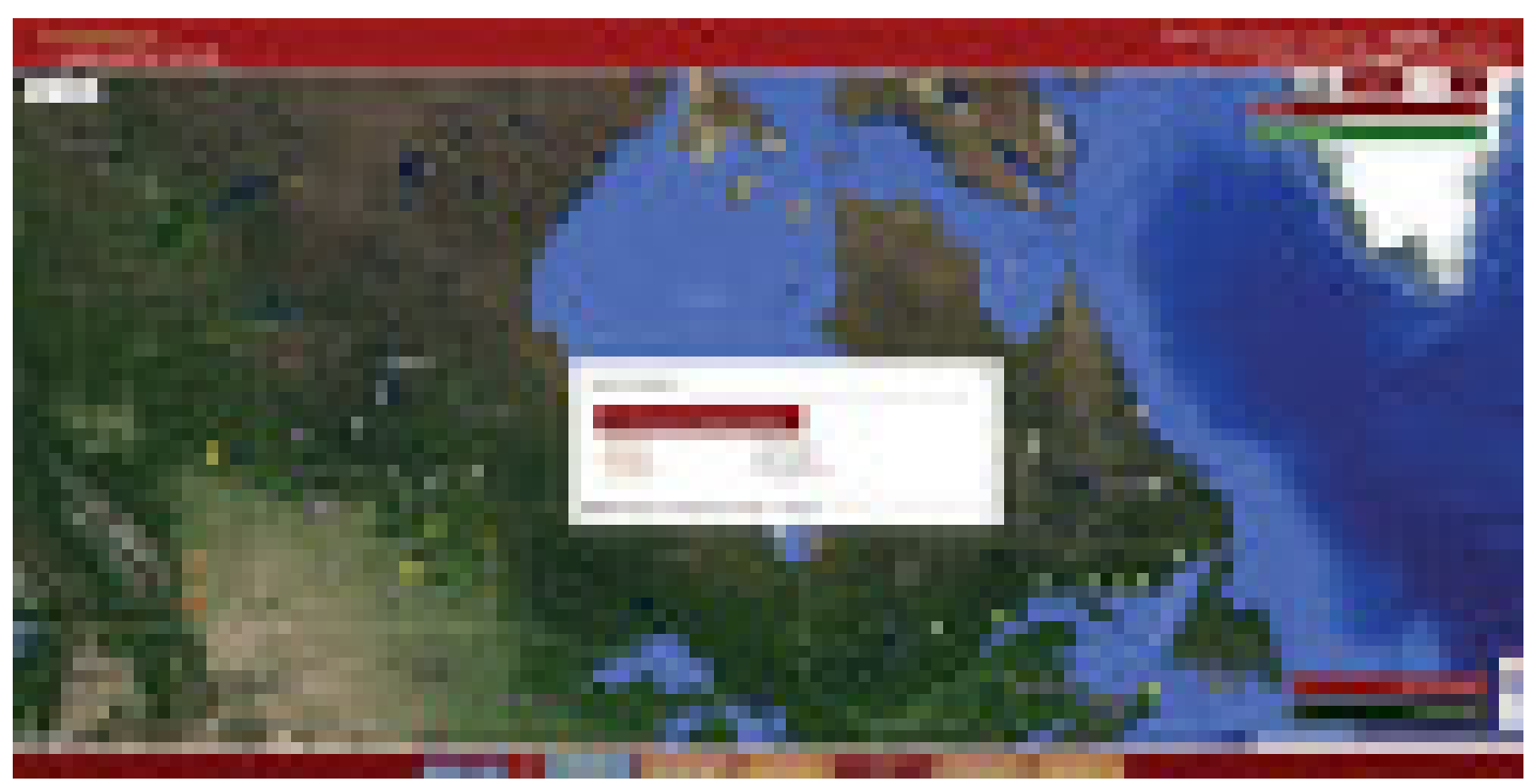

Figure 1. Screenshot of the main atlas page, with a speech sample selected Source: Map data $\odot 2017$ Google Imagery; ${ }^{\odot} 2017$ NASA, TerraMetrics.

who grew up before these technologies became available. As this study involves Indigenous contexts and languages, the term digital generation (DG) will be used instead of "digital natives," and the term mature will be used for "digital immigrants." In Schnürer, Sieber, and Çöltekin (2015), the DG includes those who were born after the year 1980, presumably when personal computers first became available. Here, the DG criterion will be changed to 1990 to reflect when the Internet first became publicly available, and personal computers, while already available to the public, became more accessible due to their decreasing cost and first true implementation of a graphical user interface (GUI; Reimer 2005).

Schnürer, Sieber, and Çöltekin (2015) indicated that DG users have intuitions about digital technologies not observed in many mature users, due to the devices available to them from an early age. Giedd (2012) noted that adolescents spend approximately 11.5 hours a day engaged with some form of device (smart phone, tablet, etc.), readily use digital devices while engaged in other tasks, and access multiple devices at the same time. Though this may mean that DG users more easily process multiple sources of data coming from a digital atlas interface, an fMRI study by Just, Keller, and Cynkar (2008) indicated that the increased cognitive load associated with attempted multitasking may cause impairment in parts of the brain responsible for the processing of space.

For the purposes of this study, the term Indigenous refers to individuals with historical, social, and ancestral backgrounds that include ties to communities of peoples who inhabited areas in what is now known as Canada prior to European colonization. This definition encompasses First Nations, Métis, and Inuit peoples, which is important to consider, as due to differing historical and legal considerations, Métis and Inuit peoples are on occasion not defined as Indigenous (for example, see Aporta and others 2014). As the atlas was created with the collaboration, assistance, and input of Indigenous peoples, represents Indigenous linguistic TK, and is primarily intended for Indigenous communities, user studies should ideally include Indigenous participants and their perspectives. At the same time, non-Indigenous users are also of interest, to gain more insight into how diverse other groups experience digital atlases.

\section{Methods \\ RECRUITMENT AND PARTICIPANTS}

A total of 23 participants completed the study, with the majority $(n=20)$ physically present in the intended laboratory settings. The remainder participated from a distance via an Internet connection $(n=2)$ or in their own spaces with the researcher present $(n=1)$. Table 1 categorizes participants according to age and indigeneity.

Indigenous DG participants ( $<30$ years of age) were recruited with a research poster in a campus Indigenous student centre, by an online version of the same poster attached to the centre's regular digital newsletter service, and by the snowballing technique. Non-Indigenous DG participants were recruited by similar physical research posters placed throughout the university campus, and via 
Mapping Experience: Age and Indigeneity as Mediating Factors in Users' Experiences with the Algonquian Linguistic Atlas

Table 1. Participants according to the study's variables of age and indigeneity $(N=23)$

\begin{tabular}{lcl}
\hline & \multicolumn{2}{c}{$n$} \\
\cline { 2 - 3 } & Age $(<30 \mathrm{y})$ & Age $(>31 \mathrm{y})$ \\
\hline Indigenous & 2 & 1 \\
Non-Indigenous & 15 & 5 \\
\hline
\end{tabular}

snowballing. Posters here only differed from those used with the Indigenous group in that potential recruits did not need to be Indigenous. Potential mature Indigenous participants were personally contacted by the researcher. Non-Indigenous participants above the age of 30 were also recruited in this manner, but also were recruited with the physical research poster and by snowballing.

\section{PROCEDURE}

If physically present in the researcher's office, participants were first provided with a gift card to a popular coffee shop. They then read a short description of the study and were prompted to ask the researcher for added clarification if necessary. Then participants signed a consent form that was subsequently also signed by the researcher. Following this briefing stage, participants began the study if they decided to stay:

(1) Atlas navigation task: Participants navigated the atlas using a desktop computer in the researcher's office. The task did not take longer than 10 minutes on the average. Though they were permitted to informally explore the atlas's features for an indefinite amount of time, they were also required to complete 12 tasks in the form of questions on a worksheet to ensure that they encountered most of the basic features the atlas has to offer. Though participants' accuracy was not individually measured, participants' overall total accuracy was recorded to better understand how intuitive the atlas is to novice users and how effective the instructions were.

(2) Questionnaire task: Upon completion of the atlas task, participants completed a questionnaire asking them to numerically rate their experiences using a Likert Scale (1-10) and to circle a response to a feature-related statement best reflecting their experiences, as shown in Table 2. This component of the questionnaire was produced as an adaptation of the usefulness, satisfaction, and ease of use (USE) questionnaire (Lund 2001). The USE questionnaire was selected because it is simple and straightforward to use while still testing subjective dimensions of user experiences, which tend to be overlooked in more conventional user studies (Lund 2001). A fourth dimension of learning ease was added to this questionnaire to focus on the process through which
Table 2. Example multiple-choice question paired with numerical rating values ${ }^{a}$

\begin{tabular}{|c|c|c|c|c|}
\hline \multicolumn{5}{|c|}{ The atlas was easy to use (circle one): } \\
\hline $\begin{array}{l}\text { Strongly } \\
\text { disagree }\end{array}$ & Disagree & Neutral & Agree & $\begin{array}{l}\text { Strongly } \\
\text { agree }\end{array}$ \\
\hline-2 & -1 & 0 & 1 & 2 \\
\hline
\end{tabular}

participants become familiar with the atlas software. For the purposes of this study, the questionnaire will thus be referred to as the USE(L). Though many user studies look at how successfully users perform various tasks on an interface in order to determine the interface's competency (for example, Laidlaw and others 2005), the primary objective here is to focus on the users themselves. The last three items were open-ended questions that prompted participants to describe what they liked about the atlas, what they did not like, and what could be improved. Though participants were given the option to leave any question they did not want to answer blank, all items were filled out on most of the questionnaires. The questionnaire did not ask for any personally identifying information, though participants were asked to disclose their age in years, any languages they were interested in or had exposure to, and where they learned about the study. Asking where they learned about the study and for their language interests was an indirect way of attempting to better understand participants' ethnic and cultural backgrounds, without explicitly asking participants to declare sensitive identifiable information.

(3) Post-questionnaire semi-structured interview: Following the questionnaire, the researcher interviewed several participants to obtain a more detailed account of their experiences. The researcher first read the questions aloud and then wrote down the participant's oral responses underneath each question. Interviewees were randomly selected from the pool of non-Indigenous participants who completed the other sections of the study, but each Indigenous participant was interviewed due to smaller groups and to place an emphasis on Indigenous perspectives. The interview questions, while similar to those in the questionnaire, were more broad and open-ended and asked participants what they thought of the potential for language atlases to be of benefit to learners in the classroom and other educational settings. Unless participants decided to spend extra time exploring the atlas' extended features, the study, including the interview, did not take more than half an hour per individual. 


\section{MEASURES}

Participant responses were measured quantitatively in the form of rating scales and a 1-10 rating scale of overall satisfaction. Experience was measured qualitatively in the form of answers to the open-ended questions in the questionnaire and subsequent interview questions. Results were placed in a Microsoft Excel file, which contained all relevant information according to each participant. Written responses to open-ended and interview questions were summarized into single-word codes expressing their main themes. The coding process consisted of deriving themes from each participant sorted by both age and Indigeneity and analysing the two most frequently occurring theme codes for each group. Any concerns voiced by participants related to the atlas were noted, to add detail to their experiential accounts and to better understand their learning processes. Finally, the researcher recorded his own experiences during the experimental process and important information to take into account when interpreting the results.

\section{Results and Discussion}

\section{STATISTICAL INTERPRETATIONS OF THE QUESTIONNAIRE DATA}

Questionnaire ratings were analysed in response to the first objective of this study, which was to assess the success of a specific digital language atlas in effectively engaging users. Because the numerical component of the questionnaire tested users' experiences through one type of scale only, descriptive statistics composed the majority of the analyses. Due to unbalanced participant groups, all participants' ratings were collapsed to obtain a global measure of how the atlas was experienced.

Overall, participants enjoyed the atlas and found it engaging, and rated their total experience as 8.3 out of 10 . Figure 2 shows participants' combined ratings for each of the four dimensions found in the questionnaire.

User Satisfaction and Learning Ease was the most positively rated dimension from the USE(L) questionnaire.
As the atlas was designed to be a user-centred way of geographically and audio-visually representing languages and showcasing related Indigenous language projects, it does not come as a surprise that user satisfaction is relatively high. As participant ratings were different between Learning Ease and Ease of Use dimensions, it can be argued that adding the dimension that focuses on the process of learning itself is appropriate and increases the scope of Lund's (2001) USE questionnaire. The Usefulness dimension was the least positively rated, as many participants felt that the material was not relevant to their own lives. This may be due in part to the majority of participants having a non-Indigenous background, which could act as a factor in determining how relevant they felt Indigenous languages were to their own lives.

Cronbach's alpha was used to assess how internally consistent each of the questionnaire's dimensions was across participants. The Usefulness scale consisted of four items $(\alpha=0.58)$, the Satisfaction scale consisted of six items ( $\alpha=0.8)$, as did the Ease-of-use scale $(\alpha=0.65)$, and the Learning Ease scale consisted of five items $(\alpha=0.71)$. However, the average across all the dimensions $(\alpha=0.69)$ was lower than the entire questionnaire's internal consistency score $(\alpha=0.87)$, indicating that the measures of internal consistency were strongly influenced by the number of items taken into account.

\section{SHORT-ANSWER RESPONSES AND SEMI-STRUCTURED INTERVIEW}

Qualitative data were obtained from three short-answer questions at the end of the questionnaire, from postquestionnaire interview responses, and from comments and observations made by the researcher during the trials themselves. In Table 3, Indigenous participants primarily focused on content-related aspects of the atlas (such as presence and delivery of languages), whereas non-Indigenous participants in Table 4 tended to focus on structure-related aspects (such as the layout and icons). However, mature

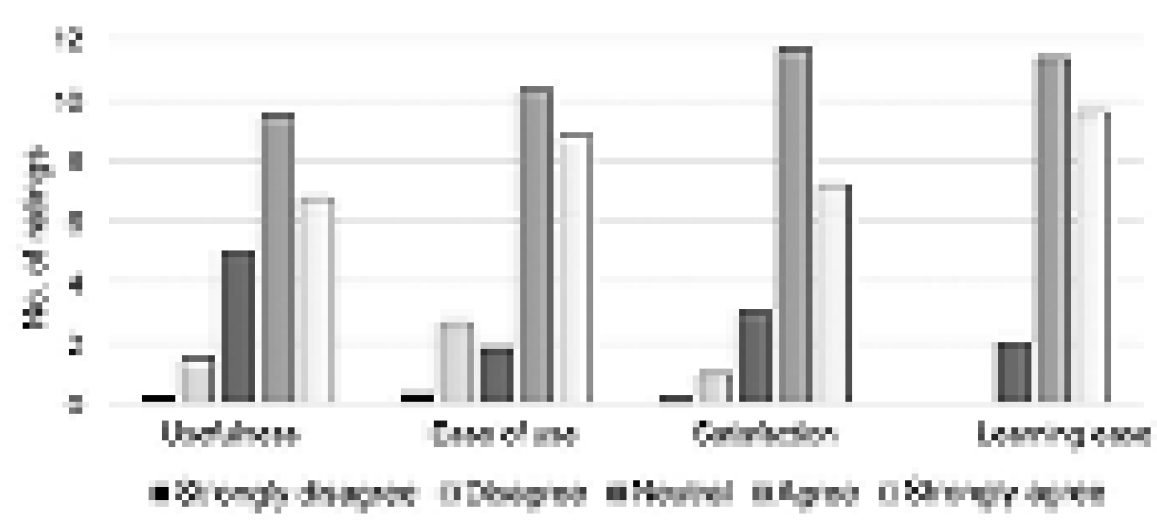

Figure 2. Collapsed ratings of the Atlas according to the four questionnaire dimensions 
Mapping Experience: Age and Indigeneity as Mediating Factors in Users' Experiences with the Algonquian Linguistic Atlas

Table 3. Indigenous participants' coded short-answer responses by frequency

\begin{tabular}{|c|c|c|}
\hline Questions & $\begin{array}{l}\text { Mature } \\
\text { Indigenous } \\
\text { responses }^{a}\end{array}$ & $\begin{array}{l}\text { DG Indigenous } \\
\text { responses }\end{array}$ \\
\hline $\begin{array}{l}\text { What did you } \\
\text { like about the } \\
\text { atlas? }\end{array}$ & Spoken media & $\begin{array}{l}\text { Provides knowledge } \\
\text { Spoken media }\end{array}$ \\
\hline $\begin{array}{l}\text { What didn't you } \\
\text { like about the } \\
\text { atlas? }\end{array}$ & $\begin{array}{l}\text { Unclear } \\
\text { components }\end{array}$ & $\begin{array}{l}\text { Unclear components } \\
\text { Few languages }{ }^{2}\end{array}$ \\
\hline $\begin{array}{l}\text { What could be } \\
\text { improved? }\end{array}$ & $\begin{array}{l}\text { More languages }{ }^{1} \\
\text { More links }^{2}\end{array}$ & $\begin{array}{l}\text { More phrases }^{1} \\
\text { More languages }^{2}\end{array}$ \\
\hline
\end{tabular}

a Single codes denote the absence of a second code, due to only one type of response being present.

and DG participants did not differ greatly in terms of what they liked and disliked about the atlas.

Participants' Backgrounds and Their Experiences with the Atlas

Indigenous participants' open-ended interview responses are emphasized due to their limited participation and subsequent representation thus far in this study. For an account of Indigenous participants' responses, see Appendix 2.

Indigenous participants tended to concentrate more on the content-related aspects of the Atlas, such as the presence, distribution, and delivery (audio or text) of language samples. Conversely, non-Indigenous participants tended to focus more on structural aspects of the Atlas, such as the placement of lists, menu items, or the legend. This may be the result of the subject matter's perceived relevance: Indigenous language information is likely more relevant to Indigenous participants in some way than to non-Indigenous participants. To further understand if relevance could have an effect, a follow-up measure of relevance in Figure 3 illustrates Indigenous and non-Indigenous participants' separately averaged total ratings on the Usefulness dimension. Using the average total ratings produces a magnitude reading of how strongly each participant group felt about the atlas's relevance.

Because the above figure is based on the combined ratings of unevenly sized groups, it serves as an exploratory illustration of how the relevance of the atlas's materials could explain the rationale behind which aspects of the atlas Indigenous and non-Indigenous participants chose to focus on. Indigenous participants had primarily positive experiences with the software, but remarked that the atlas still had a long way to go in including more languages and expanding the number of words and phrases available for translation. Indigenous participants appreciated multimedia aspects such as audio recordings, but also said that the atlas and similar projects ought to include more contextual
Table 4. Non-Indigenous participants' coded short-answer responses by frequency

\begin{tabular}{|c|c|c|}
\hline Questions & $\begin{array}{l}\text { Mature } \\
\text { non-Indigenous } \\
\text { responses }\end{array}$ & $\begin{array}{l}\text { DG } \\
\text { non-Indigenous } \\
\text { responses }\end{array}$ \\
\hline $\begin{array}{l}\text { What did you like } \\
\text { about the atlas? }\end{array}$ & $\begin{array}{l}\text { Clear }^{1} \\
\text { Visually appealing }\end{array}$ & $\begin{array}{l}\text { Multimedia } \\
\text { Provides } \\
\text { knowledge }^{2}\end{array}$ \\
\hline $\begin{array}{l}\text { What didn't you } \\
\text { like about the } \\
\text { atlas? }\end{array}$ & $\begin{array}{l}\text { Confusing } \\
\text { icons }{ }^{1} \text { Small area }\end{array}$ & $\begin{array}{l}\text { Unclear } \\
\text { components }^{1} \\
\text { Confusing icons }{ }^{2}\end{array}$ \\
\hline $\begin{array}{l}\text { What could be } \\
\text { improved? }\end{array}$ & $\begin{array}{l}\text { More multimedia }{ }^{1} \\
\text { More phrases }^{2}\end{array}$ & $\begin{array}{l}\text { Clearer } \\
\text { multimedia }{ }^{1} \\
\text { More languages }^{2}\end{array}$ \\
\hline
\end{tabular}

information to provide users with an understanding of why language samples occur where they do, and take the forms that they do. Any additional links should ideally be made to Indigenously owned sources that are in turn able to benefit in some way from the inclusion of their information in the atlas.

The amount of language-related information available in the atlas is directly dependent on how much information is volunteered from the communities themselves. As a result, any lack of representation in the atlas does not stem from a lack of recognition, but instead a lack of contact with a specific community. Though the atlas has been published online for some time, it is still very much a process undergoing continual development whereby more features and languages are added when information becomes available.

Participants' Ages and Their Experiences with the Atlas

There did not appear to be any meaningful qualitative differences between DG and mature participants' experiential accounts. Neither age group appeared to focus more on one aspect of the atlas than the other, and they had similar comments and concerns. It is worth noting that as participants in both age groups were recruited primarily from post-secondary institutional settings, both likely regularly engage with a variety of digital technologies for work purposes on a daily basis. To better understand how age might affect atlas experiences, future research should also focus on developing a representative sample of participants from outside post-secondary settings.

An unintended observation from the atlas task was that many DG participants engaged with other digital devices while performing atlas tasks, which was not observed in the mature group. Though one possibility could be that DG participants were less engaged with the atlas, a general lack of difference between the richness of DG and mature participants' open-ended responses regarding their experiences indicates that DG participants were focused 


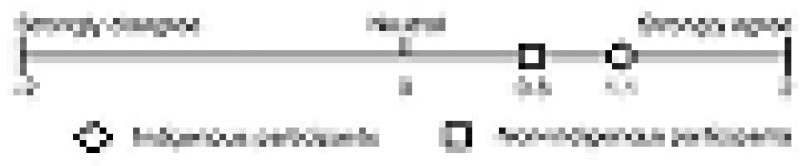

Figure 3. How highly participants rated the Atlas on the Usefulness dimension

on the tasks at hand, and wished to contribute meaningful responses. However, Giedd (2012) states that the use of multiple devices at a given time is an important characteristic of members of the DG, which may reflect underlying cognitive differences in how digital devices are approached (Prensky 2001). In this way, perhaps DG and mature participants had similar experiences of the atlas, but approached the tasks differently. Any future research ought to take this into consideration. In both age groups, the atlas was rated by participants as either very clear or unclear, meaning that there were likely additional personal factors not accounted for in this study that determined how clear the atlas's layout appeared to users.

\section{STRENGTHS AND LIMITATIONS OF THE USER STUDY}

The guiding questions used to aid participants in exploring the atlas were measured for accuracy to assess how uniform their experiences were; a relatively high score would indicate that participants were on the average able to successfully explore the variety of features, which would reflect a greater likelihood that participants had all viewed the same material. Overall, participants answered 10.5 out of $12(88 \%)$ questions correctly, meaning that while the tasks could still be made clearer, the guiding questions asked were clear for the majority of participants.

Because I used a mixed-methods approach to gather both quantitative user ratings and qualitative accounts of users' experiences, a wide range of different information could be obtained regarding how such atlas software can be experienced by a number of groups with differing perspectives and backgrounds. Participants offered no complaints to me during the atlas task, and a high percentage of accurate responses in the guiding questions component indicated that the general layout of the atlas was easy to grasp, and that participants received a relatively uniform experience. The efficacy of the 5-point rating scale used in the questionnaire requires further investigation in future studies of this kind, as 5- and 7-point scales tend to produce slightly higher average scores than would be achieved with a 10-point scale (Dawes 2012), and reveal less information (D.R.F. Taylor, personal communication, 29 March 2017). Though Cronbach's alpha was used as a direct measure of the questionnaire's internal consistency and thus an indirect measure of reliability, the entire alpha score was very different from each dimension's score, meaning that the measure was affected by the number of items as much as if not more than the consistency itself, which is a concern mentioned in Sijtsma (2009).

The short-answer questions at the end of the questionnaire were received well and yielded important experiential information, but the post-questionnaire interview questions were too open-ended, and participants either had trouble providing detailed responses or did not find the questions relevant. The benefit of writing down oral responses is that greater anonymity can be ensured than with voice recordings, while still capturing participants' messages. However, this method does sacrifice some of the detail that would otherwise be preserved in audio or video recordings. Further refinement of questions or a change in interview techniques (such as using focus groups) is thus important to improving the interview component.

Differences between specific Indigenous communities are not specified in this study, and proficiency in an Indigenous language was not a requirement. Given a larger number of Indigenous participants, separating user experiences into meaningful results by these differences would become more feasible. The small participant number is explainable in the case of Indigenous participants above the age of 30 , as the potential recruits tended to live outside the Ottawa area and were unable or too busy to attend sessions physically or via Skype. However, a markedly lower Indigenous DG participation comes as a surprise, as the most recruitment methods were employed with this population. According to the cultural liaison for the campus Indigenous student centre, the research invitations were received well and met with enthusiasm, but only two attempts at contacting the researcher were made. It is possible that the incentive for participation was not sufficiently high to engage often time-constrained Indigenous students, who on average face greater internal and external pressures than non-Indigenous populations, such as family care, financial difficulties, or being the first in the family to pursue an advanced degree (Asmar, Page, and Radloff 2011). Investigation into reasons for lower Indigenous DG participation in user studies such as this is important, and deserves a separate study of its own alongside the development of more appropriate and engaging recruitment methods for Indigenous populations in all generations.

\section{Conclusion}

A mixed-methods user study focused on the experiences of Indigenous and non-Indigenous users as they navigated the Algonquian Linguistic Atlas. By their completing a series of tasks, answering questions related to those tasks, and participating in a semi-structured interview, an experiential account of the Atlas' ability to convey useful and relevant information was obtained. By additionally focusing on how this methodology was able to obtain such experiential accounts in the first place, this investigation also functioned as an important first step in developing 
appropriate and informative evaluations of language atlas software.

While Indigenous and non-Indigenous participants of all ages enjoyed using the atlas, Indigenous participants found the atlas more relevant and useful and focused more on the content, while non-Indigenous participants focused on the layout and structure. Though there were negligible differences between DG and mature students' experiences (likely due to both groups coming from academic settings), DG participants were observed to use the atlas differently by engaging with multiple devices during the task. Indigenous participants reported enjoying the atlas experience, but advised that projects mapping Indigenous languages and knowledge should provide a greater contextual background and strive to benefit the Indigenous communities they map. To increase the efficacy of user studies of Indigenous language atlases, more detailed rating scales and more precise interview techniques should be used to better capture users' opinions and experiential accounts. More appropriate recruitment methods in a greater variety of locations would improve sample sizes, reinforcing statistical assessments of the numerical results.

To successfully teach Indigenous members of younger generations, digital atlases need to focus on making their content accessible and appealing, while also ensuring that it is relevant to the community. The study confirms that multimedia approaches work well to engaging both learners from societies with oral traditions and those who are accustomed to multiple modalities in their devices. Developers, educators, and researchers should strive to create learning interfaces that are interactive and engage learners' ears, eyes, mouths, and fingers while also working on multiple types of devices such as smartphones, computers, and tablets. In this way, traditional knowledge can remain a crucial and integral aspect of Indigenous lives in a world of rapidly changing technologies and identities.

\section{Appendix 1: Glossary of Terms as Used in This Paper}

\begin{tabular}{ll}
\hline Term & Definition \\
\hline The Algonquian language & $\begin{array}{l}\text { A grouping of related Indigenous languages spread over much of Canada and parts of the } \\
\text { United States. Examples of Algonquian languages include Cree, Ojibwe, Innu, Algonquin, } \\
\text { and Naskapi. }\end{array}$ \\
Age & One of two main variables focused on in this study. Refers to chronological age in years. \\
Atlas & A framework through which maps are presented. \\
Cognitive load & $\begin{array}{l}\text { The total amount of mental effort an individual experiences when problem solving or } \\
\text { performing a specific task. Demanding tasks have a high cognitive load, meaning that they } \\
\text { require more of an individual's attention and concentration. }\end{array}$ \\
Community & $\begin{array}{l}\text { A self-designated grouping of people who share a common heritage but may be physically } \\
\text { separated over large distances (e.g., individuals with ties to the Algonquins of Kitigan Zibi). }\end{array}$ \\
Cybercartography & $\begin{array}{l}\text { A distinctive critical map-based approach that provides a framework for dealing with } \\
\text { complex social, political, and economic phenomena. }\end{array}$ \\
Device & $\begin{array}{l}\text { A physical electronic object that allows individuals to connect to the Internet, or related } \\
\text { software. Often refers to smartphones, computers, or tablets. }\end{array}$ \\
Digital generation (DG) & $\begin{array}{l}\text { Those who are } 30 \text { years of age or younger, who are more likely to have grown up with } \\
\text { a digital device such as a personal computer, laptop, smartphone, mobile phone, and/or } \\
\text { Internet connection in their household. } \\
\text { A theory that as opposed to being strictly within an individual, knowledge is instead a } \\
\text { shared process, in which multiple individuals share their unique knowledges between each } \\
\text { other and in tools, objects, and artefacts, resulting in a greater understanding than one } \\
\text { individual could ever be capable of (Hutchins 1995). } \\
\text { Individuals in Indigenous societies who, among other things, have a wealth of information } \\
\text { regarding the culture, customs, language, and knowledge of the community. Elders do } \\
\text { not necessarily need to be elderly, but inversely most elderly community members are } \\
\text { considered as elders. }\end{array}$ \\
\hline Eistributed cognition &
\end{tabular}




\section{Appendix 1: Glossary of Terms as Used in This Paper (Continued)}

\begin{tabular}{|c|c|}
\hline $\begin{array}{l}\text { Graphical user interface } \\
\text { (GUI) }\end{array}$ & $\begin{array}{l}\text { The visually oriented layout most people encounter on their digital devices. GUls typically } \\
\text { use icons, visual menus, and animations in order to convey information, such as the } \\
\text { Windows, Linux, or Macintosh platforms. This sort of interface allows non-programmers to } \\
\text { interact with digital devices more easily. }\end{array}$ \\
\hline Indigenous & $\begin{array}{l}\text { Refers here to First Nations, Inuit, or Métis persons' cultural backgrounds, languages, } \\
\text { viewpoints, or knowledge. }\end{array}$ \\
\hline Map & $\begin{array}{l}\text { Graphic models of parts of (or phenomena related to) a physical area produced to scale for } \\
\text { decision-making purposes, and graphical representations of relationships between concepts, } \\
\text { ideas, or knowledge. They are often (but not always) contained in an atlas. }\end{array}$ \\
\hline Mature participant & $\begin{array}{l}\text { Participants } 31 \text { years of age or older, who are less likely to have grown up surrounded by } \\
\text { digital technologies such as the Internet or personal computers. }\end{array}$ \\
\hline Recruits & Those who have the potential to be included in the study but have not yet participated. \\
\hline Traditional knowledge (TK) & $\begin{array}{l}\text { Indigenous people's understandings and experience of their surroundings and lives, as } \\
\text { mediated through their worldview. This is manifested in aspects such as human rights, } \\
\text { language, culture, institutions, and social processes. }\end{array}$ \\
\hline User & $\begin{array}{l}\text { An individual who is directly interacting with a digital language atlas or map. (On occasion } \\
\text { used interchangeably with viewer.) }\end{array}$ \\
\hline Viewer & $\begin{array}{l}\text { An individual who either is reading a paper map, or is exposed to a digital map, but is not } \\
\text { necessarily actively interacting with its interfaces. }\end{array}$ \\
\hline
\end{tabular}

\section{Appendix 2: Response to Interview Questions by Indigenous Participants}

(1) Tell me about your experience using the atlas. What did you think?

The fact that there was an audio component was a great feature of the atlas, as it allowed me to hear how the words actually sound, as opposed to just written. However, I didn't know there was a zoom function or that you could change the words that appear until I played around the mouse and found them by accident. These should be made more apparent. (Mature Indigenous participant)

I like how many different communities are represented on this map with different language samples, so that you can hear how they change over a large space. I know that there are many [Indigenous] communities around [own community], but I don't see any speakers from there. It would be nice to see more samples on the map. (DG Indigenous participant)

(2) Do you think the atlas was a good way of representing different languages?

Yes, but there should also be greater consideration to what defines a language in the first place, and how it's different from a dialect. The atlas's stance on this should be made clear somewhere in the atlas itself. (Mature Indigenous participant)
It was a neat way of showing how languages change depending on where you are. It's probably not the best substitute for face-to-face learning though. (DG Indigenous participant)

(3) Do you feel the atlas taught you some new things?

The atlas would be a great resource for learning some more words from my own language, as well as learning what the same words would look like in other areas. (Mature Indigenous participant).

(4) Was there something the atlas didn't do well, or could have done better?

There could have been more links sections to other language resources, which should be local and indigenously owned. The atlas should also be constructed in such a way as to establish more relations with local communities. (Mature Indigenous participant)

(5) How useful do you think atlases could be in language education?

This atlas could be really helpful in language education, so long as it is implemented in a culturally sensitive way. Also, the atlas should take into account more contextual information. As an example, Whapmagoostui Cree speakers speak a much older dialect than in other areas, as it has been the least changed by colonialism, but this isn't mentioned directly in the atlas. (Mature Indigenous participant). 


\section{Author Information}

Adam W. Stone is a PhD student at the School of Linguistics and Language Studies at Carleton University, Ottawa, Canada. He holds a Master's of Cognitive Science and an Honours BA in Psychology and Linguistics, also from Carleton University. His research interests focus on establishing language mapping as a coherent and reflective discipline capable of constructively engaging a variety of groups including linguists, cartographers, Indigenous communities, and linguistic minorities. E-mail: adam.stone@carleton.ca.

\section{References}

Aporta, C., I. Kritsch, A. Andre, K. Benson, S. Snowshoe, W. Firth, and D. Carry. 2014. "The Gwich'in Atlas: Place Names, Maps, and Narratives." In Fraser Taylor and Lauriault 2014, 229-44.

Asmar, Christine, Susan Page, and Ali Radloff. 2011. Dispelling Myths: Indigenous Students' Engagement with University. AUSSE Research Briefings, Vol. 10. Available at https://research.acer.edu. au/ausse/2/.

Bollmann, J. 2010. "Maps and Cognition." In The Handbook of Language Mapping. Berlin: De Gruyter Mouton.

Caquard, S., and D.R.F. Taylor. 2005. "Art, Maps and Cybercartography: Stimulating Reflexivity among Map-Users." Modern Cartography Series 4: 285-307. https://doi.org/10.1016/ s1363-0814(05)80015-2.

Dawes, J.G. 2012. "Do Data Characteristics Change According to the Number of Scale Points Used? An Experiment Using 5 Point, 7 Point and 10 Point Scales." International Journal of Market Research 51(1): 61-104. https://doi.org/10.1177/147078530805000106.

Dyson, L.E. 2004. "Cultural Issues in the Adoption of Information and Communication Technologies by Indigenous Australians." In Proceedings Cultural Attitudes towards Communication and Technology, ed. C. Ess and F. Sudweeks, 58-71. Perth: Murdoch University.

Fraser Taylor, D.R., ed., and T.P. Lauriault, assoc. ed. 2014. Developments in the Theory and Practice of Cybercartography: Applications and Indigenous Mapping. Amsterdam: Elsevier.

Giedd, J.N. 2012. "The Digital Revolution and Adolescent Brain Evolution." Journal of Adolescent Health 51(2): 101-105. https:// doi.org/10.1016/j.jadohealth.2012.06.002.

Goddard, Ives. 1978. "Eastern Algonquian Languages." In Northeast, Vol. 15 of Handbook of North American Indians, ed. B.G. Trigger, 70-77. Washington, DC: Smithsonian Institution.

Hutchins, E. 1995. Cognition in the Wild. Cambridge, MA: MIT Press.

Junker, M.0., director. 2005-17. "The Algonquian Linguistic Atlas." Available at http://atlas-ling.ca/.

Junker, Marie-Odile, and Terry Stewart. 2008. "Building Search Engines for Algonquian Languages." In Papers of the 39th Algonquian
Conference, ed. K.S. Hele and R. Darnell, 59-71. London, ON: University of Western Ontario.

Just, M.A., T.A. Keller, and J. Cynkar. 2008. "A Decrease in Brain Activation Associated with Driving when Listening to Someone Speak." Brain Research 1205: 70-80. https://doi.org/10.1016/j. brainres.2007.12.075.

Keith, D., K. Crockatt, and A. Hayes. 2014. "The Kitikmeot Place Name Atlas." In Fraser Taylor and Lauriault 2014, 219-27.

Laidlaw, D.H., R.M. Kirby, C.D. Jackson, J.S. Davidson, T.S. Miller, M. Da Silva, W.H. Warren, and M.J. Tarr. 2005. "Comparing 2D Vector Field Visualization Methods: A User Study." IEEE Transactions on Visualization and Computer Graphics 11(1): 59-70. https://doi. org/10.1109/tvcg.2005.4.

A. Lameli, R. Kehrein, and S. Rabanus, ed. 2010. Language Mapping, Part 1. Vol. 2 of Language and Space: An International Handbook of Linguistic Variation. Berlin: De Gruyter Mouton.

Luebbering, C.R., K.N. Kolivras, and S.P. Prisley. 2013. "The Lay of the Language: Surveying the Cartographic Characteristics of Language Maps." Cartography and Geographic Information Science 40(5): 383-400. https://doi.org/10.1080/15230406.2013.809892.

Lund, A.M. 2001. "Measuring Usability with the USE Questionnaire." Usability Interface 8(2): 3-6. Available at https://www. researchgate.net/profile/Arnold_Lund/publication/230786746_ Measuring_Usability_with_the_USE_Questionnaire/links/ 56e5a90e08ae98445c21561c/Measuring-Usability-with-theUSE-Questionnaire.pdf.

Mühlhäusler, P. 2010. "Mapping Linguistic Typology." In Lameli, Kehrein, and Rabanus 2010, 355-87. https://doi.org/10.1515/978 3110219166.1.355.

Oguamanam, C. 2011. Intellectual Property in Global Governance: $A$ Development Question. London: Routledge.

Ormeling, F. 2010. "Visualizing Geographic Space: The Nature of Maps." In Lameli, Kehrein, and Rabanus 2010, 21-40. https://doi. org/10.1515/9783110219166.1.21.

Pannekoek, F. 2001. "Cyber Imperialism and the Marginalization of Canada's Indigenous Peoples." In The Handing Down of Culture, Smaller Societies and Globalization, ed. J.-P. Baillargeon, 64-80. Toronto: Grubstreet Editions.

Payne, C., A. Hayes, and S. Ellison. 2014. "Mapping Views from the North: Cybercartographic Technology and Inuit Photographic Encounters." In Fraser Taylor and Lauriault 2014, 191-200.

Prensky, M. 2001. "Digital Natives, Digital Immigrants: Part 1." On the Horizon 9(5): 1-6. https://doi.org/10.1108/10748120110424816.

Reimer, J. 2005. "A History of the GUI." Ars Technica 5: 1-17. Available at http://www.cdpa.co.uk/UoP/Found/Downloads/reading6. pdf.

Roberts, S., A. Parush, and G. Lindgaard. 2005. "Cognitive Theories and Aids to Support Navigation of Multimedia Information Space." In Cybercartography: Theory and Practice, Modern Cartography Series, Vol. 4, ed. D.R. Fraser Taylor, 231-56. Amsterdam: Elsevier. https://doi.org/10.1016/s1363-0814(05)80013-9. 
Schnürer, R., R. Sieber, and A. Çöltekin. 2015. "The Next Generation of Atlas User Interfaces: A User Study with 'Digital Natives'." In Modern Trends in Cartography, ed. J. Brus, A. Vondrakova, and V. Vozenilek, 23-26. Cham, Switzerland: Springer.

Sijtsma, K. 2009. "On the Use, the Misuse, and the Very Limited Usefulness of Cronbach's Alpha." Psychometrika 74:107. https:// doi.org/10.1007/s11336-008-9101-0.
Taylor, D.F. 2005. "The Theory and Practice of Cybercartography: An Introduction." Modern Cartography Series 4: 1-13.

Upton, C. 2010. "Designing Maps for Non Linguists." In Lameli, Kehrein, and Rabanus 2010, 142-66. https://doi.org/10.1515/978 3110219166.1 .142$. 
13.1 Reflections on the user study's implementation.

This section looks back at what has been done in the study of users' experiences with Algonquian Linguistic Atlas in 2018, issues encountered during the research process, and what can be done differently to augment future studies of user experience in digital language atlases.

\subsubsection{Looking back at the Algonquian Linguistic Atlas user study.}

First and foremost, the lower rate of Indigenous participation is of interest here. Despite a higher degree of advertising for the study toward Indigenous participants than toward nonIndigenous groups, few Indigenous individuals participated. In addition to the user study's speculation that Indigenous participants did not have the time to take part in the study, there are some alternative possibilities. One is that the lower participation rates among Indigenous recruits was entirely due to random chance. However, such a possibility ignores all internal and external factors, and should be treated with caution. Instead, the Indigenous recruits may have already been experiencing 'study fatigue,' as Indigenous students and other members of Indigenous communities often find themselves the subjects of studies, which even with the best of intentions, can be relentless (De Padua \& Rabbitskin, 2018, p.66).

Another important consideration is the nature of the research. Though the study aimed to be as respectful and culturally sensitive as possible, Hart (2010, p.1) notes that research involving Indigenous participation and knowledge must also be rooted in processes and approaches that arise from and are part of Indigenous cultures. This user study's design and implementation were not informed by Indigenous values and designs, nor did they involve the extended input of members of Indigenous communities. Similarly, the study was not implemented in a way that directly benefitted specific Indigenous communities (see Weber-Pillwax, 2004, p.78). Instead, 
this research indirectly benefits Indigenous communities by introducing Indigenous participants to the atlas, by informing the atlas' design, and sensitizing scholarly audiences to Indigenous issues. Instead of being a limitation specific to this user study, Moreton-Robinson and Walter (2009, p.1) note that methodologies informed by Indigenous interpretive frameworks are absent throughout social research. More recently, Ingram $(2019$, p.481) laments the same trend, and mentions that research involving Indigenous knowledge rarely reflects Indigenous worldviews or is done principally for the benefit of Indigenous communities.

Taking these considerations into account, it is important to note that there was an established deadline for the user study and finite resources on hand. As this user study was originally part of a core doctoral seminar credit course, it needed to be designed, implemented, and finalized during a single academic year. Because of this, the research needed to be developed quickly and could only feasibly include participants involved in some way in the Carleton community. While the results indicated that, overall, these participants had little difficulty using the atlas and enjoyed their experience, many noted that they would have not otherwise thought to use the atlas. As such, participants were selected on the basis of geographic proximity to the study, and not based on their interest in the atlas or experience with its thematic content. In other words, given the resources on hand, this user study focused on the experience of participants, but was not controlled to look at actual users. Given these considerations, there are a number of recommendations I can offer for future studies of user experience of this kind that focus on digital language maps and atlases. 
13.1.2 Future directions for studies of language atlas user experience.

One way of looking at future directions in this area would be to ask the question: Were research of this kind performed again with more time and resources, what would it look like?

A good place to start before conducting a study of user experience would be to look at who is using a language atlas in the first place. A descriptive account of who is engaging with atlases of interest would enable a more detailed user profile, which could then contribute to the development of more appropriate questions and study design elements. Additionally, knowing who is using an atlas and why would provide insights into how atlases are actually used in comparison to what they were originally intended for.

There are ways of obtaining this information, such as interviewing known users of the atlas at workshops or conferences, or placing web analytics on an atlas website. In a basic sense, web analytics are coded elements added to web pages that make it possible to ascertain where a user is accessing an atlas from, how they found the atlas (e.g. via search engine), or what pages they look at the most and for how long (Jansen, 2009, p.1). The information generated from web analytics on an atlas website, such as IP addresses, could indicate whether the users mostly come from a university or from elsewhere in the community. Similarly, if web analytics reports indicate that a specific interactive module (such as interactive language lessons) is being accessed more than another (such as a data visualization function), developers and authors could conclude that users consider the language lessons to be more useful. There are, of course, important ethical considerations unique to each atlas involved with using web analytics, such as the extent to which users' privacy is respected versus the extent to which usable information can be gathered to understand them better. Were I to conduct a similar study of user experience, I would consider investigating who uses the atlas before performing a study of user experience. 
To make a study of this kind more adept at understanding users' experiences with language maps, especially regarding Indigenous and/or minority knowledge and populations, I would recommend that future investigations use this model. Additionally, they could benefit from critically reflecting on their implementation, perhaps in a similar fashion to this critical analysis. Before subsequent investigations of this kind occur which involve Indigenous knowledge and participants, extensive dialogue will need to address how to appropriately build relationships with the participants and their communities, incorporate Indigenous perspectives into the study's design and implementation, be based on Indigenous input, and directly help Indigenous communities.

Whether or not a user study of this kind focuses on Indigenous knowledge or involves Indigenous participation, future research projects should also experiment with more and/or different ways of coding participant responses in the post-questionnaire semi-structured interviews. As I come from an overwhelmingly quantitative research background, I felt less 'at home' in the qualitative aspect of this research, and thus feel that a similar user study conducted by those more experienced in qualitative practices would look different and perhaps provide more detailed and contextualized evidence for user experiences.

After creating new tools and adapting existing ones for the purposes of building reflective capacity in language mapping, the following section outlines an area of work that reflective practices in language mapping and cybercartography can be of benefit in. 


\section{Essay}

Indigenous Language Education and Cybercartography 


\subsection{Indigenous Language Education and Cybercartography.}

While the majority of this dissertation focuses on the past and the present of language mapping, the following section marks a small departure into identifying a specific area in which language maps can reasonably be applied. As one chapter in a book that is itself part of a series of books focusing on the theory and applications of cybercartography (Taylor, Anonby, \& Murasugi, 2019), this published work does not produce original empirical research per se. Instead, the purpose of this chapter is to highlight the importance of language maps in Indigenous language education that emphasize the democratization of mapping technologies and promote communities' agency over their own knowledge. 


\section{CHAPTER}

\section{Cybercartography in Indigenous Language Education}

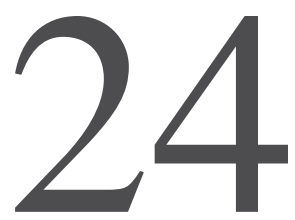

Adam Stone $^{\mathrm{a}}$, Erik Anonby ${ }^{\mathrm{b}, \mathrm{c}}$

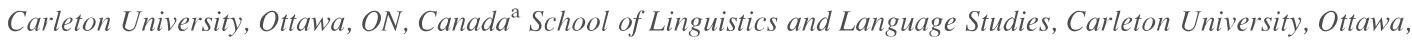
ON, Canada ${ }^{\mathrm{b}}$ Allgemeine Sprachwissenschaft, Universität Bamberg, Bamberg, Germany ${ }^{\mathrm{c}}$

\section{Chapter outline}

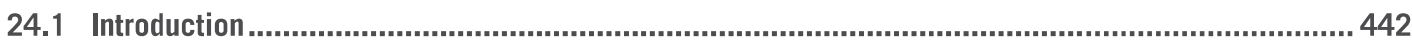

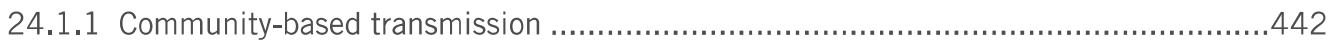

24.1.2 The colonial disruption in language transmission.........................................442

24.1.3 A brief history of Indigenous Language Education in Canada ..............................443

24.1.4 Possibilities for strengthening Indigenous Language Education............................445

24.2 Indigenous Language Education and the practice of mapmaking .............................................. 445

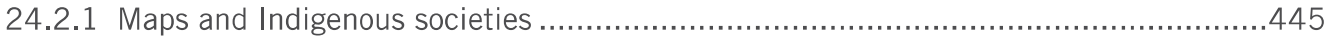

24.2.2 Interactivity, multimedia, and access over large distances..................................446

24.2.3 Maps as a means to engage learners of multiple ages .......................................447

24.2.4 The contribution of maps to Indigenous Language Education ...............................447

24.3 Cybercartography and Indigenous Language Education..................................................................448

24.3.1 The need for self-determination and autonomy ...............................................449

24.3.2 Cybercartography as a means for community voices to be heard...........................449

24.3.2.1 Volunteered geographic information and linguistic maps.............................450

24.3.2.2 Linguistic maps are a process, not product, in Cybercartography..................450

24.3.2.3 New opportunities for learning .................................................. 451

24.3.3 The role of linguistic cybercartographic atlases in Indigenous Language Education.....451

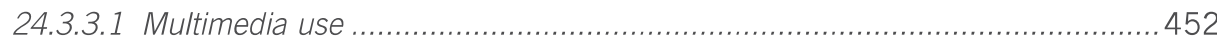

24.3.3.2 Interactivity: Students as teachers.................................................... 453

24.3.3.3 Specific learning modules............................................................... 454

24.3.3.4 Promote language learning outside of the atlas............................................455

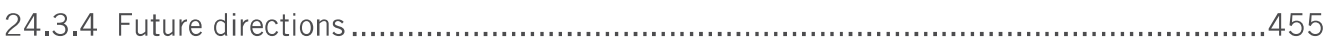

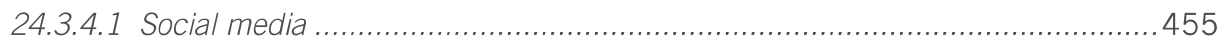

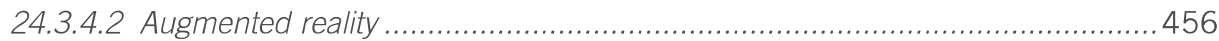

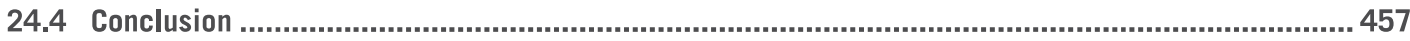

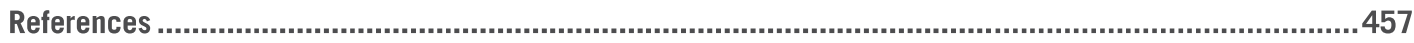

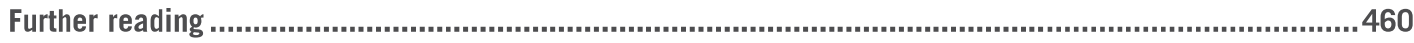

Modern Cartography Series. Volume 9. ISSN 1363-0814. https://doi.org/10.1016/B978-0-444-64193-9.00024-5 


\subsection{Introduction}

Over the last few centuries, languages in every part of the world have undergone dramatic changes in the ways they are transmitted from one generation of speakers to the next. Changes in language transmission reflect cultural shifts and, in the case of Indigenous languages, there have been major and overwhelmingly negative disruptions as colonial languages and technologies come into contact with Indigenous societies. In this article, we reflect on the community-based language transmission that has characterized Indigenous cultures, and ways in which the holistic nature of Indigenous Language Education can be strengthened in contemporary communities. The practice of Cybercartography brings communities and technology together, and places communities at the centre of their own technologyrelated activities. In particular, community-based language mapping is one of many ways that linguistic knowledge can be imparted to learners and represents an opportunity for facilitating the transmission of ancestral languages through activities in the digital sphere. Indigenous learners, who are increasingly immersed in digital technology, can consequently continue to derive benefits from being grounded in culture and tradition.

\subsubsection{Community-based transmission}

Prior to European contact, the linguistic landscape of North America displayed staggering diversity: estimates suggest that anywhere from a few hundred to nearly a thousand languages in over fifty distinct language families thrived on the continent (Brown, 2014, p. 7). Some groups tended to remain functionally monolingual (Dorais and Sammons, 2000, p. 1), whereas others were multilingual, speaking the languages of their neighbours in addition to their own (Hebert, 1984, p. 122; Brown, 2014 , p. 8). One ubiquitous factor, as is still typical of most of the world's languages today, is that language transmission from older to younger generations started in the home and was based on interactions with others from the same linguistic community. Intergenerational language transmission can be defined as an ongoing process through which a language is passed from one generation to another through interactions between children, parents, and grandparents (Chrisp, 2005, p. 150). Indigenous languages in what is now Canada were passed down through such community and familial interactions over a speaker's lifetime, and were part of the oral literacies that formed an integral part of Indigenous education. Societies in close proximity to one another tended to have similarly prestigious languages to the extent that the presence of one linguistic group would not directly contribute to the decline in speakers of another (Dixon, 1997, p. 69).

\subsubsection{The colonial disruption in language transmission}

The arrival of European explorers, followed by waves of colonists and settlers, brought with it a legacy of residential schools, the influence and even imposition of dominant European languages, and a general tendency towards overt discouragement of the use of Indigenous languages (Norris, 2004, p. 1). The introduction of these new languages was accompanied by the imposition of new domains such as formal education, writing, the church, and a centralized government in which Indigenous languages were deemed irrelevant and unwelcome. Residential schools created generations of Indigenous community members unable or unwilling to pass on their own language to their children, and this resulted in a general sense of disconnection between younger and older generations (Olthuis et al., 2013, p. 38). 
The domains in which Indigenous languages were used became increasingly restricted during the 18 th to 20 th centuries, leading in turn to the perception, by community members and outsiders alike, of many languages as less useful. While some communities did not necessary lose the traditional domains in which their language was spoken (e.g., Cree: Westman and Schreyer, 2014, p. 123), the introduction of new and foreign domains meant that community members were required to become proficient in the new majority languages in order to become educated, gain access to basic infrastructure and services, and adapt to a changing and increasingly connected world (Grenoble and Whaley, 2006, p. 9).

The damage of this history to Indigenous language communities has been profound and longlasting. Despite this reality, there have been attempts to help Indigenous language communities adapt to language change and the loss of domains. A Latin-based orthography was introduced to communities, such as the Innu in Eastern Canada, to promote written literacy in the community language (Baraby, 2002, p. 200), while the neighbouring Inuit and James Bay Cree adopted a syllabics system originally designed by missionaries (Coulmas, 2003, p. 70).

\subsubsection{A brief history of Indigenous Language Education in Canada}

Until the end of the 20th century, there was little in the way of formal Indigenous Language Education programming in Canada, and specific institutions such as the Residential School system attempted to eliminate the very existence of such languages (McBride, 2015). Though academics had already conducted language documentation research for over a century, most studies were of sole benefit to the researchers, who would store linguistic information in archives and publish academic papers (Henke and Berez-Kroeker, 2016, p. 413). Carpenter et al. (2016) note that Indigenous communities have worked for decades to maintain and strengthen their languages; however, there is a lack of literature from this time regarding language education, since this activity was viewed as subversive and needed to remain hidden in order to take place at all (p. 2). The dire situation faced by many Indigenous languages began to reach a broader academic audience following Krauss' (1992) call for action, which reported the state of endangerment among North American Indigenous languages and the need for education and revitalization practices in order, at the very least, to slow the rapid pace of language decline.

Since 1992, there has been a remarkable increase in publications that focus on Indigenous language revitalization, conservation, and education (Kruijt and Turin, 2017, p. 257). During this time, researchers have devoted efforts to creating practices in language documentation and curriculum development that are not just for communities, but with and by the communities themselves (Czaykowska-Higgins, 2009, p. 23).

Similarly, in the past two decades, legislation surrounding the right to speak and learn Indigenous languages has become more favourable towards language education. Established in 2008, the Truth and Reconciliation Commission (TRC) is an independent body that aims to oversee the process through which survivors of residential schools recover from traumatic personal experiences, as well as the loss of culture and language. In 2015, the TRC made four calls-to-action relating to languages:

- a call for the government to acknowledge that Indigenous rights include language rights;

- a call for the government to enact an Indigenous languages act and provide funding that is sufficient and is managed by Indigenous communities;

- a call for the appointment of a languages commissioner; and

- a call for post-secondary institutions to create degree and diploma programs in Indigenous languages (Truth and Reconciliation Commission of Canada, 2015, p. 2). 
In December 2016, Canadian Prime Minister Justin Trudeau announced an Indigenous Languages Act to be co-developed with Indigenous peoples, which was introduced in Parliament in June 2018 and was passed into law in 2019 (First People's Cultural Council (FPCC), 2017). In addition, the federal government promised $\$ 90$ million in 2017 to preserve, protect, and revitalize Indigenous languages (Morin, 2017), while the provincial government of British Columbia committed $\$ 50$ million in 2018 to do the same (Hunter, 2018).

During the 20 years that Indigenous Language Education has taken root in Canada, Penfield and Tucker (2011) have noted that Indigenous Language Education and revitalization programs are most commonly practiced outside the home, and may include such efforts as 'community classes, immersion camps, workshops, [and] Master-Apprentice programmes' (p. 294). When discussing these efforts, Penfield and Tucker (2011) explain that educators need to work with multiple age groups simultaneously due to a lack of funding or multiple instructors. Furthermore, though more and more Indigenous people are migrating away from their communities to larger urban centres where they comprise a minority of the overall population (Norris, 2004, p. 6), the majority of language documentation and education efforts remain in rural communities (Yamada, 2007), meaning that those who have been separated from their communities by large geographic distances often do not have access to the resources that would enable them to study and learn their traditional language.

Even in many cases where communities have access to sufficient funding and instructors, much of the linguistic information used for curriculum development and the preparation of classroom materials had originally been gathered by Western academics for their own purposes in ways that were not intended to benefit the community (Shepard, 2016, p. 461). In such cases, it may be difficult to disseminate or even use such materials due to lack of agreement between community members regarding a proper orthography, the sole availability of a poorly-made dictionary, or records of the language that are neither accessible nor culturally appropriate to the community (Hinton, 2001, p. 421). Such a discrepancy between available linguistic materials and communities' needs can compromise such groups' self-determination regarding language education, presenting a major obstacle to the fostering of new speakers (Shepard, 2016, p. 465).

Finally, if Indigenous languages are to enjoy a vitality within the communities that can approach - and even counterbalance - that of the majority language (English and French) that currently permeate much of Indigenous society in Canada, the number of domains in which the language is used will have to increase (Grenoble and Whaley, 2006, p. 9). While one's traditional language may survive in particular domains such as in ceremonies or religious rites, speakers will often find themselves required to use English to engage with any domains associated with the larger majority, such as technology, employment, or education. According to Hermes et al. (2012), even an increase in new speakers does not necessarily guarantee that a language's vitality has improved if the number of domains the language is used in has not increased (p. 397). However, many of the current efforts towards documentation and education attempt to restore Indigenous languages to a 'pure' state as it existed prior to European contact and set aside the need for such languages to be adapted to new uses and domains that have only recently come into existence (Amery, 2009, p. 139). As a result, learners only have the opportunity to learn their traditional language for specific purposes within community life, and speakers will continue to only be able to use their language in a limited set of contexts. 


\subsubsection{Possibilities for strengthening Indigenous Language Education}

To strengthen the potential for Indigenous Language Education in Canada to foster new speakers and support existing ones, successful initiatives depend on engaging learners of all ages, ensuring that linguistic materials are obtained and represented in such a way as to acknowledge self-determination, and encourage languages to be used in new and ever-changing domains. At a time when people around the world are becoming more tightly connected due to the universalization of digital technologies and the Internet, the potential for Indigenous languages to be better represented in some way in the digital sphere has become something that researchers, educators, and speakers have a responsibility to address, especially if those languages are going to survive as a means of spoken communication and thrive in all domains of life and experience.

While authors such as Grenoble and Whaley (2006) and Amery (2009) stress how important it is for speakers of Indigenous languages to reflectively respond to changes and pressures from a majority society, researchers such as Czaykowska-Higgins (2009) underscore that it is essential for such change to happen from within the communities themselves, and on their own terms. An example of a reflective, innovative community-driven effort would be the incorporation of linguistic and curricular materials into the digital sphere by way of digital mapping techniques that spatially arrange culturally meaningful linguistic information in an interactive way that facilitates language learning and use. In line with key tenets of cybercartographic theory, the focus of mapping is proceeds from direct Indigenous input, and tells a story about the language from the community's perspective (Ljubicic et al., 2014, p. 202; Taylor, 2014, p. 8).

\subsection{Indigenous Language Education and the practice of mapmaking}

In this section, we look at the role and potential of maps-and the act of mapmaking itself-for Indigenous Language Education. First, we consider maps, including language maps, as they relate to Indigenous histories and cultures (Section 24.2.1). We then look at how maps can engage learners (Section 24.2.2) and even help overcome disconnection between generations in the language transmission process (Section 24.2.3). The section concludes with a consideration of how digital maps in particular can contribute to Indigenous Language Education (Section 24.2.4).

\subsubsection{Maps and Indigenous societies}

Language itself is fundamentally oral in our evolution and individual development as humans and societies, rather than textual. Maps are an intrinsically pictorial—and likewise non-textual—means of communication and can impart significant amounts of complex information in an intuitive way, without the interference of text (Upton, 2010, p. 144). Furthermore, Upton (2010, p. 143) states that language maps are important and meaningful, and viewers are often instinctively drawn to them; and in contrast to many other media of instruction, viewers often feel they have some immediate understanding of the concepts represented. In the context of Indigenous societies in particular, the visual nature of maps as a medium echoes the traditionally oral structure of communication, knowledge and education in a way that text-based communication is not capable of doing. Herlihy and Knapp (2003) note that ' $[\mathrm{n}]$ ative communities have maintained cognitive maps that are delineated verbally using place 
names that convey place and spatial orientations' (p. 304), which provided a wealth of knowledge to Indigenous peoples in those areas as well as explorers who arrived in those areas (p. 303).

Because of their communicatory strengths and their propensity to model reality to their viewers-whatever their motivation, basis or factuality-maps have been and continue to be used as tools of power (Herlihy and Knapp, 2003, p. 303). Whoever controls depictions of a given geographical, political or 'linguistic' territory has the means of shaping a society's thoughts regarding that territory, insofar as the maps are accepted as 'top-down' representations of the group's claims to sovereignty (Ormeling, 2010, p. 28). In colonial contexts, maps were and are used as tools of ownership, in which European groups assigned names and borders to newly-created territories, hence 'claiming' them (Aporta et al., 2014; Ormeling, 2010, p. 28). Language distribution maps (see Girnth, 2010) produced by scholars perpetuate the association of language communities with European nation-state or territory-like political entities (Ormeling, 2010, p. 28) Consequences of such maps include incorrect implications that the distribution of a language is limited to permanent settlements rather than the whole area over which a society is connected to the land, and that societies speaking different languages do not share territory for the same or different land uses. It is important to acknowledge that such a legacy exists in cartography when considering the use of maps in Indigenous language documentation activities, both to offer a partial explanation as to why some communities may choose not to participate and, more importantly, to ensure that new maps do not perpetuate similar power imbalances. This is especially important in the context of Indigenous Language Education, where linguistic and cultural knowledge is situated within the community, and must remain there.

\subsubsection{Interactivity, multimedia, and access over large distances}

To address the imbalances of power that can be associated with mapping, and to ground the process of mapping within Indigenous communities, maps need to be authentic, interactive and accessible. The digital construction of maps allows for information to become more than something that is simply delivered to users. Because many different kinds of data can be presented through applications on the Internet, users can not only see linguistic information on a map, but also hear sentences being spoken, watch filmed speakers tell a story, and-most importantly-interact with the linguistic material in ways that suit their needs. In response to the issues raised in the previous section (Section 24.2.1), different types of linguistic knowledge and distribution can be distinguished. When these types of multimodal information are on a map as a data point or shape, users can engage with the linguistic material quickly and effectively (Olariu and Olariu, 2014, p. 76). Furthermore, information about a language can be conveyed and modified through animations that depict a change over time, patterns of movement, or internal dispersions of characteristics within languages themselves that may change over a geographic area or in keeping with features of how societies are constituted.

The ability for digital interfaces to superimpose maps as layers on one another means that, as opposed to more traditional forms of aggregate mapping that compared maps conveying different kinds of information side by side, these different information types can be directly placed with others, according to the viewer's initiative. In this way, the user's role shifts from that of a passive recipient of linguistic information to an active one. For more information on how digital maps can use animations and interactivity to convey dynamic aspects of language, see Kunst (2010). 


\subsubsection{Maps as a means to engage learners of multiple ages}

As discussed in Section 24.1.2, the intergenerational transmission of Indigenous languages has been disrupted by the legacy of residential schools and many other aspects of cultural domination from Canada's settler-centred majority culture. This situation underscores the importance of involving all generations in language education and raises the potential for maps as a forum for bringing speakers of different generations together.

Prensky (2001) mentions that members of different generations tend to have different needs when engaging with digital devices due to differing levels of exposure to such technologies during their formative years. Members of what Prensky (2001) describes as 'digital natives' (broadly speaking, people in North America who were born after 1985) tend to have an intuition of how to go about using unfamiliar digital devices, such as a new computer or digital application, that those born before 1985 may not have; those in this digital generation tend to learn how to engage with digital technologies more quickly even if they have had less absolute exposure to such technologies than older people (p. 2).

Still, as spatial beings that navigate a spatial world, almost all people access and identify with maps on some level (Upton, 2010, p. 144), allowing for a common point of reference even when unfamiliar topics and concepts are involved. Digital maps can serve as an effective means to bridge generational and socioeconomic gaps in user populations due to the intuitive way they present information, as discussed in the previous sections (Sections 24.2.1 and 24.2.2). A user study (Stone, 2018) of the Algonquian Linguistic Atlas (Junker et al., 2005-2019; see also the following section) found that while younger users tended to engage with the digital map differently than older users (e.g., were more prone to multitasking), users of all ages reported the map-based interface to be easy to use and intuitive.

\subsubsection{The contribution of maps to Indigenous Language Education}

Digital maps present linguistic information multimodally in ways that engage users of many ages and backgrounds. Because of this, they are ideal for use in educational programs such as immersion programs or language classes, and equally appropriate for a classroom setting as for informal, relationshipcentred educational contexts. Though there are a number of ways in which students can use digital maps to connect with linguistic materials, the Algonquian Linguistic Atlas serves as an apt example.

The Algonquian Linguistic Atlas (Junker et al., 2005-2019) is a collection of online and interactive maps of Algonquian languages from the Cree-Innu-Naskapi continuum along with Mi'kmaw, Ojibwe, Blackfoot, Algonquian, and Michif (Cenerini et al., 2017, p. 307). The main map which users encounter presents data points from a variety of Indigenous communities on a Google Maps background. When clicked, each data point presents a recording of a sentence, along with a written transcription, the name of the speaker, and their community of origin. The desired sentence can be chosen from a drop-down menu located at the top of the screen. Aside from this main language map, users can also access linguistic feature maps, which show the distribution of particular features across the dialectical continuum that comprises the Algonquian language family is composed of. Links to dictionaries, and language learning apps available for download onto mobile devices, are also provided (Fig. 24.1).

While it is important to acknowledge that such digital language maps can never fully substitute for face-to-face contact between learners and teachers, they nonetheless provide a rich base from which to draw linguistic materials in an engaging and interactive manner. Students are able to memorize important phrases in their language, as well as understand how those phrases will differ between their 


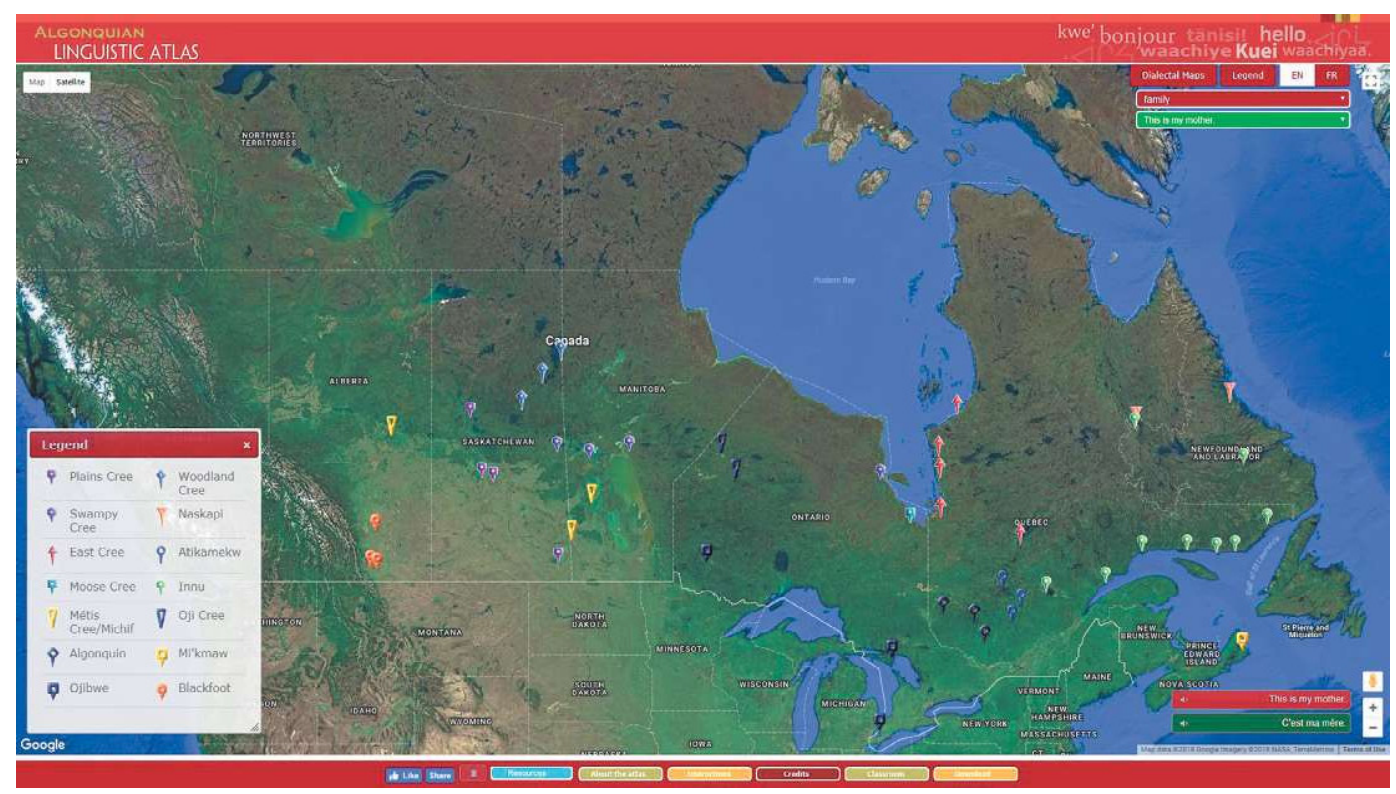

FIG. 24.1

The main page of the Algonquian Linguistic Atlas (Junker et al., 2005-2019).

community and others. In adding a spatial dimension to the material they are learning, students are able to situate themselves in the language in ways that studying texts or practicing phrases alone could not account for (Upton, 2010, p. 144). As a form of communication that has historically been limited, in many cases, to research settings, language maps are in fact ideal instruments for presenting ideas and information to language students, though caution must be taken in order to ensure that such ideas are imparted in a culturally sensitive and appropriate manner (see also Section 24.2.1).

\subsection{Cybercartography and Indigenous Language Education}

Cybercartography is defined as the 'organization, presentation, analysis and communication of spatially referenced information on a wide variety of topics of interest and use to society in an interactive, dynamic, multimedia, multisensory and multidisciplinary format' (Taylor, 2003, p. 406). As a means of representing human knowledge in a holistic manner that allows those who possess the knowledge to also map it (Taylor, 2005, p. 4), Cybercartography offers Indigenous language communities a means to represent and ultimately control their own linguistic knowledge in the digital sphere.

In this section, we look at the role of Cybercartography in producing maps which can be useful resources in Indigenous Language Education. First, we explore how Indigenous self-determination and autonomy are important in Indigenous Language Education, and how a lack of such factors is a major barrier to language learning (Section 24.3.1). We then look at how cybercartographic theory can allow for community voices to be heard (Section 24.3.2) through the use of Volunteered 
Geographic Information (Section 24.3.2.1.), how mapping as a process complements notions of language learning as a process (Section 24.3.2.2), and how cybercartographic language maps create new opportunities for learning (Section 24.3.2.3). Subsequently, we focus on the need for language maps to be produced in a cybercartographic framework (Section 24.3.3) and explore how multimedia (Section 24.3.3.1), interactivity (Section 24.3.3.2), and specific learning modules (Section 24.3.3.3) can promote language learning in other aspects of students' lives (Section 24.3.3.4). Finally, we explore some emerging technologies (Section 24.3.4) that would be advantageous to cybercartographic language learning interfaces, such as social media (Section 24.3.4.1) and augmented reality (Section 24.3.4.2).

\subsubsection{The need for self-determination and autonomy}

Broadly speaking, self-determination refers to 'the idea that [Indigenous] communities can effectively interpret their past and have a right to make decisions about the trajectory of their present and future' (Shepard, 2016, p. 460). Traditionally in academia, interacting with communities was often a primary objective of documentation, but the extent to which the community uses and controlled such materials tended to decrease once the study had been completed. In his detailed analysis, Shepard (2016) discusses how communities that perceive themselves to lack self-determination and control over content management will tend to less readily engage in documentation, revitalization, and education projects, even when such projects have been designed by researchers with the best of intentions. A major contributing factor to this lack of self-determination is in fact exemplified in such a statement, as such projects are defined as being designed by the researchers, while communities assume the role of the recipient of the products in of research. In such situations, communities assume a passive role at best and find themselves at the mercy of the rules and requirements of the ruling bodies responsible for the linguistic research.

In language education contexts specifically, this can result in a failure for learning materials to be embraced by educators, students, community members, sponsors, or some combination thereof. In order for Indigenous Language Education programs to be truly successful in engaging the community in culturally appropriate ways that promote self-determination, engagement between community members and researchers must occur from the outset and continue in each step, with community needs and wishes driving the entire project (Linn, 2014, p. 56).

\subsubsection{Cybercartography as a means for community voices to be heard}

Similar to digital linguistic mapping methods described in Sections 24.2.2 and 24.2.3, Cybercartography takes advantage of multisensory and interactive media formats to communicate to a diverse audience an array of topics in new ways that are not limited to the physical environment (Taylor, 2005 , p. 2). In taking this into account, the term 'atlas' is more apt than 'map' when applied to the cybercartographic methodology, as one of the defining characteristics of cybercartographic projects is that they are part of a greater information package, and not stand-alone devices. The discipline of Cybercartography extends the notion of what it means for languages to be a part of a mapped system, in that the human element of the information that gets presented not only cannot be ignored, but instead is essential to the map's very existence. Woodward and Lewis (1998, p. 1) claim that any definition does not account for maps as acting as social constructs fails to understand that maps have many other functions than just wayfinding devices. 
In the second edition of this book, Taylor (2014) remarks that cybercartographic atlases dealing with traditional knowledge must privilege Indigenous voices over others, as they have the potential to be compelling means of storytelling which contain multiple narratives of those who have traditionally not had a strong voice (p. 8). An important characteristic of cybercartographic maps and atlases is the emphasis on Volunteered Geographic Information, whereby contributors from the mapped communities are responsible for creating and uploading the information they wish to see online.

\subsubsection{Volunteered geographic information and linguistic maps}

More than just the perspective that information should be directly contributed to maps by community members, Volunteered Geographic Information (VGI) is a collaborative approach to digital mapping that promotes the development and use of digital software and hardware to provide the means through which such direct contribution is possible (Engler et al., 2014, p. 44). Such direct connectivity between the 'back-end' interface and community member is made possible by the use of the CouchDB document database system, which is easy-to-use due to its unstructured and schema-less composition. Such a tool allows for a flexible approach to information upload that can account for communities' changing needs, users, and data, and can accommodate new relationships between different kinds of information (Engler et al., 2014, p. 50).

In linguistic maps, this means that speakers of an Indigenous language would, following initial training by specialists, have the ability to gather their own linguistic data and upload it to the map they are in essence creating in ways that are appropriate to their communities' wishes, needs, and requirements.

Aside from affording the contributors more freedom surrounding what they wish to have displayed on a map, allowing culturally appropriate information uploads can help linguistic classroom materials more closely resemble language as it would naturally occur, simply because elicitations are both designed and recorded by those who have the most experience regarding how the language is actually used. Conventionally recorded phrasal information available in many online archives or databases is most often recorded in the form of elicitations, whereby a speaker hears a sentence or phrase in the dominant language and is required to translate it into their own (Himmelmann, 2012, p. 197). Though this form of recording is an excellent means to control exactly what information is recorded, Himmelmann (2012) also cautions that the act of extracting elicitations in a researcher-participant model creates an artificial situation that produces unnatural forms that would not occur in normal speech. By both collecting and producing linguistic information, community members can produce linguistic materials that are closer to natural speech and more realistic as a means towards language learning.

The extent to which this information can be copyrighted or belongs to the contributor depends on the nature of the linguistic information, and the objectives of the mapping project. Though issues surrounding intellectual property rights are nothing new to the disciplines of language documentation, revitalization, and education (e.g., see Newman, 2007, p. 29), the VGI infrastructures set in place in Cybercartography mean community members can upload information in such ways that may help them retain ownership over their own information, at least insofar as a language can be copyrighted.

\subsubsection{Linguistic maps are a process, not product, in Cybercartography}

While maps are considered in many other linguistic disciplines to be a product of a specific research endeavour such as an illustration of the lexical distribution of a linguistic item, atlases created within Cybercartography are viewed as an ongoing process, which change and evolve according to contributors' (e.g., speakers') shifting needs (Taylor, 2005, p. 2). Just as both the teaching and learning of any language 
are a process which can last a lifetime, linguistic cybercartographic maps in theory also never reach an 'end point' where all information that could possibly be uploaded has been exhausted. While speakers and Indigenous community members will have a dynamic set of needs and wishes, learners and educators too have their own specific sets of needs which change over time. Linguistic cybercartographic atlases which by design incorporate new information and kinds of information into their framework are able to accommodate the creation of new lessons or introduction of new vocabulary after the initial interface has already been created and put into use.

\subsubsection{New opportunities for learning}

While engaging with material already available on maps is an intuitive means of imparting linguistic information, providing learners with an opportunity to create and upload their own linguistic information is an invaluable opportunity to make language learning more important and relevant to those learners' lives. Taylor et al. (2014) describe how workshops involving the Inuit Siku (Sea Ice) Atlas allowed high school students in Cape Dorset, Igloolik, and Pangnirtung to work alongside community elders in both learning about and contributing to the atlas. During these workshops, the high school students, who had previously been trained on how to use the atlas, would show elders different aspects of the map, while the elders in turn would elaborate on the information the atlas contains. Together, they would upload new information, and explored ways in which the atlas could be further improved to suit their diverse needs.

While engaging students and elders in this example clearly allowed for more information to be contributed to the atlas, the presence of such an initiative also encouraged those involved to become more interested in how to preserve their knowledge in the digital sphere. Training offered to the high school students in the workshops provided them with new technological skills that aside from enabling them to work on the atlas themselves, also provided them with digital skills that could be used elsewhere. Furthermore, students were able to reinforce or form new relationships with elders in their communities and reported developing new and greater interests in the natural world around them (Taylor et al., 2014, p. 307).

\subsubsection{The role of linguistic cybercartographic atlases in Indigenous Language Education}

It is thus of great interest to see what the result of such cybercartographic educational initiatives would be when applied to the area of Indigenous Language Education. While the Inuit Siku (Sea Ice) Atlas contains large amounts of information, stories, and resources in Inuktitut, the objective itself of the atlas is not to function directly as a language learning resource. An atlas with important similarities to a program designed for language learning is the Inuktut Lexicon Atlas (Murasugi et al., 2016), which is a multidialectical database of the Inuit language family currently encompassing language varieties from the Northwest Territories to Labrador. In this atlas, Inuktut words are available for reference in a word list, which also contains the English equivalent, the semantic category (e.g., seasons), the grammatical type (e.g., verb), an explanation of the meaning, and the word's source. A dialect chart enables users to compare the same words across the different dialects represented in the atlas, while a community map geographically indicates where the different dialects and communities are located. In addition, the atlas makes use of a sculptionary, which is an interactive image of an Inuk sculpture that allows users to explore different parts of the body in different Inuktut dialects. Finally, a section devoted to birds enables users to learn Inuktut words for different species of birds by clicking on a variety of hand-drawn images of bird species (Fig. 24.2). 


\section{2}

CHAPTER 24 Cybercartography in Indigenous Language Education

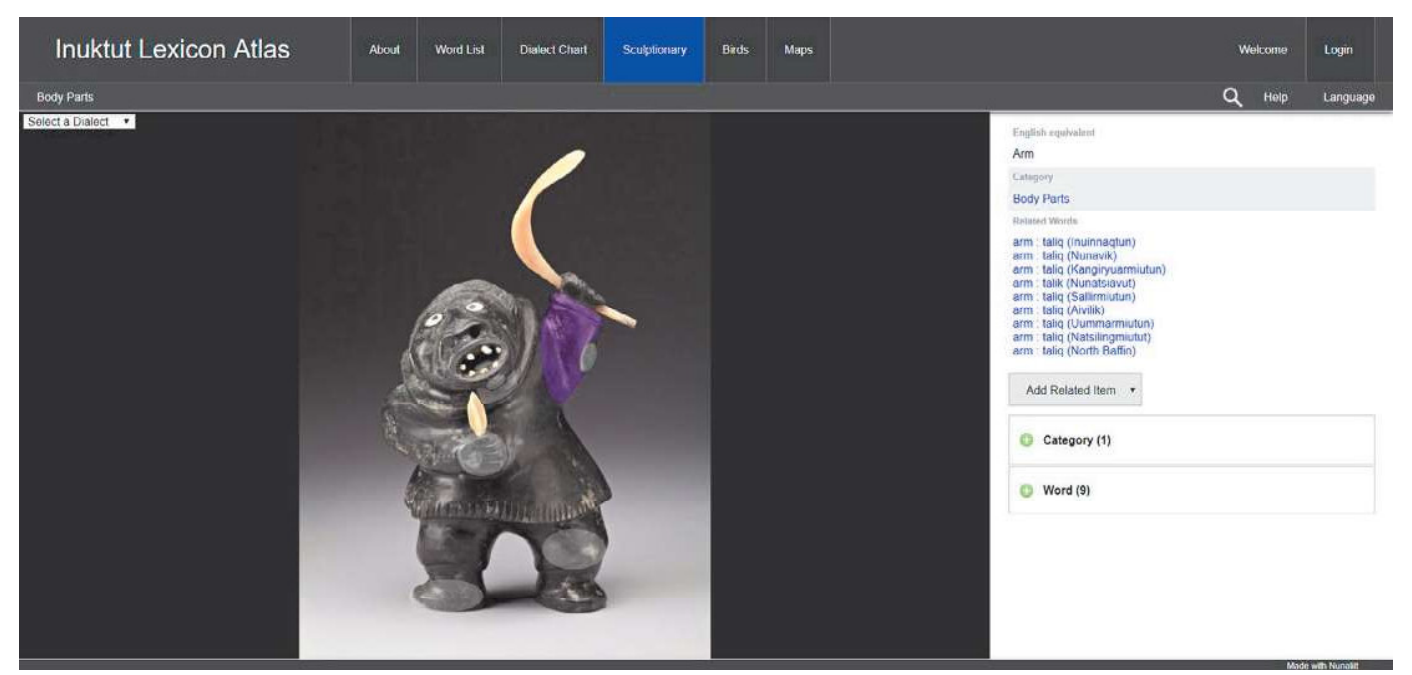

FIG. 24.2

The Pictionary section of the Inuktut Lexicon Atlas.

While this atlas is an excellent example of how word lists can be made interactive to improve accessibility and interactivity, its main goal is not to be used directly as an educational material per se. As described in the 'About' section of the atlas, '[t]he goal of this atlas is to help protect and strengthen the vitality of Inuktut dialects in Canada by documenting [own italics] their words' (from: https:// inuktutlexicon.gcrc.carleton.ca/index.html?module=module.inuktutlexicon_about). While language documentation is an essential component of gathering materials for both linguistic research and language education, it is mainly a phase in which primary and secondary linguistic materials are gathered (Himmelmann, 1998, p. 345). Language revitalization in turn is delivering such materials back to learners in ways that (in the context of language education) are suitable for learning in the specific educational settings (Penfield and Tucker, 2011, p. 294).

To date, however, no such atlas with a primary focus on educational materials has been developed. In drawing from aspects of Indigenous Language Education, conventional practices in digital linguistic mapping, and theories and practices in Cybercartography, however, it is possible to posit important requirements that an atlas project designed primarily for language learning would need to fulfil.

\subsubsection{Multimedia use}

Just as language is on one level an integration of context, timing, and what one can see and hear, a cybercartographic atlas designed as a language learning resource would need to archive and present linguistic information through multiple modalities that resemble spoken (or even signed) language. Chun (2011, p. 676) indicated that better vocabulary learning occurs when linguistic items are presented in multiple ways (e.g., pictures and text) as opposed to text alone. Furthermore, learners can engage with their language in ways that more closely resemble how it is spoken naturally, and in doing so come to experience their traditional language being used in new and emerging domains. This notion is supported in the first of six key ideas of Cybercartography posited by Taylor (2014), which states: 
'[i]ndividuals use all of their senses while observing what is around them: Cybercartography is therefore exploring the possibilities of using all [...] senses in its representations in order to make cybercartographic atlases as reflective as possible of sensory realities' (p. 3).

What might such multimedia use look like? Conventional digital language maps and interactive online archives already use speech recordings and videos to impart real-time language samples on users. However, such language samples are cited (e.g., Czaykowska-Higgins, 2009) to often be gathered by researchers who inadvertently introduce their own biases into what such language samples contain and how they are presented. If community members instead are primarily responsible for gathering and uploading multimedia linguistic information and guide each stage of dissemination thereof, then biases will still exist, but in an advantageous way by reflecting community perspectives in an appropriate manner.

In making a variety of multimedia options available to learners, many different learning styles can be accommodated. While some learners may value and derive benefit from frequent and repeated memorization of phrases or lexical items (e.g., through flash cards), others may learn more effectively by listening to conversations or watching videos containing natural speech. By having the freedom to decide how linguistic resources are accessed and used, learners have the added opportunity to address their specific needs (Taylor, 2014, p. 3), while instructors can devote their time and energy to other elements of the teaching process.

\subsubsection{Interactivity: Students as teachers}

According to Taylor (2014, p. 3), 'educational theory suggests that individuals learn best when they are actively [... involved' in any learning process. While on a user-interface level this could mean improving the interactivity of features of the atlas, which is no doubt important, interactivity here refers to enabling learners to teach others what they have learned or contribute their own information to the atlas once they have gained enough experience to do so.

Peer tutoring —or allowing learners to teach others in the same program with less expertise-has been shown to improve learners' outcomes in a variety of tasks and subjects, both in terms of overall performance and grades (Delquadri et al., 1986). Similarly, learners across different socioeconomic statuses analysed in longitudinal studies were found to engage in higher levels of academic behaviour during lessons involving peer-tutoring (Greenwood et al., 1989, p. 540), meaning that regardless of social background, learners felt more motivated to engage with the material and derived a greater value from what they were studying.

As observed in elder-student relationships formed by using the Inuit Siku (Sea Ice) Atlas (Taylor et al., 2014), understanding that learners already have expertise in other areas of their lives that is useful in the mapping process is a powerful way of motivating them both to engage with the material more enthusiastically, and help them realize that they are an integral part of the learning and teaching processes. In language education, this could prove invaluable in promoting the community language as relevant and important to speakers' lives, especially at a time when the recent legacy of linguicide remains fresh in Indigenous people's hearts. As much of the language learning (at least in the context of Cybercartography) would occur in the digital sphere, peer-tutoring could occur either in the form of learners assisting elders in recording their language into the atlas or by learners directly contributing information they have learned to the atlas in such a way as to be accessible by other learners or members of their community. 


\section{4}

CHAPTER 24 Cybercartography in Indigenous Language Education

\subsubsection{Specific learning modules}

Many cybercartographic atlases' interfaces to date are open-ended in the sense that users can browse freely through the materials without needing to complete specific tasks or modules to move on to a subsequent part of the map. Open-ended design such as this is advantageous as users can access the information they need specifically, without needing to sift through other materials that are not of use to them. This makes such atlases important as ongoing tools that can be used multiple times for users' specific purposes, such as research. For less experienced users, however, an atlas without rigid structures to act as guidance may cause them to become lost in the materials they encounter, making the atlas essentially less effective in disseminating linguistic material.

While atlases that have a rigid structure in place tend to be designed for a more limited array of purposes, such rigidity is advantageous if the atlas is produced for specific audiences or uses, such as language learning in the classroom. As an example of such rigidity, the Fifth Thule Expedition Atlas (https://thuleatlas.org/index.html) produced by the Kitikmeot Heritage Society in collaboration with the Geomatics and Cartographic Research Centre at Carleton University is principally designed to tell the story of a Danish anthropological expedition, which was the first to record traditional Inuit societies in Canada. Unlike more open-ended atlas projects that initially only present a map interface, the Expedition Atlas provides users with a window at the bottom of the screen that directly displays documents associated with the data points as they occur on the map. To the user, many such documents have specific paths to other clusters of documents, which allow particular subjects to be explored without the interference of other topics (Fig. 24.3).

In language learning situations, such a rigid structure would permit the design of lesson plans that are directly integrated into the atlas. While seeing relevant linguistic information displayed on a map, learners could also directly access documents, multimedia language material, or interactive learning

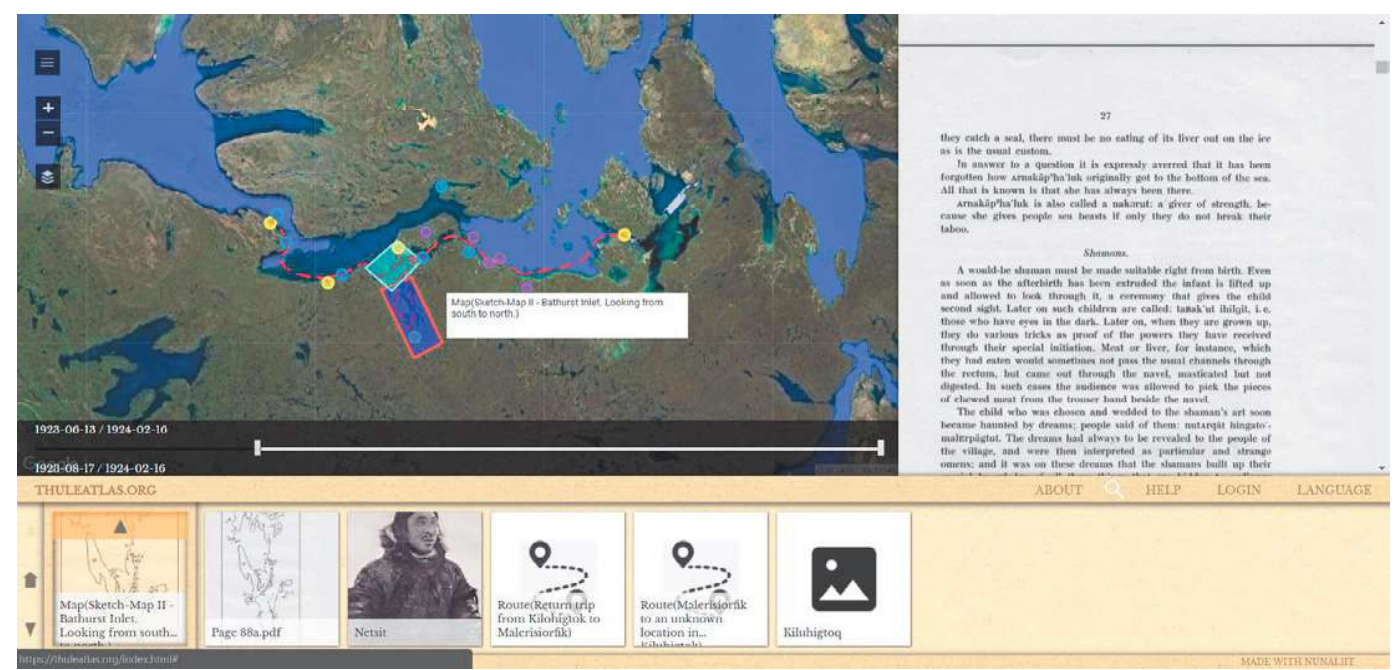

FIG. 24.3

The Fifth Thule Expedition Atlas, produced and maintained by the Kitikmeot Heritage Society in collaboration with the Geomatics and Cartographic Research Centre. 
modules in a window below in a way that facilitates a classroom experience that can be consistent and controlled. Due to the process-oriented structure of cybercartographic atlases, new lessons can easily be added to the interface over time as the first cohort of learners' proficiency improves, or new linguistic materials become available.

\subsubsection{Promote language learning outside of the atlas}

While linguistic cybercartographic atlases hold great potential in assisting educational efforts in language revitalization and introducing Indigenous languages to digital domains, it is important to remember that no amount of technological intricacy can fully substitute face-to-face interactions in the learning process. Any attempts to do so would be unsuccessful and ultimately undermine the very nature of linguistic transmission in Indigenous cultures. However, there is much that cybercartographic atlases can do to promote learning and use in the community outside the digital sphere.

As evidenced by the workshops involving the Inuit Siku Atlas, creating a common platform where community members collaborate with one another in culturally appropriate roles can establish new relationships between such members built around both the subject (e.g., their language) and their common goals (e.g., language learning and teaching). While such relationships are important to the development of new language materials and expanded lesson plans, they have the potential to also extend into the community by adding opportunities for speakers to use their language and improving learners' intrinsic motivation to speak it.

\subsubsection{Future directions}

\subsubsection{Social media}

While creating new relationships and opportunities to use the community language would likely assist in changing some communities' perspectives surrounding language use, directly adding domains in which the language could be present would also be of importance in making such language use more widespread. Gartner (2014) describes social media as an important tool for learning more about users' preferences and appraisals of cybercartographic technologies, both because users' comments on such social media platforms can be recorded, and because data mining techniques can analyse how such individuals are using the software (p. 102). While these market research-related strategies would be crucial in making such atlases more easy-to-use and better address users' needs, they also present some major challenges to communities' ability to consent informedly, especially when such members do not have a sufficient background in digital technologies to properly understand advanced principles of data mining. Social media management nonetheless is an appealing avenue for exploration for reasons related to both educational materials development and language use.

As social media platforms such as Facebook and Instagram become more ubiquitous, especially among members of more recent generations, a new fast-paced domain of instantaneous communication is becoming increasingly apparent (Kaplan and Haenlein, 2010, p. 64). While such platforms themselves are in theory undeniably useful spaces to increase the presence of Indigenous languages, they also represent an interesting means for learners to connect with an atlas' interface. For example, if learners of an Indigenous language were to join a Facebook group reserved for a particular class, connections between such learners would need not be limited by physical distance and those living separately from their linguistic communities would be afforded the chance to participate as well. Through the use of real-time chats or video communications, learners and elders/educators could 
connect and participate in language activities related to use of the atlases, such as training, exploring, or participating in discussions of the features thereof. In this way, developers and those involved in the educational process could take advantage of existing and prevalent technologies to engage in distance and engaging education, and not need to reinvent the wheel, as it were. While members of such a group could in theory use their social media connections to share and upload linguistic information to moderators who would then upload such information to an atlas, it is important for all involved parties to remain cognizant of the social media platform's regulations involving ownership of information that is uploaded through their site.

An alternative to an existing social media platform could be for the atlas to create its own social media platform, which could exist as an additional interactive feature of the atlas. Functionally, such a social media platform would not necessarily be as expansive as Twitter or Instagram or contain as many features, but instead serve as a space for developers, contributors, learners, and elders to freely communicate with one another on issues regarding use, development, or the sharing of linguistic materials. Though purely speculative, the idea of such a platform existing serves as an exciting opportunity to establish more engaging means of collaboration and communication between stakeholders, while providing learners with easier-to-grasp means to engage actively in the mapping process. Such a platform could ideally be adapted to mobile devices in such a way as to allow instant communication that is not restricted to computer labs or physical classrooms, so that learners and educators would have the ability to engage with learning activities in locations that are most convenient for them.

\subsubsection{Augmented reality}

An enticing opportunity for the use of cybercartographic mapping in everyday life has recently begun to present itself in the form of augmented reality. In contrast to Virtual Reality, where users are immersed in a 3-D visual environment composed entirely of digital stimuli, users engaged with Augmented Reality see virtual objects superimposed on the real world around them (Azuma, 1997, p. 355). As an example of 3-D language mapping, users could experience augmented reality in the form of viewing physical objects in their vicinity (e.g., through a smartphone camera), such as trees or houses, and see written translations above such objects in their community language. Assuming that particular areas have already been mapped (the information is already present in the atlas), learners could access specific information about particular landmasses in their local area, or specific objects and places that are part of their community.

Outside of Cybercartography, Wikiupedia (https://wikiupedia.com) is one such project that is currently being developed to introduce Indigenous histories into daily spheres of engagement (Bridges, 2017). Designed by Adrian Duke, the augmented reality app aims to tell stories about areas of interest to Indigenous peoples with the assistance of an animated raven, which appears on users' screens when they point their cameras at a relevant site. If applied to language learning, such an app would allow learners to introduce their community language into many aspects of the world around them in ways that allow for their immediate surroundings to act as language materials.

Along with social media, an augmented reality interface would give learners the opportunity to engage with the language materials found on a cybercartographic atlas in ways that are directly related to their own daily lives. As the linguistic materials in such atlases would already be georeferenced by definition, the addition of an augmented reality interface would not represent a new form of mapping, so much as it would represent a new means to make indexed information more accessible and relevant. 


\subsection{Conclusion}

It is important to remember that such possibilities for implementing Indigenous Language Education in Cybercartography are still mostly speculative, as no such language atlases currently exist in this kind of framework. As a young and fast-moving discipline, however, such speculations are at the very least important for driving both the development of Cybercartography and finding new endeavours for future research. As many of Canada's Indigenous languages are no longer transmitted to members of younger generations through use in the home or community, it is important that educational programs create and empower new speakers in ways that will allow their languages to survive once the elders are gone.

Cybercartography has much it can offer to improve learning outcomes for those who wish to regain the use of their traditional language due to the ways in which immersive technologies are employed to tell stories from the perspective of Indigenous peoples. In making language programs more enjoyable, engaging, and appropriate for learners and teachers alike, cybercartographic atlases have important potential in empowering all stakeholders to re-introduce their languages into both old and new spheres of existence.

\section{References}

Amery, R., 2009. Phoenix or relic? Documentation of languages with revitalization in mind. Lang. Doc. Conserv. 3 (2), 138-148. http://hdl.handle.net/10125/4436.

Aporta, C., Kritsch, I., Andre, A., Benson, K., Snowshoe, S., Firth, W., Carry, D., 2014. The Gwich'in atlas: place names, maps, and narratives. In: Developments in the Theory and Practice of Cybercartography: Applications and Indigenous Mapping, second ed. Elsevier, Amsterdam, pp. 229-244.

Azuma, R.T., 1997. A survey of augmented reality. Presence-Teleop. Virt. Environ. 6 (4), 355-385.

Baraby, A.M., 2002. The Process of Spelling Standardization of Innu-Aimun (Montagnais). In: Indigenous Languages Across the Community. Proceedings of the Annual Conference on Stabilizing Indigenous Languages, 7th, Toronto, Ontario, Canada. May 11-14, 2000.

Bridges, A., 2017. Canadian app developer seeking 600 stories for Indigenous 'Wikiupedia'. CBC News. Retrieved from, http://www.cbc.ca/news/canada/saskatoon/adrian-duke-wikiupedia-phone-app-1.3988502. on April 25, 2018.

Brown, J.F., 2014. Language matters: linguistic relativism and native American language revitalization. In: Honours Thesis in Anthropology. Whitman College.

Carpenter, J., Guerin, A., Kaczmarek, M., Lawson, K., Nathan, L.P., Turin, M., 2016. Digital access for language and culture in first nations communities. In: Knowledge Synthesis Report for the Social Sciences and Humanities Research Council (SSHRC). University of British Columbia, Vancouver, BC. retrieved from: http:// heiltsuk.sites.olt.ubc.ca/files/2017/05/Digital_Language_Access_report_May2017.pdf.

Cenerini, C., Junker, M.O., Rosen, N., 2017. Mapping dialectal variation using the Algonquian linguistic atlas. Lang. Doc. Conserv. 11, 305-324. retrieved from, http://hdi.handle.net/10125/24738.

Chrisp, S., 2005. Māori intergenerational language transmission. Pacific sociolinguistics. Int. J. Sociol. Lang. (172), 149-181. retrieved 5 Apr. 2018, from, https://doi.org/10.1515/ijs1.2005.2005.172.149.

Chun, D.M., 2011. Computer-assisted language learning. In: Handbook of Research in Second Language Teaching and Learning, Routledge, Vol. 2, pp. 663-680.

Coulmas, F., 2003. Writing Systems: An Introduction to Their Linguistic Analysis. Cambridge University Press.

Czaykowska-Higgins, E., 2009. Research models, community engagement, and linguistic fieldwork: reflections on working within Canadian Indigenous communities. Lang. Doc. Conserv. 3 (1), 15-50. on April 18, 2018 retrieved from, http://hdl.handle.net/10125/4423. 
Delquadri, J., Greenwood, C.R., Whorton, D., Carta, J.J., Hall, R.V., 1986. Classwide peer tutoring. Except. Child. $52(6), 535-542$.

Dixon, R.M., 1997. The Rise and Fall of Languages. Cambridge University Press.

Dorais, L., Sammons, S., 2000. Discourse and identity in the Baffin region. Arct. Anthropol. 37 (2), 92-110. Retrieved from, http://www.jstor.org/stable/40316532.

Engler, N.J., Scassa, T., Taylor, D.R.F., 2014. Cybercartography and volunteered geographic information. In: Developments in the Theory and Practice of Cybercartography-Applications and Indigenous Mapping, second ed. Elsevier, Amsterdam, pp. 43-57.

First People's Cultural Council (FPCC), 2017. Update on Indigenous Languages Legislation Timeline. First Peoples' Cultural Council. retrieved from: http://www.fpcc.ca/files/PDF/Language/FPCC_Update_on_Indige nous_Languages_Legislation_Timeline_2017.pdf.

Gartner, G., 2014. Advances in location-based services. In: Developments in the Theory and Practice of Cybercartography—Applications and Indigenous Mapping, second ed. Elsevier, Amsterdam, pp. 97-106.

Girnth, H., 2010. Mapping language data. In: Language and Space. De Gruyter Mouton, Berlin, pp. 98-145.

Greenwood, C.R., Delquadri, J.C., Hall, R.V., 1989. Longitudinal effects of classwide peer tutoring. J. Educ. Psychol. 81 (3), 371-383.

Grenoble, L.A., Whaley, L.J., 2006. Saving Languages: An Introduction to Language Revitalization. Cambridge University Press.

Hebert, Y., 1984. The sociopolitical context of native Indian language education in British Columbia. Can. J. Nativ. Stud. 4 (1), 121-137.

Henke, R., Berez-Kroeker, A.L., 2016. A brief history of archiving in language documentation, with an annotated bibliography. Lang. Doc. Conserv. 10, 411-457. retrieved from, http://hdl.handle.net/10125/24714.

Herlihy, P.H., Knapp, G., 2003. Maps of, by, and for the peoples of Latin America. Hum. Organ. 62 (4), 303-314.

Hermes, M., Bang, M., Marin, A., 2012. Designing Indigenous language revitalization. Harv. Educ. Rev. 82 (3), 381-402. retrieved from, https://doi.org/10.17763/haer.82.3.q8117w861241871j.

Himmelmann, N., 1998. Documentary and descriptive linguistics. Linguistics 36 (1), 161-196. retrieved from, https://doi.org/10.1515/ling.1998.36.1.161.

Himmelmann, N.P., 2012. Linguistic data types and the interface between language documentation and description. Lang. Doc. Conserv. 6, 187-207. retrieved from, http://hdl.handle.net/10125/4503.

Hinton, L., 2001. The use of linguistic archives in language revitalization: the native California language restoration workshop. In: Hinton, L., Hale, K. (Eds.), The Green Book of Language Revitalization in Practice. Academic Press, San Diego, CA, pp. 419-428.

Hunter, J., 2018. British Columbia commits \$50-million to protect Indigenous languages. In: The Globe and Mail, The Globe and Mail: Phillip Crawley. retrieved from, https://www.theglobeandmail.com/canada/british-co lumbia/article-british-columbia-commits-50-million-to-protect-indigenous-languages/ on April 19, 2018.

Junker, M.-O., MacKenzie, M., Rosen, N., Valentine, J.R., Wolvengrey, A., 2005-2017, 2019. dir. co-dir. The Algonquian Linguistic Atlas. Available at: http://www.atlas-ling.ca/\#. Last accessed: April 16, 2018.

Kaplan, A.M., Haenlein, M., 2010. Users of the world, unite! The challenges and opportunities of social media. Bus. Horiz. 53 (1), 59-68. https://doi.org/10.1016/j.bushor.2009.09.003.

Krauss, M., 1992. The world's languages in crisis. Language 68 (1), 4-10.

Kruijt, A., Turin, M., 2017. Review article. Lang. Soc. 46 (2), 257-269, retrieved from, https://doi.org.10.1017/ 50047404517000161.

Kunst, J.P., 2010. Animated maps. In: Language and Space. De Gruyter Mouton, Berlin, pp. 415-437.

Linn, M.S., 2014. Living archives: a community-based language archive model. In: Nathan, D., Austin, P.K. (Eds.), Language Documentation and Description, vol. 12, pp. 53-67. Special Issue on Language Documentation and Archiving, retrieved from, http://www.elpublishing.org/PID/137. 
Ljubicic, G.J., Pulsifer, P.L., Hayes, A., Taylor, D.R.F., 2014. The creation of the Inuit siku (sea ice) atlas. In: Developments in the Theory and Practice of Cybercartography-Applications and Indigenous Mapping, second ed. Elsevier, Amsterdam, pp. 201-218.

McBride, B., 2015. The Future of Aboriginal Languages. Canada's history.

Morin, B., 2017. Feds rushing to help save endangered Indigenous languages. CBC News. retrieved from, http:// www.cbc.ca/news/indigenous/canada-indigenous-languages-legislation-1.4285633. on April 19, 2018.

Murasugi, K., Johns, A., Taylor, D.R.F., 2016. dir Inuktut Lexicon Atlas, The Geomatics and Cartographic Research Centre, Carleton University. Available at: https://inuktutlexicon.gcrc.carleton.ca/index.html Last accessed: April 25, 2018.

Newman, P., 2007. Copyright essentials for linguists. Lang. Doc. Conserv. 1 (1), 28-43.

Norris, M.J., 2004. From generation to generation: survival and maintenance of Canada's aboriginal languages, within families, communities and cities. TESL Can. J. 21 (2), 1-16.

Olariu, F., Olariu, V., 2014. The Romanian linguistic cartography in the digitizing era: the electronic atlases. Dialectol. Geolinguis. 22 (1), 75-90. retrieved from, https://doi.org/10.1515/dialect-2014-0005.

Olthuis, M.L., Kivelä, S., Skutnabb-Kangas, T., 2013. Revitalising Indigenous Languages: How to Recreate a Lost Generation. Multilingual Matters.

Ormeling, F., 2010. Visualizing geographic space: the nature of maps. In: Language and Space. De Gruyter Mouton, Berlin, pp. 21-39.

Penfield, S.P., Tucker, B.V., 2011. From documenting to revitalizing an endangered language: where do applied linguists fit? Lang. Educ. 25 (4), 291-305. retrieved from, https://doi.org/10.1080/09500782.2011.577219.

Prensky, M., 2001. Digital natives, digital immigrants part 1. On Horiz. 9 (5), 1-6.

Shepard, M.A., 2016. The value-added language archive: increasing cultural compatibility for native American communities. Lang. Doc. Conserv. 10, 458-479. retrieved from, http://hdl.handle.net/10125/24715.

Stone, A., 2018. Mapping experience: age and indigeneity as mediating factors of users' experiences with the Algonquian linguistic atlas. Cartographica 53 (4), 229-240.

Taylor, D.R.F., 2003. The concept of cybercartography. In: Maps and the Internet. International Cartographic Association, pp. 405-420.

Taylor, D.R.F., 2005. The theory and practice of cybercartography: an introduction. In: Cybercartography: Theory and Practice, first ed. Elsevier, Amsterdam, pp. 1-14.

Taylor, D.R.F., 2014. Some recent developments in the theory and practice of cybercartography: applications in Indigenous mapping: an introduction. In: Developments in the Theory and Practice of CybercartographyApplications and Indigenous Mapping, second ed. Elsevier, Amsterdam, pp. 2-16.

Taylor, D.R.F., Cowan, C., Ljubicic, G.J., Sullivan, C., 2014. Cybercartography for education: the application of cybercartography to teaching and learning in Nunavut, Canada. In: Developments in the Theory and Practice of Cybercartography_Applications and Indigenous Mapping, second ed. Elsevier, Amsterdam. 927-324.

Truth and Reconciliation Commission of Canada, 2015. Calls to Action, Truth and Reconciliation Commission of Canada. retrieved from, http://www.trc.ca/websites/trcinstitution/file/2015/findings/calls_to_action_english2. pdf. on April 18, 2018.

Upton, C., 2010. Designing maps for non-linguists. In: Language and Space. De Gruyter Mouton, Berlin, pp. 142-166.

Westman, C., Schreyer, C., 2014. Înîhiyawîtwâw 'they are speaking Cree': Cree language use and issues in northern Alberta, Canada. Int. J. Sociol. Lang. (230), 115-140. retrieved from, https://doi.org/10.1515/ijsl-20140023. on April 5, 2018.

Woodward, D., Lewis, G.M. (Eds.), 1998. Cartography in the Traditional African, American, Arctic, Australian, and Pacific Societies. Humana Press.

Yamada, R.M., 2007. Collaborative linguistic fieldwork: practical application of the empowerment model. Lang. Doc. Conserv. 1 (2), 257-282. retrieved from, http://hdl.handle.net/10125/1717. 


\section{Further reading}

Giedd, J.N., 2012. The digital revolution and adolescent brain evolution. J. Adolesc. Health 51 (2), 101-105. Grenoble, L.A., 2009. Linguistic cages and the limits of linguists. In: Reyhner, J., Lockard, L. (Eds.), Indigenous Language Revitalization: Encouragement, Guidance \& Lessons Learned. Northern Arizona University, Flagstaff, AZ, pp. 61-69. retrieved from, http://jan.ucc.nau.edu/ jar/ILR/ILR-5.pdf.

Skutnabb-Kangas, T., 2009. The stakes: linguistic diversity, linguistic human rights and mother-tongue-based multilingual education-or linguistic genocide, crimes against humanity and an even faster destruction of biodiversity and our planet. In: Keynote Presentation at the Bamako International Forum on Multilingualism. DeGruyter, Bamako, Mali, pp. pp. 19-21. 
15.0 Part 2 conclusion: Bringing the research together.

While the research in Part 2-both published and unpublished-identifies the current state of language mapping, it also introduces a new set of tools that can be used to understand practices and technologies in a clearer and more systematic way. As first posed in the introduction, the underlying question for Part 2 asked how we can change the way we look at language mapping so that we can further our knowledge of the practice of language mapping. To do so, the four chapters propose tools that foster critical reflection in language mapping in the three domains of knowledge identified by Griffin and Fabrikant (2012, p.298) in cartography: representation, cognition, and behaviour.

First, I introduced the Evaluative Map Typology as a means to deconstruct language maps into their basic components, and showed how coding methods can be used to compare these deconstructed components to one another. In many ways, a language mapping typology performs a similar function to a competitive analysis, albeit in a more focused way. Second, the subsequent chapter hosted a competitive analysis featuring the EMT as a fundamental component, along with an impressionistic overview of a diverse array of language maps and an assessment of maps' relative strengths and weaknesses. In addition, a series of interviews with atlas producers helped contextualize the use of specific atlas features and the use of back-end databases. Together, these two studies propose a means to study the mapping technologies and cartographic practices in language mapping, which emphasize the linguistic aspects of maps and atlases.

To add a social context to reflections of what is currently available and used in digital language mapping, a user study, which I performed in 2018 studied Indigenous and nonIndigenous users of varying ages' experiences with learning how to navigate the Algonquian 
Linguistic Atlas. At the same time, this user study introduced a mixed-methods approach to studying user experience designed to be compatible with language maps. As a modification of existing methods for analyzing user experience, the USE(L) questionnaire and semi-structured post-questionnaire interviews provide a way of understanding what users value in a language map and what they wish could be improved. This study proposed reflective practices in the second domain of knowledge identified by Griffin and Fabrikant (2012), namely the users' and audiences' side of the mapping process.

Finally, a section on Cybercartography in the Nunaliit framework in Indigenous Language Education introduced an area of work that future investigation should seek to explore and understand, in which the tools introduced in this dissertation may be of assistance. In addition, this section indicated how language maps, harnessed in sustainable and culturally appropriate ways, can positively impact the groups and communities who use them. As a more forwardthinking work, this chapter focused on emerging mapmaking practices, and how tools for language mapping can be put to use.

I believe the tools here mark departure points from which more thought and analysis in language mapping is possible. As these tools are meant to be processes which change and develop with use each time, they should be used in such a way that their subject matter and their own implementation are reflected upon in a meaningful way. More tools could — and should — be created to further language mapping in theory and application; however, every tool must have an origin. As folk wisdom states, 'Begin as you wish to go on', so too should the tools which are subsequently developed be used to reflect the considerations enumerated herein. 


\subsection{Evaluating the effectiveness of this research.}

In closing this research, a final question must be addressed, which echoes one asked in the critical analysis of the user study: Has this research successfully addressed the lack of critical reflection in language mapping identified by Lameli (2010, p.567) and Luebbering (2013, p.54)? Perhaps another way of posing this question would be to ask: Does the Part 2 research in this dissertation support a capacity for reflection in language mapping?

I would contend the first three investigations help contribute to increasing self-reflection in language mapping. First, the EMT presents itself as a way of analyzing the basic components of maps and mapping techniques, and was demonstrated to be useful in developing a set of recommendations for atlas development that saves time and facilitates planning. Second, the competitive analysis using the EMT integrates existing and proposed techniques for understanding the landscape of more general digital maps into the realm of language mapping. In doing so, practices already accepted in cartography are aligned with maps and practices that involve linguistic information, which can help bridge the conceptual gap between language mapping and cartography identified by Briscoe (2009) and Luebbering (2011).

Third, the user study introduces a new method for understanding how audiences of contemporary language maps experience what they see and how they feel about the maps they encounter. As an exploratory investigation in this area, this study performed as expected in the sense that the prescribed qualitative and quantitative techniques for evaluating user experience captured what they were intended to. In addition, unanticipated observations enriched the investigation, such as younger generations' use of multiple devices during the atlas tasks. Furthermore, unbalanced participant groups suggest that future user studies of this kind would benefit from determining the composition of the actual atlas users prior to focusing on their 
experiences. In addition, future studies of this kind would more appropriately assess user experience by being shaped by user communities. As self-reflection is an emphasized element of the user study, these unanticipated results and subsequent recommendations are an integral part of the research process. 
Conclusion

Future directions in language mapping and concluding remarks. 


\subsection{Main conclusion.}

Language mapping is a loosely clustered practice of reflecting upon relationships between people, language, and space that, in its short history, has experienced an astounding amount of changes and new applications. Born out of the intricate relationships we humans hold between the languages we speak and the spaces we exist in, language maps provide ways of understanding our world that are more relatable and familiar than text. Through the use of (mostly) commonly-accepted symbols such as points, lines, and polygons, as well as legends, titles, projections, and colours, complex linguistic phenomena can be communicated in ways that allow us to grasp what is going on, at least partially. Though not a discipline in of itself, language mapping is informed by many fields that study language, space, and the interactions between language and space. As a result, language mapping as an object of study is often brushed over in favour of more cohesive and tangible disciplines.

This dissertation seeks to understand what is already known about the practice of language mapping. It also explores what can be done to expand this understanding so we are better equipped to know how languages are mapped, who does the mapping, how maps can be used, and the consequences of such practices. To do so, this dissertation asked two main questions, which formed the basis of Parts 1 and 2: What do we already know about language mapping, and what do we not? and How can we advance the way we look at language mapping so that we can further this knowledge?

To look at what language mapping is, what it is made of, how it came to be, and how it currently exists, Part 1 brought theories and research from linguistics, applied linguistics, cartography, GIS, and geolinguistics together in a multidisciplinary analysis. However, this analysis also brought forth what we currently do not know about language mapping, including a 
marked inability to be able to reflect on the practices of language mapping and their consequences. As such, Part 2 introduced and demonstrated three tools that can be used to reinforce reflectivity in language mapping, as well as a published work that details the positive impacts that language maps can have on communities that make use of them. The hybrid thesis style adopted in this dissertation thus proved useful, as it enabled both reviews of existing knowledge and the development of applied tools to occur. In this way, the research presented here is applied, in addition to being based in theory, and will ideally function as a starting point for subsequent research outside this dissertation. Additionally, the way in which this research was implemented demonstrates the use of methodologies that account for both the technical and human sides of language mapping.

These studies compiled in the dissertation made use of mixed-methods techniques in a number of ways. While the Algonquian Linguistic Atlas user study gathered qualitative and quantitative data in such a way that they strengthened and contextualized each other, the Evaluative Map Typology systematically gathered qualitative data and analyzed them qualitatively and quantitatively. I believe the use of mixed-methods in such a transdisciplinary practice as language mapping to be beneficial. Doing so accounts for the multiple perspectives and methods which go into language mapping, as well as the many people and backgrounds that contribute to its practice. As such, based on this research, I recommend that subsequent research in this field incorporate mixed-methods approaches into their design.

The work herein is meant to be observational and descriptive in nature, and not prescriptively recommend what ought to occur within the study of language mapping. Not that this research is not important — as it certainly is — but it comes later, once we have a better idea of what we are working with. However, this does not mean that the research should not be looking 
forward at the next logical steps in the development of best practices. A subsection dedicated to exploring future directions in subsequent research, while not explicitly making formal recommendations, can be included in hybrid dissertations of this type, such as those by Lotulelei (2012) and Enriquez (2012).With this knowledge, too, we can get a better idea of where language mapping is going, if not because we can see into the future, then because we have a clearer view of how cutting-edge changes to methods and technologies are unfolding. 
17.0 Proposed future directions in language mapping.

This section explores future avenues of research in the study of language mapping practices, which work toward solidifying language mapping as a cohesive and conventionalized practice. Though not exhaustive, this section discusses three strategies that are directed toward promoting cohesion: the establishment of formal conventions, best practices, and a language mapping ontology. While a set (or sets) of conventions would assist in providing formalized avenues through which interested parties could develop language maps, a set of best practices would enable those involved in language mapping to share and evaluate what they value in the mapping process. Finally, an ontology would help address the inconsistencies in terminology identified in Part 1 of this dissertation, as well as assist in delineating the definitional boundaries of what language mapping as a practice encompasses.

Before proceeding along this line of thinking, it is important to acknowledge that in addition to the numerous advantages associated with conventionalizing and formalizing language mapping, there are some potentially detrimental effects that would need to be mitigated, or at the very least accounted for. While established conventions, best practices, and other similar kinds of infrastructure can improve communication within communities of practice, the way they are made can also make communication with other related practices more difficult and prevent innovation through shared resources (Borgman, 2015, p.46). As language mapping practices are built upon traditions and perspectives from many different spheres within academia and communities of users, sustained and improved interdisciplinary communication is as crucial as improved internal cohesion. Unless features ranging from guiding principles to data formatting standards are developed in ways that account for existing standards and conventions elsewhere, any formalized system of language mapping will become more distant from practices in 
cartography, community-level initiatives, or linguistics (to name a few). In becoming an insular practice in the face of such interdisciplinarity, the impacts of this intended conventionalization risk being ineffective, if not destructive. In order to promote the communication flow and exchange of information between language mappers and practitioners in other associated areas of study and practice, Borgman (2015, p.46) recommends any conventions, best practices, ontologies, or similar methods remain open to discussion and alteration. Open source tools for organizing and implementing these methods would enable broader communities to engage with these issues, while accessible forums and platforms for education and resources would make engagement more possible for those from different backgrounds and practices.

\subsection{Conventions.}

As mapping technologies transition from textbooks and classroom walls to computers, GPS, and the Internet, linguistic data can increasingly be collected, manipulated, stored, and analyzed (Luebbering, 2013, p.50). New spheres have evolved where such information can be applied and more people can access and contribute to maps themselves. While a set of common conventions would help define the boundaries of what language mapping includes (Borgman, 2015, p.37) and allow for a degree of consistency within a genre that involves actors from many different backgrounds (Luebbering, Kolivras, \& Prisley, 2013, p.383), no such conventions currently exist (Luebbering, 2013, p.45). However, a criticism I have of such observations is that those who recognize this lack of conventions do not state what is exactly meant by 'convention' in the first place. It would seem, therefore, that before creating conventions, we should agree upon the definition of the term and the component parts it incorporates. 
Miller (1992) describes conventions as "regularities in action" (p.435) in recurring situations where an individual follows a procedure they believe others also follow, which is used to achieve a common goal (p.438). In language mapping, a recurring situation could be imagined as the act of spatially representing linguistic information, while the individuals would be the linguists, cartographers, mapmakers, and other stakeholders involved in the mapmaking process. Additionally, the procedure would be the specific techniques and tools used to create the language map (be it symbology, projection, or digital media), while the common goal would be a map that reflects the spatial reality of some linguistic phenomenon in a reliable and wellunderstood way.

While Miller (1992) considers conventions to be ubiquitous in economic and political life (p.435) and a necessary feature of social life (p.439), they are not deemed to belong to institutions or governing bodies per se, but more generally apply to any form of community of practice. Conventions may be written and instated in a form of law; they may also be unwritten mutual beliefs concerning procedures and collective ends (Miller, 1992, p.439). As such, the use of the term convention in isolation is a safe means to describe a commonly accepted procedure in order to achieve a common goal, without specifying who the creators or supporters of such procedures are. However, there is a specific avenue I believe to be appropriate to adding consistency to the area of language mapping, namely a set of best practices.

\subsection{Best practices.}

In a most basic definition, best practices are defined as "systematically developed statements" (Lohr \& Field 1990, p.8) that assist individuals in making decisions regarding which of several possible approaches is best (Bird \& Simons, 2003, p.570). Unlike more generalizable 
conventions, Lohr and Field (1990, p.8) refer to best practices as products defined by some authority, while Bird and Simons (2003, p.570) assert that best practices are necessarily based on value statements. In this, judgments on which practices are best can only be made once the values motivating the choices are clear. In turn, such values can only be established once the actors involved agree on what they are in the first place. In language mapping, the establishment of best practices would thus require a consensus between those contributing the information, map authors, cartographers, and audience members on what the most important values in the mapping process ought to be. Indeed, the Treasury Board of Canada Secretariat (1996, in Meredith, 1997, p.4) notes that sharing best practices information in an institution by recording and disseminating work methods, processes, and initiatives improves effectiveness and the audiences' satisfaction.

Bird and Simons (2003, p.570) offer such a groundwork for best practices in linguistic archiving, whereby they list a detailed array of values including accountable linguistic research, a use of consistent terminology (p.571), and easy access to stored materials (p.572). Furthermore, in non-linguistic contemporary GIS cartography, Peterson (2009) lists specific best practice recommendations for aspects of a map such as the title (p.19), legend (p.22), and authorship information (p.29). Interestingly however, authors focusing specifically on language mapping practices such as Ambrose and Williams (1991), Ormeling (1992), Luebbering (2011), Luebbering, Kolivras and Prisley (2013), and Gawne and Ring (2016) only discuss conventions and standards, and shy away from the notion of best practices. Speculatively, this could be the result of language mapping being too broadly defined as an transdisciplinary area of research which also derives inspiration from fields such as anthropology and sociology (Luebbering, 2013, p.42). 
Without a unified (or perhaps even core) disciplinary identity, a consensus on values is difficult, posing a distinct challenge to the establishment of a set of best practices in language mapping. As a slowly-growing field (Luebbering, 2013, p.42) that maintains a distance from geography (Britain, 2010, p.73) and has historically done so (Lee and Kretzschmar, 1993, p.541; Trudgill, 1974, p.215), language mapping has perhaps missed much of the systemic development associated with more general cartographic and geographic practice. For example, systems of best practices already exist for land administration systems (Williamson, 2000, p.1), natural resource management (Meredith, 1997, p.2), and language documentation (Penfield et al., 2008, p.192; Austin, 2016, p.152). In addition, the dynamic and intangible nature of language makes cartographic representation difficult in general (Luebbering, Kolivras, \& Prisley, p.383). The number and variety of approaches to dealing with issues such as uncertainty (Luebbering, 2011, p.37), multiple languages (Luebbering, 2011, p.14), and linguistic boundaries (Luebbering, 2013, p.47) challenge a unifying set of values. ${ }^{50}$

As is the case with standards (Brunsson, Rasche, \& Seidl, 2012, p.617), best practice recommendations require achieving a consensus among relevant actors to be effective (Bird \& Simons, 2003, p.570). Though Bird and Simons (2003, p.558) place a marked emphasis on value statements, they also include other comprehensive elements in their analysis with the objective of building such consensus. While their account is not a complete and exhaustive set of best practices, it does serve as an example of what considerations need to be accounted for when working with language, and acts as foundation from which best practices could be implemented.

\footnotetext{
${ }^{50}$ It should be noted that I believe that conventions or best practices should be available, but not enforced, to any language mapping project. Krygier and Wood (2016, p.195) mention that there are times when conventionalized approaches to mapping are not appropriate, and would hamper the expression, creativity, and ideas of particular projects.
} 
Before mentioning value statements, Bird and Simons (2003, p.558) review the tools and technologies used in language documentation and description. Such an introduction is important for giving their readers a common understanding of what tools (and corresponding issues) exist in the field. In language mapping, such an account would include tools used to gather spatial and linguistic information as well as GIS software, printing equipment, or related map-making paraphernalia.

Bird and Simons (2003, p.562) then detail several 'problem areas' related to such technologies and other pertinent language documentation issues. Such reflection is important in creating motivating factors for their value statements and establishing why such a set of best practices is important in the first place. In addition, Bird and Simons (2003) also use this section to introduce relevant jargon in their value statements, such as ease of access and format. Each definition is followed with a brief account of the consequences of associated issues in the area. This discussion of problem areas is then followed by the value statements themselves.

In their penultimate section, Bird and Simons (2003, p.572) introduce the Open Languages Archives Community (OLAC) as an important means to share best practice recommendations and contribute to the existing knowledge in language documentation. In their final section, Bird and Simons (2003, p.574) outline a preliminary set of best practice recommendations, which they state would require added input from others associated in some way with language documentation and does not reflect the official position of any authoritative body. It becomes evident in this final disclaimer that any set of best practices in language mapping, once created, would require the added input and consensus from others with a relevant relation to language maps, and the ultimate approval from authoritative bodies or large numbers of practitioners to be formally accepted. 


\subsection{A language mapping ontology.}

To address a lack of consensus surrounding terminology, an ontology of how terms relate to one another would be a useful tool in building terminological consensus in language mapping. This proposed language mapping ontology has a variety of purposes in line with the more general ontology features listed by Uschold and Grüninger (1996, p.7), including providing the means to structure a knowledge base from which a cohesive discipline can rise and integrating different but overlapping fields into a coherent framework. Additionally, Noy and McGuinness (2001, p.1) note that ontologies are useful for creating a common understanding of the structure of relevant information (i.e. domain knowledge), enabling the reuse and analysis of domain knowledge, making assumptions within this domain knowledge explicit, and separating such domain knowledge from operational knowledge. As opposed to some abstract notion of how concepts and ideas within an area of study should be organized, ontologies concretely and formally arrange concepts using specialized computer programs such as Protégé, Ontolingua, or Chimaera (Noy \& McGuinness, 2001, p.3).

Interestingly, this proposed research is not the first attempt at regulating specific elements of language mapping. Di Buccio, Di Nunzio, and Silvello (2013, p.1101) developed a system that makes dialectal data compatible with a variety of geolinguistic projects through data-driven models, but their research currently only covers Italian databases. Similarly, Farrar and Langendoen (2003, p.97) created the General Ontology for Linguistic Description (GOLD) to help establish an online linguistic community of practice, Frontini, Del Gratta, and Monachini (2016, p.230) made the already-existing GeoNames (placename) ontology more accessible by redesigning it with a WordNet configuration, and Scholz, Hrastnig, and Wandl-Vogt (2017, p.4) 
attempted to make geographical linguistic data more easily shared across databases by attaching formalized semantic descriptions in an ontology to linguistic information.

A similar project to the proposed ontology is an online index ${ }^{51}$ by Kretzschmar (2013) that provides detailed definitions, basic procedures, and examples of digital and paper mapmaking techniques. While this is a comprehensive and important account of many mapmaking practices, it is still narrow in scope as it only focuses on dialect and word geography and a handful of digital mapmaking technologies. Similarly, no attempt is found to situate linguistic geography within the greater terminological landscape of names for spatially representing linguistic information. Its main intent appears to be for general reference and to provide a detailed introduction for those who are new to language mapping. It is expected that Kretzschmar's (2013) accounts will remain important throughout the development process. While the importance of such a guide should not be underestimated, the limitations in scope and application mean that there is still ample room for the ontology proposed in this research to expand on emerging perspectives, technologies, and applications.

${ }^{51}$ As of July 27, 2020, this index can be accessed at https://www.uni-bamberg.de/fileadmin/engling/fs/Chapter_3/Index.html?25PresentationofLanguageDataonMa.html 


\subsection{Concluding remarks.}

As language mapping continues to evolve in the face of new technological development and spheres of use, maps' potential as well as the needs of those involved in their existence will also change. To understand these changes and the effects they have, we need to be able to look at what maps do, how they do it, and how their technologies and domains of use change alongside the resources available to us. The process of reflecting upon language mapping will never reach a conclusion, but will continue to develop, ideally in parallel with language mapping practices.

In returning to the original analogy of C.S. Lewis' Wood Between the Worlds, we have used our magic ring — this dissertation — to explore many of the pools that others have already seen but have not necessarily related to each other. In addition, we have taken this magic ring to peer into pools that others might have skimmed, but not explored in detail. While this research has hopefully made this small swathe of forest a little less murky, there are many more acres to explore and many more pools to discover. It is my hope that this research, in addition to functioning as a proverbial magic ring, can also be used as a map (to use a double analogy) through which new areas and dimensions of language mapping can be explored. 
References 


\section{References}

Agarwal, A., Xie, B., Vovsha, I., Rambow, O., \& Passonneau, R. (2011, June). Sentiment analysis of twitter data. In Proceedings of the workshop on languages in social media (pp. 30-38). Association for Computational Linguistics.

Agassiz, L. (1859). An Essay of Classification. Longmann.

Agumya, A., \& Hunter, G. J. (2002). Responding to the consequences of uncertainty in geographical data. International Journal of Geographical Information Science, 16(5), $405-417$.

Alexander, T. (2013). Object relationship diagram [map]. Retrieved from: https://files.assetweb.co.uk/scips/documentation/object_diagram.html

Ali, A. H. (2017). Framework development of cybercartography for mobile environment. American Journal of Geographic Information System, 6(1), 14-25.

Ambrose, J. E., \& Williams, C. H. (1991). Language made visible: Representation in Geolinguistics. Linguistic Minorities, Society, and Territory, (78), 298-314.

Ammar, E., Behairy, A., \& Habibi, A. (2016). GIS cartography for topographic maps series. In Proceedings of the $6^{\text {th }}$ international conference on cartography and GIS, June 13-17 (pp. 704-711).

Andrienko, G., Andrienko, N., Demsar, U., Dransch, D., Dykes, J., Fabrikant, S. I., ... \& Tominski, C. (2010). Space, time and visual analytics. International Journal of Geographical Information Science, 24(10), 1577-1600.

Anonby, E., Murasugi, K., \& Domínguez, M. (2018). Mapping language and land with the Nunaliit Atlas Framework: Past, present and future. In S. Drude, N. Ostler \& M. Moser 
(Eds.), Endangered languages and the land: Mapping landscapes of multilingualism, Proceedings of FEL XXII/2018 (Reykjavik, Iceland), 56-63. FEL \& EL Publishing. Anonby, E., \& Sabethemmatabadi, P. (2019). Representing complementary user perspectives in a language atlas. In D. R. F. Taylor, E. Anonby, \& K. Murasugi (Eds.), Further developments in the theory and practice of cybercartography (pp. 413-440). Elsevier.

Anonby, E., \& Stone, A. (2016-2019). Typology for analyzing language maps. Retrieved from https://forms.gle/vwMYwPnwGQULYHNRA

Anonby, E., Taheri-Ardali, M., et al. (eds.). (2015-2020). Atlas of the Languages of Iran. Ottawa: Geomatics and Cartographic Research Centre. Retrieved from http://iranatlas.net Aporta, C., Kritsch, I., Andre, A., Benson, K., Snowshoe, S., Firth, W., \& Carry, D. (2014). The Gwich'in atlas: Place names, maps, and narratives. In D. R. F. Taylor (Ed.), Developments in the theory and practice of cybercartography: Applications and Indigenous mapping (2nd ed., pp. 229-244). Elsevier.

Applied Linguistics and Discourse Studies (ALDS), (n.d.). Manuscript thesis option. Retrieved from https://carleton.ca/slals/wp-content/uploads/PHD-manuscript-thesis-policies.pdf

Atkinson, Q. D., \& Gray, R. D. (2006). How old is the Indo-European language family? Illumination or more moths to the flame? In P. Forster \& C. Renfrew (Eds.), Phylogenetic methods and the prehistory of languages (pp. 91-109). McDonald Institute for Archaeological Research.

Atzori, L., Iera, A., \& Morabito, G. (2010). The Internet of Things: A survey. Computer Networks, 54, 2787-2805.

Auer, P. (2013). The geography of language: Steps toward a new approach. Freiburger Arbeitspapiere zur Germanistischen Linguistik (FRAGL), 16, 1-39. 
Austin, J. L. (1975). How to do things with words. Harvard University Press.

Austin, P. K. (2016). Language documentation 20 years on. In L. Filipović \& M. Pütz (Eds.), Endangered Languages and Languages in Danger. Issues of Language Documentation, Policy, and Language Rights (pp. 147-170). John Benjamins Publishing Company.

Balchin, W. G., \& Coleman, A. M. (1966). Graphicacy should be the fourth ace in the pack. Cartographica: The International Journal for Geographic Information and Geovisualization, 3(1), 23-28.Bennett, S., Maton, K., \& Kervin, L. (2008). The 'digital natives' debate: A critical review of the evidence. British Journal of Educational Technology, 39(5), 775-786.

Bergsland, K., \& Vogt, H. (1962). On the validity of glottochronology. Current Anthropology, $3(2), 115-153$.

Bernhardsen, T. (2002). Geographic information systems: An introduction. John Wiley \& Sons. Bierswisch, M. (1999). How much space gets into language? In P. E. Bloom, M. A. Peterson, L. E. Nadel, \& M. F. Garrett (Eds.), Language and space (pp. 31-76). MIT Press.

Bird, S., \& Simons, G. (2003). Seven dimensions of portability for language documentation and description. Language, 79(3), 557-582.

Blades, M., \& Spencer, C. (1987). The use of maps by 4-6-year-old children in a large-scale maze. British Journal of Developmental Psychology, 5(1), 19-24.

Bloom, P. (2007, May). The scientific notion of language and structure [transcribed lecture]. Retrieved from https://brewminate.com/how-we-communicate-language-in-the-brainmouth-and-the-hands/

Blumson, B. (2010). Maps and meaning. Journal of Philosophical Research, 35, 123-127. 
Blust, R. (2000). Why lexicostatistics doesn't work: The 'universal constant' hypothesis and the Austronesian languages. Time Depth in Historical Linguistics, 2, 311-331.

Board, C. (2017). 2: The communication models in cartography. In A. J. Kent \& P. Vujakovic (Eds.), The Routledge handbook of mapping and cartography (pp. 29-43). Routledge.

Boberg, C. (2000). Geolinguistic diffusion and the US-Canada border. Language Variation and Change, 12(1), 1-24.

Boberg, C., Nerbonne, J., \& Watt, D. (2018). Introduction. In C. Boberg, J. Nerbonne, \& D. Watt (Eds.), The Handbook of dialectology (pp. 1-15). Wiley.

Boberg, C., Nerbonne, J., \& Watt, D. (2018). The handbook of dialectology. Wiley.

Bollmann, J. (2010). 3. Maps and cognition. In A. Lameli, R. Kehrein, \& S. Rabanus (Eds.), Language and space, Volume 2: Language mapping (pp. 40-69). De Gruyter Mouton.

Bollmann, J., \& Koch, W. G. (2001). Lexikon der Kartographie und Geomatik in zwei Bänden [Lexicon of cartography and geomatics in two volumes], Vol. 1. Spektrum.

Borchert, J. R. (1987). Maps, geography, and geographers. The Professional Geographer, 39(4), $387-389$.

Borgman, C. L. (2015). Big data, little data, no data: Scholarship in the networked world. MIT press.

Botsman, D. V. (2016). The return of the outcast(e) map: Kobe, cartography and the problem of discrimination in Modern Japan. Asia Pacific Journal: Japan Focus, 14(18), 1-8.

Bourhis, R. Y., \& Landry, R. (2012). Group vitality, cultural autonomy and the wellness of language minorities. In R. Y. Bourhis (Ed.), Decline and prospects of the Englishspeaking communities of Quebec (pp. 23-69). Centre d'études ethniques des universités montréalaises (CEETUM). 
Brasseur, V. V. (2018). Forge your future with open source: Build your skills. Build your network. Build the future of technology. Pragmatic Bookshelf.

Breton, R. J. L. (1991). Geolinguistics: Language dynamics and ethno-linguistic geography. translated and expanded by Harold Schiffman. Presses de l'Université d'Ottawa.

Brewer, C. A. (1994). Color use guidelines for mapping. In A. M. MacEachren \& D. R. F. Taylor (Eds.), Visualization in modern cartography (Vol. 2, pp. 123-148). Pergamon.

Briscoe, U. (2009). Geolinguistic GIS applications: Aspect of data quality in mapping lesserused languages. Master's Thesis, Centre for Geoinformatics.

Britain, D. (2002). Space and spatial diffusion. In J. K. Chambers, P. Trudgill, \& N. SchillingEstes (Eds.), The handbook of language variation and change (pp. 603-637). Blackwell.

Britain, D. (2010). 4. Conceptualizations of geographic space in linguistics. In A. Lameli, R. Kehrein, \& S. Rabanus (Eds.), Language and space, Volume 2: Language mapping (pp. 69-102). De Gruyter Mouton.

Bronzaft, A. L., Dobrow, S. B., \& O'Hanlon, T. J. (1976). Spatial orientation in a subway system. Environment and Behavior, 8(4), 575-594.

Brovelli, M. A., Mitasova, H., Neteler, M., \& Raghavan, V. (2012). Free and open source desktop and Web GIS solutions. Applied Geomatics, 4(2), 65-66.

Brown, G., Strickland-Munro, J., Kobryn, H., \& Moore, S. A. (2017). Mixed methods participatory GIS: An evaluation of the validity of qualitative and quantitative mapping methods. Applied Geography, 79, 153-166.

Brunn, S. D., \& Kehrein, R. (2020a). Handbook of the changing world language map. Springer. 
Brunn, S. D., \& Kehrein, R. (2020b). Introduction: State of knowledge and research challenges. In S. D. Brunn \& R. Kehrein (Eds.), Handbook of the changing world language map (pp. ix-lxxxi). Springer.

Brunsson, N., Rasche, A., \& Seidl, D. (2012). The dynamics of standardization: Three perspectives on standards in organization studies. Organization Studies, 33(5-6), 613632.

Bryant, D. J. (1997). Representing space in language and perception. Mind \& Language, 12(3-4), 239-264.

Bucholtz, M., \& Hall, K. (2005). Language and Identity. In A. Duranti (Ed.), A Companion to Linguistic Anthropology (pp. 369-394). Blackwell.

Bugayevskiy, L. M., \& Snyder, J. (2013). Map projections: A reference manual. CRC Press.

Cafolla, M. C. (2011). Map of Scots and Gaelic speakers [map]. (no scale). Retrieved from http://scotshaunbuik.co.uk/wp/?p=1219

Caquard, S. (2013). Cartography I: Mapping narrative cartography. Progress in Human Geography, 37(1), 135-144.

Carver, C. M. (1987). Pail versus bucket in Pennsylvania [map]. (no scale). In American regional dialects: A word geography. University of Michigan Press.

Cassidy, F. G., \& Hall, J. H. (Eds.). (1985). Dictionary of American regional English: Vol. 1. Belknap Press of Harvard University Press.

Cenerini, C., Junker, M. O., \& Rosen, N. (2017). Mapping dialectal variation using the Algonquian Linguistic Atlas. Language Documentation and Conservation, 11, 305-324.

Cenoz, J., \& Gorter, D. (2006). Linguistic landscape and minority languages. International Journal of Multilingualism, 3(1), 67-80. 
Chambers, J. K. \& Trudgill, P. (1998). 2. Dialect geography. In J. K. Chambers \& P. Trudgill (Eds.), Dialectology (2nd ed., pp. 13-31). Cambridge University Press.

Chambers, R. (2006). Participatory mapping and geographic information systems: whose map? Who is empowered and who disempowered? Who gains and who loses? The Electronic Journal of Information Systems in Developing Countries, 25(1), 1-11.

Chapin, M., Lamb, Z., \& Threlkeld, B. (2005). Mapping Indigenous lands. Annual Review of Anthropology, 34, 619-638.

Cheshire, J. (Ed.). (1991). English around the world: Sociolinguistic perspectives. Cambridge University Press.

Christophe, S., Duménieu, B., Turbet, J., Hoarau, C., Mellado, N., Ory, J., Loi, H., Masse, A., Arbelot., B., Vergne, R., ... \& Vanderhaeghe, D. (2016). Map style formalization: Rendering techniques extension for cartography. In Expressive 2016: The joint symposium on computational aesthetics and sketch-based interfaces and modeling and non-photorealistic animation and rendering (pp. 59-68).

Clarke, A., Johnson, A., \& Mathews, D. (2020). The gendered language of gravestones: A comparative study of central and northern Appalachian cemeteries. In S. D. Brunn \& R. Kehrein (Eds.), Handbook of the changing world language map (pp. 1839-1851). Springer.

Clarke, K. C. (1986). Advances in geographic information systems. Computers, Environment and Urban Systems, 10(3-4), 175-184.

Constant, N. (2011). Human language families [map]. (no scale). Linguistics 201 - Introduction to Linguistic Analysis. Retrieved from http://people.umass.edu/ nconstan/201/

Cook, G. (2003). Applied linguistics. Oxford University Press. 
Cotfas, L., \& Diosteanu, A. (2010). Evaluating accessibility in crowdsourcing GIS. Journal of Applied Collaborative Systems, 2(1), 45-49.

Crampton, J. W. (2011). Mapping: A critical introduction to cartography and GIS (Vol. 11). John Wiley \& Sons.

Crawford, P. V. (1973). The perception of graduated squares as cartographic symbols. The Cartographic Journal, 10(2), 85-88.

Cresswell, M. J. (1978). Prepositions and points of view. Linguistics and Philosophy, 2(1), 1-41.

Creswell, J. W., Shope, R., Plano Clarke, V. L., \& Green, D. O. (2006). How interpretive qualitative research extends mixed methods research. Research in the Schools, 13(1), 111.

Croft, W. (2002). Typology and universals. Cambridge University Press.

Croushore, D. (2011). Frontiers of real-time data analysis. Journal of Economic Literature, 49(1), 72-100.

Crutzen, P. J. (2002, November). The “Anthropocene”. In Journal de Physique IV (Proceedings) (Vol. 12, No. 10, pp. 1-5). EDP sciences.

Czaykowska-Higgins, E. (2009). Research models, community engagement, and linguistic fieldwork: Reflections on working within Canadian Indigenous communities. Language Documentation and Conservation, 3(1), 15-50.

Dagenais, D., Moore, D., Sabatier, C., Lamarre, P., \& Armand, F. (2008). Linguistic landscape and language awareness. In E. Shohamy \& D. Gorter (Eds.), Linguistic landscape: Expanding the scenery (pp. 293-309). Routledge.

Dangermond, J. (2004). Keynote address to ESRI users conference. Retrieved from http://www.esri.com 
Darkes, G. (2017). 21: An introduction to map design. In A. J. Kent \& P. Vujakovic (Eds.), The Routledge handbook of mapping and cartography (pp. 287-298). Routledge.

Davies, A. (2007). An introduction to applied linguistics: From practice to theory. Edinburgh University Press.

Davies, A., \& Elder, C. (2004). General introduction - Applied linguistics: Subject to discipline? In A. Davies \& C. Elder (Eds.), The handbook of applied linguistics (pp. 1-15). Blackwell Publishing.

Davies, M. (2011). Concept mapping, mind mapping and argument mapping: What are the differences and do they matter? Higher Education, 62(3), 279-301.

De Padua, A., \& Rabbitskin, N. (2018). Working with Indigenous leadership and Indigenous environments. In J. Wagner (Ed.), Leadership and Influencing Change in Nursing (pp. 52-72). University of Regina Press.

De Saussure, F. (2011). Course in general linguistics. Columbia University Press.

DeBoer, M. (2015). Understanding the heat map. Cartographic Perspectives, (80), 39-43.

Deckert, S. K., \& Vickers, C. H. (2011). An introduction to sociolinguistics: Society and identity. A\&C Black.

Deelman, E., Blythe, J., Gil, Y., Kesselman, C., Mehta, G., Vahi, K., ... \& Koranda, S. (2003). Mapping abstract complex workflows onto grid environments. Journal of Grid Computing, 1(1), 25-39.

Deetz, C. H., \& Adams, O. S. (1921). Elements of map projection with applications to map and chart construction (No. 68). US Government Printing Office. 
Del Casino Jr, V. J., \& Hanna, S. P. (2006). Beyond the 'binaries': A methodological intervention for interrogating maps as representational practices. ACME: An International E-Journal for Critical Geographies, 4(1), 34-56.

Delgado-Escueta, A. V., Wilson, W. A., Olsen, R. W., \& Porter, R. J. (1999). New waves of research in the epilepsies: crossing into the third millennium. Advances in Neurology, 79, 3-58.

Dell'Aquila, V. (2010). 23. GIS and sociolinguistics. In A. Lameli, R. Kehrein, \& S. Rabanus (Eds.), Language and space, Volume 2: Language mapping (pp. 458-482). De Gruyter Mouton.

Deutscher, G. (2010). Does your language shape how you think? The New York Times, 29.

Di Buccio, E. D., Di Nunzio, G. M. D., \& Silvello, G. (2014). A linked open data approach for geolinguistics applications. International Journal of Metadata, Semantics and Ontologies, 9(1), 29-41.

Di Gessa, S., Poole, P., \& Bending, T. (2008). Participatory mapping as a tool for empowerment: Experiences and lessons learned from the ILC network. Rome: ILC/IFAD, 45.

DiBiase, D., MacEachren, A. M., Krygier, J. B., \& Reeves, C. (1992). Animation and the role of map design in scientific visualization. Cartography and Geographic Information Systems, 19(4), 201-214.

Dieth, E. \& Orton, H. (1952). A questionnaire for a linguistic atlas of England. Chorley and Pickersgill.

Dixon, R. M. (1997). The rise and fall of languages. Cambridge University Press. 
Dowler, L., Carubia, J., \& Szczgiel, B. (2005). Gender and landscape: Renegotiating morality and space. In L. Dowler, J. Carubia, \& B. Szczgiel (Eds.), Gender and landscape: Renegotiating moral landscape (pp. 1-15). Routledge.

Duncan, A. L., \& Lach, D. H. (2006). Privileged knowledge and social change: Effects on different participants of using geographic information systems technology in natural resource management. Environmental Management, 38(2), 267-285.

Duranti, A. (2011). Linguistic anthropology: Language as a non-neutral medium. In R. Mesthrie (Ed.), The Cambridge handbook of sociolinguistics. Cambridge University Press.

Eddy, B. G., \& Taylor, D. R. F. (2005). 3. Exploring the concept of cybercartography using the holonic tenets of Integral Theory. In D. R. F. Taylor \& T. Lauriault (Eds.), Cybercartography: Theory and practice (1st ed., pp. 35-61). Elsevier.

Egland, S., Bartholomew, D., \& Ramos., S. C. (1978). La inteligibilidad interdialectal de las lenguas indígenas de México: Resultado de algunos sondeos [the interdialectal intelligibility of Mexico’s Indigenous languages: Results of some surveys]. Instituto Lingüístico de Verano.

Eisenstein, J., O’Connor, B., Smith, N. A., \& Xing, E. P. (2012). Mapping the geographical diffusion of new words. arXiv preprint:1210.5268, 1-13.

Engler, N. J., Scassa, T., \& Taylor, D. R. F. (2014). Cybercartography and volunteered geographic information. In D. R. F. Taylor (Ed.), Modern cartography series (Vol. 5, pp. 43-57). Academic Press.

Enriquez, J. E. (2012). Improving student engagement: An evaluation of the Latinos in Action program. Doctoral Dissertation, Brigham Young University. 
Ercegovac, Z. (1990). Proposed definitional conditions as a basis to study the concept of map author. Cataloging \& Classification Quarterly, 10(4), 19-50.

Faden, W. (1776). The attack and defeat of the American Fleet under Benedict Arnold by the King's Fleet [map]. (no scale). In the Boston public library digital map collection. Retrieved from https://collections.leventhalmap.org/search/commonwealth:q524n8916

Farrar, S., \& Langendoen, D. T. (2003). A linguistic ontology for the semantic web. GLOT International, 7(3), 97-100.

Farrelly, L. (2008). Basics architecture 01: Representational techniques (Vol. 1). AVA Publishing.

Filipponio, L., \& Nocchi, N. (2010). Figura 1: Sambuca Pistoiese rispetto alla linea La SpeziaRimini (o Carrara-Fano). [Figure 1: Sambuca Pistoiese compared to the La Spezia-Rimini (or Carrara-Fano) line.] [map]. (no scale). Diagnostica fonetica e diagnosi fonologica. Ossitoni lunghi di sillaba libera a Sambuca Pistoiese (PT) [Phonetic diagnostics and phonological diagnosis. Long free syllable oxytones in Sambuca Pistoiese (PT)]. Zurich Open Repository and Archive.

Fox, S. (2007). The demise of Cockneys? Language change among adolescents in the “traditional” East End of London. Unpublished Ph.D. dissertation, University of Essex.

Fridlund, B. (2010). The dissertation book; Should it be a monograph or compilation thesis? European Journal of Cardiovascular Nursing, 9, 144-145.

Fromkin, V., Rodman, R., \& Hyams, N. (2018). An introduction to language. Cengage Learning. Frontini, F., Del Gratta, R., \& Monachini, M. (2016, December). GeoDomain WordNet: Linking the GeoNames ontology to WordNet. In Language and Technology Conference (pp. 229242). Springer. 
Fukushima, C. (2016). Tracing real- and apparent-time language changes by comparing linguistic maps. The future of dialects: Selected papers from Methods in Dialectology XV, $1,363-375$.

Garuba, H. (2002). Mapping the land/ body/ subject: Colonial and postcolonial geographies in African narrative. Alternation, 9(1), 87-116.

Gauchat, L. (1903). Gibt es Mundartgrenzen? [Are there dialect boundaries?]. Archiv für das Studium der neueren Sprachen und Literaturen, 3, 365-403.

Gawne, L., \& Ring, H. (2016). Mapmaking for language documentation and description. Language Documentation \& Conservation, 10, 188-242.

Gee, J. P. (2010). An introduction to discourse analysis: Theory and method. Routledge.

Geisler, H., \& List, J. M. (2010). Beautiful trees on unstable ground: Notes on the data problem in lexicostatistics. Retrieved from https://core.ac.uk/download/pdf/47338237.pdf

Geoscience Australia. (2012, January 20). Section 1 - National topographic database structure and specifications. Retrieved from http://www.ga.gov.au/mapspecs/topographic/v6/section1.html

Georgiadou, Y., Kounadi, O., \& de By, R. (2020). Digital Earth Ethics. In H. Guo, M. F. Goodchild, \& A. Annoni (Eds.), Manual of Digital Earth (pp. 785-810). Springer.

Gersmehl, P. J. (1991). The language of maps. Pathway in geography series, (Title No. 1). Indiana University of Pennsylvania.

Gill, G. A. (1988). Experiments in the ordered perception of coloured cartographic line symbols. Cartographica: The International Journal for Geographic Information and Geovisualization, 25(4), 36-49.

Gilliéron, J. (1881). Petit atlas phonétique du Valais roman (sud du Rhone). H. Champion. 
Gilliéron, J., \& Edmont, E. (1902). Atlas linguistique de la France (Vol. 36). H. Champion.

Girnth, H. (2010). 5. Mapping language data. In A. Lameli, R. Kehrein, \& S. Rabanus (Eds.), Language and space, Volume 2: Language mapping (pp. 98-145). De Gruyter Mouton.

Glushkova, S., Belotserkovich, D., Morgunova, N., \& Yuzhakova, Y. (2019). The role of smartphones and the internet in developing countries. Revista Espacios, 40(27), 10-19.

Goebl, H. (1993). Dialectometry: a short overview of the principles and practice of quantitative classification of linguistic atlas data. In R. Köhler \& B. B. Rieger (Eds.), Contributions to quantitative linguistics (pp. 277-315). Springer.

Goebl, H. (2007, June). On the geolinguistic change in Northern France between 1300 and 1900: A dialectometrical inquiry. In proceedings of ninth meeting of the ACL special interest group in computational morphology and phonology (pp. 75-83).

Goeb1, H. (2018). 7. Dialectometry. In C. Boberg, J. Nerbonne, \& D. Watt (Eds.), The handbook of dialectology (pp. 123-142). Wiley.

Gomulkiewicz, R. W. (2009). Open source license proliferation: Helpful diversity or hopeless confusion? Washington University Journal of Law \& Policy, 30(1), 261-291.

Good, J. (2011). Valuing technology: Finding the linguist's place in a new technological universe. In L. A. Grenoble \& N. L. Furbee (Eds.), Language documentation: Practice and values (pp. 111-132). John Benjamins.

Goodchild, M. F. (1989). Chapter 10: Modelling error in objects and fields. In M. F. Goodchild, \& S. Gopal (Eds.), The accuracy of spatial databases (pp. 107-113). CRC Press.

Goodchild, M. F. (2009). Geographic information system. In L. Liu \& M. T. Özsu (Eds.), Encyclopedia of database systems (pp. 251-262). Springer. 
Goodchild, M. F. (2020). Mapping language in the age of GIS. In S. D. Brunn \& R. Kehrein (Eds.), Handbook of the changing world language map (pp. 3673-3682). Springer.

Goodchild, M. F., Haining, R., \& Wise, S. (1992). Integrating GIS and spatial data analysis: problems and possibilities. International Journal of Geographical Information Systems, $6(5), 407-423$.

Gorter, D. (2006). 1. Introduction: The study of the linguistic landscape as a new approach to multilingualism. In D. Gorter (Ed.), Linguistic landscape: A new approach to multilingualism (pp. 1-6). Multilingual Matters.

Goudie, A. S. (2017). The integration of Human and Physical Geography revisited. The Canadian Geographer/Le Geographe Canadien, 61(1), 19-27.

Graham, M., \& Shelton, T. (2013). Geography and the future of big data, big data and the future of geography. Dialogues in Human Geography, 3(3), 255-261.

Gregory, I., Cooper, D., Hardie, A., \& Rayson, P. (2015). Spatializing and analyzing digital texts. Corpora, GIS, and places. In D. J. Bodenhamer, J. Corrigan, \& T. M. Harris (Eds.), Spatial Narratives and Deep Maps (pp. 150-178). Indiana University Press.

Griffin, A. L., \& Fabrikant, S. I. (2012). More maps, more users, more devices means more cartographic challenges. Cartographic Journal, 49(4), 298-301.

Gudschinsky, S. C. (1956). The ABC's of lexicostatistics (glottochronology). Word, 12(2). 1-37.

Gunnemark, E. (1991). What is geolinguistics? Geolinguistics, 17, 12-13.

Haight, M., Quan-Haase, A., \& Corbett, B. A. (2014). Revisiting the digital divide in Canada: the impact of demographic factors on access to the internet, level of online activity, and social networking site usage. Information, Communication \& Society, 17(4), 503-519. 
Hall, S. (1997). Chapter 1: Representation, meaning and language. In E. Laclau (Ed.), Representation: Cultural representations and signifying practices (pp. 15-64). In association with the Open University.

Halliday, M. A. K. (2006). On Language and linguistics (Vol. 3). A\&C Black.

Hanewinkel, C., \& Losang, E. (2010). 21. Animated maps. In A. Lameli, R. Kehrein, \& S. Rabanus (Eds.), Language and space Volume 2: Language mapping (pp. 415-437). De Gruyter Mouton.

Hardy, D., Frew, J., \& Goodchild, M. F. (2012). Volunteered geographic information production as a spatial process. International Journal of Geographical Information Science, 26(7), $1191-1212$.

Harley, J. B. (1987). 1. The map and the development of the history of cartography. In J. B. Harley \& D. Woodward (Eds.), The history of cartography, volume 1: Cartography in prehistoric, ancient, and Medieval Europe and the Mediterranean (pp. 1-41). University of Chicago press.

Harley, J. B. (1988). Maps, knowledge, and power. In D. Cosgrove, \& S. Daniels (Eds.), The Iconography of Landscape (pp. 277-312). Cambridge University Press.

Harley, J. B. (1991). Can there be a cartographic ethics? Cartographic Perspectives, 10, 9-16.

Harmsworth, G. (1999). Indigenous values and GIS: A method and a framework. Business Alert, 14(1), 10-15.

Hart, M. A. (2010). Indigenous worldviews, knowledge, and research: The development of an indigenous research paradigm. Journal of Indigenous Social Development, 1(1A).

Harvey, F. (2015). A primer of GIS: fundamental geographic and cartographic concepts, second edition. Guilford Publications. 
Harvey, F., Kwan, M. P., \& Pavlovskaya, M. (2005). Introduction: critical GIS. Cartographica: The International Journal for Geographic Information and Geovisualization, 40(4), 1-4.

Haugen, E. (1966). Dialect, language, nation. American Anthropologist, 68(4), 922-935.

Hauser, M. D., Chomsky, N., \& Fitch, W. T. (2002). The faculty of language: What is it, who has it, and how did it evolve? Science, 298, 1569-1579.

Haviland, M. (1998). Guugu Yimithirr cardinal directions. Ethos, 26(1), 25-47.

Hayes, A. \& Taylor, D. R. F. (2019). 13: Developments in the Nunaliit cybercartographic atlas framework. In D. R. F. Taylor, E. Anonby, \& K. Murasugi (Eds.), Further developments in the theory and practice of cybercartography (pp. 205-217). Elsevier.

Haynie, H. J. (2014). Geography and spatial analysis in historical linguistics. Language and Linguistics Compass, 8(8), 344-357.

Head, C. G. (1984). The map as natural language: A paradigm for understanding. Cartographica: The International Journal for Geographic Information and Geovisualization, 21(1), 1-32.

Heeringa, W. J. (2004). Measuring dialect pronunciation differences using Levenshtein distance. Doctoral dissertation, University Library Groningen.

Helsper, E. J., \& Eynon, R. (2010). Digital natives: where is the evidence? British Educational Research Journal, 36(3), 503-520.

Hendry, J. (2014). Science and sustainability: Learning from Indigenous wisdom. Springer.

Henke, R., \& Berez-Kroeker, A. (2016). A brief history of archiving in language documentation, with an annotated bibliography. Language Documentation and Conservation, 10, 411457. 
Henry, D., \& Henry, K. (1969). Koyukon locationals. Anthropological Linguistics, 11(4), 136142.

Hensel, G. (1741). Africa poly-glotta scribendi modos gentium exhibens: Charts of Aethiopic (Ge'ez) and Coptic scripts [map]. (no scale). Synopsis universae philologiae: in qua miranda unitas et harmonia linguarum totius orbis terrarum occulta e literarum, syllabarum, vocumque natura \& recessibus eruitur: cum grammatica LL. orient. harmonica synoptice tractata... [Synopsis of the philology, in which a remarkable unity and harmony all the languages of the world is hidden from letters, syllables, or words. Nature recessions rescued with grammar. Orient. harmonics synoptice treated...] In commissis apud heredes Homannianos.

Herlihy, P. H., \& Knapp, G. (2003). Maps of, by, and for the peoples of Latin America. Human Organization, 62(4), 303-314.

Hickey, R. (2018). 1. Dialectology, philology, and historical linguistics. In C. Boberg, J. Nerbonne \& D. Watt (Eds.), The handbook of dialectology (pp. 23-38). Wiley. Hinton, L. (2001). The use of linguistic archives in language revitalization: The Native California language restoration workshop. In L. Hinton \& K. Hale (Eds.), The green book of language revitalization in practice (pp. 419-428). Academic Press.

Hoch, S., \& Hayes, J. J. (2010). Geolinguistics: The incorporation of Geographic Information Systems and science. Geographical Bulletin, 51(1), 23-36.

Hoefle, S. W. (2006). Eliminating scale and killing the goose that laid the golden egg? Transactions of the Institute of British Geographers, 31(2), 238-243.

Hoijer, H. (1956). Lexicostatistics: A critique. Language, 32(1), 49-60. 
Hopkin, V. D. (1992). Issues in color application. In H. Widdel \& D. L. Post (Eds.), Color in electronic displays (pp. 191-207). Springer.

Hovy, D., Rahimi, A., Baldwin, T., \& Brooke, J. (2020). Visualizing regional language variation across Europe on twitter. In S. D. Brunn \& R. Kehrein (Eds.), Handbook of the changing world language map (pp. 3719-3742). Springer.

Hruby, F., Miranda, R., \& Riedl, A. (2009). Bad globes \& better globes-multilingual categorization of cartographic concepts exemplified by "map" and "globe" in English, German and Spanish. In Proceedings of the 24th International Cartography Conference (ICC 2009), Santiago, Chile.

Huffman, N. H. (1997). Charting the other maps: Cartography and visual methods in feminist research. In S. Ahrentzen \& K. F. Al-Hindi (Eds.), Thresholds in feminist geography: Difference, methodology, representation (pp. 255-283). Rowman \& Littlefield.

Hüning, M. (2015). Language and space. An international handbook of linguistic variation. Volume 3: Dutch. De Gruyter Mouton.

Hurst, P., \& Clough, P. (2013). Will we be lost without paper maps in the digital age? Journal of Information Science, 39(1), 48-60.

Hymes, D. H. (1960). Lexicostatistics so far. Current Anthropology, 1(1), 3-44.

Ingram, R. (2019). 26: Mapping Kanyen'kéha (Mohawk) ethnophysiographical knowledge. In D. R. F. Taylor, E. Anonby, \& K. Murasugi (Eds.), Further developments in the theory and practice of cybercartography (pp. 475-506). Elsevier.

Inoue, F. (1999). Chapter 12: Subjective dialect division in Great Britain. In D. R. Preston (Ed.), Handbook of perceptual dialectology (Vol. 1, pp. 161-176). John Benjamins Publishing. 
Ish Ishwar, (2005). Languages of North America [map]. (no scale). Retrieved from https://commons.wikimedia.org/wiki/File:Langs_N.Amer.png

Jackendoff, R. (1983). Semantics and cognition (Vol. 8). MIT press.

Jackson, P. C. (2019). Introduction to artificial intelligence. Courier Dover Publications.

Jansen, B. J. (2009). Understanding user-web interactions via web analytics. Morgan \& Claypool.

Jenkins, R. (2004). Chapter 5: Embodied selves. In R. Jenkins (Ed.), Social identity (3rd ed., pp. 38-51). Routledge.

Johnson, J. M. (2001). Mapping ethnicity: Color use in depicting ethnic distribution. Cartographic Perspectives, (40), 12-31.

Johnson, J. T., Louis, R. P., \& Pramono, A. H. (2005). Facing the future: Encouraging critical cartographic literacies in Indigenous communities. ACME: An International Journal for Critical Geographies, 4(1), 80-98.

Johnson, R. B., \& Onwuegbuzie, A. J. (2004). Mixed methods research: A research paradigm whose time has come. Educational Researcher, 33(7), 14-26.

Johnson, R. B., Onwuegbuzie, A. J., \& Turner, L. A. (2007). Toward a definition of mixed methods research. Journal of Mixed Methods Research, 1(2), 112-133.Johnstone, B. (2011). Language and place. In R. Mesthrie (Ed.), The Cambridge handbook of sociolinguistics (pp. 203-217). Cambridge University Press.

Jordan, P. (2014). Thoughts on a concept of language geography. Geographica Timisiensis, $23(1), 33-46$. 
Junius, H. (1567). Nomenclator omnium rerum propria nomina, septem diversis linguis explicata indicans [Fully explained bulletin of the proper names of seven different languages]. Georg Olms Verlag.

Junker, M. O. (dir.), MacKenzie, M., Rosen, N., Valentine, J. R., \& Wolvengrey, A. (co-dir.). (2005-2020). The Algonquian Linguistic Atlas. Retrieved from http://www.atlas-ling.ca/\#

Junker, M. O., \& Torkornoo, D. (2017). Algonquian dictionaries project: Common digital infrastructure for lexicography and language documentation. Paper presented at the Canadian Society for Digital Humanities, Congress of the Social Sciences and Humanities Research Council (SSHRC).

Karlsson, F. (2018). Finnish - A comprehensive grammar. Routledge.

Keates, J. S. (1982). Understanding maps. Longman.

Kehrein, R., Lameli, A., \& Rabanus, S. (2010). Introduction. In A. Lameli, R. Kehrein, \& S. Rabanus (Eds.), Language and space, Volume 2: Language mapping (pp. xi-xxvi). De Gruyter Mouton.

Kent, A. J. (2017). Trust me, I'm a cartographer: Post-truth and the problem of acritical cartography. The Cartographic Journal, 54(3), 193-195.

Kent, A. J., \& Vujakovic, P. (2017). Introduction. In A. J. Kent \& P. Vujakovic (Eds.), The Routledge handbook of mapping and cartography (pp. 1-5). Routledge.

Kessler, F. C., \& Slocum, T. A. (2011). Analysis of thematic maps published in two geographical journals in the twentieth century. Annals of the Association of American Geographers, 101(2), 292-317. 
Kessler, F. C., \& Slocum, T. A. (2020). The map's changing role: A survey of the annals of the Association of American Geographers. In S. D. Brunn \& R. Kehrein (Eds.), Handbook of the changing world language map (pp. 289-318). Springer.

Kiik, A. (2015). Cartographic design of thematic polygons: a comparison using eye-movement metrics analysis. Master's Thesis. Student thesis series INES.

Kirk, J. M., \& Kretzschmar Jr., W. A. (1992). Interactive linguistic mapping of dialect features. Literary and Linguistic Computing, 7(3), 168-175.

Kirkness, V. J., \& Barnhardt, R. (1991). First Nations and higher education: The four R'srespect, relevance, reciprocity, responsibility. Journal of American Indian Education, 115.

Kitchel, E., \& Otto, F. (2020). Language of maps for blind and partially sighted people: Expressive and perceptive skills. In S. D. Brunn \& R. Kehrein (Eds.), Handbook of the changing world language map (pp. 319-331). Springer.

Kitchin, R. (2010). Post-representational cartography. lo Squaderno, 15, 7-12.

Kitchin, R. (2013). Big data and human geography: Opportunities, challenges and risks. Dialogues in human geography, 3(3), 262-267.

Kitchin, R., Perkins, C., \& Dodge, M. (2009). Thinking about maps. Rethinking maps: New frontiers in cartographic theory, 28, 1-25.

Klawe, J. J. (1973). Population Mapping. Cartographica: The International Journal for Geographic Information and Geovisualization, 10(1), 44-50.

Klemola, J., \& Jones, M. (1999). The Leeds corpus of English dialects-project. Leeds Studies in English, 30, 17-30. 
Kluge, S. (2000, January). Empirically grounded construction of types and typologies in qualitative social research. In Forum Qualitative Sozialforschung/Forum: Qualitative Social Research, 1(1).

Kolb, E. (1979). Atlas of English sounds. Francke.

Kraak, M. J., \& Ormeling, F. J. (2013). Cartography: visualization of spatial data. Routledge.

Kracht, M. (2002). On the semantics of locatives. Linguistics and Philosophy, 25(2), 157-232.

Kretzschmar, Jr., W. A. (2013). Computer mapping of language data. In M. Krug \& J. Schlüter (Eds.), Research methods in language variation and change (pp. 53-68). Cambridge University Press.

Kretzschmar, Jr., W. A. (2015). 2.3: Map. In Research Methods: Online accompaniment to Computer mapping of language data. In M. Krug \& J. Schlüter (Eds.), Research methods in language variation and change. Retrieved from https://www.unibamberg.de/fileadmin/eng-ling/fs/Chapter_3/Index.html?References.html

Kruijsen, T. J. W. M., \& Sijs, N. (2010). 9. Mapping Dutch and Flemish. In A. Lameli, R. Kehrein, \& S. Rabanus (Eds.), Language and space, Volume 2: Language mapping (pp. 180-202). De Gruyter Mouton.

Krygier, J., \& Wood, D. (2016). Making maps: a visual guide to map design for GIS. Guilford Publications.

Küng, L., Picard, R. G., \& Towse, R. (2008). 1: Introduction. In L., Küng, R. G., Picard, \& R. Towse, (Eds.), The internet and the mass media (pp. 1-16). Sage.

Kunst, J. P., Barbiers, S. (2010). 20. Generating maps on the internet. In A. Lameli, R. Kehrein, \& S. Rabanus (Eds.), Language and space, Volume 2: Language mapping (pp. 401-422). De Gruyter Mouton. 
Kurath, H. (1949). A word geography of the eastern United States. University of Michigan Press.

Kurzweil, R. (2004). The law of accelerating returns. In Alan Turing: Life and legacy of a great thinker (pp. 381-416). Springer.

Lameli, A. (2010). Linguistic atlases-traditional and modern. Auer and Schmidt, 567-592.

Lameli, A., Kehrein, R., \& Rabanus, S. (2010). Language and space. An international handbook of linguistic variation. Volume 2: Language mapping. De Gruyter Mouton.

Landau, B., Dessalegn, B., \& Cohen-Goldberg, A. M. (2011). Language and space: momentary interactions. In Proceedings of the annual meeting of the cognitive science society, 33.

Landry, R., \& Bourhis, R. Y. (1997). Linguistic landscape and ethnolinguistic vitality: An empirical study. Journal of Language and Social Psychology, 16(1), 23-49.

Leclerc, J. (2010). Historical map of New France made using Microsoft Paint [map]. (no scale). Retrieved from http://www.axl.cefan.ulaval.ca/francophonie/Nlle-France-Plaisance.htm

Lee, J., \& Kretzschmar, W. A. (1993). Spatial analysis of linguistic data with GIS functions. International Journal of Geographical Information Science, 7(6), 541-560.

Levine, S. S., \& Prietula, M. J. (2014). Open collaboration for innovation: Principles and performance. Organization Science, 25(5), 1414-1433.

Levinson, S. C. (1996). Language and space. Annual Review of Anthropology, 25(1), 353-382.

Levinson, S. C., Kita, S., Haun, D. B., \& Rasch, B. H. (2002). Returning the tables: Language affects spatial reasoning. Cognition, 84(2), 155-188.

Li, T. J. J., Sen, S., \& Hecht, B. (2014, November). Leveraging advances in natural language processing to better understand Tobler's first law of geography. In Proceedings of the 22Nd ACM SIGSPATIAL international conference on advances in Geographic Information Systems (pp. 513-516). ACM. 
Li, Y., Yu, M., Xu, M., Yang, J., Sha, D., Liu, Q., \& Chang, Y. (2020). Big data and cloud computing. In H. Guo, M. F. Goodchild, \& A. Annoni (Eds.), Manual of Digital Earth (pp. 325-356). Springer.

Lindsay, I., Rubinson, K. S., Greene, A. F., Hammer, E., \& Lawrence, D. (2018). Progress, problems, and possibilities of GIS in the South Caucasus: An international workshop summary. Antiquity, 92(362), 1-7.

Linn, M. (2014). Living archives: A community-based language archive model. Language Documentation and Description, 12, 53-67.

Linnaeus, C. (1735). Systema Naturae.

LL-Map. (2020). Language and location: A map annotation project. LL-Map [online]. Retrieved from http://llmap.org/.

Llamas, C. (2018). The dialect questionnaire. In C. Boberg, J. Nerbonne, \& D. Watt (Eds.), The Handbook of dialectology (pp. 253-267). Wiley.

Lohr, K. N., \& Field, M. J. (Eds.). (1990). Clinical practice guidelines: directions for a new program, 90(8). National Academies Press.

Lotulelei, S. (2012). Identifying patterns in the crucial educational leadership constructs used by the most cited authors and published works of 1990-2010. Doctoral dissertation, Brigham Young University.

Lucy, J. (2000). Introductory comments. In S. Niemeier \& R. Dirven (Eds.), Evidence for linguistic relativity (pp. ix-xxi). John Benjamins.

Luebbering, C. R. (2011). The Cartographic Representation of Language: Understanding language map construction and visualizing language diversity. Doctoral dissertation, Virginia Tech. 
Luebbering, C. R. (2013). Displaying the geography of language: The cartography of language maps. Linguistics Journal, 7(1), 39-67.

Luebbering, C. R., Kolivras, K. N., \& Prisley, S. P. (2013). The lay of the language: Surveying the cartographic characteristics of language maps. Cartography and Geographic Information Science, 40(5), 383-400.

Lyons, J., \& John, L. (1968). Introduction to theoretical linguistics (Vol. 510). Cambridge University Press.

Macaulay, R. (2018). Dialect sampling methods. In C. Boberg, J. Nerbonne, \& D. Watt (Eds.), The Handbook of dialectology (pp. 241-252). Wiley.

MacEachren, A. M. (2004). How maps work: Representation, visualization, and design. Guilford Press.

MacEachren, A. M., Roth, R. E., O'Brien, J., Li, B., Swingley, D., \& Gahegan, M. (2012). Visual semiotics \& uncertainty visualization: An empirical study. IEEE Transactions on Visualization and Computer Graphics, 18(12), 2496-2505.

Mackey, W. F. (1988). 2. Geolinguistics: Its scope and principles. In C. H. Williams (Ed.), Language in geographic context (pp. 20-46). Multilingual Matters.

Maguire, W., McMahon, A., Heggarty, P., \& Dediu, D. (2010). The past, present, and future of English dialects: Quantifying convergence, divergence, and dynamic equilibrium. Language Variation and Change, 22(1), 69-104.

Majid, A., Bowerman, M., Kita, S., Haun, D. B., \& Levinson, S. C. (2004). Can language restructure cognition? The case for space. Trends in Cognitive Sciences, 8(3), 108-114.

Maling, D. H. (2013). Coordinate systems and map projections. Elsevier.

Malinowski, B. (1935). Coral gardens and their magic. Allen \& Unwin. 
Margaryan, A., Littlejohn, A., \& Vojt, G. (2011). Are digital natives a myth or reality? University students' use of digital technologies. Computers \& Education, 56(2), 429440.

Mark, D. M. (2003). Geographic information science: Defining the field. Foundations of Geographic Information Science, 1, 3-18.

Martinet, A. (1960). Elements of general linguistics. Studies in general linguistics (Vol. 1). Translated by Elisabeth Palmer Rubbert. Faber.

McDavid Jr, R. I., \& O'Cain, R. K. (1973). Sociolinguistics and linguistic geography. The Kansas Journal of Sociology, 9(2), 137-156.

McGowan, D. (2001). Legal implications of open-source software. University of Illinois Law Review, 241-304.

McGregor, W. B. (2015). Linguistics: An introduction. Bloomsbury Publishing.

McIvor, O., \& Jacobs, P. (2017). NETOLNEW Environmental Scan (pp. 1-2, Issue brief). University of Victoria.

McKenzie, G., Janowicz, K., \& Adams, B. (2014). A weighted multi-attribute method for matching user-generated points of interest. Cartography and Geographic Information Science, 41(2), 125-137.

McLean, J. E. (2006). Foreword: From quantitative to qualitative and half way back. Research in the Schools, 13(1), iii-iv.

Meakins, F. (2011). Spaced out: Intergenerational changes in the expression of spatial relations by Gurindji people. Australian Journal of Linguistics, 31(1), 43-77.

Meng, L. (2003). Missing theories and methods in digital cartography. In Proceedings of the $21^{s t}$ International Cartographic Conference (ICC) 'Cartographic Renaissance. 
Meredith, C. (1997). Best practice in performance reporting in natural resource management. Natural Resource Management, ANZECC Working Group on National Parks and Protected Area Management.

Miestamo, M., Sinnemäki, K., \& Karlsson, F. (Eds.). (2008). Language complexity: Typology, contact, change (Vol. 94). John Benjamins Publishing.

Millardet, G. (1910). Petit atlas linguistique d'une région des Landes: contribution à la dialectologie gasconne. É. Privat.

Miller, G. A. (1951). Language and communication. McGraw-Hill Book Company Inc.

Miller, S. (1992). On conventions. Australasian Journal of Philosophy, 70(4), 435-444.

Milroy, J., \& Milroy, L. (1985). Linguistic change, social network and speaker innovation. Journal of Linguistics, 21(2), 339-384.

Misra, R. P., \& Ramesh, A. (1989). Fundamentals of cartography. Concept Publishing Company.

Mocanu, D., Baronchelli, A., Perra, N., Gonçalves, B., Zhang, Q., \& Vespignani, A. (2013). The twitter of babel: Mapping world languages through microblogging platforms. PloS one, $8(4), 1-9$.

Modern Language Association. (2010). The Modern Language Association Language map: A map of languages in the United States, MLA Language Map, retrieved from: https://www.mla.org/Resources/Research/MLA-Language-Map

Moellering, H. (2007, August). Expanding the ICA conceptual definition of a map. In Proceedings: 23rd international cartographic conference, Moscow.

Moldaschl, M., \& Weber, W. G. (1998). The "three waves" of industrial group work: Historical reflections on current research on group work. Human Relations, 51(3), 347-388. 
Molenaar, M. (1998). An introduction to the theory of spatial object modelling for GIS. CRC Press.

Monk, J. (1992). Gender in the landscape: Expressions of power and meaning. In K. Anderson \& F. Gale (Eds.), Inventing places: Studies in cultural geography. Longman Cheshire.

Monmonier, M. (1991). Ethics and map design: Six strategies for confronting the traditional onemap solution. Cartographic Perspectives, (10), 3-8.

Monmonier, M. (1993). Mapping it out: Expository cartography for the humanities and social sciences. University of Chicago Press.

Monmonier, M. (1996). How to lie with maps. University of Chicago Press.

Monmonier, M. (2005a). POMP and circumstance: Plain old map products in a cybercartographic world. In D. R. F. Taylor \& T. Lauriault (Eds.), Cybercartography: Theory and practice (1st ed., pp. 15-34). Elsevier.

Monmonier, M. (2005b). Lying with Maps. Statistical Science, 20(3), 215-222.

Montello, D.R. (2002). Cognitive map-design research in the twentieth century: Theoretical and empirical approaches. Cartography and Geographic Information Science, 29(3), 283304.

Moreton-Robinson, A., \& Walter, M. (2009). Chapter 22: Indigenous methodologies in social research. In A. Bryman, J. J. Teevan, \& E. Bell (Eds.), Social Research Methods (2nd ed., pp. 1-18). Oxford University Press.

Morris, C. W. (1938). Foundations of the theory of signs. In O. Neurath (Ed.), International encyclopedia of unified science (pp. 1-59). Chicago University Press. 
Mühlhäusler, P. (2010). 17. Mapping linguistic typology. In A. Lameli, R. Kehrein, \& S. Rabanus (Eds.), Language and space, Volume 2: Language mapping (pp. 355-387). De Gruyter Mouton.

Mulcahy, K. A., \& Clarke, K. C. (2001). Symbolization of map projection distortion: a review. Cartography and Geographic Information Science, 28(3), 167-182.

Murasugi, K. (2019). 22. Linguistic cybercartography: Expanding the boundaries of language maps. In D. R. F. Taylor, E. Anonby, \& K. Murasugi (Eds.), Further developments in the theory and practice of cybercartography (pp. 389-412). Elsevier.

Najafabadi, M. M., Villanustre, F., Khoshgoftaar, T. M., Seliya, N., Wald, R., \& Muharemagic, E. (2015). Deep learning applications and challenges in big data analytics. Journal of Big Data, 2(1), 1-21.

Nash, J. (2017). When do linguists make their best (cartographic) work? The Globe, 81, 37-41.

Native Land. (2019). The land you live on: An education guide by Native Land. Native Land.ca. Retrieved from: https://native-land.ca/wp/wpcontent/uploads/2019/03/teacher_guide_2019_final.pdf

Ndzabandzaba, C. (2020). Participatory Geographic Information System (PGIS): A discourse toward a solution to traditional GIS challenges. In S. D. Brunn \& R. Kehrein (Eds.), Handbook of the changing world language map (pp. 3825-3835). Springer.

Nerbonne, J., \& Kretzschmar Jr., W. (2003). Introducing computational techniques in dialectometry. Computers and the Humanities, 37(3), 245-255.

Neumann, A. (2011). Web mapping and web cartography. In W. Kresse \& D. M. Danko (Eds.), Springer handbook of geographic information (pp. 567-587). Springer.

Nielsen, J. (1992). The usability engineering life cycle. Computer, 25(3), 12-22. 
Norris, R. P., \& Harney, B. Y. (2014). Songlines and navigation in Wardaman and other Australian Aboriginal cultures. Journal of Astronomical History and Heritage, 17, 141148.

Noy, N. F., \& McGuinness, D. L. (2001). Ontology development 101: A guide to creating your first ontology. Stanford knowledge systems laboratory technical report KSL-01-05 and Stanford medical informatics technical report SMI-2001-0880.

Núñez, R., Cooperrider, K., Doan, D., \& Wassmann, J. (2012). Contours of time: Topographic construals of past, present, and future in the Yupno valley of Papua New Guinea. Cognition, 124(1), 25-35.

Obermeyer, N. J. (1995). The hidden GIS technocracy. Cartography and Geographic Information Systems, 22(1), 78-83.

Odell, J. (2019). How to do nothing: Resisting the attention economy. Melville House.

Ogino, T. (1980). Computational dialectology using GLAPS. Automated processing of field survey data. Proceedings of the 8th conference on computational linguistics, 605-613.

Olariu, F. \& Olariu, V. (2014). The Romanian linguistic cartography in the digitizing era: The electronic atlases. Dialectologia et Geolinguistica, 22(1), 75-90.

Onishi, T. (2010a). 16. Mapping the Japanese language. In A. Lameli, R. Kehrein, \& S. Rabanus (Eds.), Language and space, Volume 2: Language mapping (pp. 333-360). De Gruyter Mouton.

Onishi, T. (2010b). Analyzing dialectological distributions of Japanese. Dialectologia: Revista Electrònica, 123-135. 
Ooms, K., De Maeyer, P., \& Fack, V. (2014). Study of the attentive behavior of novice and expert map users using eye tracking. Cartography and Geographic Information Science, $41(1), 37-54$.

Ooms, K., De Maeyer, P., \& Fack, V. (2015). Listen to the map user: Cognition, memory, and expertise. The Cartographic Journal, 52(1), 3-19.

O'Raghallaigh, P., Sammon, D., \& Murphy, C. (2010). Map-making and theory-building: Principles for achieving presentation and content effectiveness. International Conference of Information Systems (ICIS) Conference Proceedings, 31, 1-16.

Orford, S. (2002). Valuing locational externalities: A GIS and multilevel modelling approach. Environment and Planning B: Planning and Design, 29(1), 105-127.

Ormeling, F. (1992). Methods and possibilities for mapping by onomasticians. Discussion Papers in Geolinguistics, 19, 50-67.

Ormeling, F. (2010). 2. Visualizing geographic space: The nature of maps. In A. Lameli, R. Kehrein, \& S. Rabanus (Eds.), Language and space, Volume 2: Language mapping (pp. 21-43). De Gruyter Mouton.

Pacheco, D., \& Velez, V. N. (2009). Maps, mapmaking, and a critical pedagogy: Exploring GIS and maps as a teaching tool for social change. Seattle Journal for Social Justice, 8, 273302.

Paltridge, B., \& Starfield, S. (2007). Thesis and dissertation writing in a second language: A handbook for supervisors. Routledge.

Pápay, G. (2017). 1: Introduction to map design. In A. J. Kent \& P. Vujakovic (Eds.), The Routledge handbook of mapping and cartography (pp. 9-28). Routledge.

Park, D. B. (1982). The meanings of "audience". College English, 44(3), 247-257. 
Pask, R. (2006). The blue marble: An introduction to geography (Vol. 1). Pearson Education South Asia.

Patrão, A. (2018). Linguistic relativism in the age of global lingua franca: Reconciling cultural and linguistic diversity with globalization. Lingua, 210, 30-41.

Pauwels, L. (Ed.). (2006). Visual cultures of science: Rethinking representational practices in knowledge building and science communication. University Press of New England.

Peeters, Y. J. D. (1992). The political importance of the visualisation of language contact. Discussion Papers in Geolinguistics, 19, 6-8.

Penfield, S. D., Serratos, A., Tucker, B. V., Flores, A., Harper, G., Hill, J., \& Vasquez, N. (2008). Community collaborations: Best practices for North American Indigenous language documentation. International Journal of the Sociology of Language, 2008(191), $187-202$.

Perens, B. (1999). The open source definition. Open sources: Voices from the open source revolution, $1,171-188$.

Perkins, C. (2007). Community mapping. The Cartographic Journal, 44(2), 127-137.

Perkins, C. (2018, February). Community mapping. Oxford Bibliographies. Retrieved from: http://www.oxfordbibliographies.com/view/document/obo-9780199874002/obo9780199874002-0184.xml\#firstMatch

Peterson, G. N. (2009). 3. Layout design. In G. N. Peterson (Ed.), GIS cartography: A guide to effective map design (1st ed., pp. 15-57). CRC Press.

Peterson, G. N. (2014). GIS cartography: A guide to effective map design (2nd ed.). CRC Press.

Peterson, M. (1999). Maps on stone: the web and ethics in cartography. Cartographic Perspectives, (34), 5-8. 
Peterson, M. (2007). Chapter 3: The internet and multimedia cartography. In W. Cartwright, M. P. Peterson, \& G. Gartner (Eds.), Multimedia cartography (pp. 35-50). Springer.

Petrilli, S., \& Ponzio, A. (2002). Sign vehicles for semiotic travels: Two new handbooks. Semiotica, 141(1/4), 203-350.

Peuquet, D. J. (1988). Representations of geographic space: Toward a conceptual synthesis. Annals of the Association of American Geographers, 78(3), 375-394.

Peuquet, D. J. (1999). Time in GIS and geographical databases. Geographical Information Systems, 1, 91-103.

Philo, C., \& Wolch, J. (2001). The 'three waves' of research in mental health geography: A review and critical commentary. Epidemiology and Psychiatric Sciences, 10(4), 230-244.

Pi, C. Y. T. (2006). Beyond the isogloss: isographs in dialect topography. Canadian Journal of Linguistics/Revue canadienne de linguistique, 51(2-3), 177-184.

Picaut, J., Fortin, N., Bocher, E., Petit, G., Aumond, P., \& Guillaume, G. (2019). An openscience crowdsourcing approach for producing community noise maps using smartphones. Building and Environment, 148, 20-33.

Pijanowski, B. C., Tayyebi, A., Doucette, J., Pekin, B. K., Braun, D., \& Plourde, J. (2014). A big data urban growth simulation at a national scale: configuring the GIS and neural network based land transformation model to run in a high performance computing (HPC) environment. Environmental Modelling \& Software, 51, 250-268.

Pinker, S., \& Jackendoff, R. (2009). The components of language: What's specific to language, and what's specific to humans. In M. H. Christiansen, C. Collins, \& S. Edelman (Eds.), Language universals (pp. 126-151). Oxford University Press.

Pisati, M. (2004). Simple thematic mapping. The Stata Journal, 4(4), 361-378. 
Powell, J. W. (1891). Linguistic stocks of American Indians north of Mexico [map]. (no scale). In Seventh annual report of the bureau of ethnology to the secretary of the Smithsonian Institution, 1885-1886. Retrieved from https://www.reddit.com/r/MapPorn/comments/3s3uta/linguistic_stocks_of_american_indi ans_north_of/

Prensky, M. (2001). Digital natives, digital immigrants, part 1. On the Horizon, 9(5), 1-6.

Preston, D. R. (2010). Mapping the geolinguistic spaces of the brain. In A. Lameli, R. Kehrein, \& S. Rabanus (Eds.), Language and space, Volume 2: Language mapping (pp. 121-153). De Gruyter Mouton.

Purschke, C. (forthcoming). Using crowdsourced data to explore the linguistic landscape of cities. Results from the participatory research project Lingscape. In S. D. Brunn \& R. Kehrein (Eds.), Handbook of the changing world language map. Springer.

Quesada Pacheco, M. Á. (2014). División dialectal del español de América según sus hablantes Análisis dialectológico perceptua [Dialectal division of American Spanish according to its speakers. An analysis from Perceptual Dialectology]. Boletín de Filología, 49(2), 257309.

Rabanus, S. (2011). The State of the Art in Linguistic Cartography. Studier i dialektologi och sociolingvistik [Studies in dialectology and sociolinguistics]. Föredrag vid den Nionde nordiska dialektologkonferensen [Lectures at the ninth nordic dialectologist conference], Uppsala, 31-52.

Rabanus, S. (2018). 20. Dialect maps. In C. Boberg, J. Nerbonne, \& D. Watt (Eds.), The handbook of dialectology (pp. 349-367). Wiley. 
Rabanus, S. (2020). 6. Language mapping worldwide: Methods and traditions. In S. D. Brunn \& R. Kehrein (Eds.), Handbook of the changing world language map (pp. 103-129). Springer.

Rabanus, S., Kehrein, R. (2010). Creating digital editions of historical maps. In A. Lameli, R. Kehrein, \& S. Rabanus (Eds.), Language and space, Volume 2: Language mapping (pp. 375-391). De Gruyter Mouton.

Ramisch, H. (2010). Current trends in British geolinguistics linking the past with the present. Dialectologia: Revista Electrònica, (special issue 1), 161-173.

Ramisch, H. (2010). Mapping British English. In A. Lameli, R. Kehrein, \& S. Rabanus (Eds.), Language and space, Volume 2: Language mapping (pp. 238-260). De Gruyter Mouton.

Rankin, J. W. (2016). After the map: cartography, navigation, and the transformation of territory in the twentieth century. University of Chicago Press.

Rea, J. A. (1958). Concerning the validity of lexicostatistics. International journal of American linguistics, 24(2), 145-150.

Read, D. W. (1989). Intuitive typology and automatic classification: Divergence or full circle? Journal of Anthropological Archaeology, 8(2), 158-188.

Remenyi, D. (2015). The Monograph Dissertation versus the Papers Approach. Alternation Journal, 22(1), 327-335.

Roberts, L. (2012). Mapping cultures: A spatial anthropology. In Mapping Cultures (pp. 1-25). Palgrave Macmillan.

Romaine, S. (2003). 4. Variation in language and gender. In J. Holmes \& M. Meyerhoff (Eds.), The handbook of language and gender (pp. 98-118). Blackwell Publishing. 
Roth, R. E. (2009). A qualitative approach to understanding the role of geographic information uncertainty during decision making. Cartography and Geographic Information Science, $36(4), 315-330$.

Roth, R. E., Donohue, R. G., Sack, C. M., Wallace, T. R., \& Buckingham, T. M. (2014). A process for keeping pace with evolving web mapping technologies. Cartographic Perspectives, (78), 25-52.

Roth, R. E., Quinn, C., \& Hart, D. (2015). The competitive analysis method for evaluating water level visualization tools. In J. Brus, A. Vondrakova, \& V. Vozenilek (Eds.), Modern trends in cartography (pp. 241-256). Springer.

Röthlisberger, M., \& Szmrecsanyi, B. (2020). Dialect typology: Recent advances. In S. D. Brunn \& R. Kehrein (Eds.), Handbook of the changing world language map (pp. 131-156). Springer.

Rubinstein, I. S. (2013). Big data: The end of privacy or a new beginning? International Data Privacy Law, 3(2), 74-87.

Saldaña, J. (2013). The coding manual for qualitative researchers. Sage

Salishchev, K. A. (1986). The concept of space and its presentation on maps: Theoretical aspects. Mapping Sciences and Remote Sensing, 23(3), 167-174.

Salvucci, C. (1999). Linguistic geography of the mainland United States [map]. (no scale). Retrieved from: http:/www.evolpub.com/Americandialects/AmDialMap.html Sandinska, Y. (2016). Technological principles and mapping applications of web GIS. In Proceedings of the $6^{\text {th }}$ international conference on cartography and GIS, Albena, Bulgaria, June 13-17 (pp. 287-296). 
Sawada, M., Cossette, D., Wellar, B., \& Kurt, T. (2006). Analysis of the urban/rural broadband divide in Canada: Using GIS in planning terrestrial wireless deployment. Government Information Quarterly, 23(3-4), 454-479.

Schenk, E., \& Guittard, C. (2011). Towards a characterization of crowdsourcing practices. Journal of Innovation Economics Management, (1), 93-107.

Scheuringer, H. (2010). 8. Mapping the German language. In A. Lameli, R. Kehrein, \& S. Rabanus (Eds.), Language and space, Volume 2: Language mapping (pp. 158-191). De Gruyter Mouton.

Schirmunski, V. (1956). Schwachbetonte Wortformen in den deutschen Mundarten [Weakly emphasized word forms in German dialects]. Fragen und Forschungen in Nereich und Umkreis der germanistischen Philologie. Akademie Verlag.

Schmeller, J. A. (1821). Die Mundarten Bayerns grammatisch dargestellt [Bavarian dialects represented grammatically]. Thienemann.

Schmidt, J. E., \& Auer, P. (2010). Language and space. An international handbook of linguistic variation. Volume 1 - Theories and methods. De Gruyter Mouton.

Schmidt, J. E. (2010). 19. Dynamic linguistic maps and validation. In A. Lameli, R. Kehrein, \& S. Rabanus (Eds.), Language and space, Volume 2: Language mapping (pp. 385-401). De Gruyter Mouton.

Schnürer, R., Sieber, R., \& Çöltekin, A. (2015). The next generation of atlas user interfaces: A user study with “digital natives”. In J. Brus, A. Vondrakova, \& V. Vozenilek (Eds.), Modern trends in cartography (pp. 23-36). Springer. 
Scholz, J., Hrastnig, E., \& Wandl-Vogt, E. (2017, September). A spatio-temporal Linked Data Representation for modeling spatio-temporal Dialect Data. In International conference on spatial information theory (pp. 275-282). Springer.

Scultetus, B. (1593). Lusatia Superior.

Séguy, J. (1973). La dialectométrie dans l'Atlas linguistique de la Gascogne. Société de linguistique romane.

Shimmura, I. (1904). The boundary lines between the east and west Japanese on grammar (reprinted 1993). Shimmura Izuru Memorial Foundation.

Shuy, R. (1962). The Northern-Midland dialect boundary in Illinois. American Dialect Society, 38. University of Alabama Press.

Sibata, T. (1969). Methods in linguistic geography. Chikumashobo.

Simonsen, K. (2007). Practice, spatiality and embodied emotions: An outline of a geography of practice. Human Affairs, 17(2), 168-181.

Skupin, A., \& Fabrikant, S. I. (2003). Spatialization methods: a cartographic research agenda for non-geographic information visualization. Cartography and Geographic Information Science, 30(2), 99-119.

Snyder, J. P. (1997). Flattening the earth: two thousand years of map projections. University of Chicago Press.

Snyder, J.P. (1989). Album of map projections. United States geological survey professional paper. United States Government Printing Office, 1453.

Sobel, D. (1998). Mapmaking with children: Sense of place education for the elementary years. Heinemann. 
Spiess, E. (1973). Proposal for definitions of map author. International Yearbook of Cartography, 13, 52-57.

Spinuzzi, C., \& Zachry, M. (2000). Genre ecologies: An open-system approach to understanding and constructing documentation. ACM Journal of Computer Documentation (JCD), 24(3), 169-181.

St. Laurent, A. M. (2004). Understanding open source and free software licensing: Guide to navigating licensing issues in existing \& new software. O'Reilly Media, Inc.

Starostin, S. A. (1999). O dokozatel'stve jazykovogo rodstva [On the proof of genetic relationship of languages]. In J. Rachilina (Ed.), Tipologija i teorija jazyka [Typology and language theory] (pp. 57-69). Moscow.

Starr, C., \& Rudman, R. (1973). Parameters of technological growth. Science, 182(4110), 358364.

Steffen, W., Crutzen, P. J., \& McNeill, J. R. (2007). The anthropocene: Are humans now overwhelming the great forces of nature. AMBIO: A Journal of the Human Environment, $36(8), 614-622$.

Stevens, J., Smith, J. M., \& Bianchetti, R. A. (2012). Mapping our changing world. Department of Geography, The Pennsylvania State University.

Stone, A. (2018). A typology for understanding and evaluating maps of Indigenous languages. In S. Drude, N. Ostler \& M. Moser (Eds.), Endangered languages and the land: Mapping landscapes of multilingualism, Proceedings of FEL XXII/2018 (Reykjavik, Iceland), 1925. FEL \& EL Publishing. 
Stone, A. (2018). Mapping experience: Age and indigeneity as mediating factors in users' experiences with the Algonquian Linguistic Atlas. Cartographica: The International Journal for Geographic Information and Geovisualization, 53(4), 229-240.

Stone, A., \& Anonby, E. J. (2019). The utility of cybercartography in Indigenous language education. In D. R. F. Taylor, E. Anonby, \& K. Murasugi (Eds.), Further developments in the theory and practice of cybercartography (pp. 441-460). Elsevier.

Sui, D. Z. (2008). The Wikification of GIS and its consequences: Or Angelina Jolie's new tattoo and the future of GIS. Computers, Environment and Urban Systems, 1(32), 1-5.

Swiggers, P. (2010). 13. Mapping the Romance languages of Europe. In A. Lameli, R. Kehrein, \& S. Rabanus (Eds.), Language and space, Volume 2: Language mapping (pp. 269-310). De Gruyter Mouton.

Talmy, L. (1983). How language structures space. In H. L. Pick Jr. \& L. P. Acredolo (Eds.), Spatial orientation (pp. 225-282). Springer.

Talmy, L. (1985). Lexicalization patterns: Semantic structure in lexical forms. In T. Shopen (Ed.), Language typology and syntactic description (pp. 57-149). Cambridge University Press.

Tanksley, S. D. (1993). Linkage map of chromosome [map]. (no scale). In Tanksley, S. D. (1993). Mapping polygenes. Annual review of genetics, 27(1), 205-233.

Tannen, D. (2019). Discourse analysis - What speakers do in conversation. Linguistic Society of America [web page]. Retrieved from https://www.linguisticsociety.org/resource/discourse-analysis-what-speakers-doconversation 
Tashakkori, A., \& Creswell, J. W. (2007). The new era of mixed methods. Journal of Mixed Methods Research, 1(1), 3-7.

Taylor, D. R. F. (2005). The theory and practice of cybercartography: An introduction. In D. R. F. Taylor \& T. Lauriault (Eds.), Cybercartography: Theory and practice (1st ed., pp. 114). Elsevier.

Taylor, D. R. F. (2019a). The theory and practice of cybercartography: An introduction. In D. R. F. Taylor, E. Anonby, \& K. Murasugi (Eds.), Further developments in the theory and practice of cybercartography (pp. 25-33). Elsevier.

Taylor, D. R. F. (2019b). Cybercartography revisited. In D. R. F. Taylor, E. Anonby, \& K. Murasugi (Eds.), Further developments in the theory and practice of cybercartography (pp. 3-21). Elsevier.

Taylor, D. R. F. (2019b). Some recent developments in the theory and practice of Cybercartography. In D. R. F. Taylor, E. Anonby, \& K. Murasugi (Eds.), Further developments in the theory and practice of cybercartography (pp. 55-67). Elsevier.

Taylor, D. R. F., Anonby, E., \& Murasugi, K. (Eds.). (2019). Further developments in the theory and practice of cybercartography: International dimensions and language mapping. Elsevier.

Temprano, V. (2015-2020). Native Land.ca. [map]. Okanagan Territory. Retrieved from https://native-land.ca/.

Thorndyke, P. W., \& Stasz, C. (1980). Individual differences in procedures for knowledge acquisition from maps. Cognitive psychology, 12(1), 137-175.

Thrower, N. J. (1959). Animated cartography. The Professional Geographer, 11(6), 9-12. 
Thrower, N. J. (2008). Maps and civilization: Cartography in culture and society. University of Chicago Press.

Thun, H. (2010). Pluridimensional cartography. In A. Lameli, R. Kehrein, \& S. Rabanus (Eds.), Language and space, Volume 2: Language mapping (pp. 506-523). De Gruyter Mouton.

Tisato, G. G. (2009 - 2018). Carte Toponomastische - 1 [digital map]. (no scale). In K. Jaberg \& J. Jud (1928-1940), Sprach- und Sachatlas Italiens und der Südschweiz [Linguistic and Ethnographic Atlas of Italy and Southern Switzerland], 1-8. Zofingen.

Tobler, W. R. (1963). Geographic area and map projections. Geographical Review, 53(1), 59-78.

Tobler, W. R. (1970). A computer movie simulating urban growth in the Detroit region. Economic Geography, 46(1), 234-240.

Trappes-Lomax, H. (2004). Discourse analysis. In A. Davies \& C. Elder (Eds.), The handbook of applied linguistics (pp. 133-164). Blackwell Publishing.

Trask, R. L. (2007). Language and linguistics: The key concepts. Routledge.

Trbovich, P. L., Lindgaard, G., \& Dillon, R. F. (2019). Cybercartography: A multimodal approach. In D. R. F. Taylor, E. Anonby, \& K. Murasugi (Eds.), Further developments in the theory and practice of cybercartography (pp. 36-49). Elsevier.

Trudgill, P. (1974). Linguistic change and diffusion: Description and explanation in sociolinguistic dialect geography. Language in Society, 3(2), 215-246.

Tufte, E. R., (1990). Envisioning information. Graphics Press.

Turell, M. T. (2003) Apparent and real time in studies of linguistic change and variation. Revista de Sociolingüística, 4(3), 1-10.

Tyner, J. A. (1982). Persuasive cartography. Journal of Geography, 81(4), 140-144.

Tyner, J. A. (2017). Principles of map design. Guilford Press. 
Tyner, J. A. (2020). Maps as language / The language of maps. In S. D. Brunn \& R. Kehrein (Eds.), Handbook of the changing world language map (pp. 333-339). Springer.

Upton, C. (2010). 7. Designing maps for non-linguists. In A. Lameli, R. Kehrein, \& S. Rabanus (Eds.), Language and space, Volume 2: Language mapping (pp. 142-166). De Gruyter Mouton.

Uschold, M., \& Gruninger, M. (1996). Ontologies: Principles, methods and applications. The Knowledge Engineering Review, 11(2), 93-136.

Van der Merwe, I. J., \& Van der Merwe, J. H. (2007). 1. Introduction - Language mapping. In I. J. Van der Merwe \& J. H. Van der Merwe (Eds.), Linguistic atlas of South Africa (pp. 18). African Sun Media.

Van Dijk, T. A. (2011). Introduction: The study of discourse. In Van Dijk, T.A. (Ed.), Discourse studies: A multidisciplinary introduction (pp. 1-7). Sage.

Van Elzakker, C. P. J. M., \& Wealands, K. (2007). Chapter 34: Use and users in multimedia cartography. In W. Cartwright, M. P. Peterson, \& G. Gartner, (Eds.), Multimedia cartography (pp. 487-504). Springer.

Van Genderen, J., Goodchild, M. F., Guo, H., Yang, C., Nativi, S., Wang, L., \& Wang, C. (2020). Digital Earth challenges and future trends. In H. Guo, M. F. Goodchild, \& A. Annoni (Eds.), Manual of Digital Earth (pp. 811-827). Springer.

Van Oostendorp, M. (2014). Phonological and phonetic databases at the Meertens Institute. In J. Durand, U. Gut, \& G. Kristoffersen (Eds.), The Oxford handbook of corpus phonology (pp. 546-551). Oxford University Press. 
Varanka, D. (2013). The manly map: the English construction of gender in early modern cartography. In L. Dowler, J. Carubia, \& B. Szczygiel (Eds.), Gender and landscape (pp. 235-251). Routledge.

Vasilev, S. (2007, March). 'Who is who' on the map? In Conference proceedings of $1^{\text {st }}$ international trade fair of geodesy, cartography, navigation and geoinformatics GEOS 2006, March 1st-3rd.

Vaux, B., \& Golder, S. (2003). The Harvard dialect survey. Harvard University Linguistics Department.

Vozenilek, V. (2012). Map design. International Cartographic Association, 1-10. Accessed from: https://icaci.org/files/documents/wom/04_IMY_WoM_en.pdf

Wade, T., \& Sommer, S. (2006). A to Z GIS, An illustrated dictionary of geographic information systems. ESRI Press.

Wanderley, C. (2020). Multiculturalism and multilingualism in the digital world: Toward the democratic access to information in the periphery. In S. D. Brunn \& R. Kehrein (Eds.), Handbook of the changing world language map (pp. 3853-3863). Springer.

Watt, D. (2018). Section 1 - Theory introduction. In C. Boberg, J. Nerbonne, \& D. Watt (Eds.), The handbook of dialectology (pp. 17-21). Wiley.

Weber-Pillwax, C. (2004). Indigenous researchers and Indigenous research methods: Cultural influences or cultural determinants of research methods. Pimatisiwin: A Journal of Aboriginal \& Indigenous Community Health, 2(1), 78-90.

Wenker, G. (1877). Sprachkarte der Rheinprovinz [Language map of the Rhine Province]. Das rheinische Platt: Den Lehrern des Rheinlandes gewidmet [he Rhine dialect: Dedicated to 
the teachers of the Rhineland]. Self-published. Accessed from: https://regionalsprache.de/rheinisches-platt.aspx

Weyhe, E. (2004). Some isoglosses in the Faroese language [map]. (no scale). In H. Thráinsson, H. P. Peterson, J. L. Jacobsen, \& Z. S. Hansen (Eds.), Faroese: An overview and reference grammar (p.368). Føroya Fróðskaparfelag.

Whaley, L. J. (1996). Basics of language typology. In L. J. Whaley (Ed.), Introduction to typology: The unity and diversity of language (pp. 7-17). Sage Publications.

Wiegand, P. (2006). Learning and teaching with maps. Routledge.

Wieling, M. B. (2012). A quantitative approach to social and geographical dialect variation. Doctoral dissertation, University of Groningen.

Wikle, T. A. \& Bailey, G. (2010). Mapping North American English. In A. Lameli, R. Kehrein, \& S. Rabanus (Eds.), Language and space, Volume 2: Language mapping (pp. 253-280). De Gruyter Mouton.

Williams, C. H. (1980). Language contact and language change in Wales, 1901-1971. Welsh History Review / Cylchgrawn Hanes Cymru, 10, 207.

Williams, C. H. (1988). 1. An introduction to geolinguistics. In C.H. Williams (Ed.), Language in geographic context (pp. 1-19). Multilingual Matters.

Williams, C. H., \& Ambrose, J. E. (1992). Geolinguistic developments and cartographic problems. Discussion Papers in Geolinguistics, 19, 11-32.

Williamson, I. P. (2000). Best practices for land administration systems in developing countries. In Proceedings of the international conference on land policy reform, Jakarta, Indonesia, $1-32$. 
Wilson, P., \& Hemphill, C. (2017). Supporting community-driven school-based Indigenous language development. Paper presented at the 2017 interdisciplinary centre for social and language documentation conference, Portugal.

Wood, D. (1992). The power of maps. Guilford Press.

Wood, D. (2010). Rethinking the power of maps. Guilford Press.

Wood, D. (2020). Maps/Language. In S. D. Brunn \& R. Kehrein (Eds.), Handbook of the changing world language map (pp. 341-352). Springer.

Wood, D., \& Fels, J. (1986). Designs on signs/myth and meaning in maps. Cartographica: The International Journal for Geographic Information and Geovisualization, 23(3), 54-103.

Woods, F. S. (1922). Higher geometry: An introduction to advanced methods in analytic geometry. Courier Corporation.

Woodward, D., \& Lewis, G. M. (Eds.). (1998). Cartography in the Traditional African, American, Arctic, Australian, and Pacific societies. Humana Press.

Yang, M. (2018). Study on vestiges of Japanese in fishing village language. Journal of Marine and Island Cultures, 7(1), 38-49.

Zhang, M., \& Gong, T. (2016). How many is enough? Statistical principles for lexicostatistics. Frontiers in Psychology, 7(1916). 1-12.

Zhou, K., Fu, C., \& Yang, S. (2016). Big data driven smart energy management: From big data to big insights. Renewable and Sustainable Energy Reviews, 56, 215-225. 
Appendices 
Appendix I: Accompanying Guide to the Evaluative Map Typology (Studies 1 and 2)

\section{Language Mapping Typology -}

\section{Categorized according to defining questions posited by Girnth (2010)}

\section{What is the aim of the map? - PURPOSE}

- Audience: $\quad$ The particular viewers and targets the map was intended for.

- Function: The reason the map was produced in the first place.

- Author: Who the map was created by.

- Context: The social, cultural, ethnic, and political circumstances relevant to when the map was produced.

\section{What is being mapped? - CONTENT}

- Coverage: The geographic and/or political area mapped.

Continent, Country, Province, Municipality.

- Variables: The main focus of the map.

The language, linguistic features (e.g. phonology), speaker populations, genetic (typological) relationships, ethnicity, status (vitality, officiality).

- Number:

If present, how multiple languages are represented together.

How many items are represented together?

How is each item portrayed in relation to one another?

- Uncertainty: How uncertainty or lack of information is dealt with.

Crisp and undefined boundaries, question marks, zipperlike boundaries, what legend symbols are available.

- Origin: Where the data for the maps are coming from.

Other maps, individual informants, aggregate data, linguistic data. 


\section{How is it mapped? - SYMBOLS}

- Colouring:

- Boundaries:

- Data points:

- Georeferencing

- Accompaniment:

- Prominence:

- Units:
The presence/ absence of colour, the extent to which colour is used, and how shading and dark/light contrasts are used.

How linguistic (or other) boundaries are depicted.

Solid boundary lines, "bunches" of isoglosses, quantified line symbols, or diffusion areas.

How data are presented on a map.

Points, lines, polygons, generalized and large-scale chorochromatic maps, quantified choropleth maps, chorochromatic shadings, etc.

Whether or not the data is georeferenced.

Presence/ absence of numerical/ other values alongside data points.

Features that intentionally or accidentally make symbols appear more prominent than others, such as shape or shading.

Whether or not a data point comes from one informant or an aggregation of many will affect the recorded distribution.

\section{How is it mapped? - FEATURES}

- Format:

- Medium:

- Layout:

- Language:

- Orientation:
Vector vs. Raster imaging.

Globe, digital, paper, static, dynamic, etc.

What style of mapping the map most closely resembles.

Mercator, Isodemographic, Equal-Area, Lambert's

conformal, conical projection, Albers conical projection, etc. Also the projection, if available.

The language the map is written in.

Presence/ absence and placement of items that orient viewers. Longitude and latitude lines, scales, legend, true north. 
- Terms: How clearly the map defines its terminology to allow a common understanding among viewers.

E.g. Is the difference between language and dialect clearly stated? If so, how?

- Commentary: The presence/absence of an explanation for the distributions of features (or languages).

Is there a commentary? If so, what does it explain?

\section{What mental picture does the map produce? - RESULT}

- Power:

- Truthfulness:

- Appearance:

- Other Comments:
What the layouts, borders, and languages displayed convey about the statuses and presence of different linguistic/cultural groups. Are the data, symbols, and features presented in such a way to easily convey relevant information in an understandable manner that does not mislead the viewer?

What is clear about the map? What isn't clear?

How visually appealing and attractive to viewers the map is.

Subjective in nature, but a short review here may offer insight into how engaging the map is.

Any other information that may be of use in the analysis. 
Appendix II: The Evaluative Map Typology tool as applied to Study 2

\title{
Map Reference:
}

Title:

\section{The Algonquian Linguistic Atlas}

\author{
Author(s): Marie-Odile Junker, Claire Owen, Delaisie Torkornoo \\ Source: http://www.atlas-ling.ca \\ Ycar: 2005-Current \\ Media type: Online; digital
}

\section{Annotation:}

[from the website] The goal of the project is to co-create an on-line, multimedia linguistic atlas of Algonquian languages. The creation of this atlas allows us to offer many training opportunities for sound editing and linguistic description training to aboriginal students.

One of our goals is also to create contacts between curriculum developers, language specialists and lexicographers of Algonquian languages, with a focus on on-line language resources and dictionaries.

We investigate user-friendly and culturally appropriate computing interfaces and database structures. We encourage dialogue, share our Open Source programs, and provide linguistic and computer training and technical support.

This project is a fertile qround for knowledge transfer and mutual inspiration, with all parties working in a collaborative spirit. Our ultimate goal is to make sure that the beautiful Algonquian languages and the cultures they embody will be heard and spoken by many more generations to come.

\section{What is the aim of the map? - PURPOSE}

\begin{tabular}{l|l|l|}
\cline { 2 - 2 } Audience: & Academics ; students ; community members \\
Function: & $\begin{array}{l}\text { Educate users about the both the distinctiveness found between Algonquian dialects, and the continuity in } \\
\text { features across a geographic space. } \\
\text { Recently, the atlas has started being developed to be used as a language-learning app }\end{array}$ \\
Context: & Indigenous language revitalization ; dialectical studies ; online language learning \\
\cline { 2 - 3 }
\end{tabular}




\section{What is being mapped? - CONTENT}

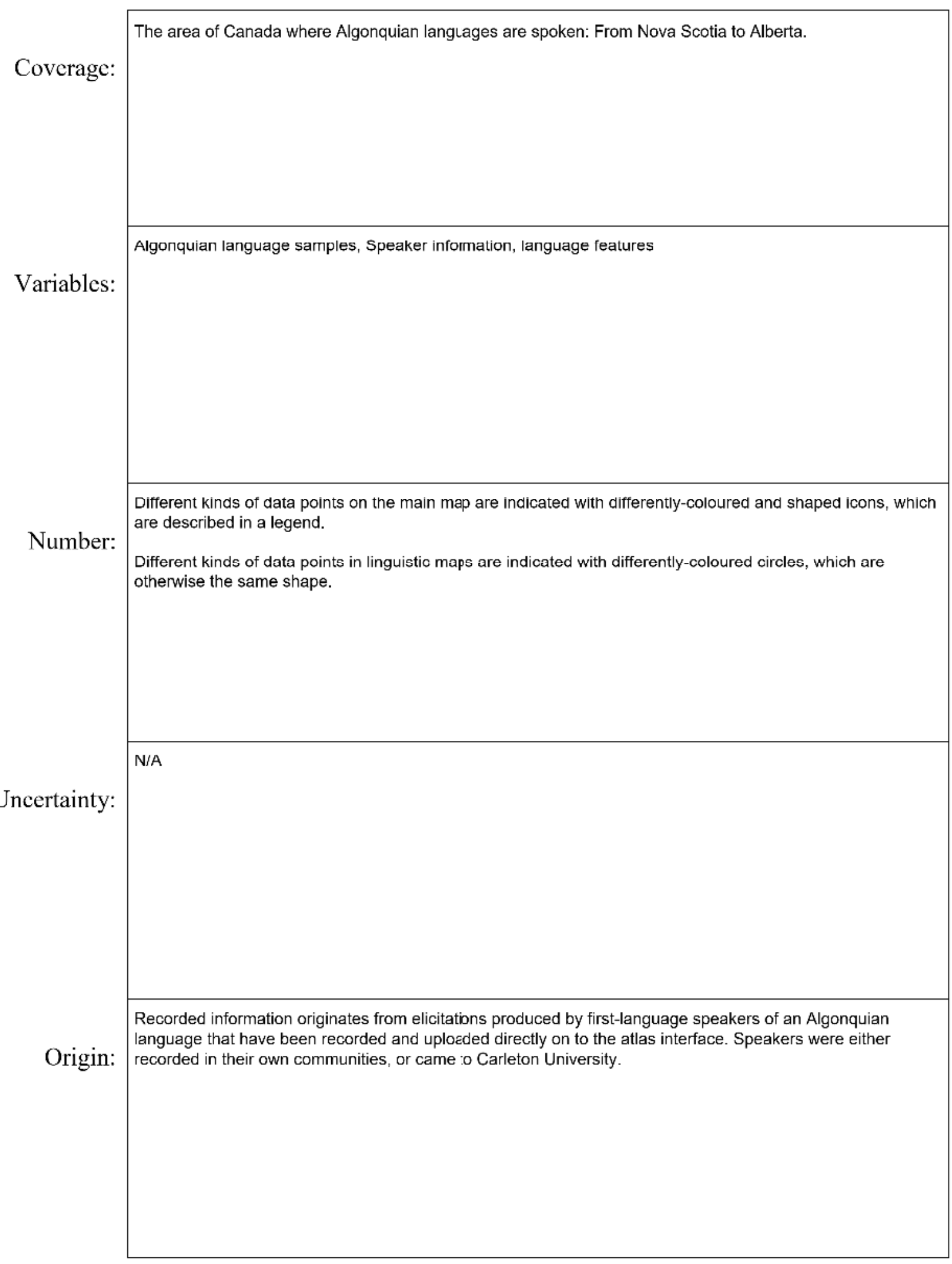




\title{
3. How is it mapped? - SYMBOLS
}

\author{
Georeferencing: \\ Data is Georeferenced \\ $\square$ Data is Not Georeferenced
}

Boundaries:

$\square$ Solid Boundary Lines

"Bunches" of Isoglosses

Other: N/A

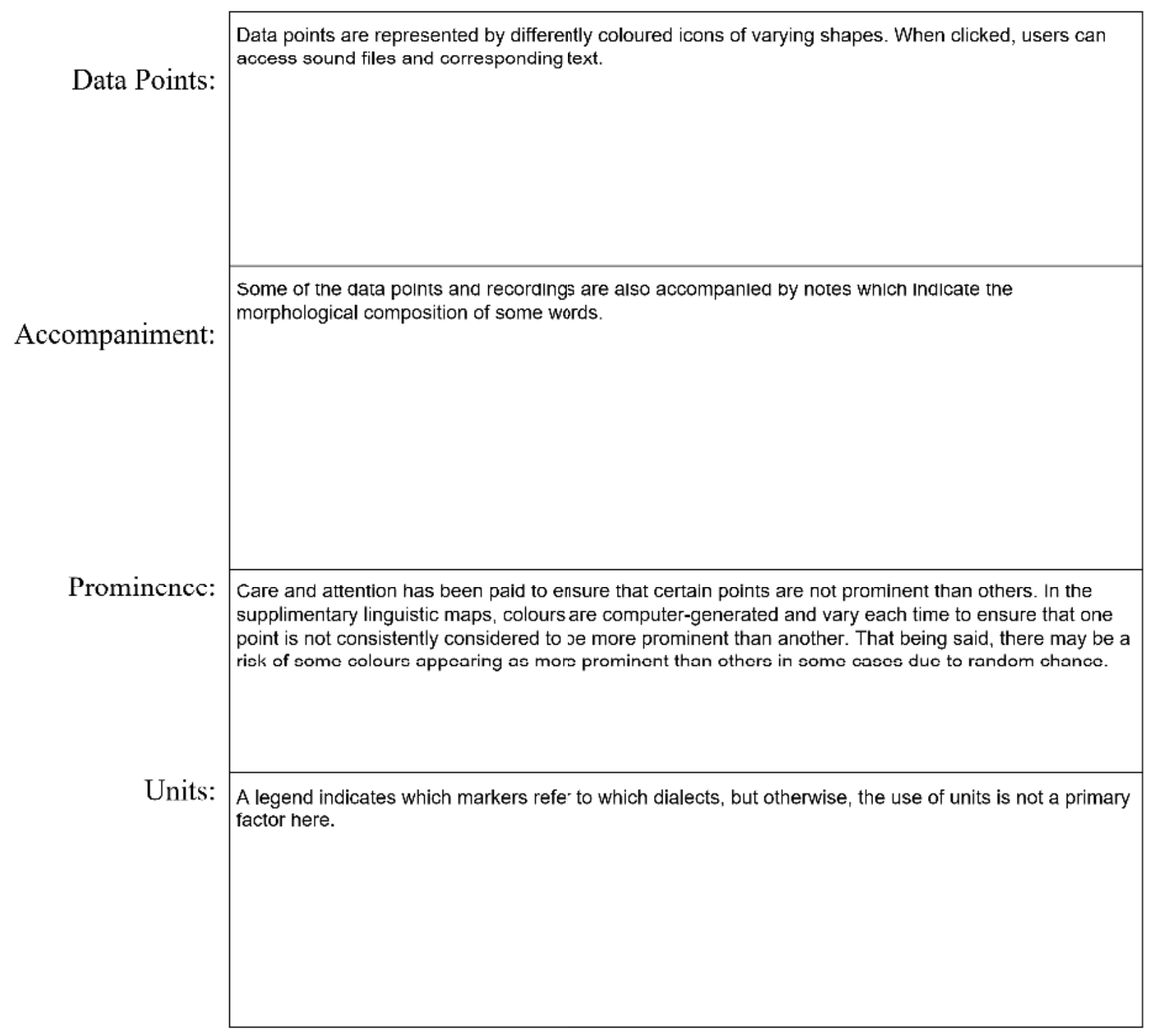




\section{How is it mapped? - FEATURES}

\begin{tabular}{|c|c|c|c|c|}
\hline Format: & $\square$ Vector Imaging & $\square$ Raster Imaging & Other: & \\
\hline Medium: & $\square$ Globe & ఐ Digital & $\square$ Online & $\square$ Static \\
\hline & $\square$ Dynamic & $\square$ Papcr & Other: & \\
\hline
\end{tabular}

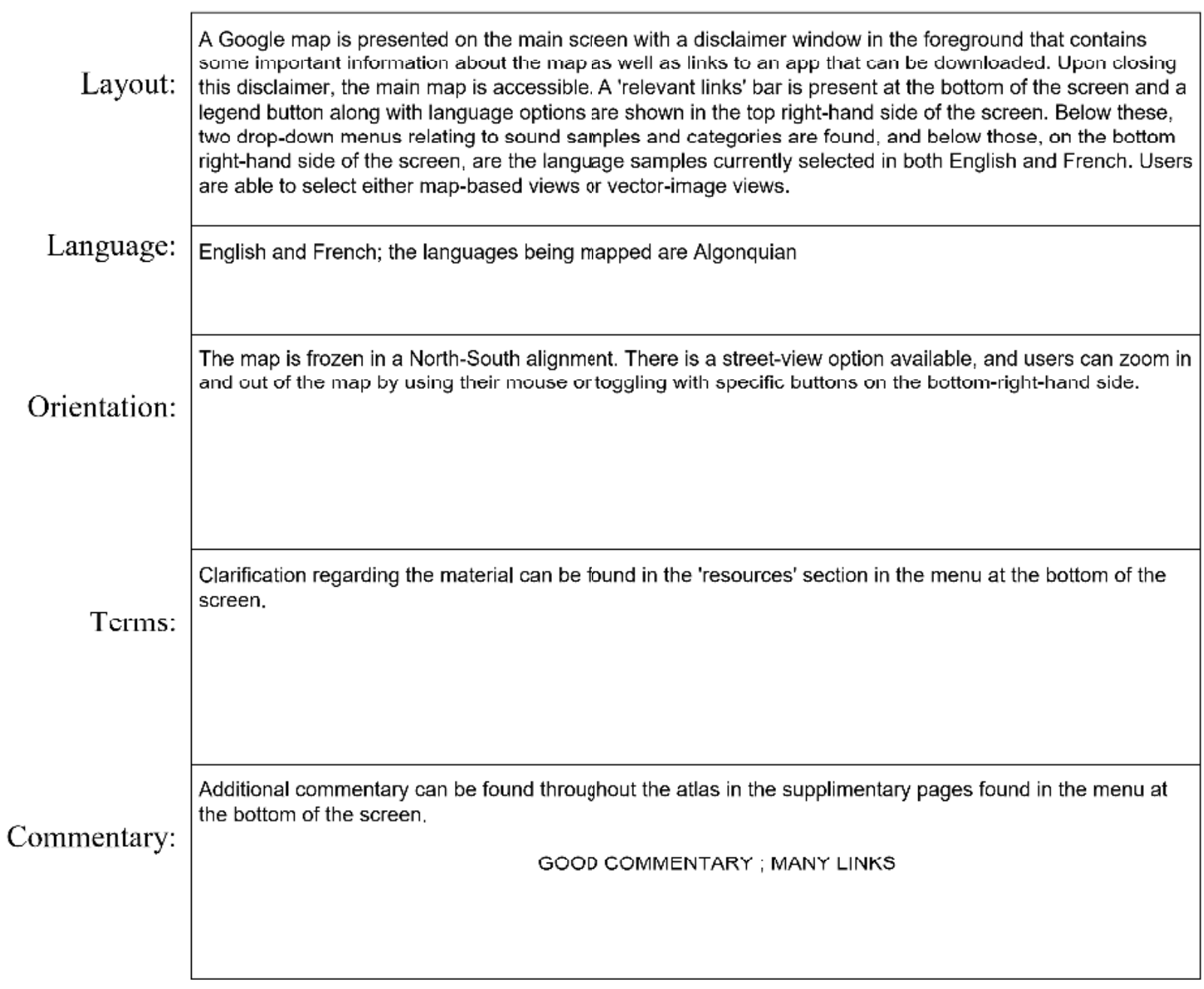




\section{What mental picture does the map produce? - RESULT}

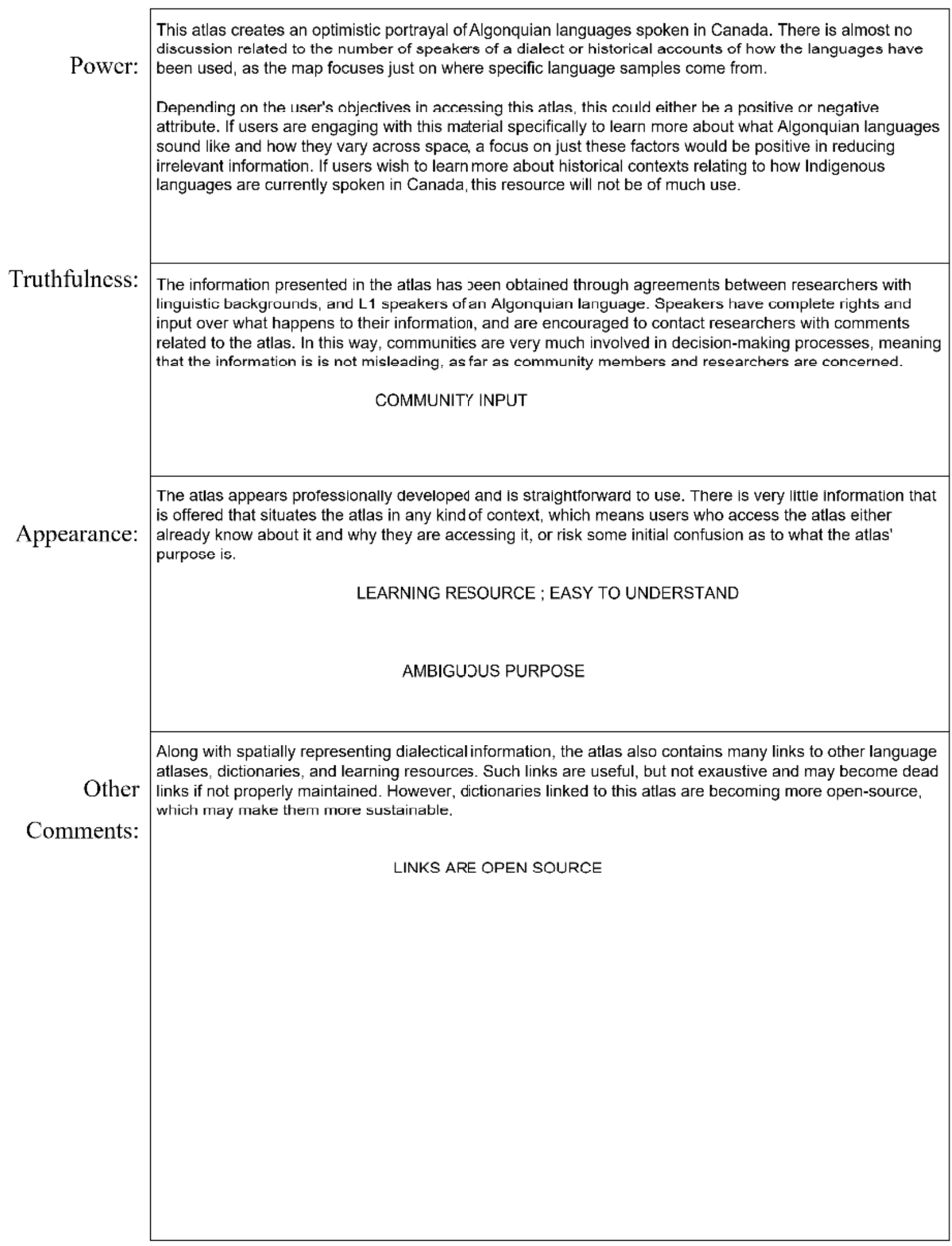




\title{
Map Reference:
}

\section{Title: The First Peoples' Language Map of B.C.}

\author{
Author(s): First Peoples' Heritage, Language, \& Cultural Council \\ Source: http://maps.fpcc.cal \\ Ycar: 2018 \\ Media typc: Online Digital Media
}

\section{Annotation:}

The First Peoples Language Map of British Columbia was initiated by First Peoples' Heritage, Language and Culture Council (FPHLCC) in 2005 with funding from the BC Ministry of Education. FPHLCC staff member and linguist, Sarah Kell, started the language map with contemporary language group boundaries provided by the Museum of Anthropology at the University of British Columbia. Adjustments were made to the language group boundaries based on Indian reserve locations and best available knowledge of language spoken by First Nations affiliated with reserve lands. Additional historical information on territories of sleeping languages was prepared for the Handbook of North American Indians (1996, revised 1999) under the supervision of Dr. Ives Goddard, and is reproduced with permission of the Smithsonian Institution. Additional map data was added from a number of sources (see Map Data Sources). The online map was created by Global CADD Systems.

In 2008, Alex Wadsworth and Towagh Behr led the expansion of the online map into a website with content on the languages, First Nations and Community Champions. The development of the web-based Drupal content management system was completed by Patrick Hayes of MapsWest. To build content for the website, Towagh completed a short series of interviews with fluent speakers from around the province. Alex, Towagh and Patrick also turned the FPHLCC Language Needs Assessments into online surveys that continue to populate the website with current statistics on language fluency and learning.

\section{What is the aim of the map? - PURPOSE}

\begin{tabular}{l|l|l|}
\hline Audience: & $\begin{array}{l}\text { Indigenous and non-Indigenous individuals interested in learning more about the distribution of Indigenous } \\
\text { languages in B.C. }\end{array}$ \\
Function: & $\begin{array}{l}\text { To showcase the linguistic diversity found in B.C. One selectable feature displays each community's level of } \\
\text { internet connectivity. An additional selectable feature allows users to view only which languages have currently } \\
\text { ceased to have any speakers, which is a powerul tool for communicating the serious situation faced by these } \\
\text { languages. Also, to compare the size of linguistic areas to their comparatively minute corresponding reserves. }\end{array}$ \\
Context: & $\begin{array}{l}\text { Linguistic diversity; language loss; linguistic distibution; linguistic/territorial boundaries in contrast to national and } \\
\text { provincial political boundaries. }\end{array}$ \\
\hline
\end{tabular}




\section{What is being mapped? - CONTENT}

\begin{tabular}{|c|c|}
\hline Coverage: & British Columbia and neighbouring provinces, states, and territories in which the languages also reside. \\
\hline Variables: & $\begin{array}{l}\text { 1. Internet Connectivity } \\
\text { 2. "Contemporary" Indigenous language spoken and their geographic coverage } \\
\text { 3. "Sleeping" Indigenous language spoken and their geographic coverage } \\
\text { 4. Individual Communities } \\
\text { 5. Reserves and their geographic coverage }\end{array}$ \\
\hline Number: & $\begin{array}{l}203 \text { First Nations Communities ; } 38 \text { languages. } \\
\text { Many languages overlap with one another, which is indicated on the map by areas of different colours being } \\
\text { superimposed on one another to create a new, darker colour. In addition, every time the pointer hovers over a } \\
\text { region, the colour disappears and is replacec by the name of the language. In areas where there is overlap, } \\
\text { every language is shown. }\end{array}$ \\
\hline Uncertainty: & Any degree of uncertainty is not accounted for on this map. \\
\hline Origin: & $\begin{array}{l}\text { The data comes from a variety of sources, which are displayed in a comprehensive bibliography on a linked } \\
\text { web page. Each bibliographic entry contains the following information: } \\
\qquad \text { ID\# ; Author(s) ; Date ; Title [with a link to the material] ; Language ; Type (e.g. web, print, etc.) } \\
\text { Sources for geographic and population-related information come primarily from Statistics Canada, and Indian } \\
\text { and Northern Affairs Canada. }\end{array}$ \\
\hline
\end{tabular}




\section{How is it mapped? - SYMBOLS}

$\square$ Data is Georeferenced

Georeferencing:

Data is Not Georeferenced

Boundaries:

- Solid Boundary Lines

"Bunches" of Isoglosses
Quantified Line Symbols

$\square$ Diffusion Areas

Other:

Overlapping regions, which blend colour

1. Linguistic regions denoted by a shaded in area of uniform colour.

Data Points: 2. Dots representing individual communities.

3. Dots representing linguistic materials, such as sound recordings.

4. Dots representing connectivity statuses for communities

5. Dots representing items of interest, such as band offices or friendship societies.

6. Dots that, when clicked, display links to websites where the user can learn more.

1. Linguistic regions display the language's name when selected.

Accompaniment: 2 . Dots representing individual communities display the community name and language when hovered over and show relevant links when clicked on.

3. Dots representing linguistic materials show relevant links when clicked on.

4. Dots representing connectivity statuses for communities show relevant links when clicked on.

Promincnce:

Regions with languages that are related to one another will appear in similar colours (though this isn't indicated. This user happens to know this because of prior linguistic knowledge). Though many colours and many shades thereof are shown, the authors did a good job of not making a particular area more prominent than another, with the exception of Cree, which is a shade of grey.

Units:

Though not explicitly stated, the data likely comes from aggregations. 


\section{How is it mapped? - FEATURES}

Format: ■ Vector Imaging घ Raster Imaging Other:

Medium:

$\begin{array}{lll}\square \text { Globe } & \square \text { Digital } & \square \text { Online } \\ \square \text { Dynamic } & \square \text { Paper } & \text { Other: }\end{array}$

Map of British Columbia using a number of different possible overlays, such as Google Street Maps, Satellite Layout: Photos, and a terrain-based raster map.

Language:

English

Zoom features are present, but users cannot navigate horizontally by dragging the map. There are arrows

Orientation: which can be clicked on to navigate in a paticular direction, but they appear not to work.

A rudimentary scale in the form of a ratio is also provided. interestingly, a legend is absent.

Terms:

An accompanying glossary page is provided, but only three items are currently present.

Commentary:

Little information regarding the context of how these languages exist in their space is offered. 


\section{What mental picture does the map produce? - RESULT}

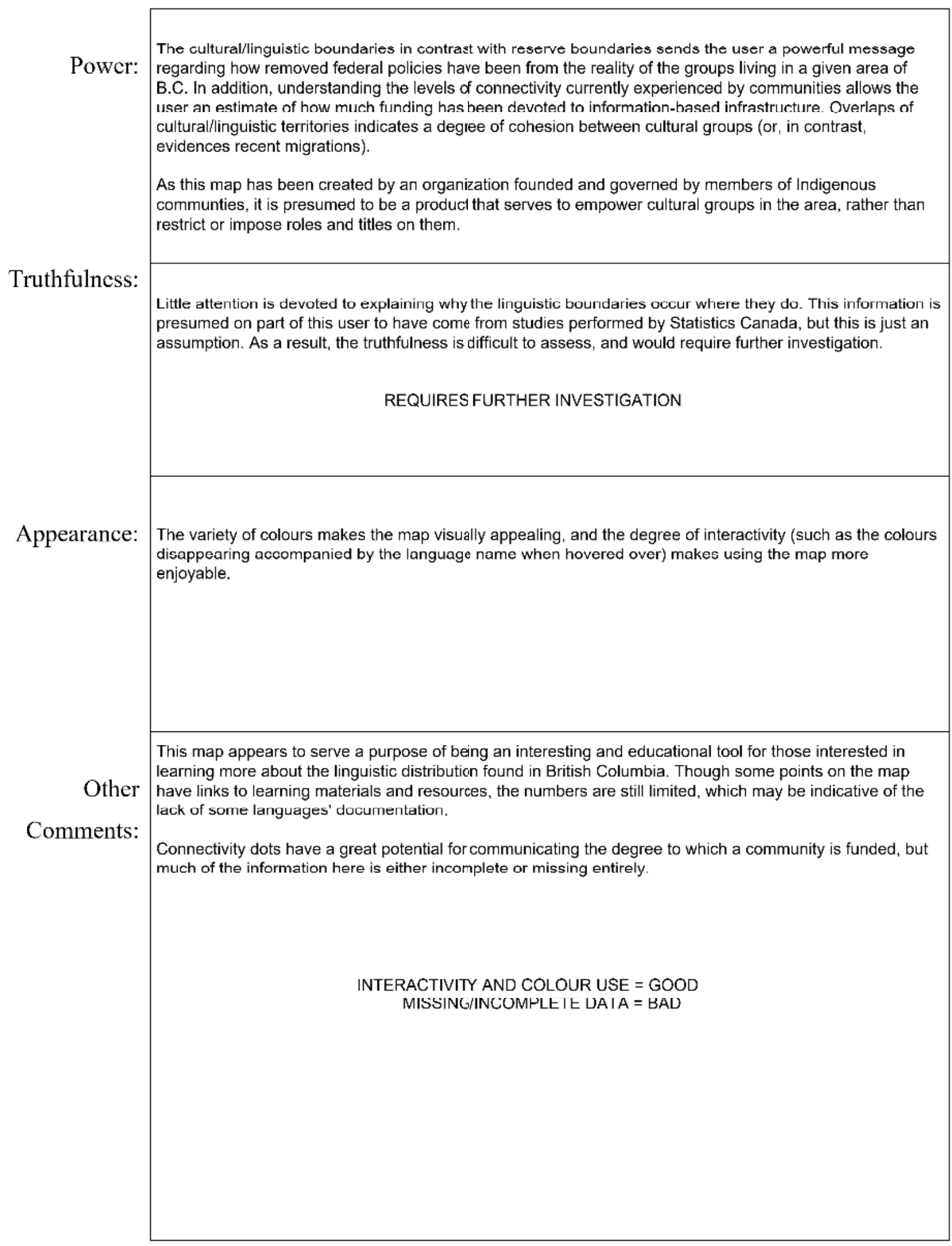




\title{
Map Reference:
}

Title:

\section{LL-MAP: Language and Location - Map Accessibility Project}

\author{
Author(s): Linguist List (no individual authors listed, though there are a number of contributing organizations) \\ Source: http://www.llmap.org/map/viewer/ \\ Ycar: not indicated \\ Media typc: Online ; Digital ; collection of multiple maps (as opposed to layers)
}

\section{Annotation:}

"LL-MAP is a project designed to integrate language information with data from the physical and social sciences by means of a Geographical Information System (GIS). The most important part of the project is a language subsystem, which relates geographical information on the area in which a language is or has been spoken to data on resources relevant to the language. Through a link to the MultiTree project, information on all proposed genetic relationships of the languages is made available and viewable in a geographic context. The system walso includes ancillary information on topography, political boundaries, demographics, climate, vegetation, and wildlife, thus providing a basis upon which to build hypotheses about language movement across territory. Some cultural information, e.g., on religion, ethnicity, and economics, is also included."

"The LL-MAP system encourages collaboration between linguists, historians, archaeologists, ethnographers, and geneticists, as they explore the relationship between lanquage and cultural adaptation and change. We hope it will elicit new insights and hypotheses, and that it will also serve as an educational resource. As a GIS, LL-MAP has the potential to be a captivating instructional tool, presenting complex data in a way accessible to all educational levels. Finally, as a free service available online, LL-MAP increases public knowledge of lesser-known languages and cultures, underlining the importance of language and linguistic diversity to cultural understanding and scientific inquiry."

\section{What is the aim of the map? - PURPOSE}

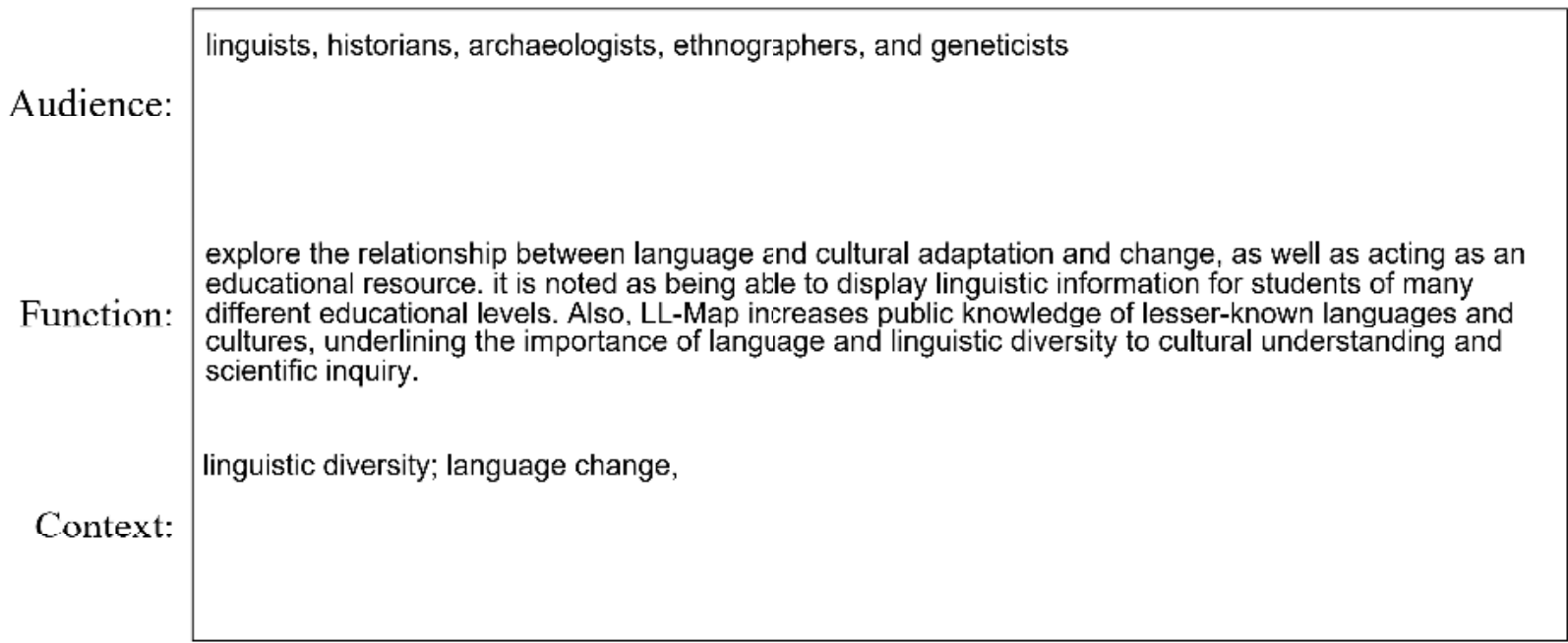




\section{What is being mapped? - CONTENT}

\begin{tabular}{|c|c|}
\hline Coverage: & localized areas depending on the specific map, but in sum the whole world. \\
\hline Variables: & $\begin{array}{l}\text { depends on the map being viewed: } \\
\text { 1. The locations of languages/dialects } \\
\text { 2. Phonological features } \\
\text { 3. Sociolinguistic variables such as the rated "pleasantness of speech" } \\
\text { 4. Lexical items } \\
\text { 5. The distribution of ethnolinguistic groups }\end{array}$ \\
\hline Number: & depends on the map \\
\hline Jncertainty: & depends on the map \\
\hline \multirow{3}{*}{ Origin: } & $\begin{array}{l}\text { Depends on the map; the data were not criginally gathered for the interactive map. Instead, if they are } \\
\text { hosted on the interactive map, they come from previous studies. }\end{array}$ \\
\hline & $\begin{array}{l}\text { Other maps are not part of the interactive map. Instead, some are static images that are linked to from } \\
\text { an atlas menu. }\end{array}$ \\
\hline & OTHER STUDIES \\
\hline
\end{tabular}




\section{How is it mapped? - SYMBOLS}

\begin{tabular}{|c|c|c|}
\hline \multirow{2}{*}{ Georeferencing: } & \multicolumn{2}{|l|}{$\square$ Data is Georeferenced } \\
\hline & \multicolumn{2}{|l|}{$\square$ Data is Not Georeferenced } \\
\hline \multirow[t]{3}{*}{ Boundaries: } & $\square$ Solid Boundary Lines & $\square$ Quantified Line Symbols \\
\hline & $\square$ "Bunches" of Isoglosses & $\square$ Diffusion Areas \\
\hline & Other: $\frac{\text { depends on the map }}{2}$ & \\
\hline
\end{tabular}

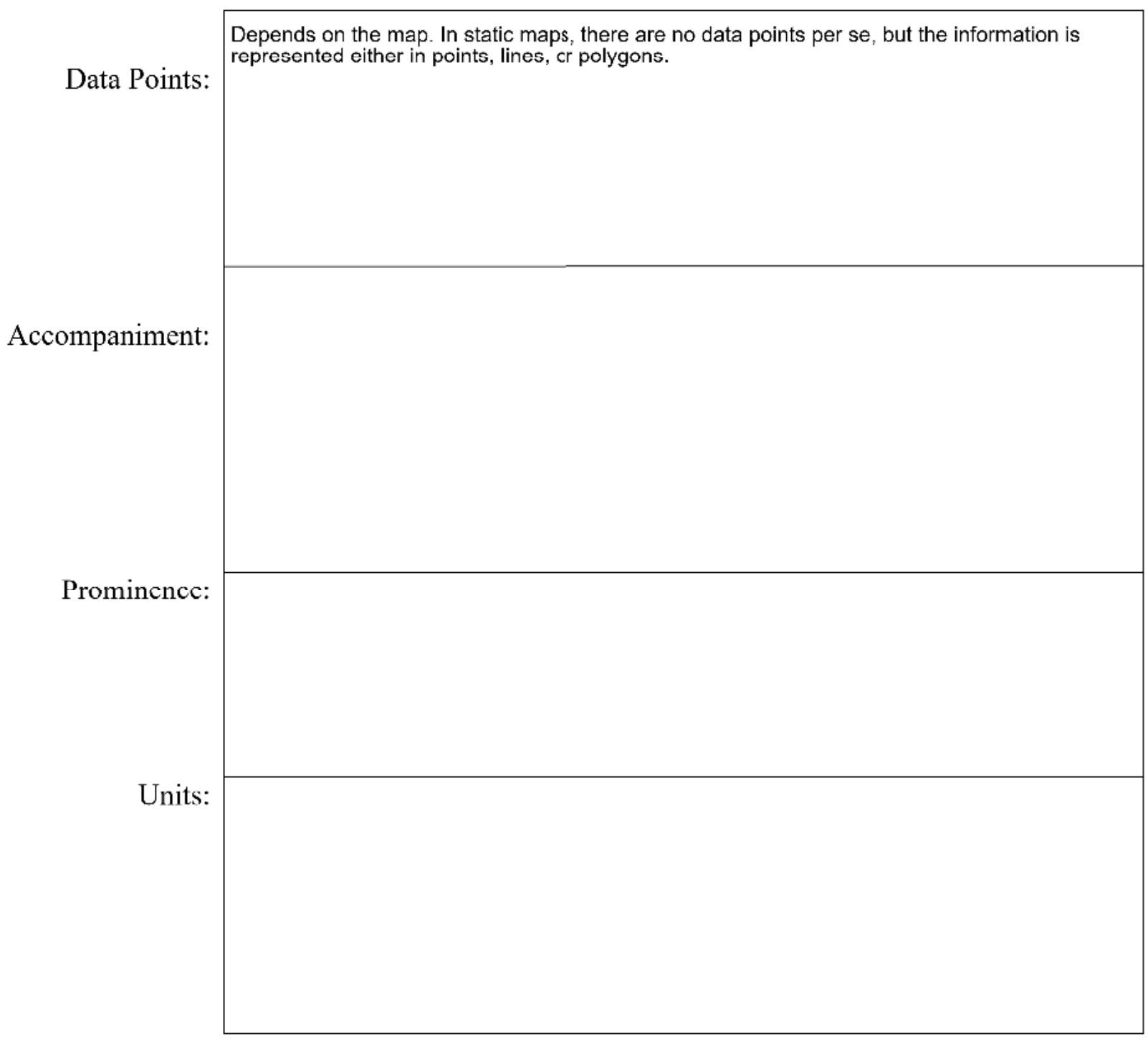




\section{How is it mapped? - FEATURES}

Format: $\square$ Vector Imaging $\square$ Raster Imaging Other:

Medium: $\square$ Globe $\square$ Digital $\square$ Online $\square$ Static

$\square$ Dynamic $\square$ Paper Other:

The main atlas interface first displays a view of OpenStreetMap, with a small button on the top left-hand side that, when clicked, opens a menu that allows users to search by language codes,

Layout: project names, or regions. There are no other features immediately available, making this map minimalist in nature.

MINIMALIST

Language:

English

fixed north-south orientation

Orientation:

FIXED

There does not appear to be a feature that explicitly defines terms

Tcrms:

Commentary:

Each map link, when double clicked on, leads to a page with a short commentary that can be read before the map is accessed. However, the commentary is not present along with the maps. 


\section{What mental picture does the map produce? - RESULT}

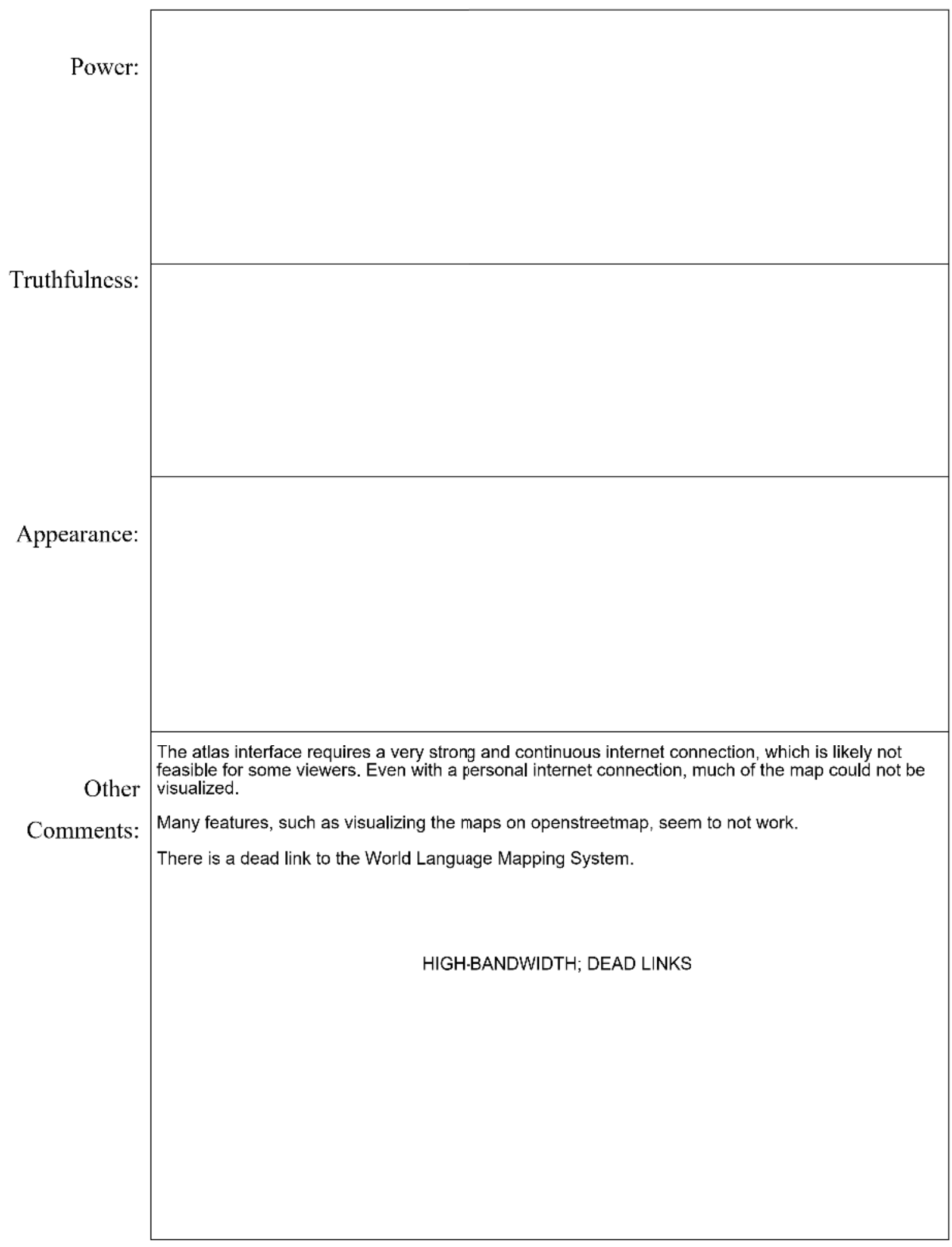




\title{
Map Reference:
}

\section{Title: Modern Language Association (MLA) map}

\author{
Author(s): Modern Language Association (MLA) \\ Source: https://www.mla.org/Resources/Research/MLA-Language-Map \\ Ycar: unknown \\ Mcdia typc: Online ; Digital ; Multilayer Digital Map
}

\section{Annotation:}

"The MLA Language Map is for students, teachers, and anyone interested in learning about the linguistic and cultural composition of the United States. The Language Map uses aggregated data from the 2006-10 American Community Survey (ACS) to display the locations and numbers of speakers of twelve languages commonly spoken in the United States."

\section{What is the aim of the map? - PURPOSE}

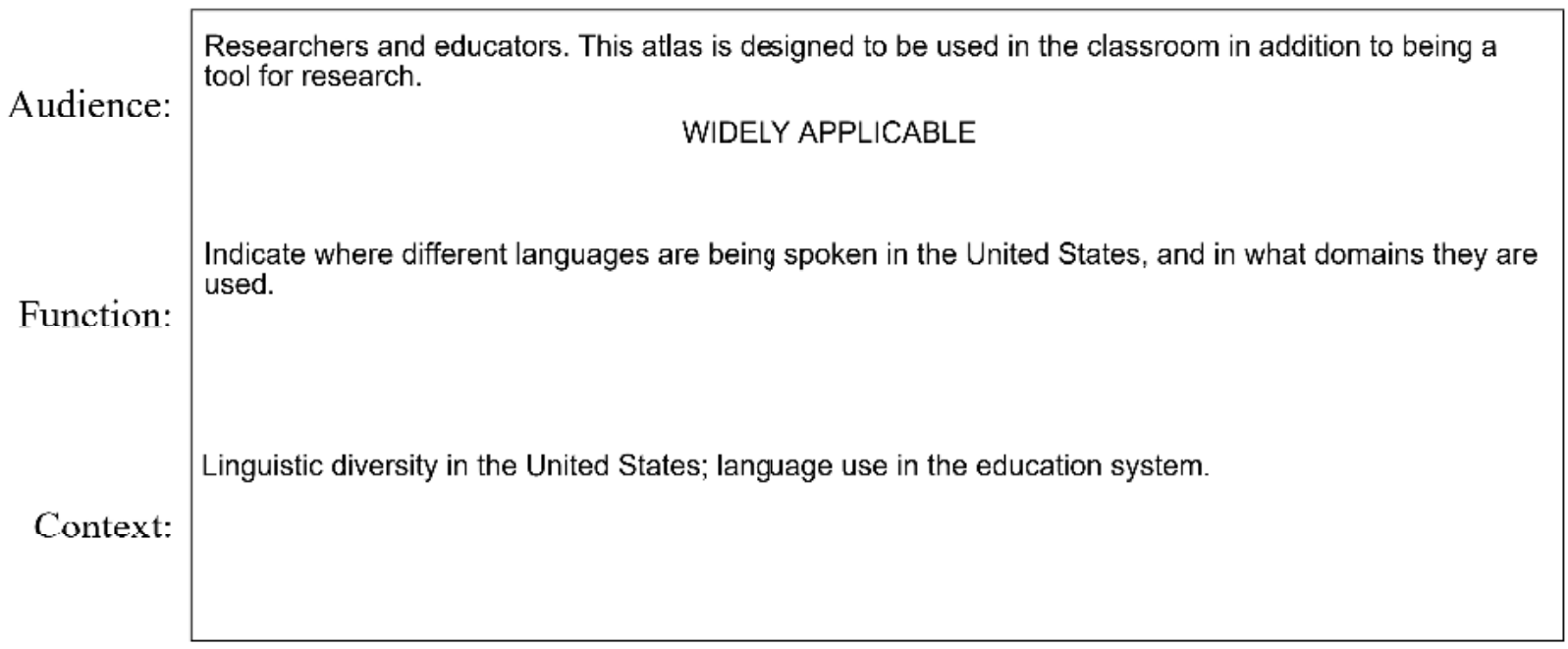




\section{What is being mapped? - CONTENT}

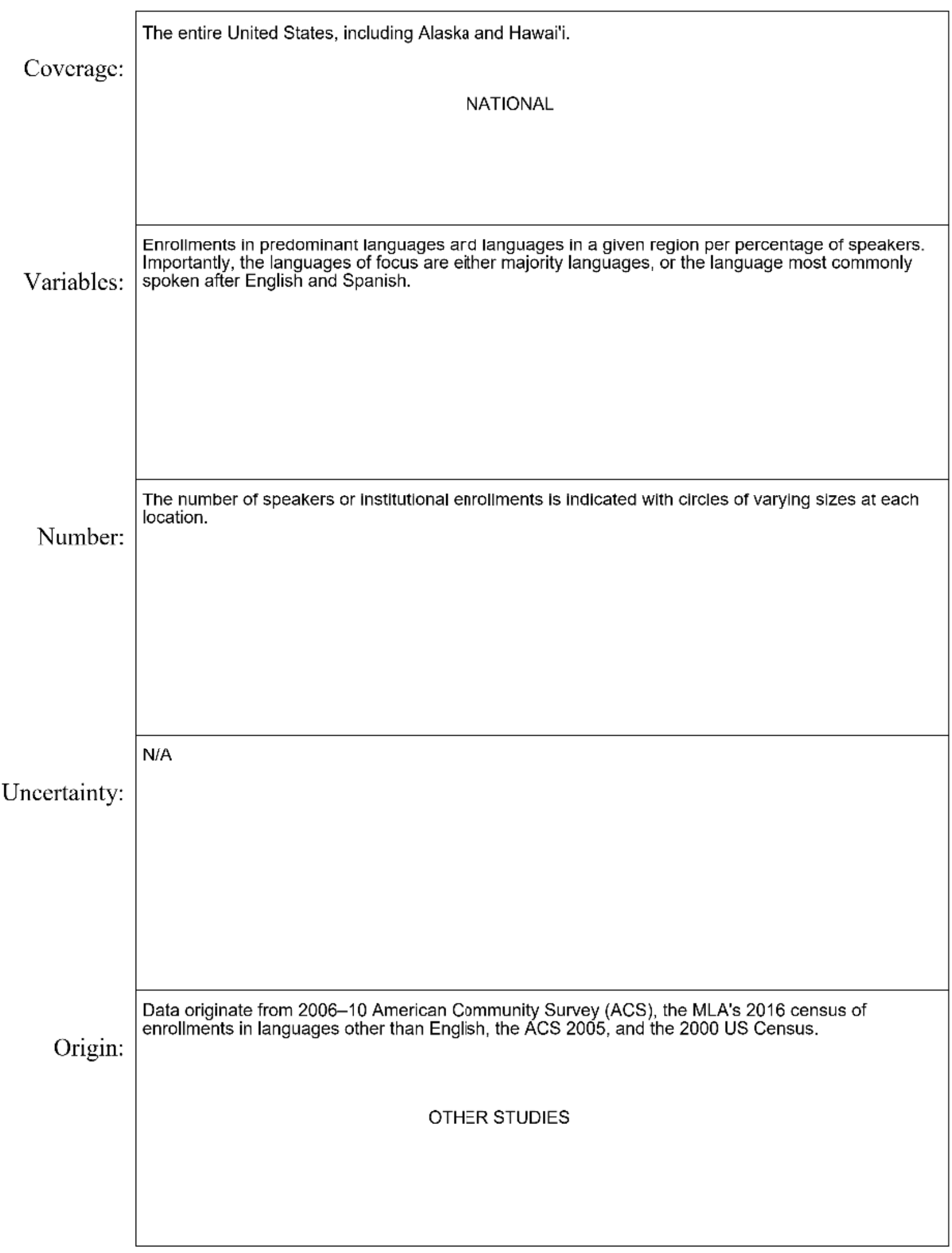




\section{How is it mapped? - SYMBOLS}

$\square$ Data is Georeferenced

Georeferencing:

\section{Data is Not Georeferenced}

Boundaries:

$\square$ Solid Boundary Lines

Quantified Line Symbols

"Bunches" of Isoglosses

Diffusion Areas

Other: N/A

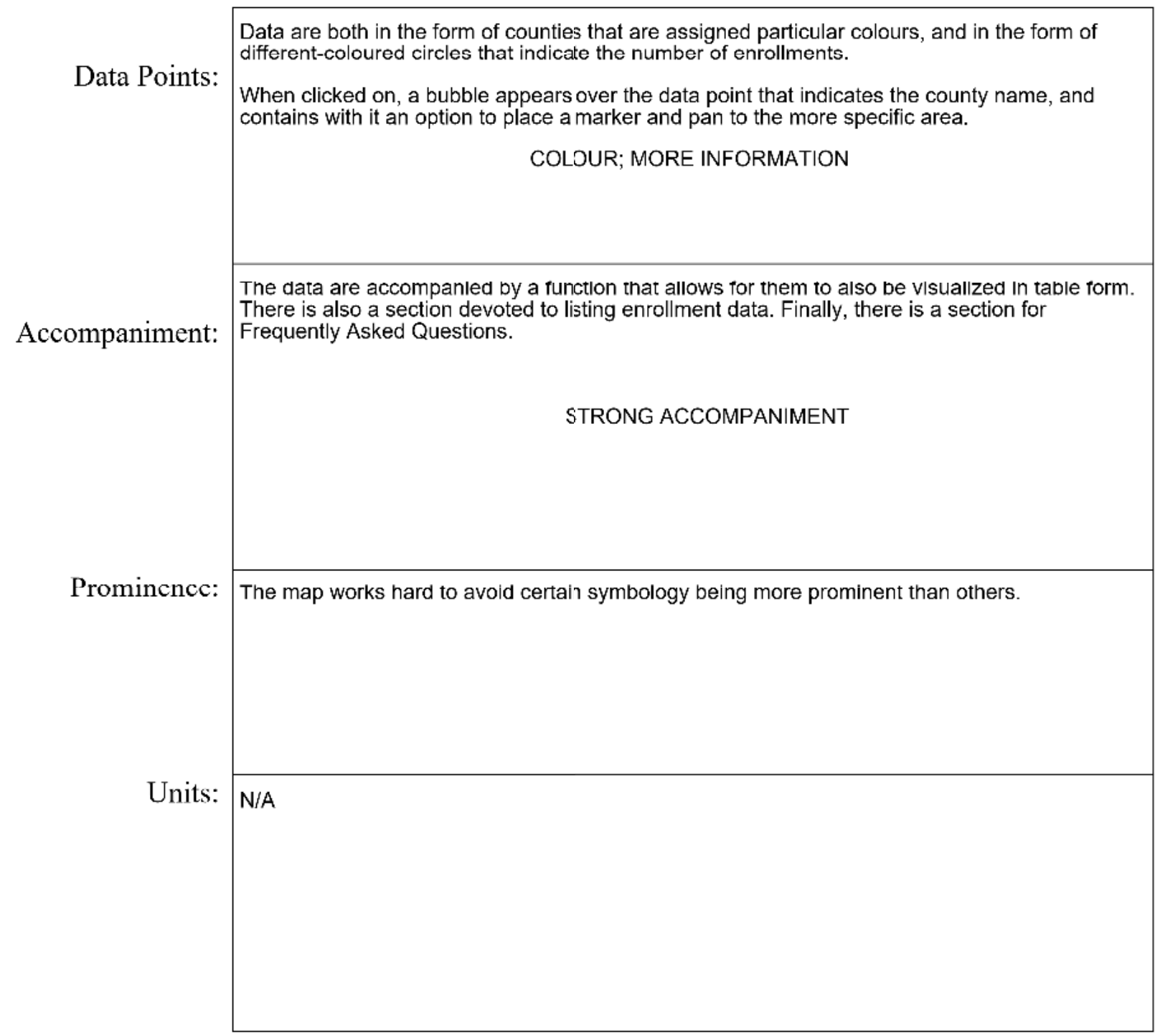




\section{How is it mapped? - FEATURES}

\begin{tabular}{|c|c|c|c|c|}
\hline Format: & $\square$ Vector Imaging & $\square$ Raster Imaging & Other: & \\
\hline Medium: & $\square$ Globe & $\square$ Digital & $\checkmark$ Online & $\square$ Static \\
\hline & $\square$ Dynamic & $\square$ Paper & Other: & \\
\hline
\end{tabular}

The map is presented on an ArcGIS web application, with the legend presented on the right side of the map and a list of tick boxes on the lower left-hand side. These tick boxes signify operational

Layout: layers, which can be expanded for more nuanced layer selection.

On the top left-hand corner, there is a short guide to using the map, which is complete with links for more information or to contact the map authors.

\section{MANY LAYERS}

\section{Language: \\ Orientation:}

English

the map is in a fixed orientation from noth to south. a zoom feature is available.

\section{Commentary:}

In place of a dedicated commentary, there is a section of the atlas website that has numerous links to related articles, databases, and descriptions about both the map and its materials. Many of these are not specific to the map, but are more generally applicable to the MLA's research on language enrollment statistics.

LINKS ELSEWHERE 


\section{What mental picture does the map produce? - RESULT}

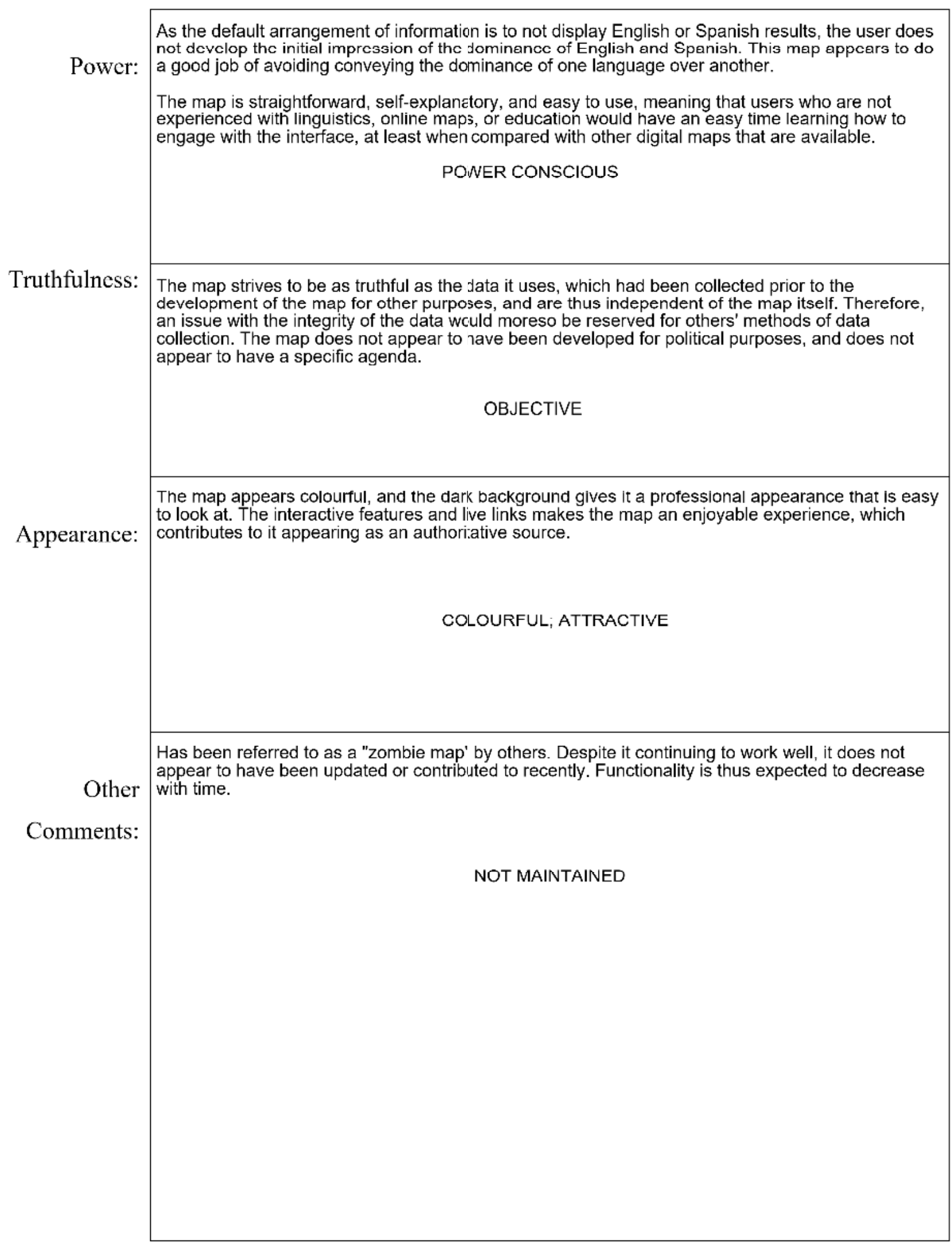




\title{
Language Mapping Typology: Initial Notes Form
}

\section{Map Reference:}

Title:

\section{Native Land}

\author{
Author(s): victor G Temprano \\ Source: http://www.native-land.ca \\ Ycar: 2015 - Present \\ Media typc: Online ; Digital ; Multilayer Digital Map
}

\section{Annotation:}

[From the website] "Native-Land.ca started in early 2015, during a time of a lot of resource development projects in British Columbia. While mapping out pipeline projects and learning more about them for the sake of public awareness, I started to ask myself whose territories all these projects were happening on. Once I started finding the geographic data and mapping... well, it just kind of expanded from there."

"I feel that Western maps of Indigenous nations are very often inherently colonial, in that they delegate power according to imposed borders that don't really exist in many nations throughout history. They were rarely created in good faith, and are often used in wrong ways. I am open to criticism about this project and I welcome suggestions and changes."

"I'm Victor. I am a settler, born in traditional Katzie territory and raised in the Okanagan. I am concerned about many of the issues raised by using maps and colonial ways of thinking when it comes to maps. For instance, who has the right to define where a particular territory ends, and another begins? Who should I speak to about such matters, anyway?"

\section{What is the aim of the map? - PURPOSE}

\begin{tabular}{l|l|l|}
\hline Audience: & $\begin{array}{l}\text { Primarily members of non-Indigenous societies who wish to learn more about Indigenous territories and } \\
\text { become engaged in Indigenous issues. However, this map may also be of use to Indigenous } \\
\text { communities for specific purposes. }\end{array}$ \\
Function: & $\begin{array}{l}\text { ANDIGENOUS; PUBLIC } \\
\text { Ownership of information }\end{array}$ \\
Context: & Colonial domination of maps, publicly available knowledge about territory and land \\
\hline
\end{tabular}




\section{What is being mapped? - CONTENT}

\begin{tabular}{|c|c|}
\hline Coverage: & $\begin{array}{l}\text { Indigenous territories, treaty areas, and lenguages in Canada, Australia, New Zealand, South } \\
\text { America, and some Pacific islands. } \\
\qquad \text { GLOBAL }\end{array}$ \\
\hline Variables: & Indigenous territories, languages, and treaty areas \\
\hline Number: & Number is not explicitly accounted for. Each item on the map is expressed with a polygon. \\
\hline Uncertainty: & $\begin{array}{l}\text { While uncertainty isn't reflected directly in the map, the author explains that this map is a work in } \\
\text { progress, notes that he is not an expert, and encourages users to think critically about what they see. } \\
\text { UNCERTAINTY ACCOUNTED FOR }\end{array}$ \\
\hline Origin: & $\begin{array}{l}\text { A large list of contributors is mentioned elsewhere on the atlas website. The list is long, varied, and } \\
\text { subject to change when new contributors are added. } \\
\text { MULTIPLE SOURCES }\end{array}$ \\
\hline
\end{tabular}




\title{
3. How is it mapped? - SYMBOLS
}

\author{
Georeferencing: \\ Data is Georeferenced \\ $\square$ Data is Not Georeferenced
}

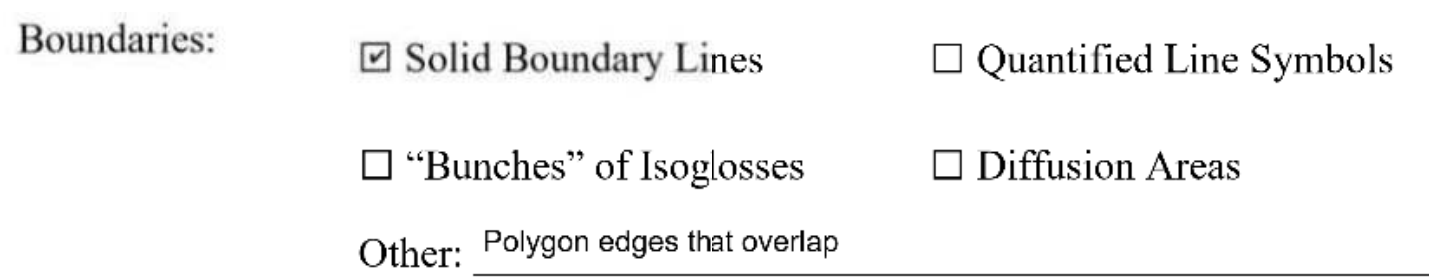

\begin{tabular}{|c|c|}
\hline Data Points: & $\begin{array}{l}\text { In polygon form: each polygon is a different colour and transparent as to show overlap between } \\
\text { territories/areas. } \\
\text { OVERLAPPING POLYGONS }\end{array}$ \\
\hline Accompaniment: & $\begin{array}{l}\text { Names of places/languages accompany each polygon. When cllcked, a Ink to the people who } \\
\text { traditionally occupied the area appears on the left-hand side of the screen, which, when } \\
\text { followed, links to another page with even more links to websites that have more information. } \\
\text { LINKED TO INFORMATION }\end{array}$ \\
\hline Prominence: & $\begin{array}{l}\text { While some colours are in theory more prominent than others (e.g. red), the polygons' } \\
\text { transparency diminishes much of the inequality in prominence that could occur. An option is } \\
\text { provided to remove colours entirely from the polygons. } \\
\text { EQUAL PROMINENCE }\end{array}$ \\
\hline Units: & N/A \\
\hline
\end{tabular}




\section{How is it mapped? - FEATURES}

\begin{tabular}{|c|c|c|c|c|}
\hline Format: & v Vector Imaging & $\square$ Raster Imaging & Other: & \\
\hline Medium: & $\square$ Globe & ఐ Digital & $\checkmark$ Online & $\square$ Static \\
\hline & $\square$ Dynamic & $\square$ Papcr & Other: & \\
\hline
\end{tabular}

Main map takes up the majority of the screen. When first accessed, a disclaimer appears on the main screen that the map is a work in progress, and provides links to access more information. On Layout: $\begin{aligned} & \text { the left-hand side, there is a box that allows users to select layers: Territory, Language, or Treaty, } \\ & \text { along with an option to enter an address to find out what communities are nearby. In a box below } \\ & \text { this, }\end{aligned}$ this, users can select specific territories, languages, or treaty areas.

\section{USER LED (or) GUIDED TOUR}

Language:
Orientation:

Available in English or French

North-to-South. The Mercator projection system used causes land masses at the polar extremes to be larger than those toward the equator

While terms are not explicitly defined, names of lands/languages have links to web pages that

Terms: describe the people's histories in greater detail.

\section{Commentary:}

\section{LINKED TO INFO}

Commentary is not provided directly in the map. However, it is provided elsewhere in the atlas website. 


\section{What mental picture does the map produce? - RESULT}

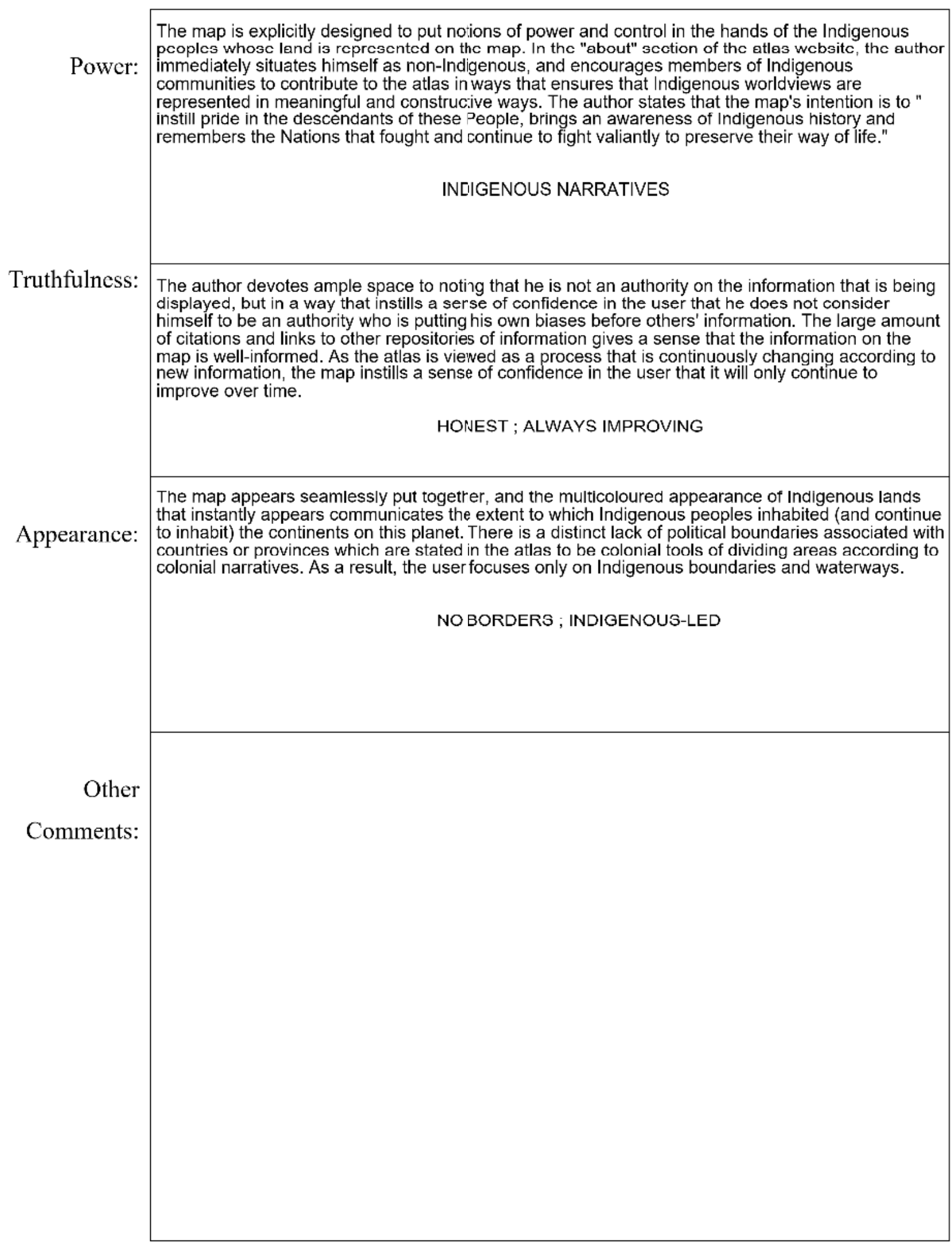




\title{
Map Reference:
}

\section{Title: PHOIBLE}

\author{
Author(s): Moran, Steven \& McCloy, Daniel \\ Source: https://phoible.org/ \\ Ycar: 2019 \\ Media typc: Online ; Digital ; Multilayer Digital Map
}

\section{Annotation:}

[from the website]

"PHOIBLE is a repository of cross-linguistic phonological inventory data, which have been extracted from source documents and tertiary databases and compiled into a single searchable convenience sample. Release 2.0 from 2019 includes 3020 inventories that contain 3183 segment types tound in 2186 distinct languages."

\section{What is the aim of the map? - PURPOSE}

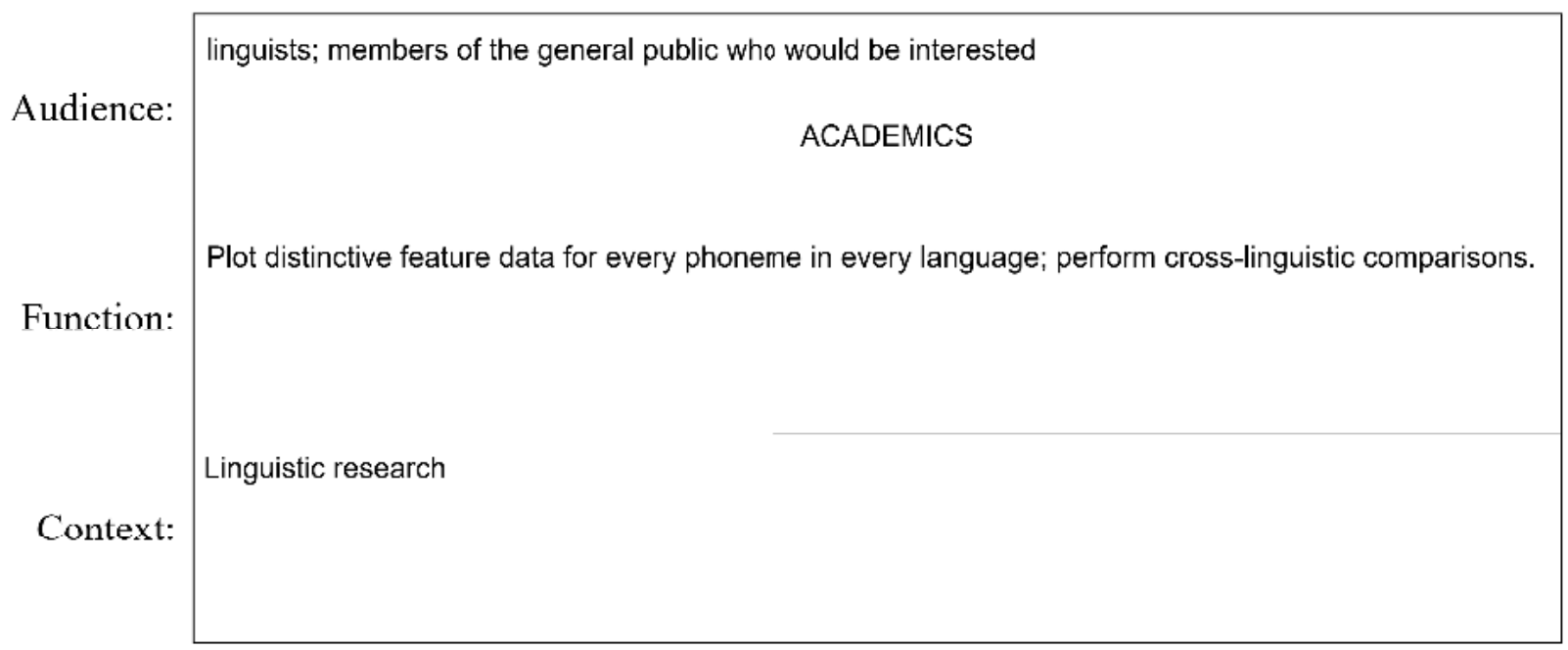




\section{What is being mapped? - CONTENT}

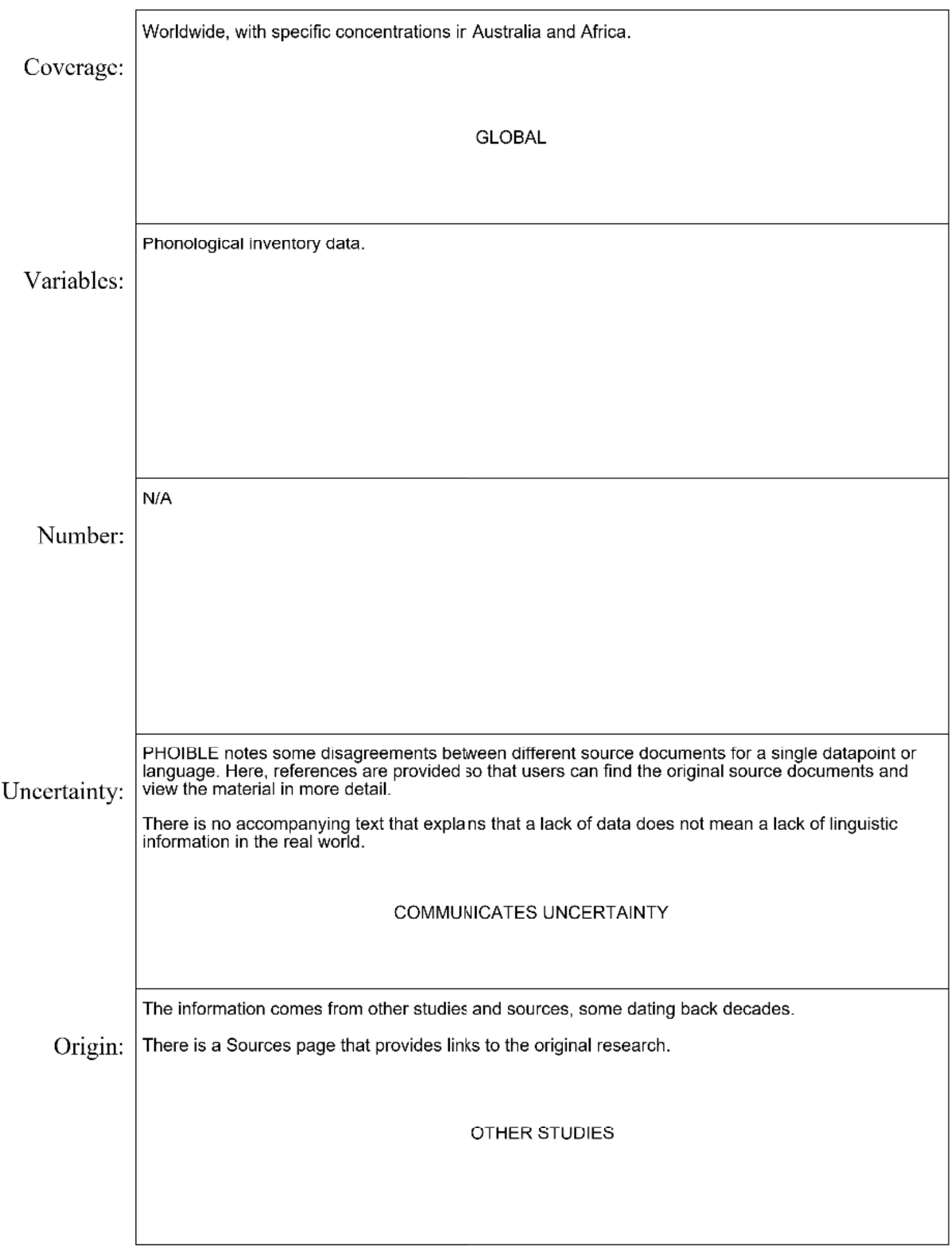




\section{How is it mapped?-SYMBOLS}

$\square$ Data is Georeferenced

Georeferencing:

\section{Data is Not Georeferenced}

Boundaries:

$\square$ Solid Boundary Lines

Quantified Line Symbols

"Bunches" of Isoglosses

Diffusion Areas

Other: N/A

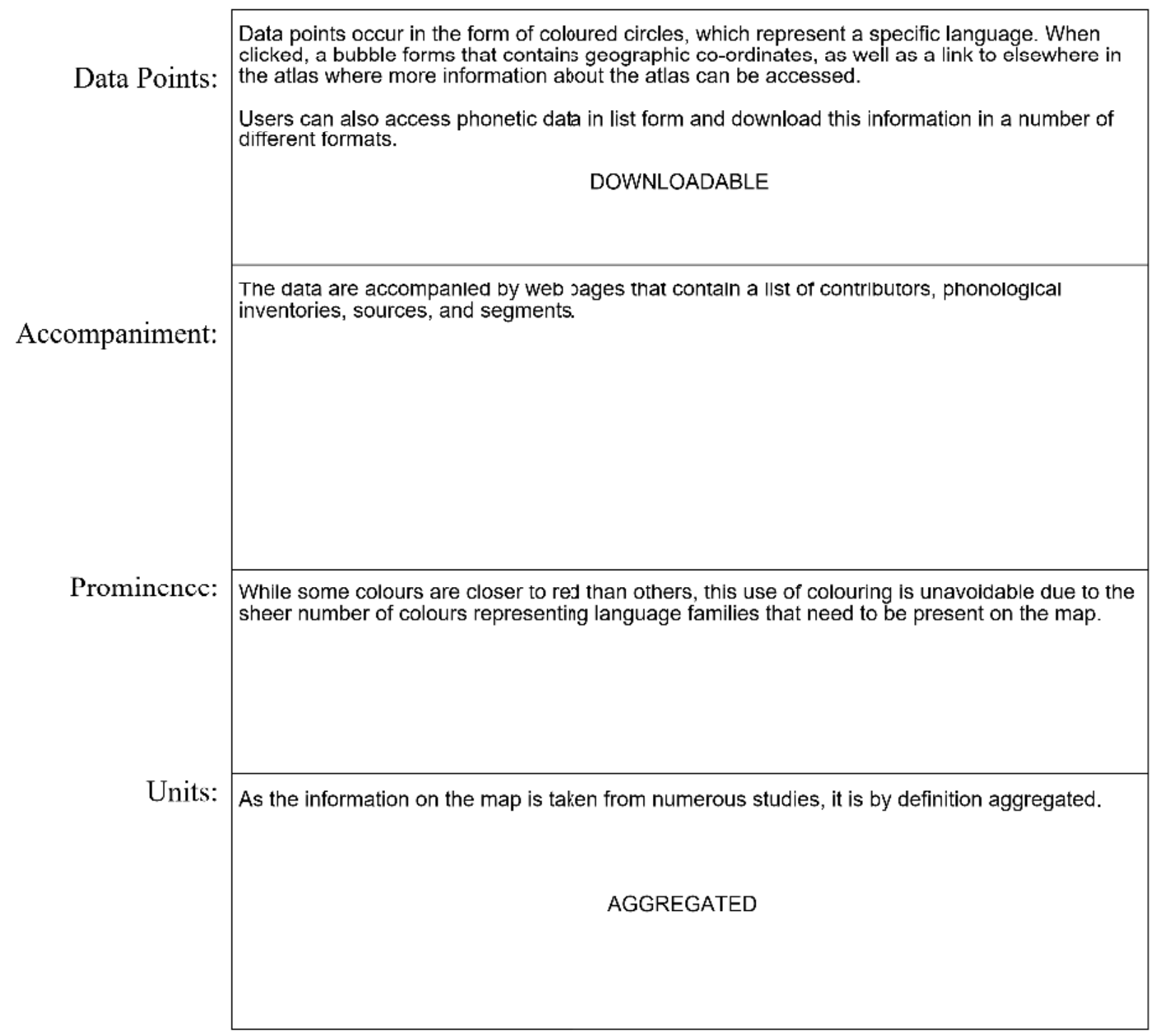




\section{How is it mapped? - FEATURES}
Format:
Vector Imaging
Raster Imaging
Other:
Medium:
$\square$ Globe
Digital
$\square$ Online
Static
Dynamic
Paper
Other:OpenStreetMap

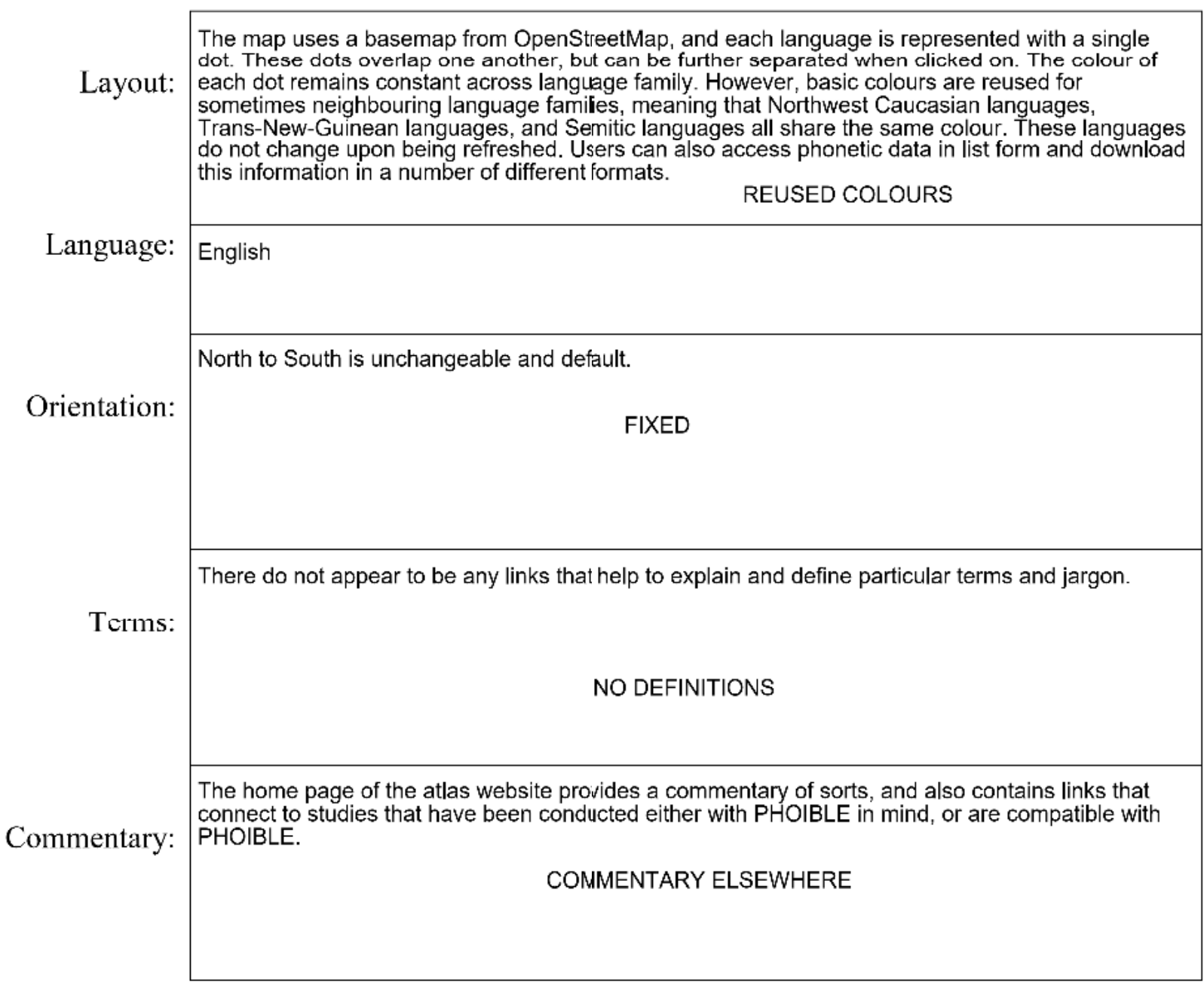




\section{What mental picture does the map produce? - RESULT}

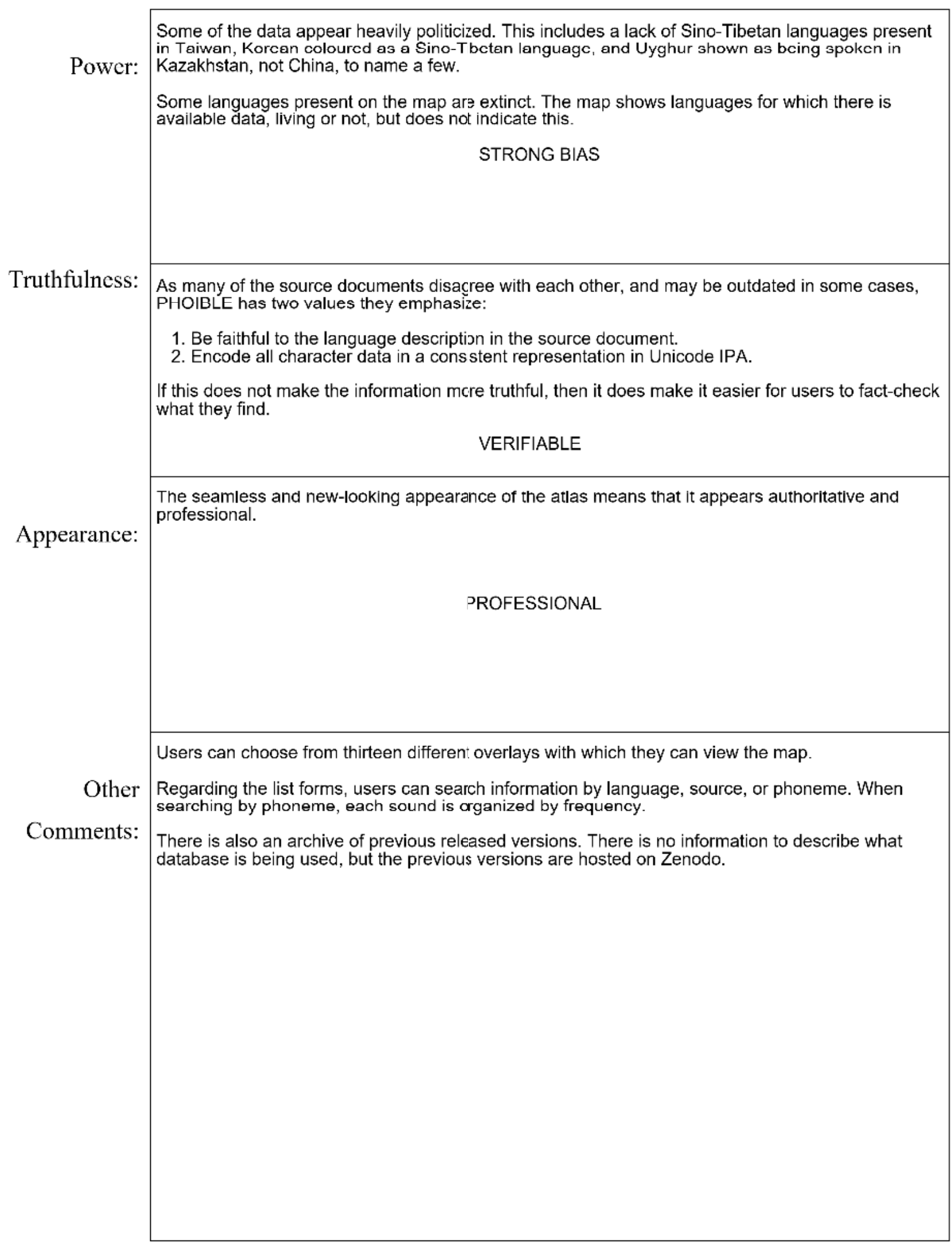




\title{
Map Reference:
}

\section{Title: Sprachatlas Niederbayern}

\author{
Author(s): Prof. Dr. Rüdiger Harnisch \\ Source: https://sprachatlasniederbayern.bayerische-landesbibliothek-online.de/ \\ Ycar: 2009 - present \\ Media type: Online ; Digital ; Multilayer Digital Map
}

\section{Annotation:}

"The around 6,000 audio files from 207 Lower Bavarian and 22 Czech locations have been compiled and academically evaluated by the chair for German linguistics of the University of Passau within the framework of various linguistic-geographical projects in the course of several years. The Bayerische Staatsbibliothek (BSB) has integrated the audio files into 32 digital maps, thereby making the exciting service accessible to the public via Bayerische Landesbibliothek Online, and additionally ensuring the long-time archiving of the recordings."

\section{What is the aim of the map? - PURPOSE}

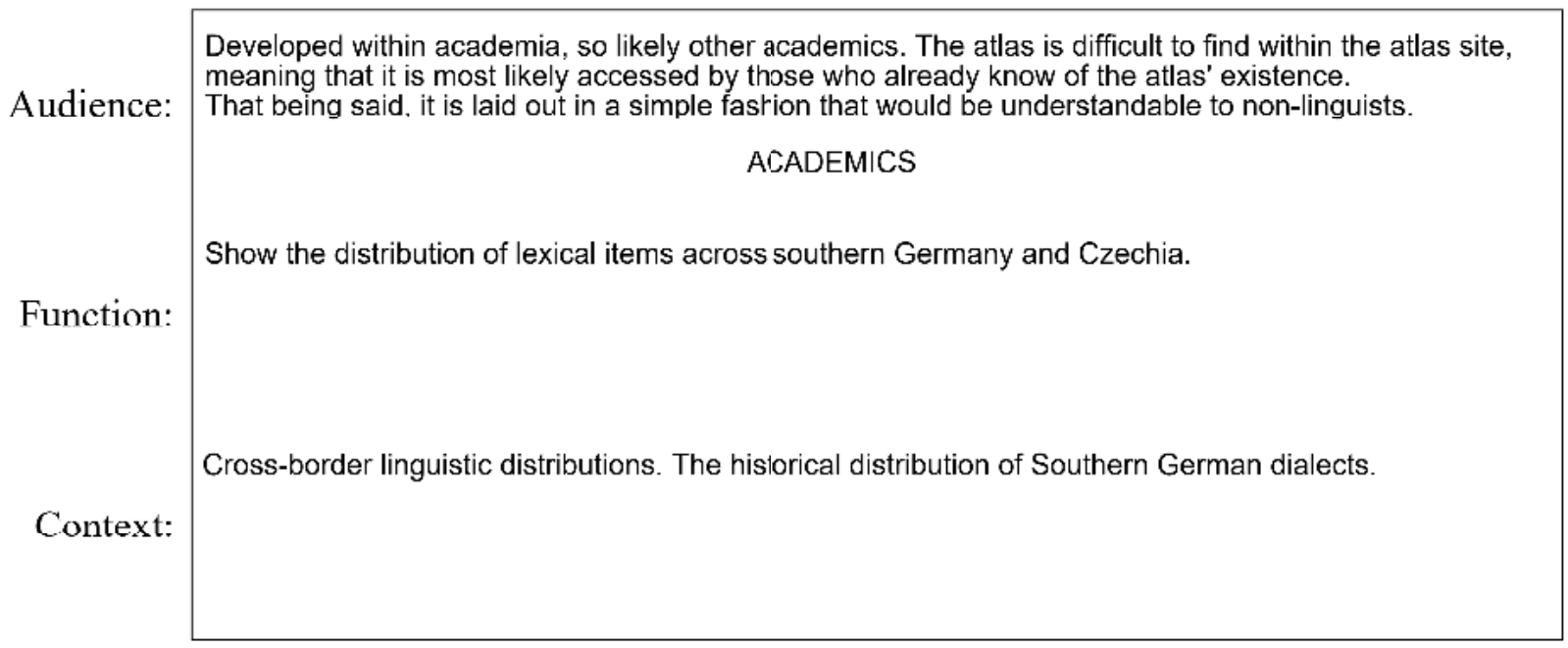




\section{What is being mapped? - CONTENT}

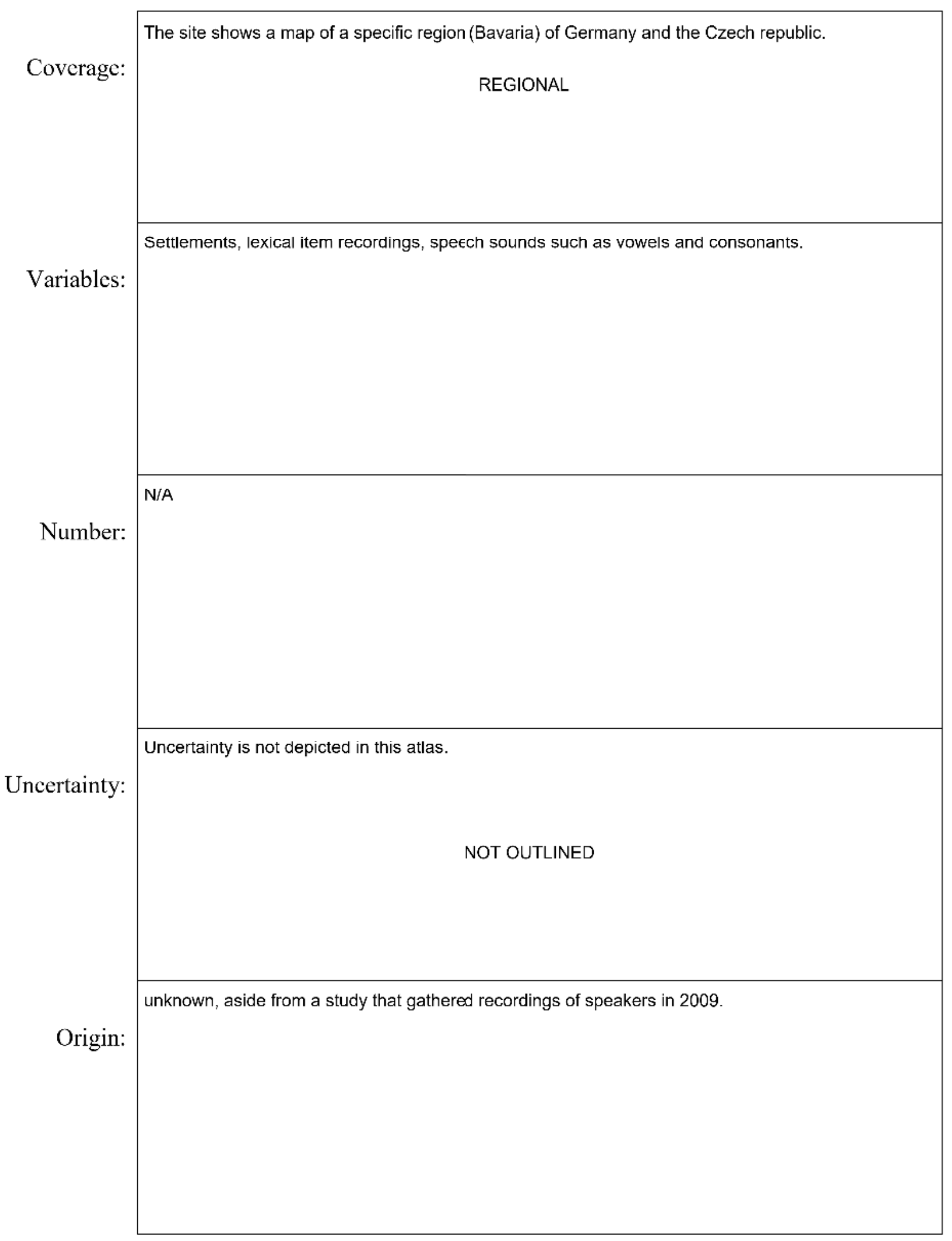




\section{How is it mapped? - SYMBOLS}

$\begin{array}{ll}\text { Georeferencing: } \quad & \square \text { Data is Georeferenced } \\ & \square \text { Data is Not Georeferenced } \\ \text { Boundaries: } \quad \square \text { Solid Boundary Lines } \quad \square \text { Quantified Line Symbols } \\ \\ \square \text { "Bunches" of Isoglosses } \quad \square \text { Diffusion Areas } \\ \text { Other: }{ }_{\text {discrete boundaries between polygons, also crosshatching }}\end{array}$

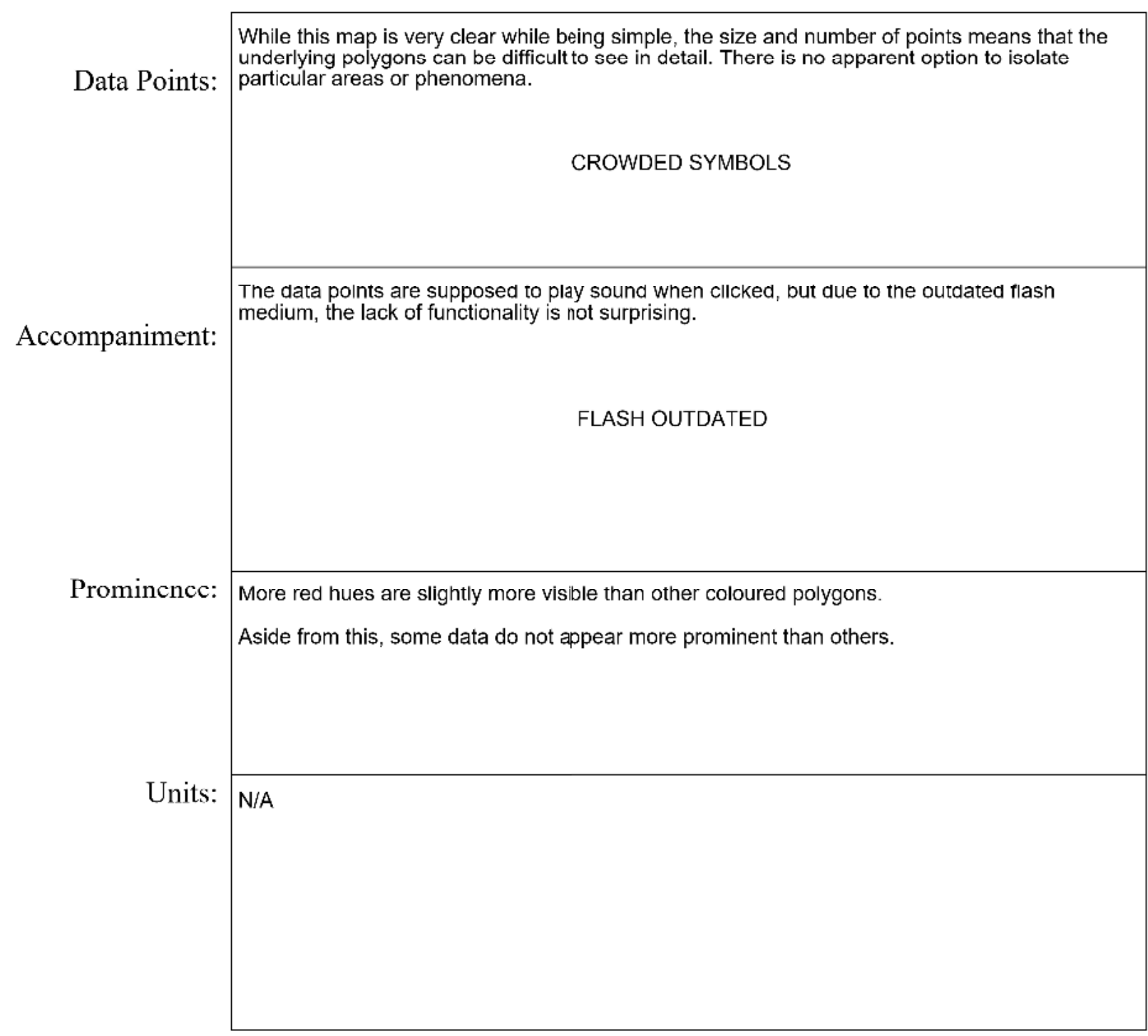




\section{How is it mapped? - FEATURES}

\begin{tabular}{|c|c|c|c|c|}
\hline Format: & $\square$ Vector Imaging & $\square$ Raster Imaging & Other: & \\
\hline Medium: & $\square$ Globe & ఐ Digital & $\square$ Online & $\square$ Static \\
\hline & $\square$ Dynamic & $\square$ Papcr & Other: & \\
\hline
\end{tabular}

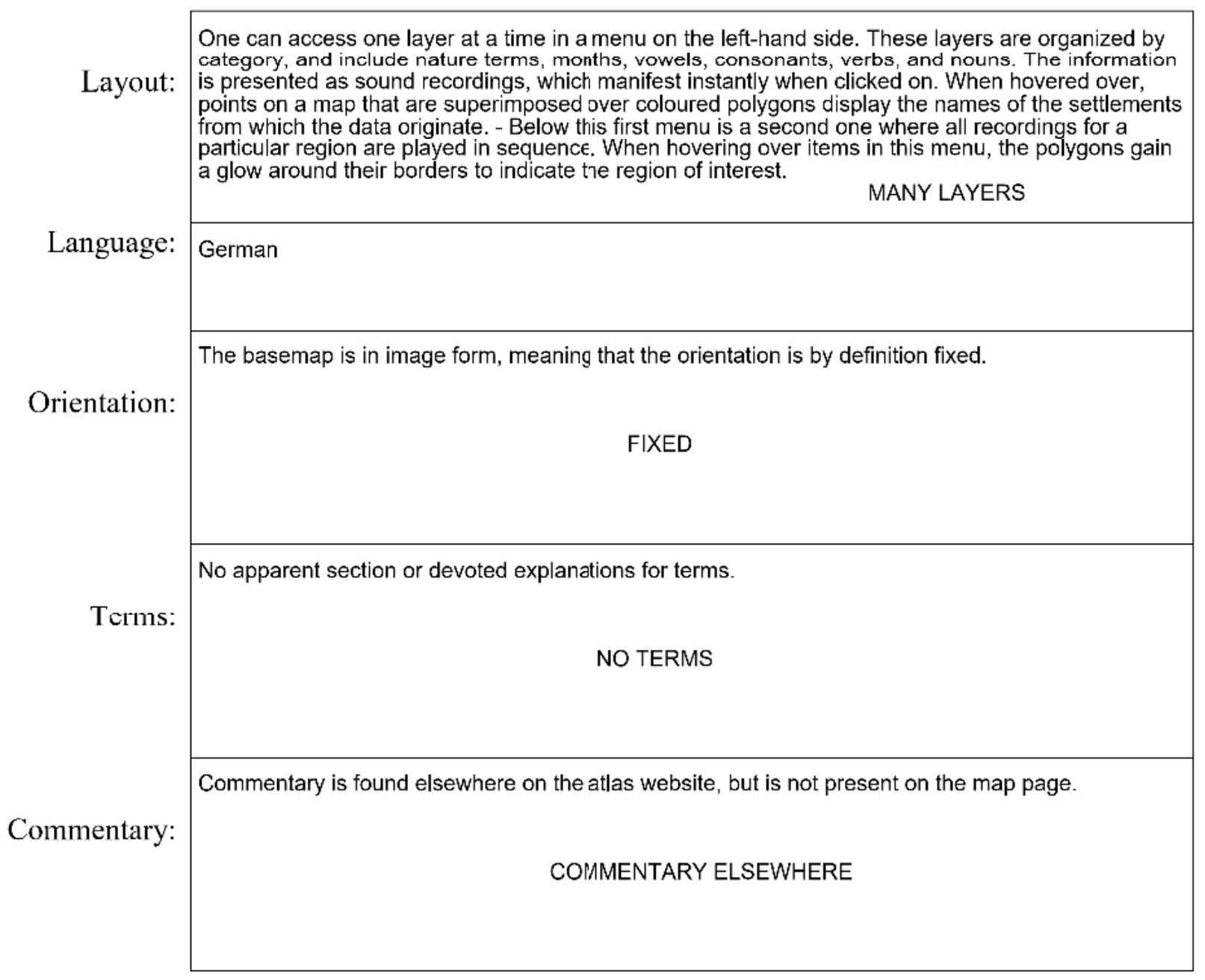




\section{What mental picture does the map produce? - RESULT}

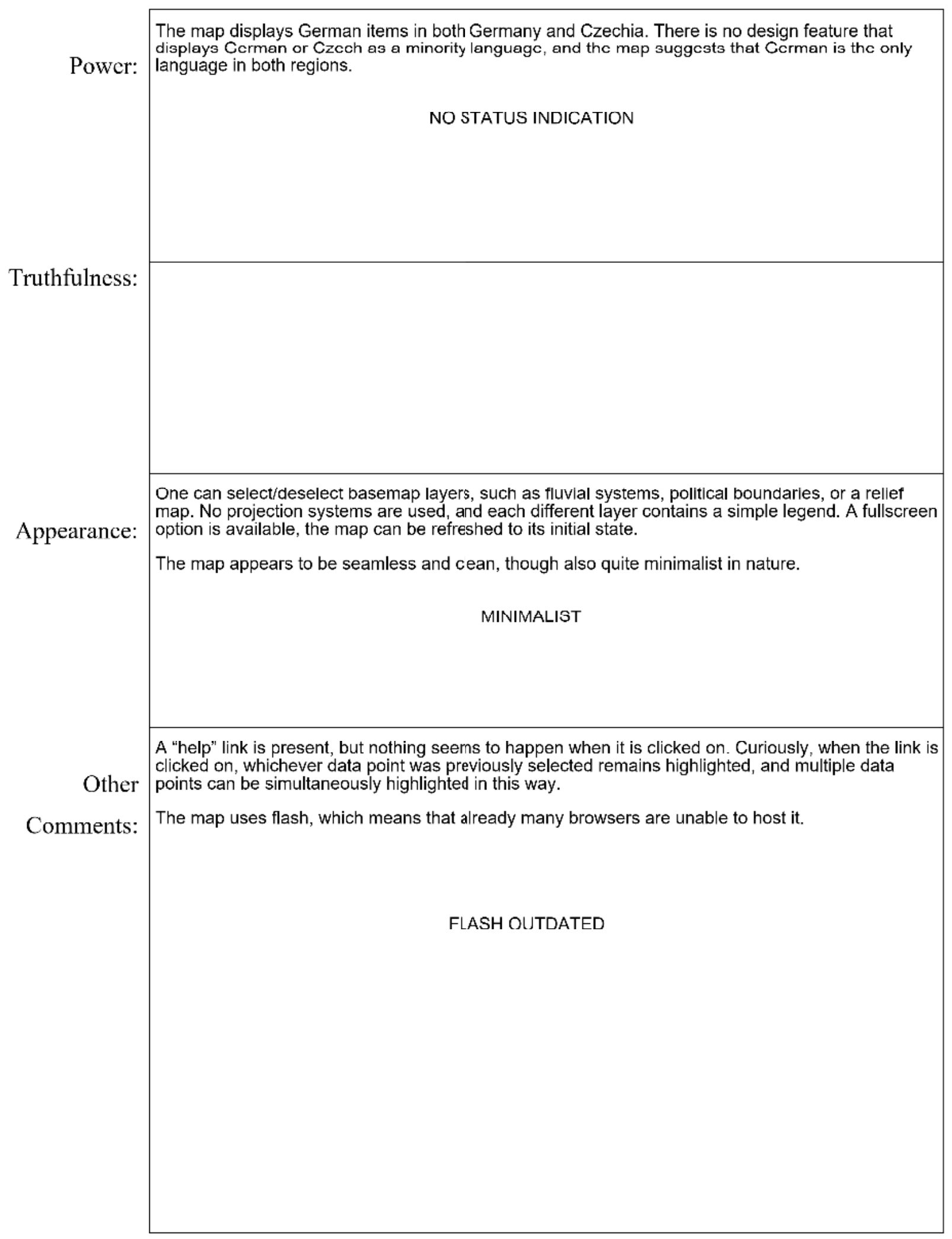




\title{
Map Reference:
}

\section{Title: Atlas of North American English (ANAE)}

\author{
Author(s): William Labov, Maciej Baranowski, Janet Barrow, Sharon Ash, Charles Boberg; TELSUR project \\ Source: https://www.ling.upenn.edu/phono_atlas/home.html \\ Ycar: 2005 - ? \\ Media type: Online
}

\section{Annotation:}

" The Telsur Project is a survey of linguistic changes in progress in North American English, supported by the National Science Foundation and the National Endowment for the Humanities. It is the creator of the Atlas of North American English [ANAE], (formerly, the Phonological Atlas of North America). The Atlas will be published in 2005 by Mouton/de Gruyter. It will be accompanied by a CD-ROM version developed by a team headed by Prof. Jurgen Handke at the University of Marburg, creators of The Mouton Interactive Introduction to Phonetics and Phonology. The maps and analyses presented on these pages include early studies that provide the background of the Atlas, and new studies continue the work of the Atlas."

"The sampling of the major urbanized areas of the United States is complete, and the phonemic categories of 700 subjects have been analyzed on the basis of minimal pairs and other elicited forms. Maps of these results appear below in the Maps section. Acoustic analysis of 417 speakers has been completed and draft chapters in the Atlas 11 and 18 are based on the 140,000 measurements involved.

Regional maps of the Inland North are available where the phonological characteristics of the Northern Cities Shift may be examined in detail, with identification of each community and speaker. Clicking on any symbol will show the basic merger data for that speaker, and for the six speakers shown with stars instead of circles, the personal data sheet also lists words with characteristic vowel qualities. Clicking on these words will enable to hear the sounds of the Northern Cities Shift. For all speakers, the sounds of sample words illustrate the advanced pronunciations of short a, short $e$, short

\section{What is the aim of the map? - PURPOSE}

\begin{tabular}{l|l|l|}
\cline { 2 - 2 } Audience: & Linguists and the general public \\
Function: & $\begin{array}{l}\text { LINGUISTS: GENERAL PUBLIC } \\
\text { to respond to the question, "How many dialects of American English are there?" and "Where are the } \\
\text { boundaries located?" }\end{array}$ \\
Context: & Linguistic change in North America \\
\end{tabular}




\section{What is being mapped? - CONTENT}

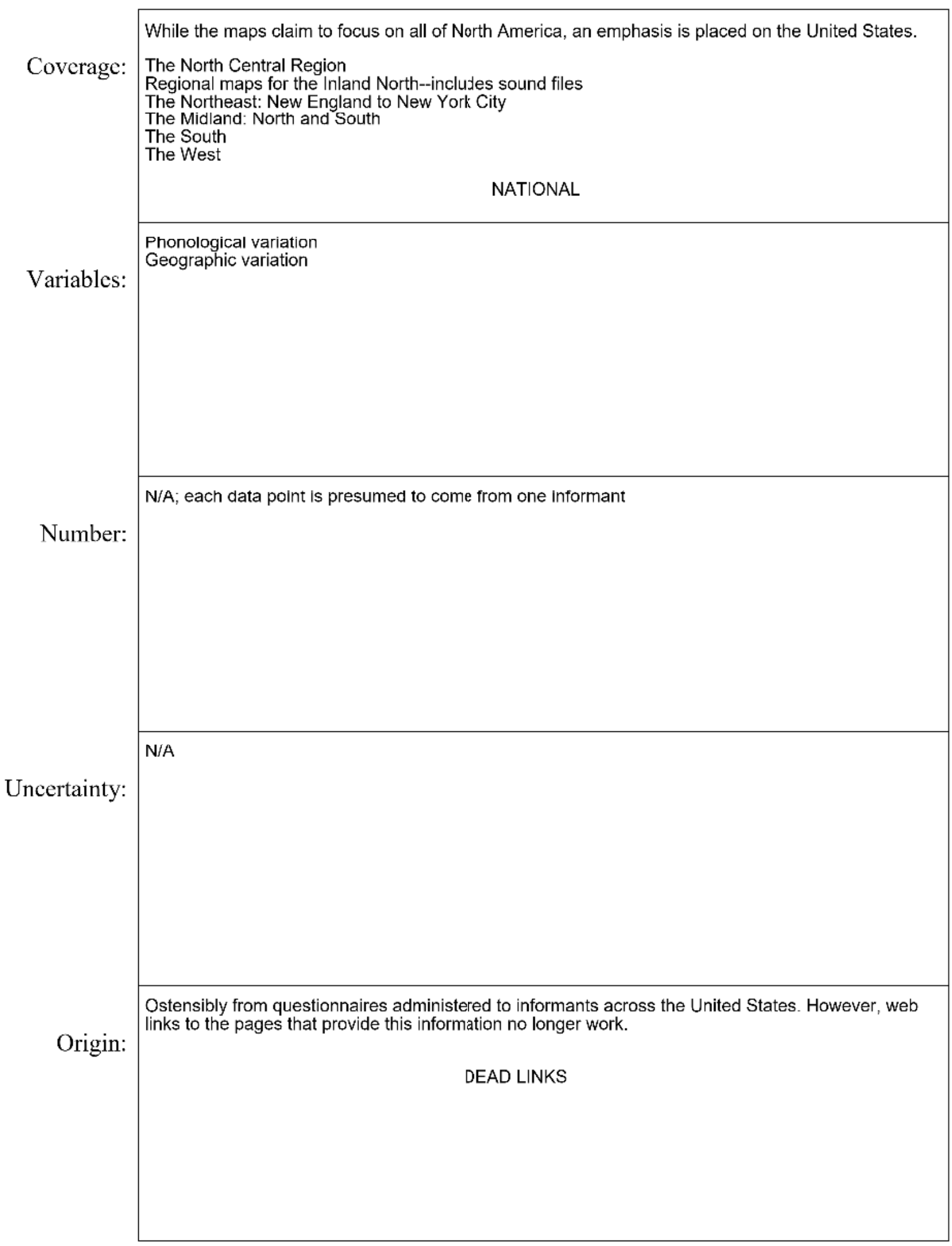




\title{
3. How is it mapped? - SYMBOLS
}

\author{
Georeferencing: \\ Data is Georeferenced \\ $\square$ Data is Not Georeferenced
}

Boundaries:

$\square$ Solid Boundary Lines

Quantified Line Symbols

"Bunches" of Isoglosses

Diffusion Areas

Other:

\begin{tabular}{|c|c|}
\hline Data Points: & $\begin{array}{l}\text { Identical symbols are used for each different sound. The number and orientation of symbols } \\
\text { differs according to different sounds. } \\
\text { IDENTICAL SYMBOLOGY }\end{array}$ \\
\hline Accompaniment: & $\begin{array}{l}\text { Each data point is accompanied by the name of the location/settlement the data were recorded } \\
\text { from. Each dialectal region is also labelled, in larger and bolder font. } \\
\text { LOCATION DATA }\end{array}$ \\
\hline Prominence: & $\begin{array}{l}\text { The greater numbers of identical symbol for some phonological varieties over others gives the } \\
\text { impression that some data points are more important or noteworthy than others. } \\
\text { PROMINENT SYMBOL OUANTITIES }\end{array}$ \\
\hline Units: & $\begin{array}{l}\text { Each datapoint comes from one informant. In theory, one would be able to click on each data } \\
\text { point to be able to access information about that informant. However, these links are now dead. } \\
\text { DEAD LINKS }\end{array}$ \\
\hline
\end{tabular}




\section{How is it mapped? - FEATURES}

\begin{tabular}{|c|c|c|c|c|}
\hline Format: & $\square$ Vector Imaging & $\square$ Raster Imaging & Other: & \\
\hline Medium: & $\square$ Globe & Digital & $\square$ Online & $\square$ Static \\
\hline & $\square$ Dynamic & $\square$ Papcr & Other: & \\
\hline
\end{tabular}

The maps have few features; no scale bar, legend, accompanying commentary, title, north arrow, or metadata. This information is presumed to exist elsewhere on the website or in published volumes,

Layout: but these are no longer accessible.

Each map contains the region of interest, along with datapoints accompanied by settlement names. State lines are present, along with primary and secondary isogloss lines indicated by differing thickness.

MINIMALIST; ISOGLOSSES

Language:

All resources and materials are in English.

While no north arrow is present, the map is presumed to run from north to south, and is

Orientation: unambiguous due to the clear appearance of continental landforms and state lines.

FIXED

While no guide to terms or jargon are present on the atlas website, links to published works based

Terms: on the dialectal investigations each define specific terms to some extent.

INDIRECT EXPLANATIONS

Commentary is found in the form of research articles that are linked to the website.

Commentary: 


\section{What mental picture does the map produce? - RESULT}

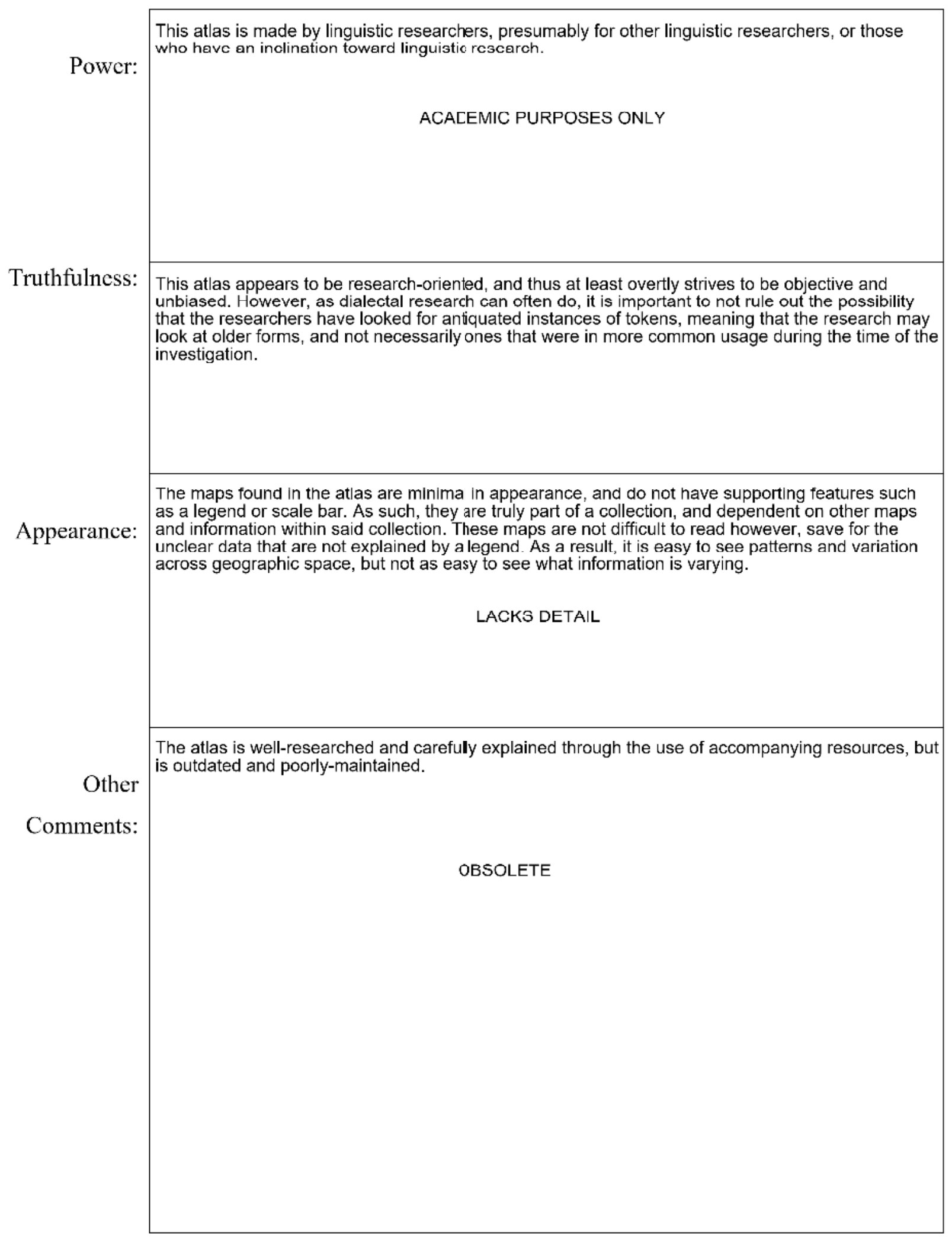




\title{
Map Reference:
}

\section{Title: UNESCO Atlas of the World's Languages in Danger}

\author{
Author(s): UNESCO \\ Source: http://www.unesco.org/languages-atlas/ \\ Ycar: $2001-2016$ \\ Media type: Online ; Digital ; Multilayer Digital Map
}

\section{Annotation:}

"UNESCO's Atlas of the World's Languages in Danger is intended to raise awareness about language endangerment and the need to safeguard the world's linguistic diversity among policy-makers, speaker communities and the general public, and to be a tool to monitor the status of endangered languages and the trends in linguistic diversity at the global level."

\section{What is the aim of the map? - PURPOSE}

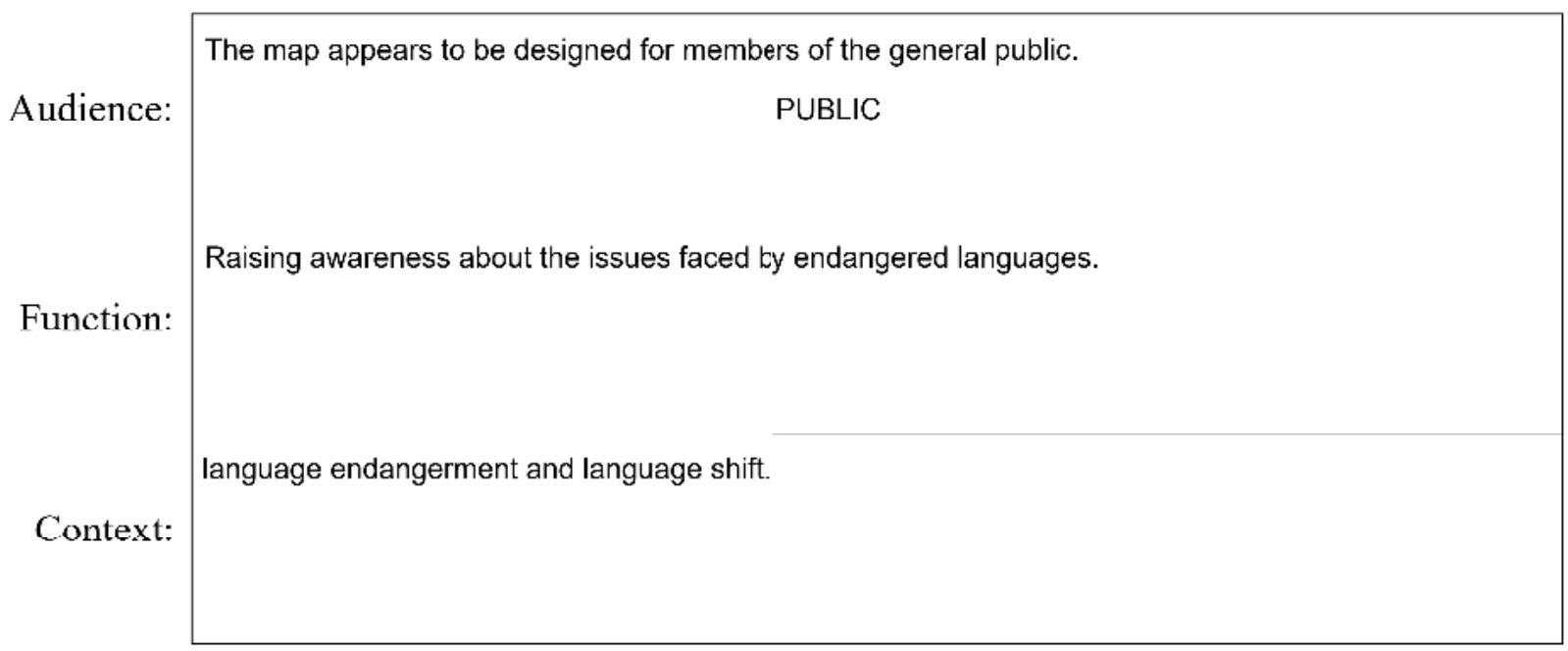




\section{What is being mapped? - CONTENT}

\begin{tabular}{|c|c|}
\hline Coverage: & WORLDWIDE \\
\hline Variables: & Country, language name, number of spezkers, vitality, or ISO code. \\
\hline Number: & N/A \\
\hline Uncertainty: & Uncertainty does not appear to be accounted for \\
\hline Origin: & $\begin{array}{l}\text { The page that would contain that information is no longer registered, making ascertaining this } \\
\text { information impossible. That being said, it is mentioned on the main Interactive Atlas page that users } \\
\text { can suggest languages to be included. } \\
\text { DEAD LINKS }\end{array}$ \\
\hline
\end{tabular}




\section{How is it mapped? - SYMBOLS}

\begin{tabular}{|c|c|c|}
\hline \multirow{2}{*}{ Georeferencing: } & \multicolumn{2}{|l|}{$\square$ Data is Georeferenced } \\
\hline & Data is Not Georeference & \\
\hline \multirow[t]{3}{*}{ Boundaries: } & $\square$ Solid Boundary Lines & $\square$ Quantified Line Symbols \\
\hline & $\square$ "Bunches" of Isoglosses & $\square$ Diffusion Areas \\
\hline & Other: ${ }^{N / A}$ & \\
\hline
\end{tabular}

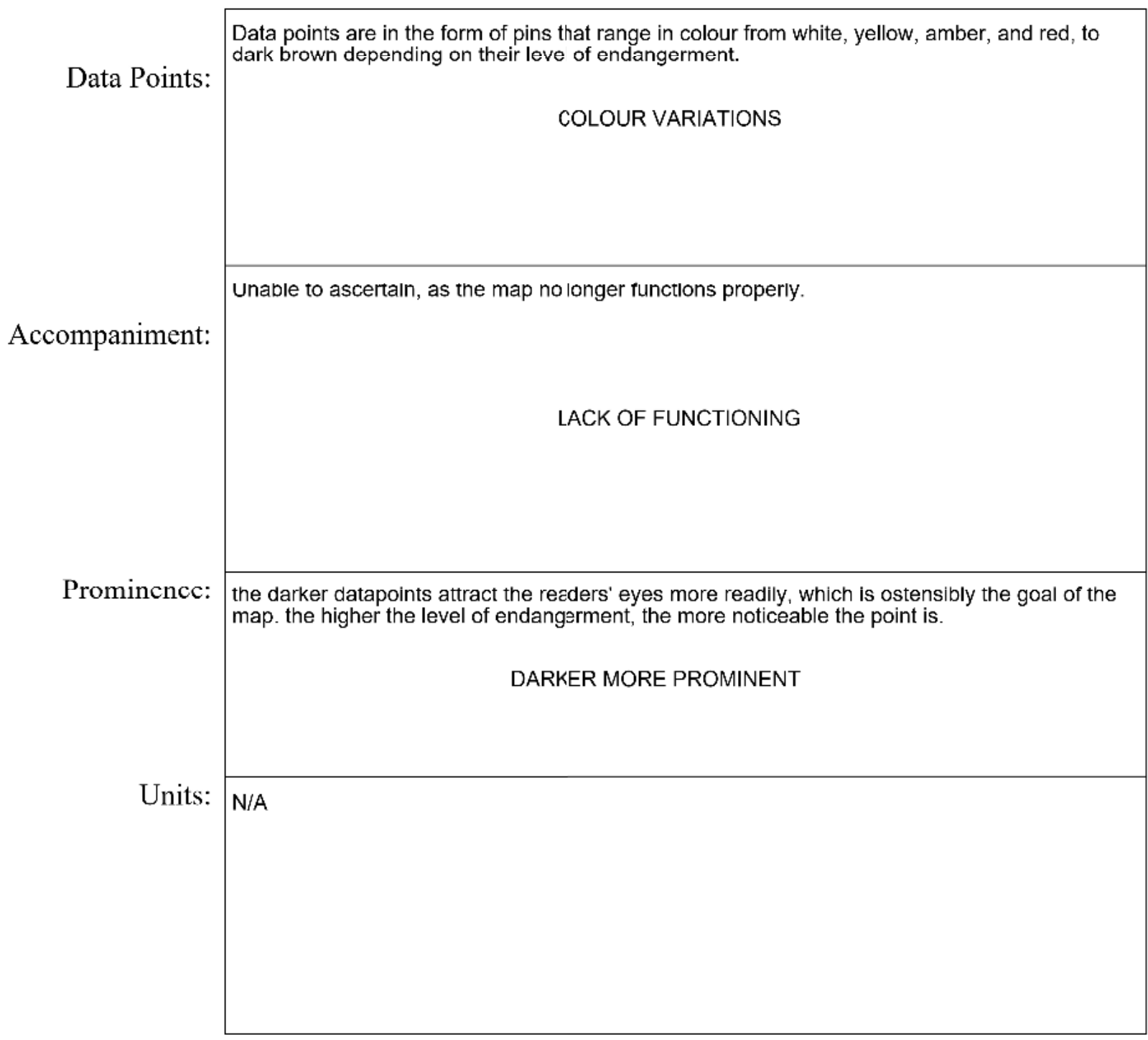




\section{How is it mapped? - FEATURES}

\begin{tabular}{|c|c|c|c|c|}
\hline Format: & $\square$ Vector Imaging & $\square$ Raster Imaging & Other: & \\
\hline Medium: & $\square$ Globe & $\square$ Digital & $\checkmark$ Online & $\square$ Static \\
\hline & $\square$ Dynamic & $\square$ Paper & Other: & \\
\hline
\end{tabular}

There is a list on the left-hand side of the map that indicate the most recent updates and changes to

Layout: the atlas site. No changes after 2017.

Language:
Orientation:

The atlas is available in English, French, and Spanish.

Fixed, from North to South.

\begin{tabular}{|c|c|}
\hline Orientation: & FIXED \\
\hline Terms: & $\begin{array}{l}\text { When language vitality is discussed, it is surprising that "safe" and "data deficient" languages are } \\
\text { grouped together. Their corresponding situations are likely the opposite of each other. } \\
\text { CONFUSING GROUPINGS }\end{array}$ \\
\hline Commentary: & $\begin{array}{l}\text { There is a detailed description of the map's features below the map itself, including a guide on how } \\
\text { to use the map and a list of degrees of endangerment. While this guide covers everything that will } \\
\text { need to be known in order to use the atlas, it takes a long time to read. It would be advantageous for } \\
\text { the map to be laid out in a more intuitive way. } \\
\text { There are ample and easily-accessible links to more resources, such as a list of authors, credits, } \\
\text { terms of use, downloadable datasets, and statistics. } \\
\text { DETAILED COMMENTARY }\end{array}$ \\
\hline
\end{tabular}




\section{What mental picture does the map produce? - RESULT}

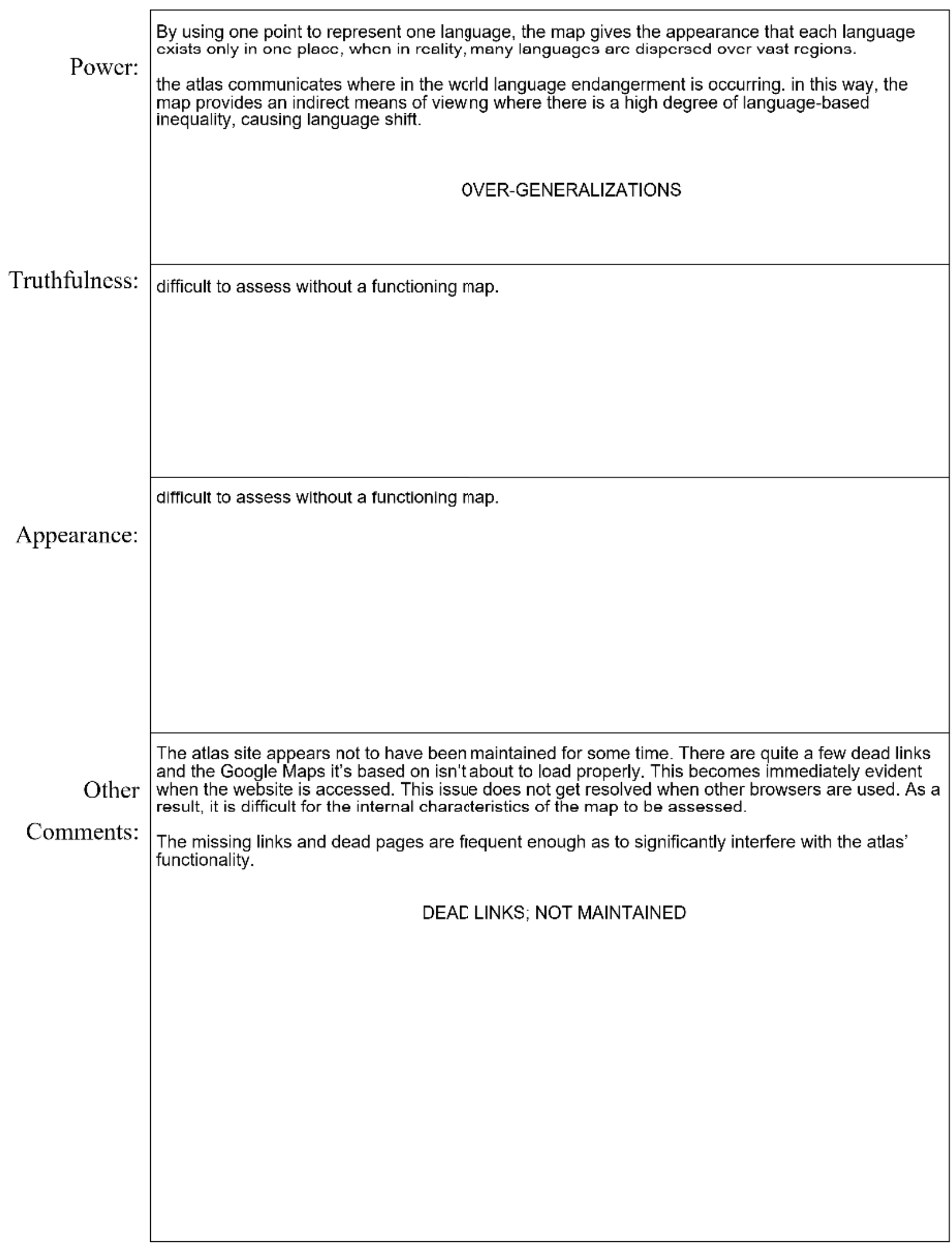




\title{
Map Reference:
}

\section{Title: The World Atlas of Language Structures (WALS)}

\author{
Author(s): Max Planck Institute for Evolutionary Anthropology \\ Source: http://wals.info/ \\ Ycar: 2005, 2011, 2013 \\ Media type: Online, digital, downloadable
}

\section{Annotation:}

"The World Atlas of Language Structures (WALS) is a large database of structural (phonological, grammatical, lexical) properties of languages gathered from descriptive materials (such as reference grammars) by a team of 55 authors.

The first version of WALS was published as a book with CD-ROM in 2005 by Oxford University Press. The first online version was published in April 2008. The second online version was pubished in April 2011.

The 2013 edition of WALS corrects a number of coding errors especially in Chapters 1 and 3 . A full list of changes is available here.

Starting with this edition of WALS, there will not be specific editicns every two or three years, but we will be updating it whenever corrections or additions are made. Changes in value assignment will be made transparent by showing a history on the respective pages.

WALS Online is a publication of the Max Planck Institute for Evclutionary Anthropology. It is a separate publication, edited by Dryer, Matthew S. \& Haspelmath, Martin (Leipzig: Max Planck Institute for Evolutionary Anthropology, 2013) The main programmer is Robert Forkel."

\section{What is the aim of the map? - PURPOSE}

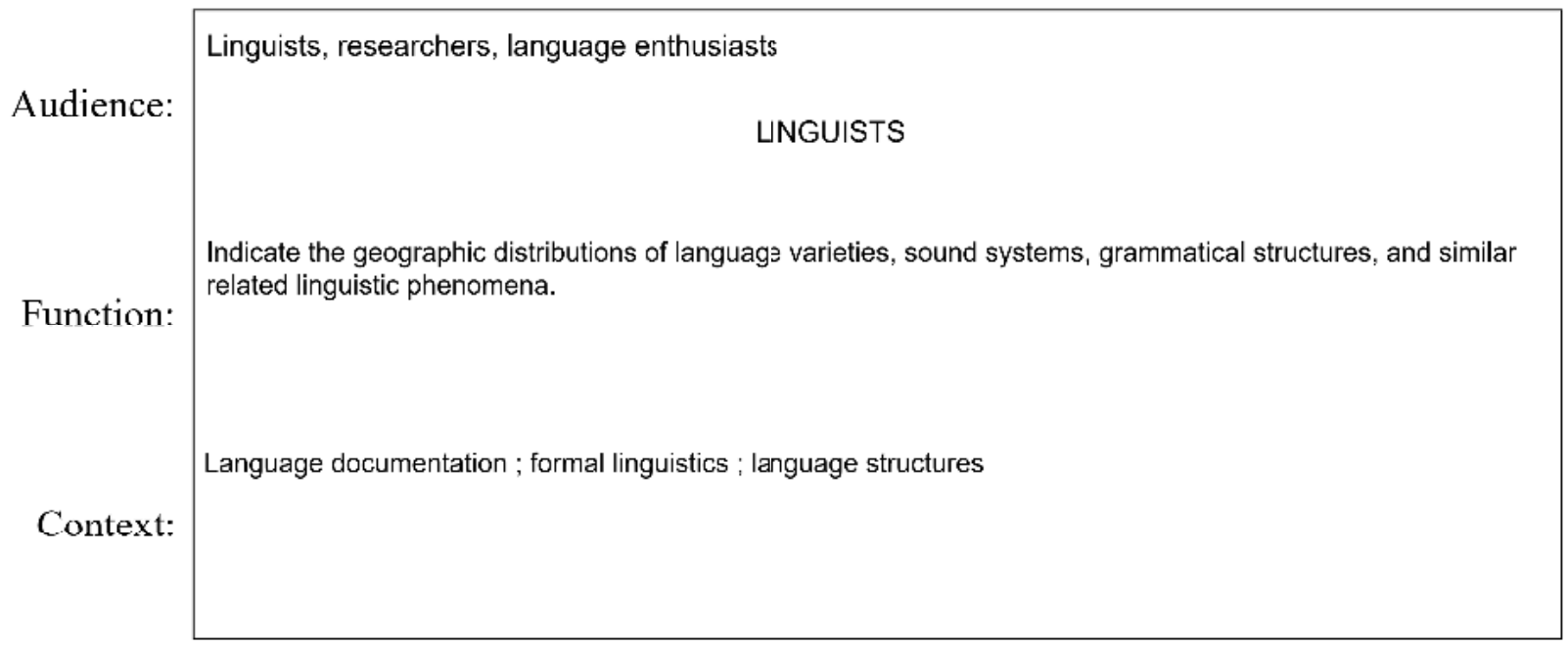




\section{What is being mapped? - CONTENT}

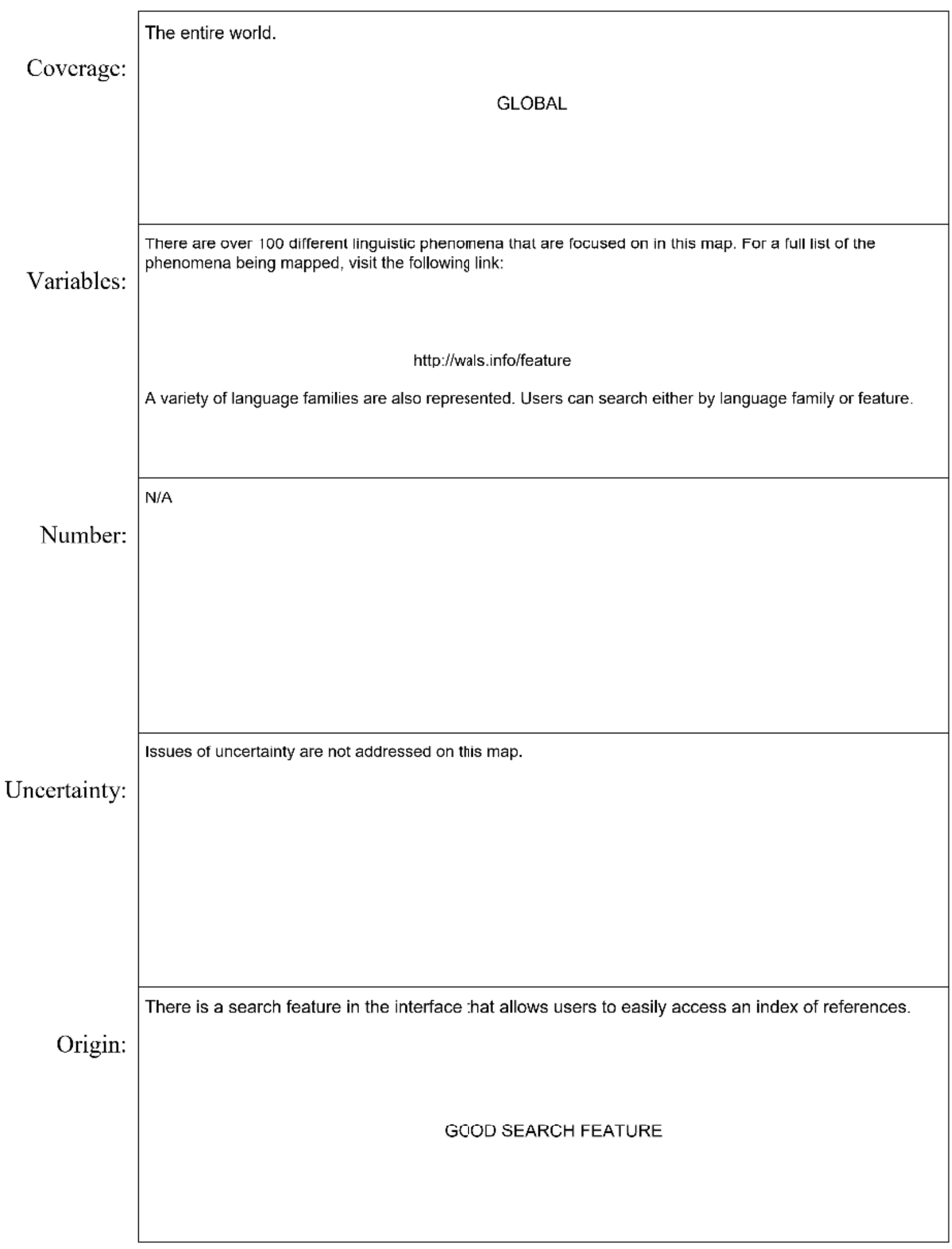




\section{How is it mapped? - SYMBOLS}

$\square$ Data is Georeferenced

Georeferencing:

\section{Data is Not Georeferenced}

Boundaries:

$\square$ Solid Boundary Lines

Quantified Line Symbols

"Bunches" of Isoglosses

Diffusion Areas

Other: N/A

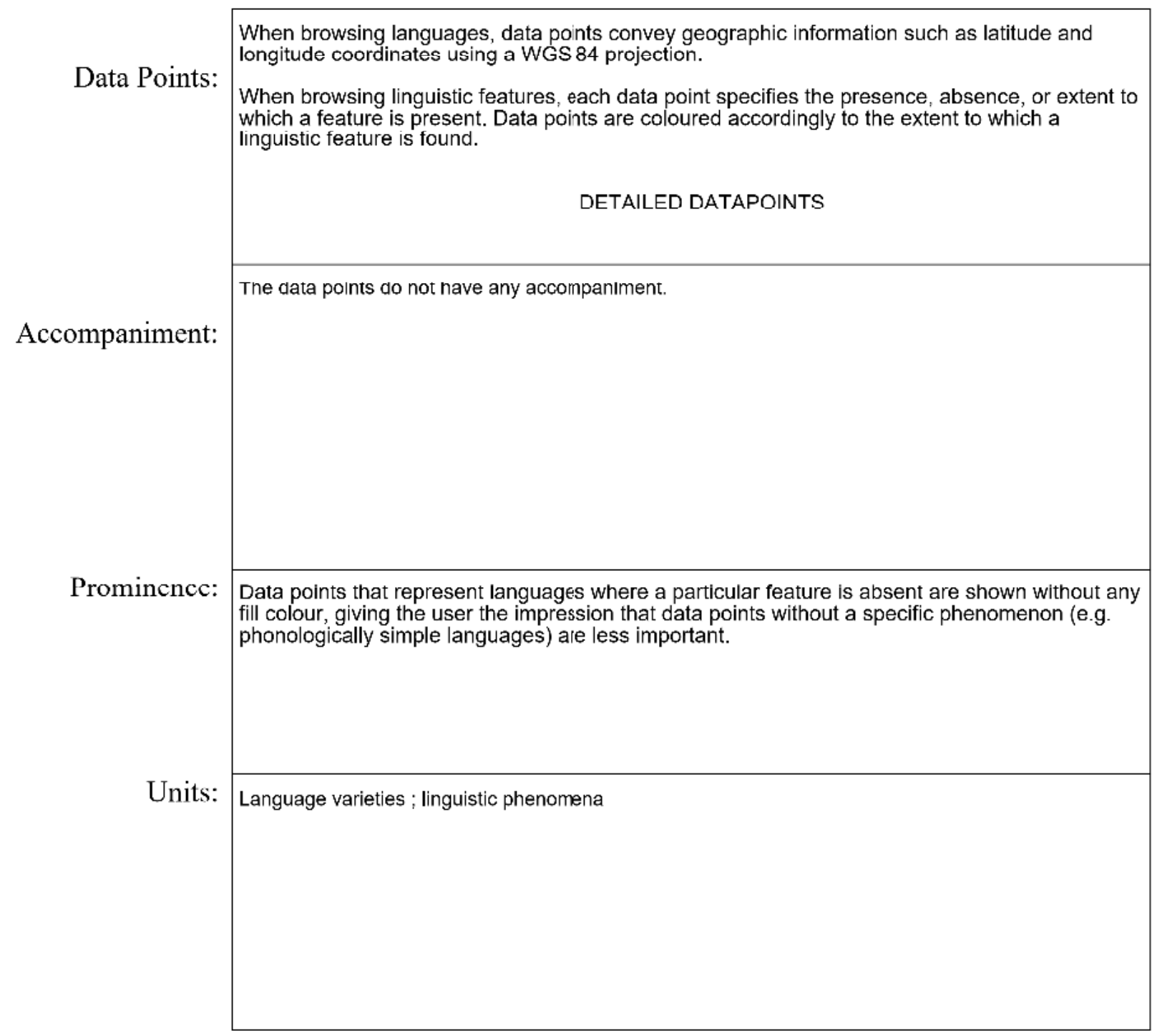




\section{How is it mapped? - FEATURES}

\begin{tabular}{|c|c|c|c|c|}
\hline Format: & $\square$ Vector Imaging & $\square$ Raster Imaging & Other: & \\
\hline Medium: & $\square$ Globe & ఐ Digital & $\square$ Online & $\square$ Static \\
\hline & $\square$ Dynamic & $\square$ Papcr & Other: & \\
\hline
\end{tabular}

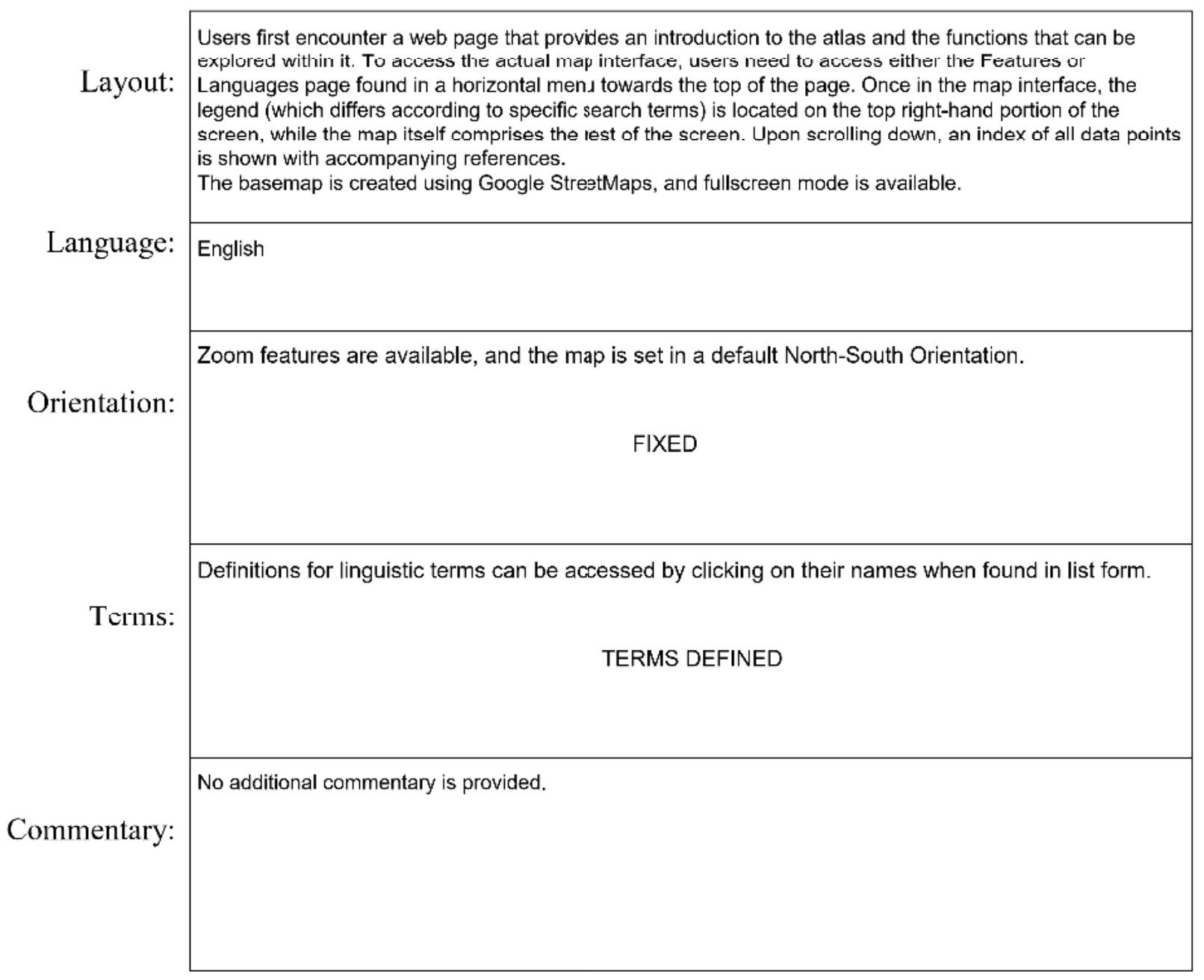




\section{What mental picture does the map produce? - RESULT}

\begin{tabular}{|c|c|}
\hline Power: & $\begin{array}{l}\text { This atlas is evidently made by academics for other academics in a research setting. On one hand } \\
\text { this could mean that socio-cultural variaoles are not included or explored, meaning that the map } \\
\text { does not attempt to place one history over another. That being said, much of the data originates from } \\
\text { studies that have been done on linguistis communities that have not actually benefited those } \\
\text { communities. } \\
\text { RESEARCH ON COMMUNITIES }\end{array}$ \\
\hline Truthfulness: & $\begin{array}{l}\text { The data are accompanied with relevant references and sources, and have been compiled by } \\
\text { multiple professionals involved in a research position. The data thus gives an appearance of being } \\
\text { reliable and valid. } \\
\text { VALID/RELIABLE }\end{array}$ \\
\hline Appearance: & $\begin{array}{l}\text { The map does not directly contain large amounts of information, and mostly represents information found } \\
\text { elsewhere in the corresponding database geographically. This allows users to access as much or as little } \\
\text { information as they require, which is a powerful tool in ensuring that the user does not become } \\
\text { ovorwhelment. } \\
\text { EASY ACCESS TO/ INTEGRATED WITH DATABASE }\end{array}$ \\
\hline $\begin{array}{l}\text { Other } \\
\text { Comments: }\end{array}$ & $\begin{array}{l}\text { The atlas gives a strong appearance of being useful primarily as a tool for research that can be used as } \\
\text { many times are required by researchers. As opposed to telling a story, the data points here give more of an } \\
\text { impression of being an addition of a spatial dimension to data that already exists. Because of this, the atlas } \\
\text { gives an impression of being a useful toul to linguistic researchers, but not particularly accessible or of use to } \\
\text { members of the general public. } \\
\text { Accessing the map interface is not intuitive, and requires a certain amount of arbitary exploration to find in } \\
\text { first-time use. } \\
\text { TOOL FOR RESEARCHERS } \\
\text { DIFFICULT TO FIND ON WEBSITE }\end{array}$ \\
\hline
\end{tabular}




\title{
Map Reference:
}

\section{Title: The World Atlas of Transitivity Pairs (WATP)}

\author{
Author(s): National Institute for Japanese Language and Linguistics. \\ Source: http://watp.ninjal.ac.jp/en/ \\ Ycar: 2014 - 2016 \\ Media typc: Online ; Digital ; Multilayer Digital Map
}

\section{Annotation:}

[from the website]

"The World Atlas of Transitivity Pairs (WATP) is a geo-typological database of morphologically related transitivity pairs such as ak-u 'to open (intransitive)': ak-e-ru 'to open (transitive)' in Japanese, which participate in the causative alternation (doa-ga aita [The door opened] vs. Taro-ga doa-o aketa [Taro opened the door]). It consists of primary data from about 80 languages contributed by about 50 researchers, which can be downloaded for research purpose."

"WATP offers visual representation of the geographical distribution of the formal relationship between the members of transitivity pairs from the two perspectives proposed in Haspelmath (1993), viz. (a) preferences of individual verb pairs for different expression types ('map' interface) and (b) preferences of individual languages for different expression types ('chart' interface). It also permits users to test the validity of the 'iconic' explanation pertaining to the direction of derivation between the members of transitivity pairs."

\section{What is the aim of the map? - PURPOSE}

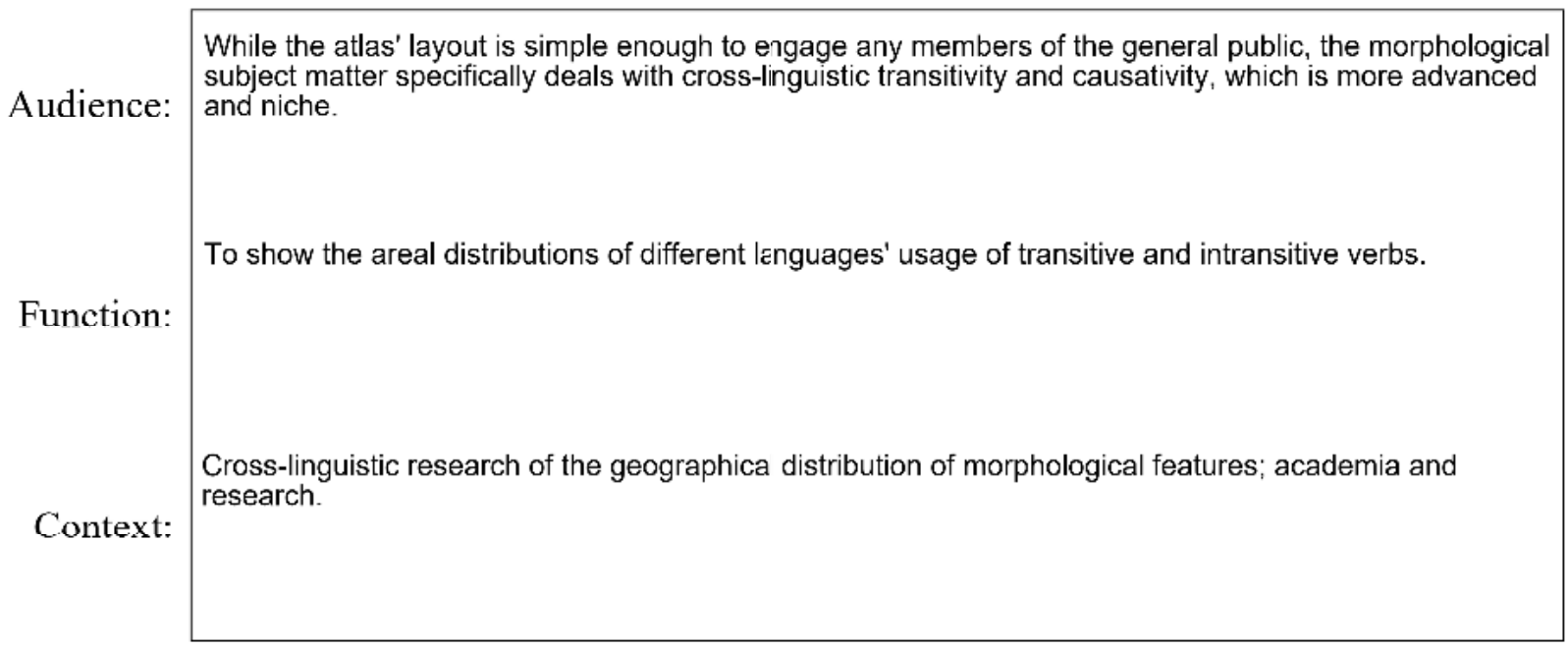




\section{What is being mapped? - CONTENT}

\begin{tabular}{|c|c|}
\hline Coverage: & $\begin{array}{l}31 \text { transitivity verb pairs proposed in Haspelmath (1993) from over } 50 \text { languages (including dialects) } \\
\text { around the world. } \\
\text { GLOBAL }\end{array}$ \\
\hline Variables: & $\begin{array}{l}\text { Different languages and dialects; five types of transitivity pairs: } \\
\text { 1. Anticausative } \\
\text { 2. Causative } \\
\text { 3. Equipollent } \\
\text { 4. Labile } \\
\text { 5. Suppletive }\end{array}$ \\
\hline Number: & Each point reflects a distinct study \\
\hline Uncertainty: & $\begin{array}{l}\text { When two studies on one language yield differing results for a language's transitivity, they are shown } \\
\text { side by side. } \\
\text { As the number of languages surveyed is explicitly mentioned, any lack of languages on the map is not } \\
\text { presumed to be the result of those languages not existing; simply, these languages have not yet been } \\
\text { introduced to the atlas. }\end{array}$ \\
\hline Origin: & $\begin{array}{l}\text { Each datapoint represents findings from a different study. Links to these studies and their citations are } \\
\text { provided in a separate section on the atlas website. }\end{array}$ \\
\hline
\end{tabular}




\section{How is it mapped?-SYMBOLS}

Georeferencing:

\author{
Data is Georeferenced
}

Data is Not Georeferenced

Boundaries:

$\square$ Solid Boundary Lines

Quantified Line Symbols

"Bunches" of Isoglosses

Diffusion Areas

Other: No boundaries present.

\begin{tabular}{|l|l|}
\hline Data Points: & $\begin{array}{l}\text { The data points occur in the form of letters that are in coloured circles, depending on what kind } \\
\text { of transitivity pair they are. When clicked, these data points yield a bubble that gives example } \\
\text { verb pairs. }\end{array}$ \\
Accompaniment: & $\begin{array}{l}\text { POINTS AS LETTERS } \\
\text { The data are accompanled by several pages on the atlas website that describe the theoretical } \\
\text { There are sections devoted to dowrloading the data, and visualizing them in different ways, } \\
\text { including colourful tables, "slopegraohs," and charts, which users can manipulate and add } \\
\text { information to. }\end{array}$ \\
\hline Promincncc: & ACCOMPANYING VISUALS \\
\hline Units: & $\begin{array}{l}\text { The causative pairs, represented with a C in a red circle are the most prominent, both due to the } \\
\text { use of the colour red and because these symbols comprise the majority of what is found on the } \\
\text { atlas. Indeed, } 73.9 \% \text { of the surveyed languages are causative. Perhaps then, the prominence is } \\
\text { not undue, and may be a conscious move on part of the atlas authors. }\end{array}$ \\
\hline N/A & \\
\hline
\end{tabular} 


\section{How is it mapped? - FEATURES}
Format:
Vector Imaging
Raster Imaging
Other:
Medium:
$\square$ Globe
Digital
$\square$ Online
Static
Dynamic
Paper
Othcr: Gooale Mans

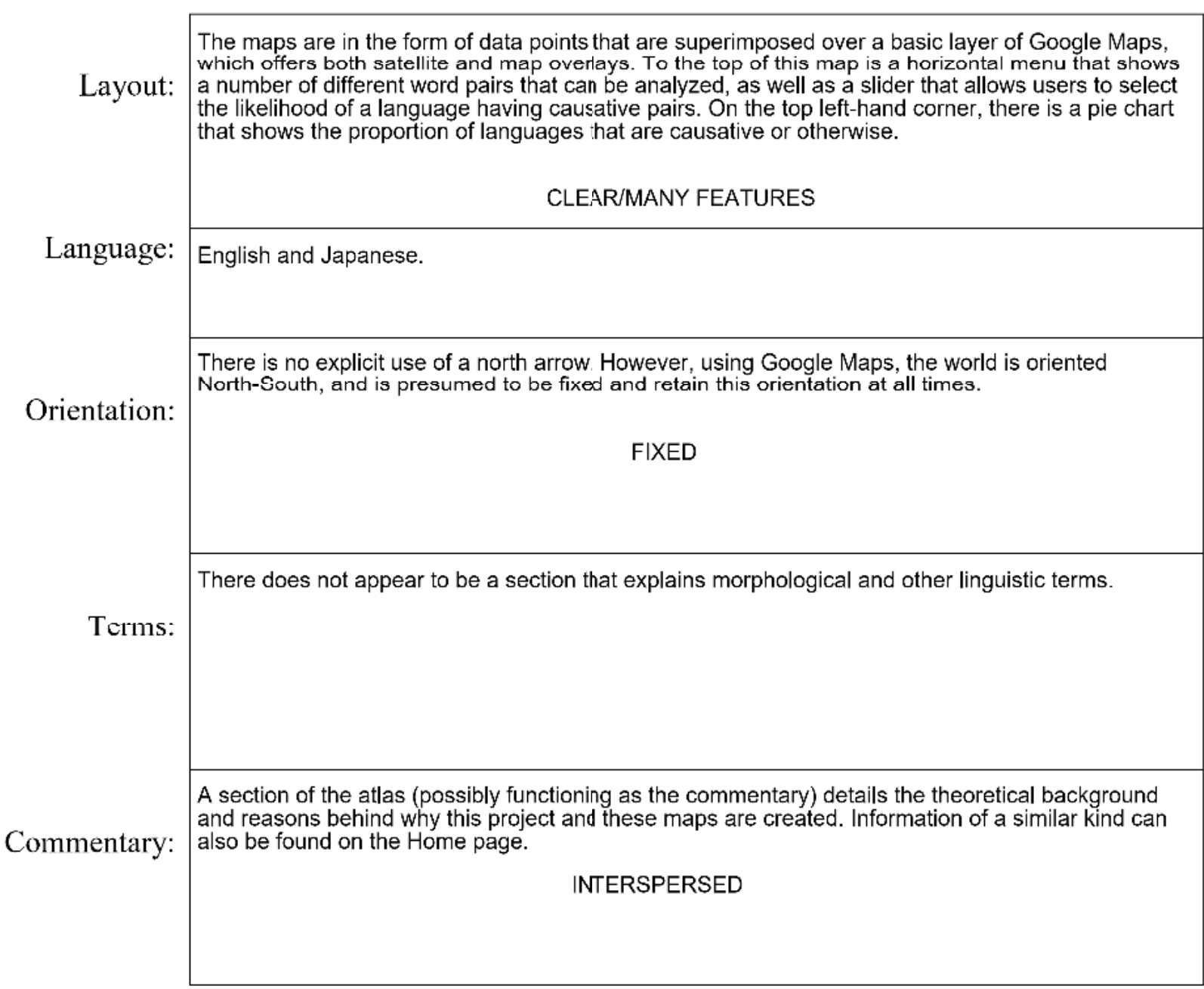




\section{What mental picture does the map produce? - RESULT}

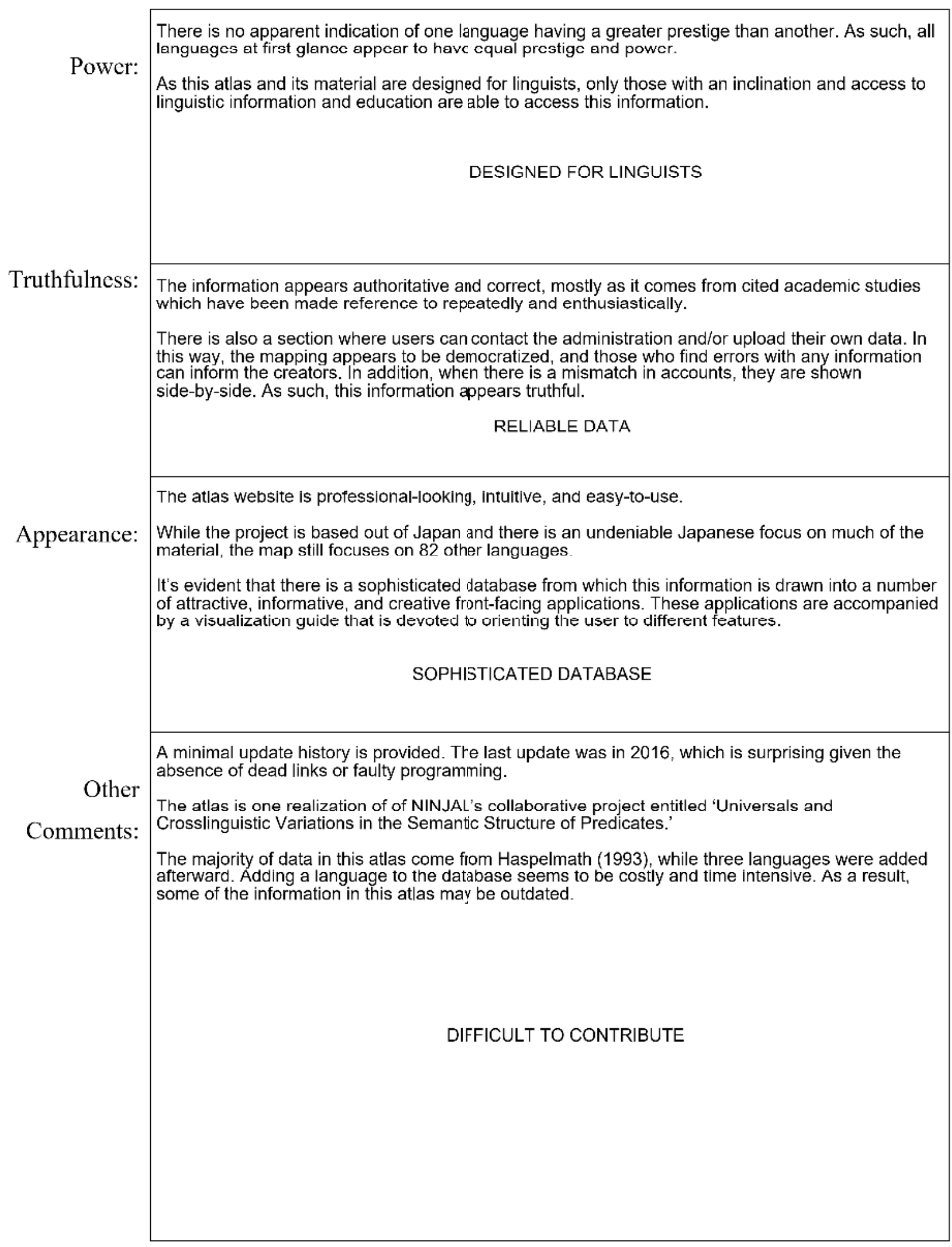


Appendix III: Anonby and Stone’s (2016-2019) typology for analyzing language maps.

\section{Typology for analyzing language maps}

Erik Anonby and Adam Stone, 2016-2019

(updated 2019-09-14)

This document provides a way to approach, understand, analyze and describe a language map.

Provide the relevant information for each point as well as answers to the accompanying questions. Include your own critiques and observations whenever they're relevant. Concentrate on the parts that interest you most, but keep in mind that all of the sections can provide insight into the nature and significance of the map. Complete what you can. It's fine to say "I don't know" if you've thought about the answer and you're still not sure. In some cases, to answer a question you might need to do further analysis, carry out library research, or contact the author of the map.

This document is continuously being updated and refined, so don't hesitate to suggest clarifications of existing elements or additions of other elements that you find valuable for analyzing language maps.

\section{Technical set-up}

\subsection{Dimensionality}

2-dimensional image accompanied by text? 3-dimensional image or model? Sound? Augmented reality? Other sensory channels? Shows change over time (the "4th dimension")?

\subsection{Media type}

Physical (paper? model? other?) or digital?

If physical: what size?

\subsection{Digital aspects}

(digital maps only)

\subsubsection{Geocoded (GIS)?}

1.3.2 Constructed with what type of platform, and using what program(s) or app(s)? Local computer platform (QGIS, Paint, Word, PowerPoint, etc.)? Distributed/web platform (Nunaliit, Google Maps, etc.)? Hybrid (ArcGIS)? Scanned representation of a physical map?

\subsubsection{Information structure}


1.3.3.1 Files, folders, directories?

1.3.3.2 File format (.jpg, .svg, .pdf, .png, etc.)

1.3.3.3 Size (MB)

1.3.3.4 Resolution (in pixels)

1.3.4 Static, or dynamic (i.e., visualizations can change)?

1.3.5 Interactivity (user-directed)

1.3.5.1 Interactive view? (describe)

1.3.5.2 Modifiable content? (describe)

1.3.5.3 Modifiable code (open source)?

\subsection{Accessibility}

Public domain? Open access but subject to copyright (and is the copyright license specified)? By purchase or subscription only?

Is the map fully accessible through its intended medium? (e.g., for books: are they in print? for web pages, are features, visualizations and links maintained and up-to-date?) Is it set up for colour-blind users or audiences with other limitations?

\subsection{Reasons for and effects of the technical set-up}

What are possible reasons for this technical set-up? What are some effects and consequences (whether intentional or unintentional)? Are these effects different for different people (scholars; people who are being mapped; general public)?

\section{Context and theme}

\subsection{Scientific context}

\subsubsection{Participants}

\subsubsection{Author}

Single author? Team? Co-creation by author(s) and users?

\subsubsection{Users (Audience)}

Who are the intended users? What might they consult and use the map for? What might be intended consequences of their use of the map?

What other groups of people might also consult the map? What might they consult and use it for? Why? When unintended audiences use this map, what might some unintended consequences be?

Are people likely to use the map once (e.g., for general reference) or many times (e.g., as an ongoing tool)? 


\subsubsection{People whose language is featured in the map}

Are these people an intended audience? In what ways have they been involved in the creation of the map, and in its outlook?

\subsubsection{Relevant reference details}

Authors, date of creation/publication, place of publication, publisher, internet link, etc. (see the course page for examples of bibliographic references for maps)

Are these details formally included as digital metadata?

\subsubsection{Bibliographic context}

Is it part of a larger work (e.g., atlas, book, website, report, etc.)? Are there articles or reviews that treat this map?

\subsubsection{Research context}

Does it fit within certain subfield(s) of linguistic geography, or another wider academic or societal discussion or discourse?

\subsection{Map language(s)}

(Language(s) used in explanatory text on the map, not the languages treated in the map.)

\subsection{Map title}

\subsection{Accompanying text}

Caption? Commentary?

Is the accompanying text found on the map, beside it, or elsewhere?

Insert all text here, or as an appendix if necessary.

\subsection{Legend}

Is there a legend?

If so, describe its appearance, and placement: on the main part of the map, and/or outside it?

Give an inventory of elements and describe how they are presented.

What are some expected elements that are not included in the legend?

What symbols or symbologies are used in the maps but not explained in a legend?

If there is no legend, why not? How is the map able to avoid this, and what are the consequences?

\subsection{Theme/topic}

Specified by the author? Or does is need to be deduced on the basis of the elements in sections 2.1-2.4 and other observations? Give your assessment about this. 


\subsection{Selection and effects of context}

What are possible reasons that the author situates the map within this larger context, and frames it with these specific elements? What are some effects and consequences (whether intentional or unintentional)?

\section{General type of language map}

Language distribution map? Linguistic structure map? Other? A combination of more than one type of language map?

\subsection{Language varieties treated}

\subsubsection{Identification of language varieties}

What are the actual names used for the language varieties in the map? Are there scientific or social implications related to the choice of these language variety names?

\subsubsection{Categorization of language varieties}

Are language varieties are associated with the label of "language family", "language", "dialect", "accent", or another label? Or is it left unspecified? What does the choice of label ("language family", "language", "dialect", etc.) indicate or suggest about prestige, history, community size, and other non-linguistic characteristics of the language variety?

\subsubsection{Delimitation of language varieties}

Does the map include entire language communities, or does it show only a subset (for example, in a delimited region)?

\subsubsection{Classification of language varieties (describe)}

\subsection{Linguistic structure maps}

For linguistic structure maps only, indicate what kind(s):

- lexicon (words and their meaning)

- morphosyntax (grammar, including morphology, syntax, discourse-level grammar)

- sound system (phonemic data, phonetic data)

- hybrid (e.g., historical phonetics in the lexicon)

- other types of linguistic structure? (describe)

\subsection{Other types of language maps}

For other kinds of language maps, indicate what kind(s):

- semantic maps and mind maps

- language classification taxonomies

- linguistic networks 
- diachronic extension: change over time

What do the data indicate about the origin of given linguistic forms? About language-internal change, and change due to contact between language varieties?

- hybrid (e.g., social distribution of linguistic usage)

- other types of linguistic structure maps? (describe)

\section{The data}

\subsection{Characteristics of key data}

Describe the key thematic data communicated by the map.

\subsection{Data sources}

Where do the data shown on the map come from? Is this indicated somewhere? Are the raw data available to the reader, and verifiable?

\subsubsection{The author's knowledge}

Who is the author? Is the map prepared based on their general impressions, their expertise in a particular domain, knowledge as a native speaker, something else, or a combination of these possibilities?

\subsubsection{Empirical research}

Are the data gathered using a questionnaire or other research tool? Is the expertise and contribution of each contributor indicated (to the degree possible according to research ethics)? Are other contextual elements included (e.g., frame sentences, explanations, or questionnaire images) to ensure the reliability and regularity of the data?

\subsubsection{Research conducted with individuals}

Are data from research conducted with individuals presented as if they represent a wider group of people?

\subsubsection{Research conducted with groups}

Do the data represent all members of a group, or a general tendency within the group?

\subsubsection{References to publications}

Does the map make an explicit reference to the source of the data? Is this reference found on or beside the map, in other accompanying text, or in some supplementary resource?

Is the ultimate source of the data (whether from the author's knowledge or from empirical research) actually indicated in the cited publications?

\subsection{Data selection}

\subsubsection{Choice of data}

How and why has this data set been chosen? 


\subsubsection{Data grouping}

How have the specific data categories been grouped? What elements of variation have been set aside?

\subsection{Other observations on the data}

Here, provide any other observations on the nature and quality of the data.

\section{Visualisations}

What are possible reasons behind each representational feature, or absence of a given feature, in this section? What are the effects and consequences (whether intentional or unintentional) of these representational choices?

\subsection{Border}

Is there a visual border? (describe: no border, solid line, artistic frame, etc.)

\subsection{Superimposed cartographic elements}

\subsubsection{Scale $(1: 1,000,000$, etc. $)$}

5.2.2 Scale bar (km, miles, other; intervals $(10,50,100)$; shape; etc.)

5.2.3 Cardinal directions (north, south, east, west)

5.2.4 Coordinates (numbers, lines)

\subsubsection{Others}

\subsection{Canvas: background and spatialization}

\subsubsection{Abstract (not related to physical space)?}

Mind map, flowchart, timeline, visualization of linguistic distance, or other abstract canvas?

\subsubsection{Spatialization}

5.3.2.1 Projection (Mercator, Spherical, etc.)

5.3.2.2 Coordinate system (WGS 84, EPSG:3857, etc.)

5.3.2.3 Map edge coordinates (e.g., for four corners of the map)

\subsubsection{Human geography}

\subsubsection{Administrative divisions and borders}

What political level/units are shown? Are differences of border levels represented visually? Which borders are shown?

\subsubsection{Populated places (cities and villages)}

Which ones? Are differences in status or size represented?

\subsubsection{Transport routes (roads, railways, paths, etc.)}




\subsubsection{Other characteristics of human geography}

\subsubsection{Natural environment}

5.3.4.1 Waterbodies and watercourses (which ones?)

5.3.4.2 Elevation (relief lines, relief colour, hillshade, triangles, drawings, etc.)

5.3.4.3 Other characteristics of the natural environment (vegetation, sea ice, other?)

\subsection{Visualisation of key data}

\subsubsection{Points}

What is represented by each point? One, or many speakers? One, or many linguistic data tokens?

\subsubsection{Polygons}

How precise are the polygons? How are spaces between data collection sites accounted for? Are sparsely inhabited or uninhabited places included in the polygons?

\subsubsection{Colours}

What are the coloured elements? Which colours are used? Are the colours meaningfully associated with data features (for example, to express linguistic similarity)?

\subsubsection{Textures}

What are the textured elements? Which textures are used? Are the textures meaningfully associated with data features (for example, to express linguistic similarity)?

\subsubsection{Resolution of visual complexity}

What does the author do when the represented data are too complex for the basic visual theme?

\subsubsection{Zoom (describe)}

\subsubsection{Inset map}

\subsubsection{Separate map(s)}

\subsubsection{Overlap of points/polygons}

Is there partial or complete overlap? How is it shown? E.g., for points: clustering; for polygons: transitional colours or superimposed patterns

\subsubsection{Other}

\subsection{Other means of representation}

Tactile 3-dimensional image? Sound? Other?

\subsection{Effects of visualization and other means of representation}


Does the use (or absence) of these visualizations (and possible other representations) help or hinder the effectiveness of the map? Concentrate on the choices that are most helpful, and least helpful.

\section{Critique}

\subsection{Possible motivations and bias}

Why would the author have chosen to make a map on this topic, and in this way? Advancement of knowledge about the languages of the world, or linguistics? Use in educational contexts? Language policy planning? Political reasons? Commercial interest? Expression of social identity and pride? As an art form? Simple interest in the topic? Something else, or a combination of these reasons?

Do you think that the author's background introduces bias into the map? In what ways?

For both of these issues - motivation and bias - support your suggestions with evidence (from the map's context, other sources, or your personal observations) when possible.

\subsection{Direct and indirect messages}

Does the objective that the author specifies for the map line up with the messages that the map communicates (directly as well as indirectly)?

How do the author's motivations influence the representations that they choose?

What balance of power is communicated in relation to a) the author, b) map users, and c) people featured in the map? What is the relative balance of power between different subgroups of people featured on the map?

Are the messages communicated by the map easy to assimilate (to understand in an intuitive way) based on the visualization, or are these messages lost in the details of the visualization?

\subsection{Limitations}

\subsubsection{Limitations of scope}

6.3.1.1 Limitations of scope which are acknowledged

6.3.1.2 Limitations of scope which are not mentioned

6.3.2 Clarity and ambiguity

\subsubsection{Distortion}

6.3.3.1 Distortions, sometimes unintended, resulting from representational choices

\subsubsection{False representations}

\subsubsection{Other limitations}

\subsection{Other maps on the same topic}

Are there other maps dealing with the same topic? What are the differences? 


\subsection{Other observations}

Do you have other observations about the map that are not addressed by the topics and questions in this document?

\subsection{Summary and conclusion}

In conclusion: In what ways does this map succeed in making a contribution to the science of linguistic geography? What are its major gaps and weaknesses? How could the map be improved? 
Appendix IV: First observations of digital language atlases, tools, and technologies (Study 2).

Online maps

WALS - World Atlas of Language Structures

Accessed at: https://wals.info/

Dryer, M.S. \& Haspelmath, M. (eds.). (2013). The World Atlas of Language Structures Online. Leipzig: Max Planck Institute for Evolutionary Anthropology.

Resource type: Web mapping - Interactive

\section{Basic Description:}

"The World Atlas of Language Structures (WALS) is a large database of structural (phonological, grammatical, lexical) properties of languages gathered from descriptive materials (such as reference grammars) by a team of 55 authors."

Own impressions:

- This is an online atlas - collection of many maps, web pages, and links to original research.

- The website is quite easy to use and navigate - but that's because I am already trained in linguistics. No explanation appears to be offered as to terminology. If explanations are present, they are difficult to find, especially for a first-time user.

- The maps offer global summaries of phonological, typological, syntactic, and morphological research performed on a varying number of languages.

- Links to the original research are offered below each map. There is also a page on the online atlas that is just a list of references, studies, and authors.

- Some of the findings are based off of one study (often older), while others are based off of multiple studies performed by a number of authors. This means that there is considerable inconsistency in methodologies, reliability, and contemporaneous theoretical backing.

- The ability to alter what the legend shows and the size of icons is a strong and important feature. However, this was found by accident, and is not listed clearly as a possible feature in the atlas.

- The ability to combine data from other maps and export everything allows WALS to be used as an ongoing research tool. 
Native Land

Accessed at: https://native-land.ca/

Temprano, V. (2015-2020). Native Land.ca. Okanagan Territory.

Resource type: Web mapping - Interactive

Basic description:

Native-Land.ca is not an organization (yet) and is run by Victor G Temprano, whose company, Mapster, funds the website. This is not an academic or professional survey of Indigenous territories, and the maps are constantly being refined from user input. These are meant more for the sake of helping people get interested and engaged.

Own impressions:

- $\quad$ The maps are easy-to-use, colourful, and engaging.

- There is ample space devoted to admitting that the map is still very much a process, and is always expanding and getting new information. A disclaimer at the beginning of the map indicates that the map should not be considered authoritative, but instead just used for general reference.

- A slider on the top-left side of the map allows the user to switch between the language map and other maps.

- A teacher's guide allows for the material in the atlas to be imparted in a structured way to students using lesson plans and activities. Guiding and critical questions are also provided.

- There is a distinct lack of political boundaries on the map, with ample room devoted to explaining that the map is now Indigenous-led and operated.

- A roadmap is offered that allows users to look at the progress of the atlas project, what it has done well, what it has done in the first place, what hasn't happened according to the plan, and what it needs to do in the future.

- The timeline offered for the information in this atlas is unclear. Do the linguistic boundaries presented reflect the current situation, the pre-colonial situation, or some combination thereof? There is also little commentary that describes current, recent, or traditional migration patterns.

- API keys are offered in a devoted section of the atlas.

- Traditional lands do not always correspond to language regions. This may resemble the actual situation, but this is not commented on anywhere.

- While some original sources are provided, they aren't detailed, and so it is difficult to verify the information that is presented on the maps.

- There is a section that explains how to make a territorial acknowledgment. 
Indigenous languages of Canada (Canadian Geographic)

Accessed at: https://www.canadiangeographic.ca/article/mapping-indigenous-languagescanada

Brackley, C. (2017). Indigenous languages of Canada [Map]. In Mapping Indigenous languages in Canada (December 2017). Ottawa, ON: Canadian Geographic.

Resource type: Web mapping - Static

Basic description:

"The data used for this map* illustrates "Aboriginal language spoken at home," either as a first or secondary language. In Canada, nearly 229,000 people rely on one or more of these languages, including those who have retained their mother tongue, who have become fluent in their ancestral language later in life, or who have learned the languages of the Indigenous community or region they eventually settled in."

Own impressions:

- The visual layout of the map is visually appealing and colourful, making it easy to view the information on the map in its intended way.

- The information on the map provides a very basic depiction on the distribution of Indigenous languages in Canada, but does not provide more information, such as the ages of speakers, the extent to which they are being actively used, the vitality of such languages, or the proportions of speakers within a community.

- The attached commentary is only speculative at best but does clarify some of the information that the rest of the map lacks.

- The atlas relies heavily on census data without any other kinds of data for contextualization purposes.

- It remains ambiguous as to what precisely qualifies a speaker.

- The census data are not necessarily reliable. There was a forest fire in Ontario at the time the 2016 data were collected, meaning that the Northern Ontario information is likely inaccurate.

- The linguistic divisions indicate typological relatedness, but not relationships between Indigenous groups or historical patterns. 
Atlas Linguistique de la France

Accessed at: http://lig-tdcge.imag.fr/cartodialect3/carteTheme

Gilliéron, J., \& Edmont, E. (1902-1910). Atlas linguistique de la France [atlas]. Paris: Honoré Champion.

Resource type: Uploaded paper map

Basic description:

« L'Atlas Linguistique de la France (ALF) a été réalisé par Jules Gilliéron et Edmond Edmont. Entre 1897 et 1901 Edmond Edmont a effectué des enquêtes de terrain dans 638 communes de la France. Pour ce faire, il a utilisé un questionnaire composé de notions qu'il a posé auprès de 735 informateurs »

Own impressions :

- Especially for the time it was produced, the ALF is very impressive and rich in information.

- The transcription conventions are difficult and unintuitive to grasp but can be learned with enough time and effort. This is unsurprising given the atlas was completed long before the establishment of the IPA.

- The resolution of the uploaded maps is high, allowing the user to zoom in on fine details of the map. While the atlas does exist in print form, it is certainly more easily accessed and safely stored through an online medium.

- The online Cartodialect interface is simple, with a zoom feature and the ability to pan over areas with a pointer. More features would likely be unnecessary, given that the maps are static, having been uploaded from their original paper versions.

- A map scrolling function at the top of each map page makes it easy to navigate between maps.

- The online version of the atlas has a page devoted to explaining its history and features, and a search function that allows for each of the many terms the atlas covers to be found quickly.

- Only varieties of "French" are covered. This term is up to interpretation, and includes Catalan, likely for sovereignty-related purposes.

- There is a credits section that acknowledges the original researchers as well as those involved in the web-mapping-based process. 


\section{Ethnologue Maps}

Accessed at: https://www.ethnologue.com/browse/maps

[no reference accessible]

Resource type: Web mapping - Static

Basic description:

A series of maps available (sort of) on the main Ethnologue website. The maps are of the distribution of languages and language families, and cover the area of countries, regions of countries (e.g. north Congo), or clusters of small countries in the same region.

Own impressions:

- I was able to view the first three maps. Afterward, the screen was blocked with a note prompting me to subscribe to view more maps.

- Each map is watermarked, meaning that it cannot be reproduced without prior (likely paid) consent from Ethnologue and SIL International.

- The maps all appear to be created in paint. However, the website indicates that that more recent maps are created using ArcGIS.

- There is an attached page to the index of maps which explains the parameters surrounding the inclusion of linguistic regions in each map.

- The projections for each map are included in this attached page.

- Academic licenses of these maps are available for a fee.

- The researchers seem sure enough of themselves that an absence of information on the map indicates an absence of any language in that region. This differs from most other language maps, where an absence of information means that the data have not been collected yet.

- The maps appear designed for general reference or academic illustration, and use linguistic boundary lines, typological organizations, and naming conventions associated with academic and Western scholarly circles.

- A scale bar, list of language numbering codes, and rudimentary legend are included.

- There is no indication of how many speakers are present, or the vitality status.

- Solid demarcation lines infer that there is no overlap or multilingualism per region.

- Provincial boundaries are indicated, but not national or other political boundaries, or settlements.

- The names of seas are included.

- The map is at once overcrowded with numbers and corresponding arrow-lines, and minimalist, with only two simple colours being shown.

- It is not clear when the information for these different regions was gathered.

- It is important to remember the origins and motivations of SIL International: a missionary-oriented organization. 
UNESCO Atlas of the World's Languages in Danger

Accessed at: http://www.unesco.org/languages-atlas/

Moseley, C. (ed.). (2010). Atlas of the World's Languages in Danger, 3rd edn. Paris, UNESCO Publishing.

Resource type: Web mapping - Interactive

Basic description:

'UNESCO's Atlas of the World's Languages in Danger is intended to raise awareness about language endangerment and the need to safeguard the world's linguistic diversity among policy-makers, speaker communities and the general public, and to be a tool to monitor the status of endangered languages and the trends in linguistic diversity at the global level."

Own impressions:

- The atlas site appears not to have been maintained for some time. There are quite a few dead links and the Google Maps it's based on isn't about to load properly. This becomes immediately evident when the website is accessed. This issue does not get resolved when other browsers are used. As a result, it is difficult for the internal characteristics of the map to be assessed.

- There is a detailed description of the map's features below the map itself, including a guide on how to use the map and a list of degrees of endangerment. While this guide covers everything that will need to be known in order to use the atlas, it takes a long time to read. It would be advantageous for the map to be laid out in a more intuitive way.

- The search function can be found at the top of the map and looks quite sophisticated. It's a shame the map doesn't work!

- There are ample and easily-accessible links to more resources, such as a list of authors, credits, terms of use, downloadable datasets, and statistics.

- When language vitality is discussed, it is surprising that "safe" and "data deficient" languages are grouped together. Their corresponding situations are likely the opposite of each other.

- The missing links and dead pages are frequent enough as to significantly interfere with the atlas' functionality.

- This does not indicate the poor maintenance of the atlas site so much as reflect the many and serious challenges associated with maintaining such a large and complex online atlas.

- There is a list on the left-hand side of the map that indicate the most recent updates and changes to the atlas site. No changes after 2017. 
AfricaMap / WorldMap Project

Accessed at: http://worldmap.harvard.edu/africamap/

Blier, S., \& Bol, P. (n.d.). AfricaMap [map]. Center for Geographic Analysis at Harvard University, Harvard, Massachusetts.

Resource type: Web mapping - Interactive

Basic description:

"Africamap is housed at the Center for Geographic Analysis at Harvard University with an initial grant from the Harvard Provosts Fund for Innovative Computing and ongoing support from the W. E. B. Du Bois Institute, the Department of African and African American Studies and the Committee for African Studies at Harvard University."

Own impressions:

- When accessing the map, the user is first greeted by an "about" popup that explains the affiliates of the mapping project and where it originated from. There is no information about the map itself.

- The map uses OpenStreetView and is visually appealing with vivid colours that are distinct from one another.

- The map displays different language families within the African continent.

- A legend on the left-hand side is detailed, and doubles as a list of layers that can be selected or deselected. These layers are detailed and numerous and include historical maps. Importantly, these layers can be clicked and dragged above or below other layers, allowing the user to not only be able to choose which layers to view, but in what order. Each layer/legend item also contains a rich description that appears when hovered over with the pointer.

- The map appears to be designed for reference or academic research purposes.

- Other layers regarding ethnicity, health and demographics, world heritage sites, religions, and the slave trade offer contextualizing information for the linguistic distributions.

- Discrete boundaries lead to the impression of solid linguistic boundaries and monolingualism.

- There are political boundaries and settlement names, but they are under the other layers and thus obscured by default.

- There is an option for users to add their own layers, and the map can be viewed using a number of overlays, such as Google Earth Satellite, Google maps, etc.

- There is a street view option for the map, but it is either broken or not yet available. 
A Linguistic Atlas of Early Middle English 1150 to 1325

Accessed at: http://www.lel.ed.ac.uk/ihd/laeme2/laeme2.html

Laing, M. (2008-2019). A linguistic atlas of Early Middle English 1150 to 1325 [atlas]. Scotland: University of Edinburgh.

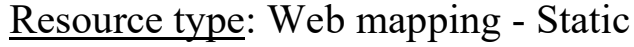

Basic description:

"There are four types of resources in LAEME: (1) a corpus of 'Tagged Texts' (CTT); (2) an 'Index of Sources' containing records which describe the sources of the tagged texts; (3) a set of maps which display the geographical distributions of a series of linguistic features attested in the texts; (4) and a set of documents, including an 'Introduction' to LAEME."

Own impressions:

- When attempting to access the atlas online, one has to first go through quite a few pages and links. These pages don't contain very much of immediate importance, but instead just have graphics or version numbers written down.

- The atlas website is very minimal and looks like it has been written in old HTML.

- There is an overview section that denotes what features are available on the atlas, what information can be found, how to access the content, and how the website is arranged. This section is very verbose and long-winded, and unless the user has a vested interest in all aspects of the atlas, they will likely quickly lose interest.

- The guide that instructs the user how to perform certain tasks provides no links to such mentioned features.

- Maps appear to only be one feature of this atlas, and extensive documentation is provided for other things, such as item lists, county lists, dictionaries, corpus files, and an index of sources.

- The maps are not visually appealing, contain an overly rudimentary legend without any explanations, and do not have any accompanying information such as a commentary or title. The maps in essence do nothing to tell a story.

- The legend does not explain why dots are different sizes. The maps are useless to those who do not commit time to first reading the how-to guide.

- It is unclear exactly whom this map is written for. Likely academics, but any academics who access this information would benefit from having the same skillset, experience, and interests as the author.

- The search feature has too many options, and search results are impossible to decode.

- There is an option of making a county list (for some reason), but no explanation is given as to why and how, and then when a "task key" is hovered over with a pointer, a very long and confusing explanation of *something* appears, and disappears if the pointer gets moved. 
The atlas of North American English

Accessed at: http://www.atlas.mouton-content.com/

Labov, W., Ash, S., Boberg, C. (2006). The atlas of North American English: Phonetics, phonology and sound change. [atlas]. Berlin and New York: Mouton de Gruyter.

Resource type: Web mapping - Interactive

Basic description:

"The Atlas of North American English: Phonetics, Phonology and Sound Change (abbreviated ANAE; formerly, the Phonological Atlas of North America) is an overview of the pronunciation patterns (accents) in all the major urbanized regional dialects of the English language spoken in the United States and Canada."

Own impressions:

- Not the most attractive of atlas layouts. Likely designed for other scholars in a similar field. The functionality and available features on the other hand, are absolute art.

- Each area (i.e. dialectology, phonology, etc.) has very clear and easy-to-understand definitions that help orient less-experienced users.

- Each section also comes with a detailed account of the methods used to gather the data. This includes explanations of techniques such as questionnaires, and examples of what questions were asked in the development of the questionnaires. These accounts are detailed and general enough that they would serve as a good reference in research outside of this atlas!

- The maps are interactive and visually appealing, but use Flash, meaning that they will likely become obsolete quite soon.

- The maps are actively fun, and I enjoyed exploring many of their features.

- Users can select a number of different lexical items and listen to them as they differ from region to region. This feature is available through a number of different browsers, including in In Cognito mode. Not all of the sound files work however.

- $\quad$ There is a page using drop-down menus devoted to finding the desired map, making what would otherwise be difficult-to-find maps a little easier to find.

- Specific maps can be accessed, or the flash module can allow the user to look at dialectal distributions in ways that make the most sense for them.

- A legend is present on the map but hidden by default as to not confuse the users.

- The section devoted to maps first contains an overview that helps prepare and orient the user to the maps. It is not overly verbose or long.

- Each page within the atlas that is accessed opens up a new browser window, as to allow the reader to easily navigate back to the main pages. 
Linguistic Atlas Project

Accessed at: http://www.lap.uga.edu/

[no reference available]

Resource type: Web mapping - Interactive

Basic description:

"The Linguistic Atlas Project (LAP) consists of a set of survey research projects about the words and pronunciation of everyday American English, the largest project of its kind in the country."

Own impressions:

- This appears to be more of an umbrella project for a series of related atlases throughout the United States.

- There doesn't appear to be any publicly available maps that show the distribution of linguistic features. The only map found as of making this point is one that catalogues the informants for each project.

- All data used in this project is also available for download in a variety of formats.

- This would be useful if one were to create their own maps based on geographic data.

- Despite a graphical user interface claiming to take users to more usable data (presumably a map), it just introduces users to more lists.

- It appears this atlas contains every single kind of geographic information presented in every single way except for a map. It serves as an excellent database, but without any meaningful spatial front end. This serves to hamper accessibility for those who are not experienced with the project or database system.

- The link to upload information is dead.

- The "download centre" is a simple file directory, which is unintuitive to those who are not experienced with file directories.

- There is a tutorials page that offers to clarify some of the more puzzling aspects of the database. This page is under construction and currently does not offer any information. This is the same situation for the "help" page.

- The atlas contains data gathered from 1930 to 1980, along with resources from linguists who have performed dialectological research in this area.

- A link to the "old site" presents a notification of an internal server error.

- While the term "atlas" is in the name of this project, it would be more appropriately designated as a database or corpus. The majority of links to external sources are indeed either databases, archives, or corpora.

- The archive is old, fraying at the edges, and not practical to use. The most recent news post is from 2011. 


\section{LL-MAP: Language and Location - A Map Annotation Project}

Accessed at: http://llmap.org/

[no reference available]

Resource type: Web mapping - Interactive

Basic description:

"LL-MAP is a project designed to integrate language information with data from the physical and social sciences by means of a Geographical Information System (GIS). The LL-MAP system encourages collaboration between linguists, historians, archaeologists, ethnographers, and geneticists, as they explore the relationship between language and cultural adaptation and change."

Own impressions:

- The atlas allows for maps (the results of sub-projects within LL-map) to be accessed either from a text-based list or from a menu with an attached OpenStreetMaps interface where the data is displayed. These maps can be displayed on the main master map simultaneously as layers.

- Information from these individual sub-projects can be searched through a number of ways, including language codes, project names, or by region.

- There is a surprising number of maps that are available as part of this project. It is the result of collaborations with related organizations, such as WALS, the LINGUIST list, and is hosted at Indiana State University.

- Technical development of the atlas is offered by The Institute for Geospatial Research and Education at Eastern Michigan University.

- $\quad$ There is a dead link to the World Language Mapping System.

- References to the authors of the original research are only available when the maps are accessed individually. The research originates from inconsistent sources, such as Wikipedia, researchers, or old archival documents. Each map, when accessed individually however, contains useful metadata, disclaimers, a description of the research, and the date that the information was digitized, if necessary.

- The map viewer is easy-to-use and intuitive.

- While the map is designed to be used as an educational and research resource, and it contains an astounding number of maps, it is not operationalized in such a way as to be used in classrooms. 
Linguistic Atlas of Dolomitic Ladinian and Neighbouring Dialects

Accessed at: http://ald.sbg.ac.at/ald/ald-i/index.php?id=0001\&lang=en

Bauer, R. (1990). Sprachatlas des Zentralrätoromanischen. In: Informat 2, 48.

Resource type: Web mapping - Static

Basic description:

"The goal of the speaking linguistic atlas ALD-I is to make available to the users an acoustic equivalent to the four volumes of the printed version of the ALD-I. Since 2005, the speaking linguistic atlas is available in two versions:

1. The internet version (simpler)

2. The DVD version (more complex)."

Own impressions:

- Despite being readily available on Google, the actual atlas containing the maps was quite difficult to find and required navigating through a number of pages in a variety of languages. Once it was found however, a page offered a simplified version of all four of the atlases.

- A striking number of maps are present, and each entered linguistic item has a corresponding sound file. This sound file is not embedded in the map and needs to be accessed from a list of java applets below.

- The quality of sound recordings is good, but in mp3 form.

- A set of research sites remains consistent/constant throughout the map.

- The phonetic transcription conventions appear to be similar to those found in the Atlas Linguistique de la France.

- The maps are minimalist in nature, and do not contain any features such as a legend, scale bar, north arrow, or sets of coordinates.

- The maps are static and non-interactive. This is not surprising given that the main focus of the project took place in the early nineties. This web version is quite simple and much more recent than the original atlas.

- The atlas is available in several languages, including Ladin, French, English, Italian, and German. However, the English version is not entirely in English, and still contains many sections that are in Italian. Fitting, as the project does not involve a single native English speaker and is not about an English-speaking area.

- The atlas primarily focuses on comparing lexical items to one another. Not for the purposes of lexicostatistical comparisons it seems, but just for illustrative purposes.

- Lexical items include verbs and nouns, by all appearances.

- It is difficult to see whom this atlas is designed for, but likely for the Ladin people themselves in terms of linguistic rights and recognition, and researchers who focus on linguistics in the immediate region. 
The World Atlas of Transitivity Pairs (WATP)

Accessed at: http://watp.ninjal.ac.jp/en/

The World Atlas of Transitivity Pairs (2014). Tokyo: National Institute for Japanese

Language and Linguistics. (Available online at: http://verbpairmap.ninjal.ac.jp).

Resource type: Web mapping - Interactive

Basic description:

"WATP offers visual representation of the geographical distribution of the formal relationship between the members of transitivity pairs from the two perspectives proposed in Haspelmath (1993), viz. (a) preferences of individual verb pairs for different expression types ('map' interface) and (b) preferences of individual languages for different expression types ('chart' interface)."

Own impressions:

- The atlas website is professional-looking, intuitive, and easy-to-use.

- While the project is based out of Japan and there is an undeniable Japanese focus on much of the material, the map still focuses on 82 other languages.

- A section of the atlas (possibly functioning as the commentary?) details the theoretical background behind why this project and these maps are created.

- There are sections devoted to downloading the data, and visualizing them in different ways, including colourful tables, "slopegraphs," and charts, which users can manipulate and add information to.

- It's evident that there is a sophisticated database from which this information is drawn into a number of attractive, informative, and creative front-facing applications. These applications are accompanied by a visualization guide that is devoted to orienting the user to different features.

- Links to a user's manual are ubiquitous throughout the atlas website.

- There is a section where users contact the administration to upload their own data.

- The basemap used in the spatial component is Google Earth.

- The atlas is evidently designed for other linguists, and includes jargon.

- A minimal update history is provided. The last update was in 2016, which is surprising given the absence of dead links or faulty programming.

- The atlas is one realization of of NINJAL's collaborative project entitled 'Universals and Crosslinguistic Variations in the Semantic Structure of Predicates.'

- The majority of data in this atlas come from Haspelmath (1993), while three languages were added afterward. Adding a language to the database may be costly and time intensive. As a result, some of the information in this atlas may be outdated.

- An account of the operating environment informs readers which browsers best support the atlas interface.

- English and Japanese versions of the atlas are both available. 
National Institute for Japanese Language Studies language map database

Accessed from: https://www2.ninjal.ac.jp/hogen/dp/ladp/ladb_index.html

Takeda, A. (2011). "Overview of language map database" Takuichiro Onishi Masako Yoshida, Atsuko Takeda, Kane Takamizu, Saeko Arai, Izumi Konishi, Chie Takagi, Mizuho Hidaka, Reiko Tsukigi, Masahiro Matsumaru, "National Dialect Survey for Elucidation of the Formation Process of Dialects-Report on" Preliminary Research "- (National Japanese Language Institute Joint Research Report 10-03).

Resource type: Uploaded paper map

\section{Basic description:}

[translated from Japanese] "It is an image database of language maps targeted by the language map catalog database. While browsing each language map, citations in articles etc. and use in GIS software are also possible. It will be added one after another after the permission procedure of the author and editor."

Own impressions:

- A basic and minimalist design. First page in the atlas is an introductory page with a list of maps.

- The maps contained within the atlas are in zipped PDF format or TIFF format (important for uploading to GIS programs) and appear to all involve the distribution of lexical items for dialectological purposes.

- Certain items have associated geotags, allowing for a more seamless transition into GIS software.

- It appears that this atlas attempts to function as rudimentary database more than an atlas in of itself and is oriented toward having its information used in more sophisticated programs.

- As opposed to an atlas, the project describes itself as a set of two databases: one is a linguistic map catalog database and the other is a linguistic map image database.

- The majority of maps were surveyed and created in the early latter half of the twentieth century.

- The maps are of varying types and styles depending on their original author.

- Some entries are entire atlases unto themselves as opposed to just maps. 
The Linguistic Atlas of French Polynesia / Atlas Linguistique de la Polynésie Française

Accessed from: http://alex.francois.online.fr/AF-LAFP_e.htm

Charpentier, J.M., \& François, A. (2015). Atlas Linguistique de la Polynésie Française Linguistic Atlas of French Polynesia. Berlin, Papeete: de Gruyter \& Université de la Polynésie Française.

Resource type: Web mapping - Static

\section{Basic description:}

"Organized by topics (body, life, individual and society, culture and technology, flora and fauna), these lexical maps are supplemented by explanatory notes and indexes in French, English, Tahitian. Text chapters in French and English present the social profile and the historical dynamics of the territory's languages, which are all endangered to various extents."

Own impressions:

- As opposed to an online atlas, this is a print atlas that is publicly available in both English and French. However, the atlases also offer Tahitian as the third medium.

- The majority of the atlas is a collection of maps. The first portion contains a disclaimer and detailed commentary of the motivations for the study, considerations that need to be taken into account, and a background of the islands and their cultures within French Polynesia.

- Each map is basic, with the same geographic area represented. Each linguistic group/region has the specific lexical item noted beside it, and in some cases transliterations for each term are provided below the map.

- There are no north arrows, scale bars, legends, or other accompanying features.

- Items on the map are numbered and correspond to a list of terms that is provided after the commentary. 
The tower of Babel: Evolution of human language project

Accessed from: http://starling.rinet.ru/maps/maps.php?lan=en

Starostin, S. (2005). The tower of babel [atlas]. [rest is unclear]

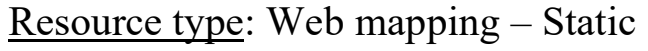

Basic description:

"The Tower of Babel (ToB) is an international, Web-based project on historical and comparative linguistics - so far, the biggest and most comprehensive of its kind to be found on the Internet."

Own impressions:

- Likely not the biggest and most comprehensive online atlas to be found.

- The genetic relatedness depicted between languages on various maps ranges from being highly unlikely to utterly absurd. Many language families and macrofamilies appear made up or are based on discredited hypotheses (e.g. Basque and Chinese both belong to the "Dene-Caucasian" language superfamily).

- What is referred to as an "interactive map" is in fact a static map. Links to other web pages with one or two sentences explaining each language depicted on the map in detail are provided below.

- There is a confusing query option in the lists of information below each map. While there is a how-to guide, it is not linked to this information and takes effort to find.

- The database appears rich in proto-reconstructions and serves as a dictionary.

- The website is available in both English and Russian.

- Databases that the maps are based off of (apparently) are available to download directly from the atlas website.

- There is a special focus in many sections of the atlas on Russian linguistics and dialectology (unsurprisingly).

- There is a section devoted to the bibliography of much of the background research on the information in the atlas.

- A main focus is placed on etymology in the non-Russian cases.

- There are some dead links, which is unsurprising, given that the website is old, outdated, and posthumously adapted from the author's personal website. Despite the website having an outdated appearance, it was last been edited in January 2019.

- A program designed by the atlas author named "Starling" is available for download. This program claims to be a linguistic database software package, but I have not been brave enough to download it...

- In the Russian section, text corpora, dictionaries, and morphological data are available for download.

- There are so many different and disparate areas that this atlas addresses that it is difficult to assess the purpose of this atlas project in the first place. 
The language families of the world

Accessed from: http://webspace.ship.edu/cgboer/languagefamilies.html

Boeree, C.G. (2003). The language families of the world [map series].

Resource type: Web mapping - Static

Basic description:

"The following maps and classification are based on Merritt Ruhlen's book A Guide to the World's Languages (Stanford University Press, 1987), which in turn is strongly influenced by the work of the great linguist Joseph Greenberg, who died May 7, 2001. Please understand that both the maps and statistics below are approximations. All errors are mine."

Own impressions:

- Similar to the tower of babel atlas (18), this atlas (appears more to be a simple series of maps) seems to have been made in honour of a linguist who had died.

- This series of maps doesn't seem to tell any story per se, only lists major language families and languages found throughout continents.

- The maps appear to be made in Microsoft paint. Instead of a legend, each language region has a corresponding label in a blank space (usually the ocean) beside it.

- The language families seem poorly researched and out of date. For example, Wakashan languages appear under the "Almosan" language family, which is in fact Algonquian. Similarly, Haida is considered an Athabaskan language.

- The same language distributions and their names vary wildly depending on which maps they appear on (e.g. Na-Dene and Athabaskan are used interchangeably).

- The coloured regions on the map are very basic, and do not indicate any overlapping regions or multilingualism.

- Each map has a corresponding description, which details approximately how many languages exist in the language family, how many speakers there are, and some notable examples. No references or evidence are provided to bolster these claims, and there is no contextual information on their political statuses or vitality. 
Linguistic maps from Muturzikin.com

Accessed from: http://www.muturzikin.com/countries.htm

[no reference available]

Resource type: Web mapping - Static

Basic description:

"Linguistic maps of Basque Country, Africa, America, Asia, Europe \& Oceania. I provide useful and practical information for thousands of languages displayed on specific geographic areas."

Own impressions:

- $\quad$ The variety and complexity of language maps offered on this website is staggering.

- The maps and atlas are available in 13 languages.

- The author of these maps is not represented or a part of an academic institution, professional body, or organization, and so in some senses in an amateur. However, the maps are not amateurish, and these maps are used in academic settings.

- The maps available through this website vary in quality, resolution, and language, but this collection of maps is probably the most comprehensive on found so far.

- The information is not linked to any database.

- There is a separate page devoted to explaining the maps' intellectual property rights, as well as the main sources of information on the maps. These sources are organized into "Sites with neutral points of view," "Websites with religious bias," and "Organizations". Neutrality appears to be an important value in this website. This section also contains frequently asked questions, which act as a commentary to the author's mapping aims in general.

- The maps openly give Indigenous languages priority over majority or non-Indigenous languages. However, no explanation is provided as to what counts as "Indigenous".

- No information is provided as to the vitality of any language or what time period the information has come from. There is no linguistic overlap, suggesting that regions are monolingual. Linguistic regions which I know only have 2 or 3 speakers are seen to be disproportionately large, giving a false impression of a stronger existence.

- Each language/linguistic region has information in its/their own language(s). The legend too is similarly multilingual. A more detailed legend below the map can be expanded by the user. This legend contains links in a variety of languages.

- Each language family has its own corresponding cluster of similar colours. While language regions often appear to coincide with political borders, this is not the rule.

- Languages are further separated into major dialects.

- Maps of Canada are presented through a variety of ways, some of which focus on political boundaries, and some of which do not. 
TITUS: Thesaurus Indogermanischer Text- und Sprachmaterialien

Accessed from: http://titus.uni-frankfurt.de/didact/karten/turk/turklm.htm

Gippert, J. (2010). Turkic languages [map]. In H. Glück (ed.), Metzler Lexikon Sprache, Stuttgart / Weimar: Metzler 1993.

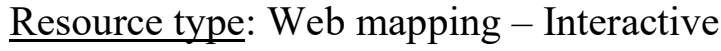

\section{Basic description:}

"The primary objective of the TITUS project was, as stated earlier, to co-ordinate the collection of relevant original texts of ancient Indo-European languages. Of course, the idea of "entrusting" such texts to the new electronic medium, which at the time was rather laboriously enforced, was not really new..."

Own impressions:

- The map is technically interactive, in the sense that one can click on various points on the map, and depending on which square is selected, a zoomed-in version will appear.

- The zoom function usually works and covers most of the area of Turkic languages in Eurasia. There are some sections that one cannot zoom into.

- The map appears to have been made in Microsoft paint, meaning no projection system is relevant.

- The website interface (where these maps are held) is basic and in HTML form and is prone to regular crashes during its use. Fitting, as the website hasn't been updated since 2003, or the maps since 2010 .

- The majority of the website is available in German, and inconsistently elsewhere in English, French, and Spanish.

- While each linguistic region has an attaching label to denote which language is spoken, there is no accompanying legend to explain different textures such as crosshatching.

- The colours of the linguistic regions do not appear to correspond to one another and appear to have been chosen arbitrarily.

- No information appears to be supplied regarding the source of the map's information. If it exists on the website, it is well-hidden behind misleading or dead links.

- Multilingualism may or may not be conveyed on the map, but it appears to not be the case (it is difficult to assess).

- There are no overlapping regions, and linguistic regions often (but not always) coincide with political boundaries. 
Many Tongues — Ancient Tales

Accessed from: http://www.alaskool.org/language/manytongues/ManyTongues.html

Many Tongues - Ancient Tales, Michael E. Krauss, September 1988, pp. 144-150 from Crossroads of Continents: Cultures of Siberia and Alaska, William W. Fitzhugh and Aron Crowell, Smithsonian Institution.

Resource type: Web mapping - Static

\section{Basic description:}

"From the earliest days of European discovery in this part of the world, linguists have considered possible genetic relationships between American language families and ones in Asia, whence they must have come. Though debate is as lively today as ever before, still no proof of genetic relationship of any native American language family to any Asiatic language family has yet been offered that satisfies even a majority of linguists who have carefully studied such subjects."

Own impressions:

- The web page consists of a map of languages in the North Pacific region made from research in 1900 (apparently). Below this map is a chart of different languages (also functions as a legend) with information on speaker populations taken in 1980.

- Eurasian information comes from Soviet census work, whereas North American information comes from Krauss (1983).

- The distributions of languages and their data are misleading, as the linked data are 80 years apart from each other.

- Each linguistic region is separated by solid boundaries, and there is no overlap between linguistic regions.

- There is a commentary below the map and chart that explains each of the language families and their contexts in detail. Much of this context relates to describing genetic relatedness between different language varieties.

- This commentary is separated into different sections that correspond to language families. Below this is a paragraph regarding the practices of language policies.

- The map, chart, and commentary appear to be rather outdated both in their information, and the writing style that has been applied. The author takes the role of a clinical and somewhat indifferent scholar who is more concerned with cataloguing linguistic information than the actual placement and situations faced by the languages and how they have changed in the $20^{\text {th }}$ century.

- There are interesting descriptions of loanwords and loaning processes. 
Map of Jewish Languages

Accessed from: https://www.jewishlanguages.org/map

[no reference available]

Resource type: Web mapping - Interactive

Basic description:

"This map represents the diversity of Jewish languages around the world and throughout history. You can click on an arrow for more information about a language. Note that the interactive features of this map may not work on mobile devices."

Own impressions:

- The map is part of a larger website that focuses on the worldwide distribution of Jewish languages. The rest of this site includes video recordings, dictionaries, a lexicon project, sound and text samples, and bibliographies.

- The map is interactive to the extent that there are labels with arrows to each of the relevant linguistic regions, which when clicked, link the user to other pages with added information.

- These pages of added information often (but not always) also feature a bibliography.

- There is some linguistics terminology present in these commentaries, but it is used in a way accessible to non-linguists.

- A list of quick facts is available here.

- Clicking elsewhere on the map leads the user back to the homepage.

- On the map, each area where a Jewish language was/is spoken is denoted with a specific colour. These colours do not relate to one another in any systematic way and were likely chosen arbitrarily.

- The basemap looks standard, with no specific projection system chosen.

- The arrows on the map are misleading at first, as the chosen arrow style is more commonly associated with migrations or movements on maps, so it appears at first glance as if Judeo-French, for example, has moved from Greenland to France.

- Some of the colours are reused for different languages, creating the false impression that they bear more resemblance to one another than they actually do.

- Some varieties on the map (e.g. Lithuanian varieties of Yiddish or Judeo-Georgian) are present, yet not indicated with a link, name, or arrow.

- Users cannot determine what information they would like to see presented on the map. 
Accessed from: https://jakubmarian.com/map-of-languages-and-language-families-ofeurope/

[no reference available]

Resource type: Web mapping - Static

\section{Basic description:}

"The following map shows the language families of Europe (distinguished by colour) and languages within those families. Note that the terms "language" and "dialect" are not mutually exclusive, and some of the languages shown in the map may be considered dialects of others."

Own impressions:

- The map is artistically produced and aesthetically pleasing.

- There is no legend. Instead, each language is labelled directly on the map, and a commentary attached below explains some of the more nuanced features of the map.

- There is extensive crosshatching and overlap between linguistic regions, but only a maximum of two languages coincide at a time. The crosshatched regions represent the most commonly spoken minority language.

- There is no overt projection system chosen for this map.

- An attached commentary explores the differences between languages and dialects, and explains what language families are.

- There is no information that describes where this information has come from, and as this is a static image of a map, there is no data that is linked by a database. Similarly, no data is available for download.

- This map is likely built for general reference.

- Each language family is colour coded.

- While some language boundaries correspond to political borders, this is often not the case.

- This map is part of a larger series of maps focusing on different aspects of Europe such as economic and cultural differences. 
Indian scheduled languages map

Accessed from: https:/www.mapsofindia.com/culture/indian-languages.html

[no reference available]

Resource type: Web mapping - Interactive

Basic description:

"India, a potpourri of different cultures, religions and beliefs, is home to not just one or two languages but to an uncountable number of different lingual families.

Languages belonging to the two major language families - Indo Aryan and Dravidian are spoken by more than $90 \%$ of the people of India. Know more about the languages of India with India Language Map."

Own impressions:

- This map is part of a larger website that features maps on a variety of Indian issues, such as political borders with Pakistan or economics.

- The map is colourful, and each state is coloured according to its dominant language.

- The map is relatively interactive: when users hover their pointer over a linguistic region, a small popup appears that contains information such as the state name, capital, and population, and then the total number of speakers of the official language. This is important, as users can immediately compare the state's population to its number of official language speakers.

- It is difficult to access the popups for smaller regions, as the click radius is tiny and relegated to the landforms.

- Below the map is a commentary that gives a general overview of the quantity and distribution of languages (official and otherwise) in India.

- Below this commentary is a detailed list of the top 29 most spoken languages in India by population.

- Below this list is another list which details the "strength" of each language. Strength is assessed as a measure of percentage of the total population of India that speaks a given language. This measure is problematic as it doesn't account for strength per region or ethnic population.

- The interactive component is minimalist but important in its minimalism, as the user does not need to intuit too much of the system.

- The legend is simple, yet more information is not needed here, it seems.

- There is no scale, north arrow, or stated projection.

- This map and its corresponding information serve as a rich and informative introduction to Indian languages, but would likely not work well as an ongoing research tool. 


\section{PHOIBLE}

Accessed from: https://phoible.org/

Moran, Steven \& McCloy, Daniel (eds.) 2019. PHOIBLE 2.0. Jena: Max Planck Institute for the Science of Human History.

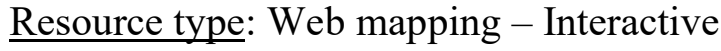

Basic description:

"PHOIBLE is a repository of cross-linguistic phonological inventory data, which have been extracted from source documents and tertiary databases and compiled into a single searchable convenience sample. Release 2.0 from 2019 includes 3020 inventories that contain 3183 segment types found in 2186 distinct languages."

Own impressions:

- Seems at first glance to be a database that has a front-facing map.

- PHOIBLE immediately addresses an important database issue on its homepage: remaining faithful to the collected data while encoding all information in a standard way (Unicode IPA).

- The map uses a basemap from OpenStreetMaps, and each language is represented with a single dot. These dots overlap one another, but can be further separated when clicked on. The colour of each dot remains constant across language family. However, basic colours are reused for sometimes neighbouring language families, meaning that Northwest Caucasian languages, Trans-New-Guinean languages, and Semitic languages all share the same colour.

- Some regions, such as Australia, are quite detailed, while others such as northern and eastern North America are quite sparse. There is no accompanying text that explains that a lack of data does not mean a lack of linguistic information in the real world.

- Each datapoint is accompanied by latitude and longitude co-ordinates.

- Some of the data appear heavily politicized. This includes a lack of Sino-Tibetan languages present in Taiwan, Korean coloured as a Sino-Tibetan language, and Uyghur shown as being spoken in Kazakhstan, not China, to name a few.

- Some languages present on the map are extinct. The map shows languages for which there is available data, living or not, but does not indicate this.

- Users can also access phonetic data in list form and download this information in a number of different formats.

- Users can choose from thirteen different overlays with which they can view the map.

- Regarding the list forms, users can search information by language, source, or phoneme. When searching by phoneme, each sound is organized by frequency.

- There is also an archive of previous released versions. There is no information to describe what database is being used, but the previous versions are hosted on Zenodo. 
The Atlas of the Languages of the World (ALW) series

Accessed from: http://lingvarium.org/raznoe/publications/alw_intro.shtml

[no references available]

Resource type: Web mapping - Static

Brief description:

"Before the first appearance of the Atlas of the World's Languages in 1993, all the world's languages had never been accurately and completely mapped. The Atlas depicts the location of every known living language, including languages on the point of extinction."

Own impressions:

- The actual maps are difficult to access, but this site should be accounted for nonetheless due to its Russian origins.

- Lingvarium, the project/organization these maps are a part of, attempts to provide a Russian-based catalogue of all the world's languages.

○ Other reference data is also available here, including a glossary, dictionaries, and some downloadable statistics on a country-by-country basis.

- The website is in Russian and made with a very basic and minimalist layout. Maps that are in English are indicated in a different colour than Russian maps.

- There is a detailed list of maps. However, many are not accessible, or links are dead.

- One map that is viewable appears to be made in Microsoft Paint by the University of Colorado at Boulder, and itself is thus not of Russian origin.

○ In this map, no overlap between linguistic regions is indicated, overly suggesting monolingualism and linguistic dominance, that may in fact not be the case. The map is from 2007, and the current linguistic situation is likely quite different. There is however a colouration devoted to regions that have no dominant language. In these sections, there is no information regarding what a potential lingua franca may be.

- The map does have a Russian bent: individual regions and their subregions are indicated, but not in the Caucasus Mountains, such as Georgia or Azerbaijan.

- Separatist regions of Georgia are indicated on the map.

- Other maps are findable given enough digging and appear to be made in Microsoft Paint or with some undisclosed GIS software (e.g. some maps use point data).

- The metadata of the maps is inconsistent, and often lacks information such as projection system, contributors, or origin of the data.

- While some maps are said to be available in English, they are in fact in French.

- Many maps present in this atlas/website are very detailed, but there is no background information readily available regarding where this information has come from.

- Some of the pages are fraught with loading errors and cannot be properly accessed. 
Indochina Ethnolinguistic Groups

Accessed from: https://legacy.lib.utexas.edu/maps/indochina_atlas/

[no references available]

Resource type: Uploaded paper map

Basic description:

“"Indochina Atlas", published in October 1970 by the Directorate of Intelligence, Office of Basic and Geographic Intelligence, U.S. Central Intelligence Agency."

Own impressions:

- Part of the "Perry-Castañeda Library Map Collection"

- The map is in print and covers Laos, Cambodia, and Vietnam.

- While linguistic groups are covered, there is also a focus on the regional religious practices. The focus on only three religions (found only in Vietnam) is slightly puzzling, and there is a significant proportion of the map that does not contain any religious information. Perhaps this is due to the political focus on Vietnam during the 1970s.

- A high resolution of detail means that many linguistic enclaves and exclaves give an impression of linguistic diversity and multilingualism. Similarly, overlapping regions are indicated with crosshatching.

- There is a small disclaimer stating that the names and boundary representations on the map are not necessarily authoritative. This comes off as rather vague and does not inspire confidence in the map's integrity, though it is also understood to be a means of not claiming responsibility for erroneous information.

- Ethnic groups are represented along with the linguistic regions.

- The legend contains information on the language (families) being spoken, tribal and ethnic groups, religions, and a small disclaimer describing the Chinese diaspora.

- A scale bar offers measurements in both kilometres and miles.

- Certain linguistic groups have their macro-family designations, while others do not. Similarly, there appears to be a "tribal" designation for many languages (i.e. MonKhmer vs. Tribal Mon-Khmer).

- Lines demarcating latitude and longitude values are present.

- This map was likely intended for use with Intelligence or the military at the time of creation. Since then however, it is likely most frequently accessed by scholars. 
Atlas Lingüistico (Y Etnográfico) De Castilla - La Mancha

Accessed from: http://www.linguas.net/alecman/

García Mouton, P., Moreno Fernández, F., Molina Martos, I., Moreno Fernández, J. E., \& Moreno Fernández, F. (2003). Atlas lingüístico (y etnográfico) de Castilla-La Mancha.

Resource type: Web mapping - Static

\section{Basic description:}

[Translated from Spanish] "We give you the "Atlas Lingüístico (y etnográfico) de Castilla y La Mancha' Project, which summarizes Spanish regions such as Andalusia, the Canary Islands, Aragón, and Navarra and Rioja, and is in the process of surveying Santander."

Own impressions:

- The atlas contains quite a few static maps, all of which appear to be made in Microsoft Paint. However, a disclaimer in another part of the site/atlas describes a specific program developed for the project that automates much of the mapping process.

- Atlante: "automatic cartography and linguistic database"

- Very basic: allows for symbol insertion, label insertion, and legend creation.

- There is a strong emphasis on identifying the informants in every part of the mapping process, and this is done in such a way as to provide informants with the amount of recognition deemed to be sufficient.

- The atlas/site is designed in a basic manner to be specifically available across multiple and varied operating systems.

- The mapping project originates academically: La Universidad de Alcalá, España.

- Most materials on the atlas site are lexically based and compare a set number of communities' terms for something to one another, as well as their phonologies.

- The maps appear to use their own transcription conventions and not the IPA. There is a guide available on the site that explains each symbol using IPA descriptions, interestingly.

- A section of the site also contains published literature related to the atlas.

- A commentary discusses major points of inquiry surrounding the subject matter.

- This includes relative population densities, informant demographics, and technologies used in the geolinguistic investigations.

- More general information about the regions being surveyed is also provided.

- There does not appear to be a section on the site where data can be downloaded.

- While the questionnaire used in the investigation is discussed in detail, the actual questionnaire is not provided. 
Atlas Linguístico-Etnográfico dos Açores

Accessed from: http://www.culturacores.azores.gov.pt/alea/Default.aspx

[no reference available]

Resource type: Web mapping - Static

Basic description:

"[translated from Portuguese] The Knowledge Center of the Azores (CCA) is the result of a project presented by the Regional Directorate for Culture in 2004 under the Regional Program for Innovative Actions (PRAI-Açores). The project focused on: promoting and disseminating Azorean culture through new technologies and providing public access to their knowledge."

Own impressions:

- The geographic aspect of the atlas is at first difficult to find and can only be accessed once multiple links have been followed.

- The remainder of the website/atlas has information regarding informants, map authors, investigators, publications, supplementary information, and a general map of the localities that are covered.

- The atlas is featured on the Government of the Azores website, under the "culture" section, suggesting that it is designed for the purposes of educating potential visitors.

- Despite being a small portion of a government website, there are lots of acknowledgments and funding packages that suggest the atlas is (part of) a large and impactful project.

- Lexical and morphological maps are available for browsing on the "map" page, as well as a main search map, which does not specify if it is lexical or morphological.

- As opposed to being greeted with a map, users are greeted with a search menu where they can either type in keywords or "search all"

- When a word from the "search all" list is accessed, a new tab appears containing an embedded PDF document explaining the etymology of the term in question on the left, and a static map showing the different pronunciations on the right.

- The phonetic transcriptions are made using a script designed for the atlas' umbrella project and loosely based off the IPA. On the maps, only the phonetic transcriptions are present, meaning that users need to be familiar with the specific transcriptions in order to be able to understand the maps' messages.

- The maps all follow the same basic layout of the Azores, and only the data on them change.

- Each map has a page number in the top-right corner, meaning that these maps are likely linked to a print atlas somewhere.

- I am not entirely sure who this map is made for. It is overly unintuitive to use, difficult to find, and requires familiarity with the rest of the project to understand. 
Interactive atlas of the prosody of Portuguese

Accessed from: http://labfon.letras.ulisboa.pt/InAPoP/

Frota, S. and M. Cruz (Coords). (2012-2015) Interactive Atlas of the Prosody of Portuguese Webplatform [http://labfon.letras.ulisboa.pt/InAPoP/]

Resource type: Web mapping - Interactive

Basic description:

"Research on prosodic, intonational and rhythmic variation in Portuguese is presently very limited. This project aims at developing research and applications in this domain, within a program of international scope that proposes a system for prosodic analysis of speech corpora, together with a set of methodological procedures that enable cross-linguistic work on prosodic variation in language. It arises as a natural consequence of much ongoing work in the last decade within the Phonetics Laboratory of the University of Lisbon and other collaborating groups."

Own impressions:

- This atlas does not appear to actually exist yet and is only being proposed at this time. This is a little worrying as the last time it was modified was in 2015. Its proposed coverage is ambitious: Maps of

- The majority of the Lusophone sphere

- The distribution of varieties as to the observed phrasing preferences

- Tonal density

- Intonational typology

- Annotated sentences for intonation and phrasing

- Prototypical examples of the different sentence types from different regions

- Corresponding F0 traces and spectrograms

- Training materials

- Methodological information

- A list of resources for research on Portuguese intonation

- While the project is based in Lisbon, the majority (if not entirety) of the website/atlas is in English.

- While this is not an atlas or a map that can be reviewed in detail, it is still something that should be kept track of, especially given its extensive documentation. 
MADISON - A Sound Map for Portuguese Dialects

Accessed from: http://cards-fly.clul.ul.pt/teitok/madison/en/index.php?action=home

[no references available]

Resource type: Web mapping - Interactive

Basic description:

"The Dialectal Sound Map (MADISON, «Mapa Dialetal Sonoro») is a sample of the Portuguese dialectal landscape from the last quarter of the 20th century. It consists of a collection of extracts drawn from the dialectal interviews that were recorded for several linguistic atlases undertaken by the Dialectology Group from the Center of Linguistics of the University of Lisbon (CLUL)."

Own impressions:

- This atlas is an overarching project that appears to contain the previous project as well.

- This atlas features an interactive sound map, which uses OpenStreetMap as a basemap, and can also be viewed in Fullscreen.

- There are three main sections to the map, including mainland Portugal, Madeira, and the Azores.

- The website is available in both English and Portuguese.

- Data are represented using points, which when clicked, bring the user to a new page (same tab) where they can then access each recording individually. The sound does not automatically play, but instead, a new page opens up containing information such as the date of recording, the location, equipment, informant details, and transcriptions of the recordings. The actual embedded recordings are cumbersome to use. This atlas features more information about the data than the data themselves!

- The map does not reset when navigated back to.

- The information is based off of informants that are elderly, non-mobile, rural, and born in the area they are interviewed in.

- There are questionnaires that were given to the informants, but these are not available for reference. There are also recorded interviews of spontaneous speech, which are stated to be intended for future syntactic research.

- There is a search function named "Corpus search" which is slightly misleading as the term "corpus" is not offered elsewhere. This search function includes a query builder, an option for visualizing the information (link appears dead), and more options in refining the search.

- The "Collaborators" link appears to be dead.

- A login page for members is offered, but there does not appear to be any other information specifying what sorts of things members have access to. 
Atlas linguistique et ethnographique de l'Italie et de la Suisse méridionale (Linguistic and Ethnographic Atlas of Italy and Southern Switzerland)

Accessed from : http://www3.pd.istc.cnr.it/navigais-web/

Jaberg, K. and Jud, J., "Sprach- und Sachatlas Italiens und der Südschweiz”, Vol.1-8, Zofingen, Bern, 1928-1940. [Reprint edition by: Kraus Reprint, Nendeln, Liechtenstein, 1971-1981. Kraus Reprint, New York].

$\underline{\text { Resource type: Uploaded paper map }}$

Basic description:

"Notwithstanding the venerable old age, the Sprach- und Sachatlas Italiens und der Südschweiz (AIS) (Linguistic and Ethnographic Atlas of Italy and Southern Switzerland), is still one of the most useful instruments in linguistic geography, and in study of Romance and Italian dialectology. Moreover, the atlas turns out to be an inexhaustible mine of ethnographic information."

Own impressions:

- The atlas was originally in print form and was uploaded using a well-documented procedure. What is discussed here focuses on the web version. A large (2 GB) atlas navigator is also available for download.

- The atlas is designed in much of the same way as the ALF, and at first glance appears to take advantage of the same transcription conventions.

- The maps are separated according to Northern Italy and Switzerland, and Southern Italy, including Sardinia and Sicily.

- The maps are written in German, even though the majority of commentary about the atlas is written in French.

- On the online interface, users can select the map they wish to view with a dropdown menu. Another dropdown menu allows users to search for information by research site. This interface is claimed to have been built solely using Javascript technology.

- There is no projection system or topography, and the legend is very basic.

- The main website offers a commentary regarding both the linguistic situations of Italy at the time of publication (1928) and the processes through which the documents were scanned in the first place. This allows for documentation of the process of transferring paper maps into the digital sphere.

- The main site contains a manual of how to use the downloadable software. This software allows users to perform tasks such as setting the interface language, choosing whether or not to display the map background, alternating between volumes, and setting the map directories. Maps have a zoom and print feature.

- A restricted area for members is also present on the main website.

- The data do not appear to be separately available in spreadsheet form, but are still embedded in the maps. 
The TELSUR project

Accessed from: https://www.ling.upenn.edu/phono_atlas/home.html

[no references available]

Resource type: Web mapping - Interactive

Basic description:

"The Telsur Project is a survey of linguistic changes in progress in North American English, supported by the National Science Foundation and the National Endowment for the Humanities. It is the creator of the Atlas of North American English [ANAE], (formerly, the Phonological Atlas of North America)."

Own impressions:

- The website is basic and minimalist and starts with a description of the maps found in the atlas. No maps are found on the initial page, and need to be navigated to elsewhere in the site.

- Several maps are available in the atlas corresponding to regions in the United States.

- The maps are basic, and do not have a corresponding legend, scalebar, or north arrow.

- The atlas aims to focus on the distribution of dialects of North American English in the United States by focusing on chain shifts and low short vowels.

- Political boundaries are present, and each linguistic region is separated with solid isogloss boundaries and has attached labels.

- The data are informant-focused, and one of the available features is to click on points on the map to access more information about the informants and their speech varieties. However, the links are now dead.

- This atlas was likely designed as a pilot project, and is stated to be a predecessor to the "National Map of the Regional Dialects of American English" mentioned earlier in this document. This might mean that it was not intended to be maintained.

- While the atlas is no longer active, it is the basis for numerous publications that have resulted in subsequent atlas projects.

- There does not appear to be any data available for download.

- A guide to the sampling methods is available on the main atlas page. 
Linguistic Atlas of Kansas German Dialects

Accessed from: http://www2.ku.edu/ germanic/LAKGD/Atlas_Intro.shtml

[no references available]

Resource type: Web mapping - Interactive

[no basic description on website]

Own impressions:

- The map is present on the home page of the atlas site and is relatively basic and monochrome.

- The top of the home page contains an artistically-written history of German in Kansas.

- The map is minimally interactive, and sound recordings can be accessed by clicking on darker counties that indicate the presence of speakers.

- When the active sound file links are clicked, a new page appears on the same tab that contains a map of the county in question, the German speaker population, and a link to a list of sound recordings that opens up in a new tab. Lists have 40 entries.

- A list of questionnaires used in the research is provided, as well as a privacy disclaimer.

- On another page in the site/atlas, there is a static map that indicates the distribution of dialects of Kansas German.

- On this site, a link to an additional map is provided that indicates the relative importance of German settlements in Kansas. This map appears to be much older than the other maps present on the site, but may have been produced using older techniques.

- Another supplementary map offered is of "German homeland" dialects in Europe, as well as a much older map of Volga German dialects in Russia.

- An additional section on the site/atlas focuses on the history of the distribution of German dialects in Kansas, including older census summaries and statistics related to German being used in public spheres.

- Many of the maps appear to not have originated with the project, but are brought in from other sources.

- Much of the information offered on this site appears to have come from the 2000 U.S census.

- There is an additional list of miscellaneous recordings on the site.

- A simple database search allows users to look up sentences by clicking on various items on a dropdown menu.

- Unclear who the intended audience of this atlas is, but it is designed for either scholars or for general reference. 
Atlas Lingüístico de Puerto Rico

Accessed from: http://www.alpr.info/

[no references available]

Resource type: Uploaded paper map

Basic description:

"[translated from Spanish] Firstly, the creation of a webpage for the Altas Lingüístico de Puerto Rico (ALPR) is important as the atlas in question is the first of its kind in the Hispanic domain (both Spanish and Latin American) which has been brought to light."

Own impressions:

- The original atlas was first published in 1948, and involved research spanning as far back as 1927.

- The atlas is immediately stated as being important to the general public as a detailed account of how Spanish terms are used and differ in Puerto Rico can help clarify ambiguities and miscommunications, such as in legal proceedings.

- Informants' information such as their names, occupations, and locations are given in such a way as to recognize informants' contributions and contextualize their language use.

- The atlas comes with a disclaimer and examples that explain the phonetic transcription method, the use of symbols, the limitations of the solid lines and boundaries that are used, and the fact that only one informant was used per region, meaning that internal diversity cannot be accounted for. This is quite rare in an atlas, and greatly appreciated!

- The maps are based on the main island of Puerto Rico, which is separated into its different municipalities in a style the authors cite as resembling the Atlas Linguistique de la France. The maps and transcriptions indeed appear to be inspired by the ALF.

- A small account describes the history of Spanish in Puerto Rico and the factors that contributed to the interesting amount of dialectal diversity that is present.

- A questionnaire was used that contained 445 different questions related to pronunciation, morphology, syntax, and vocabulary.

- The atlas page no longer seems to be able to be hosted directly on the website, but a link to a Flickr account with each of the maps listed is provided.

- The maps appear to have been hand-drawn, and dotted lines form isoglosses that separate regions that differ from one another. Different symbols (i.e. squares vs. triangles) also help to emphasize these differences.

- Some maps have their transcriptions directly embedded, while others have numbers which refer to transcriptions that are found on a list on a different page.

- Many of the terms are colloquialisms or words that differ from standard Spanish. 
Mapa lingüístico de América Latina

Accessed from: http://spanport.utoronto.ca/spa321/Index.htm

[no references available]

Resource type: Web mapping - Interactive

Basic description:

"[translated from Spanish] This site allows users to listen to recordings of Spanish speakers from different countries [in Latin America]. This site also imparts basic characteristics of Castilian Spanish spoken in each of the countries. For the moment, we only have information and recordings from Argentina, Chile, and the Dominican Republic. To start, click on each of the three countries."

Own impressions:

- The atlas site and the map are very basic. One short description, and a single map that appears to have been made in Microsoft Paint. From here, one can click on one of the three highlighted countries.

- When clicked on, a new page for each country is accessed using the same tab, meaning that users must navigate away from the main atlas.

- The fonts used in the transcriptions are not supported by Unicode, meaning that they do not appear properly in browsers.

- Here, a short list of some distinguishing dialectal (mostly phonological) phenomena is present, along with a menu that allows users to access things such as recordings and texts. These links are dead.

- There are no other commentary, acknowledgments, credits, or maps sections. 
The Toronto Language Map

Accessed from: https://www.socialplanningtoronto.org/languages_map

[no references available]

Resource type: Web mapping - Interactive

Basic description:

"The Toronto Language Map is an interactive resource that allows users to explore Toronto's evolving linguistic diversity over the past decade. Using 2006, 2011 and 2016 census data, this resource reveals the changing linguistic makeup of the city based on the languages that residents speak most often at home."

Own impressions:

- The map is easy to find, colourful, simple, and intuitive to use.

- While there is no legend per se, there is a menu on the right-hand side of the map that allows one to isolate particular languages for use on the map.

- The map page and other pages on the atlas are colourful and organized.

- The authors' bias comes through in number of subtle ways, including a separation between the "Chinese" and "Cantonese" languages, and the fact that English is indicated on the map as grey (colourless), and is not found in the menu. Similarly, no statistics are available for English, while basic statistics are available for other languages.

- Census data are available and easily interchangeable from 2006, 2011, and 2016.

- While no direct statistics are available apart from percentage of speakers in an area and the number of participants used, darker shades on the map indicate greater percentages of speakers.

- It is unclear whether these different languages comprise a majority of the area, or if they are the most commonly spoken languages after English.

- Specific locations can be manually searched using a tool at the bottom of the screen. This tool is not at first evident and requires some effort to find.

- When each region is hovered over, the speaker number and percentage come up. When clicked, the relative rankings of other languages also appear.

- An accompanying page contains statistics related to language use in the home and how it has changed in the 10 recorded years.

- This map appears to have been designed for general reference, likely mostly amongst residents of Toronto. 
Langscape

Accessed from: http://langscape.umd.edu/map.php

[no references available]

Resource type: Web mapping technology

Basic description:

"Langscape is a developing online resource for mapping, integrating and making discoverable a wide range of information about the world's languages as well as for raising public awareness of language diversity. Langscape allows users with interests ranging from recreational to academic to discover the world's languages via interactive tools and access to established research."

Own impressions:

- The data originates from Glottolog, which is a database that contains "comprehensive reference information for the world's languages, especially the lesser known languages."

- The interactive map is created using Esri technologies.

- Users can choose from two layers to view the maps with, including official/majority languages, and unofficial/minority/Indigenous languages.

- Each smaller/unofficial language is represented with a point on the map, which provides users with links to more information in a window that appears below.

- This information includes links to Wikipedia in a number of different languages, as well as genetic relatedness to other languages and in some instances speech recordings.

- None of this information details how many people speak the language, its linguistic boundaries, or its vitality.

- All language points are the same colour, and do not indicate any other information such as status or relatedness.

- Many of the languages represented on the map are extinct and have been for centuries.

- Base maps are available in four different settings, such as satellite and topological.

- There is a search feature that allows users to find a specific language.

- Other features on the map include a language identification tool (where users can paste text and find out what language it is), lists of other resources, a mission statement, and an account of intended future directions.

- The atlas is created by an academic institution and is most likely to be used by other academics or institutions.

- A GIS-based language game is also linked to the site, but this link appears to be down

- There is a section that allows users to contribute more information, or funds.

- The map site has a blog, which has not been updated since 2015. 


\section{$\underline{\text { Glottolog }}$}

Accessed from: https://glottolog.org/

Hammarström, Harald \& Forkel, Robert \& Haspelmath, Martin. 2019. Glottolog 4.0.

Jena: Max Planck Institute for the Science of Human History. (Available online at http://glottolog.org, Accessed on 2019-07-08.)

Resource type: Web mapping - Interactive

Basic description:

"Glottolog provides a comprehensive catalogue of the world's languages, language families and dialects. It assigns a unique and stable identifier (the Glottocode) to (in principle) all languoids, i.e. all families, languages, and dialects."

Own impressions:

- While this is not an atlas or map in of itself, it is an important online database with a spatial component that should be accounted for.

- Data are organized according to languages, or families, and are coded for name, toplevel family, ISO number, Macro-Area, child dialects, and geographic co-ordinates.

- Language data are available for download in a number of different formats

- The original language data come from studies performed by a variety of researchers from a variety of dates, some older than a hundred years.

- This means that while a lot of citable information is present, significant change has likely occurred since the time of original research and present representation on the map. Similarly, researchers likely all come from different backgrounds, meaning that the research was probably performed differently for each study.

- These references are coded for the area of linguistics they are a part of (e.g. phonology), making sources easy to find. There is also a separate reference search feature which further simplifies this process.

- This database is maintained by the Max Planck institute, and thus likely has ongoing funding. 
Modern Language Association (MLA) map

Accessed from: https://www.mla.org/Resources/Research/MLA-Language-Map

[no references available]

Resource type: Web mapping - Interactive

Basic description:

"The MLA Language Map is for students, teachers, and anyone interested in learning about the linguistic and cultural composition of the United States. The Language Map uses aggregated data from the 2006-10 American Community Survey (ACS) to display the locations and numbers of speakers of twelve languages commonly spoken in the United States."

Own impressions:

- $\quad$ The map is created using the ESRI technology ArcGIS.

- The map first shows the most commonly spoken language per county not including English or Spanish. Each county is coloured according to the resident language, which is detailed in a legend on the right-hand side of the screen. There are circles of varying size present that represent the number of enrollments in the language.

- A short paragraph detailing how to use the map is present in the top left-hand corner of the screen, complete with links to a FAQ section, the source of the data, and contact information (the author's e-mail account).

- On the bottom-left-hand corner, there is a layers section, which is separate from the legend. Two layers are present at first (Enrollments and Predominant Languages; Languages by percentage of speakers), but these are collapsed, and when opened have many more selectable/deselectable sublayers to choose from.

- In enrollments and predominant languages, one can also choose a sublayer that also includes English or Spanish as the primary language.

- In the Languages by percentage of speakers, one can choose which language they would like to see present. One or multiple languages can be selected.

- Once selected, these languages indicate what percentage of residents speak the particular language, and percentages are related to the statistics.

- There is also an option on this site to create tables of the data, whose resolution can be on a state, region, or census subdivision level.

- Language enrolment data from 1958 to 2016 is downloaded in various formats.

- There is finally a section where one can access many of the primary documents, such as original reports and datasets.

- The site is minimalist in many ways and aesthetically pleasing. This map does not appear to be part of an atlas, but instead one feature of a site that contains many different ways of focusing on data. 
Algonquian Linguistic Atlas

Accessed from: http://www.atlas-ling.ca

Junker, M-O. (dir.), MacKenzie, M., Rosen, N., Valentine, J. R., \& Wolvengrey, A. (codir.). (2005-2020). The Algonquian Linguistic Atlas.

Resource type: Web mapping - Interactive

Basic description:

"The goal of the project is to co-create an on-line, multimedia linguistic atlas of Algonquian languages. The creation of this atlas allows us to offer many training opportunities for sound editing and linguistic description training to aboriginal students." Own impressions:

- The atlas site begins practically with a popup window that details the browser requirements for use along with links to apps available for smartphone usage.

- Unlike websites, this interface is very much a digital atlas, and is strongly based with the map as the medium, not just one feature of a much larger site.

- The main map that appears when the atlas is first accessed is a satellite map of Canada featuring points, which play recordings of the language local to the area.

- These recordings can be selected and changed in a set of two drop-down menus in the top right-hand corner of the screen.

$\circ$ When the recordings are accessed from the point on the map, a bubble appears that shows informant information, such as name, community, and dialect.

- Each point is a different colour and shape, making it easy to discern dialects.

$\circ$ While the legend is not first visible, it can easily be accessed and then closed.

- There are also sections in the main atlas devoted to instructions, more information about the atlas, credits to both authors and informants, a language learning module, and a downloadable version of the atlas.

$\circ \quad$ The language learning module currently offers lessons in PDF form.

- Next to a legend is another feature, which brings the user to a page where they can alternate between a number of dialectal maps.

- These maps also come with a set of instructions, a legend, a disclaimer, the option to switch between English and French as the main content language, and a button to navigate back to the main atlas page.

- The instructions, legend, and disclaimer do not appear on a new and separate page, but instead appear in a popup window.

- Sound files and conversation manuals are available for download, as well as conversation apps which users can use directly on their phones. 
First Peoples Language map of British Columbia

Accessed from: https://maps.fpcc.ca/languages

First Peoples' Map of British Columbia. (2020). Languages. [map]. First Peoples' Cultural Council.

Resource type: Web mapping - Interactive.

Basic description:

"British Columbia is home to 203 First Nations communities and an amazing diversity of Indigenous languages; approximately $60 \%$ of the First Peoples' languages of Canada are spoken in B.C. You can access indexes of all the languages, First Nations and Community Champions through the top navigation on all pages of this website"

Own impressions:

- A welcome screen appears when the atlas is first accessed, which prompts users to select a language map, a map of arts initiatives, and points of interest related to cultural heritage. The map appears to be well-organized and intuitive to use.

- Information from all three maps is shown at the same time.

- The language map is colourful and visually appealing, and related language varieties are coloured similarly to each other.

- While the map focuses on languages spoken in British Columbia, many languages are also covered in Alberta, as well as Washington, Idaho, and Montana, likely because many linguistic groups transcend political boundaries in the region.

- The atlas is hosted on the MapBox platform, while the basemap is OpenStreetMap.

- There is a list on the left-hand side of the map that allows one to click on a language community. When clicked, the map zooms to the specific region, and a number of options become available. These include audio recordings of the language's name, as well as a greeting in the language. Links to language learning resources are also present, as well as links to related information in other map layers, such as communities, artists, and points of interest.

- Alternate names and numbers for total population, fluent speakers, learning speakers, and those who partially understand the language are also present.

- The same lists and functions can be accessed by clicking on the coloured polygons superimposed on the basemap used to denote where a language is spoken.

- Across the top of the map, there is a search bar, a link to a popup window showing relevant upcoming events, a link to a login page, and a button that expands the menu to include a page where one can order maps, learn about the atlas' terms of use, a help page, and a "contact us" page.

- Users are encouraged to add their own information to the maps, and are able to make changes to their own information.

- There is an available feature for these maps to be shared/embedded on other websites. 
The Atlas of the Languages of Iran

Accessed from: http://iranatlas.net/index.html

Anonby, Erik, Mortaza Taheri-Ardali, et al. (eds.). (2015-2019). Atlas of the Languages

of Iran (ALI). Geomatics and Cartographic Research Centre, Carleton University.

Resource type: Web mapping - Interactive

Basic description:

"Rather than communicating a single view of Iran's languages and dialects, the Atlas allows users to enrich their own perspectives on language distribution with location-based language data."

Own impressions:

- The atlas appears minimalist at first glance, with a Google Maps overlay and points on a map focused on Iran that indicate a site where data have been gathered.

- When one hovers over a datapoint, information about the datapoint such as settlement name and the recorded language varieties present appears.

- When one clicks on the datapoint, a more detailed window appears in the right-hand side of the map that contains information such as the site's population, location, alternate pronunciations, and metadata.

- Much of the information present in this section also appears to be designed for use in other systems. In this way, the atlas is designed to be an ongoing resource for other mapping projects as well.

- There are additional features available in this atlas, including:

- Supplementary language maps, which are in both static and interactive form, that focus on language distributions on a provincial level as well as distributions of particular linguistic features.

- Two language classification trees: one is a classical static classification tree that catalogs language varieties in list form, and one is a dynamic and interactive visual tree that plots out how different language varieties are related to one another.

- Atlas resources including a bibliography, questionnaires, and transcription conventions used throughout the atlas

- This atlas devotes ample time and space to enabling users to conduct their own research, and if possible, contribute to the atlas themselves. This is an important feature of bottom-up mapping, as it ensures that users of the atlas are also able to play a significant role in geolinguistic research.

- There is a section that explicitly outlines these values and how they are important in research and cultural empowerment more generally. 
Language Map of British Columbia

Accessed from: https://maps.fpcc.ca/

[no references available]

Resource type: Web mapping - Interactive

Basic description:

"British Columbia is home to 203 First Nations communities and an amazing diversity of Indigenous languages; approximately $60 \%$ of the First Peoples' languages of Canada are spoken in BC. You can access indexes of all the languages, First Nations and Community Champions through the top navigation on all pages of this website."

Own impressions:

- The map page is owned and operated by the First Peoples' Cultural Council, meaning that it has been produced with Indigenous perspectives and interests in mind.

- The map contains colourful polygons displaying the language name when hovered on.

- When clicked on, a bubble appears with the language name and a placeholder where sound recordings are intended to go.

- When the names in the bubbles are clicked, the user is brought to a page, which discusses the specific language's information in greater detail along with links to relevant resources (e.g. keyboards, dictionaries, etc.).

- There are many placeholders for information that is not present yet, suggesting that there are plans for this atlas project to be expanded.

- There are other pages as part of the site where Indigenous languages and communities are listed, each of which link to specific points on the main map.

- There is a separate page devoted to placenames that contains a main map displaying all placename data, as well as a series of smaller-sized maps below that display each language and their locality. Difficult to believe that each language has exactly one point on the map however, and while it is interesting to see each language instantly, the decision to use multiple maps in this way is a little confusing.

- An additional page offers a list of phrases in a variety of Indigenous languages found in British Columbia. This list is not organized according to any parameters however, and looks quite prototypical in nature.

- There is a search feature available at the top of this page, which is minimalist and allows one to search by phrase or language.

- A help section offers information regarding the language map project, links to order printed maps, a glossary of terms, data sources, and an ability to create a language map account. There are also links to download a Language Needs Assessment file. This help section appears to basically mirror additional pages in the "Home" section.

- While there is a section that discusses where data have come from, no links are offered and the citation style is minimal and inconsistent. 
Sprachatlas Niederbayern

Accessed from: https://sprachatlasniederbayern.bayerische-landesbibliothek-online.de/

Der "Sprechende Sprachatlas von Niederbayern und angrenzendem Böhmerwald" entstand ab 2009 als Kooperationsprojekt der Universität Passau und der Bayerischen Staatsbibliothek unter der wissenschaftlichen Leitung des Lehrstuhls für Deutsche Sprachwissenschaft an der Universität Passau (Prof. Dr. Rüdiger Harnisch).

Resource type: Web mapping - Interactive

Basic description:

"The around 6,000 audio files from 207 Lower Bavarian and 22 Czech locations have been compiled and academically evaluated by the chair for German linguistics of the University of Passau within the framework of various linguistic-geographical projects in the course of several years. The Bayerische Staatsbibliothek (BSB) has integrated the audio files into 32 digital maps, thereby making the exciting service accessible to the public via Bayerische Landesbibliothek Online, and additionally ensuring the long-time archiving of the recordings."

Own impressions:

- The map uses flash, which means that already many browsers are unable to host it.

- When accessed, the site immediately shows a map of Bavaria and the Czech republic.

- The site is only available in German, which makes sense as it's a German project.

- One can access one layer at a time in a menu on the left-hand side. These layers are organized by category, and include nature terms, months, vowels, consonants, verbs, and nouns. The information is presented as sound recordings, which manifest instantly when clicked on. When hovered over, points on a map that are superimposed over coloured polygons display the names of the settlements from which the data originate.

- Below this first menu is a second one where all recordings for a particular region are played in sequence. When hovering over items in this menu, the polygons gain a glow around their borders to indicate the region of interest.

- While this map is very clear while being simple, the size and number of points means that the underlying polygons can be difficult to see in detail. There is no apparent option to isolate particular areas or phenomena.

- One can select/deselect basemap layers, such as political boundaries or a relief map.

- No projection systems are used, and each different layer contains a simple legend. A fullscreen option is available, the map can be refreshed to its initial state.

- A "help" link is present, but nothing seems to happen when it is clicked on. When the link is clicked on, whichever data point was previously selected remains highlighted, and multiple data points can be simultaneously highlighted in this way. 


\section{L’Atlas linguistique de la Wallonie (ALW)}

Accessed from: http://alw.philo.ulg.ac.be/

[references available as per volume]

Resource type: Uploaded paper map

Basic description:

“[translated from French] L'Atlas linguistique de la Wallonie (ALW) is an ascomplete-as-possible presentation of Wallon, Picard, Lorrain, and Champenois speakers in Belgium. Of the twenty volumes which comprise the entire publication (as well as a general index), ten are currently published."

Own impressions:

- The maps available on the website are at first difficult to find, and are a limited collection of the maps that are available in the published volumes. At this time, sample maps from volumes 1, 2, 6, and 17 are available for viewing.

- The maps that are available for viewing are good-quality uploads, and contain accompanying commentary and observations.

- The maps are hand-drawn, and consist of multiple point-based observations superimposed on a single base map, which is similar to the ALF.

- The goal of this site appears not to be to host maps per se, but to provide a preview of what the actual atlas contains.

- Unlike the ALF, data points are represented with differently shaped symbols, which correspond to a legend on the bottom-left corner of the map. These symbols also have numbers which correspond to a list above the map.

- There are some places where there is a textured background, similar to a polygon, which I assume is just another kind of symbology.

- The accompanying commentary discusses overall trends observed on the map for each linguistic phenomenon.

- Each map does not contain colour but is complete with a title (to the left of Wallonie, not on top of the map), and below that a simple legend without any background or border. Below this is a scale bar. There is also information corresponding to which map and volume is present.

- While strong and definite boundaries represent national borders, the discrete boundaries between regions of various phenomena are dotted lines, suggesting to a certain extent that the differences are perhaps less discrete. 
IranCarto - Cartographic studies on Iran and the Iranian world

Accessed from: http://www.irancarto.cnrs.fr/index.php?l=en

[no reference available]

Resource type: Uploaded paper map

Basic description:

“- A permanent Atlas of Iran and the Iranian World online

- A Census of the Maps of Iran and the Iranian World on Internet

- Online Publication of Atlas (Iran, Tehran, ethnography)"

Own impressions:

- The online atlas in fact appears to be a repository of other atlases that are all hosted on the same site.

- The layout is minimal yet elegant. A map is hosted on the main page, and users can toggle between other maps as well. Below the map is a simple description of IranCarto, and to the left is a menu containing links to other atlases, historical commentary, and information about the people, politics, society, and economics.

- The maps present in the atlases on the site are static, but this site contains perhaps the richest commentaries of any site reviewed thus far.

- Most maps viewed appear to be made electronically with some GIS software, with basemaps made of both vector and raster images. Some are hand drawn.

- Many of these maps contain scale bars, legends, titles, and co-ordinates on the sides of the map.

- As these maps are part of atlas compilations, many of them have different mapping and spelling conventions, especially it comes to Romanized Persian spelling.

- Each map has a devoted section to metadata, and a devoted section to external links.

- There is a sitewide search feature available, which is basic, but fast and offers easilyparsable results.

- There is a section of the site purporting to analyze maps of Iran found elsewhere on the Internet. However, no information shows up here.

- The site is also available in French and Farsi. Both French and Farsi language versions of the site have content in comparable detail, but the maps are still mostly either in English or French, or bilingual with Farsi.

- Links to the sponsoring organizations are offered at the bottom of the home page.

- Overall, this site is easy-to-use, and a wealth of information. As this website is a repository of historical information and documents, it does not directly contain any geospatial data linked to anything and is not a front end to anything. 
Isfahan xxi. Provincial Dialects

Accessed from: http://www.iranicaonline.org/articles/isfahan-xxi-provincial-dialects

[no reference available]

Resource type: Web mapping - Static

Basic description:

"The Encyclopædia Iranica is a comprehensive research tool dedicated to the study of Iranian civilization in the Middle East, the Caucasus, Central Asia, and the Indian subcontinent."

Own impressions:

- The maps are present as a series of images hosted in an online encyclopedia article written by Donald Stilo.

- Among the very few maps of actual linguistic data of Iran's languages.

- The objective of this article and the maps within it is to indicate different groupings of Iranian and non-Iranian languages and dialects. These are demonstrated through maps that show semantic shifts, lexical shifts, and phonological changes.

- Subsequent comments will focus on the maps:

- The maps are very minimalist and basic in appearance, and do not contain any basemaps/ backgrounds, north arrows, or scalebars. A rudimentary legend is present, which shows a numbered list, where terms correspond to numbers which are actually present on the map.

- The map gives the appearance of being an early example of computergenerated mapping.

- These maps are quite technical in nature and require careful reading (and likely a strong background knowledge) to be understood, leading to the assumption that they are either created for other researchers, or as a prototype which is intended to be expanded upon later. It is nearly impossible to understand these maps without either a map of the Isfahan area for reference, or an intimate knowledge of the region.

- Despite the minimal nature of the maps, there is extensive usage of isoglosses; both overlapping and side-by-side.

- Some of the maps are truly impossible to decipher without an accompanying guide to specific abbreviations. An important feature of linguistic maps is that they are able to summarize large amounts of data in an easily understandable way that quickly leads a reader to a particular conclusion. These maps do not seem to do such a thing. 
Tübinger Atlas des Vorderen Orients (TAVO)

Orywal, Erwin (ed.). 1988. Karte 10, Vorderer Orient: Sprachen und Dialekte [Map 10, Middle East: Languages and Dialects]. Tübinger Atlas des Vorderen Orients (TAVO), series A, vol. 8. Wiesbaden: Reichert.

Resource type: Uploaded paper map

Basic description:

"A masterpiece of academic work, the TAVO atlas consists of close to 400 maps on 295 sheets, presenting the largest cartographical project on the Near and Middle East ever. A bilingual publication in German and English, the TAVO atlas covers the Near and Middle East from the European Parts of Turkey down to Sudan, from the Arab Peninsula up to Central Asia."

Own impressions:

- The maps are visually appealing and colourful with an ostensibly high amount of detail.

- The maps employ polygons with discrete boundaries.

- Crosshatching:

- A maximum of two languages appear representable on the map and are denoted using diagonal crosshatching.

- Languages that are closely related to one another are also shown with diagonal crosshatching, leading to the assumption that there is one "main" language that has a number of offshoots.

- The difference between the two crosshatching styles is the related dialects are represented using thin black lines while the linguistic overlap is represented with diagonal lines using the colours of each language.

- While political borders are used in some cases, they are difficult to see in others and look the same as linguistic boundaries.

- There is a legend present on the left-hand side and is complex enough that it spans the length of the page in two columns.

- Languages on the map are not necessarily related to one another but are ones that occupy the same geographic area.

- The map is complete with metadata, a scalebar, co-ordinates in the border surrounding the map, and the names of large bodies of water.

- Point symbols:

- Especially surrounding the Iran-Iraq border, settlements are shown using dots.

- Settlement-specific dialects are shown using rectangles and triangles.

- There are so many colours used on the map that many are confusable with one another. 
Web Mapping Technologies

Apple Maps

https://mapsconnect.apple.com/

“Apple Maps (or simply Maps) is a web mapping service developed by Apple Inc. It is the default map system of iOS, macOS, and watchOS. It provides directions and estimated times of arrival for automobile, pedestrian, and public transportation navigation. Apple Maps also features Flyover mode, a feature that enables a user to explore certain densely populated urban centers and other places of interest in a 3D landscape composed of models of buildings and structures. On September 19, 2012, Apple released its mapping service in iOS, replacing Google Maps as the default mapping service for Apple operating systems."

Price: Included with Apple products

ArcGIS Online $\quad$ https://www.esri.com/en-us/arcgis/products/arcgis-online/overview

"Part of the Esri Geospatial Cloud, ArcGIS Online enables you to connect people, locations, and data using interactive maps. Work with smart, data-driven styles and intuitive analysis tools that deliver location intelligence. Share your insights with the world or specific groups.

Price: Paid subscription

D3JS

https://d3js.org/

"D3.js is a JavaScript library for manipulating documents based on data. D3 helps you bring data to life using HTML, SVG, and CSS. D3's emphasis on web standards gives you the full capabilities of modern browsers without tying yourself to a proprietary framework, combining powerful visualization components and a data-driven approach to DOM manipulation."

Price: Free

GeoNode $\quad$ http://geonode.org/

"GeoNode is a web-based application and platform for developing geospatial information systems (GIS) and for deploying spatial data infrastructures (SDI). It is designed to be extended and modified, and can be integrated into existing platforms."

Price: Free 
GeoServer http://geoserver.org/

"GeoServer is an open source server for sharing geospatial data. Designed for interoperability, it publishes data from any major spatial data source using open standards. GeoServer is a OGC compliant implementation of a number of open standards such as Web Feature Service (WFS), Web Map Service (WMS), and Web Coverage Service (WCS). Additional formats and publication options are available including Web Map Tile Service (WMTS) and extensions for Catalogue Service (CSW) and Web Processing Service (WPS).”

Price: Free

Google Maps http://www.maps.google.com/

"Google Maps is a web mapping service developed by Google. It offers satellite imagery, aerial photography, street maps, $360^{\circ}$ panoramic views of streets, real-time traffic conditions, and route planning for traveling by foot, car, bicycle and air, or public transportation."

Price: Free

Here Maps https://www.here.com/

"Here WeGo is a web mapping and navigation service, operated by Here Technologies. Originally developed by Nokia as Here Maps, the mapping software application was first released for Windows Phone and the World Wide Web in 2013 as a revamped version of Nokia Maps. The Here Maps application was then released for the Android platform on 10 December 2014 and later for iOS on 11 March 2015, and the name was changed to Here WeGo in July 2016. The data is provided in-house by Here, which includes satellite views, traffic data, and other location services. Maps are updated at two- to three-month intervals."

Mapbox $\quad$ https://www.mapbox.com/

"Mapbox is a developer platform used across industries to create custom applications that solve problems with maps, data, and spatial analysis. Mapbox's tools are building blocks that support every part of the web and mobile map-making process."

Price: Paid subscription 
OpenStreetMap https:/www.openstreetmap.org/

"OpenStreetMap powers map data on thousands of web sites, mobile apps, and hardware devices. OpenStreetMap is built by a community of mappers that contribute and maintain data about roads, trails, cafés, railway stations, and much more, all over the world. OpenStreetMap emphasizes local knowledge. Contributors use aerial imagery, GPS devices, and low-tech field maps to verify that OSM is accurate and up to date.

Price: $\underline{\text { Free }}$

TurfJS

https://turfjs.org/

"Modular, simple-to-understand JavaScript functions that speak GeoJSON. Turf is a collection of small modules, you only need to take what you want to use. Takes advantage of the newest algorithms and doesn't require you to send data to a server."

Price: Free

WorldMap http://worldmap.harvard.edu/

"Harvard WorldMap is an Open Source mapping platform developed to lower barriers for scholars who wish to explore, visualize, edit, and publish geospatial information." Basic definitions of what WorldMap defines as "Layers" and a "map" in the first place help define core principles of web mapping before projects even begin. Multilingual user manuals help to orient and train the prospective user. This is a great means for allowing greater access for web mapping in ways that overtake GIS, due to their ostensible ease of use and free nature. WorldMap is working on joining forces with GeoNode, which is part of the OSGeo project. The software is hosted on the Massachusetts Open Cloud (MOC) network. All the open source code used to make maps is available online thorough GitHub. The project offers a roadmap of their past and current development, and where they hope to go in the future. New maps can be created using this platform, and existing maps can be viewed for research and inspiration.

Price: Free 


\section{GIS Software}

ArcGIS https://www.esri.com/en-us/arcgis/products/arcgis-pro/overview

"Mash up multiple data sources to make maps that are as smart as they are beautiful. Use powerful 2D and 3D analytical tools to identify patterns and trends. Then put this information to good use by sharing it within your organization, online, and through mobile apps."

Price: Paid subscription

Capaware! http://www.capaware.org/

"Capaware! es un framework de desarrollo de entornos 3D geográficos multicapa. La potencia de poder realizar aplicativos directamente sobre un terreno $3 \mathrm{~d}$ abre un amplio abanico de aplicaciones a Capaware!. Nada mejor para hacerse una composición de lugar del escenario de trabajo que poder visualizarlo en tres dimensiones. Capaware! permite la vusualización[sic] de grandes extensiones de terrenos ya que usa en todo momento la mínima cantidad de información necesaria para mostrar la porción de territorio observada. Aquellas partes que no son visibles no se procesan, por lo que las cargas al procesador son las minimas necesarias en todo momento. »

[Own translation] "Capaware! It is a multilayer geographic $3 D$ environment development framework. The power of being able to make applications directly on a $3 D$ terrain opens up a wide range of functions to Capaware!. What better way to make a composition of the workplace scenario than to visualize it in three dimensions? Capaware! enables the visualization of large land areas as it uses the minimum amount of information necessary show the portion of territory observed. Regions not visible are not processed, so the processing loads are as low as possible."

Price : Free

\section{GRASS GIS https:/grass.osgeo.org/}

"GRASS GIS, commonly referred to as GRASS (Geographic Resources Analysis Support System), is a free and open source Geographic Information System (GIS) software suite used for geospatial data management and analysis, image processing, graphics and maps production, spatial modeling, and visualization. GRASS GIS is currently used in academic and commercial settings around the world, as well as by many governmental agencies and environmental consulting companies. It is a founding member of the Open Source Geospatial Foundation (OSGeo)."

Price: $\underline{\text { Free }}$ 
"gvSIG is known for having a user-friendly interface, being able to access the most common formats, both vector and raster ones. It features a wide range of tools for working with geographic-like information (query tools, layout creation, geoprocessing, networks, etc.)."

Price: $\underline{\text { Free }}$

ILWIS https://gisgeography.com/ilwis-integrated-land-and-water-informationmanagement/

"Over the years, ILWIS has improved significantly. Notably, its visualization had a massive upgrade in version 3.8 for overall performance in maps. The most significant upgrade is how the performance of its drawing speed increased. As part of this upgrade, maps have the capability to display transparencies at many levels (whole maps, selections, individual elements or properties). Furthermore, almost all elements can be made transparent." 3D visualization and drawing.

Price: $\underline{\text { Free }}$

MapWindowGIS https://www.mapwindow.org/

"The MapWindow GIS project includes a free and open source desktop geographic information system (GIS) with an extensible plugin architecture, a GIS ActiveX control, and C\# GIS programmer library called DotSpatial. We would be happy to have you join our project. MapWindow4, MapWindow5 and MapWinGIS are connected with each other and have their own team of developers. DotSpatial and HydroDesktop are also connected and have their own team."

Price: $\underline{\text { Free }}$

OpenJUMP http://www.openjump.org/

"OpenJUMP is an open source Geographic Information System (GIS) written in the Java programming language. It is developed and maintained by a group of volunteers from around the globe. OpenJUMP started as JUMP GIS designed by Vivid Solutions."

Price: $\underline{\text { Free }}$ 
SAGA GIS $\quad$ http://www.saga-gis.org/en/index.html

"System for Automated Geoscientific Analyses (SAGA GIS) is a geographic information system (GIS) computer program, used to edit spatial data. It is free and open-source software, developed originally by a small team at the Department of Physical Geography, University of Göttingen, Germany, and is now being maintained and extended by an international developer community. SAGA GIS is intended to give scientists an effective but easily learnable platform for implementing geoscientific methods. This is achieved by the application programming interface (API). SAGA has a fast-growing set of geoscientific methods, bundled in exchangeable module libraries."

Price: $\underline{\text { Free }}$

QGIS http://www.qgis.org

"QGIS is a user friendly Open Source Geographic Information System (GIS) licensed under the GNU General Public License. QGIS is an official project of the Open Source Geospatial Foundation (OSGeo). It runs on Linux, Unix, Mac OSX, Windows and Android and supports numerous vector, raster, and database formats and functionalities." Price: $\underline{\text { Free }}$

WhiteBox GAT https://jblindsay.github.io/ghrg/Whitebox/index.html

"The Whitebox GAT project began in 2009 and was conceived as a replacement for the Terrain Analysis System (TAS). Whitebox GAT was intended to have a broader focus than its predecessor, positioning it as an open-source desktop GIS and remote sensing software package for general applications of geospatial analysis and data visualization. Whitebox GAT is intended to provide a platform for advanced geospatial data analysis with applications in both environmental research and the geomatics industry more broadly. It was envisioned from the outset as providing an ideal platform for experimenting with novel geospatial analysis methods. Equally important is the project's goal of providing a tool that can be used for geomatics-based education."

Price: $\underline{\text { Free }}$ 


\section{GIS Resources}

GDAL

https://gdal.org/

"GDAL is a translator library for raster and vector geospatial data formats that is released under an X/MIT style Open Source License by the Open Source Geospatial Foundation. As a library, it presents a single raster abstract data model and single vector abstract data model to the calling application for all supported formats. It also comes with a variety of useful command line utilities for data translation and processing."

Price: Free

GeoNetwork https://geonetwork-opensource.org/

"GeoNetwork is a catalog application to manage spatially referenced resources. It provides powerful metadata editing and search functions as well as an interactive web map viewer. It is currently used in numerous Spatial Data Infrastructure initiatives across the world. GeoNetwork provides an easy to use web interface to search geospatial data across multiple catalogs. The search provides full-text search as well as faceted search on keywords, resource types, organizations, scale. Users can easily refine the search and quickly get to the records of interest. GeoSpatial layers, but also services, maps or even non-geographic datasets can be described in the catalog. Easily navigate across records and find sources or services publishing a dataset.

Price: Free

"GMT is an open-source collection of command-line tools for manipulating geographic and Cartesian data sets (including filtering, trend fitting, gridding, projecting, etc.) and producing PostScript illustrations ranging from simple $\mathrm{x}-\mathrm{y}$ plots via contour maps to artificially illuminated surfaces and 3D perspective views. It supports many map projections and transformations and includes supporting data such as coastlines, rivers, and political boundaries and optionally country polygons."

Price: Free 
FalconView https://www.falconview.org

"FalconView is a mapping system created by the Georgia Tech Research Institute. It was initially developed for the Windows family of operating systems; however, versions for Linux and mobile operating systems are under development. It displays various types of maps and geographically referenced overlays. Many types of maps are supported, but the primary ones of interest to most users are aeronautical charts, satellite images and elevation maps. FalconView also supports a large number of overlay types that can be displayed over any map background. The current overlay set is targeted toward military mission planning users and is oriented towards aviators and aviation support personnel."

Price: Paid subscription

Kalypso https://kalypso.bjoernsen.de/

"Kalypso is an open source modelling program. It focuses on numerical simulations in water management and ecology such as the generation of inundation and flood risk maps by hydrologic and hydrodynamic models and GIS functionality. The Kalypso software system has been collaboratively developed in a joint project by the company Björnsen Consulting Engineers (BCE) and the department for river and coastal engineering at Hamburg University of Technology, Germany. It is available for download at SourceForge.net under the GNU LGPL."

Price: Free

MultiTree http://multitree.org/search

"MultiTree is a searchable database of hypotheses on language relationships: Compare language trees and access bibliographical information on them; see a graphical representation of every scholarly hypothesis on language relationships; view information on every language; share comments on hypotheses and add new hypotheses (as a registered user); and access an interactive map of the language or family of your choice through LLMap"

This site shows a different side of mapping that instead looks at degrees of relatedness outside of physical geography. When one submits a query (name, code, author, or year), a list of different sources appears. When one clicks on one of the sources, a language tree appears that reflects the connections hypothesized in the particular study. There are two main ways of visualizing this information, which correspond to different versions of the system. The library does not offer commentary regarding which relatedness trees are more correct than others. Language trees can be combined with others to make a composite tree. In this case, they are regarded as primary sources. If just a single tree is used however, it is regarded as a secondary source. There is a detailed help page that provides users with guide to using the module. ISO 639-3 codes are used throughout, and 
where they are not applicable, codes determined by the Linguist List are used. Each time a search is queried, a new tab in the browser opens, as to ensure the user does not lose their place on the original page.

Price: Free

\section{TerraGIS http://www.terragis.net/}

"Terra GIS provides expertise in Geographical Information Systems, consulting for social and environmental issues, and subjects related to sustainable development. Services include environmental research, field work and reporting, spatial analysis, cartography, implementation of web mapping and information systems, and the creation of specialized tools related to conservation, natural resources management, and public and social engagement issues." More of a consultancy service.

Price: $\underline{\text { Consultancy fee }}$

World GeoDatasets http://worldgeodatasets.com/language/index.html

"The World Language Mapping System (WLMS) Version 19 is the most comprehensive, up-to-date, and trusted geographic dataset of the point and area (polygon) locations of the world's nearly 7,100 living language groups." Language locations are provided in both point and polygon form, and the basemaps and data need to be purchased. The organization was bought by SIL International, meaning that the proceeds of this data will go to missionary organizations; information gathering does appear to be in partnership with Global Mapping International, which is an organization of Christian ministries. The last time these datasets were updated was 2016, and the newest version includes immigrant, extinct, and signed languages. Other basemap products are available as well and can be combined with the linguistics dataset. There doesn't appear to be any information regarding where these data were collected from, but the languages use ISO codes.

Price: Basemaps require purchasing 
Appendix V: Codes used in the strengths/weaknesses analysis of language atlases (Study 2).

\begin{tabular}{|c|c|c|}
\hline Code & Definition & Related Codes \\
\hline MINIMALIST & $\begin{array}{l}\text { Maps/atlases that provide the most information } \\
\text { with the fewest items. Only what is necessary is } \\
\text { used. }\end{array}$ & -- \\
\hline VERIFIABLE & $\begin{array}{l}\text { There is a means to assess the extent to which the } \\
\text { presented information resembles the information } \\
\text { gathered originally. }\end{array}$ & Sources \\
\hline CLEAR & $\begin{array}{l}\text { The subject matter of the map/atlas is easy-to- } \\
\text { understand, and if it is online and/or digital, the } \\
\text { interface is easy to learn and use. }\end{array}$ & $\begin{array}{l}\text { Intuitive; Easy- } \\
\text { to-use; } \\
\text { Detailed } \\
\text { Commentary }\end{array}$ \\
\hline ATTRACTIVE & $\begin{array}{l}\text { Involves an emotional reaction, similar to looking } \\
\text { at a work of art. }\end{array}$ & $\begin{array}{l}\text { Visually- } \\
\text { appealing }\end{array}$ \\
\hline OBJECTIVE & $\begin{array}{l}\text { Though bias cannot be completely removed, the } \\
\text { map/atlas attempts to avoid as much bias and } \\
\text { subjectivity as feasible, or makes evident } \\
\text { subjective viewpoints. }\end{array}$ & $\begin{array}{l}\text { Unbiased; } \\
\text { Admits Bias }\end{array}$ \\
\hline SELF-AWARE & $\begin{array}{l}\text { The map/atlas is reflective of its own practices, } \\
\text { uses, and impacts. }\end{array}$ & -- \\
\hline
\end{tabular}




\begin{tabular}{|c|c|c|}
\hline DEAD LINKS & $\begin{array}{l}\text { Links to other pages, data, or websites do not } \\
\text { work or lead to pages which do not exist. }\end{array}$ & $\begin{array}{l}\text { Broken links; } \\
\text { Ambiguous } \\
\text { links }\end{array}$ \\
\hline OUTDATED & $\begin{array}{l}\text { The digital map/atlas either contains information } \\
\text { that is no longer commonly accepted, or uses } \\
\text { platforms that are no longer supported by internet } \\
\text { browsers or computer software. }\end{array}$ & $\begin{array}{l}\text { System Errors; } \\
\text { Old Info. }\end{array}$ \\
\hline NOT MAINTAINED & $\begin{array}{l}\text { The map/atlas has not recently had any input or } \\
\text { updating. }\end{array}$ & -- \\
\hline MISSING INFO. & $\begin{array}{l}\text { Data points or other similar important } \\
\text { information have incomplete or missing } \\
\text { information, which becomes evident to the user. }\end{array}$ & -- \\
\hline BANDWIDTH ISSUES & $\begin{array}{l}\text { A digital interface requires a strong internet } \\
\text { connection to load properly, meaning that it } \\
\text { cannot be accessed using weaker connections. }\end{array}$ & -- \\
\hline POLITICAL BIAS & $\begin{array}{l}\text { There is an evident political bias, such as the } \\
\text { placement of borders or naming conventions, that } \\
\text { is not explicitly accounted for in the map/atlas. }\end{array}$ & -- \\
\hline
\end{tabular}


Appendix VI: Copy of the request to atlas authors for an interview.

\section{Dear [author/developer name(s)],}

My name is Adam Stone, and I am a PhD Candidate at Carleton University, in Ottawa, Canada. My dissertation investigates how online linguistic atlases and their databases are used in linguistic research. I am impressed by your language atlas and am interested in learning more about how it was designed. If possible, I would appreciate your time in answering several questions related to database use in your project (may I ask that these questions get passed along to the individual or team responsible for managing the database, if this is easier for you?):

- What kind of database do you use for your project (e.g. object-oriented, documentoriented, etc.)?

- What is the name and version of the specific database software you are using?

- Why did you choose this form of database to work with?

- Why did you choose this specific database program to work with?

- Is this the first database software and version you have used in this project, or did you use a previous version or program at the beginning of the project?

- What do you see the benefits of using this form of database over others being?

- Are there any shortcomings of this database you have encountered in the development and implementation of your atlas?

Thank you for your anticipated time in this matter. I will be happy to share the results of my analysis with you and your team upon completion.

Sincerely, Adam 
END

Adam Stone, September 18, 2020 